\title{
Spektroskopische Untersuchungen zur Kinetik und Produktbildung bei Reaktionen von zyklischen und offenkettigen Kohlenwasserstoff-Radikalen
}

\author{
Dissertation \\ zur Erlangung des Doktorgrades \\ der Mathematisch-Naturwissenschaftlichen Fakultäten \\ der Georg-August-Universität zu Göttingen
}

vorgelegt von

Jörg Nothdurft

aus Nürnberg

Göttingen 2006 
D 7

Referent: Prof. Dr. K. Hoyermann

Korreferent: Prof. Dr. M. Buback

Tag der mündlichen Prüfung: 04. Mai 2006 


\section{THE ROAD NOT TAKEN}

„Two roads diverged in a yellow wood,

And sorry I could not travel both

And be one traveller, long I stood

And looked down one as far as I could

To where it bent in the undergrowth;

Then took the other, as just as fair;

And having perhaps the better claim,

Because it was grassy and wanted wear;

Though as for that the passing there

Had worn them really about the same,

And both that morning equally lay

In leaves no step had trodden black.

Oh, I kept the first for another day!

Yet knowing how way leads on to way,

I doubted if I should ever come back.

I shall be telling this with a sigh

Somewhere ages and ages hence:

Two roads diverged in a wood, And I-

I took the one less travelled by,

And that has made all the difference."

(Robert Frost) 


\section{Danksagung}

Mein besonderer Dank gilt Herrn Prof. Dr. K. Hoyermann für die Förderung dieser Arbeit, die wertvollen Ratschläge und die anregenden Diskussionen.

Bei Herrn Prof. Dr. M. Buback bedanke ich mich für die Übernahme des Korreferats.

Dem EGS „Microstructural Control in Free Radical Polymerisation“ danke ich für die finanzielle Förderung dieser Arbeit und dessen Mitgliedern für fruchtbare Diskussionen.

Allen Mitgliedern und ehemaligen Mitgliedern der Arbeitsgruppe, namentlich Herrn Dr. Markus Hold, Herrn Sven Maarfeld, Herrn Dr. Jens Wehmeyer und Herrn Dr. Thomas Zeuch danke ich für die kollegiale Zusammenarbeit. Besonderer Dank gilt dabei Herrn Dr. Thomas Zeuch für die große Hilfe bei Fragen zur FTIR-Analyse und zu kinetischen Simulationsrechnungen. Bei Herrn Dr. Markus Hold bedanke ich mich besonders für die intensive Unterstützung bei der GCMS-Apparatur und allgemeinen physikalisch-chemischen Problemen. Herrn Prof. Dr. M. Olzmann danke ich für die gute und fruchtbare Zusammenarbeit.

Allen Mitarbeitern der Werkstätten unter der Leitung der Herren A. Knorr, V. Meyer und H. Schlette danke ich für die schnelle Lösung von apparatur-spezifischen Problemen.

Meiner Freundin Janina danke ich für überabzählbar schöne Stunden, die die Arbeit unbezahlbar erleichtert haben und für die Unterstützung und Hilfe, die sie mir stets ist.

Meiner Mutter, meinem Vater und meinem Bruder danke ich für die immerwährende Unterstützung während der Studien- und Promotionszeit und dafür, dass ich immer willkommen bin.

Robert, Jaane, Anne, Tilmann, Jörg, Birte, Frederike, Melli, Jens, Andreas und Susa danke ich für die Zeit neben der Arbeit, die das Studium und die Promotion bereichert und sicherlich auch erfolgreicher gemacht haben. 


\section{Inhaltsverzeichnis}

1 Einleitung 1

2 Theoretische Grundlagen 11

2.1 Physikalisch-Chemische Grundlagen . . . . . . . . . . . . . . . . . . 11

2.1.1 Spektroskopische Grundlagen . . . . . . . . . . . . . . . . 11

2.1 .2 Kinetische Grundlagen . . . . . . . . . . . . . . . . . 13

2.1.2.1 Parallelreaktionen: Kombination vs. Disproportionierung • 13

2.1.2.2 Druckabhängigkeit chemischer Reaktionen . . . . . . . . . 16

2.2 Mathematische Grundlagen der (Fast)-Fourier-Transformation . . . . . . . 17

3 Beschreibung der experimentellen Bedingungen 25

3.1 FTIR Apparatur . . . . . . . . . . . . . . . . . . . . . . . 25

3.1.1 Schematischer Aufbau der Versuchsanordnung . . . . . . . . . . 25

3.1 .2 Laser-Photolyse . . . . . . . . . . . . . . . . . . . . 27

3.1.3 Nachweis und Signalverarbeitung . . . . . . . . . . . . . . 28

3.1 .4 Computersteuerung . . . . . . . . . . . . . . . . . . . . . . . 29

3.2 Radikalvorläufer . . . . . . . . . . . . . . . . . . . . . . . . . . 29

3.2.1 Die Reaktion Alkylradikal + Sauerstoffatom . . . . . . . . . . . . 29

3.2.2 Die Reaktion Alkylradikal + Alkylradikal . . . . . . . . . . . . . . . 30

3.2.3 Die Reaktion Allylradikal + Allylradikal . . . . . . . . . . . . . . 31

3.2.4 Durchführung und Auswertung von Messungen . . . . . . . . . . . 32

3.2.4.1 Qualitative Analyse . . . . . . . . . . . . 33

3.2.4.2 Quantitative Analyse . . . . . . . . . . . . . . 37

3.3 Reinheit der verwendeten Substanzen . . . . . . . . . . . . . . . . . . . 41 
4.1 Untersuchung der Reaktionen zyklischer Kohlenwasserstoff-Radikale mit

Sauerstoff-Atomen . . . . . . . . . . . . . . . . 43

4.1 .1 Die Reaktion Cyclopentyl + O . . . . . . . . . . . . . . . . . 46

4.1.1.1 Einleitung . . . . . . . . . . . . . . . 46

4.1.1.2 Qualitative Analyse . . . . . . . . . . . . . . . . 46

4.1.1.3 Quantitative Analyse . . . . . . . . . . . . . . . . 50

4.1.1.4 Simulation . . . . . . . . . . . . . . 55

4.1.1.5 Quantenchemische Rechnungen . . . . . . . . . . . 58

4.1.1.6 Diskussion . . . . . . . . . . . . . . . . . . 60

4.1 .2 Die Reaktion Cyclohexyl $+\mathrm{O}$. . . . . . . . . . . . . . . . . 62

$4.1 .2 .1 \quad$ Einleitung . . . . . . . . . . . . . . . . . 62

4.1.2.2 Qualitative Analyse . . . . . . . . . . . . . 63

4.1.2.3 Quantitative Analyse . . . . . . . . . . . . . . . . 65

4.1.2.4 Simulation . . . . . . . . . . . . . . . . . . . 68

4.1.2.5 Quantenchemische Rechnungen . . . . . . . . . . . 71

4.1 .2 .6 Diskussion . . . . . . . . . . . . . . . . . . . . . 74

4.1 .3 Die Reaktion Cyclooktyl + O . . . . . . . . . . . . . . 77

4.1.3.1 Einleitung . . . . . . . . . . . . . . . . . 77

4.1.3.2 Qualitative Analyse . . . . . . . . . . . . 78

4.1.3.3 Quantitative Analyse . . . . . . . . . . . . . 80

4.1.3.4 Quantenchemische Rechnungen . . . . . . . . . . 80

4.1.3.5 Diskussion . . . . . . . . . . . . . . . . . 85

4.2 Untersuchung der Reaktion eines zyklischen Alkens mit Sauerstoff-Atomen 86

4.2.1 Die Reaktion Cyclopenten $+\mathrm{O} \ldots \ldots$. . . . . . . . 86

$4.2 .1 .1 \quad$ Einleitung . . . . . . . . . . . . . . . 86

4.2.1.2 Qualitative Analyse . . . . . . . . . . . . . . . 87

4.2.1.3 Quantitative Analyse . . . . . . . . . . . . . . . 90

4.2 .1 .4 Diskussion . . . . . . . . . . . . . . . . . . . . . 91

4.3 Untersuchung der Reaktionen offenkettiger Kohlenwasserstoffradikale mit

Sauerstoff-Atomen . . . . . . . . . . . . . . . . . . 94 
4.3 .1 Die Reaktion Methyl $+\mathrm{O}$. . . . . . . . . . . . . . . . . . . 95

4.3.1.1 Einleitung . . . . . . . . . . . . . . . . 95

$4.3 .1 .2 \quad$ Qualitative Analyse . . . . . . . . . . . . . 96

4.3.1.3 Quantitative Analyse . . . . . . . . . . . . . . . 98

4.3.1.4 Simulation . . . . . . . . . . . . . . . . . . . . . 99

4.3.1.5 Diskussion . . . . . . . . . . . . . . . . . . . 101

$4.3 .2 \quad$ Die Reaktion Ethyl $+\mathrm{O}$. . . . . . . . . . . . . . . . 102

4.3.2.1 Einleitung . . . . . . . . . . . . . . . . 102

4.3.2.2 Qualitative Analyse . . . . . . . . . . . . . . . 103

4.3.2.3 Quantitative Analyse . . . . . . . . . . . . . 105

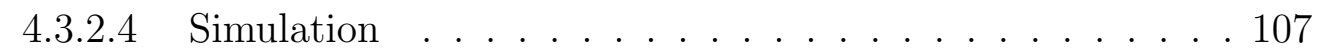

4.3.2.5 Diskussion . . . . . . . . . . . . . . . . . . . 110

$4.3 .3 \quad$ Die Reaktion Allyl + O . . . . . . . . . . . . . . . 112

$4.3 .3 .1 \quad$ Einleitung . . . . . . . . . . . . . . . . . . . 112

4.3 .3 .2 Qualitative Analyse . . . . . . . . . . . . . 113

4.3.3.3 Quantitative Analyse . . . . . . . . . . . . . . . 123

4.3.3.4 Simulation . . . . . . . . . . . . . . . . 128

4.3.3.5 Quantenchemische Rechnungen . . . . . . . . . . . . 131

4.3.3.6 Diskussion . . . . . . . . . . . . . . . . . . . . . . 132

4.4 Untersuchung der Reaktionen offenkettiger Kohlenwasserstoffradikale . . . 135 4.4 .1 Die Reaktion Methyl + Methyl . . . . . . . . . . . . . . . . . . . 135

$4.4 .1 .1 \quad$ Einleitung . . . . . . . . . . . . . . . . . . . . 135

$4.4 .1 .2 \quad$ Qualitative Analyse . . . . . . . . . . . . 136

4.4.1.3 Quanitative Analyse . . . . . . . . . . . . . . . 137

4.4.1.4 Diskussion . . . . . . . . . . . . . . . . . . . . . . . . 138

4.4 .2 Die Reaktion Ethyl + Ethyl . . . . . . . . . . . . . . . . . . . 139

$4.4 .2 .1 \quad$ Einleitung . . . . . . . . . . . . . . . . . . . . . . . . 139

4.4.2.2 Qualitative Analyse . . . . . . . . . . . . . . 139

4.4.2.3 Quantitative Analyse . . . . . . . . . . . . . . . . . . 141

4.4.2.4 Diskussion . . . . . . . . . . . . . . . . . . . . . . . . . 143

4.4 .3 Die Reaktion 1-Propyl + 1-Propyl . . . . . . . . . . . . . . . . . . . 144 
4.4 .3 .1 Einleitung . . . . . . . . . . . . . . . . . . . . . 144

$4.4 .3 .2 \quad$ Qualitative Analyse . . . . . . . . . . . . . . . . 145

4.4 .3 .3 Quantitative Analyse . . . . . . . . . . . . . . . . . . 149

4.4 .3 .4 Diskussion . . . . . . . . . . . . . . . . . . . . 153

4.4 .4 Die Reaktion 2-Propyl + 2-Propyl . . . . . . . . . . . . . . . 154

4.4.4.1 Einleitung . . . . . . . . . . . . . . . . . . 154

4.4.4.2 Qualitative Analyse . . . . . . . . . . . . . . 155

4.4.4.3 Quantitative Analyse . . . . . . . . . . . . . . . 158

4.4.4.4 Diskussion . . . . . . . . . . . . . . . . . . . . . . . . . . 159

4.4.5 Die Reaktion 1-Butyl + 1-Butyl $\ldots \ldots \ldots$. . . . . . . . . . 160

4.4 .5 .1 Einleitung . . . . . . . . . . . . . . . . . 160

4.4.5.2 Qualitative Analyse . . . . . . . . . . . . . . . 161

4.4.5.3 Quantitative Analyse . . . . . . . . . . . . . . . . . 163

4.4.5.4 Diskussion . . . . . . . . . . . . . . . . . . . 163

4.4 .6 Die Reaktion Allyl + Allyl . . . . . . . . . . . . . . . . . . . . . 164

4.4 .6 .1 Einleitung . . . . . . . . . . . . . . . . . . 164

4.4.6.2 Qualitative Analyse . . . . . . . . . . . . . . . 164

4.4.6.3 Quantitative Analyse . . . . . . . . . . . . . . . . . . 172

4.4.6.4 Druckabhängigkeit . . . . . . . . . . . . . . . 176

4.4.6.5 Diskussion . . . . . . . . . . . . . . . . 177

4.5 Untersuchung der Kreuzreaktionen von Methylradikalen mit Kohlenwas-

serstoffradikalen . . . . . . . . . . . . . . . . . . . . . . . . . 180

$4.5 .1 \quad$ Die Reaktion Methyl + Ethyl $\ldots \ldots \ldots$. . . . . . . . . . 180

4.5.1.1 Qualitative Analyse . . . . . . . . . . . . . . . . 181

4.5.1.2 Quantitative Analyse . . . . . . . . . . . . . . . . . 184

4.5 .1 .3 Simulation . . . . . . . . . . . . . . . . 185

4.5.1.4 Diskussion . . . . . . . . . . . . . . . . . . . . 187

4.5.2 Die Reaktion Methyl + 1-Propyl … . . . . . . . . . . . . . 189

4.5.2.1 Qualitative Analyse _. . . . . . . . . . . . . . . . 190

$4.5 .2 .2 \quad$ Quantitative Analyse . . . . . . . . . . . . . . . . . 197

4.5 .2 .3 Simulation . . . . . . . . . . . . . . . . . 201 
4.5.2.4 Diskussion . . . . . . . . . . . . . . . . . . . 204

4.5.3 Die Reaktion Methyl + 2-Propyl . . . . . . . . . . . . . . . . . 205

4.5.3.1 Qualitative Analyse . . . . . . . . . . . . 206

4.5.3.2 Quantitative Analyse . . . . . . . . . . . . . . . 209

4.5 .3 .3 Simulation . . . . . . . . . . . . . . . 211

4.5.3.4 Diskussion . . . . . . . . . . . . . . . . . . 214

4.5.4 Die Reaktion Methyl + 1-Butyl . . . . . . . . . . . . . . . . . 215

4.5.4.1 Einleitung . . . . . . . . . . . . . . . . . 215

4.5.4.2 Qualitative Analyse . . . . . . . . . . . . . 215

4.5.4.3 Quantitative Analyse . . . . . . . . . . . . . . . 218

4.5.4.4 Simulation . . . . . . . . . . . . . . . . . . . 219

4.5.4.5 Diskussion . . . . . . . . . . . . . . . . . . . 222

4.6 Die Reaktionen von $\mathrm{CH}_{2} \mathrm{~F}$ mit $\mathrm{CHF}_{2}, \mathrm{CH}_{3}$ und $\mathrm{C}_{2} \mathrm{H}_{5}$ Radikalen . . . . . . 223

$\begin{array}{lll}5 & \text { Ausblick } & 227\end{array}$

5.1 Die Reaktion Methyl + 2-Butyl . . . . . . . . . . . . . . . . . . . 227

5.2 Die Reaktion Methyl + 1-Pentyl . . . . . . . . . . . . . . . . . . 230

$\begin{array}{lll}6 & \text { Zusammenfassung } & 233\end{array}$

6.1 Reaktionen von zyklischen Alkylradikalen und vom Allylradikal mit Sauerstoffatomen . . . . . . . . . . . . . . . . . . 233

6.2 Geschwindigkeitskoeffizienten für die Selbstreaktionen der Alkylradikale $\mathrm{CH}_{3}$ und $\mathrm{C}_{2} \mathrm{H}_{5}$ sowie des Allylradikals . . . . . . . . . . . . . 235

6.3 Mechanismus der Reaktion der primären und sekundären Alkylradikale $\mathrm{C}_{n} \mathrm{H}_{2 n+1}(\mathrm{n}=1,2,3,4)$ und des Allylradikals . . . . . . . . . 235

6.4 Kreuzreaktionen kleiner Alkylradikale mit Methylradikalen und teilfluorierten Alkylradikalen . . . . . . . . . . . . . . . . . 237

$\begin{array}{lll}7 & \text { Anhang } & 239\end{array}$

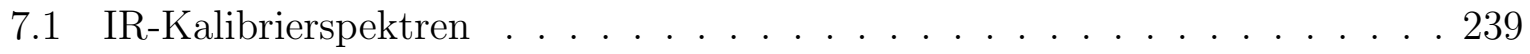

7.2 Integrale Absorptionskoeffizienten . . . . . . . . . . . . . . . . 269 
INHALTSVERZEICHNIS

INHALTSVERZEICHNIS 


\section{Abbildungsverzeichnis}

1.1 Peroxy-Initiatoren . . . . . . . . . . . . . . . . . . . 3

1.2 Zerfall von Di-tert-Butylperoxy . . . . . . . . . . . . . . . . . . 4

2.1 Schematischer Aufbau Michelson-Interferometer . . . . . . . . . . . . . 21

3.1 Schematischer Aufbau der FTIR-Apparatur . . . . . . . . . . . . 27

3.2 Qualitative Analyse der Photolyse von Aceton- $\mathrm{d}_{6}$. . . . . . . . . . . 35

3.3 Beispiel zur Bestimmung des integralen Absorptionskoeffizienten . . . . . . 38

3.4 Zeitliche Absorbanzzunahme am Beispiel von 4-Pentenal . . . . . . . . . . 39

4.1 Reaktion $\mathrm{c}_{-} \mathrm{C}_{5} \mathrm{H}_{9}+\mathrm{O}$, Reaktionsspektrum . . . . . . . . . . . 48

4.2 Reaktion c- $\mathrm{C}_{5} \mathrm{H}_{9}+\mathrm{O}$, qualitative Analyse, Kanäle Cyclopentoxyzerfall . . 49

4.3 Reaktion $\mathrm{c}^{-} \mathrm{C}_{5} \mathrm{H}_{9}+\mathrm{O}$, qualitative Analyse, Abstraktionsprodukt . . . . . . 50

4.4 Reaktion $\mathrm{c}_{-} \mathrm{C}_{5} \mathrm{H}_{9}+\mathrm{O}$, quantitative Analyse, O-Atom-Unterschuss . . . . 52

4.5 Reaktion $\mathrm{c}_{-} \mathrm{C}_{5} \mathrm{H}_{9}+\mathrm{O}$, quantitative Analyse, O-Atom-Überschuss . . . . . 53

4.6 Reaktion $\mathrm{c}^{-} \mathrm{C}_{5} \mathrm{H}_{9}+\mathrm{O}$, Verhältnis Cyclopentanon zu 4-Pentenal . . . . . . 54

4.7 Reaktion $\mathrm{c}_{-} \mathrm{C}_{5} \mathrm{H}_{9}+\mathrm{O}$, Simulation der Konzentrations-Zeit-Profile . . . . . 56

4.8 Reaktion $\mathrm{c}_{-} \mathrm{C}_{5} \mathrm{H}_{9}+\mathrm{O}$, Einfluss der Startkonzentrationen auf die Simulationsrechnungen . . . . . . . . . . . . . . . . . 5 58

4.9 Reaktion $\mathrm{c}_{-} \mathrm{C}_{5} \mathrm{H}_{9}+\mathrm{O}$, Energiediagramm (quantenchemischen Rechnungen) 59

4.10 Reaktion c- $\mathrm{C}_{5} \mathrm{H}_{9}+\mathrm{O}$, Geschwindigkeit des Cyclopentoxyzerfalls (RRKM) 60

4.11 Reaktion $\mathrm{c}^{-} \mathrm{C}_{6} \mathrm{H}_{11}+\mathrm{O}$, Reaktionsspektrum . . . . . . . . . . . 63

4.12 Reaktion $\mathrm{c}^{-} \mathrm{C}_{6} \mathrm{H}_{11}+\mathrm{O}$, qualitative Analyse, Kanäle Cyclohexoxyzerfall . 64

4.13 Reaktion $\mathrm{c}_{-} \mathrm{C}_{6} \mathrm{H}_{11}+\mathrm{O}$, quantitative Analyse Cyclohexoxyzerfall . . . . . 67

4.14 Reaktion $\mathrm{c}^{-} \mathrm{C}_{6} \mathrm{H}_{11}+\mathrm{O}$, Simulation der Konzentrations-Zeit-Profile . . . . . 70 
4.15 Reaktion c- $\mathrm{C}_{6} \mathrm{H}_{11}+\mathrm{O}$, Energiediagramm (quantenchemische Rechnungen) 71

4.16 Reaktion $\mathrm{c}^{-} \mathrm{C}_{6} \mathrm{H}_{11}+\mathrm{O}$, Geschwindigkeit des Cyclohexoxyzerfalls (RRKM) $\quad 72$

4.17 Reaktion $\mathrm{c}^{-} \mathrm{C}_{6} \mathrm{H}_{11}+\mathrm{O}$, Geschwindigkeit des Hexanal-6-yl-Zerfalls (RRKM) 73

4.18 Reaktion $\mathrm{c}-\mathrm{C}_{8} \mathrm{H}_{15}+\mathrm{O}$, qualitative Analyse . . . . . . . . . . . . . 79

4.19 Reaktion $\mathrm{c}_{-} \mathrm{C}_{8} \mathrm{H}_{15}+\mathrm{O}$, Energiediagramm (quantenchemische Rechnungen) 81

4.20 Reaktion c- $\mathrm{C}_{8} \mathrm{H}_{15}+\mathrm{O}$, Geschwindigkeit des Cyclooctoxyzerfalls (RRKM) . 82

4.21 Reaktion $\mathrm{c}-\mathrm{C}_{8} \mathrm{H}_{15}+\mathrm{O}$, Geschwindigkeit des Oktanal-8-yl (RRKM) . . . . 83

4.22 Reaktion $\mathrm{c}-\mathrm{C}_{5} \mathrm{H}_{8}+\mathrm{O}$, Reaktionsspektrum . . . . . . . . . . . 88

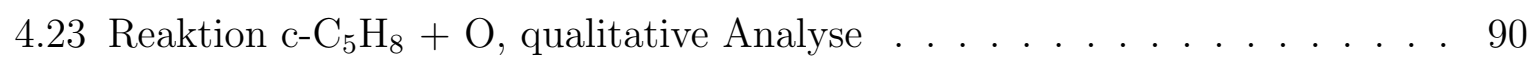

4.24 Reaktion $\mathrm{c}-\mathrm{C}_{5} \mathrm{H}_{8}+\mathrm{O}$, quantitative Analyse . . . . . . . . . . . . 91

4.25 Reaktionssystem $\mathrm{CH}_{3} / \mathrm{O}$, Reaktionsspektrum Vorläufer Aceton / $\mathrm{SO}_{2}$. . 97

4.26 Reaktionssystem $\mathrm{CH}_{3} / \mathrm{O}$, qualitative Analyse Vorläufer Aceton / $\mathrm{SO}_{2}$. . 97

4.27 Reaktionssystem $\mathrm{CH}_{3}$ / O, Simulation der Konzentrations-Zeit-Profile . . . 100

4.28 Reaktionssystem $\mathrm{C}_{2} \mathrm{H}_{5} / \mathrm{O}$, Reaktionsspektrum, Vorläufer Ethyliodid . . . 104

4.29 Reaktionssystem $\mathrm{C}_{2} \mathrm{H}_{5} / \mathrm{O}$, qualitative Analyse . . . . . . . . . . 105

4.30 Reaktionssystem $\mathrm{C}_{2} \mathrm{H}_{5} / \mathrm{O}$, quantitative Analyse . . . . . . . . . 106

4.31 Reaktionssystem $\mathrm{C}_{2} \mathrm{H}_{5} / \mathrm{O}$, Simulation der Konzentrations-Zeit-Profile . . 109

4.32 Reaktion Allyl + O, Reaktionsspektrum, Vorläufer Diallyl . . . . . . . . . 115

4.33 Reaktion Allyl + O, qualitative Analyse, Vorläufer Diallyl . . . . . . . . . 117

4.34 Reaktion Allyl + O, Reaktionsspektrum, Vorläufer Allylbromid . . . . . . 117

4.35 Reaktion Allyl + O, qualitative Analyse, Vorläufer Allylbromid . . . . . . 120

4.36 Reaktion Allyl + O, Reaktionsspektrum, Vorläufer Allylchlorid . . . . . . . 120

4.37 Reaktion Allyl + O, qualitative Analyse, Vorläufer Allylchlorid . . . . . . . 122

4.38 Reaktion Allyl + O, quantitative Analyse, Vorläufer Diallyl . . . . . . . . . 123

4.39 Reaktion Allyl + O, quantitative Analyse, Vorläufer Allylbromid . . . . . . 125

4.40 Reaktion Allyl + O, quantitative Analyse, Vorläufer Allylchlorid . . . . . . 126

4.41 Reaktion Allyl + O, Simulation der Konzentrations-Zeit-Profile . . . . . . 129

4.42 Reaktion Allyl + O, RRKM-Rechnungen . . . . . . . . . . . . 132

4.43 Reaktion $\mathrm{CD}_{3}+\mathrm{CD}_{3}$, Reaktionsspektrum . . . . . . . . . . 136

4.44 Reaktion $\mathrm{CD}_{3}+\mathrm{CD}_{3}$, qualitative Analyse . . . . . . . . . . . . 136

4.45 Reaktion $\mathrm{CD}_{3}+\mathrm{CD}_{3}$, quantitative Analyse . . . . . . . . . . . 138 
4.46 Reaktion $\mathrm{C}_{2} \mathrm{H}_{5}+\mathrm{C}_{2} \mathrm{H}_{5}$, Reaktionsspektrum . . . . . . . . . . . 140

4.47 Reaktion $\mathrm{C}_{2} \mathrm{H}_{5}+\mathrm{C}_{2} \mathrm{H}_{5}$, qualitative Analyse . . . . . . . . . . . . 141

4.48 Reaktion $\mathrm{C}_{2} \mathrm{H}_{5}+\mathrm{C}_{2} \mathrm{H}_{5}$, quantitative Analyse $\ldots \ldots \ldots$. . . . . . 142

4.49 Reaktion $1-\mathrm{C}_{3} \mathrm{H}_{7}+1-\mathrm{C}_{3} \mathrm{H}_{7}$, Reaktionsspektrum, Vorläufer Propyliodid . . 146

4.50 Reaktion 1- $\mathrm{C}_{3} \mathrm{H}_{7}+1-\mathrm{C}_{3} \mathrm{H}_{7}$, qualitative Analyse, Vorläufer Propyliodid . . 147

4.51 Reaktion $1-\mathrm{C}_{3} \mathrm{H}_{7}+1-\mathrm{C}_{3} \mathrm{H}_{7}$, Reaktionsspektrum, Vorläufer Dipropylketon . 148

4.52 Reaktion $1-\mathrm{C}_{3} \mathrm{H}_{7}+1-\mathrm{C}_{3} \mathrm{H}_{7}$, qualitative Analyse, Vorläufer Dipropylketon . 149

4.53 Reaktion $1-\mathrm{C}_{3} \mathrm{H}_{7}+1-\mathrm{C}_{3} \mathrm{H}_{7}$, quantitative Analyse, Vorläufer Propyliodid . . 150

4.54 Reaktion $1-\mathrm{C}_{3} \mathrm{H}_{7}+1-\mathrm{C}_{3} \mathrm{H}_{7}$, quantitative Analyse, Vorläufer Dipropylketon 152

4.55 Reaktion $2-\mathrm{C}_{3} \mathrm{H}_{7}+2-\mathrm{C}_{3} \mathrm{H}_{7}$, Reaktionsspektrum, Vorläufer Isopropyliodid . 156

4.56 Reaktion $2-\mathrm{C}_{3} \mathrm{H}_{7}+2-\mathrm{C}_{3} \mathrm{H}_{7}$, qualitative Analyse, Vorläufer Isopropyliodid . 157

4.57 Reaktion $2-\mathrm{C}_{3} \mathrm{H}_{7}+2-\mathrm{C}_{3} \mathrm{H}_{7}$, quantitative Analyse, Vorläufer Isopropyliodid 159

4.58 Reaktion $1-\mathrm{C}_{4} \mathrm{H}_{9}+1-\mathrm{C}_{4} \mathrm{H}_{9}$, Reaktionsspektrum . . . . . . . . . . . . 161

4.59 Reaktion $1-\mathrm{C}_{4} \mathrm{H}_{9}+1-\mathrm{C}_{4} \mathrm{H}_{9}$, qualitative Analyse . . . . . . . . . . . . . 162

4.60 Reaktion $\mathrm{C}_{3} \mathrm{H}_{5}+\mathrm{C}_{3} \mathrm{H}_{5}$, Reaktionsspektrum, Vorläufer Diallyl . . . . . . . 165

4.61 Reaktion $\mathrm{C}_{3} \mathrm{H}_{5}+\mathrm{C}_{3} \mathrm{H}_{5}$, qualitative Analyse, Vorläufer Diallyl . . . . . . . 166

4.62 Reaktion $\mathrm{C}_{3} \mathrm{H}_{5}+\mathrm{C}_{3} \mathrm{H}_{5}$, Vergleich Reaktionsspektrum, Vorläufer Diallyl . . 167

4.63 Reaktion $\mathrm{C}_{3} \mathrm{H}_{5}+\mathrm{C}_{3} \mathrm{H}_{5}$, Reaktionsspektrum, Vorläufer Allylbromid . . . . 168

4.64 Reaktion $\mathrm{C}_{3} \mathrm{H}_{5}+\mathrm{C}_{3} \mathrm{H}_{5}$, qualitative Analyse, Vorläufer Allybromid . . . . . 169

4.65 Reaktion $\mathrm{C}_{3} \mathrm{H}_{5}+\mathrm{C}_{3} \mathrm{H}_{5}$, Reaktionsspektrum, Vorläufer Allylchlorid . . . . 170

4.66 Reaktion $\mathrm{C}_{3} \mathrm{H}_{5}+\mathrm{C}_{3} \mathrm{H}_{5}$, qualitative Analyse, Vorläufer Allychlorid . . . . . 171

4.67 Reaktion $\mathrm{C}_{3} \mathrm{H}_{5}+\mathrm{C}_{3} \mathrm{H}_{5}$, quantitative Analyse, Vorläufer Diallyl . . . . . . 172

4.68 Reaktion $\mathrm{C}_{3} \mathrm{H}_{5}+\mathrm{C}_{3} \mathrm{H}_{5}$, quantitative Analyse, Vorläufer Allylbromid . . . 173

4.69 Reaktion $\mathrm{C}_{3} \mathrm{H}_{5}+\mathrm{C}_{3} \mathrm{H}_{5}$, quantitative Analyse, Vorläufer Allylchlorid . . . . 174

4.70 Reaktion $\mathrm{C}_{3} \mathrm{H}_{5}+\mathrm{C}_{3} \mathrm{H}_{5}$, Druckabhängigkeit . . . . . . . . . . . . 176

4.71 Reaktionssystem $\mathrm{CH}_{3} / \mathrm{C}_{2} \mathrm{H}_{5}$, Reaktionsspektrum . . . . . . . . . . 182

4.72 Reaktionssystem $\mathrm{CH}_{3} / \mathrm{C}_{2} \mathrm{H}_{5}$, qualitative Analyse . . . . . . . . . . . 183

4.73 Reaktionssystem $\mathrm{CH}_{3} / \mathrm{C}_{2} \mathrm{H}_{5}$, Simulation der Konzentrations-Zeit-Profile . 186

4.74 Reaktionssystem $\mathrm{CH}_{3} / \mathrm{C}_{2} \mathrm{H}_{5}$, Einfluss der Radikalanfangskonzentrationen auf die Simulationsrechnungen . . . . . . . . . . . . . . . 187 
4.75 Reaktionssystem $\mathrm{CH}_{3} / 1-\mathrm{C}_{3} \mathrm{H}_{7}$, Reaktionsspektrum Vorläufer Aceton- $\mathrm{d}_{6} /$ Propyliodid . . . . . . . . . . . . . . . . . . . . . 191

4.76 Reaktionssystem $\mathrm{CH}_{3} / 1-\mathrm{C}_{3} \mathrm{H}_{7}$, qualitative Analyse, Vorläufer Aceton- $\mathrm{d}_{6}$ / Propyliodid . . . . . . . . . . . . . . . . . . . . . 192

4.77 Reaktionssystem $\mathrm{CH}_{3} /$ 1-C $_{3} \mathrm{H}_{7}$, Reaktionsspektrum, Vorläufer Aceton- $\mathrm{d}_{6}$ / Dipropylketon . . . . . . . . . . . . . . . . . . . . 193

4.78 Reaktionssystem $\mathrm{CH}_{3} / 1-\mathrm{C}_{3} \mathrm{H}_{7}$, qualitative Analyse, Vorläufer Aceton- $\mathrm{d}_{6}$ / Dipropylketon . . . . . . . . . . . . . . . . . . . . . 194

4.79 Reaktionssystem $\mathrm{CH}_{3} /{ }_{1}-\mathrm{C}_{3} \mathrm{H}_{7}$, Reaktionsspektrum, Vorläufer Methylpropylketon . . . . . . . . . . . . . . . . . . . . 195

4.80 Reaktionssystem $\mathrm{CH}_{3} / 1-\mathrm{C}_{3} \mathrm{H}_{7}$, qualitative Analyse, Vorläufer Methylpropylketon . . . . . . . . . . . . . . . . . . . 196

4.81 Reaktionssystem $\mathrm{CH}_{3} / 1-\mathrm{C}_{3} \mathrm{H}_{7}$, quantitative Analyse, Vorläufer Propyliodid 197 4.82 Reaktionssystem $\mathrm{CH}_{3} / 1-\mathrm{C}_{3} \mathrm{H}_{7}$, quantitative Analyse, Vorläufer Dipropylketon . . . . . . . . . . . . . . . . . . . . . . . . . 198

4.83 Reaktionssystem $\mathrm{CH}_{3} / \mathrm{1}_{3} \mathrm{C}_{7}$, quantitative Analyse, Vorläufer Dipropylketon . . . . . . . . . . . . . . . . . . . . . 200

4.84 Reaktionssystem $\mathrm{CH}_{3} / 1-\mathrm{C}_{3} \mathrm{H}_{7}$, Einfluss der Radikalanfangskonzentrationen auf die Simulationsrechnungen . . . . . . . . . . . . . . . . . 201

4.85 Reaktionssystem $\mathrm{CH}_{3} / \mathrm{1}_{3} \mathrm{H}_{7}$, Einfluss der Kreuzkombinationsgeschwindigkeit auf die Simulationsrechnungen . . . . . . . . . . . . . . . . 203

4.86 Reaktionssystem $\mathrm{CH}_{3} / 2-\mathrm{C}_{3} \mathrm{H}_{7}$, Reaktionsspektrum, Vorläufer Aceton- $\mathrm{d}_{6}$ / Isopropyliodid . . . . . . . . . . . . . . . . . . 206

4.87 Reaktionssystem $\mathrm{CH}_{3} / 2-\mathrm{C}_{3} \mathrm{H}_{7}$, Reaktionsspektrum, Vorläufer Aceton- $\mathrm{d}_{6}$ / Diisopropylketon . . . . . . . . . . . . . . . . . . 207

4.88 Reaktionssystem $\mathrm{CH}_{3} / 2-\mathrm{C}_{3} \mathrm{H}_{7}$, qualitative Analyse, Vorläufer Aceton- $\mathrm{d}_{6}$ / Isopropyliodid . . . . . . . . . . . . . . . . . . . . . 208

4.89 Reaktionssystem $\mathrm{CH}_{3} / 2-\mathrm{C}_{3} \mathrm{H}_{7}$, qualitative Analyse, Vorläufer Aceton- $\mathrm{d}_{6}$ / Diisopropylketon . . . . . . . . . . . . . . . . . . 209

4.90 Reaktionssystem $\mathrm{CH}_{3} / 2-\mathrm{C}_{3} \mathrm{H}_{7}$, quantitative Analyse, Vorläufer Aceton- $\mathrm{d}_{6}$ / Isopropyliodid . . . . . . . . . . . . . . . . . . . . 210 
4.91 Reaktionssystem $\mathrm{CH}_{3} / 2-\mathrm{C}_{3} \mathrm{H}_{7}$, Einfluss der Kreuzkombinationsgeschwindigkeit . . . . . . . . . . . . . . . . . . . . 212

4.92 Reaktionssystem $\mathrm{CH}_{3} / 2-\mathrm{C}_{3} \mathrm{H}_{7}$, Einfluss der Radikalanfangskonzentrationen 213 4.93 Reaktionssystem $\mathrm{CH}_{3} / 1-\mathrm{C}_{4} \mathrm{H}_{9}$, Reaktionsspektrum . . . . . . . . . . 216

4.94 Reaktionssystem $\mathrm{CH}_{3} / 1-\mathrm{C}_{4} \mathrm{H}_{9}$, qualitative Analyse . . . . . . . . . . . 217

4.95 Reaktionssystem $\mathrm{CH}_{3} / 1-\mathrm{C}_{4} \mathrm{H}_{9}$, Einfluss der Kreuzkombinationsgeschwindigkeit . . . . . . . . . . . . . . . . . . . . 220

4.96 Reaktionssystem $\mathrm{CH}_{3} / 1-\mathrm{C}_{4} \mathrm{H}_{9}$, Einfluss der Radikalanfangskonzentrationen 221

4.97 Reaktionssystem $\mathrm{CH}_{2} \mathrm{~F} / \mathrm{CHF}_{2}$, Simulation der Konzentrations-Zeit-Profile 224

4.98 Reaktionssystem $\mathrm{CH}_{2} \mathrm{~F} / \mathrm{CH}_{3}$, Simulation der Konzentrations-Zeit-Profile. 225

4.99 Reaktionssystem $\mathrm{CH}_{2} \mathrm{~F} / \mathrm{C}_{2} \mathrm{H}_{5}$, Simulation der Konzentrations-Zeit-Profile 226

5.1 Reaktionssystem $\mathrm{CH}_{3} / 2-\mathrm{C}_{4} \mathrm{H}_{9}$, Reaktionsspektrum . . . . . . . . . . 228

5.2 Reaktionssystem $\mathrm{CH}_{3} / 2-\mathrm{C}_{4} \mathrm{H}_{9}$, qualitative Analyse . . . . . . . . . . . . 229

5.3 Reaktionssystem $\mathrm{CH}_{3} / 1-\mathrm{C}_{5} \mathrm{H}_{11}$, Reaktionsspektrum . . . . . . . . . . 230

5.4 Reaktionssystem $\mathrm{CH}_{3} / 1-\mathrm{C}_{5} \mathrm{H}_{11}$, qualitative Analyse . . . . . . . . . . 231

7.1 Kalibrierspektrum 1-Buten . . . . . . . . . . . . . . . . . . 239

7.2 Kalibrierspektrum 1,3-Butadien . . . . . . . . . . . . . . . . 240

7.3 Kalibrierspektrum 2,3-Dimethyl-2-buten . . . . . . . . . . . . . . . 240

7.4 Kalibrierspektrum 2-Hexanon . . . . . . . . . . . . . . . . . . . . . 241

7.5 Kalibrierspektrum 2-Methyl-Cyclopentanon . . . . . . . . . . . . . . 241

7.6 Kalibrierspektrum 2-Pentanon . . . . . . . . . . . . . . . . . . . . . . . . 242

7.7 Kalibrierspektrum 2-trans-Buten . . . . . . . . . . . . . . . . 242

7.8 Kalibrierspektrum 4-Pentenal . . . . . . . . . . . . . . . . . . . . 243

7.9 Kalibrierspektrum 5 -Hexenal . . . . . . . . . . . . . . . . . 243

7.10 Kalibrierspektrum Acetaldehyd . . . . . . . . . . . . . . . . . . 244

7.11 Kalibrierspektrum Aceton . . . . . . . . . . . . . . . . . . . . . . 244

7.12 Kalibrierspektrum Aceton- $\mathrm{d}_{6}$. . . . . . . . . . . . . . . . . . . 245

7.13 Kalibrierspektrum Acrolein . . . . . . . . . . . . . . . . . . 245

7.14 Kalibrierspektrum Allen . . . . . . . . . . . . . . . . . 246

7.15 Kalibrierspektrum Allylbromid . . . . . . . . . . . . . . 246 
7.16 Kalibrierspektrum Bicyclohexyl … . . . . . . . . . . . . . . 247

7.17 Kalibrierspektrum Butan . . . . . . . . . . . . . . . . . . . 247

7.18 Kalibrierspektrum Butanal . . . . . . . . . . . . . . . . . . . 248

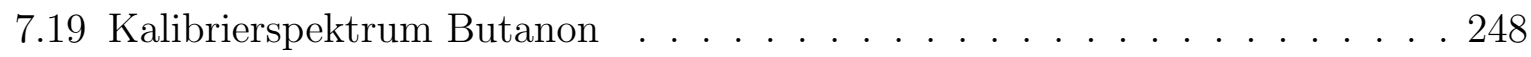

7.20 Kalibrierspektrum Butyliodid ． . . . . . . . . . . . . . . . . . 249

7.21 Kalibrierspektrum Cyclohexan . . . . . . . . . . . . . . . . . . . . 249

7.22 Kalibrierspektrum Cyclohexanon … . . . . . . . . . . . 250

7.23 Kalibrierspektrum Cyclohexen . . . . . . . . . . . . . . . . . 250

7.24 Kalibrierspektrum Cyclohexenon … . . . . . . . . . . . . . 251

7.25 Kalibrierspektrum Cyclooktanon … . . . . . . . . . 251

7.26 Kalibrierspektrum Cyclookten . . . . . . . . . . . . . . . . . 252

7.27 Kalibrierspektrum Cyclopentan … . . . . . . . . . . . . . 252

7.28 Kalibrierspektrum Cyclopentancarboxyaldehyd . . . . . . . . . . . . 253

7.29 Kalibrierspektrum Cyclopentanon . . . . . . . . . . . . . . . . . 253

7.30 Kalibrierspektrum Cyclopenten … . . . . . . . . . . . . . 254

7.31 Kalibrierspektrum Cyclopentenoxid . . . . . . . . . . . . . . . . . 254

7.32 Kalibrierspektrum Diallyl . . . . . . . . . . . . . . . . 255

7.33 Kalibrierspektrum Dicyclopropylketon … . . . . . . . . . . 255

7.34 Kalibrierspektrum Diisopropyl . . . . . . . . . . . . . . . . . . 256

7.35 Kalibrierspektrum Diisopropylketon . . . . . . . . . . . . . 256

7.36 Kalibrierspektrum Dipropylketon … . . . . . . . . . . 257

7.37 Kalibrierspektrum Di-tert-Butylketon … . . . . . . . . . . . 257

7.38 Kalibrierspektrum Ethan $\ldots \ldots \ldots \ldots . \ldots \ldots$

7.39 Kalibrierspektrum Ethan- $\mathrm{d}_{6} \ldots \ldots \ldots \ldots \ldots$

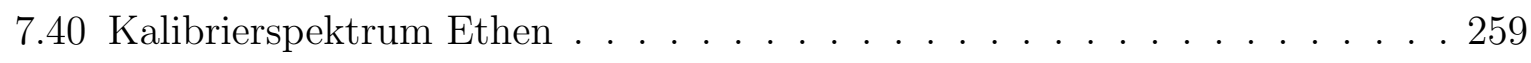

7.41 Kalibrierspektrum Ethin . . . . . . . . . . . . . . . . . . . 259

7.42 Kalibrierspektrum Ethyliodid ． . . . . . . . . . . . . . . 260

7.43 Kalibrierspektrum Formaldehyd . . . . . . . . . . . . . . . . 260

7.44 Kalibrierspektrum Hexan . . . . . . . . . . . . . . . . . . . . 261

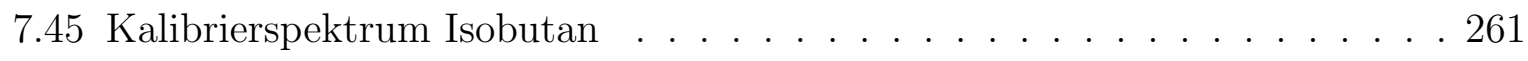

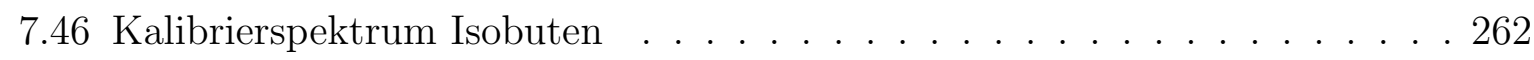


7.47 Kalibrierspektrum Isopropyliodid . . . . . . . . . . . . . . . . . . 262

7.48 Kalibrierspektrum Methan . . . . . . . . . . . . . . . . 263

7.49 Kalibrierspektrum Methyliodid . . . . . . . . . . . . . . 263

7.50 Kalibrierspektrum Methyl-Propyl-Keton . . . . . . . . . . . . . . . . 264

7.51 Kalibrierspektrum Pentanal . . . . . . . . . . . . . . . 264

7.52 Kalibrierspektrum Pentyliodid . . . . . . . . . . . . . . 265

7.53 Kalibrierspektrum Pivaldehyd . . . . . . . . . . . . . . . 265

7.54 Kalibrierspektrum Propan . . . . . . . . . . . . . . . . . 266

7.55 Kalibrierspektrum Propen . . . . . . . . . . . . . . . . 266

7.56 Kalibrierspektrum Propin . . . . . . . . . . . . . . . 267

7.57 Kalibrierspektrum Propionaldehyd _ . . . . . . . . . . . . . . 267

7.58 Kalibrierspektrum Propyliodid . . . . . . . . . . . . . . . . . . 268 
ABBILDUNGSVERZEICHNIS

ABBILDUNGSVERZEICHNIS 


\section{Tabellenverzeichnis}

3.1 Tabelle der verwendeten Substanzen . . . . . . . . . . . . . . . . . . . . . 42

4.1 Reaktion $\mathrm{c}_{-} \mathrm{C}_{5} \mathrm{H}_{9}+\mathrm{O}$, Geschwindigkeitskoeffizienten für Reaktionen primärer Produkte mit $\mathrm{O}$. . . . . . . . . . . . . . . . . 51

4.2 Reaktion $\mathrm{c}_{-} \mathrm{C}_{5} \mathrm{H}_{9}+\mathrm{O}$, Messwerte O-Atom-Unterschuss . . . . . . . . . 51

4.3 Reaktion $\mathrm{c}_{-} \mathrm{C}_{5} \mathrm{H}_{9}+\mathrm{O}$, Messwerte O-Atom-Überschuss . . . . . . . . . . . 52

4.4 Reaktion $\mathrm{c}_{-} \mathrm{C}_{5} \mathrm{H}_{9}+\mathrm{O}$, Parameter für die Simulationsrechnungen . . . . . . 55

4.5 Reaktion $\mathrm{c}^{-} \mathrm{C}_{5} \mathrm{H}_{9}+\mathrm{O}$, Vergleich Experiment vs. Simulationsrechnungen . . 57

4.6 Reaktion $\mathrm{c}^{-} \mathrm{C}_{6} \mathrm{H}_{11}+\mathrm{O}$, Messwerte $4 \mathrm{mbar} \ldots \ldots 6$

4.7 Reaktion $\mathrm{c}^{-} \mathrm{C}_{6} \mathrm{H}_{11}+\mathrm{O}$, Parameter für die Simulationsrechnungen . . . . . 69

4.8 Reaktion $\mathrm{c}_{-} \mathrm{C}_{6} \mathrm{H}_{11}+\mathrm{O}$, Vergleich Experiment vs. Simulationsrechnungen . 70

4.9 Reaktion $\mathrm{c}^{-} \mathrm{C}_{6} \mathrm{H}_{11}+\mathrm{O}$, Geschwindigkeitskoeffizienten aus RRKM . . . . . 74

4.10 Reaktion c- $\mathrm{C}_{6} \mathrm{H}_{11}+\mathrm{O}$, Kanalverzweigung aus Experiment und Rechnung . 77

4.11 Reaktion $\mathrm{c}^{-} \mathrm{C}_{8} \mathrm{H}_{15}+\mathrm{O}$, Wahrscheinlichkeit Zerfall vs. $Z_{L J}[M] \ldots$. . . . 84

4.12 Vergleich der Reaktionen Cycloalkyl + O . . . . . . . . . . . . . 85

4.13 Reaktion $\mathrm{c}_{-} \mathrm{C}_{5} \mathrm{H}_{8}+\mathrm{O}$, Produktverhältnis . . . . . . . . . . . . . . 91

4.14 Reaktion $\mathrm{c}^{-} \mathrm{C}_{5} \mathrm{H}_{8}+\mathrm{O}$, Messwerte . . . . . . . . . . . . . . 91

4.15 Reaktion $\mathrm{c}^{-} \mathrm{C}_{5} \mathrm{H}_{8}+\mathrm{O}$, Produktverhältnisse im Vergleich . . . . . . . . . 92

4.16 Reaktionssystem $\mathrm{CH}_{3} / \mathrm{O}$, Parameter für Simulation . . . . . . . . . . . . 99

4.17 Reaktionssystem $\mathrm{CH}_{3} / \mathrm{O}$, Verhältnis $\mathrm{C}_{2} \mathrm{H}_{6} / \mathrm{H}_{2} \mathrm{CO}$ vs. Geschwindigkeit . 101

4.18 Reaktionssystem $\mathrm{C}_{2} \mathrm{H}_{5} / \mathrm{O}$, Messwerte . . . . . . . . . . . 107

4.19 Reaktionssystem $\mathrm{C}_{2} \mathrm{H}_{5} / \mathrm{O}$, Parameter für Simulation . . . . . . . . . . 108

4.20 Reaktionssystem $\mathrm{C}_{2} \mathrm{H}_{5} / \mathrm{O}$, Verhältnis $\mathrm{H}_{2} \mathrm{CO} /$ Butan vs. $\mathrm{C}_{2} \mathrm{H}_{5} / \mathrm{O}$. . . 109

4.21 Reaktion Allyl + O, Vorläufer Diallyl, Messwerte . . . . . . . . . . . . . . 124 
4.22 Reaktion Allyl + O, Vorläufer Allylbromid, Messwerte . . . . . . . . . . 125

4.23 Reaktion Allyl + O, Vorläufer Allylchlorid, Messwerte . . . . . . . . . . . . 126

4.24 Reaktion Allyl + O, Vergleich der Produktanteile . . . . . . . . . . . . . 128

4.25 Reaktionssystem $\mathrm{C}_{3} \mathrm{H}_{5} / \mathrm{O}$, Parameter für Simulation . . . . . . . . . . 130

4.26 Reaktion Allyl + O, Vergleich Experiment mit Simulation . . . . . . . . . 131

4.27 Reaktion $\mathrm{CD}_{3}+\mathrm{CD}_{3}$, Vorläufer Aceton- $\mathrm{d}_{6}$, Messwerte . . . . . . . . . . 137

4.28 Reaktion $\mathrm{C}_{2} \mathrm{H}_{5}+\mathrm{C}_{2} \mathrm{H}_{5}$, Vorläufer Ethylidodid, Messwerte . . . . . . . . . 142

4.29 Reaktion 1- $\mathrm{C}_{3} \mathrm{H}_{7}+1-\mathrm{C}_{3} \mathrm{H}_{7}$, Vorläufer Propyliodid, Messwerte . . . . . . . 151

4.30 Reaktion 1- $\mathrm{C}_{3} \mathrm{H}_{7}+1-\mathrm{C}_{3} \mathrm{H}_{7}$, Vorläufer Dipropylketon, Messwerte . . . . . . 152

4.31 Reaktion $2-\mathrm{C}_{3} \mathrm{H}_{7}+2-\mathrm{C}_{3} \mathrm{H}_{7}$, Vorläufer Isopropyliodid, Messwerte . . . . . . 158

4.32 Reaktion $1-\mathrm{C}_{4} \mathrm{H}_{9}+1-\mathrm{C}_{4} \mathrm{H}_{9}$, quantitative Analyse . . . . . . . . . . 163

4.33 Reaktion $\mathrm{C}_{3} \mathrm{H}_{5}+\mathrm{C}_{3} \mathrm{H}_{5}$, Vorläufer Diallyl, Messwerte . . . . . . . . . . . 172

4.34 Reaktion $\mathrm{C}_{3} \mathrm{H}_{5}+\mathrm{C}_{3} \mathrm{H}_{5}$, Vorläufer Allylbromid, Messwerte . . . . . . . . 173

4.35 Reaktion $\mathrm{C}_{3} \mathrm{H}_{5}+\mathrm{C}_{3} \mathrm{H}_{5}$, Vorläufer Allylchlorid, Messwerte . . . . . . . . . 174

4.36 Reaktion $\mathrm{C}_{3} \mathrm{H}_{5}+\mathrm{C}_{3} \mathrm{H}_{5}$, Vergleich der Vorläufer . . . . . . . . . . . . 175

4.37 Reaktionssystem $\mathrm{CH}_{3} / \mathrm{C}_{2} \mathrm{H}_{5}$, quantitative Auswertung . . . . . . . . . 184

4.38 Reaktionssystem $\mathrm{CH}_{3} / \mathrm{C}_{2} \mathrm{H}_{5}$, Parameter für die Simulationsrechnungen. . 185

4.39 Reaktionssystem $\mathrm{CH}_{3} / \mathrm{C}_{2} \mathrm{H}_{5}$, Vergleich Experiment mit Simulation . . . . 186

4.40 Reaktionssystem $\mathrm{CH}_{3} / \mathrm{C}_{2} \mathrm{H}_{5}$, Vorläufersensitivität . . . . . . . . . . . 187

4.41 Reaktionssystem $\mathrm{CH}_{3} / 1-\mathrm{C}_{3} \mathrm{H}_{7}$, Vorläufer Propyliodid, Messwerte . . . . . 197

4.42 Reaktionssystem $\mathrm{CH}_{3} / 1-\mathrm{C}_{3} \mathrm{H}_{7}$, Vorläufer Dipropylketon, Messwerte . . . . 199

4.43 Reaktionssystem $\mathrm{CH}_{3} / 1-\mathrm{C}_{3} \mathrm{H}_{7}$, Parameter für die Simulationsrechnungen 204

4.44 Reaktionssystem $\mathrm{CH}_{3} / 2-\mathrm{C}_{3} \mathrm{H}_{7}$, Vorläufer Isopropyliodid, Messwerte . . . 210

4.45 Reaktionssystem $\mathrm{CH}_{3} / 2-\mathrm{C}_{3} \mathrm{H}_{7}$, quantitative Analyse . . . . . . . . . . 211

4.46 Reaktionssystem $\mathrm{CH}_{3} / 2-\mathrm{C}_{3} \mathrm{H}_{7}$, Parameter für die Simulationsrechnungen 214

4.47 Reaktionssystem $\mathrm{CH}_{3} / 1-\mathrm{C}_{4} \mathrm{H}_{9}$, quantitative Analyse . . . . . . . . . . 218

4.48 Reaktionssystem $\mathrm{CH}_{3} / 1-\mathrm{C}_{4} \mathrm{H}_{9}$, quantitative Analyse (normiert) . . . . . . 218

4.49 Reaktionssystem $\mathrm{CH}_{3} / 1-\mathrm{C}_{4} \mathrm{H}_{9}$, Parameter für die Simulationsrechnungen 222

4.50 Reaktionssystem $\mathrm{CH}_{2} \mathrm{~F} / \mathrm{CHF}_{2}$, Parameter für die Simulationsrechnungen 224

4.51 Reaktionssystem $\mathrm{CH}_{2} \mathrm{~F} / \mathrm{CH}_{3}$, Parameter für die Simulationsrechnungen . 225

4.52 Reaktionssystem $\mathrm{CH}_{2} \mathrm{~F} / \mathrm{C}_{2} \mathrm{H}_{5}$, Parameter für die Simulationsrechnungen . 225 
6.1 Unimolekularer Zerfall zyklischer Alkoxyradikale . . . . . . . . . . . . . . . 234

6.2 Hochdruckgrenzwerte für unimolekularen Zerfall . . . . . . . . . . . . . . . 234

6.3 Selbstreaktionen von Alkylradikalen . . . . . . . . . . . . . . . 236

6.4 Kreuzreaktionen kleiner Alkylradikale mit Methylradikalen . . . . . . . . . 237

7.1 Integale Absorptionskoeffizienten . . . . . . . . . . . . . . . . 269 
TABELLENVERZEICHNIS

TABELLENVERZEICHNIS 


\section{Kapitel 1}

\section{Einleitung}

Kohlenwasserstoffe und ihre reinen und teiloxidierten Radikale nehmen eine herausragende Stellung in der Chemie ein. Unbewußt wird das Leben des Menschen erleichtert durch Produkte von Kohlenwasserstoff-Reaktionen. Beim Einkauf von Joghurt, Getränken oder abgepackten Lebensmitteln, überall finden sich Verpackungsprodukte, deren Erzeugung über die Reaktionen von Kohlenwasserstoffen erfolgt. Hier handelt es sich um Polymerisationsprodukte wie Polypropylen (PP) oder Polyethylen (PE), die aus Kohlenwasserstoffeinheiten (Monomeren) aufgebaut sind. Neben diesen, das tägliche Leben einfacher und sicherer machenden Produkten, spielen die Reaktionen von Kohlenwasserstoffradikalen in einem anderen Bereich eine so bedeutende Rolle, dass eine Industrienation wie Deutschland ohne diese Reaktionen kaum vorstellbar wäre: Die Verbrennung von Kohlenwasserstoffen und der Aufbau von höheren und ungesättigten aus niedermolekularen Kohlenwasserstoffen. Es werden zwar Bestrebungen vorangetrieben, den „Energiehunger“ industrieller Staaten durch Energiegewinnung aus erneuerbaren Energiequellen zu stillen, doch werden momentan noch $86 \%$ der weltweiten Energie durch Verbrennung von fossilen Brennstoffen wie Schweröl, Gas und Kohle erzeugt [Ann04].

Durch die Pyrolyse von Schwerölen und die Umsetzung von niedermolekularen gesättigten Kohlenwasserstoffen, insbesondere von Methan aus Erdgas, werden niedermolekulare ungesättigte Kohlenwasserstoffe als veredelte Grundchemikalien hergestellt, wobei die Disproportionierungs- / Kombinationsreaktionen der Kohlenwasserstoffradikale von zentraler Bedeutung sind. 
Zwei Reaktionstypen stehen im Mittelpunkt dieser Arbeit:

Typ 1: Die Bildung von teiloxidierten Kohlenwasserstoffradikalen $\mathrm{C}_{n} \mathrm{H}_{m} \mathrm{O}$ über die Reaktionen der Kohlenwasserstoffradikale $\mathrm{C}_{n} \mathrm{H}_{m}$ mit Sauerstoffatomen, gefolgt von den unimolekularen Reaktionen des chemisch aktivierten Intermediats $\mathrm{C}_{n} \mathrm{H}_{m} \mathrm{O}^{\star}$ nach dem Mechanismus Stabilisierung / Isomerisierung / Zerfall.

$$
\begin{aligned}
C_{n} H_{m}+\mathrm{O} & \longrightarrow C_{n} H_{m} O^{\star} \\
& \longrightarrow C_{n} H_{m-1}+O H \\
C_{n} H_{m} O^{\star} & \longrightarrow \text { Produkte } \\
& \longrightarrow \text { Produkte }
\end{aligned}
$$

Typ 2: Die bimolekularen Reaktionen von in der Struktur verschiedenen Kohlenwasserstoffradikalen $\mathrm{R}_{1}$ und $\mathrm{R}_{2}$ mit den Mechanismen Kombination und Disproportionierung nach Selbst- und Kreuzreaktion.

$$
\begin{aligned}
R_{1}+R_{1} & \longrightarrow R_{1}-R_{1} \\
& \longrightarrow R_{1} H+\text { Alken } \\
R_{1}+R_{2} & \longrightarrow R_{1}-R_{2} \\
& \longrightarrow R_{1} H+\text { Alken }
\end{aligned}
$$

In dieser Einleitung soll die Bedeutung derselben Reaktionstypen (Typ 1 und 2) für die sehr verschiedenen Anwendungsgebiete, die Chemie der radikalischen Polymerisation und die Chemie der Kohlenwasserstoffoxidation / -pyrolyse, mit ihren spezifischen Aspekten aufgezeigt werden.

\section{Radikalische Polymerisation}

Durch radikalische Polymerisation von olefinen Verbindungen wie Vinylchlorid, Ethen oder Styrol werden ein Großteil der benötigten Polymerprodukte gewonnen [Lec93]. Dabei wird durch den photolytischen oder thermischen Zerfall von geeigneten Initiatormolekülen eine Radikalbildung gestartet. Die Reaktion dieser Radikale mit Monomeren führt durch Addition des Radikals an die Doppelbindung des Monomers zu einem Monomer-Radikal. Ein Kettenwachstum wird gestartet, welches durch fortlaufende Addition neuer Monomere an die Radikalstelle zur Bildung von Makroradikalen führt und schließlich durch 
Terminierung zum Abbruch kommt. In der Startphase der radikalischen Polymerisation hat die Selbstreaktion der Initiatorradikale, die bei dem Zerfall des Initiators zumeist in Paaren gebildet werden, einen wesentlichen Einfluss auf die Initiatoreffizienz. Der dabei auftretende Käfigeffekt (cage effect) führt dazu, dass Initiatorradikale vorrangig durch Selbstreaktion verbraucht werden [Moa95]. Diese Radikal-Radikal-Reaktionen sind denen in der Gasphase ähnlich. Daher können Resultate von Radikalreaktionen in der Gasphase auf die radikalische Polymerisation übertragen werden, falls dabei keine Diffusionskontrolle vorliegt. Als Initiatoren für die radikalische Polymerisation kommen häufig Peroxide oder Peroxyester zum Einsatz, da diese durch thermische oder photolytische bzw. photochemische Einwirkung zur Radikalbildung angeregt werden können [Bub05, Moa95]. Typische Peroxy-Initiatoren sind in Abbildung 1.1 dargestellt:

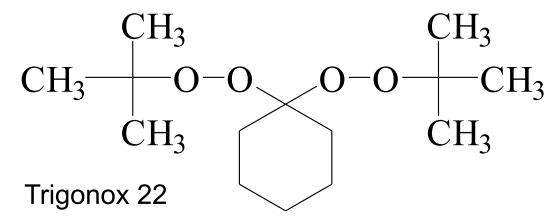<smiles>CC(C)(C)OOC(C)(C)C</smiles>

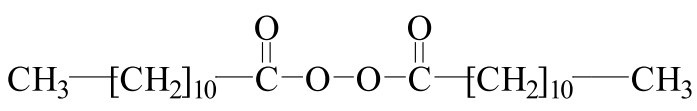

Laurox<smiles>CC(C)(C)C1CCC(OC(=O)OOC(=O)OC2CCC(C(C)(C)C)CC2)CC1</smiles>

Perkadox 16

Abbildung 1.1: Peroxy-Initiatoren. Thermische oder photochemische Einwirkung führt zur Bildung von Radikalen, die ein Kettenwachstum starten können.

Dabei führt die photolytische oder thermische Spaltung der Initiatormoleküle vorrangig zum O-O-Bindungsbruch. Durch Folgereaktionen wie Decarboxylierung oder $\beta$-C-CBindungsbruch kann zum Einen ein Wechsel von einem O-zentrierten Initiatorradikal zu einem C-zentrierten Radikal erfolgen, wie es bei dem weiteren Zerfall von Di-tertButylperoxyestern der Fall ist, zum Anderen kann es zur Stabilisierung des primären Radikals und Bildung kleinerer Radikale kommen [Bub00, Bub05]. Die beiden möglichen Folgereaktionen sind exemplarisch für den Zerfall des Di-tert-Butylperoxyesters in Abbildung 1.2 dargestellt. 


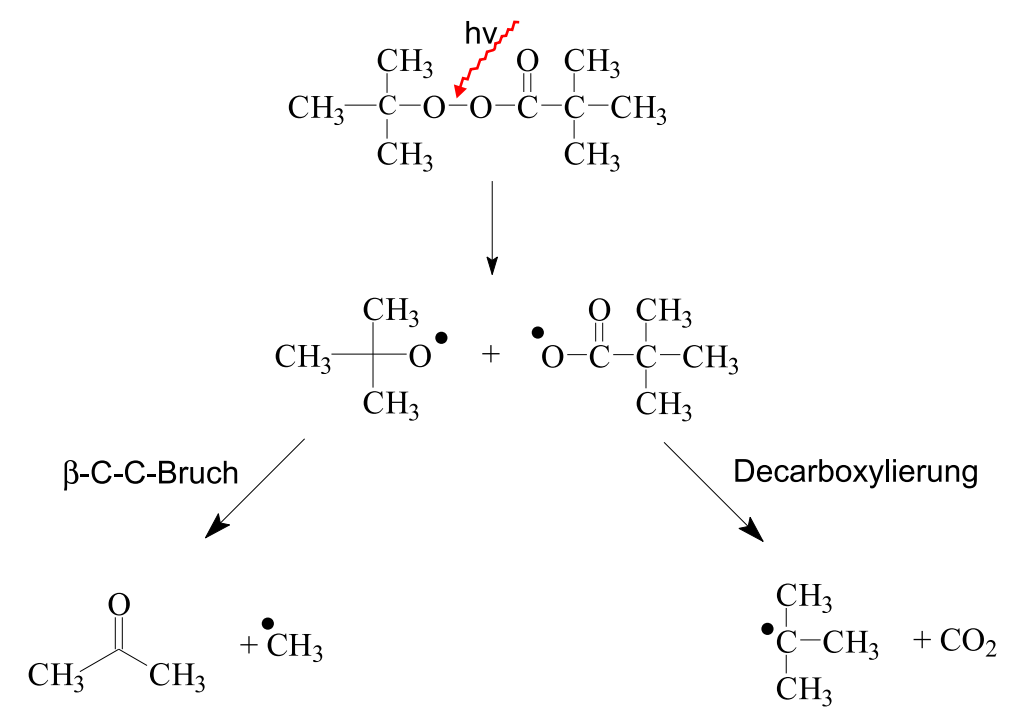

Abbildung 1.2: Der Zerfall von Di-tert-Butylperoxy. Die Photolyse führt zu einem O-OBindungsbruch. Sekundäre Zerfälle führen zur Bildung von Aceton und Methylradikalen ( $\beta$ $C$-C-Bindungsbruch) und zum Wechsel von einem O-zentrierten zu einem $C$-zentrierten Radikal (Decarboxylierung).

Sowohl der Wechsel von einem O-zentrierten zu einem C-zentrierten Initiatorradikal als auch ein $\beta$-C-C-Bindungsbruch haben Auswirkungen auf die Initiatoreffizienz $f$ und den Charakter des Polymerisationsprodukts, bedingt durch die unterschiedlichen Reaktionen, die O-zentrierte oder C-zentrierte Radikale vorzugsweise eingehen [Bub05]. In diesem Zusammhang spielen $H$-shifts und $\beta$-C-C-Bindungsbrüche der Radikale eine wesentliche Rolle. Kommt es zum Zerfall des primären Initiatorradikals unter Bildung kleinerer Alkylradikale, so hat das Verhältnis von Kombinations- zu Disproportionierungsgeschwindigkeit dieser Alkylradikale Auswirkungen auf die Initiierung des Polymerwachstums und auf die Endgruppen eines Polymers, die durch Kombination oder Disproportionierung eines Makroradikals mit einem Initiatorradikal von dem gewünschten Polymerendgruppen abweichen können.

Eine wichtige Frage ist, ob der Zerfall von Initiatormolekülen, wie Peroxydicarbonate, über einen schrittweisen oder konzertierten Prozess abläuft. Neuere theoretische Untersuchungen deuten darauf hin, dass Initiatormoleküle mit Elektronen anziehenden Alkylgruppen über schrittweise Zerfälle ablaufen. Dabei zerfallen die Initiatormoleküle sowohl über einen simultanen Bruch der O-O-Einfachbindung und den Bruch einer R-C-Einfachbindung mit anschließendem Zerfall des $\mathrm{R}-\mathrm{C}(\mathrm{O}) \mathrm{O}$-Radikals als auch über die Bildung von zwei Carboxyl-Radikalen, die in einem Folgeschritt zwei Alkylradikale bilden. Bei dem ers- 
ten Prozess kommt es zunächst zur Bildung eines O-zentrierten und eines C-zentrierten Radikals, wohingegen der zweite Reaktionsweg zunächst über die Bildung von zwei Ozentrierten Radikalen abläuft. Initiatormoleküle mit Elektronen „schiebenden“ Alkylgruppen laufen nur über den Prozess ab, bei dem im ersten Schritt zwei Bindungsbrüche erfolgen [Gu05]. Experimentelle Untersuchungen und quantenchemische Rechnungen wurden für den Zerfall organischer Peroxide im Hinblick auf die Geschwindigkeit der Decarboxylierung und den Reaktionsmechanismus mittels Ultra-Kurzzeitspektroskopie von BUBACK et al. in Lösungen durchgeführt [Abe03, Bub04].

Die Untersuchung des Zerfalls O-zentrierter Radikale stellt einen Schwerpunkt der vorliegenden Arbeit dar. Dabei wurden die Reaktionen hinsichtlich $H$-shifts und $\beta-C$ - $C$ - bzw. C-H-Bindungsbrüchen analysiert. Die saubere Herstellung von langkettigen O-zentrierten Radikalen, die über andere chemische und photochemische Reaktionen nicht oder nur schwierig erzeugt werden können, wurde durch Oxidation zyklischer Kohlenwasserstoffradikale mit Sauerstoffatomen erreicht, wobei die chemisch aktivierten Cycloalkoxyradikale primär unter $\beta$-C-C-Ringbruch zu langkettigen Alkoxyradikalen isomerisieren.

Dies ist in einem Mechanismus für das chemisch aktivierte $\mathrm{c}^{-} \mathrm{C}_{5} \mathrm{H}_{9} \mathrm{O}^{\star}$-Radikal aufgezeigt, das in dieser Arbeit studiert wurde.

$$
\begin{array}{rlr}
\mathrm{c}-\mathrm{C}_{5} \mathrm{H}_{9}+\mathrm{O} & \longrightarrow \mathrm{c}-\mathrm{C}_{5} \mathrm{H}_{9} \mathrm{O}^{\star} & \text { Aktivierung } \\
\mathrm{c}-\mathrm{C}_{5} \mathrm{H}_{9} \mathrm{O}^{\star} & \longrightarrow \mathrm{c}-\mathrm{C}_{5} \mathrm{H}_{8} \mathrm{O}+\mathrm{H} & \beta \text {-C-H-Bruch } \\
& \longrightarrow \text { Cyclopentenoxid }+\mathrm{H} & \text { C-H-Bruch } \\
\mathrm{c}-\mathrm{C}_{5} \mathrm{H}_{9} \mathrm{O}^{\star} & \longrightarrow 1-\mathrm{C}_{5} \mathrm{H}_{9} \mathrm{O}^{\star} & \text { Isomerisierung } \\
1-\mathrm{C}_{5} \mathrm{H}_{9} \mathrm{O}^{\star} & \longrightarrow \mathrm{C}_{4} \mathrm{H}_{7} \mathrm{CHO}+\mathrm{H} & \beta \text {-C-H-Bruch } \\
& \longrightarrow \mathrm{C}_{3} \mathrm{H}_{4} \mathrm{O}+\mathrm{C}_{2} \mathrm{H}_{4}+\mathrm{H} & \text { C-C-Bruch } \\
& \longrightarrow 2 \mathrm{C}_{2} \mathrm{H}_{4}+\mathrm{H}+\mathrm{CO} & \beta \text {-C-C-Bruch } \\
\mathrm{c}-\mathrm{C}_{5} \mathrm{H}_{9}+\mathrm{O} & \longrightarrow \mathrm{c}-\mathrm{C}_{5} \mathrm{H}_{8}+\mathrm{OH} & H \text {-Abstraktion }
\end{array}
$$

Durch die quantitative Bestimmung der Produktausbeuten nach den Reaktionskanälen zusammen mit begleitenden quantenchemischen Rechnungen und RRKM-Modellierungen wurden dann die molekularen Parameter wie Energiebarrieren und Frequenzfaktoren ermittelt, die Grundlage für die Ermittlung von Geschwindigkeitskoeffizienten und ihrer 
Temperaturabhängigkeit (Aktivierungsenergie) für thermisch aktivierte Reaktionen sind. Durch den Einsatz von zyklischen Radikalvorläufern von c- $\mathrm{C}_{5}$ - bis $\mathrm{c}_{-} \mathrm{C}_{8}$-Alkanen stehen primäre langkettige Oxyradikale von $\mathrm{l}_{-} \mathrm{C}_{5} \mathrm{H}_{9} \mathrm{O}$ bis $\mathrm{l}_{-} \mathrm{C}_{8} \mathrm{H}_{15} \mathrm{O}$ für die Untersuchung zur Verfügung.

Neben dem für die Initiierung von radikalischen Polymerisationen wichtigen Zerfall Ozentrierter Radikale wurden Reaktionen von Alkylradikalen mit Alkylradikalen im Hinblick auf die Auswirkung der Kettenlänge und der Verzweigung der Alkylradikale auf das Verhältnis von Kombination zu Disproportionierung eingehend untersucht. Diese Untersuchungen sollen das Verständnis von Prozessen, welche bei der Initiierung von radikalischen Polymerisationen ablaufen, vertiefen und leisten damit einen Beitrag zum Graduiertenkolleg European Graduate School (EGS): „Microstructural Control in Free Radical Polymerisation" mit dem speziellen Forschungsschwerpunkt „Reactions of Hydrocarbon Radicals in the Gasphase: Rates, Primary Products, Mechanisms“. Dieser Reaktionstyp Typ 2 wird im Zusammenhang mit der Kohlenwasserstoffoxidation / -pyrolyse weiter unten erläutert.

\section{Kohlenwasserstoffoxidation / -pyrolyse}

Die Relevanz der Reaktionen von Kohlenwasserstoffradikalen, ihre Selbstreaktionen und die Reaktionen mit Sauerstoff-Atomen, für die Verbrennung und die Pyrolyse soll hier kurz erläutert werden.

Vor Allem im Hinblick auf den wachsenden asiatischen Markt, insbesondere die Entwicklung Chinas und die Motorisierung der Bevölkerung, wird der Energiebedarf innerhalb der nächsten Jahre drastisch steigen. Im Zeitraum von 1994 bis 2003 ist der primäre Energieverbrauch Chinas um circa 34 \% gestiegen, wohingegen der Vebrauch in den Vereinigten Staaten von Amerika „nur“ um $10 \%$ und in Europa um $14 \%$ angestiegen ist. Der globale Verbrauch erhöhte sich im betreffenden Zeitraum um $18 \%$ Ann04].

Deshalb scheint es dringender denn je geboten, neben der technischen Entwicklung von neuen energiesparenden Motoren, durch Aufklärung von verbrennungsrelevanten Reaktionsmechanismen allgemein die Verbrennungsprozesse zu optimieren. Dafür sind die quantitative Bestimmung der Reaktionsprodukte und deren Reaktionskanäle und Geschwindigkeitskoeffizienten nötig. Eine Optimierung sollte dafür sorgen, dass der Kraftstoff- 
verbrauch möglichst gering ist und möglichst wenig umweltschädliche Produkte $\left(\mathrm{NO}_{x}\right.$, CO, Rußpartikel) bei der Verbrennung enstehen. „Rußpartikel“ ist dabei ein Sammelbegriff für PAH (polycyclic aromatic hydrocarbons), kondensierte Kohlenwasserstoffe und hochkondensierte wasserstoffhaltige C-Verbindungen. Ihr Aufbau erfolgt über (Radikal + Radikal)- und (Radikal + Alken, Alkin)-Reaktionen [Hoy04. In diesem Zusammenhang sei die hochaktuelle Problematik des Feinstaubs und der Beitrag seiner Bildung durch Dieselmotoren in deutschen Innenstädten genannt. Neben dem Einbau von Feinpartikelfiltern soll die Entwicklung neuer Motoren und dafür „maßgeschneiderte“ Kraftstoffe zur Reduktion dieser Probleme führen.

Einen aktuellen Schwerpunkt bildet daher weiterhin die Entwicklung von HCCI (Homogenous Charge Compression Ignition) Motoren, für deren Betrieb die Bereitstellung neuer selbstzündender (Benzin)-Treibstoffe mit den oben genannten Eigenschaften eine entscheidende Rolle spielt [Kim04].

In der vorliegenden Arbeit wurden experimentell Elementarreaktionen untersucht, die bei der Verbrennung gewöhnlicher Treibstoffe auftreten, beziehungsweise die für die Vorhersage von Modelltreibstoffen für HCCI Motoren von Bedeutung sind. WestBrook [Wes99] betont, dass die gegenwärtige Herausforderung in der Reduktion der Schadstoffe bei gleichzeitiger Steigerung der Treibstoffeffizienz liegt, und dass für diese Herausforderung die Modellierung der Verbrennungsprozesse unerlässlich ist. Die Bestimmung der verbrennungsrelevanten Reaktionen und deren Geschwindigkeitskoeffizienten ist damit auch im Hinblick auf die Modellierung von aktuellem Interesse.

Gegenstand dieser Arbeit sind daher Reaktionen von zyklischen Kohlenwasserstoffradikalen mit Sauerstoffatomen, die direkt in die Modellierung der chemischen Prozesse bei der motorischen Verbrennung eingehen und indirekt, wie bereits oben erläutert, einen eleganten Zugang zur Untersuchung von langkettigen primären Alkoxyradikalen bieten. Zyklische Kohlenwasserstoffe kommen vor allem in gewöhnlichen Treibstoffen wie Diesel oder Kerosin (Jet A, Jet A-1, JP-8, etc.) mit Anteilen von über 50\% vor [Edw03, Pot98]. Daher ist das Wissen um Kanalverzweigung und Geschwindigkeit für die Reaktionen von zyklischen Kohlenwasserstoffradikalen mit Sauerstoffatomen essentiell, um die oben genannten Ziele wie Schadstoffreduktion und Leistungsoptimierung zu erreichen. Die Kon- 
zentration von molekularem Sauerstoff bei Verbrennungen liegt zwar meistens weit über der Konzentration von atomarem Sauerstoff, doch ist die Reaktion von Alkylradikalen mit atomarem Sauerstoff und anderen Radikalen $\left(\mathrm{OH}, \mathrm{CH}_{3}\right)$ um bis zu zwei Größenordungen schneller [Gri95]. Der Abbau von Kohlenwasserstoffen wird daher vorzugsweise über diese, in der Arbeit betrachteten, Reaktionswege verlaufen.

Neben den Reaktionen von Kohlenwasserstoffradikalen mit Sauerstoffatomen ist die Pyrolyse von Kohlenwasserstoffen unter Hochtemperaturbedingungen wichtig, um Verbrennungsprozesse adäquat beschreiben zu können. Hierbei spielen Selbst- und Kreuzreaktionen von kleinen Kohlenwasserstoffradikalen eine herausragende Rolle, da $\mathrm{C}_{1^{-}}$bis $\mathrm{C}_{5^{-}}$ Spezies in hohen Konzentrationen vorliegen. Auf Grund dieser Bedeutung, neben der Relevanz für die radikalische Polymerisation, beschäftigt sich der zweite Teil dieser Arbeit mit den Selbstreaktionen von Kohlenwasserstoffradikalen und Kreuzreaktionen von Kohlenwasserstoffradikalen von verschiedener Struktur: primäre, sekundäre und ungesättigte; insbesondere mit der Reaktion von Allylradikalen. Letztere ist von besonderem Interesse, da es sich bei dem Allylradikal um eines der kleinsten resonanzstabilisierten Radikale handelt und es daher bei Verbrennungsprozessen in hohen Konzentrationen vorkommt. Außerdem wurde die Reaktion von Allylradikalen mit Sauerstoffatomen in das Untersuchungsprogramm aufgenommen, da diese Reaktion gut als Referenzreaktion für Allylradikal-Reaktionen $\left(\mathrm{C}_{3} \mathrm{H}_{5}+\mathrm{C}_{3} \mathrm{H}_{5}, \mathrm{CH}_{3}+\mathrm{C}_{3} \mathrm{H}_{5}\right.$ etc. $)$ geeignet ist, in der Literatur jedoch widersprüchliche Daten über Mechanismus und Geschwindigkeit vorliegen.

Die Reaktion von zwei Alkylradikalen lässt sich durch folgenden Mechanismus allgemein beschreiben:

$$
\begin{array}{rlr}
\mathrm{R}_{1}+\mathrm{R}_{2} & \longrightarrow \mathrm{R}_{1} \mathrm{R}_{2}^{\star} & \text { Addition } \\
\mathrm{R}_{1} \mathrm{R}_{2}^{\star} & \longrightarrow \mathrm{R}_{1}+\mathrm{R}_{2} & \text { Re-Dissoziation } \\
& \longrightarrow \text { Alken }+ \text { Alkan } & \text { Disproportionierung } \\
\mathrm{R}_{1} \mathrm{R}_{2}^{\star}+\mathrm{M} & \longrightarrow \mathrm{R}_{1} \mathrm{R}_{2}+\mathrm{M} & \text { Kombination }
\end{array}
$$

Auf Grundlage der experimentell bestimmten Kanalverzweigungen wurden mittels Simulationsrechnungen mit einfachen Reaktionsmechanismen Geschwindigkeitskoeffizienten bestimmt und diese mit Vorhersagen der geometric mean rule verglichen. Die geometric 
mean rule (GMR) wird häufig als Abschätzungsmethode für Geschwindigkeitskoeffzienten der Kreuzkombination von den Radikalen $A$ und $B$ herangezogen, wenn die Geschwindigkeitskoeffizienten der Selbstreaktionen der beiden Radikale $A$ und $B$ bekannt sind:

$$
\begin{aligned}
& A+A \longrightarrow \text { Produkte }\left(k_{A A}\right) \\
& B+B \longrightarrow \text { Produkte }\left(k_{B B}\right) \\
& A+B \longrightarrow \text { Produkte }\left(k_{A B}\right)
\end{aligned}
$$

Die geometric mean rule lautet:

$$
k_{A B}=2 \cdot \sqrt{k_{A A} k_{B B}}
$$

wobei $k_{A B}$ den Geschwindigkeitskoeffizienten der Kreuzreaktion $A+B$ bezeichnet und $k_{A A}$ bzw. $k_{B B}$ für den Geschwindigkeitskoeffizienten der Selbstreaktion $A+A$ bzw. $B+B$ steht.

Ziel der Untersuchung dieser Radikal-Radikal-Reaktionen ist die direkte experimentelle Bestimmung von Geschwindigkeitskoeffizienten und Mechanismen, die dann auch eine Überprüfung der geometric mean rule und eine Abschätzung ihrer Anwendungsgrenzen zulassen. 
Die Arbeit gliedert sich wie folgt.

Nach dieser Einleitung werden in Kapitel 2 die physikalisch-chemischen und mathematischen Grundlagen dargestellt. In Kapitel 3 erfolgt eine Erläuterung der verwendeten Apparatur und eine Beschreibung der Radikalerzeugung sowie allgemeine Angaben zur qualitativen und quantitativen Analyse von FTIR-Spektren. In Kapitel 4 werden die Ergebnisse dieser Arbeit präsentiert. Dabei werden zuerst die Reaktionen von zyklischen Kohlenwasserstoffradikalen mit Sauerstoffatomen vorgestellt. Danach erfolgt eine Darstellung der Ergebnisse für die Reaktionen von kleinen Alkylradikalen mit Sauerstoffatomen. In den darauf folgenden Abschnitten werden die Ergebnisse für Selbst- und Kreuzreaktionen von Alkylradikalen erläutert. Am Ende dieses Kapitels erfolgt kurz die Darstellung der Ergebnisse der Simulationsrechnungen zu den Reaktionen von teilfluorierten Alkylradikalen. Abschließend erfolgt in Kapitel 5 ein Ausblick über weitere Forschungsansätze und in Kapitel 6 eine Zusammenfassung der Ergebnisse. Im Anhang befinden sich die für die qualitative und quantitative Analyse benötigten Eichkoeffizienten bzw. FTIR-Spektren.

Teile dieser Arbeit wurden bereits publiziert oder sind dafür vorgesehen:

1. Beiderhase, T.; Hoyermann, K.; Nothdurft, J.; Olzmann, M.: Gas-Phase Kinetics of the Reactions of the $\mathrm{CH}_{2} \mathrm{~F}$ Radicals with the Radicals $\mathrm{CHF}_{2}, \mathrm{CH}_{3}$, and $\mathrm{C}_{2} H_{5}$, Z. Phys. Chem. 218, 493 (2004)

2. Hoyermann, K.; Nothdurft, J.; Olzmann, M.; Wehmeyer, J.; Zeuch, T.: Formation and decomposition of chemically activated cyclopentoxy radicals from the $c-C_{5} H_{9}+O$ reaction, J. Phys. Chem. A, 110, 3165 (2006)

3. Hoyermann, K.; MaArfeld, S.; Nacke, F.; Nothdurft, J.; Olzmann, M.; Wehmeyer, J.; Welz, O.; Zeuch, T.: Experimental and Theoretical Studies on the Formation and Decomposition of Chemically Activated Cycloalkoxy Radicals, Proc. Combust. Institute, zur Veröffentlichung eingereicht

4. Hoyermann, K.; Nothdurft, J.; Olzmann, M.; Wehmeyer, J.; Zeuch, T.: Formation and decomposition of chemically activated cyclopentoxy radicals from the $c-C_{5} H_{9}+O$ reaction, $18^{\text {th }}$ International Symposium on Gas Kinetics, Bristol (2004) 


\section{Kapitel 2}

\section{Theoretische Grundlagen}

\subsection{Physikalisch-Chemische Grundlagen}

\subsubsection{Spektroskopische Grundlagen}

Fällt ein Lichtstrahl mit einer Intensität $I_{0}$ auf eine Probe, so kann die Strahlung reflektiert, gestreut oder absorbiert werden. Damit hat der aus der Probe austretende Strahl eine um diese Prozesse verringerte Intensität $I$. Unter der Lichtintensität wird im Hinblick auf die Teilcheneigenschaft die Anzahl der Photonen verstanden, die mit einer gegebenen Energie pro Photon auf eine Fläche pro Zeiteinheit auftreffen. Unter Berücksichtigung der Welleneigenschaft des Lichts wird unter der Intensität die Energie, welche pro Zeiteinheit durch eine zur Ausbreitungsrichtung der Welle senkrechte Flächeneinheit transportiert wird, verstanden. Sie hängt quadratisch von der Amplitude der Wellenlänge des eingestrahlten Lichts ab. Bei der IR-Spektroskopie wird ausgenutzt, dass die Absorbanz molekülspezifisch und eine Funktion der Wellenlänge $A=f(\lambda)$ ist.

Die logarithmische Intensitätsdifferenz ist proportional zur Schichtdicke bzw. zur Probendicke $d$ und zur Konzentration der in der Probe befindlichen Stoffe $c$. Diesen Zusammenhang beschreibt das Lambert-Beersche Gesetz (siehe dazu z.B. [Wed97]), wobei jedoch die Konzentration $c$ nicht beliebig hoch sein darf. Es gilt:

$$
I=I_{0} \cdot e^{-\epsilon \cdot c \cdot d} \quad \text { oder } \quad I=I_{0} \cdot 10^{-\tilde{\epsilon} \cdot c \cdot d}
$$


Dabei bezeichnet

$$
\begin{aligned}
& I \quad \text { die austretende Strahlungsintensität, } \\
& I_{0} \quad \text { die eintretende Strahlungsintensität, } \\
& d \quad \text { die Schicht- oder Probendicke, } \\
& c \quad \text { die Konzentration der Probe und } \\
& \epsilon, \tilde{\epsilon} \quad \text { die molaren Absorptionskoeffizienten. }
\end{aligned}
$$

Umformung bezüglich der Konzentration liefert:

$$
\begin{aligned}
& \frac{I}{I_{0}}=e^{-\epsilon \cdot c \cdot d} \\
\Leftrightarrow & \epsilon \cdot c \cdot d=\ln \frac{I_{0}}{I}=: A \\
\Leftrightarrow & c=\frac{A}{\epsilon \cdot d} .
\end{aligned}
$$

Der Quotienten $\frac{I}{I_{0}}$ wird als die Durchlässigkeit oder Transmission T, $A$ als die Absorbanz bezeichnet. Der Absorptionskoeffizient $\epsilon$ ist eine stoffspezifische Größe, die von der Art des Moleküls und den damit verbundenen Möglichkeiten zur Rotation, Translation und Vibrationsschwingungen sowie der Frequenz des eingestrahlten Lichts abhängt.

Da sich die Absorptionsbanden der untersuchten Substanzen über einen Frequenzbereich erstrecken, muss für die Berechnung der Konzentration, beziehungsweise des Partialdrucks die integrale Intensität bezüglich eines betreffenden Frequenzbereichs betrachtet werden. Folglich wird statt des molaren Absorptionskoeffizienten $\epsilon$ ein Koeffizient benötigt, der in funktionalem Zusammenhang mit der Frequenz steht. Es ergibt sich der integrale Absorptionskoeffizient:

$$
\omega=\int \epsilon(\nu) d \nu
$$

Dieser wird teilweise auch totaler Absorptionskoeffizient genannt. Dabei beschreibt $\epsilon(\nu)$ eine von der Frequenz abhängige Funktion.

Besitzt die Reaktionszelle im Experiment ein konstantes Volumen, so kann von der Konzentration zum Partialdruck übergegangen werden. Verhalten sich die Gase in dem untersuchten Druckbereich zudem ideal, vereinfacht sich das Problem wie im Folgenden dargestellt. Diese Annahme ist bei den im Rahmen dieser Arbeit durchgeführten Experimenten zulässig, da bei allen Experimenten der Gesamtdruck $\leq 1000$ mbar war. Umformung der idealen Gasgleichung liefert

$$
p \cdot V=n \cdot R \cdot T
$$




$$
\begin{aligned}
& \Leftrightarrow \quad p=\frac{n}{V} \cdot R \cdot T \\
& \Leftrightarrow \quad p=c \cdot k \quad \text { mit einer Konstanten } k \text { für } T \text { konstant, }
\end{aligned}
$$

wobei

$$
\begin{array}{ll}
p & \text { den Partialdruck, } \\
V & \text { das Zellvolumen, } \\
n & \text { die Stoffmenge, } \\
R & \text { die allgemeine Gaskonstante und } \\
T & \text { die absolute Temperatur }
\end{array}
$$

bezeichnet. Unter Berücksichtigung des integralen Absorptionskoeffizienten folgt

$$
\begin{aligned}
& \frac{I}{I_{0}}=\exp \left(-\int \epsilon(\nu) d \nu \frac{p_{i}}{R \cdot T} d\right) \\
\Rightarrow & \underbrace{\ln \left(\frac{I_{0}}{I}\right)}_{:=A_{i}}=p_{i} \cdot \underbrace{\frac{\int \epsilon(\nu) d \nu \cdot d}{R \cdot T}}_{:=\Omega_{i}}
\end{aligned}
$$

Zur Berechnung des Partialdrucks ergibt sich folgende Formel:

$$
p_{i}=\frac{A_{i}}{\Omega_{i}},
$$

wobei $A_{i}$ die integrale Absorbanz der Komponente $i$ über denselben Integrationsbereich bezeichnet, den der integrale Absorptionskoeffizient $\omega$ umfasst.

\subsubsection{Kinetische Grundlagen}

Wie in der Einleitung dargestellt, ist für die Optimierung von Verbrennungsprozessen im Sinne eines möglichst effizienten und sauberen Prozesses, die Kenntnis der Reaktionsprodukte und der zu den Reaktionen gehörigen Geschwindigkeitskoeffizienten elementar. Für die Beschreibung und Vorhersage der bei einer Polymerisation ablaufenden Prozesse werden ebenfalls Geschwindigkeiten für Radikal-Radikal-Reaktionen benötigt. Dies gilt besonders für die Beschreibung und Vorhersage der Initiatoreffizienz $f$ und von Produkten, die durch Kombination oder Disproportionierung eines Makroradikals mit einem Initiatorradikal gebildet werden.

\subsubsection{Parallelreaktionen: Kombination vs. Disproportionierung}

In der vorliegenden Arbeit werden Prozesse untersucht bei denen sowohl Parallel- als auch Folgereaktionen auftreten. Dabei handelt es sich in der Regel um Reaktionen, die durch 
einen dritten Stoßpartner, also meistens durch das Badgas, beeinflusst werden können.

Wird zunächst die Elementarreaktion

$$
A+B \longrightarrow \text { Produkte }
$$

betrachtet, so ergibt sich folgende Geschwindigkeitsgleichung:

$$
\frac{d[A]}{d t}=-k[A][B]
$$

wobei $[\mathrm{A}]$ und $[\mathrm{B}]$ die Konzentrationen der Spezies A und B bezeichnen. Dabei wird ein konstantes Volumen, wie es in der Reaktionszelle stets vorliegt, vorausgesetzt. Die Umformung der Differentialgleichung mit Seperationsansatz liefert

$$
\int_{[A]_{0}}^{[A]_{t}} \frac{d[A]}{[A]}=-k \int_{t_{0}}^{t}[B] d \tau .
$$

Integration über den Bereich $\tau=0, \ldots, t$ ergibt

$$
\ln \left(\frac{[A]_{t}}{[A]_{0}}\right)=-k \int_{0}^{t}[B] d \tau \text {. }
$$

Für eine stationäre Konzentration von $\mathrm{B}$, dies gilt in guter Näherung für $[B]_{0} \gg[A]_{0}$, folgt ein Zeitgesetz pseudo-erster Ordnung und es ergibt sich:

$$
\ln \left(\frac{[A]_{t}}{[A]_{0}}\right)=-k[B]_{0} t
$$

mit $k[B]_{0}=: k_{B}$ folgt für die Konzentration von $A$ zum Zeitpunkt $t$

$$
[A]_{t}=[A]_{0} e^{-k_{B} t}
$$

Werden nun Parallelreaktionen (Elementarreaktionen) betrachtet, die zum Einen zur Bildung des Kombinationsprodukts $B$ und zum Anderen zu den Disproportionierungsprodukten $C$ und $D$ führen

$$
\begin{array}{ll}
A+A & \stackrel{k_{1}}{\longrightarrow} B \\
A+A & \stackrel{k_{2}}{\longrightarrow} C+D,
\end{array}
$$

so liegen zwei Reaktionen 2. Ordnung vor. Die Abnahme an $A$ kann durch die Geschwindigkeitsgleichung

$$
r=-\frac{1}{2} \frac{d[A]}{d t}=k[A]^{2}=k_{1}[A]^{2}+k_{2}[A]^{2}
$$


beschrieben werden, wobei $k=k_{1}+k_{2}$ gilt. $k_{1}$ bzw. $k_{2}$ bezeichnen den Geschwindigkeitskoeffizienten der Kombination bzw. den der Disproportionierung. Integration liefert in diesem Fall

$$
\begin{aligned}
& \int_{[A]_{0}}^{[A]_{t}} \frac{d[A]}{[A]^{2}}=-2 k \int_{0}^{t} d \tau \\
\Leftrightarrow & -\left(\frac{1}{[A]_{t}}-\frac{1}{[A]_{0}}\right)=-2 k \int_{0}^{t} d \tau \\
\Leftrightarrow & \frac{1}{[A]_{t}}-\frac{1}{[A]_{0}}=2 k \cdot t \\
\Leftrightarrow & {[A]_{t}=\frac{1}{\frac{1}{[A]_{0}}+2 k \cdot t} . }
\end{aligned}
$$

Für die Bestimmung der Geschwindigkeitskoeffizienten ist das Verhältnis der Konzentration des Kombinationsprodukts $B$ zu den Konzentrationen der Disproportionierungsprodukte $C$ und $D$ relevant. Dabei gelten für die Produkte die Zeitgesetze

$$
\begin{aligned}
& \frac{d[B]}{d t}=k_{1}[A]^{2}=k_{1}\left(\frac{1}{[A]_{0}}+2 k \cdot t\right)^{-2} \\
& \frac{d[C]}{d t}=k_{2}[A]^{2}=k_{2}\left(\frac{1}{[A]_{0}}+2 k \cdot t\right)^{-2}
\end{aligned}
$$

Nach Integration über die Zeit für die Konzentrationen der Stoffe $B$ und $C$ folgt:

$$
\begin{aligned}
& {[B]_{t}=\frac{k_{1}}{2 k} \cdot\left([A]_{0}-\frac{1}{\frac{1}{[A]_{0}}+2 k \cdot t}\right)} \\
& {[C]_{t}=\frac{k_{2}}{2 k} \cdot\left([A]_{0}-\frac{1}{\frac{1}{[A]_{0}}+2 k \cdot t}\right)}
\end{aligned}
$$

Damit gilt für das Verhältnis der Konzentrationen:

$$
\frac{[B]}{[C]}=\frac{k_{1}}{k_{2}}
$$

Ist der Geschwindigkeitskoeffizient der Gesamtreaktion oder einer der beiden Teilreaktionen bekannt, so ist durch Bestimmung des Konzentrationsverhältnisses $[B] /[C]$ eine Berechnung der Geschwindigeitskoeffizienten beider Teilreaktionen möglich.

Liegt neben dem Stoff A noch ein anderes Edukt vor, das sowohl mit A als auch mit sich selbst reagieren kann, so ergibt sich unter der Voraussetzung, dass nur Kombinationsund Disproportionierungsreaktionen auftreten und dabei eine der beiden möglichen Disproportionierungsreaktionen stark bevorzugt ist, folgender Reaktionsmechanismus:

$$
A+A \stackrel{k_{1}}{\longrightarrow} B
$$




$$
\begin{array}{lll}
A+A & \stackrel{k_{2}}{\longrightarrow} & C+D \\
A+B & \stackrel{k_{3}}{\longrightarrow} & E \\
A+B & \stackrel{k_{4}}{\longrightarrow} & F+G \\
B+B & \stackrel{k_{5}}{\longrightarrow} & H \\
B+B & \stackrel{k_{6}}{\longrightarrow} & I+K
\end{array}
$$

Eine einfache analytische Herleitung des Verhältnisses der Geschwindigkeitskoeffizienten aus den Konzentrationen der Produkte ist für diesen Mechanismus nicht mehr möglich. Daher wurden die Geschwindigkeitskoeffzienten durch Simulationsrechnungen bestimmt, bei denen die Geschwindigkeitskoeffizienten der Selbstreaktionen experimentell bestimmt oder der Literatur entnommen wurden.

\subsubsection{Druckabhängigkeit chemischer Reaktionen}

Bei der Untersuchung von einigen Reaktionen konnte eine Druckabhängigkeit der Kanalverteilung beobachtet werden. Daher wird in diesem Zusammenhang der Ansatz für die Beschreibung von druckabhängigen Reaktionen kurz dargestellt.

Kann eine Druckabhängkeit für eine Reaktion gefunden werden, so ist davon auszugehen, dass die Reaktion in diesem Bereich nicht durch eine einfache Elementarreaktion erklärt werden kann. Einen Ansatz zur Beschreibung dieser Druckabhängigkeit stellt der Lindemann-Hinshelwood Mechanismus dar, der hier unter Einbeziehung von Kombination und Disproportionierung am Beispiel der (Alkyl + Alkyl)-Reaktion erläutert wird. Im eigentlichen Sinne geht der Lindemann-Hinshelwood Mechanismus von einer thermischen Aktivierung aus, sodass es sich hierbei korrekterweise nur um eine Anlehnung an den Mechanismus handelt.

Ausgehend von der Reaktion zweier Alkylradikale $R$, die in einem ersten Schritt einen aktivierten Übergangszustand $R R^{\star}$ bilden, und dass der Reaktionsweg zum Kombinationsprodukt durch Stöße mit einem dritten Partner M stabilisiert wird, ergibt sich das nachfolgende Reaktionsschema. Darstellungen zu diesem komplexen Bildungsmechanismus finden sich z.B. in [Tro94].

$$
\begin{aligned}
R+R & \stackrel{k_{A}}{\longrightarrow} R R^{\star} \\
R R^{\star} & \stackrel{k_{R}}{\longrightarrow} R+R
\end{aligned}
$$




$$
\begin{aligned}
R R^{\star} & \stackrel{k_{D}}{\longrightarrow} \text { Alken }+ \text { Alkan } \\
R R^{\star}+M & \stackrel{k_{K}}{\longrightarrow} R R+M
\end{aligned}
$$

Weiterhin wird von einer quasistationären Konzentration des aktivierten Komplexes $R R^{\star}$ ausgegangen $\left(\frac{d\left[R R^{\star}\right]}{d t}=0\right)$. Es folgt dann das Zeitgesetz für den Verbrauch der Alkylradikale $R:$

$$
-\frac{1}{2} \frac{d[R]}{d t}=k[R]^{2}=\frac{k_{D}+k_{K}[M]}{k_{R}+k_{D}+k_{K}[M]} k_{A}[R]^{2},
$$

wobei $k$ den beobachteten Geschwindigkeitskoeffizienten bezeichnet. Die Geschwindigkeitskoeffizienten der Redissoziation $k_{R}$ und der Disproportionierung $k_{D}$ hängen von der mittleren Anregungsenergie des Komplexes ab. Des Weiteren gilt $k_{K}[M] \propto p$. Für den Fall, dass $k_{D}+k_{K}[M] \gg k_{R}$ vereinfacht sich der Ausdruck zu:

$$
-\frac{1}{2} \frac{d[R]}{d t}=k[R]^{2}=k_{A}[R]^{2} .
$$

In diesem Fall ist die Bestimmung der Kombinations- bzw. Disproportionierungsgeschwindigkeit auf Grundlage des Konzentrationsverhältnisses möglich, wie im vorhergehenden Abschnitt dargestellt wurde.

\subsection{Mathematische Grundlagen der (Fast)-Fourier- Transformation}

Die Ausführungen zu den mathematischen Grundlagen der Fouriertransformation stützen sich im wesentlichen auf JAMES [Jam95] und Beschreibungen von ZEUCH [Zeu03].

Bei der Betrachtung der Welleneigenschaft des Lichts kann Licht als Überlagerung unendlich vieler periodischer Funktionen mit unterschiedlicher Periodenlänge (Wellenlänge) dargestellt werden. Mathematisch lässt sich dies durch eine Linearkombination der periodischen Sinus- und Cosinusfunktion zu einer Grundfrequenz $\nu_{0}$, die sich aus einer Grundperiodenlänge $T_{0}$ ergibt, ausdrücken. Damit lässt sich eine Funktion aufstellen, die von der Grundfrequenz $\nu_{0}$ und von der Zeit $t$ abhängt. Diesen Prozess nennt man Fouriersynthese. Man erhält die Fourierreihe:

$$
F(t)=\sum_{n=-\infty}^{\infty} a_{n} \cos \left(2 \pi \nu_{0} n t\right)+b_{n} \sin \left(2 \pi \nu_{0} n t\right) .
$$


Dabei bezeichnen $a_{n}$ und $b_{n}$ konstante Faktoren, die die Amplitude bestimmen.

Unter der Berücksichtigung, dass es sich bei der Cosinusfunktion um eine gerade und bei der Sinusfunktion um eine ungerade Funktion handelt, erhält man:

$$
F(t)=\frac{A_{0}}{2}+\sum_{n=1}^{\infty} A_{n} \cos \left(2 \pi \nu_{0} n t\right)+B_{n} \sin \left(2 \pi \nu_{0} n t\right),
$$

wobei $A_{n}=a_{n}+a_{-n}$ und $B_{n}=b_{n}-b_{-n}$ gilt. Da in den meisten Fällen die Kurve $F(t)$ durch ein Experiment bestimmt wurde und so $A_{n}$ und $B_{n}$ unbekannt sind, ist es von Interesse, die Amplituden $A_{n}$ und $B_{n}$ für jedes $\mathrm{n}$ zu bestimmen, sodass mit den Amplituden $A_{n}$ und $B_{n}$ auch die Intensitäten des n-fachen der Grundfrequenz berechnet werden können. Liegt eine Funktion $F(t)$ vor, so können mittels einer Fourieranalyse die Amplituden berechnet werden. Dabei wird die Orthogonalitätseigenschaft der Sinus- und Cosinusfunktion ausgenutzt. Es gilt:

$$
\begin{aligned}
& \left.\begin{array}{l}
\int_{t=0}^{P} \cos \left(2 \pi k \nu_{0} t\right) \cdot \cos \left(2 \pi l \nu_{0} t\right) d t=0 \\
\int_{t=0}^{P} \sin \left(2 \pi k \nu_{0} t\right) \cdot \sin \left(2 \pi l \nu_{0} t\right) d t=0
\end{array}\right\} \text { für } k \neq l \\
& \int_{\substack{t=0 \\
P}}^{P} \sin \left(2 \pi k \nu_{0} t\right) \cdot \cos \left(2 \pi l \nu_{0} t\right) d t=0 \quad \text { für alle } k, l \\
& \int_{t=0}^{P} \cos ^{2}\left(2 \pi k \nu_{0} t\right) d t=\int_{t=0}^{P} \sin ^{2}\left(2 \pi k \nu_{0} t\right) d t=\frac{P}{2}
\end{aligned}
$$

Multiplikation von $F(t)$ mit $\sin \left(2 \pi m \nu_{0} t\right)$ und Integration über eine Periodenlänge $P=\frac{1}{\nu_{0}}$ ergibt:

$$
\begin{aligned}
& \int_{t=0}^{P} F(t) \sin \left(2 \pi m \nu_{0} t\right) d t \\
= & \int_{t=0}^{P}\left(\frac{A_{0}}{2}+\sum_{n=1}^{\infty} A_{n} \cos \left(2 \pi n \nu_{0} t\right)+B_{n} \sin \left(2 \pi n \nu_{0} t\right)\right) \sin \left(2 \pi m \nu_{0} t\right) d t \\
= & \int_{t=0}^{P} \frac{A_{0}}{2} \sin \left(2 \pi m \nu_{0} t\right) d t+\int_{t=0}^{P} \sum_{n=1}^{\infty}\left(A_{n} \cos \left(2 \pi n \nu_{0} t\right)+B_{n} \sin \left(2 \pi n \nu_{0} t\right)\right) \sin \left(2 \pi m \nu_{0} t\right) d t \\
= & \sum_{n=1}^{\infty} \int_{t=0}^{P} A_{n} \cos \left(2 \pi n \nu_{0} t\right) \sin \left(2 \pi m \nu_{0} t\right)+B_{n} \sin \left(2 \pi n \nu_{0} t\right) \sin \left(2 \pi m \nu_{0} t\right) d t \\
= & \int_{t=0}^{P} B_{m} \sin ^{2}\left(2 \pi m \nu_{0} t\right) d t=B_{m} \int_{t=0}^{P} \sin ^{2}\left(2 \pi m \nu_{0} t\right) d t=\frac{B_{m}}{2 \nu_{0}}
\end{aligned}
$$


Damit folgt also für die Konstanten $B_{m}$ und $A_{m}$, wobei man letztere durch analoge Rechnung erhält:

$$
\begin{aligned}
& B_{m}=\frac{2}{P} \int_{t=0}^{P} F(t) \sin \left(2 \pi m \nu_{0} t\right) d t \\
& A_{m}=\frac{2}{P} \int_{t=0}^{P} F(t) \cos \left(2 \pi m \nu_{0} t\right) d t
\end{aligned}
$$

Bei gegebener Funktion $F(t)$ und der Grundfrequenz $\nu_{0}$ können die Koeffizienten der Fourierreihe durch die Integrale (2.8) und (2.9) bestimmt werden.

Da sich mit komplexen Exponentialfunktionen einfacher rechnen lässt als mit trigonometrischen Polynomen, überführt man die Funktion wie folgt, unter Verwendung der Gleichungen

$$
\begin{aligned}
\cos \left(2 \pi m \nu_{0} t\right) & =\frac{1}{2}\left(e^{2 \pi i m \nu_{0} t}+e^{-2 \pi i m \nu_{0} t}\right), \\
\sin \left(2 \pi m \nu_{0} t\right) & =\frac{1}{2 i}\left(e^{2 \pi i m \nu_{0} t}-e^{-2 \pi i m \nu_{0} t}\right)
\end{aligned}
$$

in eine Exponentialfunktion:

$$
\begin{aligned}
F(t) & =\frac{A_{0}}{2}+\sum_{n=1}^{\infty} A_{n} \cos \left(2 \pi n \nu_{0} t\right)+B_{n} \sin \left(2 \pi n \nu_{0} t\right) \\
& =\frac{A_{0}}{2}+\sum_{n=1}^{\infty} \frac{A_{n}}{2}\left(e^{2 \pi i n \nu_{0} t}+e^{-2 \pi i n \nu_{0} t}\right)+\frac{B_{n}}{2 i}\left(e^{2 \pi i n \nu_{0} t}-e^{-2 \pi i n \nu_{0} t}\right) \\
& =\frac{A_{0}}{2}+\sum_{n=1}^{\infty} \frac{1}{2}\left(\left(A_{n}+\frac{B_{n}}{i}\right) e^{2 \pi i n \nu_{0} t}+\left(A_{n}-\frac{B_{n}}{i}\right) e^{-2 \pi i n \nu_{0} t}\right) \\
& =\frac{A_{0}}{2}+\frac{1}{2} \sum_{n=1}^{\infty} \frac{1}{i^{2}}\left(i^{2} A_{n}+i B_{n}\right) e^{2 \pi i n \nu_{0} t}+\frac{1}{i^{2}}\left(i^{2} A_{n}-i B_{n}\right) e^{-2 \pi i n \nu_{0} t} \\
& =\frac{A_{0}}{2}+\frac{1}{2} \sum_{n=1}^{\infty}\left(A_{n}-i B_{n}\right) e^{2 \pi i n \nu_{0} t}+\left(A_{n}+i B_{n}\right) e^{-2 \pi i n \nu_{0} t} \\
& =\sum_{n=-\infty}^{\infty} C_{n} e^{2 \pi i n \nu_{0} t}
\end{aligned}
$$

mit

$$
C_{n}=\left\{\begin{array}{ccc}
\frac{A_{n}-i B_{n}}{2} & : & n \geq 1 \\
\frac{A_{n}+i B_{n}}{2} & : & n \leq-1 \\
\frac{A_{0}}{2} & : & n=0 .
\end{array}\right.
$$


Im Falle der Infrarotspektroskopie soll die Absorption oder Transmission der in der Reaktionszelle befindlichen Stoffe in Abhängigkeit von der Frequenz angeben werden. Dazu wird eine Transformation der periodischen Funktion in eine Funktion auf dem interessierenden Frequenzbereich benötigt. Diese Überführung leistet die Fouriertransformation. Dabei kann jede, insbesondere auch jede nicht periodische Funktion transformiert werden, indem für die Grundperiodenlänge $T_{0} \rightarrow \infty$ und damit für die Grundfrequenz $\nu_{0} \rightarrow 0$ gilt. Die Summe lässt sich an dieser Stelle durch ein Integral ersetzen, da es sich bei der Funktion um den Grenzwert einer Riemannschen Summe der Exponentialfunktionen aus Gleichung (2.10) handelt (siehe zum Beispiel [For99, Seite 182]):

$$
F(t)=\int_{-\infty}^{\infty}(a(\nu) \cos (2 \pi \nu t)+b(\nu) \sin (2 \pi \nu t)) d \nu
$$

Mit Gleichung (2.11) kann die Funktion $F(t)$ nun wie folgt ausgedrückt werden:

$$
F(t)=\int_{-\infty}^{\infty} \Phi(\nu) e^{2 \pi i \nu t} d \nu
$$

Dabei ist $\Phi(\nu)$ äquivalent zu $C_{n}$ und kann damit ebenfalls komplex sein. $\Phi(\nu)$ enthält Informationen über die Amplitude und die Phasenverschiebung für die Frequenz $\nu$ von der vorherigen Grundfrequenz $\nu_{0}$.

Gleichung (2.11) und (2.12) zeigen, wie sich bei bekannten Amplituden bezüglich der Frequenz die Schwingungsdarstellung aus dem Frequenzspektrum berechnen lässt.

Handelt es sich bei $F(t)$ um eine zu der Amplitudenachse symmetrische Funktion, die Funktion ist also gerade und sinusanteilfrei, so folgt aus Gleichung (2.11):

$$
F(t)=\int_{-\infty}^{\infty} a(\nu) \cos (2 \pi \nu t) d \nu
$$

Das besondere an der Fouriertheorie ist, dass die Fourieranalyse dieselbe Form wie die Synthese besitzt:

$$
\Phi(\nu)=\int_{-\infty}^{\infty} F(t) e^{-2 \pi i \nu t} d t
$$

$F(t)$ und $\Phi(\nu)$ können durch dieselbe Transformation ineinander überführt werden und bilden ein Fourierpaar. 
Der Prozess der Fouriertransformation wird durch die im Folgenden näher beschriebene Apparatur ermöglicht.

\section{Michelson-Interferometer:}

Um eine bestimmte Schwingung in einer gegebenen Anzahl gleicher Moleküle anzuregen, benötigt man Licht mit einer bestimmten Intensität und Wellenlänge.

Bei der FT-IR-Spektroskopie wird nun durch Strahlteilung und Variation des Laufweges eines Teilstrahls die Intensität bezüglich der Wellenlängen nach Wiederzusammenführung gesteuert. Durch die Interferenz der Teilstrahlen entsteht ein Interferenzmuster, das gerade die Fouriertransformierte des Wellenlängenspektrums des einfallenden Strahls ist. Der Prozess der Strahlteilung, Variation des Laufweges und Wiedervereinigung erfolgt im Herzstück des FT-IR-Spektrometers, dem Michelson-Interferometer. Die folgenden Darstellungen orientieren sich wieder an dem Buch von JAMES [Jam95, Kapitel 5]. Der einfal-

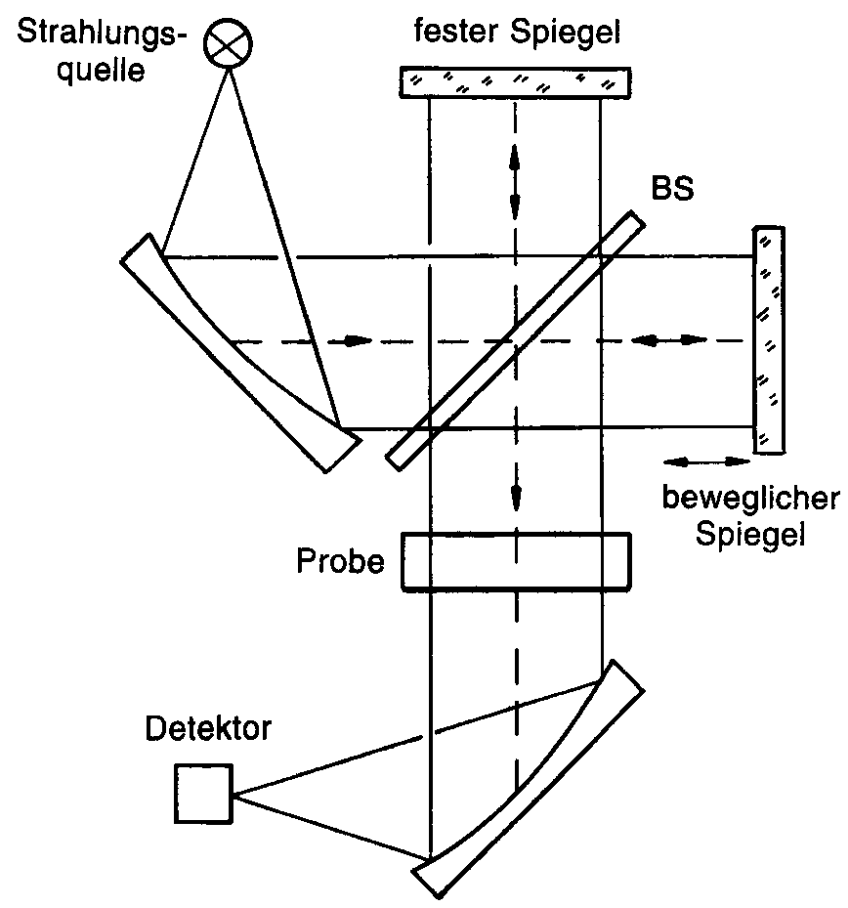

Abbildung 2.1: Schematischer Aufbau des Michelson-Interferometers aus [Gue96, Seite 74]

lende Strahl wird dabei an einem halbdurchlässigen Spiegel auf zwei senkrecht zueinander stehende Wege gelenkt. Im Idealfall sollte der Strahlteiler Licht mit der Amplitude $\frac{A(\nu)}{2}$ auf jeden Arm lenken, sodass der Strahl in beide Richtungen die Hälfte der ursprünglichen 
Intensität besitzt. Die Lichtstrahlen werden am Ende eines jeden Weges an einem Spiegel reflektiert, am Strahlteiler wiedervereinigt und verlassen das Interferometer senkrecht zum einfallenden Strahl in Richtung Probe. Einer der beiden Spiegel ist auf einem Luftbett beweglich gelagert und kann so durch Auslenkung Laufwegunterschiede im Bereich von 0 bis $x_{\max }$ ermöglichen (Abbildung 2.1).

Betrachtet man den einfacheren Fall einer monochromatischen Strahlungsquelle, so kann die Amplitude der stehenden Welle, welche die Strahlung beschreibt, in komplexer Darstellung durch

$$
A \cdot e^{2 \pi i \tilde{\nu} d}
$$

beschrieben werden, wobei

$$
\begin{aligned}
& A \text { die Amplitude und } \\
& d \quad \text { die Phase bezeichnet. }
\end{aligned}
$$

Unter der Annahme, dass jeder Teilstrahl die Hälfte der Ausgangsintensität besitzt, kann die Amplitude nach Wiedervereinigung durch die Linearkombination der beiden Teilstrahlen wie folgt ausgedrückt werden:

$$
\begin{aligned}
& \frac{A}{2} \cdot e^{2 \pi i \tilde{\nu} d_{1}}+\frac{A}{2} \cdot e^{2 \pi i \tilde{\nu} d_{2}} \\
& =\frac{A}{2}\left(e^{2 \pi i \tilde{\nu} d_{1}}+e^{2 \pi i \tilde{\nu} d_{2}}\right) .
\end{aligned}
$$

Dabei bezeichnen $d_{1}$ und $d_{2}$ den Abstand zwischen dem Strahlteiler und dem betreffenden Spiegel. Die Intensität des wiedervereinigten Strahls ist dann gegeben durch:

$$
\begin{aligned}
I_{T} & =\left|\left(\frac{A}{2}\right)\left(e^{2 \pi i \tilde{\nu} d_{1}}+e^{2 \pi i \tilde{\nu} d_{2}}\right)\right|^{2} \\
& =\frac{A A^{*}}{4}\left|e^{2 \pi i \tilde{\nu} d_{1}}+e^{2 \pi i \tilde{\nu} d_{2}}\right|^{2} \\
& =\frac{A A^{*}}{4}\left|\left(e^{2 \pi i \tilde{\nu} d_{1}}+e^{2 \pi i \tilde{\nu} d_{2}}\right)\left(e^{-2 \pi i \tilde{\nu} d_{1}}+e^{-2 \pi i \tilde{\nu} d_{2}}\right)\right| \\
& =\frac{A A^{*}}{4}\left|\left(e^{2 \pi i \tilde{\nu}\left(d_{1}-d_{2}\right)}+e^{-2 \pi i \tilde{\nu}\left(d_{1}-d_{2}\right)}+2\right)\right| \\
& =\frac{A A^{*}}{2}\left|\left(\cos \left(2 \pi \tilde{\nu}\left(d_{1}-d_{2}\right)\right)+1\right)\right| .
\end{aligned}
$$

Die Intensität bezüglich eines monochromatischen Lichtstrahls lässt sich durch

$$
I_{T}=\frac{I_{0}}{2}(1+\cos (2 \pi \tilde{\nu} \Delta))
$$


beschreiben. Dabei steht

$\Delta$ für die Wegdifferenz $2\left(d_{1}-d_{2}\right)$ und

$I_{0}$ für die Intensität des eingestrahlten Lichts $A A^{*}$.

Mit Hilfe der die Strahlteilung beschreibenden Funktion kann man die Intensität $I_{T}$ zwischen $I_{0}$ (für $2 \pi \tilde{\nu} \Delta=k 2 \pi, k \in \mathbf{Z}$ ) und 0 (für $2 \pi \tilde{\nu} \Delta=(2 l+1) \pi, l \in \mathbf{Z}$ ) variieren.

Bei Licht mit verschiedenen Wellenlängen oder bei einem kontinuierlichem Spektrum erfolgt durch das Michelson-Interferometer eine „Aufschlüsselung“ dieser Strahlung in Cosinusfunktionen mit bekannten Amplituden, die sich als Fourierreihe schreiben lassen. Die Fourierreihe ist anstelle der Zeit eine Funktion von der Auslenkung des Spiegels. Wird die oben dargestellte Fouriertransformation auf die Reihe angewendet, so erhält man eine Fourieranalyse der Form

$$
S(\tilde{\nu})=\int_{-\infty}^{\infty} I_{T}(\Delta) \cos (2 \pi \tilde{\nu} \Delta) d \Delta .
$$

Diese ist im reellen Fall äquivalent zu Gleichung (2.14). Die Formel (2.17) erlaubt eine Berechnung der Intensität bezüglich jeder Wellenzahl des eingestrahlten Lichts.

Da nur symmetrische Funktionen zu den Amplitudenachsen vorliegen, wäre ein symmetrisches Wellenzahlspektrum zu erwarten. In der Praxis treten auf Grund optischer und elektronischer Effekte Phasenverschiebungen auf, sodass das erhaltene Interferogram unsymmetrisch und somit eine komplexe Fouriertransformation notwendig ist. 
KAPITEL 2. THEORETISCHE GRUNDLAGEN

2.2. MATHEMATISCHE GRUNDLAGEN DER (FAST)-FOURIER-TRANSFORMATION 


\section{Kapitel 3}

\section{Beschreibung der experimentellen Bedingungen}

Für die Produktuntersuchung der Gasphasenreaktionen von Kohlenwasserstoffradikalen mit Sauerstoffatomen und für die Kreuzreaktion verschiedener Kohlenwasserstoffradikale hat sich die verwendete FTIR-Apparatur als äußerst effizient und verlässlich erwiesen. Der apparative Aufbau ist in verschiedenen Veröffentlichungen (siehe z.B. [Hac05], Hoy06a]) und Dissertationen innerhalb der Arbeitsgruppe (z. B. [Ker99], [Trö97], [Zeu03]) dargestellt. Der Photolyselaser wurde aber, anders als in früheren Arbeiten, direkt in die Zelle eingekoppelt, sodass im Folgenden eine ausführliche Beschreibung erfolgt.

\subsection{FTIR Apparatur}

Im Folgenden wird die verwendete FTIR-Apparatur ausführlich dargestellt. Die FTIRApparatur besteht im Wesentlichen aus drei Hauptkomponenten, der Reaktionszelle mit optischer Bank, dem Excimerlaser und dem FTIR-Spektrometer mit Detektor.

\subsubsection{Schematischer Aufbau der Versuchsanordnung}

Für die qualitative und quantitative Analyse stabiler Produkte von Gasphasenreaktionen und für die Erstellung dazu benötigter Reinspektren wurde ein kommerziell erhältliches Fourier-Transform-Spektrometer (Bruker IFS 66) verwendet. Auf Grund des schnellen MCT-Detektors erlaubte die Apparatur zeitaufgelöste Messungen bis zu einer Zeitauf- 
lösung von $5 \mu s$. Die gute Zeitauflösung des Detektors wurde bei der Produktanalyse nicht benötigt, diese fand Verwendung im sogenannten „Step-Scan“ Modus. Zur Optimierung des Signal-Rausch-Verhältnisses befand sich das Spektrometer auf einem aktiv schwingungsgedämpften Tisch (Fa. Halcyonics), welcher aus einer Wabenplatte und zwei Isolationsmodulen aufgebaut war. Der im Spektrometer generierte IR-Strahl wurde über zwei planparallele Spiegel in die Multireflektionszelle eigekoppelt. Innerhalb der Reaktionszelle wurde der IR-Strahl mit Hilfe einer White`schen Spiegelanodnung 32-mal reflektiert, bevor er aus der Zelle ausgekoppelt und mittels eines weiteren planparallelen und Ellipsoidspiegels auf den Detektor fokussiert wurde.

Bei der Reaktionszelle handelte es sich um einen Eigenbau mit 6 CF 100 Flanschöffnungen aus VA-Stahl (VAB Elmshorn VA 1.4311), die ein Zellvolumen von ca. 6 L hatte. Der IR-Strahl wurde senkrecht zum Photolyselaser horizontal eingekoppelt. Über den oben liegenden Flansch wurden der Reaktionsvorläufer $\mathrm{SO}_{2}$ sowie das Badgas Argon der Reaktionszelle zugeführt. Die Zelle war über den unteren Flansch mit einer Vakuumpumpe (Brand, RD 15), der eine Kühlfalle vorgeschaltet war, verbunden. Mit der verwendeten Vakuumpumpe ließ sich ein Enddruck von $1 \cdot 10^{-6}$ bar erreichen. An einer Verlängerung des Flanschs waren weiterhin zwei Druckmessköpfe (MKS, Baratron 0-10 mbar und 11000 mbar) angeschlossen, die eine durchgehende Bestimmung des Gesamtdrucks im Bereich von $1 \cdot 10^{-6}$ bis 1 bar ermöglichten. Über den horizontalen Flansch, der dem Flansch zur Ein- und Auskoppelung des IR-Strahls gegenüber lag, wurden der Kohlenwasserstoff und der in einigen Versuchen zur Radikalbildung nötige Fluor-Chlor-Kohlenwasserstoff $\left(\mathrm{CFCl}_{3}\right)$, bzw. die verschiedenen Kohlenwasserstoffe der Reaktionszelle zugeführt. Die zur Justage der Innenspiegel notwendigen Stellschrauben befanden sich ebenfalls an dem Flansch. Abbildung 3.1 zeigt eine schematische Darstellung der verwendeten Apparatur. 


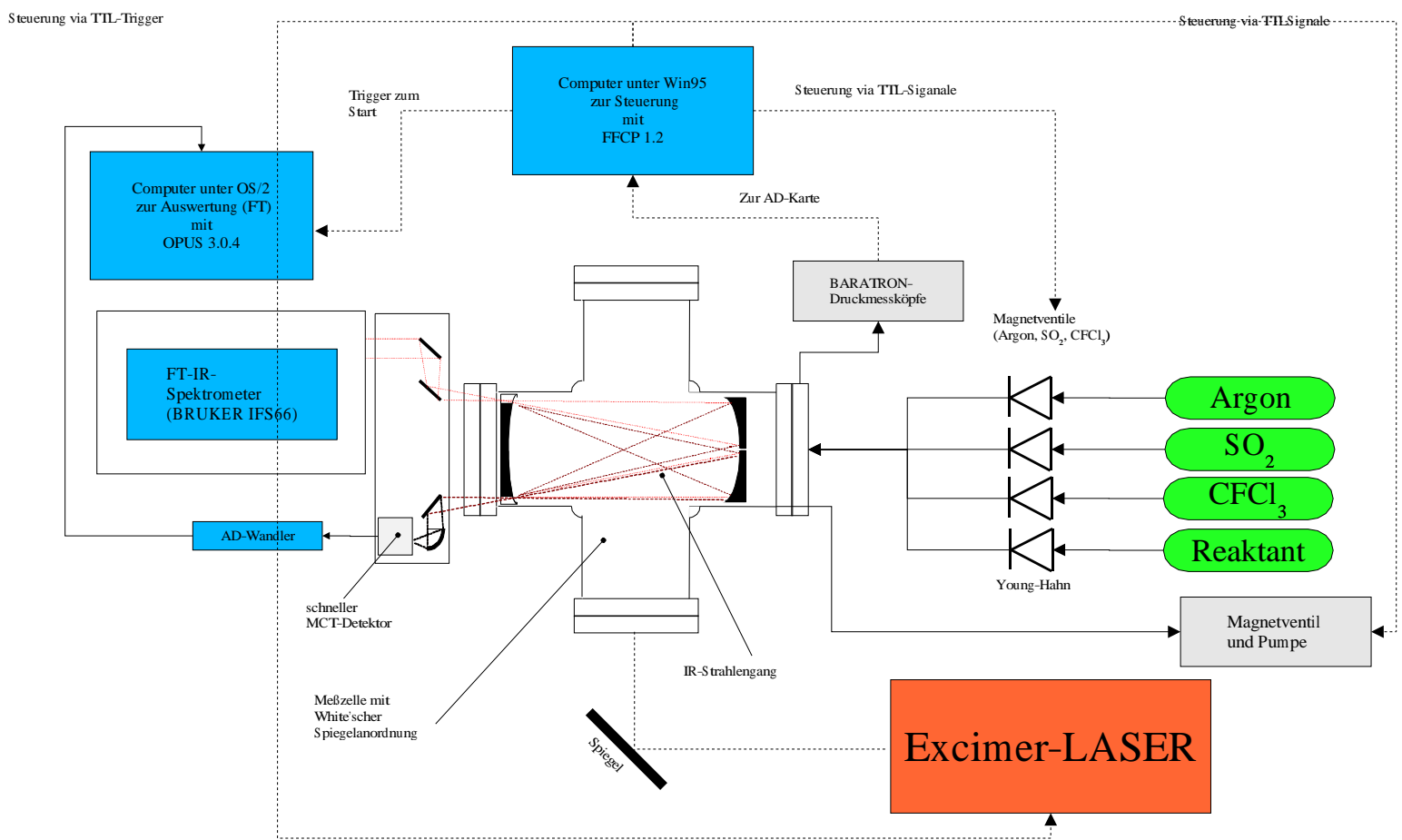

Abbildung 3.1: Schematischer Aufbau der FTIR-Apparatur

\subsubsection{Laser-Photolyse}

Zur Erzeugung der Reaktionspartner aus den Reaktionsvorläufern wurde ein Excimerlaser (Lambda Physik, Compex 102) verwendet, wobei der Betrieb mit einer Argon-FluorFüllung erfolgte. Die emittierte Wellenlänge war daher 193 nm. Die maximale Laserenergie betrug vor dem Umbau der Apparatur vor Einkoppellung in die Zelle ca. 60 mJ. Nach dem Umbau strahlte der Laser direkt in die Reaktionszelle, wodurch eine Laserenergie vor Eintritt in die Zelle von 140 mJ erzielt wurde. Für die Bestimmung der Produktausbeute und für Simulationsrechnungen war es notwendig, sowohl die Sauerstoffatomkonzentration als auch die absolute Radikalkonzentration bzw. die Konzentration der zur Radikalbildung benötigten Cl-Atome zu bestimmen. Dazu wurden von beiden Substanzen der gerätespezifische Absorptionskoeffizient ermittelt.

Der Laser wurde bei allen Experimenten mittels einer von M. Hold entwickelten Software (FCCP 1.3) [Hol01] extern getriggert, womit neben der Laserfrequenz (0.1 bis $20 \mathrm{~Hz})$ 
und Photolysenanzahl auch alle Pumpen und Magnetventile gesteuert werden konnten. Bei der Befüllung der Zelle wurde auf die Verwendung des Programms verzichtet und die entsprechenden Partialdrücke manuell unter Benutzung von (temperaturbeständigen) Young-Hähnen eingeleitet. Diese ließen eine genauere Dosierung als das Programm zu, da die verwendeten Magnetventile eine gewisse Ansprechzeit besaßen.

Um die effektive Laserenergie zu erhöhen, wurde im Rahmen dieser Doktorarbeit, anders als in der schematischen Darstellung gezeigt, der Laser so angeordnet, dass der Strahl direkt in die Zelle eigekoppelt werden konnte. Die ursprüngliche Anordnung unter Verwendung von Umlenkspiegeln führte zu einem prozentualen Energieverlußt von ca. 60\%. Aus diesem Grund erschien eine Neuanordnung der Apparatur sinnvoll. Die Neuanordnung erlaubte bei gleicher Photolysenanzahl einen deutlich höheren Umsatz. Der entscheidende Vorteil bestand in der Tatsache, dass in dem Reaktionsvolumen durch eine größere Laserenergie ein höherer Anteil an Reaktanden erzeugt wurde, wodurch das Verhältnis von Radikalen zu Vorläufern im Reaktionsvolumen erheblich gesteigert wurde. Damit konnten Reaktionen von Radikalen mit Vorläufern noch stärker unterdrückt werden.

\subsubsection{Nachweis und Signalverarbeitung}

Zur Aufnahme der IR-Spektren wurde das beschriebene FTIR-Spektrometer (Bruker, IFS 66) verwendet. Die Signalumwandlung mittels Fourier-Transformation wurde durch die mitgelieferte Software (Bruker, Opus 3.04 auf Pentium III (OS2)) nach Aufnahme und Mittelung über die gewünschte Anzahl von Einzelspektren durchgeführt. Für die Analyse wurden im Allgemeinen Spektren im Wellenzahlbereich von $4000-600 \mathrm{~cm}^{-1}$ aufgenommen. Das Spektrometer erlaubte eine maximale Auflösung von $0.12 \mathrm{~cm}^{-1}$, wobei im standardmäßigen Betrieb eine Aufösung von $1 \mathrm{~cm}^{-1}$ verwendet wurde. Aufnahme, Mittelung und Fourier-Transformation von 100 Einzelspektren konnte durch Software und Rechenleistung in weniger als einer Minute erreicht werden.

Zur eigentlichen Signalaufnahme diente ein mit flüssigem Stickstoff gekühlter MCT-Detektor $(\mathrm{Hg} / \mathrm{Cd} / \mathrm{Te})$, der eine maximale Zeitauflösung von $5 \mu s$ besaß. Die gute Zeitauflösung war Grundvoraussetzung für den Betrieb des Spektrometers im sogenannten „Step-Scan“Modus, bei dem, auf Grund der festen Spiegelposition des Michelson-Interferrometers, 
einzig die Zeitkonstante des Detektors der begrenzende Paramter ist.

\subsubsection{Computersteuerung}

Wie schon in Abschnitt 3.1.3 beschrieben, konnte das FTIR-Spektrometer mittels der mitgelieferten Software „OPUS“ bedient werden. Das Programm erlaubte, neben gerätetechnischen Einstellungen wie Blendendurchmesser, Spiegelgeschwindigkeit, Spektralbereich und Ähnlichem, die direkte Auswertung und Bearbeitung der aufgenommenen Interferogramme. Eine detaillierte Darstellung zur Auswertung von Spektren erfolgt im Abschnitt über die Durchführung und Auswertung von Messungen (3.3).

Die Magnetventile, gekoppelt mit der Vakuumpumpe, ließen sich mit dem bereits erwähnten Programm „FCCP 1.3“ von M. HolD steuern [Hol01]. Mit Hilfe des Programms konnte die Zelle durch simultane Schaltung des Auslassventils und der Vakuumpumpe evakuiert werden. Das Programm ermöglichte zudem die Befüllung der Zelle mit Reaktanden unter Berücksichtigung der Einströmungsgeschwindigkeit, d.h. bei Erreichen eines frei wählbaren Drucks (gewöhnlich 80\% des gewünschten Drucks), erfolgte der Befehl zum Schließen des Magnetventils, sodass, zusammen mit der Ansprechzeit des Ventils, der gewünschte Druck erreicht wurde. Wie schon erwähnt wurde der Excimerlaser ebenfalls mit Hilfe des Programms getriggert.

\subsection{Radikalvorläufer}

Im Folgenden werden die zum Einsatz gekommenen Radikalvorläufer vorgestellt und deren Vor- und Nachteile diskutiert. Neben der Reaktion von zyklischen und offenkettigen Kohlenwasserstoffradikalen mit Sauerstoffatomen wurden Kreuzreaktionen von Kohlenwasserstoffradikalen untersucht. Aus diesem Grund wird auf die Radikalvorläufer in separaten Abschnitten eingegangen.

\subsubsection{Die Reaktion Alkylradikal + Sauerstoffatom}

Für die Untersuchung der Reaktion von zyklischen und offenkettigen Kohlenwasserstoffradikalen mit Sauerstoffatomen wurde eine Vorläufer-Mischung des entsprechenden Kohlenwasserstoffs mit $\mathrm{SO}_{2}$ und $\mathrm{CFCl}_{3}$ benutzt. Die verwendete UV-Strahlung mit der Wel- 
lenlänge von $193 \mathrm{~nm}$ führte zum Einen zu einer photolytischen Spaltung von $\mathrm{SO}_{2}$ zu $\mathrm{SO}$ und $\mathrm{O}\left({ }^{3} \mathrm{P}\right)$ und zum Anderen zur Bildung von Chlor-Atomen. Die Chlor-Atome reagierten dann mit dem Kohlenwasserstoff in einer schnellen Abstraktion zu dem gewünschten Kohlenwasserstoffradikal und $\mathrm{HCl}$ :

$$
\begin{aligned}
\mathrm{SO}_{2} & \stackrel{h \nu}{\longrightarrow} \mathrm{SO}+\mathrm{O} \\
\mathrm{CFCl}_{3} & \stackrel{h \nu}{\longrightarrow} \mathrm{CFCl}_{2}+\mathrm{Cl} \\
\mathrm{R}-\mathrm{H}+\mathrm{Cl} & \longrightarrow \mathrm{R}+\mathrm{HCl}
\end{aligned}
$$

Für die Untersuchung der Reaktion von offenkettigen Kohlenwasserstoffen mit Sauerstoffatomen wurden, neben der erwähnten Möglichkeit Radikale über eine HCl-Abstraktion zu erzeugen, teiliodierte beziehungsweise teilbromierte Kohlenwasserstoffe und Dialkylketone verwendet. Der Reaktionsmechanismus dieser Radikalvorläufer wird im folgenden Abschnitt dargestellt.

\subsubsection{Die Reaktion Alkylradikal + Alkylradikal}

Die Kreuzreaktion verschiedener Kohlenwasserstoffradikale wurde anhand unterschiedlicher Radikalvorläufer untersucht. Es wurden sowohl iodierte beziehungsweise bromierte Kohlenwasserstoffe als auch symmetrische und asymmetrische Dialkylketone eingesetzt. Bei der Photolyse der jeweiligen Vorläufer liefen folgende (idealisierte) Reaktionen ab:

$$
\begin{aligned}
\mathrm{RI} & \stackrel{h \nu}{\longrightarrow} \mathrm{R}+\mathrm{I} \\
\mathrm{R}_{1} \mathrm{COR}_{1} & \stackrel{h \nu}{\longrightarrow} 2 \mathrm{R}_{1}+\mathrm{CO} \\
\mathrm{R}_{1} \mathrm{COR}_{2} & \stackrel{h \nu}{\longrightarrow} \mathrm{R}_{1}+\mathrm{R}_{2}+\mathrm{CO}
\end{aligned}
$$

Die Verwendung von Dialkylketonen hatte den entscheidenden Vorteil, dass es bei der verwendeten Wellenlänge vorrangig zur Spaltung der C-C-Bindungen am Carbonyl-C-Atom kam (Norrish Typ I Spaltung), wobei neben zwei Alkylradikalen das chemisch inerte Kohlenmonoxid entstand. Für kleine Alkylradikale stellte die Photolyse der entsprechenden Ketone somit eine saubere Quelle dar. Bei größeren Dialkylketonen erfolgte neben der Norrish Typ I Spaltung ein $\beta$-C-C-Bindungsbruch mit anschließendem $H$-shift vom $\gamma$-C-Atom unter Bildung eines Methyl-Alkylketons und einem Alken (Norrish Typ III Spaltung) [Cal66]. 
Für die Untersuchung von Alkylradikalreaktionen erwies sich dabei der Einsatz einer Reaktionsmischung aus einem undeuteriertem und einem vollständig deuteriertem Dialkylketon als besonders geeignet. Mit diesen war eine einfache Unterscheidnung der Kreuz-Reaktionsprodukte von den Selbstreaktions-Produkten des deuterierten Alkylradikals möglich, da im IR-Spektrum die auftretenden C-D-Schwingungen eindeutig von den übrigen C-H-Schwingungen unterscheidbar waren.

Die Verwendung einer Reaktionsmischung aus Kohlenwasserstoffen und $\mathrm{CFCl}_{3}$ hatte den Nachteil der unspezifischen Bildung von Radikalen, da zum Beispiel die Photolyse einer Mischung aus Propan und $\mathrm{CFCl}_{3}$ neben 1-Propylradikalen auch die Bildung von 2Propylradikalen zur Folge hatte. Ein Vorteil bestand darin, dass es sich um eine relativ saubere $\mathrm{Cl}$-Atom Quelle handelte, da $\mathrm{CFCl}_{2}$ nicht in die betrachtete Reaktion eingriff, beziehungsweise Produkte aus dieser Reaktion leicht von den Produkten der untersuchten Reaktionen unterscheidbar waren. Auf Grund des erwähnten Nachteils wurde zur Untersuchung der Kreuzreaktionen auf den Einsatz dieser Alkylradikalquelle verzichtet, da bei der Kreuzreaktion eine Vielzahl von Reaktionen ablaufen, deren Anzahl durch die Bildung verschiedener Alkylradikale noch weiter erhöht worden wäre.

Der Einsatz iodierter Kohlenwasserstoffe hatte dagegen den Nachteil, dass neben der erwünschten Iod-Abspaltung eine schnelle HI-Elimination zur Bildung eines Alkens führte. Bei Iod-Atomen handelt es sich im Vergleich zu den übrigen Radikalen um chemisch wenig reaktive Reaktionspartner, aber die Reaktion von Alkyliodiden mit Sauerstoffatomen ist relativ schnell, und Produkte für diese Reaktionen sind bisher wenig erforscht. Der Anteil der HI-Elimination musste bei Verwendung von iodierten Radikalvorläufern separat bestimmt werden, um bei der Betrachtung von Kreuzreaktionen die tatsächliche Radikalkonzentration angeben zu können. Für die Untersuchung von Kreuzreaktionen stellen sie aber neben der Verwendung von Dialkylketonen eine geeignete Alternative dar.

\subsubsection{Die Reaktion Allylradikal + Allylradikal}

Für die Untersuchung der Reaktion von Allylradikalen mit Sauerstoffatomen stand neben der Photolyse der Vorläufermischungen $\mathrm{C}_{3} \mathrm{H}_{5} \mathrm{X} / \mathrm{SO}_{2}(\mathrm{X}=\mathrm{Br}, \mathrm{Cl})$ noch ein weiterer Radikalvorläufer zur Verfügung. Die Photolysen von Diallyl (1,5-Hexadien) führten bei der verwendeten Wellenlänge vorrangig zur Bildung des Allylradikals, sodass Diallyl eine 
geeignete Quelle für die Untersuchung der Reaktion darstellt. Problematisch ist bei der Verwendung dieses Vorläufers die sehr schnelle Reaktion von Diallyl mit Sauerstoffatomen. So konnte bei Verwendung von Diallyl als Allylradikalquelle eine direkte Reaktion mit Sauerstoffatomen unter Bildung von 5-Hexenal beobachtet werden, die ausschließlich aus der direkten Reaktion von Diallyl mit Sauerstoffatomen stammen kann.

\subsubsection{Durchführung und Auswertung von Messungen}

Im Folgenden wird das Verfahren bei der Untersuchung einer Reaktion dargestellt.

$\mathrm{Zu}$ Beginn einer Messung wurden die Radikalvorläufer sowie das Badgas durch Einlasshähne sukzessive bis zum gewünschten Druck in die Zelle eingeleitet. Dabei erwies es sich als vorteilhaft, vor den Radikalvorläufern die Zelle so mit Argon zu befüllen, dass nach Einleiten der Radikalvorläufer der Gesamtdruck erreicht wurde. Bei Wahl der umgekehrten Reihenfolge zeigte sich das Phänomen der Kondensation der Edukte auf den innenliegenden Spiegeln und der damit verbundenen Intensitätszunahme in Abhängigkeit von der Zeit noch stärker ausgeprägt. Auf dieses Phänomen und auf die Tatsache, dass nach gründlicher Säuberung des Zelleninnenraums durch mehrtägige Evakuierung statt einer Intensitätszunahme nun eine Intensitätsabnahme in Abhängikeit von der Zeit zu beobachten war, wird bei der Beschreibung der quantitativen Analyse detailliert eingegangen (Abschnitt 3.3.2) und wird daher hier nur am Rande bemerkt. Um Sekundärreaktionen zu unterdrücken und eine hohe Ausbeute für die gewünschte Reaktion zu erzielen, wurden bei der Befüllung der Zelle verschiedene Mischungen getestet und deren Anteile so lange variiert, bis eine optimale Vorläuferzusammensetzung gefunden wurde.

Nach der Befüllung der Zelle mit allen Komponenten wurde ein Referenzspektrum aufgenommen. Dabei wartete man so lange, bis sich das Signal stabilisiert hatte, d.h. eine Zunahme (beziehungsweise Abnahme) nicht mehr stattfand. Dann wurde mit der CoPhotolyse der Reaktionsmischung begonnen. Nach jeweils 100 Photolysen wurde (nach Neuanordnung der Lasers 50) ein aus 100 Einzelspektren gemitteltes FTIR-Spektrum aufgenommen. Die maximale Photolysenanzahl betrug 600 beziehungsweise 300 bei direkter Einkoppelung des Excimerlasers, was einem Vorläuferumsatz von ca. 15\% entsprach. Die erhaltenen Spektren wurden dann wie im folgenden Abschnitt beschrieben ausgewertet. Nach Erreichen der maximalen Photolysenanzahl wurde die Zelle vollständig evakuiert. 
Dabei wurde im Falle einer beabsichtigten Weiteruntersuchung mittels GCMS das Reaktionsgemisch in einer der Vakuumpumpe vorgeschalteten Kühlfalle ausgefroren. Zur Kühlung wurde flüssiger Stickstoff verwand. Es wurde zudem der Versuch unternommen, die Reaktionsmischung gestaffelt auszufrieren. Dazu wurden je eine mit einer Methanol / $\mathrm{CO}_{2(\mathrm{~s})}$-Mischung (Kühlfalle 1) und eine mit flüssigem Stickstoff (Kühlfalle 2) beladene Kühlfalle hintereinander geschaltet. Diese Anordnung lieferte aber in Kühlfalle 1 für eine weitere Anaylse nicht ausreichend viel Produkt. Daher wurde in weiteren Versuchen nur mit einer Kühlfalle gearbeitet.

Die GCMS-Analysen von ausgefrorenen Reaktionsmischungen lieferten auf Grund hoher Vorläuferanteile keine brauchbaren Ergebnisse. Daher wird in dieser Arbeit auf die weitere Darstellung der GCMS-Analyse verzichtet.

\subsubsection{Qualitative Analyse}

Bei den vorgestellten Experimenten wurden mittels Co-Photolyse der verschiedenen Radikalvorläufer zum Einen die Reaktionen von Alkylradikalen mit Sauerstoffatomen und zum Anderen die Kreuzreaktion von verschiedenen Alkylradikalen untersucht. Dazu erstellte man von den stabilen Endprodukten nach einer definierten Photolysenanzahl FTIRSpektren. Dass es sich auch bei der relativ hohen Photolysenanzahl um Endprodukte der primären Reaktion handelte, wurde durch folgende experimentelle Bedingungen sichergestellt:

Zum Einen wurden die Anteile der zu photolysierenden Reaktionsmichungen so gewählt (Sauerstoffunterschuss, relativ geringe Cl-Konzentration, hohe Alkankonzentration), dass Sekundärreaktionen gebildeter Produkte mit O- und Cl-Atomen unterdrückt wurden. Zum Anderen gewährleistete eine auf einen Ausschnitt der Reaktionszelle begrenzte Photolysenquerschnittsfläche die Diffusion gebildeter primärer Produkte aus derselbigen, bevor der nächste Laserpuls (Laserfrequenz $2 \mathrm{~Hz}$ ) erfolgte. Die Radikalkonzentration war gegenüber den Vorläuferkonzentrationen in dem Reaktionsvolumen zudem sehr hoch. Des Weiteren hatten die gewählten Vorläufer gegenüber den Produkten hohe Absorptionsquerschnitte, sodass eine Sekundärphotolyse nur für sehr wenige Produkte (wie z.B. Aceton) mit vergleichbaren Absorptionsquerschnitten $\sigma$ relevant war.

Die verwendete FTIR-Apparatur war hierbei zur quantitativen Endproduktanalyse ge- 
eignet. Dies wurde durch vergleichende Simulationsrechnungen bestätigt. Des Weiteren wurde bei quantitativen Analysen der Produkte eine Kohlenwasserstoffmassenbilanz vorgenommen, mit der für alle Experimente mindestens 85\% des Alkylvorläuferumsatzes erklärt werden konnte.

Die qualitative Analyse eines Reaktionsspektrums wird im Folgenden am Beispiel der Photolyse von Aceton- $\mathrm{d}_{6}$ erläutert. Die qualitative und quantitative Auswertung der Photolyse von Aceton- $\mathrm{d}_{6}$ war besonders einfach und ist daher als einführendes Beispiel gut geeignet. Es zeigt in außerordentlicher Weise die Eignung der FTIR-Apparatur für die Produktanalyse und die ausgereifte Methode der quantitativen Analyse von Reaktionsspektren. 

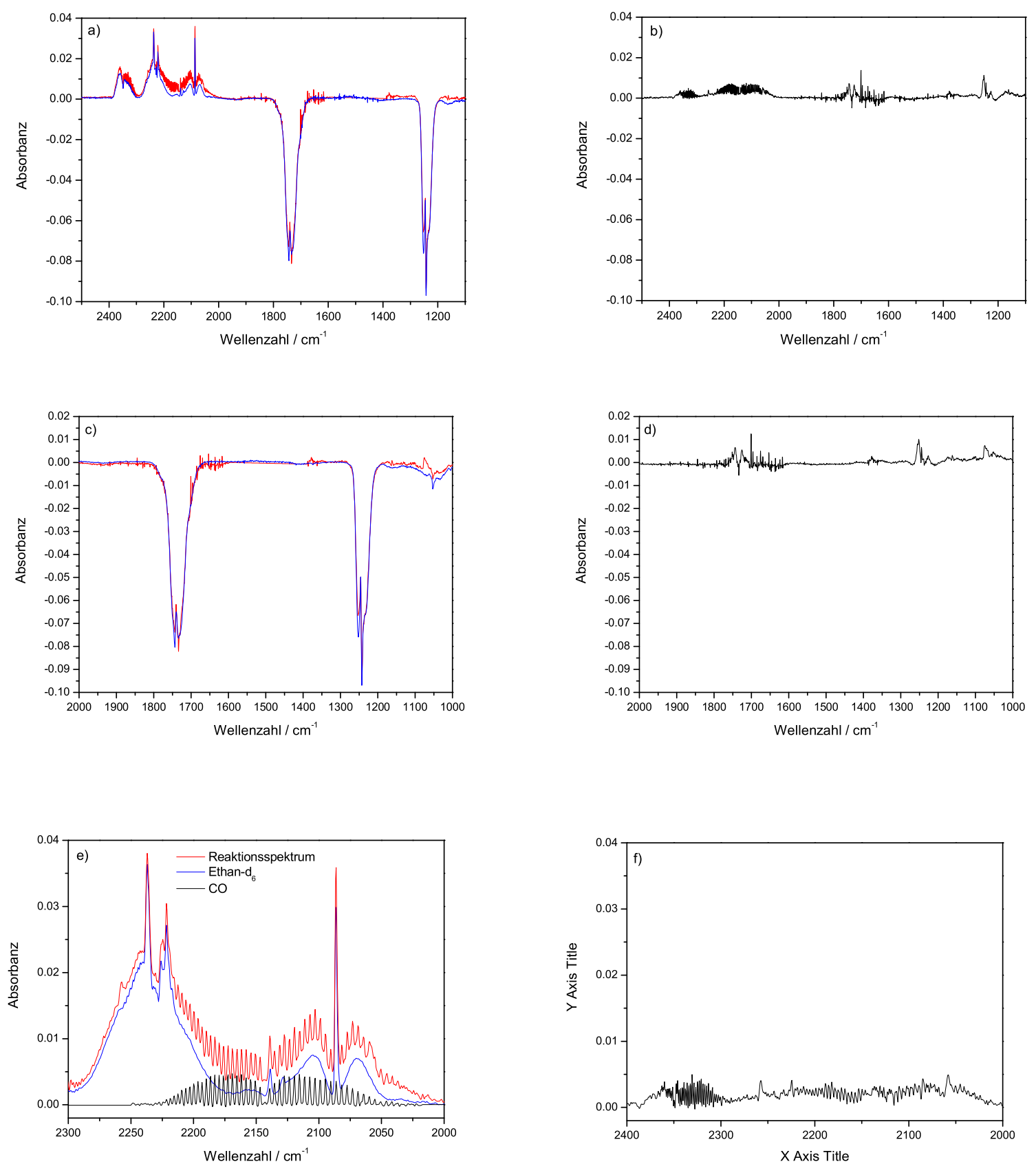

Abbildung 3.2: Auswertung der Photolysen von Aceton- $d_{6}\left(p\left(\right.\right.$ Aceton- $\left._{6}\right)=0,2$ mbar, $p_{\text {ges }}=$ 4 mbar, 400 Photolysen); a) und b) zeigen im Vergleich das Reaktionsspektrum und das additive Spektrum von Ethan- $d_{6}$ und Aceton- $d_{6}$ beziehungsweise das Residuum; c), d), e) und f) zeigen Ausschnitte des Reaktionsspektrums im Vergleich mit den Spektren der Reinsubstanzen (c) mit Aceton-d 6 , e) mit Ethan-d 6 und CO; das rot dargestellte Spektrum ist jeweils das Reaktionsspektrum, das blau dargestellte das Spektrum der Reinsubstanz und das schwarze das des Residuums.

Da auf Grund fehlender $\gamma$-C-Atome bei der Photolyse von Aceton- $\mathrm{d}_{6}$ keine Norrish Spaltung III, also kein $H$-shift, auftreten kann, wurde als einziges Photolyseprodukt das deu- 
terierte Methylradikal gebildet. Die Methylradikale reagierten in einer Kombinationsreaktion ausschließlich zu Ethan- $\mathrm{d}_{6}$. Die Abnahme an Aceton- $\mathrm{d}_{6}$ wird durch den Vergleich mit dem Aceton- $\mathrm{d}_{6}$-Reinspektrum in Abbildung 3.2 c) nachgewiesen, die Zunahme an Ethan$\mathrm{d}_{6}$ durch den Vergleich mit dem entsprechenden Reinspektrum in Abbildung 3.2 e). Das Residuum (Abbildung 3.2 d)) zeigt, dass die Banden exakt übereinstimmen. Die kleinen positiven Banden, die bei der Subtraktion dennoch verbleiben, können durch nichtlineares Verhalten (Kondensationseffekte) erklärt werden. Abbildung 3.2 f) zeigt die nach Subtraktion von Ethan- $\mathrm{d}_{6}$ deutlich auftretende, rotationsaufgelöste CO-Bande. CO konnte neben Ethan- $\mathrm{d}_{6}$ als einziges Produkt der Photolyse von deuteriertem Aceton nachgewiesen werden.

Für die qualitative Analyse wurde, wie in Abbildung 3.2 dargestellt, das Reaktionsspektrum mit den Reinspektren möglicher Produkte verglichen. Dazu wurde zunächst vom Reaktionsspektrum das Reinspektrum des Vorläufers, beziehungsweise die Reinspektren der Vorläufer abgezogen, sodass in den entprecheden Wellenzahlbereichen keine negativen Banden mehr auftraten. Produkte verursachten positive IR-Banden in bestimmten Wellenzalbereichen. Daher war beim Abzug der Vorläuferspektren eine „Nulllinie“ nicht das Ziel.

Für eine eindeutige Identifikation wurden nun für jedes Reaktionsspektrum verschiedene IR-Bandenbereiche und charakteristische Peaks herangezogen. So zeigen Carbonylverbindungen auf Grund der C=O-Streckschwingung im Bereich von $1840-1660 \mathrm{~cm}^{-1}$ eine starke Bande, die in genauer Lage und Form meist eine eindeutige Identifikation der Carbonylsubstanz zulässt. Mit steigender C-Atom-Anzahl ähneln die Carbonylverbindungen aber einander, sodass für eine Identifikation der Bereich der symmetrischen und asymetrischen C-H-Streckschwingung (3000 - $2800 \mathrm{~cm}^{-1}$ ) hinzugenommen wurde. Aldehyde unterscheiden sich nun von Ketonen durch die Ausbildung eines Bandendoppels bei 2900 - $2800 \mathrm{~cm}^{-1}$ und $2780-2680 \mathrm{~cm}^{-1}$ auf Grund der zufälligen Entartung der 1. Oberschwingung $2 \delta(\mathrm{OCH})$ mit der $\mathrm{C}-\mathrm{H}-$ Valenzschwingungsfrequenz $\nu(\mathrm{C}-\mathrm{H})$ der Aldehydgruppe. Bei dem ersten Oberton handelt es sich um eine $\mathrm{O}=\mathrm{C}-\mathrm{H}$ Winkeldeformation. Diese zufällige Entartung nennt man Fermi-Resonanz (siehe z.B. Gue96]). Als Folge der zufälligen Entartung kommt es zur Abstoßung der Energiezustände und damit zu einem Auseinan- 
derrücken der Frequenzen beider Schwingungen. Die schwächere Oberschwingung „borgt“ sich von der Fundamentalschwingung Energie und damit kommt es zur Ausbildung von zwei ähnlich starken Banden. Liegen weiterhin ungesättigte Aldehyde vor, so verschiebt sich die Lage des Bandendoppels durch konjugative Effekte.

Die Identifikation kleiner Alkane und Alkene liess sich ebenfalls anhand von ausgewählten charakteristischen Peaks durchführen. Kleine Moleküle wie Methan, Ethan, Ethen, Ethin, Propen und Formaldehyd besitzen zudem eine rotationsaufgelöste Struktur, aus der die P-, Q- und R-Zweige ersichtlich sind und somit eindeutig identifiziert werden können (siehe dazu z.B. Abbildung $3.2 \mathrm{e})$ ).

Nach eindeutiger Identifikation eines Produkts wurde das Reinspektrum mit einem Faktor so multipliziert, dass es in der Intensität der charakteristischen Bande oder dem charakteristischen Peak mit dem Reaktionsspektrum übereinstimmte. Dann wurde das multiplizierte Reinspektrum von dem Reaktionsspektrum subtrahiert und die Identifikation weiterer Produkte vorgenommen. Die Vorgehensweise ist hierfür analog.

\subsubsection{Quantitative Analyse}

Die quantitative Analyse wird am Beispiel der Reaktion von Cyclopentylradikalen mit Sauerstoffatomen vorgestellt. Die Untersuchung der Reaktion wurde schon im Rahmen der Examensarbeit von NothduRfT vorgenommen [Not03. Nach Fertigstellung der Examensarbeit wurden noch Änderungen in der Verteilung der Produktkanäle entdeckt, die bei intensiverer Untersuchung verschiedener Vorläuferverhältnisse aufgetreten sind. Die Ergebnisse dieser Untersuchungen sind veröffentlicht Hoy06a.

Wie schon beschrieben wurden Photolysen von einer optimalen Vorläufergemischzusammensetzung durchgeführt. Nachdem man eine qualtitative Analyse durchgeführt hatte, und keine unidentifizierten Produkte mehr vorhanden waren, ging man zur quantitativen Analyse des Reaktionsspektrums über. Für die quantitative Analyse benötigte man ein Maß, das angibt, welche Bandenintensität einem bestimmten Partialdruck der Substanz entspricht. Dieses Maß stellt der in Abschnitt 2.1.1 beschriebene integrale Absorptionskoeffizient dar.

Den integralen Absorptionskoeffizienten erhielt man aus einer Auftragung der integralen 
Absorbanz gegen den eingefüllten Partialdruck. Dabei ist entscheidend, dass die integrale Absorbanz immer bezüglich eines definierten Wellenzahlbereiches verstanden werden muss und sowohl eine Funktion der Konzentration als auch der Lichtweglänge durch die Probe ist (siehe dazu auch Abschnitt 2.1.1.). Der integrale Absorptionskoeffizient ist somit auch immer an den Wellenzahlbereich und die jeweilige Apparatur gebunden. Abbildung 3.3 zeigt eine Auftragung zur Ermittlung des integralen Absorptionskoeffzienten am Beispiel von Allylbromid.

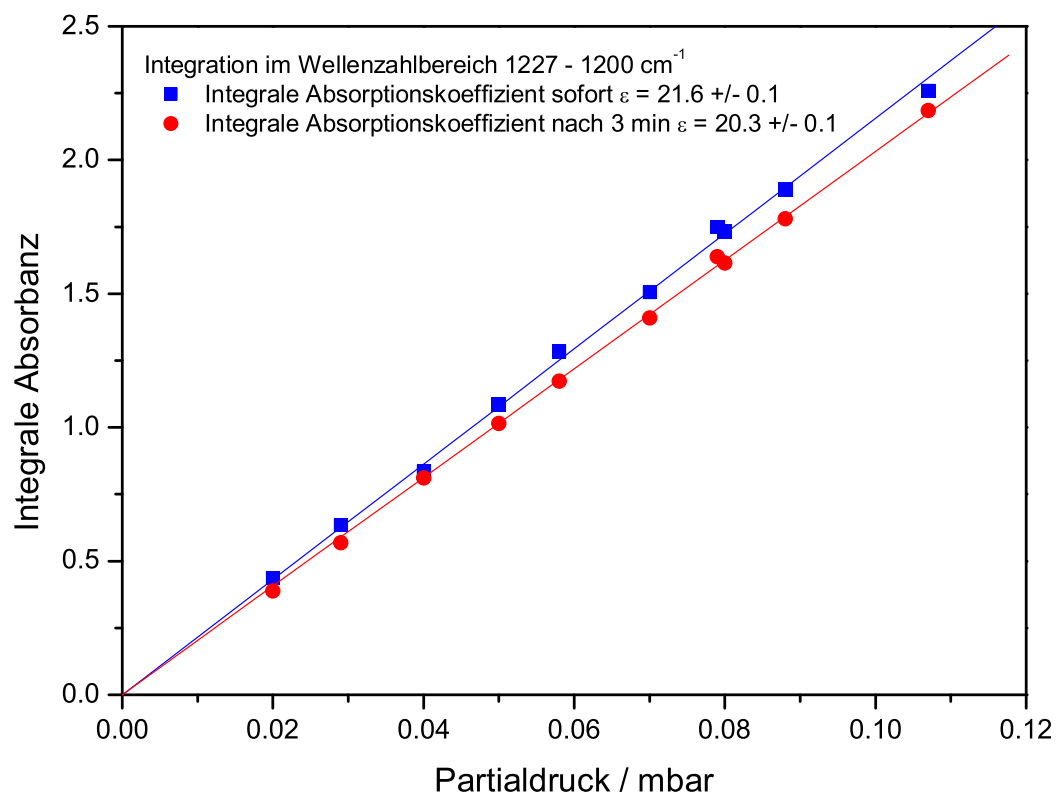

Abbildung 3.3: Beispiel zur Bestimmung des integralen Absorptionskoeffizienten anhand der Auftragung integrale Absorbanz vs. Partialdruck für Allylbromid. Die zwei Geraden stellen die lineare Regression der Datenpunkte unter der Bedingung einer Nullpunktsgerade dar. Die blau dargestellte(n) Punkte / Gerade entsprechen dem Integral, das man bei Integration der Absorptionsbande direkt nach Befüllung erhielt. Die rote(n) Punkte / Gerade erhielt man aus dem Spektrum, das 3 Minuten nach der Befüllung der Zelle aufgenommen wurde. Eine Erklärung der unterschiedlichen Steigungen ist im Text gegeben.

Für die Bestimmung des Koeffizienten wurden in einem Partialdruckbereich von etwa 0,01 bis 0,1 mbar bis zu acht Kalibrierspektren jeweils sofort nach Einfüllen in die Zelle und nach 3 Minuten aufgenommen. Diese Reinspektren wurden in dem vorher definierten Wellenzahlbereich integriert und die erhaltenen Wertepaare wurden zwecks Bestimmung des Koeffizienten aufgetragen. Eine lineare Regression unter der Voraussetzung, dass es sich um eine Nullpunktsgerade handelte, lieferte dann den integralen Absorptionskoeffizienten. 
Eine Aufstellung aller bestimmten Absorptionskoeffizienten befindet sich in Tabelle 7.1 im Anhang der Arbeit in Abschnitt 7.2.

Die Reinspektren der qualitativen Analyse wurden mit einem Faktor so multipliziert, dass sie in Intensität mit dem Reaktionsspektrum übereinstimmten und dann über den festgelegten Wellenzahlbereich integriert. Der erhaltene Wert musste dann zur Partialdruckbestimmung durch den integralen Absorptionskoeffizienten dividiert werden.

Im Rahmen der Examensarbeit [Not03] wurde festgestellt, dass sich die Intensitäten der Absoptionsbanden im Verlauf der Zeit ändern. Verglich man das Spektrum, das fünf Minuten nach Einfüllen in die Zelle aufgenommen wurde mit dem, welches sofort nach Einfüllen von der Substanz aufgenommen wurde, so stellte sich heraus, dass bei einigen Substanzen eine Verdoppelung der integralen Absorbanz stattgefunden hatte. Diesen Sachverhalt zeigt Abbildung 3.4 für 4-Pentenal.

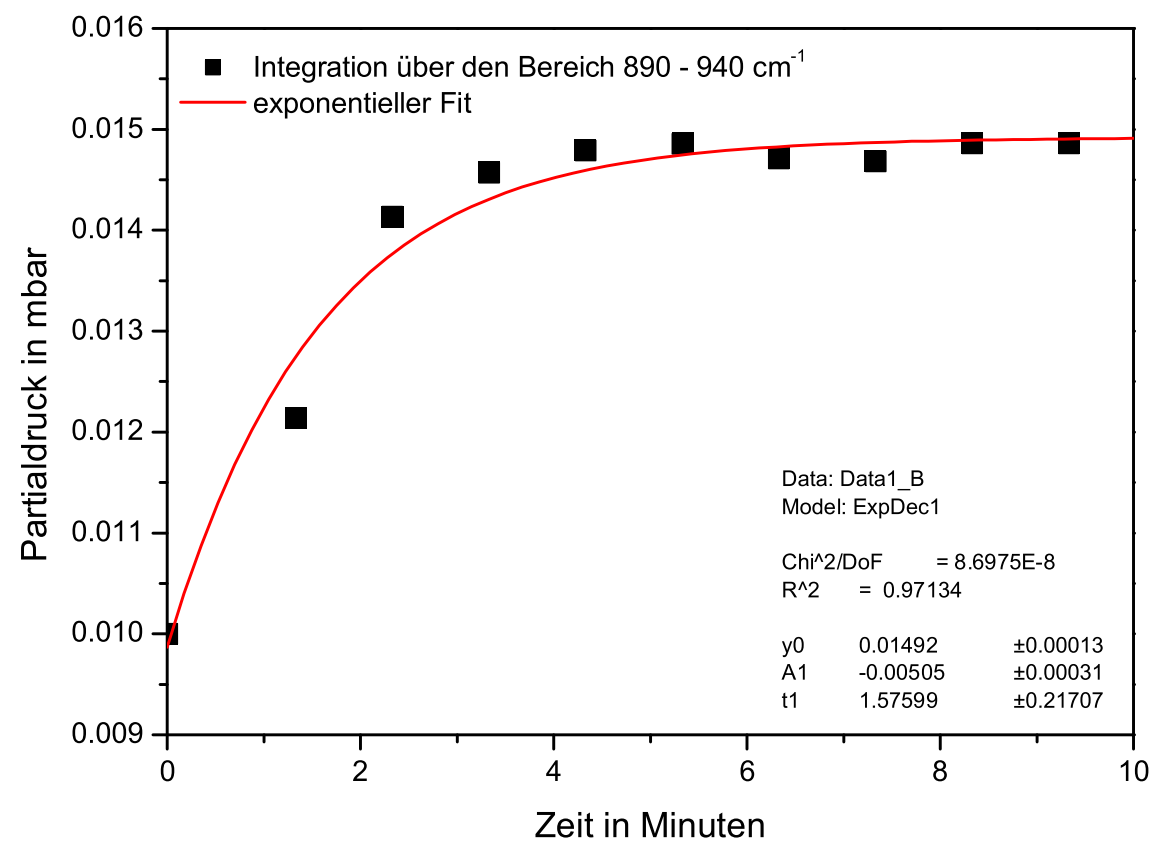

Abbildung 3.4: Zeitliche Absorbanzzunahme am Beispiel von 4-Pentenal. Dabei handelt es sich um die Auftragung des Partialdrucks gegen die Zeit nach Einfüllen der Substanz. Für die Auftragungen wurde eine bestimmte Menge Substanz in die Zelle gefüllt und die Zelle auf 4 mbar aufgefüllt. Dann wurden im Abstand von ca. 60 Sekunden IR-Spektren der Füllung aufgenommen, ohne deren Menge zu ändern. Die Abbildung zeigt deutlich, dass nach circa 3 Minuten keine signifikante Änderung mehr auftritt, bis dahin der Partialdruck aber anscheinend um den Faktor 1,5 zunimmt. 
Bei der Bestimmung der Absorptionskoeffizienten nach mehrtägiger Evakuierung der Zelle zeigte sich dagegen ein genau umgekehrtes Bild. Die integrale Absorbanz in einem gegebenen Wellenzahlbereich nahm mit der Zeit ab. Eine Stabilisierung war aber nach circa drei Minuten gegeben. Eine detaillierte Beschreibung der Intensitätsabnahme mit steigender Verweilzeit in der Reaktionszelle ist in der Examensarbeit von Sven MAARFELd [Maa06] in der Arbeitsgruppe Hoyermann beschrieben.

Die beiden gegensätzlichen Tendenzen lassen sich wie folgt erklären. Eine Zunahme der integralen Absorbanz ließ sich bis zu dem Zeitpunkt beobachten, an dem eine mehrtätgige Evakuierung der Zelle gestartet wurde. Die mehrtägige Evakuierung der Zelle wurde auf Grund des stark in der Zelle haftenden Cyclooktanons vorgenommen. Bis zu diesem Tag war wahrscheinlich durch die große Anzahl an Experimenten die innere Oberfläche der Zelle, welche aus Edelstahl besteht, mit einer Schicht verschiedenster Substanzen belegt, sodass die neu zu untersuchenden Substanz nicht mehr auf der inneren Zellenoberfläche anhaften konnte. Eine Kondensation bzw. Anlagerung der Substanz auf den Goldspiegeln, welche für den langen Lichtweg des IR-Strahl sorgen, war dahingegen noch möglich, wodurch eine Zunahme der integralen Absorbanz mit der Zeit beobachtet werden konnte. Nach der mehrtägigen Evakuierung der Zelle war die innere Oberfläche der Zelle von einem Großteil der vorher anhaftenden Moleküle befreit. Dadurch konnte bei der Untersuchung einer Substanz nach dem Einfüllen ein gewisser Anteil an die Oberfläche der innenliegenden Wände adsorbiert werden, wodurch der Partialdruck der entsprechenden Substanz sank. Diese Erniedrigung des Partialdrucks ist also auf eine Verminderung an Molekülteilchen im IR-Strahlengang gegenüber der sofortigen Bestimmung zurückzuführen. 


\subsection{Reinheit der verwendeten Substanzen}

\begin{tabular}{|c|c|c|}
\hline Substanz & Reinheit in \% & Hersteller / Verkäufer \\
\hline 1-Buten & $\geq 99 \%$ & Aldrich \\
\hline 1-Penten & $\geq 95 \%$ & Aldrich \\
\hline 2-Butyliodid & $\geq 98 \%$ & Fluka \\
\hline 2-Methyl-Cyclopentanon & $\geq 98 \%$ & Aldrich \\
\hline 2-Pentanon & $\geq 99 \%$ & Aldrich \\
\hline 4-Pentenal & $\geq 97 \%$ & Lancaster \\
\hline 5-Hexenal & $\geq 96 \%$ & eigene Herstellung \\
\hline Acetaldehyd & $\geq 99,5$ & Fluka \\
\hline Aceton & $\geq 99 \%$ & Fluka \\
\hline Aceton- $\mathrm{d}_{6}$ & $\geq 99,9 \%$ & Aldrich \\
\hline Acrolein & $\geq 95 \%$ & Fluka \\
\hline Allen & $\geq 97 \%$ & Aldrich \\
\hline Allylbromid & $\geq 99 \%$ & Aldrich \\
\hline Allylchlorid & $\geq 99 \%$ & Aldrich \\
\hline Argon & $\geq 99,998$ & Messer-Griesheim \\
\hline Butan & $\geq 99$ & Fluka \\
\hline Butanal & $\geq 99$ & Fluka \\
\hline Butanon & $\geq 99,5$ & Fluka \\
\hline Butyliodid & $\geq 99 \%$ & Aldrich \\
\hline Cyclohexan & $\geq 99,5 \%$ & Merck \\
\hline Cyclohexanon & $\geq 99,5 \%$ & Fluka \\
\hline Cyclohexen & $\geq 99 \%$ & Aldrich \\
\hline Cyclohexenon & $\geq 98 \%$ & Fluka \\
\hline Cyclooctan & $\geq 98 \%$ & Fluka \\
\hline Cyclooctanon & $\geq 98 \%$ & Aldrich \\
\hline Cyclookten & $\geq 95 \%$ & Aldrich \\
\hline Cyclopentan & $\geq 99,8 \%$ & Merck \\
\hline Cyclopentancarboxyaldehyd & $\geq 97 \%$ & Aldrich \\
\hline Cyclopentanon & $\geq 99,9 \%$ & Fluka \\
\hline Cyclopenten & $\geq 96 \%$ & Aldrich \\
\hline Cyclopentenoxid & $\geq 98 \%$ & Aldrich \\
\hline Diallyl & $\geq 97 \%$ & Aldrich \\
\hline
\end{tabular}


KAPITEL 3. BESCHREIBUNG DER EXPERIMENTELLEN BEDINGUNGEN

3.3. REINHEIT DER VERWENDETEN SUBSTANZEN

\begin{tabular}{|l||l|l|}
\hline Diisopropyl & $\geq 98 \%$ & Aldrich \\
\hline Diisopropylketon & $\approx 98$ & Fluka \\
\hline Dipropylketon & $\approx 98$ & Fluka \\
\hline Ethan & $\geq 99,5$ & Messer-Griesheim \\
\hline Ethan-d 6 & $\geq 99 \%$ & Sigma-Aldrich \\
\hline Ethen & $\geq 99,7$ & Merck \\
\hline Ethyliodid & $\geq 99 \%$ & Fluka \\
\hline Fluor / Helium & $\geq 99,995 / 98 \%$ & Messer-Griesheim \\
\hline Helium & $\geq 99,996$ & Messer-Griesheim \\
\hline Hexan & $\geq 99$ & Merck \\
\hline Isobutan & $\geq 99,6$ & Fluka \\
\hline Isobuten & $\geq 99$ & Fluka \\
\hline Isopropyliodid & $\geq 97$ & Fluka \\
\hline Kohlenmonoxid & $\geq 98$ & Messer-Griesheim \\
\hline Methan & $\geq 99,995$ & Messer-Griesheim \\
\hline Methyl-Propylketon & $\geq 99$ & Fluka \\
\hline Pentyliodid & $\geq 98 \%$ & Aldrich \\
\hline Pivaldehyd & $\geq 97$ & Fluka \\
\hline Propan & $\geq 99$ & Aldrich \\
\hline Propen & $\geq 99,95$ & Linde \\
\hline Propionaldehyd & $\approx 98$ & Fluka \\
\hline Propyliodid & $\geq 98$ & Fluka \\
\hline Schwefeldioxid & Messer-Griesheim \\
\hline Trichlorfluormethan & Fluka \\
\hline & $\geq 99,5$ & \\
\hline
\end{tabular}

Tabelle 3.1: Tabelle der verwendeten Substanzen 


\section{Kapitel 4}

\section{Messergebnisse und Diskussion}

\subsection{Untersuchung der Reaktionen zyklischer Kohlen- wasserstoff-Radikale mit Sauerstoff-Atomen}

Der Zerfall von sauerstoffzentrierten Kohlenwasserstoffradikalen ist, wie schon in der Einleitung dargestellt, in verschiedenen Gebieten chemischer Forschung, Entwicklung und Produktion, wie z. B. der kinetischen Modellierung der Kohlenwasserstoff Oxidation (Verbrennung) oder der Polymerchemie von Interesse. Um die Bedeutung dieser Reaktionen zu verdeutlichen, werden zwei Aspekte des Zerfalls O-zentrierter Kohlenwasserstoffradikale hier nochmals dargestellt:

(i) Der thermische oder photochemische Zerfall von Peroxyester-Bindungen führt zur Bildung von O-zentrierten Alkoxyradikalen und Carbonyloxyradikalen. Da Peroxyester in vielen Fällen als Initiatoren im Bereich der freien radikalischen Polymerisation genutzt werden, werden Informationen über weitere mögliche Zerfälle der primär gebildeten O-zentrierten Radikale benötigt. Unter $\beta$-C-H-Bindungsbruch wird der Charakter des Initiatorradikals von einem O-zentrierten in ein C-zentriertes Radikal umgewandelt. Mit diesem Wechsel sinkt die Initiatoreffizienz und neben der Kettenstartreaktion kann die Mikrostruktur der Polymerketten beeinflusst werden Bub05.

Zwar bestehen zwischen den Reaktionsbedingungen, bei denen eine radikalische Polymerisation abläuft (hohe Viskosität / Teilchendichte, hoher Grad an Wechsel- 
wirkungen) und der Reaktion in der Gasphase gravierende Unterschiede, die eine direkte Übertragung der in der Gasphase gewonnen Ergebnisse auf die Radikalische Polymerisation nur bedingt zulassen. Doch liefern Experimente in der Gasphase Hinweise auf Zerfallsreaktionen und -wege, die unter Berücksichtigung des Käfigeffekts für die Polymerisation Aufschluß über Initiatoreffizienz, $\beta$-C-H-, $\beta$-C-C-Brüche und Übertragungsreaktionen geben.

(ii) Da der Zerfall von Alkoxyradikalen zu einer Vielzahl verschiedener Produkte führen kann, sind für die Beschreibung der Hochtemperatur Oxidation kohlenwasserstoffbasierter Treibstoffe und der Atmosphärenchemie Verzweigungsverhältnisse und die zugehörigen Geschwindgkeitskoeffizienten der unterschiedlichen Reaktionskanäle notwendig. In diesem Zusammenhang werden Alkoxyradikale als wichtige Zwischenprodukte bei der Reaktion von Alkylradikalen mit O-Atomen gebildet. Der Hauptreaktionsweg für den Verbrauch von Alkanen unter Bildung von Alkylradikalen (R) besteht in der Reaktion mit Atomen $(\mathrm{H}, \mathrm{O})$ und Radikalen $\left(\mathrm{OH}, \mathrm{CH}_{3}\right)$, welche hauptsächlich bei der Hochtemperatur vorhanden sind. Obwohl die Konzentration von O-Atomen oft weit unter der Konzentration von $\mathrm{O}_{2}$ liegt, gewinnt die Reaktion $\mathrm{R}+\mathrm{O}$ unter mageren Bedingungen (wenig Treibstoff, viel Luft) an Bedeutung, da für viele Radikale die Geschwindigkeitskoeffizienten der Reaktionen $\mathrm{R}+\mathrm{O}$ unter mageren Bedingungen die der Reaktionen $\mathrm{R}+\mathrm{O}_{2}$ um mehrere Größenordnungen übersteigen [Gri95].

Praktische Treibstoffe bestehen dabei aus einer komplexen Mischung verschiedener Kohlenwasserstoffe. Eine Beschreibung der Zusammensetzung typischer Treibstoffe findet sich z. B. in [Edw03]. Da diese Treibstoffe zu komplex sind um sie direkt zu modellieren, benutzt man Modell-Treibstoffe für die Reaktionsmechanismen und somit Geschwindigkeitskoeffizienten durch eine möglichst große Anzahl an Experimenten validiert sind. In diesem Zusammenhang steigt das Interesse an zyklischen Alkanen, insbesondere an Cyclohexan und dessen Derivaten, da Modell-Treibstoffe, die zyklische Kohlenwasserstoffe enthalten, Kandidaten für die Modellierung der Verbrennung von Kerosin sind [Dag02]. Der Anteil zyklischer Alkane und deren Derivate ist des Weiteren in gewöhnlichen Treibstoffen wie Diesel oder Kerosin (Jet A, Jet A-1, JP-8, ...) oft größer als 50\% [Edw03, Pot98]. Schließlich stellt die Benzin- 
selbstzündungsverbrennung (HCCI, Homogeneous Charge and Compression Ignition) besonders hohe Ansprüche an die Treibstoffe und erfordert so neue ModellTreibstoffe. Hierbei berücksichtigt die aktuelle Forschung zyklische, ungesättigte und aromatische Verbindungen.

Für die Reaktion von zyklischen Kohlenwasserstoffradikalen $\mathrm{R}$ mit O-Atomen sind die beiden folgenden Reaktionswege denkbar:

$$
\begin{aligned}
& \mathrm{R}+\mathrm{O} \longrightarrow[\mathrm{R}-\mathrm{O}]^{\star} \longrightarrow \text { Produkte } \\
& \mathrm{R}+\mathrm{O} \longrightarrow \text { Cycloalken }+\mathrm{OH}
\end{aligned}
$$

Das durch Addition eines O-Atoms (1) gebildete hoch angeregte Intermediat R-O^ zerfällt in einem Folgeschritt unter Abspaltung eines H-Atoms zu sauerstoffhaltigen Produkten (Ketone, Aldehyde) oder zu kleineren Radikalen durch einen weiteren C-C-Bruch. Durch verschiedene $H$-shifts können außerdem Isomere gebildet werden, deren weiterer Zerfall zu anderen Kohlenwasserstoffen führen kann. Eine Darstelllung möglicher Reaktionswege für die Oxidation von Alkylradikalen findet sich unter Anderem in Hoy79a oder [Sla88]. Der Zerfall des hoch angeregten Zwischenprodukts aus Reaktionsweg (1) erfolgt primär unter Ringbruch ( $\beta$-C-C-Bindungsbruch) oder durch Elimination des verbliebenen H-Atoms am $\alpha$-C-Atom ( $\beta$-C-H-Bruch), wodurch es zur Bildung eines Cycloketons kommt. Analog zu der Reaktion von offenkettigen Kohlenwasserstoffen kann die Abstraktion eines $\beta$-HAtoms unter Bildung eines Cycloalkens (2) auch bei der Reaktion von Cycloalkylradikalen mit O-Atomen beobachtet werden. Dabei wird für den Abstraktionskanal ein direkter Prozess ohne Ausbildung des hoch angeregten Zwischenprodukts (1) angenommen.

Im Rahmen dieser Dissertation werden die Untersuchungen zu den Reaktionen von Cyclopentyl, Cyclohexyl, Cyclooctcyl und Cyclopenten mit O-Atomen vorgestellt. Die Reaktion von Cyclooctyl mit O-Atomen wurde hierbei schwerpunktmäßig im Rahmen einer Examensarbeit von Sven MaArfeld untersucht [Maa06]. Für alle Reaktionen wurde eine mögliche Druckabhängigkeit im Bereich von 4 mbar bis 1000 mbar analysiert. In dem durch die Apparatur zugänglichen Druckbereich sind Reaktionen dieses Typs jedoch zumeist druckunabhängig. Für die Reaktion Cyclooctyl + O konnte bereits in dem durch die 
Apparatur zugänglichen Druckbereich eine Änderung des Reaktionsmechanismus beobachtet werden. Diese Druckabhängigkeit wurde auch durch quantenchemische Rechnungen von M. Olzmann und O. Welz bestätigt; diese sind zusammen mit den experimentellen Ergebnissen zur Veröffentlichung eingereicht Hoy06b.

\subsubsection{Die Reaktion Cyclopentyl + O}

\subsubsection{Einleitung}

Die Ergebnisse der Untersuchung der Reaktion Cyclopentyl + O sind zusammen mit quantenchemischen Rechnungen von M. OlzMann für den Zerfall des Cyclopentoxyradikals veröffentlicht Hoy06a. Für diese Reaktion ist der im vorausgehenden Abschnitt beschriebene Reaktionsmechanismus Grundlage, welcher in folgende Reaktionskanäle mündet:

$$
\begin{array}{rlr}
\mathrm{c}-\mathrm{C}_{5} \mathrm{H}_{9}+\mathrm{O} & \longrightarrow \mathrm{c}-\mathrm{C}_{5} \mathrm{H}_{9} \mathrm{O}^{\star} & (a) \\
\mathrm{c}-\mathrm{C}_{5} \mathrm{H}_{9} \mathrm{O}^{\star} & \longrightarrow \mathrm{c}-\mathrm{C}_{5} \mathrm{H}_{8} \mathrm{O}+\mathrm{H} & \left(a_{1}\right) \\
\mathrm{c}-\mathrm{C}_{5} \mathrm{H}_{9} \mathrm{O}^{\star} & \longrightarrow \mathrm{CH}_{2}\left(\mathrm{CH}_{2}\right)_{3} \mathrm{CHO} & \left(a_{3}\right) \\
\mathrm{CH}_{2}\left(\mathrm{CH}_{2}\right)_{3} \mathrm{CHO} & \longrightarrow \mathrm{C}_{4} \mathrm{H}_{7} \mathrm{CHO}+\mathrm{H} & \left(a_{4}\right) \\
& \longrightarrow \mathrm{C}_{3} \mathrm{H}_{4} \mathrm{O}+\mathrm{C}_{2} \mathrm{H}_{4}+\mathrm{H} & \left(a_{5}\right) \\
& \longrightarrow \mathrm{C}_{2} \mathrm{H}_{4}+\mathrm{H}+\mathrm{CO} & \left(a_{6}\right) \\
\mathrm{c}-\mathrm{C}_{5} \mathrm{H}_{9}+\mathrm{O} & \longrightarrow \mathrm{Cyclopentenoxid}+\mathrm{H}-\mathrm{C}_{5} \mathrm{H}_{8}+\mathrm{OH} & (b)
\end{array}
$$

Ein möglicher Weg führt dabei über einen Abstraktionsprozess zur Bildung von Cyclopenten und $\mathrm{OH}$ (b). Der zweite Reaktionsweg, der über das energetisch hoch angeregte Cyclopentoxyradikal ( $\mathrm{E} \approx 377 \mathrm{~kJ} / \mathrm{mol}$ ) verläuft $(\mathrm{a})$, liefert verschiedene Oxidationsprodukte. Diese sind Folge der verschiedenen C-C- beziehungsweise C-H-Bindungsbrüche oder H-shifts.

\subsubsection{Qualitative Analyse}

Die Analyse stabiler Endprodukte und deren Kanalverteilung wurde mittels der in Abschnitt 3.1.1 beschriebenen FTIR-Apparatur durchgeführt. Die Cyclopentylradikale wur- 
den mittels Laser-Photolyse einer Mischung aus Cyclopentan und $\mathrm{CFCl}_{3}$ generiert.

Für diese Reaktion wurden eine Vielzahl von Messungen durchgeführt, wobei die Anfangskonzentrationen in einem großen Bereich variiert wurden. Sowohl das Verhältnis der Vorläufer $\left[\mathrm{c}-\mathrm{C}_{5} \mathrm{H}_{10}\right]_{0}:[\mathrm{O}]_{0}$ wurde von einem Sauerstoffunterschuss bis zu einem starken Sauerstoffüberschuss verändert als auch die Cl-Atom-Konzentration durch Variation der $\mathrm{CFCl}_{3}$-Anfangskonzentration im Vergleich zu der Cyclopentan-Anfangskonzentration.

Alle Messungen wurden bei Zimmertemperatur durchgeführt. Für die Ermittlung einer Druckabhängigkeit wurden neben Messungen bei einem Gesamtdruck von 4 mbar auch Experimente bei einem Gesamtdruck von 500 mbar und 1 bar durchgeführt. Diese lieferten aber keine eindeutige Veränderung der Kanalverzweigung, weshalb die Reaktion in dem durch die Apparatur zugänglichen Druckbereich als druckunabhängig angesehen werden kann. Mit Hilfe der beschriebenen FTIR-Apparatur wurden 100-fach gemittelte Spektren nach jeweils 100 Photolysen bei 193 nm aufgenommen. Die maximale Anzahl an Photolysen mit einer Gasmischung betrug 600. Dabei wurden bis zu 15\% des Vorläufers Cyclopentan verbraucht. Um Sekundärphotolysen der primären Produkte zu vermeiden, stellte dies die obere Grenze des Umsatzes dar.

Die Produktspektren wurden wie in Abschnitt 3.3.1 beschrieben ausgewertet. Die Abbildung 4.1 zeigt ein Reaktionsspektrum nach 400 Photolysen bei einer Vorläuferzusammensetzung von $\mathrm{p}\left(\mathrm{c}-\mathrm{C}_{5} \mathrm{H}_{10}\right)=0,2 ; \mathrm{p}\left(\mathrm{SO}_{2}\right)=0,1 ; \mathrm{p}\left(\mathrm{CFCl}_{3}\right)=0,2$ mbar und Abbildung 4.2 vergrößerte Ausschnitte des Reaktionsspektrums im Vergleich mit den Spektren der entsprechenden Reinsubstanzen. 


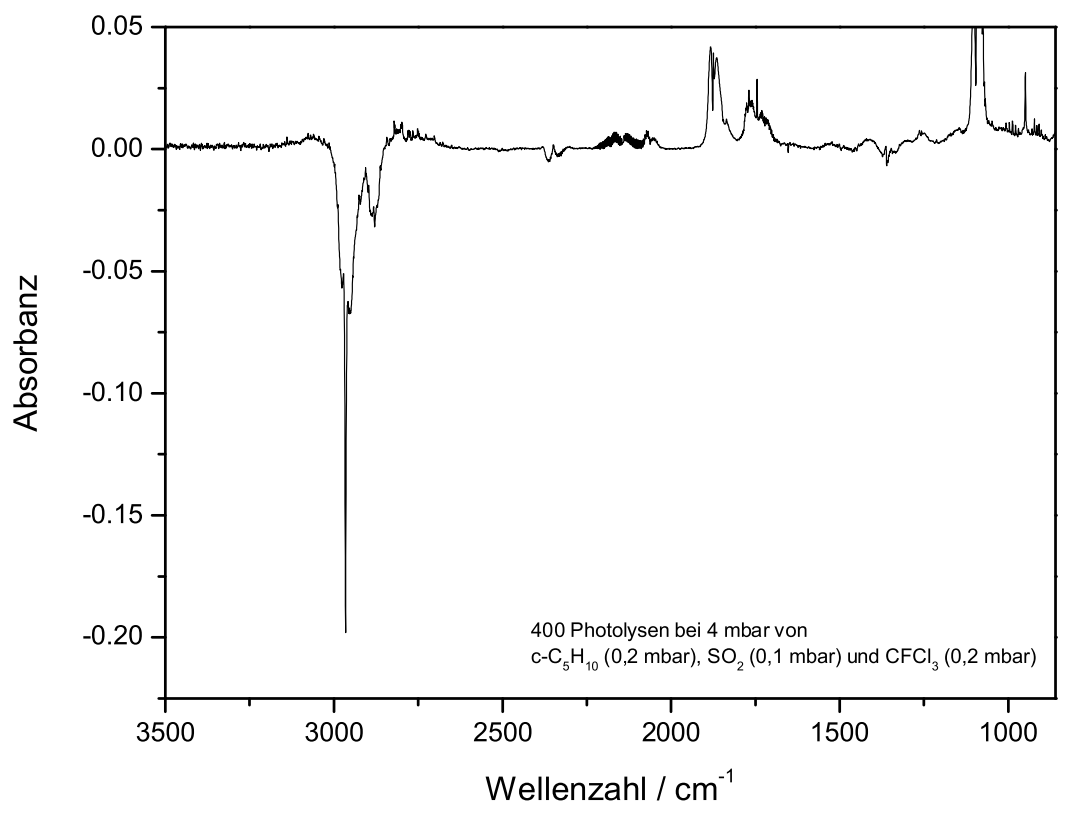

Abbildung 4.1: Reaktion $c-C_{5} H_{9}+O$, Reaktionsspektrum. $p\left(c-C_{5} H_{10}\right)=0,2 ; p\left(S_{2}\right)=0,1$; $p\left(\mathrm{CFCl}_{3}\right)=0,2$ mbar; $p_{\text {ges }}=4$ mbar, $T=298 \mathrm{~K} ; 400$ Photolysen 

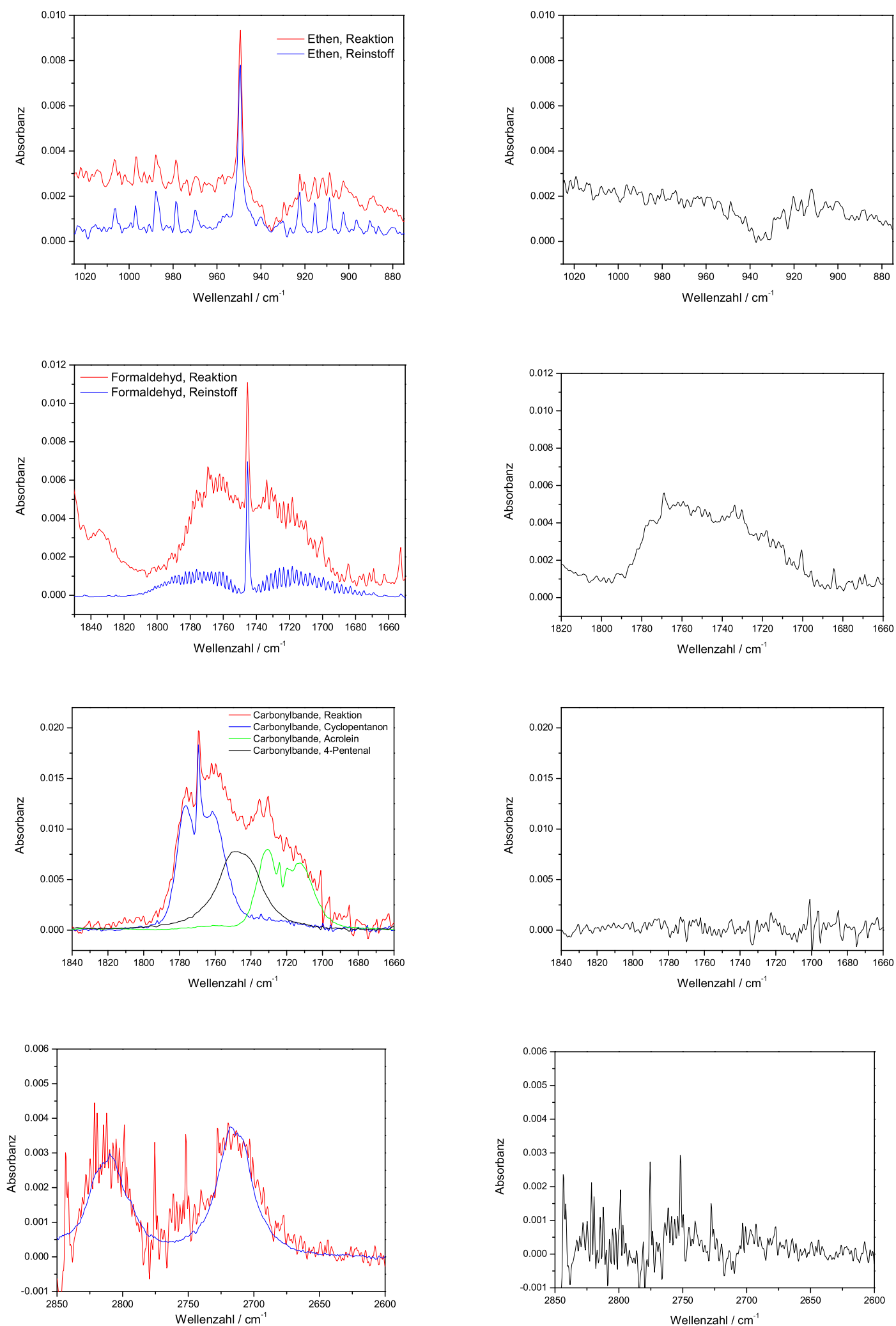

Abbildung 4.2: Reaktion c- $\mathrm{C}_{5} \mathrm{H}_{9}+\mathrm{O}$, Vergleich des Reaktionsspektrums mit Spektren der Reinsubstanzen in vergrößerten Ausschnitten; in der linken Spalte ist jeweils das Reaktionsspektrum zusammen mit dem Spektrum der Reinsubstanz beziehungsweise der Reinsubstanzen dargestellt; das Residuum ist jeweils in schwarz in der rechten Spalte dargestellt. 
Die Abbildung 4.2 zeigt als Produkte der Reaktion c- $\mathrm{C}_{5} \mathrm{H}_{9}+\mathrm{O}$ eindeutig Ethen, Cyclopentanon, 4-Pentenal, Acrolein und Formaldehyd. Der Vergleich des Reaktionsspektrums mit den Spektren der Reinsubstanzen im Bereich der Carbonyl-Streckschwingung beweist, dass die Carbonyl-Bande des Reaktionsspektrums durch eine Linearkombination der Reinstoffe vollständig erklärt werden kann. Die Bildung weiterer carbonylhaltiger Substanzen kann damit ausgeschlossen werden. Die Identifikation des Abstraktionsprodukts Cyclopenten wird in Abbildung 4.3 gezeigt. Weitere Produkte konnten nicht gefunden werden.
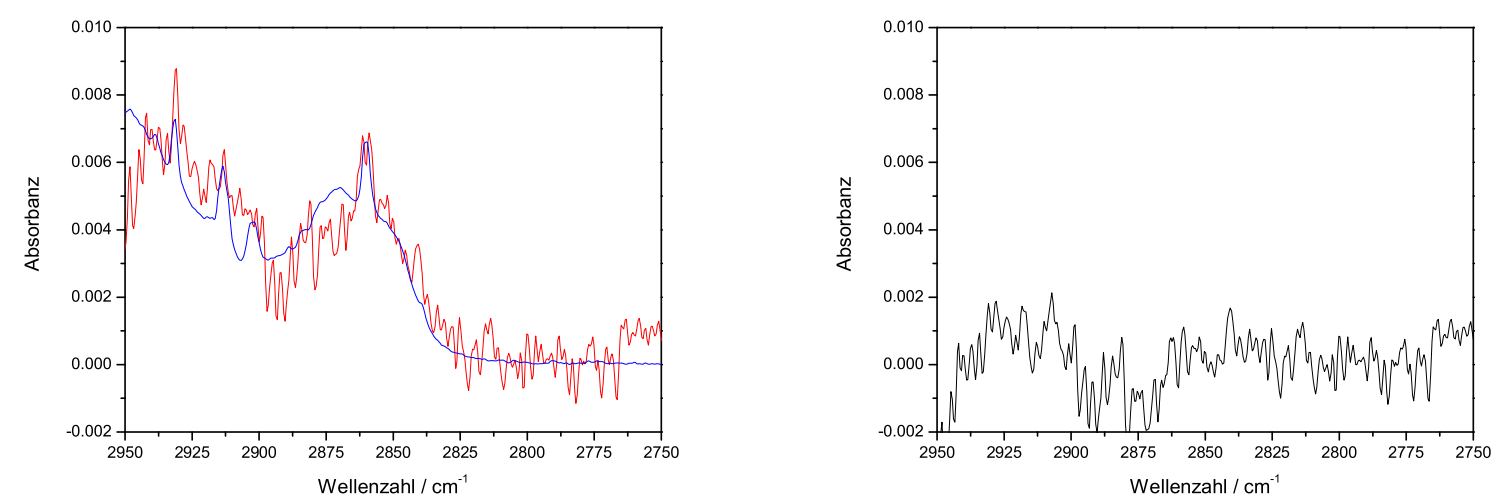

Abbildung 4.3: Reaktion c- $\mathrm{C}_{5} \mathrm{H}_{9}+\mathrm{O}$, qualitative Analyse. Die Abbildung zeigt das Reaktionsspektrum im Vergleich mit dem Abstraktionsprodukt $c-C_{5} H_{8}$. Die Übereinstimmung in den Peaks bei 2860, 2912 und $2931 \mathrm{~cm}^{-1}$ und die charakteristische Form lassen eine eindeutige Identifikation zu. Auf der linken Seite sind das Reaktionsspektrum (rot) und das Reinsspektrum von Cyclopenten (blau) im Vergleich und auf der rechten Seite das dazugehörige Residuum zu sehen.

\subsubsection{Quantitative Analyse}

Die quantitative Analyse wurde wie in Abschnitt 3.3.2 dargestellt vorgenommen. Für die identifizierten Substanzen wurden bei einem Gesamtdruck von 4 mbar Reinspektren aufgenommen. Dabei wurde der Partialdruck der jeweiligen Substanz von 0,01 mbar bis 0,1 mbar variiert. Nach Wahl eines geeigneten Integrationsbereichs wurde aus einer Auftragung Integrale Absorbanz vs. Partialdruck der integrale Absorptionskoeffizient bestimmt. Mit diesen Absorptionskoeffizienten wurde der Partialdruck der Produkte aus den Reaktionsspektren bestimmt.

Wie in der qualitativen Analyse dargestellt, wurde Cyclopenten als primäres Produkt der Reaktion von Cyclopentyl + O identifiziert. Da die Reaktion von Cyclopenten mit O im Vergleich mit weiteren primären Produkten und der Reaktion Cyclopentyl + O sehr schnell ist, wurde diese Reaktion seperat untersucht (siehe dazu Abschnitt 4.1.4). Eine 
Übersicht über die Geschwindigkeitskoeffizienten der primären Produkte der betrachten Reaktion mit O-Atomen im Vergleich mit der Titelreaktion gibt Tabelle 4.1. Aus dieser wird ersichtlich, dass die Reaktion von Cylopenten mit O-Atomen wesentlich schneller als die Reaktion der übrigen Produkte mit O-Atomen ist.

\begin{tabular}{|l|c|}
\hline Substanz & $\mathrm{k} /\left[\mathrm{cm}^{3} /(\mathrm{mol} \cdot \mathrm{s})\right]$ \\
\hline \hline $\mathrm{c}-\mathrm{C}_{5} \mathrm{H}_{9}$ & $1,73 \cdot 10^{14}$ \\
\hline $\mathrm{c}-\mathrm{C}_{5} \mathrm{H}_{8}$ & $1,25 \cdot 10^{13}$ \\
\hline $\mathrm{c}-\mathrm{C}_{5} \mathrm{H}_{10}$ & $7,40 \cdot 10^{10}$ \\
\hline $\mathrm{c}-\mathrm{C}_{5} \mathrm{H}_{8} \mathrm{O}$ & $1,07 \cdot 10^{10}$ \\
\hline $\mathrm{C}_{2} \mathrm{H}_{4}$ & $5,48 \cdot 10^{11}$ \\
\hline
\end{tabular}

Tabelle 4.1: Reaktion $c_{-} \mathrm{C}_{5} \mathrm{H}_{9}+\mathrm{O}$, Geschwindigkeitskoeffizienten primärer Produkte für die Reaktion mit O-Atomen im Vergleich mit der Reaktion Cyclopentyl + O. Die angegebenen Werte gelten für $298 \mathrm{~K}$ und einem Druck von ca. 1 mbar. Die Werte wurden der Online Datenbank Nist [Nis05] entnommen.

Auf Grund der schnellen Folgereaktion von Cyclopenten mit Sauerstoffatomen wurde für die Reaktion Cyclopentyl + O eine Abhängigkeit von der O-Atom-Konzentration intensiv untersucht. Eine starke Abhängigkeit des Verhältnisses von Cyclopentanon zu 4-Pentenal konnte dabei festgestellt werden. Abbildung 4.4 zeigt die Partialdrücke der identifizierten Substanzen in Abhängigkeit von dem Verbrauch des Cyclopentylvorläufers Cyclopentan bei O-Atom-Unterschuss und Abbildung 4.5 bei O-Atom-Überschuss. Die zugehörigen Messdaten finden sich in Tabelle 4.2 beziehungsweise Tabelle 4.3 .

\begin{tabular}{|c||c|c|c|c|c|c|c|}
\hline $\begin{array}{c}\text { Verbrauch } \\
\mathrm{c}-\mathrm{C}_{5} \mathrm{H}_{10}\end{array}$ & $\begin{array}{c}\mathrm{p}(\mathrm{CO}) \\
{[\mathrm{mbar}]}\end{array}$ & $\begin{array}{c}\mathrm{p}\left(\mathrm{H}_{2} \mathrm{CO}\right) \\
{[\mathrm{mbar}]}\end{array}$ & $\begin{array}{c}\mathrm{p}\left(\mathrm{C}_{2} \mathrm{H}_{4}\right) \\
{[\mathrm{mbar}]}\end{array}$ & $\begin{array}{c}\mathrm{p}\left(\mathrm{c}-\mathrm{C}_{5} \mathrm{H}_{8} \mathrm{O}\right) \\
{[\mathrm{mbar}]}\end{array}$ & $\begin{array}{c}\mathrm{p}\left(\mathrm{C}_{5} \mathrm{H}_{8} \mathrm{O}\right) \\
{[\mathrm{mbar}]}\end{array}$ & $\begin{array}{c}\mathrm{p}\left(\mathrm{C}_{3} \mathrm{H}_{4} \mathrm{O}\right) \\
{[\mathrm{mbar}]}\end{array}$ & $\begin{array}{c}\mathrm{p}\left(\mathrm{c}-\mathrm{C}_{5} \mathrm{H}_{8}\right) \\
{[\mathrm{mbar}]}\end{array}$ \\
\hline \hline 0,0041 & 0,0009 & 0,0004 & 0,0013 & 0,0009 & 0,0013 & 0,0004 & 0,0014 \\
\hline 0,0078 & 0,0013 & 0,0007 & 0,0025 & 0,0019 & 0,0024 & 0,0006 & 0,0029 \\
\hline 0,0112 & 0,0014 & 0,0009 & 0,0030 & 0,0023 & 0,0030 & 0,0009 & 0,0029 \\
\hline 0,0139 & 0,0015 & 0,0011 & 0,0040 & 0,0029 & 0,0033 & 0,0009 & 0,0047 \\
\hline 0,0175 & 0,0016 & 0,0013 & 0,0050 & 0,0032 & 0,0036 & 0,0008 & 0,0059 \\
\hline 0,0209 & 0,0018 & 0,0013 & 0,0060 & 0,0035 & 0,0040 & 0,0010 & 0,0059 \\
\hline
\end{tabular}

Tabelle 4.2: Reaktion $c-C_{5} H_{9}+O$, Messwerte O-Atom-Unterschuss. $p\left(c-C_{5} H_{10}\right)=0,1$ mbar; $p\left(\mathrm{SO}_{2}\right)=0,01$ mbar; $\mathrm{p}\left(\mathrm{CFCl}_{3}\right)=0,3 \mathrm{mbar}, \mathrm{p}_{\text {ges }}=4 \mathrm{mbar}, \mathrm{T}=298 \mathrm{~K}$, Badgas Argon. 


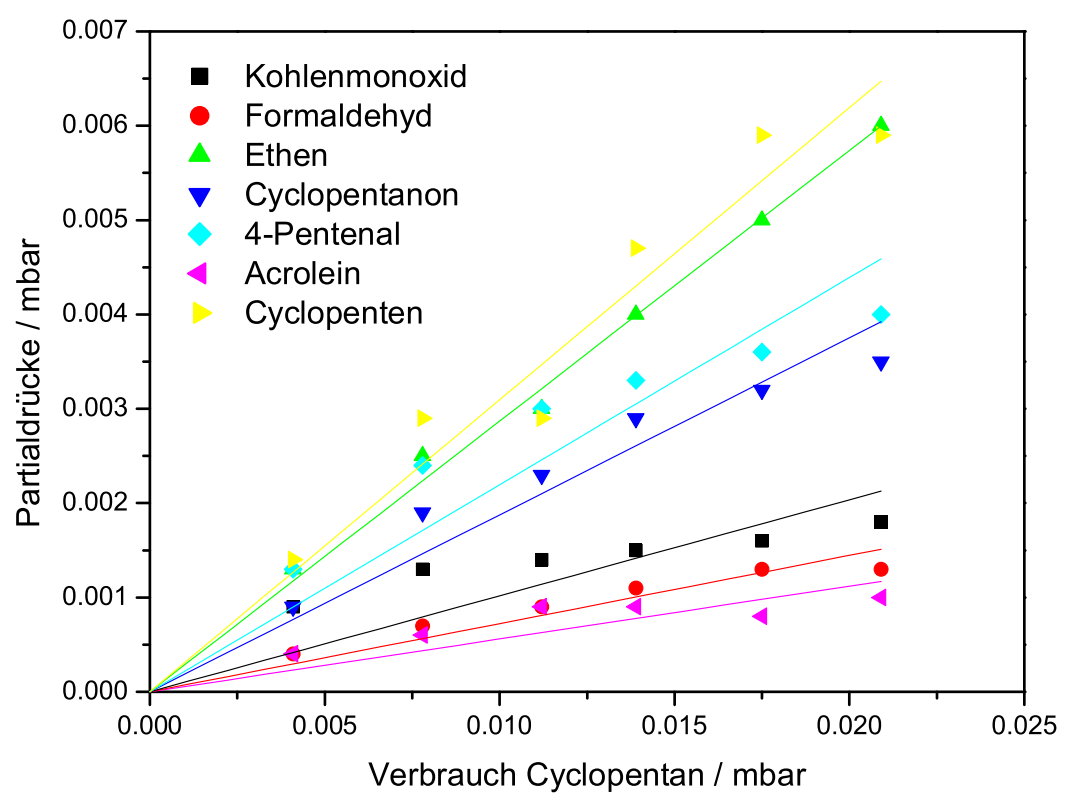

Abbildung 4.4: Reaktion $c-C_{5} H_{9}+O$, Produkte vs. Vorläuferverbrauch; $p\left(c-C_{5} H_{10}\right)=0,1$ mbar; $p\left(\mathrm{SO}_{2}\right)=0,01$ mbar; $\mathrm{p}\left(\mathrm{CFCl}_{3}\right)=0,3 \mathrm{mbar}, \mathrm{p}_{\text {ges }}=4 \mathrm{mbar}, \mathrm{T}=298 \mathrm{~K}$, Badgas Argon.

Wie aus Abbildung 4.4 hervor geht, zeigen alle Substanzen bezüglich des Cyclopentanverbrauchs ein annähernd lineares Verhalten. Eine signifikante Sekundärphotolyse primär gebildeter Produkte kann somit ausgeschlossen werden, da in diesem Fall mit steigendem Cyclopentanverbrauch eine starke Abweichung vom anfänglich linearen Anstieg des Produktpartialdrucks hin zu einem schwächeren Anstieg oder einem konstanten / abnehmenden Partialdruck beobachtbar sein müsste. Aus der Abbildung 4.4 wird ersichtlich, dass der Partialdruck an Cyclopenten, gefolgt von Ethen, 4-Pentenal und Cyclopentanon am größten ist.

\begin{tabular}{|c||c|c|c|c|c|c|c|}
\hline $\begin{array}{c}\text { Verbrauch } \\
\mathrm{c}-\mathrm{C}_{5} \mathrm{H}_{10}\end{array}$ & $\begin{array}{c}\mathrm{p}(\mathrm{CO}) \\
{[\mathrm{mbar}]}\end{array}$ & $\begin{array}{c}\mathrm{p}\left(\mathrm{H}_{2} \mathrm{CO}\right) \\
{[\mathrm{mbar}]}\end{array}$ & $\begin{array}{c}\mathrm{p}\left(\mathrm{C}_{2} \mathrm{H}_{4}\right) \\
{[\mathrm{mbar}]}\end{array}$ & $\begin{array}{c}\mathrm{p}\left(\mathrm{c}-\mathrm{C}_{5} \mathrm{H}_{8} \mathrm{O}\right) \\
{[\mathrm{mbar}]}\end{array}$ & $\begin{array}{c}\mathrm{p}\left(\mathrm{C}_{5} \mathrm{H}_{8} \mathrm{O}\right) \\
{[\mathrm{mbar}]}\end{array}$ & $\begin{array}{c}\mathrm{p}\left(\mathrm{C}_{3} \mathrm{H}_{4} \mathrm{O}\right) \\
{[\mathrm{mbar}]}\end{array}$ & $\begin{array}{c}\mathrm{p}\left(\mathrm{c}-\mathrm{C}_{5} \mathrm{H}_{8}\right) \\
{[\mathrm{mbar}]}\end{array}$ \\
\hline \hline 0,0099 & 0,0018 & 0,0012 & 0,005 & 0,0025 & 0,0016 & 0,0016 & 0,0029 \\
\hline 0,0140 & 0,0023 & 0,0016 & 0,007 & 0,0034 & 0,0021 & 0,0023 & 0,0029 \\
\hline 0,0175 & 0,0025 & 0,0022 & 0,0085 & 0,0041 & 0,0019 & 0,0025 & 0,0029 \\
\hline 0,0235 & 0,0030 & 0,0028 & 0,01 & 0,0050 & 0,0019 & 0,0029 & 0,0047 \\
\hline 0,0264 & 0,0038 & 0,0026 & 0,012 & 0,0061 & 0,0031 & 0,0032 & 0,0036 \\
\hline
\end{tabular}

Tabelle 4.3: Reaktion $c-C_{5} H_{9}+O$, Messwerte O-Atom- Überschuss. $p\left(c-C_{5} H_{10}\right)=0,1$ mbar; $p\left(\mathrm{SO}_{2}\right)=0,3$ mbar; $p\left(\mathrm{CFCl}_{3}\right)=0,3$ mbar, $p_{\text {ges }}=4$ mbar, $\mathrm{T}=298 \mathrm{~K}$, Badgas Argon. 


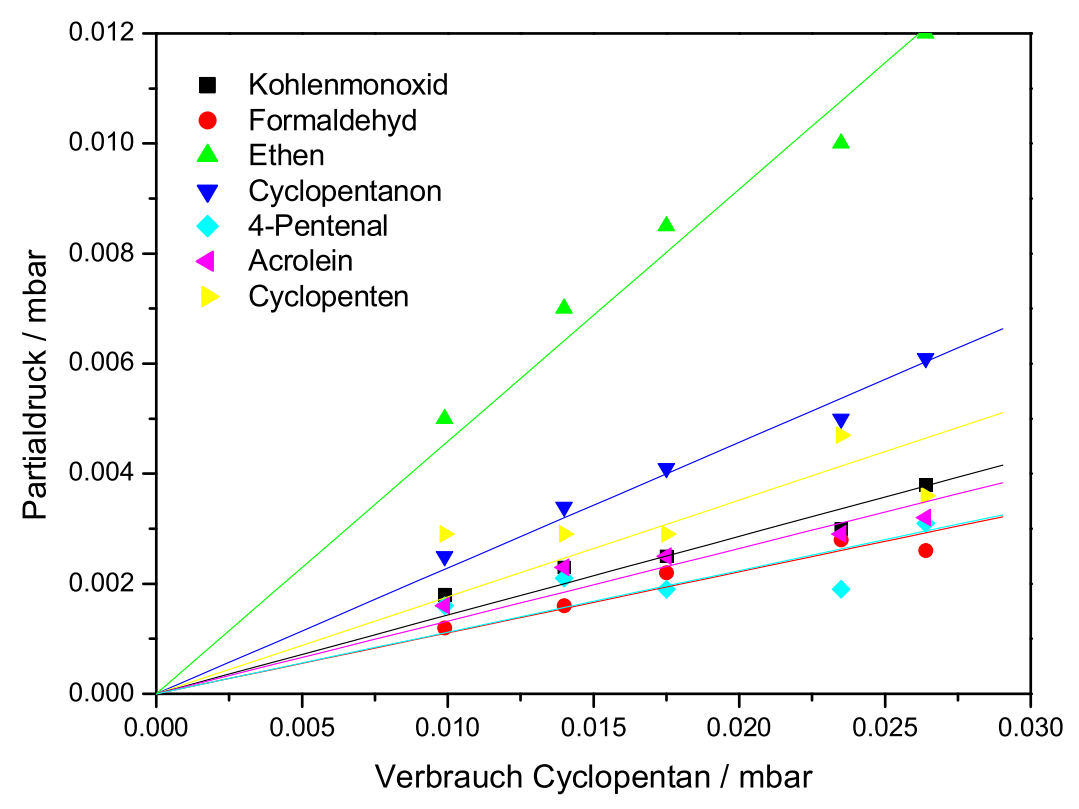

Abbildung 4.5: Reaktion $c-C_{5} H_{9}+O$, Produkte vs. Vorläuferverbrauch; $p\left(c-C_{5} H_{10}\right)=0,1$ mbar; $p\left(\mathrm{SO}_{2}\right)=0,3$ mbar; $\mathrm{p}\left(\mathrm{CFCl}_{3}\right)=0,3$ mbar; $p_{\text {ges }}=4$ mbar, $\mathrm{T}=298 \mathrm{~K}$, Badgas Argon.

Abbildung 4.5 zeigt, dass im Fall eines $\mathrm{SO}_{2}$ Überschusses ebenfalls mit steigender Photolysenanzahl und damit steigendem Cyclopentanverbrauch die Partialdrücke der Produkte linear zunehmen. Dies trifft allerdings nicht auf die Bildung von Cyclopenten zu, dessen Partialdruck mit zunehmender Photolysenanzahl nur gering anwächst. In diesem Fall kann von einer Sekundärreaktion von Cyclopenten mit O-Atomen ausgegangen werden. Im Gegensatz zum Sauerstoffunterschuss dominiert hier der Partialdruck von Ethen, gefolgt von Cyclopentanon und Cyclopenten.

Wie den Abbildungen 4.4 und 4.5 zu entnehmen ist, hängt das Verhältnis von Cyclopentanon zu 4-Pentenal somit stark von der O-Atom-Konzentration ab. Aus diesem Grund wurde das Verhältnis der Vorläufersubstanzen $\left[\mathrm{SO}_{2}\right]_{0}:\left[\mathrm{c}^{-} \mathrm{C}_{5} \mathrm{H}_{10}\right]_{0}$ variiert. Abbildung 4.6 zeigt das Verhältnis von Cyclopentanon zu 4-Pentenal in Abhängigkeit von dem Partialdruck des O-Atom-Vorläufers $\mathrm{SO}_{2}$. 


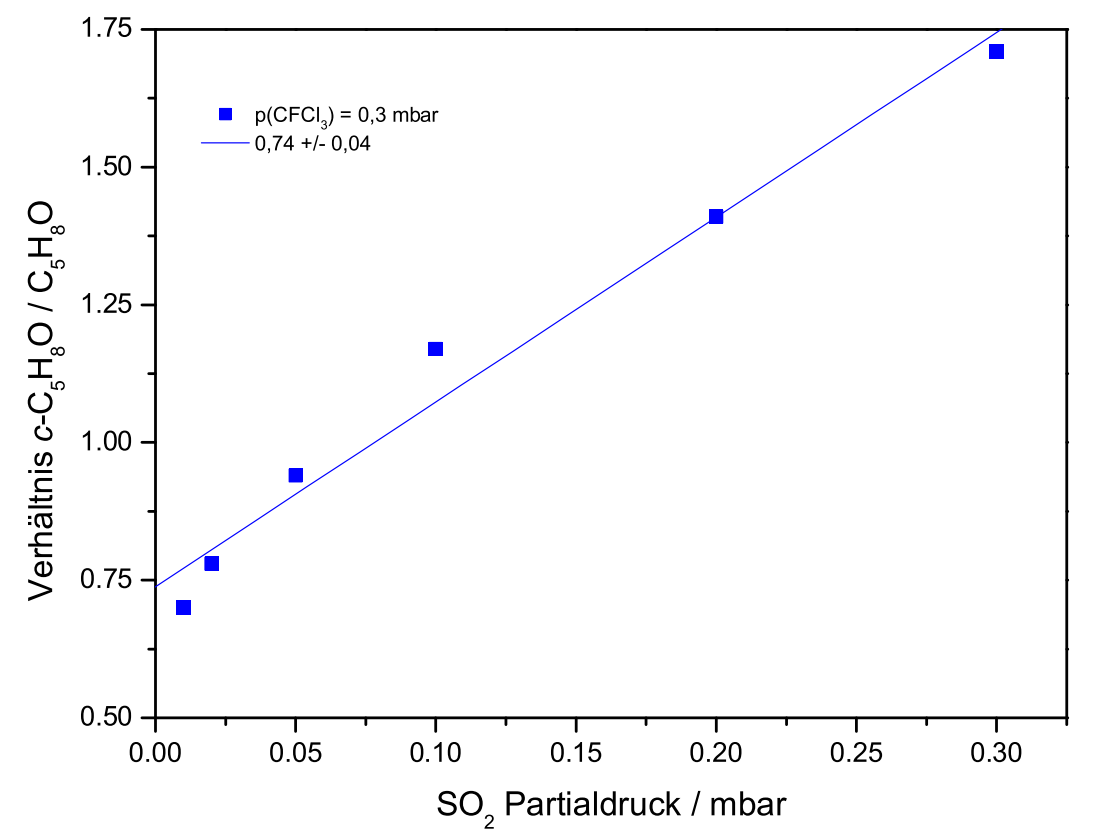

Abbildung 4.6: Reaktion c- $C_{5} H_{9}+O$, Verhältnis Cyclopentanon zu 4-Pentenal in Abhängigkeit von dem Partialdruck des O-Atom-Vorläufers $\mathrm{SO}_{2}$ bei konstantem Cyclopentan und $\mathrm{CFCl}_{3}$ Partialdrücken. $p_{\text {ges }}=4$ mbar, $\mathrm{T}=298 \mathrm{~K}, 400$ Photolysen.

Ihr kann man entnehmen, dass mit steigender O-Atom-Konzentration das Verhältnis von Cyclopentanon zu 4-Pentenal zunimmt. Dies hat zwei Gründe:

1. Bei steigender O-Atom-Konzentration nimmt die Wahrscheinlichkeit, dass primäre Produkte in einer Reaktion mit O-Atomen weiterreagieren, beziehungsweise zerfallen zu. Für diese Annahme spricht eine Zunahme der Ethen-Konzentration und eine absolute Abnahme der 4-Pentenal-Konzentration bei hoher O-Atom-Konzentration gegenüber einer niedrigen O-Atom-Konzentration.

2. Bei hoher O-Atom-Konzentration spielt die Reaktion des primären Produkts Cyclopenten mit O-Atomen eine größere Rolle, da diese Reaktion, wie oben gezeigt, sehr schnell verläuft. Dementsprechend ist ein Zuwachs an Produkten aus dieser Reaktion zu erwarten. Hauptprodukte dieser Reaktion sind Cyclopentanon und Ethen und Acrolein. Alle drei Produkte nehmen bei hoher O-Atom-Konzentration stark zu. 


\subsubsection{Simulation}

Um den Einfluss sekundärer Reaktionen auf die gemessene Produktverteilung zu ermitteln und Fehlergrenzen für die Kanalverzweigung abzuschätzen, wurde eine vollständige Simulation der Konzentrations-Zeit-Profile durchgeführt.

\begin{tabular}{|c|c|c|c|c|c|}
\hline \multicolumn{5}{|l|}{ Reaktion } & \multirow{2}{*}{$\begin{array}{l}\frac{\mathrm{cm}^{3} /(\mathrm{mol} \cdot \mathrm{s})}{2,00 \cdot 10^{13}} \\
\end{array}$} \\
\hline $\mathrm{OH}$ & $+\mathrm{O}$ & $\rightarrow \mathrm{O}_{2}$ & $+\mathrm{H}$ & + & \\
\hline $\mathrm{OH}$ & $+\mathrm{H}$ & $\rightarrow \mathrm{H}_{2} \mathrm{O}$ & $+\mathrm{H}$ & + & $1,00 \cdot 10^{10}$ \\
\hline$c-\mathrm{C}_{5} \mathrm{H}_{9}$ & $+\mathrm{O}$ & $\rightarrow \mathrm{c}-\mathrm{C}_{5} \mathrm{H}_{8}$ & $+\mathrm{OH}$ & + & $5,54 \cdot 10^{13}$ \\
\hline c- $\mathrm{C}_{5} \mathrm{H}_{9}$ & $+\mathrm{O}$ & $\rightarrow \mathrm{c}-\mathrm{C}_{5} \mathrm{H}_{8} \mathrm{O}$ & $+\mathrm{H}$ & + & $3,63 \cdot 10^{13}$ \\
\hline $\mathrm{c}-\mathrm{C}_{5} \mathrm{H}_{9}$ & $+\mathrm{O}$ & $\rightarrow 2 \mathrm{C}_{2} \mathrm{H}_{4}$ & $+\mathrm{HCO}$ & + & $1,90 \cdot 10^{13}$ \\
\hline $\mathrm{HCO}$ & + & $\rightarrow \mathrm{H}$ & $+\mathrm{CO}$ & + & $7,00 \cdot 10^{14}$ \\
\hline c- $\mathrm{C}_{5} \mathrm{H}_{9}$ & $+\mathrm{O}$ & $\rightarrow \mathrm{C}_{4} \mathrm{H}_{7} \mathrm{CHO}$ & $+\mathrm{H}$ & + & $4,67 \cdot 10^{13}$ \\
\hline c- $\mathrm{C}_{5} \mathrm{H}_{9}$ & $+\mathrm{O}$ & $\rightarrow \mathrm{C}_{2} \mathrm{H}_{4}$ & \multicolumn{2}{|c|}{$+\mathrm{C}_{3} \mathrm{H}_{4} \mathrm{O}+\mathrm{H}$} & $1,38 \cdot 10^{13}$ \\
\hline c- $\mathrm{C}_{5} \mathrm{H}_{9}$ & $+\mathrm{H}$ & $\rightarrow \mathrm{c}-\mathrm{C}_{5} \mathrm{H}_{10}$ & + & + & $8,50 \cdot 10^{12}$ \\
\hline c- $\mathrm{C}_{5} \mathrm{H}_{9}$ & $+\mathrm{OH}$ & $\rightarrow$ Produkte & + & + & $2,00 \cdot 10^{13}$ \\
\hline c- $\mathrm{C}_{5} \mathrm{H}_{9}$ & $+\mathrm{Cl}$ & $\rightarrow \mathrm{c}-\mathrm{C}_{5} \mathrm{H}_{8}$ & $+\mathrm{HCl}$ & + & $2,74 \cdot 10^{14}$ \\
\hline $\mathrm{c}-\mathrm{C}_{5} \mathrm{H}_{10}$ & $+\mathrm{Cl}$ & $\rightarrow \mathrm{c}^{-} \mathrm{C}_{5} \mathrm{H}_{9}$ & $+\mathrm{HCl}$ & + & $1,96 \cdot 10^{14}$ \\
\hline $\mathrm{c}-\mathrm{C}_{5} \mathrm{H}_{10}$ & $+\mathrm{O}$ & $\rightarrow \mathrm{c}-\mathrm{C}_{5} \mathrm{H}_{9}$ & $+\mathrm{OH}$ & + & $7,40 \cdot 10^{10}$ \\
\hline $\mathrm{c}-\mathrm{C}_{5} \mathrm{H}_{10}$ & $+\mathrm{H}$ & $\rightarrow \mathrm{c}-\mathrm{C}_{5} \mathrm{H}_{9}$ & $+\mathrm{H}_{2}$ & + & $3,44 \cdot 10^{09}$ \\
\hline $\mathrm{c}-\mathrm{C}_{5} \mathrm{H}_{10}$ & $+\mathrm{OH}$ & $\mathrm{I} \rightarrow \mathrm{c}-\mathrm{C}_{5} \mathrm{H}_{9}$ & $+\mathrm{H}_{2} \mathrm{O}$ & + & $2,89 \cdot 10^{12}$ \\
\hline c- $\mathrm{C}_{5} \mathrm{H}_{8}$ & $+\mathrm{O}$ & \multicolumn{2}{|c|}{$\rightarrow$ Cyclopentenoxide +} & + & $2,50 \cdot 10^{12}$ \\
\hline $\mathrm{c}-\mathrm{C}_{5} \mathrm{H}_{8}$ & $+\mathrm{O}$ & $\rightarrow \mathrm{c}-\mathrm{C}_{5} \mathrm{H}_{8} \mathrm{O}$ & + & + & $3,25 \cdot 10^{12}$ \\
\hline c- $\mathrm{C}_{5} \mathrm{H}_{8}$ & $+\mathrm{O}$ & $\rightarrow \mathrm{C}_{2} \mathrm{H}_{4}$ & \multicolumn{2}{|c|}{$+\mathrm{C}_{3} \mathrm{H}_{4} \mathrm{O}+$} & $2,00 \cdot 10^{12}$ \\
\hline $\mathrm{c}-\mathrm{C}_{5} \mathrm{H}_{8}$ & $+\mathrm{O}$ & $\rightarrow \mathrm{C}_{4} \mathrm{H}_{7} \mathrm{CHO}$ & + & + & $6,25 \cdot 10^{11}$ \\
\hline c- $\mathrm{C}_{5} \mathrm{H}_{8}$ & $+\mathrm{OH}$ & $\rightarrow$ Produkte & + & + & $3,40 \cdot 10^{13}$ \\
\hline c- $\mathrm{C}_{5} \mathrm{H}_{8} \mathrm{O}$ & $+\mathrm{O}$ & $\rightarrow$ Produkte & $+\mathrm{OH}$ & + & $1,07 \cdot 10^{10}$ \\
\hline $\mathrm{c}-\mathrm{C}_{5} \mathrm{H}_{8} \mathrm{O}$ & $+\mathrm{OH}$ & $\rightarrow$ Produkte & + & + & $1,77 \cdot 10^{12}$ \\
\hline $\mathrm{c}-\mathrm{C}_{5} \mathrm{H}_{8} \mathrm{O}$ & $+\mathrm{Cl}$ & $\rightarrow$ Produkte & $+\mathrm{HCl}$ & + & $2,86 \cdot 10^{13}$ \\
\hline $\mathrm{C}_{2} \mathrm{H}_{4}$ & $+\mathrm{O}$ & $\rightarrow$ Produkte & + & + & $5,48 \cdot 10^{11}$ \\
\hline $\mathrm{C}_{2} \mathrm{H}_{4}$ & $+\mathrm{H}$ & $\rightarrow \mathrm{C}_{2} \mathrm{H}_{3}$ & $+\mathrm{H}_{2}$ & + & $6,03 \cdot 10^{3}$ \\
\hline $\mathrm{C}_{2} \mathrm{H}_{4}$ & $+\mathrm{H}$ & $\rightarrow \mathrm{C}_{2} \mathrm{H}_{5}$ & + & + & $6,59 \cdot 10^{11}$ \\
\hline $\mathrm{C}_{2} \mathrm{H}_{4}$ & $+\mathrm{OH}$ & $\rightarrow$ Produkte & + & + & $5,66 \cdot 10^{12}$ \\
\hline $\mathrm{C}_{2} \mathrm{H}_{3}$ & $+\mathrm{H}$ & $\rightarrow \mathrm{C}_{2} \mathrm{H}_{4}$ & + & + & $1,21 \cdot 10^{14}$ \\
\hline
\end{tabular}

Tabelle 4.4: Reaktion c- $\mathrm{C}_{5} \mathrm{H}_{9}+\mathrm{O}$, Reaktionen und Geschwindigkeitskoeffizienten für die Simulation des Reaktionssytems (Startkonzentrationen siehe Text). 
Auf der Basis eines empirischen Reaktionsmechanismus mit einem Satz von 28 Reaktionen wurde das Reaktionssystem Cyclopentyl / Cyclopentan / O / Cl unter den im Experiment vorherrschenden Bedingungen simuliert. Die verwendeten Reaktionen und Geschwindigkeitskoeffizienten sind in Tabelle 4.4 aufgeführt.

Für die eigentliche Reaktion c- $\mathrm{C}_{5} \mathrm{H}_{9}+\mathrm{O}$ wurden die in der Arbeit ermittelten kinetischen Daten benutzt. Dabei wurde der von WeHMEYer bestimmte Geschwindigkeitskoeffizient $\mathrm{k}\left(\mathrm{c}-\mathrm{C}_{5} \mathrm{H}_{9}+\mathrm{O} \rightarrow\right.$ Produkte $)=1,73 \times 10^{14} \mathrm{~cm}^{3} /(\mathrm{mol} \cdot \mathrm{s}) \mathrm{zu}$ Grunde gelegt Weh02. Die Ergebnisse der im Rahmen dieser Arbeit untersuchten Reaktion $\mathrm{c}_{-} \mathrm{C}_{5} \mathrm{H}_{8}+\mathrm{O}$ sind ebenfalls eingeflossen. Die Anfangskonzentrationen der O- und Cl-Atome wurden über die Abnahme der Laserenergie und den spezifischen Absorptionskoeffizienten von $\mathrm{SO}_{2}$ und $\mathrm{CFCl}_{3}$ ermittelt. Die für die Simulation bestimmten Anfangskonzentrationen der Radikale und die von Cyclopentan betrugen $[\mathrm{O}]_{0}=3,4 \times 10^{-10} \mathrm{~mol} / \mathrm{cm}^{3},[\mathrm{Cl}]_{0}=5,6 \times 10^{-10} \mathrm{~mol} / \mathrm{cm}^{3}$, $\left[\mathrm{c}_{-} \mathrm{C}_{5} \mathrm{H}_{10}\right]_{0}=2,0 \times 10^{-8} \mathrm{~mol} / \mathrm{cm}^{3}$. Die Konzentrations-Zeit-Profile der Hauptprodukte sind in Abbildung 4.7 dargestellt.

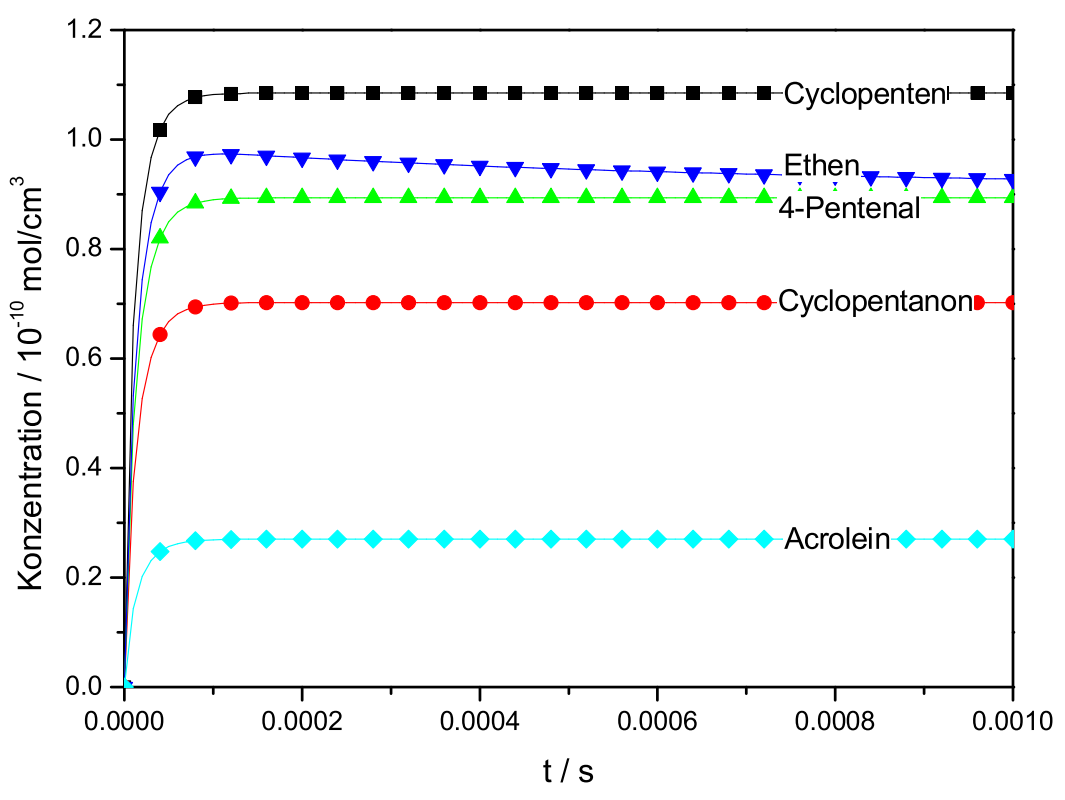

Abbildung 4.7: Reaktion c- $\mathrm{C}_{5} \mathrm{H}_{9}+\mathrm{O}$, Simulation der Konzentrations-Zeit-Profile; $[\mathrm{O}]_{0}=3,4 \times$ $10^{-10} \mathrm{~mol} / \mathrm{cm}^{3},[\mathrm{Cl}]_{0}=5,6 \times 10^{-10} \mathrm{~mol} / \mathrm{cm}^{3},\left[\mathrm{c}_{-} \mathrm{C}_{5} \mathrm{H}_{10}\right]_{0}=2,0 \times 10^{-8} \mathrm{~mol} / \mathrm{cm}^{3}$ (die verwendeten Reaktionen und Geschwindigkeitskoeffizienten sind in Tabelle 4.4 gegeben).

Ein Vergleich der experimentell gefundenen Kanalverzweigung mit den Daten, die aus der Simulation der Reaktion gewonnen wurden, zeigt innerhalb der experimentellen Fehler- 
grenzen eine gute Übereinstimmung. Daher wird das experimentell bestimmte Kanalverzweigungsverhältnis für den O-Atom-Unterschuss als eine gute Basis für einen Vergleich mit den im folgenden Abschnitt dargestellten quantenchemischen Rechnungen angesehen. Die experimentellen Daten sind zusammen mit den Werten der Simulation in Tabelle 4.5 nochmals angegeben.

\begin{tabular}{lcc}
\hline Substanz & Experiment & Simulation \\
\hline $\mathrm{c}-\mathrm{C}_{5} \mathrm{H}_{8}$ & $27 \pm 4$ & 28 \\
$\mathrm{C}_{4} \mathrm{H}_{7} \mathrm{CHO}$ & $23 \pm 5$ & 23 \\
$\mathrm{C} \mathrm{C}_{5} \mathrm{H}_{8} \mathrm{O}$ & $18 \pm 2$ & 18 \\
$\mathrm{C}_{2} \mathrm{H}_{4}$ & $25 \pm 5$ & 24 \\
$\mathrm{C}_{3} \mathrm{H}_{4} \mathrm{O}$ & $7 \pm 5$ & 7 \\
\hline
\end{tabular}

Tabelle 4.5: Reaktion c- $\mathrm{C}_{5} \mathrm{H}_{9}+\mathrm{O}$, Vergleich der experimentell bestimmten Produktausbeuten mit den Daten der Simulation. Startkonzentrationen und verwendete Geschwindigkeitskoeffizienten für die Simulation sind in Tabelle 4.4 bzw. im Text gegeben.

Bei den in Tabelle 4.5 angegebenen Werten handelt es sich um die relativen Anteile bezogen auf die Summe aller Produkte. Die Kanalverzweigung wurde aus diesen unter Berücksichtigung des Reaktionsmechanismus berechnet.

Abbildung 4.8 zeigt die Abhängigkeit der Produktverteilung von der Sauerstoffatomkonzentration. Neben der experimentell bestimmten Sauerstoffkonzentration ist dabei eine Simulation für eine um den Faktor 3 höhere Sauerstoffkonzentration dargestellt. Aus der Abbildung wird ersichtlich, dass in diesem Fall die Reaktion von Cyclopenten mit Sauerstoffatomen eine entscheidende Rolle spielt. Der Verbrauch an Cyclopenten führt zu höheren Anteilen der übrigen Produkte, da diese bei der Reaktion Cyclopenten + O gebildet werden. 


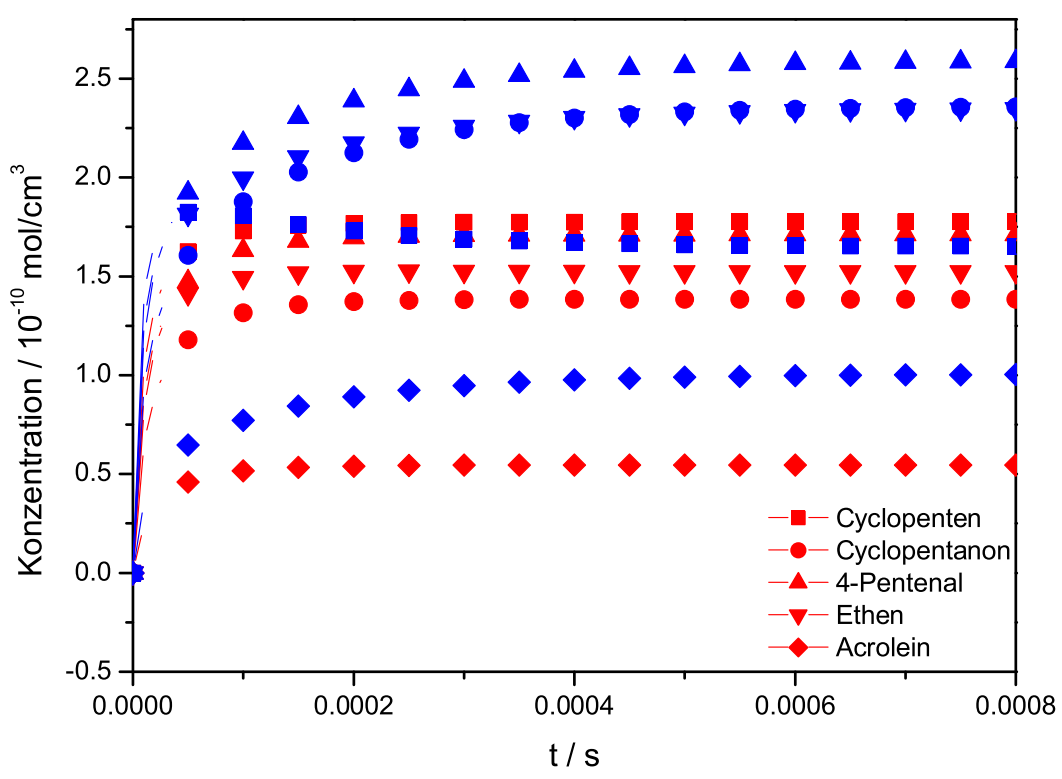

Abbildung 4.8: Reaktion $c_{-} \mathrm{C}_{5} \mathrm{H}_{9}+\mathrm{O}$, Simulation der Konzentrations-Zeit-Profile für verschiedene Sauerstoffkonzentrationen; $[\mathrm{Cl}]_{0}=5,6 \times 10^{-10} \mathrm{~mol} / \mathrm{cm}^{3},\left[\mathrm{c}-C_{5} H_{10}\right]_{0}=2.0 \times 10^{-8} \mathrm{~mol} / \mathrm{cm}^{3}$; rot: $[O]_{0}=3,4 \times 10^{-10} \mathrm{~mol} / \mathrm{cm}^{3}$, blau: $[O]_{0}=1,2 \times 10^{-9} \mathrm{~mol} / \mathrm{cm}^{3}$, (die verwendeten Reaktionen und Geschindigkeitskoeffizienten sind in Tabelle 4.4 gegeben)

\subsubsection{Quantenchemische Rechnungen}

Wie in der Einleitung dieses Abschnittes erwähnt, wurden die quantenchemischen Rechnungen im Rahmen einer Kooperation mit M. Olzmann (TU Karlsruhe) durchgeführt [Hoy06a]. Dabei beziehen sich die Rechnungen ausschließlich auf den Zerfall des Cyclopentoxyradikals. Da für den unimolekularen Zerfall des Cyclopentoxyradikals keine thermochemischen Daten für die Rechnungen vorlagen, wurden die Energiebarrieren mittels des Gaussian 98 Programm Pakets mit dem Basissatz G2(MP2) berechnet. Die Ergebnisse der Rechnungen sind in der Abbildung 4.9 zusammenfassend dargestellt. 


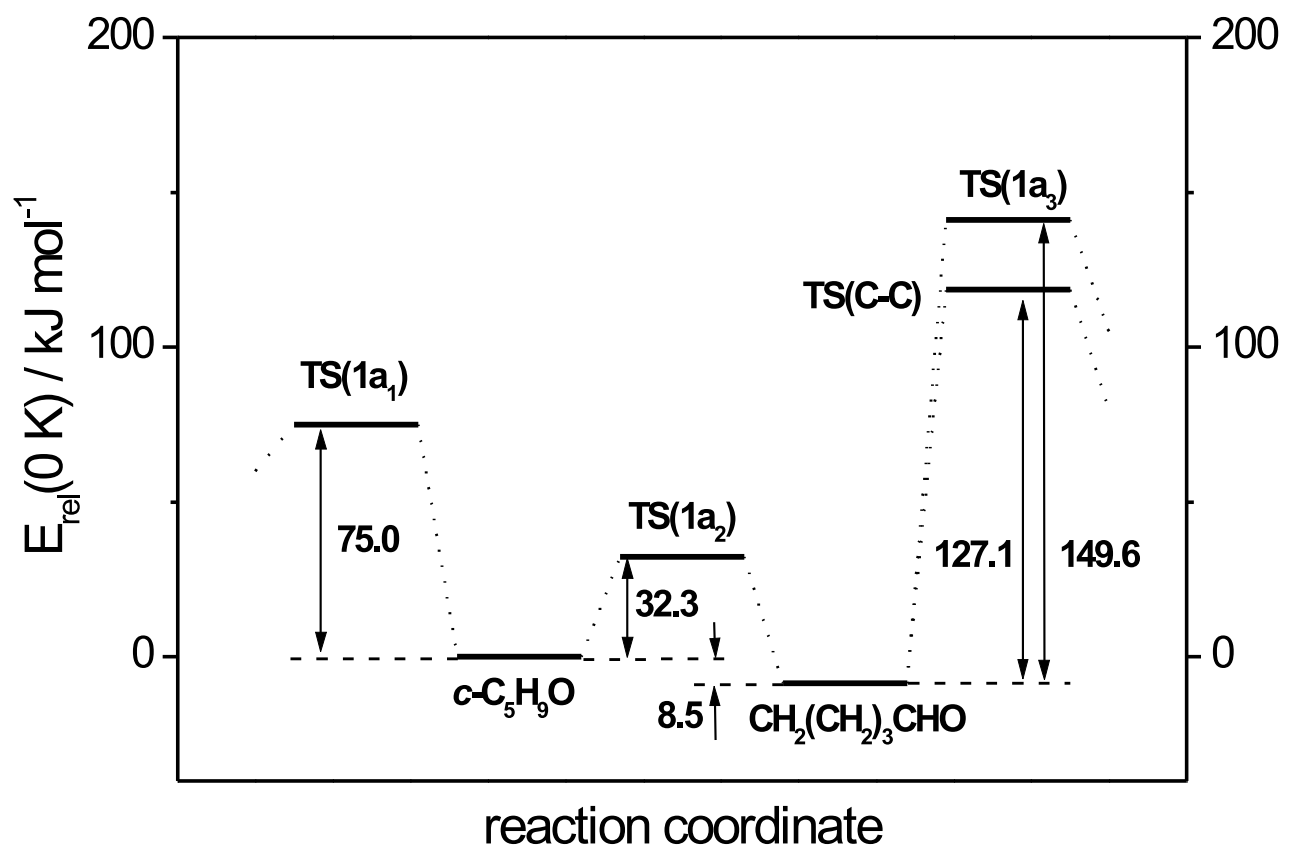

Abbildung 4.9: Reaktion c- $\mathrm{C}_{5} \mathrm{H}_{9}+\mathrm{O}$, Energiediagramm für den unimolekularen Zerfall des $\mathrm{Cy}$ clopentoxyradikals auf G2(MP2) Niveau; TS(1 $\left.a_{1}\right)$ steht für den ersten Übergangszustand der Abspaltung eines H-Atoms unter Bildung von Cyclopentanon, TS $\left(1 \mathrm{a}_{2}\right)$ für den ersten Übergangszustand der Ringöffnung, TS(C-C) bezeichnet den ersten Übergangszustand entlang des Kanals unter Ringöffnung (Bildung von Ethen, Acrolein und $H$ ) und TS(1 $\left.\mathrm{a}_{3}\right)$ den ersten Übergangszustand für die Bildung von 4-Pentenal unter H-Abspaltung.

Wie in Abbildung 4.9 deutlich wird, liegt die Aktivierungsenergie für die Ringöffnung $(32,3 \mathrm{~kJ} / \mathrm{mol})$ weit unter der Aktivierungsenergie für die Abspaltung eines H-Atoms (75,0 kJ/mol). Bei der der Ringöffnung folgenden Stabilisierungsreaktion liegt die Schwellenenergie für einen C-C-Bindungsbruch unter der Energiebarriere die zur Bildung des 4Pentenal gehört.

Für die Berechnung der Verzweigungsverhältnisse wurden die spezifischen Geschwindigkeitskoeffizienten mittels RRKM Theorie [Mar51, Mar52] unter Verwendung von skalierten Schwingungsfrequenzen und Rotationskonstanten auf Basis von ab initio Rechnungen auf MP2/6-31G(d) Niveau durchgeführt. Die so erhaltenen Geschwindigkeitskoeffizienten sind in Abhängigkeit von der Anregungsenergie des Cyclopentoxyradikals in Abbildung 4.10 visualisiert. 


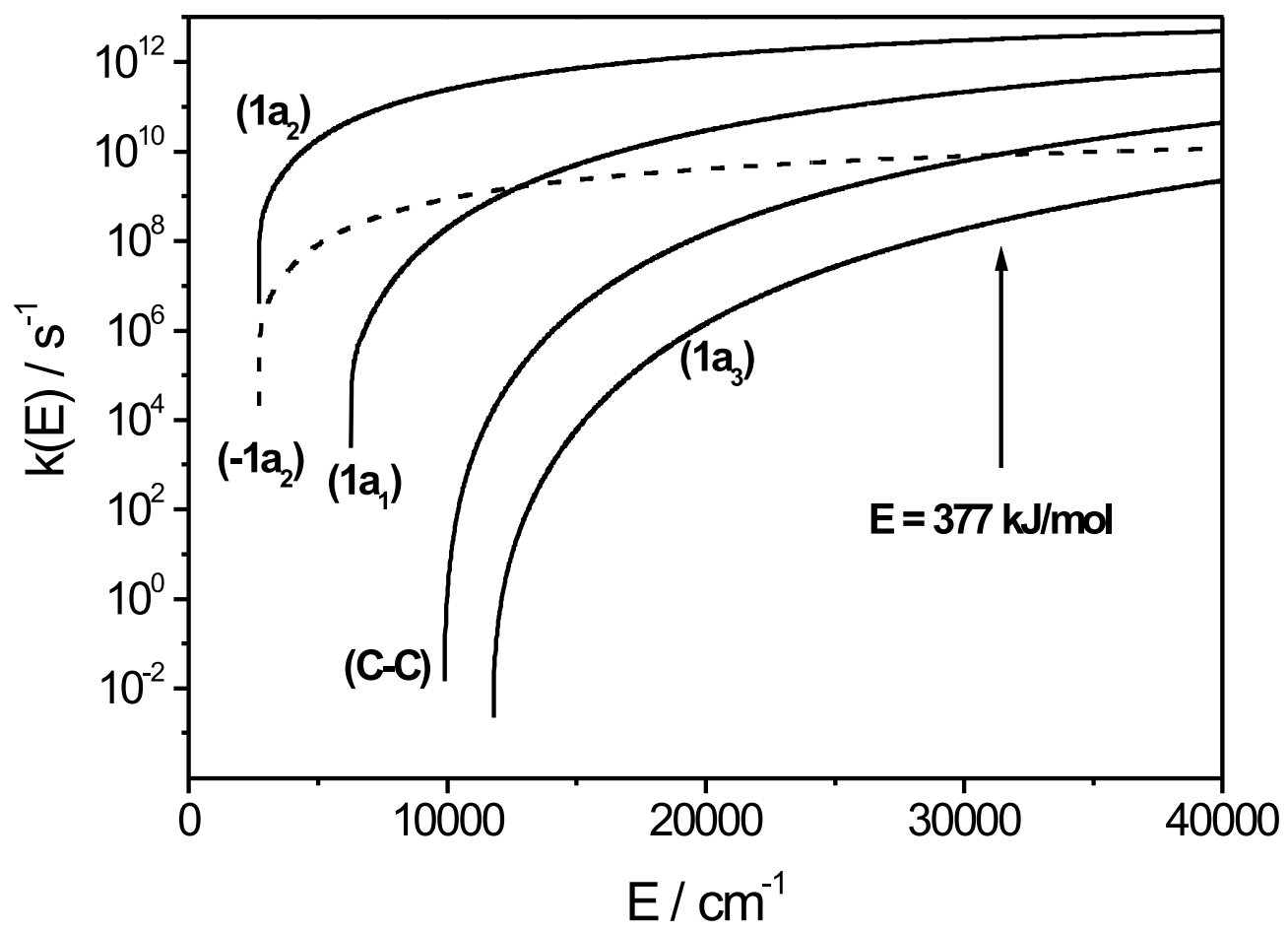

Abbildung 4.10: Reaktion $c-C_{5} H_{9}+O$, spezifische Geschwindigkeitskoeffizienten der RRKM Rechnung für den unimolekularen Zerfall des Cyclopentoxyradikals. (1 $\left.\mathrm{a}_{1}\right)$ entspricht der $\mathrm{H}$ Abspaltung unter Bildung von Cyclopentanon, $\left(1 \mathrm{a}_{2}\right)$ bzw (-1 $\left.\mathrm{a}_{2}\right)$ steht für die Ringöffnung bzw. Ringschluss, ( $\left.1 \mathrm{a}_{3}\right)$ für die Bildung von 4-Pentenal unter $H$-Abspaltung und $(C-C)$ für einen weiteren $C$-C-Bruch nach Ringöffnung. Die eingezeichnete Energie $E=377 \mathrm{~kJ} / \mathrm{mol}$ stellt die ursprüngliche Anregungsenergie dar (für Voraussetzungen und Basissatz siehe Text).

Die RRKM Rechnungen liefern als Resultat, dass der C-C-Bindungsbruch unter Ringöffnung die vorherrschende Reaktion ist (87\%). Die Bildung von Cyclopentanon wird unter den gegebenen Bedingungen zu 13\% angegeben (experimenteller Wert: 31\%). Der auf den Ringbruch folgende C-C-Bruch, der zur Bildung von Ethen, Acrolein und H führt, wird zu 84\% berechnet (experimentelles Ergebnis: 29\%). Demzufolge wird für die Bildung von 4-Pentenal ein Anteil von nur 3\% vorausgesagt, wobei dem gegenüber ein experimenteller Wert von $40 \%$ gefunden wurde.

\subsubsection{Diskussion}

Die Reaktion Cyclopentyl $+\mathrm{O}$ wurde innerhalb der Arbeitsgruppe schon von RoHDE [Roh88] und im Rahmen einer Examensarbeit von NothduRFT untersucht [Not03]. 
RoHDE verwendete für die Untersuchung der Reaktion ein Strömungssystem und massenspektrometrische Untersuchungsmethoden. Eine Unterscheidung auf Grund sich überlagernder Massen machte eine qualitative und quantitative Analyse und damit die Bestimmung der Produktkanalverteilung nur bedingt möglich. RoHDE hält eine Bildung des Abstraktionsprodukts Cyclopenten für wenig wahrscheinlich. Ebenso sieht RoHDE, im Gegensatz zu den hier dargestellten Ergebnissen, den Substitutionskanal zu Cyclopentanon als untergeordnet. Die Bildung von Pentenal wird vermutet, wobei eine eindeutige Unterscheidung von 4-Pentenal und 2-Pentenal mit der verwendeten Apparatur nicht möglich war. An dieser Stelle konnte die vorliegende Arbeit eine eindeutige Aufklärung zugunsten des 4-Pentenals liefern.

TAKAGi et al. [Tak81] berichten über die Bildung von Cyclopentanon bei der Photooxidation von $c-\mathrm{C}_{5} \mathrm{H}_{10}$ in einem NO/ $\mathrm{H}_{2} \mathrm{O} /$ Luft-System. Auf Grund der Komplexität des Systems laufen in diesem aber eine Vielzahl verschiedener Elementarreaktionen ab.

ORLANDO et al. [Orl00] diskutieren in ihrer Untersuchung nur den Kanal unter Ringöffnung als weitere Reaktion des Cyclopentoxyradikals. Dabei gehen sie wie in dieser Arbeit von einer Cl-initiierten Oxidation von Cyclopentan aus.

Wie ein Vergleich der experimentell gefundenen Werte mit den Werten der Simulationsrechnung zeigt, spielen Sekudärreaktionen bei den verwendeten experimentellen Bedingungen (Sauerstoff-Atom-Unterschuss) keine Rolle. Die Werte weichen lediglich innerhalb der Fehlergrenzen voneinander ab. Simulationen der Konzentrations-Zeit-Profile unter Sauerstoff-Atom-Überschuss zeigen hingegen einen deutlichen Einfluss der Oxidationsreaktionen auf die Produktverteilung. Dabei wird vor allem deutlich, dass die Reaktion von Cyclopenten mit Sauerstoff-Atomen bei höheren Sauerstoff-Atom-Konzentrationen eine entscheidende Rolle spielt. Die quantenchemischen Rechnungen und die experimentellen Werte stimmen in sofern überein, dass der Ringbruch den stärksten Kanal darstellt. Dabei wird ein weiterer Zerfall des linearen Pentoxyradikals bei den Rechnungen als Hauptkanal vorhergesagt, was experimentell nicht gefunden wurde. Die Stabilisierung des Ringes unter Bildung von Cyclopentanon wird in den Rechnungen unterschätzt.

Für die Reaktion von Cyclopentyl $+\mathrm{O}$ ergibt sich aus der qualitativen und quantitativen Analyse der IR-Spektren die folgende Produktkanalverteilung. Dabei wird für die 
Kanalverteilung ein O-Atom-Unterschuss, wie in der quantitativen Analyse dargestellt, zugrunde gelegt, da in diesem Fall sekundäre Reaktionen unterdrückt wurden:

$$
\begin{array}{rlr}
\mathrm{c}-\mathrm{C}_{5} \mathrm{H}_{9}+\mathrm{O} & \longrightarrow \mathrm{c}-\mathrm{C}_{5} \mathrm{H}_{9} \mathrm{O}^{\star} & \\
\mathrm{c}-\mathrm{C}_{5} \mathrm{H}_{9} \mathrm{O}^{\star} & \longrightarrow \mathrm{c}-\mathrm{C}_{5} \mathrm{H}_{8} \mathrm{O}+\mathrm{H} & (21 \pm 3) \% \\
& \longrightarrow \mathrm{C}_{4} \mathrm{H}_{7} \mathrm{CHO}+\mathrm{H} & (27 \pm 3) \% \\
& \longrightarrow \mathrm{C}_{3} \mathrm{H}_{4} \mathrm{O}+\mathrm{C}_{2} \mathrm{H}_{4}+\mathrm{H} & (8 \pm 2) \% \\
& \longrightarrow 2 \mathrm{C}_{2} \mathrm{H}_{4}+\mathrm{H}+\mathrm{CO} & (11 \pm 2) \% \\
\mathrm{c}-\mathrm{C}_{5} \mathrm{H}_{9}+\mathrm{O} & \longrightarrow \mathrm{c}-\mathrm{C}_{5} \mathrm{H}_{8}+\mathrm{OH} &
\end{array}
$$

\subsubsection{Die Reaktion Cyclohexyl + O}

\subsubsection{Einleitung}

Analog zu der Reaktion von Cyclopentyl mit O-Atomen sind für die Reaktion Cyclohexyl + $\mathrm{O}$ eine H-Abstraktion zu Cyclohexen (b) und eine O-Atom Addition zu einem hochangeregten Cyclohexoxyradikal (a), welches dann unter H-Abspaltung oder Ringbruch weiter zerfällt, wahrscheinlich. Das nachfolgende Schema verdeutlicht die im Vergleich mit quantenchemischen Rechnungen gewonnenen Prozesse:

$$
\begin{array}{rlr}
\mathrm{c}-\mathrm{C}_{6} \mathrm{H}_{11}+\mathrm{O} & \longrightarrow \mathrm{c}-\mathrm{C}_{6} \mathrm{H}_{11} \mathrm{O}^{\star} & (a) \\
\mathrm{c}-\mathrm{C}_{6} \mathrm{H}_{11} \mathrm{O}^{\star} & \longrightarrow \mathrm{c}-\mathrm{C}_{6} \mathrm{H}_{10} \mathrm{O}+\mathrm{H} & \left(a_{1}\right) \\
& \longrightarrow \mathrm{CH}_{2}\left(\mathrm{CH}_{2}\right)_{4} \mathrm{CHO} & \left(a_{2}\right) \\
\mathrm{CH}_{2}\left(\mathrm{CH}_{2}\right)_{4} \mathrm{CHO} & \longrightarrow \mathrm{C}_{5} \mathrm{H}_{9} \mathrm{CHO}+\mathrm{H} & \left(a_{4}\right) \\
& \longrightarrow \mathrm{C}_{2} \mathrm{H}_{4}+\mathrm{C}_{4} \mathrm{H}_{6} \mathrm{O}+\mathrm{H} & \left(a_{5}\right) \\
& \longrightarrow \mathrm{C}_{2} \mathrm{H}_{4}+\mathrm{CH}_{2} \mathrm{CHO} & \left(a_{6}\right) \\
& \longrightarrow \mathrm{C}_{2} \mathrm{H}_{5}+\mathrm{C}_{4} \mathrm{H}_{6} \mathrm{O} & \left(a_{7}\right) \\
& \longrightarrow \mathrm{C}_{5} \mathrm{H}_{10}+\mathrm{H}+\mathrm{CO} \\
\mathrm{c}-\mathrm{C}_{6} \mathrm{H}_{11}+\mathrm{H} \mathrm{H}_{7}+\mathrm{C}_{3} \mathrm{H}_{4} \mathrm{O} & \left(a_{8}\right)
\end{array}
$$


Als weiteres Produkt ist Formaldehyd zu erwarten, welches nach Ringbruch vorrangig durch Oxidation von Ethyl- und Propyl-Radikalen gebildet werden kann.

\subsubsection{Qualitative Analyse}

Die in Abschnitt 3.1.1. dargestellte FTIR-Apparatur wurde zur Endproduktanalyse und zur Bestimmung der Kanalverzweigungsverhältnisse verwendet. Zur Erzeugung der Cyclohexylradikale und Sauerstoff-Atome wurde eine Mischung aus c- $\mathrm{C}_{6} \mathrm{H}_{12} / \mathrm{CFCl}_{3} / \mathrm{SO}_{2}$ mit Laserlicht bei $\lambda=193 \mathrm{~nm}$ photolysiert.

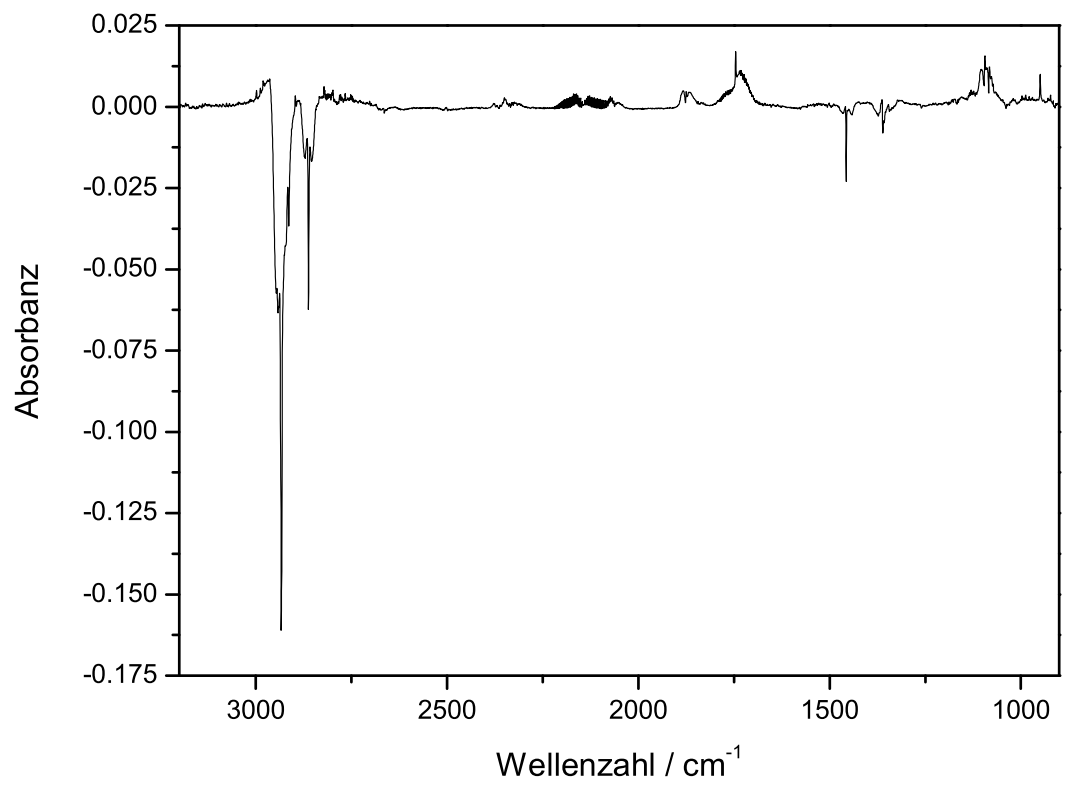

Abbildung 4.11: Reaktion $c-C_{6} H_{11}+O$, Reaktionsspektrum nach 400 Photolysen

Wie auch bei der Untersuchung der Reaktion von Cyclopentyl + O wurde das Verhältnis der Konzentrationen der Radikal- / Atomverläufer $\left(\left[\mathrm{c}-\mathrm{C}_{6} \mathrm{H}_{10}\right]_{0} /\left[\mathrm{SO}_{2}\right]_{0}\right)$ von starkem Sauerstoffunterschuss bis -überschuss variiert.

Alle Messungen wurden bei Zimmertemperatur vorgenommen. Für die Untersuchung einer möglichen Druckabhängigkeit wurde der Gesamtdruck in dem durch die Apparatur zugänglichen Bereich von 4 mbar bis 1000 mbar verändert. Die Reaktionsspektren wurden nach jeweils 100 Photolysen mittels der in Abschnitt 3.1.1 beschriebenen FTIR-Apparatur aufgenommen, wobei ein Reaktionsspektrum aus 100 Einzelspektren gemittelt wurde. Die maximale Anzahl an Photolysen betrug hierbei 600, um eine Sekundärphotolyse primärer 
Endprodukte zu vermeiden. Der Vorläuferumsatz blieb dabei unter $15 \%$.

Die Reaktionsspektren wurden wie in Abschnitt 3.3.1 beschrieben ausgewertet. Die Abbildung 4.11 zeigt ein Reaktionsspektrum nach 400 Photolysen.

Vergrößerte Ausschnitte aus dem Reaktionsspektrum sind in Abbildung 4.12 dargestellt.
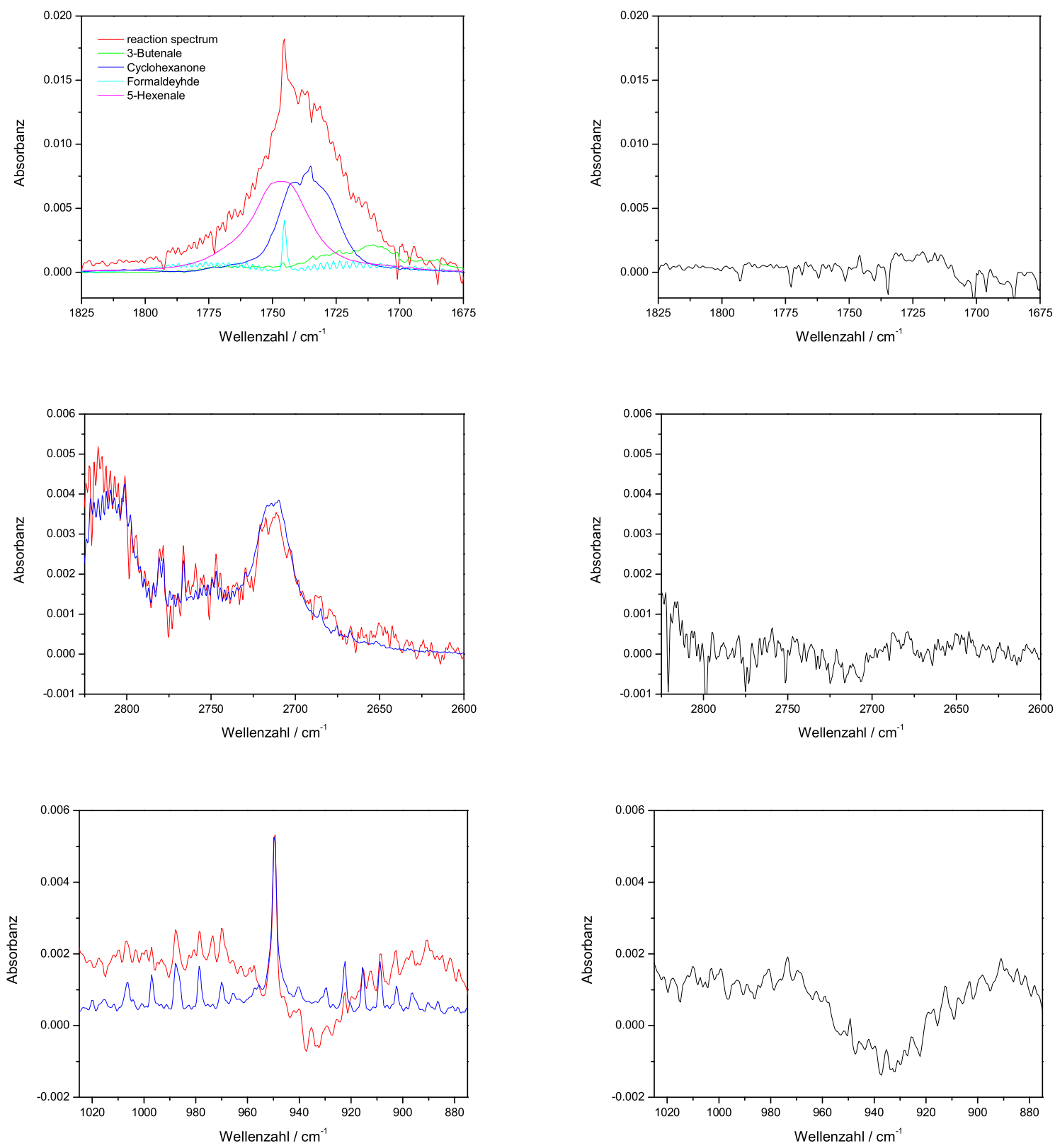

Abbildung 4.12: Reaktion c- $C_{6} H_{11}+O$, Vergleich des Reaktionsspektrums mit Spektren der Reinsubstanzen in vergrößerten Ausschnitten. In der linken Spalte ist jeweils das Reaktionsspektrum zusammen mit dem Spektrum der Reinsubstanz beziehungsweise der Reinsubstanzen dargestellt; das Residuum ist jeweils in schwarz in der rechten Spalte dargestellt.

Der Vergleich des Reaktionsspektrums mit den Reinspektren der idenitifizierten Substan- 
zen in Abbildung 4.12 ermöglicht eine eindeutige Identifizierung von 5-Hexenal, Cyclohexanon, Acrolein, 3-Butenal, Formaldehyd und Ethen. Die Bildung des Abstraktionsprodukts Cyclohexen konnte nicht festgestellt werden. Eindeutig läßt sich die Bildung dieses Produktes aber auf Grund von Überlagerungen im Bereich der symmetrischen und asymmetrischen C-H-Valenzschwingung nicht ausschließen, der Anteil ist aber $\leq 10 \%$. $\mathrm{Zu}$ erwähnen ist an dieser Stelle, dass es sich bei dem Kalibrierspektrum von 3-Butenal nicht um das Spektrum einer kommerziell erhältlichen Substanz handelt. Vielmehr wurde dies aus der Reaktion von 1,3-Butadien mit O-Atomen im Überschuss erhalten. Wie ein Vergleich des Reinspektrums von 5-Hexenal, welches in der Arbeitsgruppe DE MEIJERE am Institut für Organische und Biomolekulare Chemie der Universität Göttingen hergestellt wurde, mit dem Spektrum der Reaktion 1,5-Hexadien + O im O-Atom Überschuss zeigt, findet bei den endständigen Dienen bevorzugt eine Addition des O-Atoms an eine der endständigen Doppelbindungen statt, womit die Oxidation von Dienen eine geeignete Quelle zur Bildung der ungesättigten Aldehyde zu sein scheint. Die Reaktionen der Diene mit atomaren Sauerstoff wurde im Rahmen der Dissertation für 1,3-Butadien, 1,5-Hexadien und 1,7-Octadien untersucht.

\subsubsection{Quantitative Analyse}

Die quantitative Analyse wurde wie in Abschnitt 3.3.2 beschrieben durchgeführt. Da die Reaktion sowohl bei einem Gesamtdruck von 4 mbar als auch bei einem Gesamtdruck von 1 bar untersucht wurde, wurden die Reinspektren bei diesen Drücken aufgenommen. Die Partialdrücke der Substanzen wurden dabei in einem Partialdruckbereich von 0,01 bis 0,1 mbar variiert.

Für die Reaktion c- $\mathrm{C}_{6} \mathrm{H}_{11}+\mathrm{O}$ konnte in dem zugänglichen Druckbereich keine Veränderung des Mechanismus bzw. des Verhältnisses der Reaktionskanäle gefunden werden. Die Reaktion kann damit in diesem Druckbereich als druckunabhängig angesehen werden. Bei einem Gesamtdruck von 4 mbar wurden die in Tabelle 4.6 aufgeführten Partialdrücke bestimmt. Die angegebenen Werte sind dabei durch mehrere Experimente gewonnen worden, bei denen das Verhältnis der Vorläuferkonzentrationen aber konstant war. 


\begin{tabular}{|c|c|c|c|c|c|c|}
\hline $\begin{array}{l}\text { Verbrauch } \\
\text { c- } \mathrm{C}_{5} \mathrm{H}_{10}\end{array}$ & $\begin{array}{c}\mathrm{p}\left(\mathrm{H}_{2} \mathrm{CO}\right) \\
{[\mathrm{mbar}]}\end{array}$ & $\begin{array}{c}\mathrm{p}\left(\mathrm{c}-\mathrm{C}_{6} \mathrm{H}_{10} \mathrm{O}\right) \\
{[\mathrm{mbar}]}\end{array}$ & $\begin{array}{c}\mathrm{p}\left(\mathrm{C}_{5} \mathrm{H}_{9} \mathrm{CHO}\right) \\
{[\mathrm{mbar}]}\end{array}$ & $\begin{array}{c}\mathrm{p}\left(\mathrm{C}_{2} \mathrm{H}_{4}\right) \\
{[\mathrm{mbar}]}\end{array}$ & $\begin{array}{c}\mathrm{p}\left(1-\mathrm{C}_{5} \mathrm{H}_{10}\right) \\
{[\mathrm{mbar}]}\end{array}$ & $\begin{array}{c}\mathrm{p}\left(\mathrm{C}_{4} \mathrm{H}_{6} \mathrm{O}\right) \\
{[\mathrm{mbar}]}\end{array}$ \\
\hline 0,0135 & 0,0012 & 0,0028 & 0,0052 & 0,003 & 0,005 & 0,003 \\
\hline 0,012 & $7,5 \mathrm{E}-4$ & 0,0021 & 0,0044 & 0,0023 & 0,0029 & 0,0024 \\
\hline 0,0076 & $5 \mathrm{E}-4$ & 0,0011 & 0,0039 & 0,0015 & 0,0029 & 0,0015 \\
\hline 0,017 & 0,0016 & 0,0034 & 0,0084 & 0,0037 & 0,0048 & 0,0037 \\
\hline 0,0157 & 0,0012 & 0,0022 & 0,0062 & 0,0028 & 0,0038 & 0,0028 \\
\hline 0,011 & $8,9 \mathrm{E}-4$ & 0,0015 & 0,0045 & 0,00184 & 0,003 & 0,00184 \\
\hline 0,0093 & $5,4 \mathrm{E}-4$ & 0,0016 & 0,0029 & 0,0016 & 0,0019 & 0,0016 \\
\hline 0,0059 & $3 \mathrm{E}-4$ & 0,0012 & 0,0021 & $9,2 \mathrm{E}-4$ & 0,0018 & $9,8 \mathrm{E}-4$ \\
\hline 0,0037 & $2 \mathrm{E}-4$ & $8,4 \mathrm{E}-4$ & 0,0019 & $5,8 \mathrm{E}-4$ & $9,5 \mathrm{E}-4$ & $6 \mathrm{E}-4$ \\
\hline 0,0027 & $1,3 \mathrm{E}-4$ & $3,5 \mathrm{E}-4$ & $9,3 \mathrm{E}-4$ & $3,5 \mathrm{E}-4$ & $7 \mathrm{E}-4$ & $4,5 \mathrm{E}-4$ \\
\hline 0,0041 & $2,4 \mathrm{E}-4$ & $5,2 \mathrm{E}-4$ & 0,0014 & $8 \mathrm{E}-4$ & 0,0014 & $9 \mathrm{E}-4$ \\
\hline
\end{tabular}

Tabelle 4.6: Reaktion c- $\mathrm{C}_{6} \mathrm{H}_{11}+\mathrm{O}$, Messwerte 4 mbar. $p\left(c-\mathrm{C}_{5} \mathrm{H}_{10}\right): p\left(\mathrm{SO}_{2}\right): p\left(\mathrm{CFCl}_{3}\right):=$ 3:1:3; $p\left(c-C_{6} H_{12}\right)=0,05-0,2$ mbar; $T=298 \mathrm{~K}$; Badgas Argon. Die Werte sind Ergebnisse verschiedener Experimente, die alle bei dem angegebenen Vorläuferverhältnis durchgeführt wurden.

Die in Tabelle 4.6 angegebenen Werte sind in Abbildung 4.13 in der Form „Zunahme Produkte gegen Verbrauch des Vorläufers c- $\mathrm{C}_{6} \mathrm{H}_{12}$ " dargestellt. Aus der Abbildung geht hervor, dass die Produkte alle linear mit der Abnahme des Vorläufers ansteigen. Somit kann ein hoher Anteil an Sekundärphotolyse ausgeschlossen werden. Nach Abzug der weiteren Produkte verbleibt im Bereich von 3000 - $2950 \mathrm{~cm}^{-1}$ eine positive Absorptionsbande, die auf die Bildung einer $\mathrm{C}_{5}$ Spezies hindeutet. Eine eindeutige Zuordnung ist aber nicht möglich. Mögliche Kandidaten sind dabei 1-Penten und Methylcyclopentanon. Im Falle von Methylcyclopentanon deutet aber ein Vergleich des Reaktionsspektrums mit dem von Methylcyclopentanon im Bereich der $\mathrm{C}=\mathrm{O}$ Valenzschwingung auf einen geringen Beitrag dieses Reaktionskanals zur Gesamtreaktion hin. 


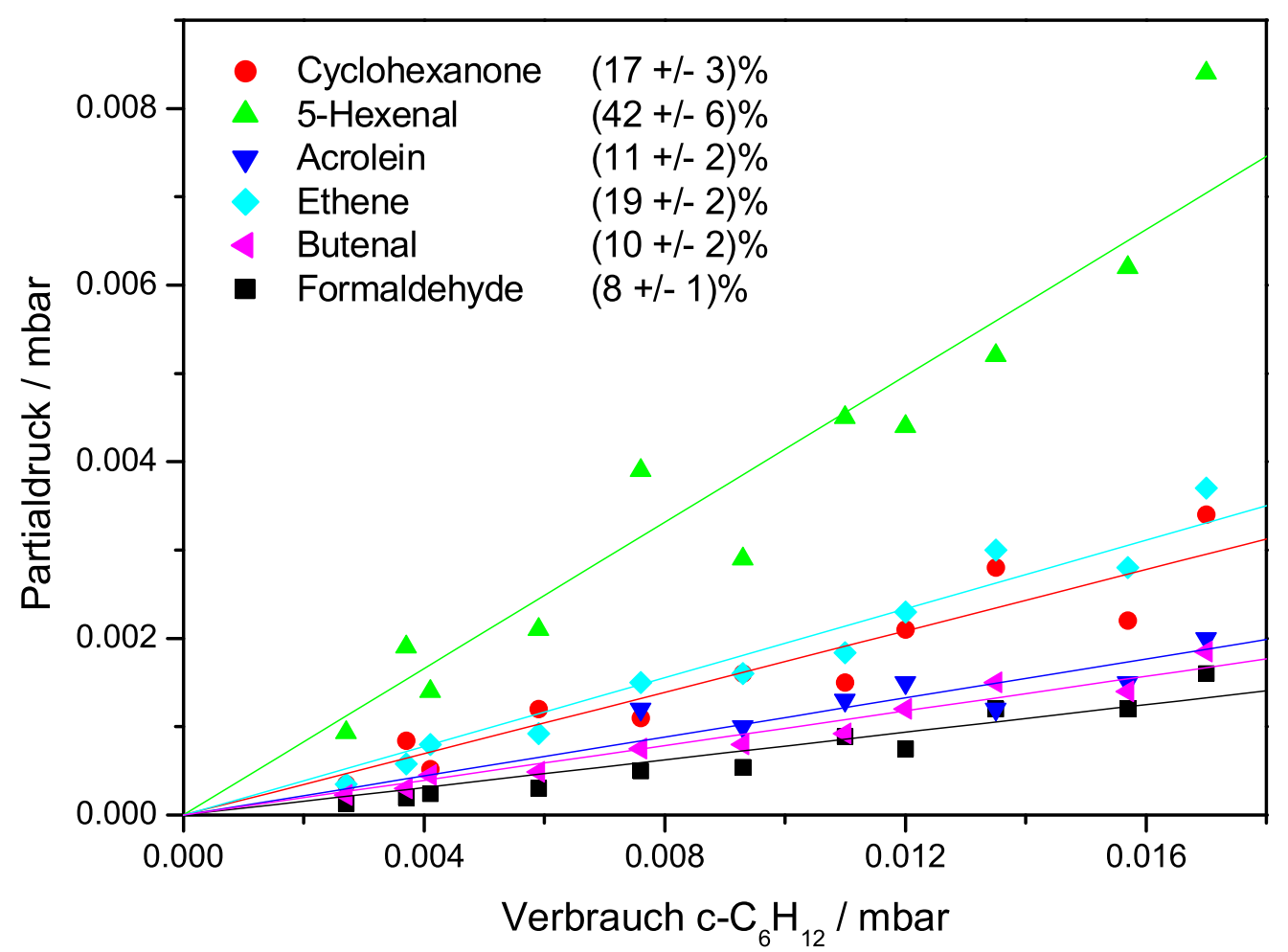

Abbildung 4.13: Reaktion $c-C_{6} H_{11}+O$, Partialdruck der Produkte in Abhängigkeit vom Vorläuferverbrauch; $p\left(c-C_{6} H_{12}\right): p\left(S_{2}\right): p\left(C_{F C l}\right):=3: 1: 3 ; p\left(c-C_{6} H_{12}\right)=0,05-0,2$ mbar; $p_{\text {ges }}=4$ mbar $\mathrm{T}=298 \mathrm{~K}$; Badgas Argon.

Die Ausbeuten wurde auf Grund der absoluten Produktkonzentrationen in Bezug auf den eingangs dargestellten Reaktionsmechanismus $\left(a_{1}\right)$ - $\left(a_{8}\right)$ berechnet. Für die quantitative Bestimmung der stabilen Produkte ergab sich folgendes relatives Verzweigungsverhältnis für den Zerfall des Cyclohexoxyradikals: $\left(\mathrm{a}_{1}\right) /\left(\mathrm{a}_{2}\right)=(19 \pm 3) /(81 \pm 9)$. Dementsprechend ist die Ringbruch-Reaktion unter $\beta$-C-C-Bindungsbruch $\left(\mathrm{a}_{2}\right)$ der Hauptkanal, wobei die H-Abspaltungs-Reaktion $\left(\mathrm{a}_{1}\right)$ ebenfalls eine Rolle zu spielen scheint. Die Detektion von 5-Hexenal $\left(\mathrm{C}_{5} \mathrm{H}_{9} \mathrm{CHO}\right)$, 3-Butenal $\left(\mathrm{C}_{4} \mathrm{H}_{6} \mathrm{O}\right)$ und Acrolein $\left(\mathrm{C}_{3} \mathrm{H}_{4} \mathrm{O}\right)$ deuten auf weitere unimolekulare Reaktionen des offenkettigen $\mathrm{C}_{6} \mathrm{H}_{11} \mathrm{O}$ Radikals hin. Dies sind zum Einen ein $\beta$-C-H-Bindungsbruch $\left(\mathrm{a}_{3}\right)$ und zum Anderen Reaktionen, die durch einen $\beta$-C-C-Bruch initiiert werden $\left(\left(a_{4}\right)\right.$ und $\left.\left(a_{5}\right)\right)$, durch einen 1,4-H-shift $\left(\left(a_{6}\right)\right.$ und $\left.\left(a_{7}\right)\right)$ und einen 1,5-Hshift $\left(\mathrm{a}_{8}\right)$.

Dabei wurden folgende Verzweigungsverhältnisse bestimmt: $\left[\mathrm{C}_{5} \mathrm{H}_{9} \mathrm{CHO}\right] /\left[\mathrm{C}_{4} \mathrm{H}_{6} \mathrm{O}\right] /\left[\mathrm{C}_{3} \mathrm{H}_{4} \mathrm{O}\right]$ $=\left(\mathrm{a}_{3}\right) /\left(\left(\mathrm{a}_{4}\right)+\left(\mathrm{a}_{6}\right)\right) /\left(\mathrm{a}_{8}\right)=1 / 0,23 / 0,28$. 
Der Anteil der Reaktionskanäle zu 1-Penten und Methylcyclopentanon ist durch Vergleich mit der Abnahme des Vorläufers Cyclohexan auf $21 \%$ beschränkt. Für die weitere Betrachtung wird der Beitrag des Reaktionskanals zum 1-Penten $\left(a_{7}\right)$ auf einen Wert von $(10 \pm 5) \%$ abgeschätzt. Damit lassen sich die Verzeigungsverhältnisse für den Zerfall des Cyclohexoxy Radikals wie folgt zusammenfassen: $\left(a_{1}\right) /\left(a_{3}\right) /\left(a_{4}\right)+\left(a_{6}\right) /\left(a_{7}\right) /\left(a_{8}\right)=(19 \pm$ $3) \% /(47 \pm 6) \% /(11 \pm 2) \% /(10 \pm 5) \% /(13 \pm 2) \%$.

Um die Druckabhängigkeit der Reaktion zu untersuchen, wurden Experimente auch bei einem Gesamtdruck von 1 bar durchgeführt. Dazu wurden Reaktionsspektren bei denselben Partialdruckverhältnissen der Vorläufer wie bei einem Gesamtdruck von 4 mbar aufgenommen, wobei zuerst die Vorläufer eingefüllt wurden und dann Argon bis zu dem gewünschten Druck hinzugegeben wurde. Die Reaktionsspektren zeigen keine zusätzlichen Produkte und keine Änderung der Verzweigungsverhältnisse innerhalb der experimentellen Fehlergrenzen. Für die quantitative Bestimmung wurden dabei, wie für die Untersuchungen bei 4 mbar, Kalibrierspektren bei einem Gesamtdruck von 1 bar aufgenommen und daraus die integralen Absorptionskoeffizienten bestimmt.

\subsubsection{Simulation}

Um den Einfluss von Sekundärreaktionen auf das Verzweigungsverhältnis zu bestimmen und die Fehlergrenzen für die Reaktionskanäle zu verifizieren, wurde eine Simulation der Konzentrations-Zeit-Profile anhand eines empirischen Mechanismus durchgeführt. Für die Simulation wurde ein Satz von 25 Reaktionen verwendet, in der die Ergebnisse der Experimente eingearbeitet wurden. Dabei wurde der von NACKE bestimmte, nahe$\mathrm{zu}$ temperaturunabhängige Geschwindigkeitskoeffizient $\mathrm{k}\left(\mathrm{c}-\mathrm{C}_{6} \mathrm{H}_{11}+\mathrm{O} \rightarrow\right.$ Produkte $)=$ $(1,32 \pm 0,1) \times 10^{14}(T / 298 K)^{(0,09 \pm 0,13)}$ in angepasster Form verwendet [Nac98]. Die verwendeten Reaktionen zusammen mit den Geschwindigkeitskoeffizienten sind in Tabelle 4.7 dargestellt. Die Simulationsrechnungen zeigen, dass der Einfluss der Sekundärreaktionen das Verhältnis der Hauptproduktkanäle nur im Rahmen der Fehlergrenzen verändert. Dieser geringe Einfluss der Sekundärreaktionen auf das Kanalverzweigungsverhältnis unter Sauerstoff-Atom-Unterschuss zeigte sich auch bei der Simulation anderer Reaktionssysteme wie z.B. $\mathrm{c}-\mathrm{C}_{5} \mathrm{H}_{9}+\mathrm{O}$. Auf Grund der guten Übereinstimmung von Experiment 
und Simulation scheinen die experimentell bestimmten Verzweigungsverhältnisse für einen Vergleich mit theoretischen Vorhersagen geeignet zu sein.

\begin{tabular}{|c|c|c|c|c|c|}
\hline Reaktion & & & & & $\mathrm{cm}^{3} /(\mathrm{mol} \cdot \mathrm{s})$ \\
\hline $\mathrm{OH}$ & $+\mathrm{O}$ & $\rightarrow \mathrm{O}_{2}$ & $+\mathrm{H}$ & + & $2,00 \cdot 10^{13}$ \\
\hline $\mathrm{OH}$ & $+\mathrm{H}$ & $\rightarrow \mathrm{H}_{2} \mathrm{O}$ & $+\mathrm{H}$ & + & $1,00 \cdot 10^{10}$ \\
\hline $\mathrm{HCO}$ & + & $\rightarrow \mathrm{H}$ & $+\mathrm{CO}$ & + & $7,00 \cdot 10^{14}$ \\
\hline $\mathrm{c}-\mathrm{C}_{6} \mathrm{H}_{11}$ & $+\mathrm{O}$ & $\rightarrow \mathrm{c}-\mathrm{C}_{6} \mathrm{H}_{10} \mathrm{O}$ & $+\mathrm{H}$ & + & $2,51 \cdot 10^{13}$ \\
\hline $\mathrm{c}-\mathrm{C}_{6} \mathrm{H}_{11}$ & $+\mathrm{O}$ & $\rightarrow \mathrm{C}_{5} \mathrm{H}_{9} \mathrm{CHO}$ & $\mathrm{O}+\mathrm{H}$ & + & $6,20 \cdot 10^{13}$ \\
\hline $\mathrm{c}-\mathrm{C}_{6} \mathrm{H}_{11}$ & $+\mathrm{O}$ & $\rightarrow \mathrm{C}_{4} \mathrm{H}_{6} \mathrm{O}$ & $+\mathrm{C}_{2} \mathrm{H}_{4}$ & $+\mathrm{H}$ & $1,45 \cdot 10^{13}$ \\
\hline $\mathrm{c}-\mathrm{C}_{6} \mathrm{H}_{11}$ & $+\mathrm{O}$ & $\rightarrow 1-\mathrm{C}_{5} \mathrm{H}_{10}$ & $+\mathrm{HCO}$ & + & $1,32 \cdot 10^{13}$ \\
\hline $\mathrm{c}-\mathrm{C}_{6} \mathrm{H}_{11}$ & $+\mathrm{O}$ & $\rightarrow \mathrm{C}_{3} \mathrm{H}_{7}$ & $+\mathrm{C}_{3} \mathrm{H}_{4} \mathrm{C}$ & + & $1,72 \cdot 10^{13}$ \\
\hline c- $\mathrm{C}_{6} \mathrm{H}_{12}$ & $+\mathrm{Cl}$ & $\rightarrow \mathrm{c}-\mathrm{C}_{6} \mathrm{H}_{11}$ & $+\mathrm{HCl}$ & + & $1,85 \cdot 10^{14}$ \\
\hline $\mathrm{c}-\mathrm{C}_{6} \mathrm{H}_{12}$ & $+\mathrm{O}$ & $\rightarrow \mathrm{c}-\mathrm{C}_{6} \mathrm{H}_{11}$ & $+\mathrm{OH}$ & + & $5,84 \cdot 10^{10}$ \\
\hline $\mathrm{c}-\mathrm{C}_{6} \mathrm{H}_{12}$ & $+\mathrm{H}$ & $\rightarrow \mathrm{c}-\mathrm{C}_{6} \mathrm{H}_{11}$ & $+\mathrm{H}_{2}$ & + & $2,80 \cdot 10^{12}$ \\
\hline $\mathrm{c}-\mathrm{C}_{6} \mathrm{H}_{12}$ & $+\mathrm{OH}$ & $\rightarrow \mathrm{c}-\mathrm{C}_{6} \mathrm{H}_{11}$ & $+\mathrm{H}_{2} \mathrm{O}$ & + & $4,63 \cdot 10^{12}$ \\
\hline $\mathrm{c}-\mathrm{C}_{6} \mathrm{H}_{10} \mathrm{O}$ & $+\mathrm{OH}$ & $\rightarrow$ Produkte & + & + & $3,85 \cdot 10^{12}$ \\
\hline $\mathrm{c}-\mathrm{C}_{6} \mathrm{H}_{10} \mathrm{O}$ & $+\mathrm{Cl}$ & $\rightarrow$ Produkte & $+\mathrm{HCl}$ & + & $4,22 \cdot 10^{13}$ \\
\hline $\mathrm{c}-\mathrm{C}_{6} \mathrm{H}_{10} \mathrm{O}$ & $+\mathrm{O}$ & $\rightarrow$ Produkte & + & + & $1,40 \cdot 10^{10}$ \\
\hline $\mathrm{C}_{2} \mathrm{H}_{4}$ & $+\mathrm{O}$ & $\rightarrow$ Produkte & + & + & $5,48 \cdot 10^{11}$ \\
\hline $\mathrm{C}_{2} \mathrm{H}_{4}$ & $+\mathrm{H}$ & $\rightarrow \mathrm{C}_{2} \mathrm{H}_{3}$ & $+\mathrm{H}_{2}$ & + & $6,03 \cdot 10^{03}$ \\
\hline $\mathrm{C}_{2} \mathrm{H}_{4}$ & $+\mathrm{H}$ & $\rightarrow \mathrm{C}_{2} \mathrm{H}_{5}$ & + & + & $6,59 \cdot 10^{11}$ \\
\hline $\mathrm{C}_{2} \mathrm{H}_{4}$ & $+\mathrm{OH}$ & $\rightarrow$ Produkte & + & + & $5,66 \cdot 10^{12}$ \\
\hline $\mathrm{C}_{2} \mathrm{H}_{3}$ & $+\mathrm{H}$ & $\rightarrow \mathrm{C}_{2} \mathrm{H}_{4}$ & + & + & $1,21 \cdot 10^{14}$ \\
\hline $\mathrm{C}_{2} \mathrm{H}_{3}$ & $+\mathrm{H}$ & $\rightarrow \mathrm{C}_{2} \mathrm{H}_{2}$ & $+\mathrm{H}_{2}$ & + & $1,21 \cdot 10^{13}$ \\
\hline $\mathrm{CH}_{3}$ & $+\mathrm{CH}_{3}$ & $\rightarrow \mathrm{C}_{2} \mathrm{H}_{6}$ & + & + & $2,40 \cdot 10^{13}$ \\
\hline $1-\mathrm{C}_{5} \mathrm{H}_{10}$ & $+\mathrm{O}$ & $\rightarrow$ Produkte & + & + & $1,78 \cdot 10^{12}$ \\
\hline $1-\mathrm{C}_{5} \mathrm{H}_{10}$ & $+\mathrm{H}$ & $\rightarrow$ Produkte & + & + & $8,31 \cdot 10^{11}$ \\
\hline $1-\mathrm{C}_{5} \mathrm{H}_{10}$ & $+\mathrm{O}$ & $\rightarrow$ Produkte & + & + & $1,88 \cdot 10^{13}$ \\
\hline
\end{tabular}

Tabelle 4.7: Reaktion $c-\mathrm{C}_{6} \mathrm{H}_{11}+\mathrm{O}$, Reaktionen und Geschwindigkeitskoeffizienten für die Simulationsrechnungen. $\left[c-C_{6} H_{12}\right]_{0}=2 \times 10^{-8} \mathrm{~mol} / \mathrm{cm}^{3} ;[O]_{0}=4 \times 10^{-10} \mathrm{~mol} / \mathrm{cm}^{3} ;[\mathrm{Cl}]_{0}=$ $4 \times 10^{-10} \mathrm{~mol} / \mathrm{cm}^{3}$.

Die aus der Simulation gewonnenen Konzentrations-Zeit-Profile sind in Abbildung 4.14 dargestellt. Ein Vergleich der experimentell bestimmten Produktanteile der Reaktion mit 
den aus der Simulation gewonnen Daten ist in Tabelle 4.8 aufgeführt. Der niedrigere Ethenanteil bei der Simulation resultiert aus der Verwendung von Reaktionsgleichungen, die zu einem Abbau an Ethen führen. Möglicherweise wurden dabei die Geschwindigkeitskoeffizienten zu hoch angesetzt. Des Weiteren kann der Ethenanteil im Experiment auf Grund der möglichen Sekundärreaktion $\mathrm{C}_{2} \mathrm{H}_{5}+\mathrm{O} \rightarrow \mathrm{C}_{2} \mathrm{H}_{4}+\mathrm{OH}$ erhöht sein.

\begin{tabular}{lcc}
\hline Substanz & Experiment & Simulation \\
\hline $\mathrm{C}_{5} \mathrm{H}_{9} \mathrm{CHO}$ & $42 \pm 6$ & 47 \\
$\mathrm{c}_{-} \mathrm{C}_{6} \mathrm{H}_{10} \mathrm{O}$ & $17 \pm 3$ & 19 \\
$\mathrm{C}_{2} \mathrm{H}_{4}$ & $19 \pm 2$ & 11 \\
$\mathrm{C}_{4} \mathrm{H}_{6} \mathrm{O}$ & $10 \pm 2$ & 11 \\
$\mathrm{C}_{3} \mathrm{H}_{4} \mathrm{O}$ & $11 \pm 2$ & 13 \\
\hline
\end{tabular}

Tabelle 4.8: Reaktion $c_{-} \mathrm{C}_{6} \mathrm{H}_{11}+\mathrm{O}$, Vergleich der experimentell bestimmten Produktanteile mit den Daten der Simulation. Startkonzentrationen und verwendete Geschwindigkeitskoeffizienten für die Simulation sind in Tabelle 4.7 gegeben.

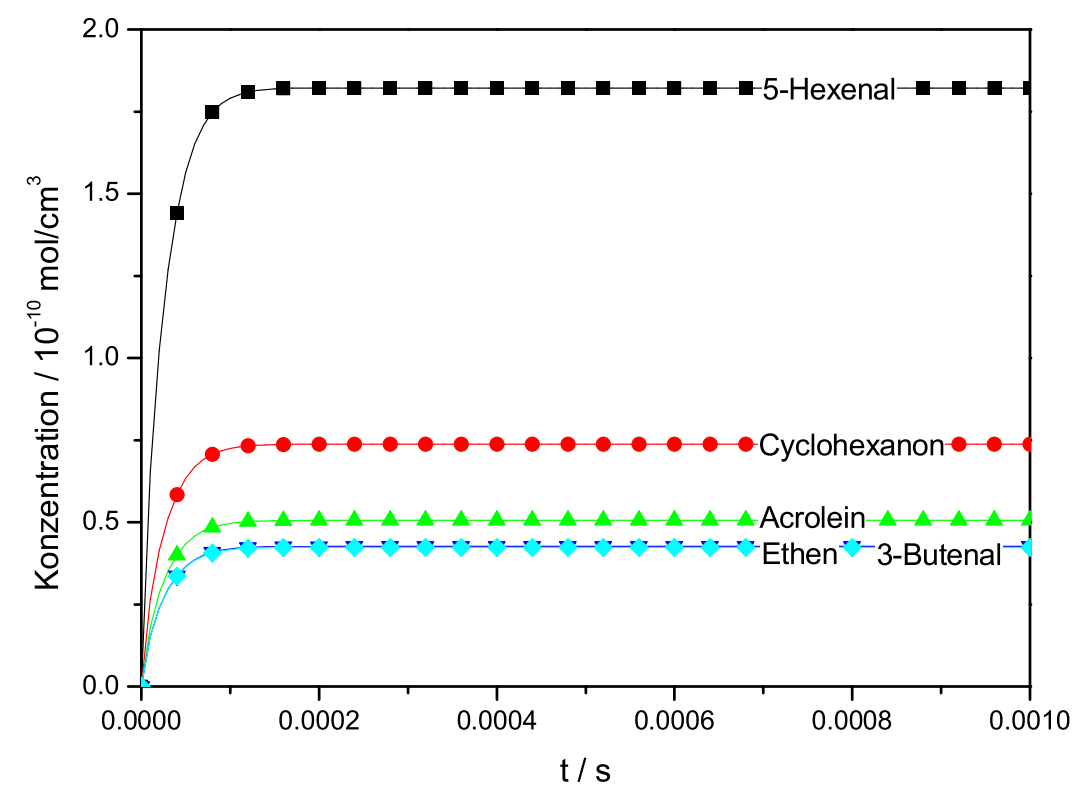

Abbildung 4.14: Reaktion $c_{-} \mathrm{C}_{6} \mathrm{H}_{11}+\mathrm{O}$, Simulation der Konzentrations-Zeit-Profile; $[\mathrm{O}]_{0}=$ $4 \times 10^{-10} \mathrm{~mol} / \mathrm{cm}^{3},[\mathrm{Cl}]_{0}=4 \times 10^{-10} \mathrm{~mol} / \mathrm{cm}^{3},\left[\mathrm{c}-C_{6} H_{11}\right]_{0}=2.0 \times 10^{-8} \mathrm{~mol} / \mathrm{cm}^{3}$ (die verwendeten Reaktionen und Geschindigkeitskoeffizienten sind in Tabelle 4.7 gegeben). 


\subsubsection{Quantenchemische Rechnungen}

Das Kanalverzweigungsverhältnis wird im Folgenden auf der Grundlage der Reaktionskanäle $\left(a_{1}\right)-\left(a_{8}\right)$ diskutiert. Die Rechnungen wurden von M. Olzmann und O. Welz im Rahmen einer zur Veröffentlichung eingereichten Arbeit [Hoy06b] an der TU Karlsruhe durchgeführt.

Die Energie wichtiger Zwischenprodukte und der Übergangszustände wurde auf dem G3MP2B3 Niveau berechnet. Die Geometrien und deren Frequenzen (skaliert mit dem Faktor 0,961) wurde auf B3LYP/6-31G(d) Niveau unter Verwendung des Gaussian 03 Programm Pakets bestimmt. Dabei wurden die in Abbildung 4.15 dargestellten Energiebarrieren für den Zerfall des chemisch aktivierten Cyclohexoxyradikals ( $\mathrm{E} \approx 375 \mathrm{~kJ} / \mathrm{mol}$ ) bestimmt.

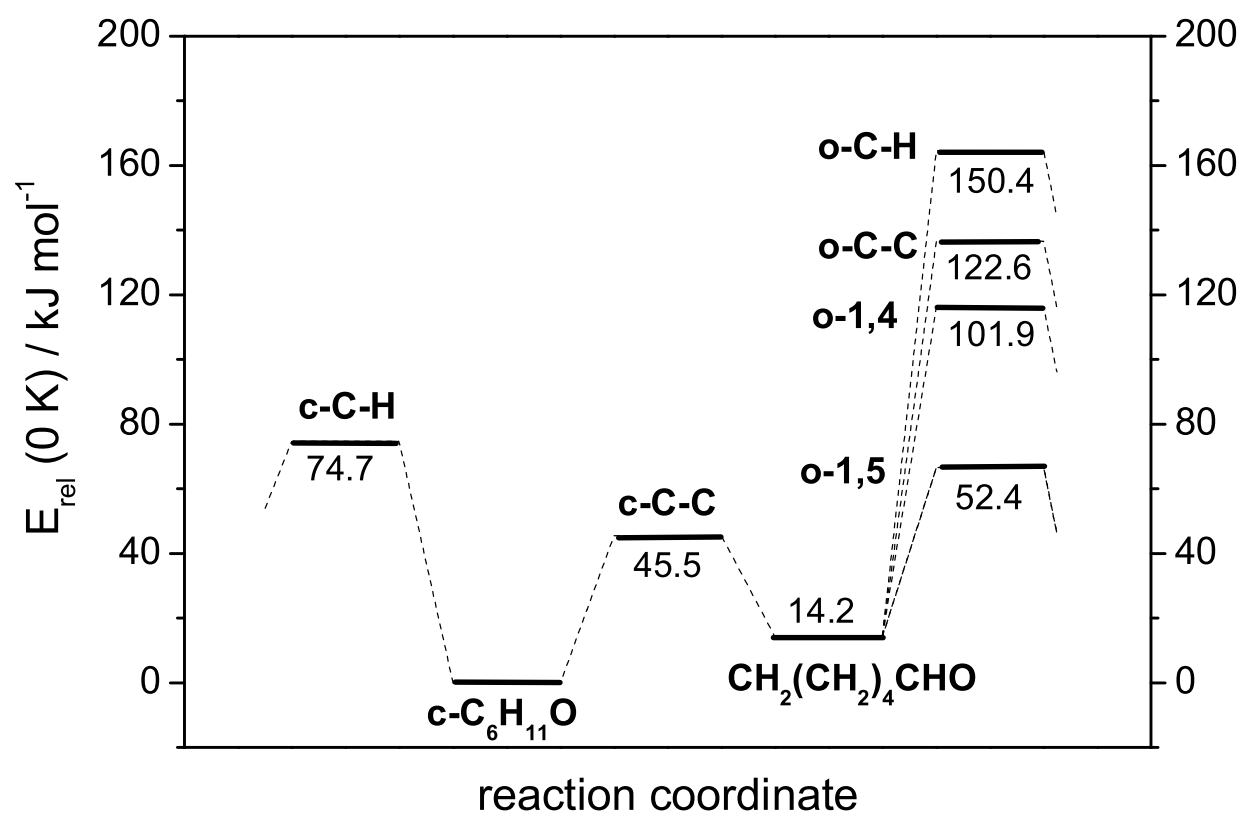

Abbildung 4.15: Reaktion c- $C_{6} H_{11}+O$, Energiediagramm erhalten aus den quantenchemischen Rechnungen (für Basissatz und Funktionen siehe Text). Die angegebenen Werte für die Energiebarrieren sind Grenzenergien. Dabei bedeutet: $c:=$ cyclisch, o:= offenkettig, o-1,5:=1,5-H-shift des offenkettigen Isomers, usw.

Für die Berechnungen wurde als stabilste Struktur des Cyclohexoxyradikals der äquatoriale Konformer angenommen. Wie die Abbildung zeigt, ist der $\beta$-C-C-Ringbruch $(\Delta \mathrm{E}=$ $45,5 \mathrm{~kJ} / \mathrm{mol})$ energetisch günstiger als der $\beta$-C-H-Bindungsbruch $(\Delta \mathrm{E}=74,7 \mathrm{~kJ} / \mathrm{mol})$. Des 
Weiteren wird als Folgereaktion für den Zerfall des offenkettigen Isomers ein 1,5-H-shift favorisiert. Ein weiterer C-C-Bindungsbruch, ohne vorherigen $H$-shift, wurde als energetisch ungünstig berechnet. Die mittels RRKM-Rechnungen erhaltenen Geschwindigkeitskoeffizienten für den Zerfall des Cyclohexoxyradikals sind in Abbildung 4.16 in Abhängigkeit von der Anregungsenergie dargestellt. Dabei sind ausgehend von dem stabilsten Konformer (äquatorialer Sessel) die Geschwindigkeitskoeffizienten für den $\beta$-C-H-Bindungsbruch und die C-C-Ringöffnung dargestellt.

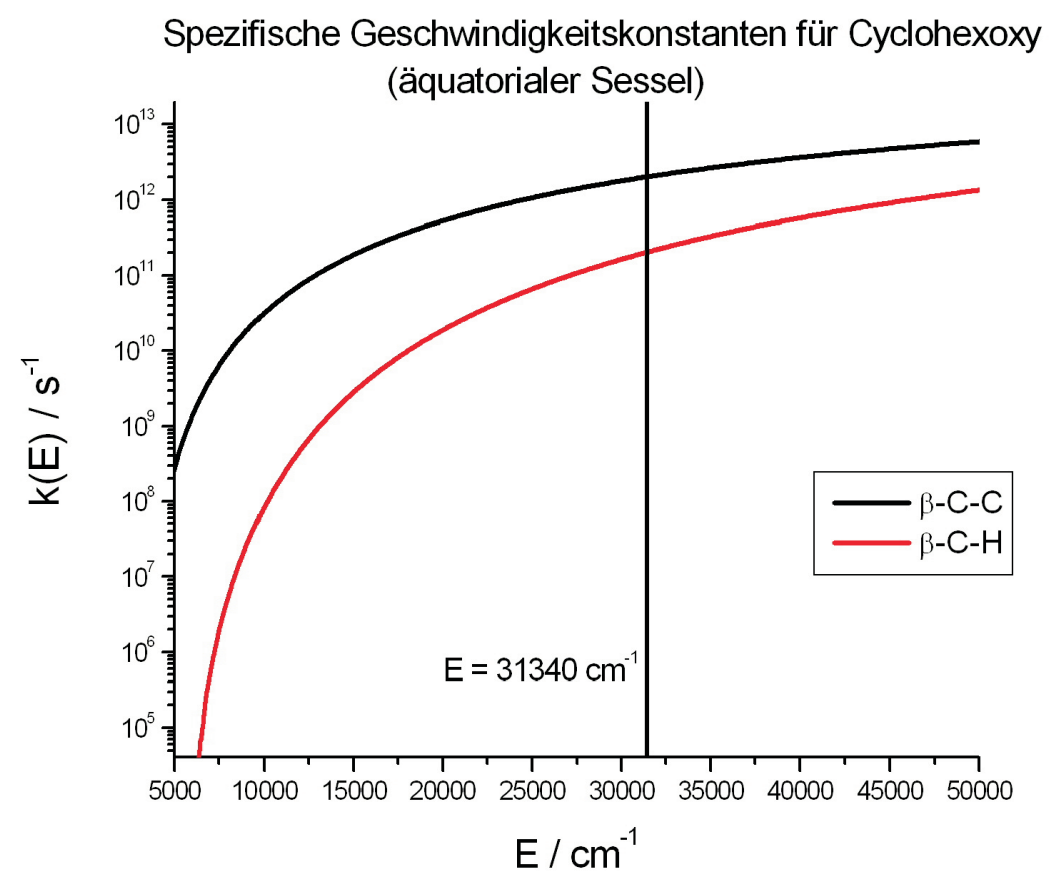

Abbildung 4.16: Reaktion c- $\mathrm{C}_{6} \mathrm{H}_{11}+\mathrm{O}$, Geschwindigkeit des Cyclohexoxyzerfalls aus $\mathrm{RRKM}$ Rechnungen in Abhängigkeit von der Anregungsenergie des Radikals. Die eingezeichnete Energie $E=31340 \mathrm{~cm}^{-1}$ entspricht der Energie des chemisch angeregten Intermediats aus der Reaktion $c-C_{6} H_{11}+O$.

In Abbildung 4.15 und 4.16 wird deutlich, dass der Ringbruch der vorrangige Reaktionskanal ist. Daher wurden für den weiteren Zerfall des offenkettigen Hexanal-6-yl-Radikals die Geschwindigkeitskoeffizienten mittels RRKM Theorie berechnet. Dabei wurden für den Zerfall des Hexanal-6-yl-Radikals die in der Abbildung 4.17 dargestellten Geschwindigkeitskoeffizienten in Abbhängigkeit von der im Isomer noch vorhandenen Energie erhalten. Diese Energie kann durch Stoßstabilisierung gemindert werden. Die Geschwindigkeitskoeffizienten $\mathrm{k}(\mathrm{i})(\langle E\rangle, J)$ beziehen sich dabei auf die mittlere Rotationsanregungsenergie (J $=60$ ) bei $\mathrm{T}=300 \mathrm{~K}$. Die Abbildung zeigt, dass aus RRKM-Rechnungen der weitere Zer- 
fall des offenkettigen Radikals unter $\beta$-C-C-Bindungsbruch der schnellste Reaktionsweg ist. Die möglichen $H$-shifts und die Rückreaktion unter Ringschluss sind zudem schneller als der Kanal zum 5-Hexenal. Letzterer wurde dagegen bei den Experimenten als dominant gefunden.

Aus der Abbildung wird ebenfalls ersichtlich, dass die Geschwindigkeiten im bzw. über dem Bereich der Geschwindigkeit der Stoßstabilisierung liegen und somit keine wesentliche Druckabhängigkeit zu erwarten ist.

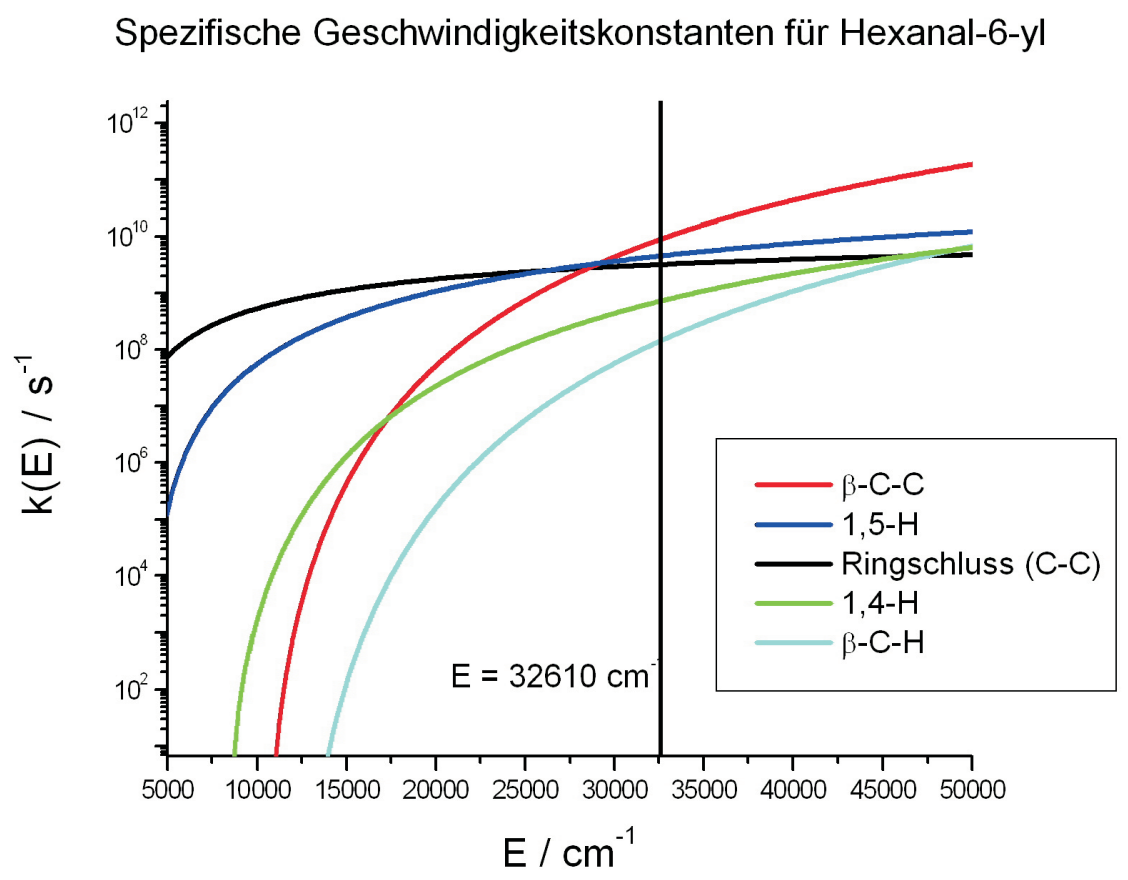

Abbildung 4.17: Reaktion c- $C_{6} H_{11}+O$, Geschwindigkeit des Hexanal-6-yl-Zerfalls aus RRKMRechnungen in Abhängigkeit von der Energie des Radikals. Die Energie $E=32610 \mathrm{~cm}^{-1}$ entspricht der mittleren Anregungsenergie des Hexanal-6-yl-Radikals.

Zusammenfassend wurden mit Hilfe der vorgestellten Ergebnisse der RRKM-Rechnungen die folgenden Geschwindigkeitskoeffizienten für die Reaktionskanäle bestimmt: 


\begin{tabular}{lr}
\hline Reaktion & Geschwindigkeit \\
\hline $\mathrm{a}_{1}$ & $2,0 \times 10^{11} \mathrm{~s}^{-1}$ \\
$\mathrm{a}_{2}$ & $4,1 \times 10^{12} \mathrm{~s}^{-1}$ \\
$-\mathrm{a}_{2}$ & $3,0 \times 10^{9} \mathrm{~s}^{-1}$ \\
o- 1,5 & $3,7 \times 10^{9} \mathrm{~s}^{-1}$ \\
o-C-C & $1,6 \times 10^{9} \mathrm{~s}^{-1}$ \\
o- 1,4 & $4,5 \times 10^{8} \mathrm{~s}^{-1}$ \\
o-C-H & $5,9 \times 10^{7} \mathrm{~s}^{-1}$ \\
\hline
\end{tabular}

Tabelle 4.9: Reaktion $c_{-} C_{6} H_{11}+O$, mittels $R R K M$ Theorie berechnete Geschwindigkeitskoeffizienten für den Zerfall des Cyclohexoyradikals bei $E \approx 375 \mathrm{~kJ} / \mathrm{mol}$ und $\mathrm{k}(\mathrm{i})(<E\rangle, J)$ bei $J=$ $60(T=300 \mathrm{~K})$.

Der Ringbruch stellt somit die dominierende Reaktion dar (>80\%) und die komplementäre Reaktion zum Cyclohexanon nimmt einen Anteil von kleiner als 20\% ein.

\subsubsection{Diskussion}

Die Ergebnisse der Reaktion Cyclohexyl $+\mathrm{O}$ werden im Folgenden mit verschiedenen Resultaten aus der Literatur verglichen.

Heinemann-Fiedler et al. [Hei90] haben die Reaktion mittels Massenspektrometrie untersucht. Dabei fanden sie Cyclohexen, 5-Hexenal und Cyclohexanon als Produkte der Reaktion. Das Verhältnis der drei Produktkanäle wurde abgeschätzt zu Cyclohexen : 5Hexenal : Cyclohexanon $=1: 1,8: 2,3$. Auf Grund massenspektrometrischer Überlagerungen müssen dabei aber große Fehlergrenzen angenommen werden und die Identifikation weiterer Produkte war nicht möglich. Insbesondere stand kein Referenzspektrum für 5Hexenal zur Verfügung.

WASHIDA et al. [Was82] benutzten für die Untersuchung der Reaktion einen StrömungsReaktor mit Photoionisations-Massenspektrometrie. Im Gegensatz zu dieser Arbeit verwendeten sie einen Sauerstoffüberschuss $\left([\mathrm{O}]_{0}>2 \times\left[\mathrm{c}-\mathrm{C}_{6} \mathrm{H}_{12}\right]_{0}\right)$, da die Cyclohexylradikale mittels der Reaktion $\mathrm{c}_{-} \mathrm{C}_{6} \mathrm{H}_{12}+\mathrm{O} \rightarrow \mathrm{c}_{-} \mathrm{C}_{6} \mathrm{H}_{11}+\mathrm{OH}$ gebildet wurden. So liegt den Experimenten von WASHIDA et al. folgender Reaktionsmechanismus zu Grunde:

$$
\begin{aligned}
& \mathrm{c}-\mathrm{C}_{6} \mathrm{H}_{12}+\mathrm{O} \rightarrow \mathrm{c}^{-} \mathrm{C}_{6} \mathrm{H}_{11}+\mathrm{OH} \\
& \mathrm{c}-\mathrm{C}_{6} \mathrm{H}_{11}+\mathrm{O} \rightarrow \mathrm{c}-\mathrm{C}_{6} \mathrm{H}_{10}+\mathrm{OH} \quad\left(\mathrm{k}_{a b s}\right)
\end{aligned}
$$




$$
\mathrm{c}-\mathrm{C}_{6} \mathrm{H}_{11}+\mathrm{O} \rightarrow \mathrm{c}-\mathrm{C}_{6} \mathrm{H}_{11} \mathrm{O} \quad\left(\mathrm{k}_{\text {add. }}\right)
$$

Das Verhältnis vom H-Abstraktionsprodukt Cyclohexen $\left(\mathrm{k}_{a b s .}\right)$ zum O-Atom AdditionsProdukt $\left(\mathrm{k}_{\text {add. }}\right)$ wurde bestimmt $\mathrm{zu}\left(\mathrm{k}_{a b s .}\right) /\left(\mathrm{k}_{a d d}\right)=52 / 48$. Dabei wurde von WASHIDA et al. lediglich eine Ausbeute von 3\% Cyclohexanon festgestellt. Demnach zerfallen 45\% des angeregten Cyclohexoxyradikals unter Ringbruch.

Um den möglichen Einfluss des Sauerstoffatom-Überschusses auf den Reaktionsmechanismus zu überprüfen, wurden die Reaktionsbedingungen der Experimente von WASHIDA et al. kopiert. D.h. es wurden Photolysen von c- $\mathrm{C}_{6} \mathrm{H}_{12} / \mathrm{SO}_{2}$ Gemischen in Abwesenheit von $\mathrm{CFCl}_{3}$ vorgenommen, bei denen ein hoher $\mathrm{SO}_{2}$ Überschuss vorlag. Eine Analyse der Reaktionsspektren dieser Vorläuferbedingungen zeigte, im Gegensatz zu der Arbeit von WAshida et al., einen Anstieg von Cyclohexanon und einen Abfall an 5-Hexenal gegenüber den Reaktionsbedingungen mit $\mathrm{CFCl}_{3}$. Die beschriebene Bildung von Cyclohexen konnte auch hier nicht nachgewiesen werden. Folglich ist zu vermuten, dass die Photolyse in $\mathrm{Abwesenheit}$ von $\mathrm{CFCl}_{3}$ den Reaktionsmechanismus zwar ändert, aber nicht in der von Washida et al. berichteten Weise. D.h. Erhöhung des Ringbruchkanals und überwiegende Bildung des H-Abstraktionsproduktes Cyclohexen. Simulationsrechnungen zu den Reaktionsbedingungen von WASHIDA et al. deuten auf einen wesentlichen Einfluss der Reaktion von Cyclohexylradikalen mit molekularem Sauerstoff hin. WASHIDA berichtet, dass die Reaktion von dabei gebildeten Cyclohexylperoxy-Radikalen mit atomarem Sauerstoff zu Cyclohexen, $\mathrm{OH}$ und $\mathrm{O}_{2}$ zu einem Anteil von 25\% stattfindet. Dies kann eine mögliche Erklärung für den hohen Cyclohexenanteil sein, da auf Grund der Bildung von Sauerstoffatomen aus molekularem Sauerstoff mittels Mikrowellenentladung der $\mathrm{O}_{2^{-}}$ Partialdruck bei allen Experimenten sehr hoch war.

Platz et al. [Pla99] haben den Zerfall des Cyclohexoxyradikals im Rahmen der Reaktion c- $\mathrm{C}_{6} \mathrm{H}_{11}+\mathrm{O}_{2} / \mathrm{NO} / \mathrm{NO}_{2}$ untersucht. Bei $296 \mathrm{~K}$ und einem Gesamtdruck von $1 \mathrm{~atm}$ Luft fanden sie für die Reaktion zum Einen den Zerfallskanal $\left(\mathrm{k}_{\text {dec. }}\right)$ und zum Anderen Produkte der Reaktion mit molekularem Sauerstoff (Bildung von Cyclohexanon $\left(\mathrm{k}_{\text {reac. }}\right)$ ). Das Verhältnis der beiden Kanäle wurde $\mathrm{zu}\left(\mathrm{k}_{\text {dec. }}\right) /\left(\mathrm{k}_{\text {reac. }}\right)=61 / 39$ bestimmt. Dabei wurde die Möglichkeit der Bildung von Cyclohexanon anhand direkter H-Abspaltung des Cyclohexoyradikals allerdings nicht berücksichtigt. 
Für die Reaktion Cyclohexyl + O ergibt sich aus den vorliegenden Messungen folgendes Kanalverzweigungsverhältnis:

$$
\left.\begin{array}{rlrl}
\mathrm{c}-\mathrm{C}_{6} \mathrm{H}_{11}+\mathrm{O} & \longrightarrow \mathrm{c}-\mathrm{C}_{6} \mathrm{H}_{11} \mathrm{O}^{\star} & \\
\mathrm{c}-\mathrm{C}_{6} \mathrm{H}_{11} \mathrm{O}^{\star} & \longrightarrow \mathrm{c}-\mathrm{C}_{6} \mathrm{H}_{10} \mathrm{O}+\mathrm{H} & (19 \pm 3) \% \\
& \longrightarrow \mathrm{CH}_{2}\left(\mathrm{CH}_{2}\right)_{4} \mathrm{CHO} & \\
\mathrm{CH}_{2}\left(\mathrm{CH}_{2}\right)_{4} \mathrm{CHO} & \longrightarrow \mathrm{C}_{5} \mathrm{H}_{9} \mathrm{CHO}+\mathrm{H} & (47 \pm 6) \% \\
& \longrightarrow \mathrm{C}_{2} \mathrm{H}_{4}+\mathrm{C}_{4} \mathrm{H}_{6} \mathrm{O}+\mathrm{H} \\
& \longrightarrow \mathrm{C}_{2} \mathrm{H}_{5}+\mathrm{C}_{4} \mathrm{H}_{6} \mathrm{O}
\end{array}\right\}\left(\begin{array}{r}
(11 \pm 2) \% \\
(10 \pm 5) \% \\
\end{array}\right.
$$

Im Vergleich zu den quantenchemischen Rechnungen zeigt sich, dass die Reaktionskanäle qualitativ übereinstimmend beschrieben werden können. Eine quantitative Übereinstimmung ist aber nicht gegeben. Wie auch bei der Reaktion Cyclopentyl + O weichen Experiment und quantenchemische Rechnung vor allem in der Beschreibung des Kanals $\left(a_{3}\right)$ unter Bildung von 5-Hexenal bzw. 4-Pentenal voneinander ab. Um die Übereinstimmung und Abweichung von Experiment und Quantenchemischer Rechnung deutlich zu machen, sind die Ergebnisse nochmals abschließend in Tabelle 4.10 dargestellt. 


\begin{tabular}{|c|c|c|c|c|}
\hline Kanal & & Rechnung & Experiment & Identifiziert \\
\hline c-C-H & $\left(a_{1}\right)$ & 6.9 & $19 \pm 3$ & c-Hexanon \\
\hline $\mathrm{c}-\mathrm{C}-\mathrm{C}$ & $\left(a_{2}\right)$ & 93.1 & & \\
\hline $\mathrm{o}-\mathrm{C}-\mathrm{H}$ & $\left(a_{3}\right)$ & 1.0 & $47 \pm 6$ & 5-Hexenal \\
\hline o-C-C & $\begin{array}{l}\left(a_{4}\right) \\
\left(a_{5}\right)\end{array}$ & 26.3 & $11 \pm 2-x$ & 3-Butenal \\
\hline \multirow{2}{*}{ o- 1,4} & $\left(a_{6}\right)$ & 3.1 & $\mathrm{x}$ & 3-Butenal \\
\hline & $\left(a_{7}\right)$ & 4.2 & $10 \pm 5$ & 1-Penten \\
\hline $\mathrm{o}-1,5$ & $\left(a_{8}\right)$ & 58.5 & $13 \pm 2$ & Acrolein \\
\hline Summe & & 100 & 100 & \\
\hline
\end{tabular}

Tabelle 4.10: Berechnete und experimentell bestimmte relative Kanalverzweigung für den unimolekularen Zerfall der zyklischen (c-) und offenkettigen (o-) Radikalspezies.

\subsubsection{Die Reaktion Cyclooktyl + O}

\subsubsection{Einleitung}

Die Reaktion Cyclooktyl + O wurde im Rahmen der Examensarbeit von S. MAARFELD untersucht [Maa06]. Die Ergebnisse sind zusammen mit den Resultaten der Reaktion Cyclohexyl $+\mathrm{O}$ zur Veröffentlichung eingereicht [Hoy06b]. Auf Grund der im Vergleich zum Cyclopentyl- oder Cyclohexylradikal großen C-Atomanzahl sind für die Reaktion eine Vielzahl von Reaktionskanälen denkbar. Diese hohe Anzahl an Reaktionskanälen macht die Analyse, neben Kondensationseffekten auf Grund der geringen Sättigungsdampfdrücke der Produkte, gegenüber den vorhergehenden Experimenten schwierig. Mögliche Reaktionskanäle für die Oxidation des Cyclooktylradikals sind unter Berücksichtigung der quantenchemischen Rechnungen im Folgenden dargestellt: 


$$
\begin{array}{rlr}
\mathrm{c}-\mathrm{C}_{8} \mathrm{H}_{15}+\mathrm{O} & \longrightarrow \mathrm{c}-\mathrm{C}_{8} \mathrm{H}_{15} \mathrm{O}^{\star} & (a) \\
\mathrm{c}-\mathrm{C}_{8} \mathrm{H}_{15} \mathrm{O}^{\star} & \longrightarrow \mathrm{c}-\mathrm{C}_{8} \mathrm{H}_{14} \mathrm{O}+\mathrm{H} & \left(a_{1}\right) \\
& \longrightarrow \mathrm{CH}_{2}\left(\mathrm{CH}_{2}\right)_{6} \mathrm{CHO} & \left(a_{2}\right) \\
\mathrm{CH}_{2}\left(\mathrm{CH}_{2}\right)_{6} \mathrm{CHO} & \longrightarrow \mathrm{C}_{7} \mathrm{H}_{13} \mathrm{CHO}+\mathrm{H} \\
& \longrightarrow \mathrm{C}_{2} \mathrm{H}_{4}+\mathrm{C}_{6} \mathrm{H}_{10}+\mathrm{H} & \left(a_{4}\right) \\
& \longrightarrow 2 \mathrm{C}_{2} \mathrm{H}_{4}+\mathrm{C}_{4} \mathrm{H}_{6} \mathrm{O}+\mathrm{H} & \left(a_{5}\right) \\
& \longrightarrow \mathrm{C}_{2} \mathrm{H}_{5}+\mathrm{C}_{6} \mathrm{H}_{10} \mathrm{O} & \left(a_{6}\right) \\
& \longrightarrow \mathrm{C}_{5} \mathrm{H}_{10}+\mathrm{C}_{3} \mathrm{H}_{4} \mathrm{O}+\mathrm{H} & \left(a_{7}\right) \\
& \longrightarrow \mathrm{C}_{3} \mathrm{H}_{7}+\mathrm{C}_{5} \mathrm{H}_{8} \mathrm{O} & \left(a_{8}\right) \\
\mathrm{C}_{6} \mathrm{H}_{12}+\mathrm{CH}_{2} \mathrm{CHO} & \left(a_{9}\right) \\
\mathrm{c}-\mathrm{C}_{6} \mathrm{H}_{11}+\mathrm{O} & \longrightarrow \mathrm{C}_{4} \mathrm{H}_{9}+\mathrm{C}_{4} \mathrm{H}_{6} \mathrm{O} & \left(a_{10}\right) \\
& \longrightarrow \mathrm{C}_{7} \mathrm{H}_{14}+\mathrm{C}_{8} \mathrm{H}_{14}+\mathrm{OH} & \left(a_{11}\right) \\
& & (b)
\end{array}
$$

Das Reaktionsschema macht die Komplexität dieser Reaktion deutlich. Doch auch hier können die Reaktionen in den Abstraktionsprozess, unter Bildung von Cyclookten $+\mathrm{OH}$ (b), und Zerfälle des hoch angeregten Cyclooktoxyradikals (a) zusammengefasst werden. Für den Zerfall des angeregeten Cyclooktoxyradikals ( $\mathrm{E} \approx 375 \mathrm{~kJ} / \mathrm{mol}$ ) kommt zum Einen ein $\beta$-C-H-Bindungsbruch $\left(\mathrm{a}_{1}\right)$ unter Bewahrung der Ringstruktur und zum Anderen eine Ringöffnung $\left(\mathrm{a}_{2}\right)$ mit anschließenden C-C- und C-H-Bindungsbrüchen in Frage. Des Weiteren sind verschiedene $H$-shifts zu erwarten, die zur Bildung stabilerer Isomere führen, welche dann weiter zerfallen können.

\subsubsection{Qualitative Analyse}

Die Analyse stabiler Endprodukte und deren quantitative Bestimmung wurde mit der in Abschnitt 3.1.1 beschriebenen FTIR Apparatur vorgenommen. Dabei wurden die Cyclooktylradikale durch die Reaktion von Cycloktan mit Cl Atomen, welcher aus der photolytischen Spaltung von $\mathrm{CFCl}_{3}$ bei $193 \mathrm{~nm}$ gewonnen wurden, erzeugt. Die Sauerstoffatome wurden durch Co-photolyse bei derselben Wellenlänge aus $\mathrm{SO}_{2}$ hergestellt. 
Das experimentelle Verfahren entspricht im Wesentlichen dem in dem vorhergehendem Kapitel beschriebenem.

Die Reaktionsspektren wurden wie in Abschnitt 3.3.1 angegeben ausgewertet. Eine Darstellung der qualitativen wie auch der quantitativen Analyse erfolgt auf Grund der ausführlichen Darstellung bei [Maa06] nur kurz. Um die Komplexität der Produktanalyse zu demonstrieren, sind hier die identifizierten Substanzen im Bereich der Carbonylstreckschwingung um $1730 \mathrm{~cm}^{-1}$ und das Reaktionsspektrum sowie die Summe der Reinspektren dargestellt. Die Abbildung 4.18 (entnommen [Maa06]) zeigt die gute Übereinstimmung im Bereich der Carbonylschwingung.

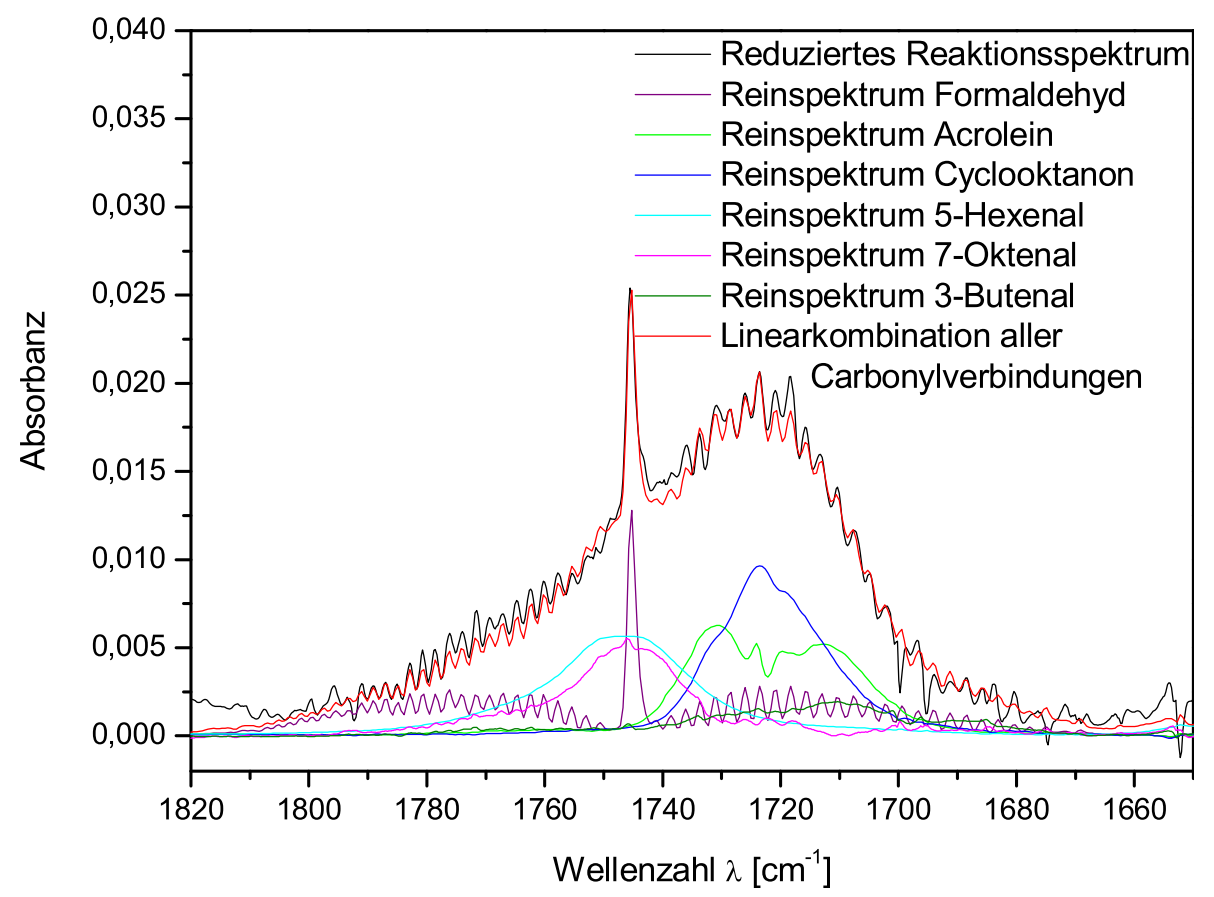

Abbildung 4.18: Reaktion c- $\mathrm{C}_{8} \mathrm{H}_{15}+\mathrm{O}$, qualitative Analyse. Die Abbildung zeigt die gute Übereinstimmung im Bereich der Carbonylschwingung von Reaktionsspektrum und der Summe der Reinspektren. Die Abbildung wurde der Examensarbeit von S. MAARFELD [Maa06] entnommen.

In der Arbeit von MAARFELD wird insbesondere die hier nicht beschriebene Identifikation von Cyclookten gezeigt. Außerdem wird in dieser Arbeit auf die Problematik der qualitativen und quantitativen Identifikation der offenkettigen Alkene eingegangen, deren eindeutige Unterscheidung auf Grund übereinstimmender IR-Spektren nicht möglich war. Ebenso kann die Bildung von 5-Hexenal aus diesem Grund nicht ausgeschlossen werden, da das entsprechende Spektrum größtenteils mit dem 7-Oktenalspektrum übereinstimmt. 
Da 7-Oktenal nicht kommerziell erhältlich war, wurde dies, wie auch 4-Butenal, aus der Reaktion des endständigen Diens mit Sauerstoffatomen erzeugt.

\subsubsection{Quantitative Analyse}

Im Folgenden werden die quantitativen Ergebnisse für die Reaktion Cyclooktyl $+\mathrm{O}$ verkürzt dargestellt. Auf der Grundlage von Auftragungen der Form Partialdruck Produkt vs. Abnahme an Vorläufer wurden die Daten linear approximiert. Die so erhaltenen Partialdrücke wurden auf die Summe der Gesamtproduktausbeuten bezogen, woraus unter Berücksichtigung aller identifizierten Substanzen die Kanalverzweigung bestimmt wurde. Dementsprechend erhielt man für die Reaktion Cyclooktyl + O unter Verwendung des in der Einleitung dieses Abschnittes dargestellten Reaktionsschemas folgende Kanalverzweigung:

$$
\begin{aligned}
& \mathrm{c}-\mathrm{C}_{8} \mathrm{H}_{15}+\mathrm{O} \longrightarrow \mathrm{c}-\mathrm{C}_{8} \mathrm{H}_{15} \mathrm{O}^{\star} \\
& \mathrm{c}-\mathrm{C}_{8} \mathrm{H}_{15} \mathrm{O}^{\star} \longrightarrow \mathrm{c}-\mathrm{C}_{8} \mathrm{H}_{14} \mathrm{O}+\mathrm{H} \quad(16 \pm 2) \% \\
& \longrightarrow \mathrm{CH}_{2}\left(\mathrm{CH}_{2}\right)_{6} \mathrm{CHO} \\
& \mathrm{CH}_{2}\left(\mathrm{CH}_{2}\right)_{6} \mathrm{CHO} \longrightarrow \mathrm{C}_{7} \mathrm{H}_{13} \mathrm{CHO}+\mathrm{H} \quad(18 \pm 2) \% \\
& \left.\longrightarrow \begin{array}{l}
\mathrm{C}_{2} \mathrm{H}_{4}+\mathrm{C}_{6} \mathrm{H}_{10} \mathrm{O}+\mathrm{H} \\
\mathrm{C}_{2} \mathrm{H}_{5}+\mathrm{C}_{6} \mathrm{H}_{10} \mathrm{O}
\end{array}\right\}(18 \pm 2) \% \\
& \longrightarrow 2 \mathrm{C}_{2} \mathrm{H}_{4}+\mathrm{C}_{4} \mathrm{H}_{6} \mathrm{O}+\mathrm{H} \quad(8 \pm 2) \% \\
& \longrightarrow \mathrm{C}_{5} \mathrm{H}_{10}+\mathrm{C}_{3} \mathrm{H}_{4} \mathrm{O}+\mathrm{H} \quad(15 \pm 2) \%
\end{aligned}
$$

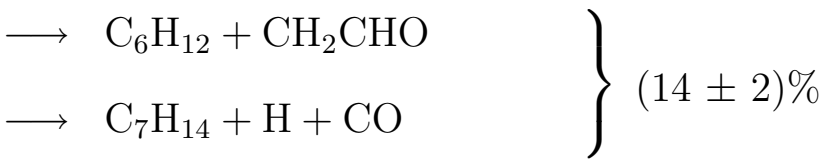

$$
\begin{aligned}
& \mathrm{c}-\mathrm{C}_{8} \mathrm{H}_{15}+\mathrm{O} \longrightarrow \mathrm{c}-\mathrm{C}_{8} \mathrm{H}_{14}+\mathrm{OH} \quad(11 \pm 2) \%
\end{aligned}
$$

\subsubsection{Quantenchemische Rechnungen}

In einer Zusammenarbeit mit der TU Karlsruhe wurden für den Zerfall des Cyclooktoxyradikals quantenchemische Rechnungen von M. Olzmann und O. Welz durchgeführt. Die experimentellen Ergebnisse sind zusammen mit den Resultaten dieser quantenchemischen Rechnungen zur Veröffentlichung eingereicht [Hoy06b]. 
Für die Rechnungen wurde das in der Einleitung des Abschnittes dargestellte Reaktionsschema zu Grunde gelegt. Die Energien relevanter Zwischenprodukte und derer Übergangszustände wurde auf Basis des G3MP2B3 Niveaus berechnet. Die Geometrien und deren Frequenzen wurden mit der Methode bzw. mit dem Basissatz B3LYP/6-31G(d) mit Hilfe des Gaussian 03 Programm Pakets berechnet. Auf Grund der höheren Ringspannung gegenüber dem $\mathrm{C}_{6}$-Ring wurde als stabilster Konformer der axiale Typ angenommen. Die aus den quantenchemischen Rechnungen resultierenden Energiebarrieren für den Zerfall des Cyclooktoxyradikals sind in Abbildung 4.19 dargestellt. Die potentielle Energie ist dabei gegen die Reaktionskoordinate aufgetragen.

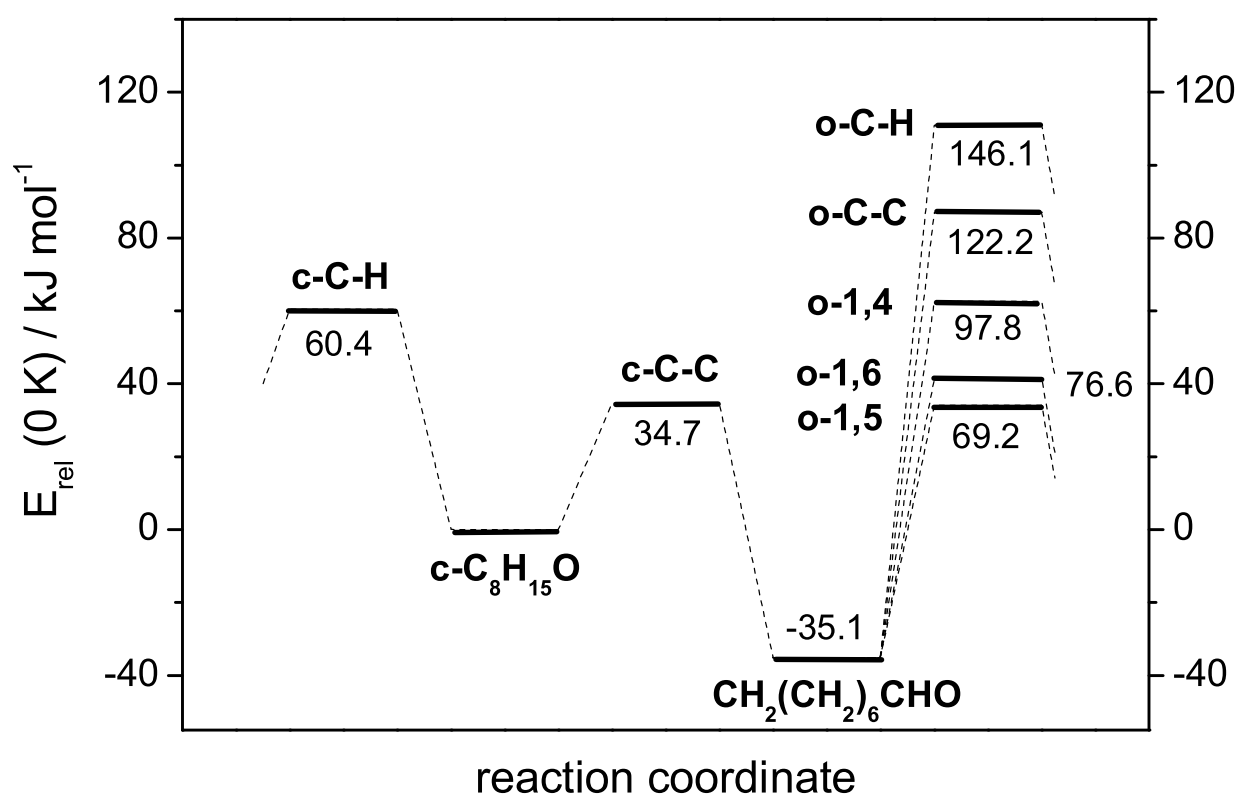

Abbildung 4.19: Reaktion $c-C_{8} H_{15}+O$, Energiediagramm erhalten aus den quantenchemischen Rechnungen (für Basissatz und Funktionen siehe Text). Die angegebenen Werte für die Energiebarrieren sind Grenzenergien. Dabei bedeutet: $c:=$ zyklisch, o:= offenkettig, o-1,5:=1,5-H-shift des offenkettigen Isomers, usw.

Die Abbildung 4.19 zeigt deutlich, dass der $\beta$-C-C-Bindungsbruch, der zur Bildung des offenkettigen Isomers führt, der energetisch günstigste Kanal ist. Sowohl die Schwellenenergie $(\Delta \mathrm{E}=34,7 \mathrm{~kJ} / \mathrm{mol})$ als auch die Energie des Übergangszustandes $(\mathrm{E}=-35,1$ $\mathrm{kJ} / \mathrm{mol})$ weisen diesen Kanal als favorisiert gegenüber dem $\beta$-C-H-Bruch $(\Delta \mathrm{E}=60,4$ kJ/mol) aus. Für den weiteren Zerfall des offenkettigen Radikals werden H-shifts als besonders günstig berechnet (in der absteigenden Reihenfolge $1,5>1,6>1,4$ ). Die direkte 
Abspaltung des $\beta$-H-Atoms oder eines $\beta$-C-Atoms wird dagegen als energetisch ungünstig berechnet $(122,2 \mathrm{~kJ} / \mathrm{mol}$ bzw. 146,1 kJ/mol).

Ausgehend von einer mittleren Anregungsenergie $\langle E\rangle=375 \mathrm{~kJ} / \mathrm{mol}$ wurden mittels RRKM Theorie die Geschwindigkeitskoeffzienten für den Zerfall des Cyclooktoxyradikals und des primär gebildeten offenkettigen Isomers berechnet. Die Geschwindigkeitskoeffizienten für den Zerfall des Cyclooktoxyradikals in Abhängigkeit von der Anregungsenergie sind in Abbildung 4.20 dargestellt. Aus dieser wird ersichtlich, dass der $\beta$-C-CBindungsbruch, unabhängig von der Anregungsenergie, immer schneller als der $\beta$-C-HBindungsbruch ist. Die Energie $\mathrm{E}=30000 \mathrm{~cm}^{-1}$ entspricht der Anregungsenergie des Cyclooktoxyradikals bei chemischer Aktivierung.

$k(E)$ für Reaktionen von Cyclooctoxy (axiale Krone)

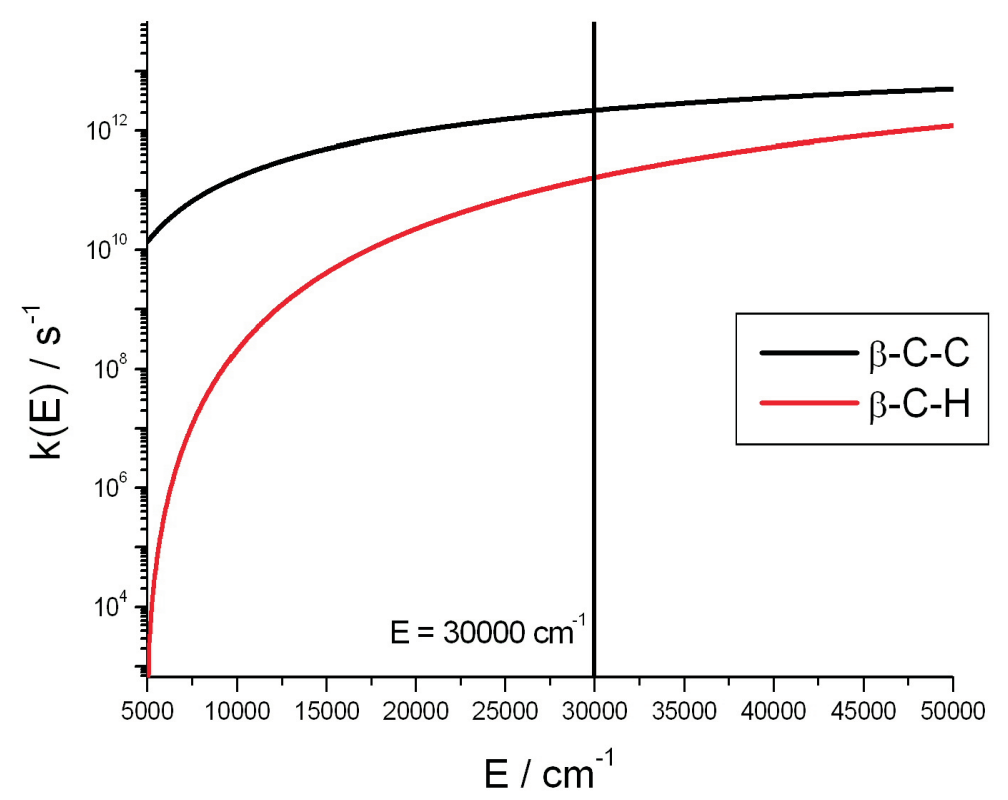

Abbildung 4.20: Reaktion $c-C_{8} H_{15}+O$, Geschwindigkeit des Cyclooctoxyzerfalls aus RRKM Rechnungen in Abhängigkeit von der Anregungsenergie des Radikals. Die Energie $E=30000$ $\mathrm{cm}^{-1}$ entspricht der Anregungsenergie durch chemische Aktivierung.

Da der $\beta$-C-C-Bindungsbruch, der zur Bildung des offenkettigen Isomers führt, die vorrangige Reaktion darstellt, wurden die Geschwindigkeiten für den weiteren Zerfall des Isomers berechnet. Für diese Zerfälle sind die Geschwindigkeitskoeffizienten in Abhängigkeit von der im Isomer noch vorhandenen Energie in Abbildung 4.21 gegeben. 


\section{Spezifische Geschwindigkeitskonstanten Octanal-8-yl}

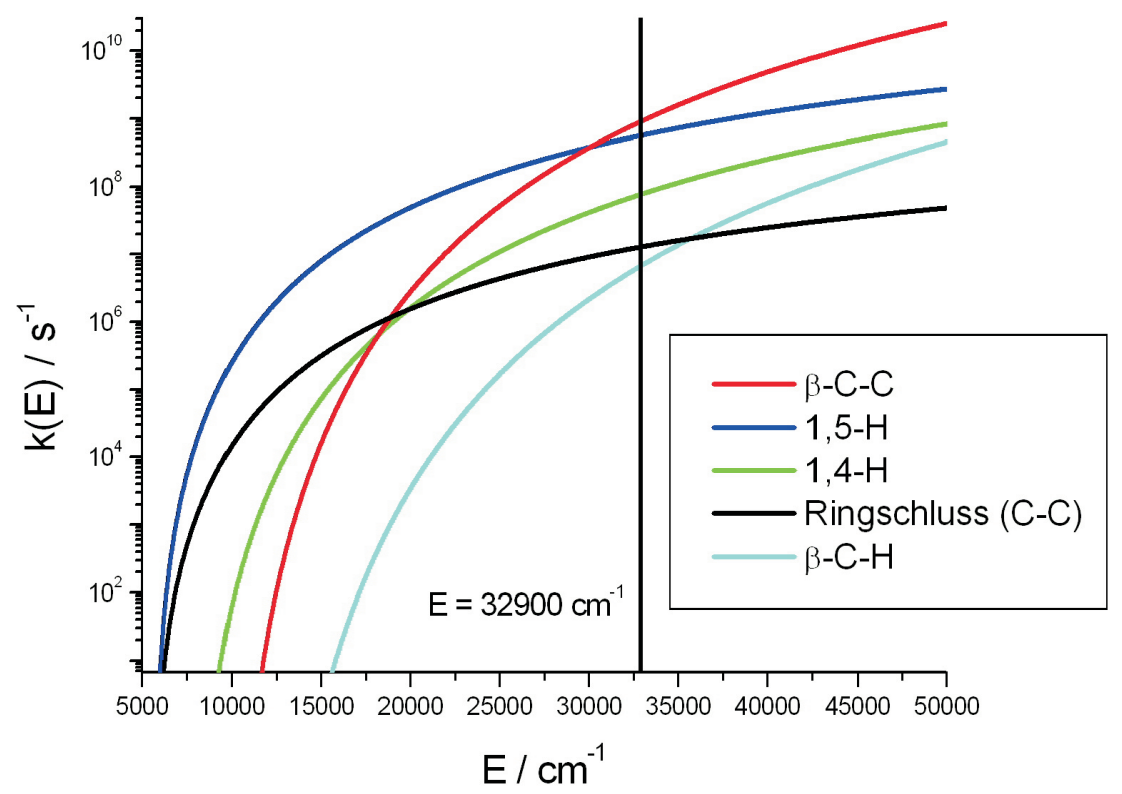

Abbildung 4.21: Reaktion c- $\mathrm{C}_{8} \mathrm{H}_{15}+\mathrm{O}$, Geschwindigkeit des Oktanal-8-ylzerfalls aus RRKM Rechnungen in Abhängigkeit von der Anregungsenergie des Radikals. Die Energie E = 32900 $\mathrm{cm}^{-1}$ entspricht der im Isomer vorhandenen Energie.

Die Abbildung verdeutlicht mehrere wichtige Aspekte des Zerfalls des Oktanal-8-yl Radikals. Bei der mittleren Energie von $32900 \mathrm{~cm}^{-1}$ ist der $\beta$-C-C-Bindungsbruch die schnellste Reaktion, gefolgt von einem 1,5- bzw. 1,4-H-shift. Die Reaktionen, die zur Rückbildung des zyklischen Isomers bzw. zur Stabilisierung unter $\beta$-C-H-Bruch führen, sind wesentlich langsamer. Weiterhin kreuzen sich die $\mathrm{k}(\mathrm{E})$-Kurven des $\beta$-C-C-Bruchs und des 1,5- $H$ shifts in einem Bereich der Geschwindigkeit von ca. $5 \times 10^{8} \mathrm{~s}^{-1}$. Geht man von einem Gesamtdruck von 1 bar aus, so erhält man einen Lennard-Jones Stoßfrequenzfaktor $\mathrm{Z}_{L J}[\mathrm{M}]$ $\approx 2 \times 10^{10} s^{-1}$, wobei [M] für die Konzentration des Stoßpartners steht, in diesem Fall für das Badgas Argon. Auf Grundlage der großen Differenz zwischen der Geschwindigkeit der Stoßstabilisierung und der Geschwindigkeit des Zerfalls wird für die Reaktion bei einem Gesamtdruck von 1 bar die Stoßstabilisierung zur Absenkung der mittleren Energie der Oktanal-8-yl Radikale führen. Dadurch wird die Geschwindigkeit des $\beta$-C-C Bruchs im Verhältnis zu den weiteren Reaktionen überproportional sinken und somit ist eine Änderung der Kanalverzweigung zu erwarten. Ausgehend von einer Senkung der mittleren Energie um $300 \mathrm{~cm}^{-1}$ pro Stoß wurde die Wahrscheinlichkeit des $\beta$-C-C-Bindungsbruchs in Abhängigkeit vom Gesamtdruck durch einen einfachen Ansatz berechnet. Dabei wird 
davon ausgegangen, dass das Radikal entweder zerfallen oder mit einem Badgasteilchen stoßen kann. Dafür wurde die k(E)-Kurve des $\beta$-C-C-Bindungsbruchs im interessierenden Druckbereich durch einen Potenzreihenansatz approximiert. Die Approximation lautet:

$$
k\left(E_{i}\right)=10^{-0,90547+4,69428 \cdot 10^{-4} \cdot E_{i}-5,03871 \cdot 10^{-9} \cdot E_{i}^{2}}
$$

Damit folgt für die Wahrscheinlichkeit des Zerfalls nach i Stößen:

$$
P\left(E_{i}\right)=\frac{k\left(E_{i}\right)}{k\left(E_{i}\right)+Z_{L J}[M]},
$$

wobei $E_{i}$ die im Radikal verbliebene Energie nach i Stößen und $Z_{L J}[M]$ die Geschwindigkeit eines Stoßes mit einem Badgasteilchen bezeichnet. Ist man nun an der Wahrscheinlichkeit des Zerfalls interessiert, muss über alle Ereignisse summiert werden. Dabei bezeichnet Ereignis 1 den Zerfall ohne Stoß, Ereignis 2 den Zerfall nach einem Stoß, Ereignis 3 den Zerfall nach zwei Stößen usw.. Die Wahrscheinlichkeit für den Zerfall ergibt sich damit zu:

$$
P=\sum_{j=1}^{\infty}\left(Z_{L J}[M]\right)^{j-1} k\left(E_{1}\right) \prod_{i=1}^{j}\left(k\left(E_{i}\right)+Z_{L J}[M]\right)^{-1} .
$$

In Abhängigkeit von dem Gesamtdruck erhält man nun die Wahrscheinlichkeit für einen Zerfall. Die obige Summe konvergiert sehr schnell, sodass für die Berechnung der Wahrscheinlichkeit als oberer Grenzwert 10 ausreicht. In der Tabelle 4.11 sind Wahrscheinlichkeiten für verschiedene Lennard-Jones Stoßfrequenzen angegeben.

\begin{tabular}{lc}
\hline$Z_{L J}[M]$ & $\mathrm{P}$ (Zerfall) \\
\hline \hline $1 \cdot 10^{9} s^{-1}$ & 0,74 \\
$2 \cdot 10^{10} s^{-1}$ & 0,31 \\
$5 \cdot 10^{10} s^{-1}$ & 0,15 \\
$9 \cdot 10^{10} s^{-1}$ & 0,09 \\
\hline
\end{tabular}

Tabelle 4.11: Reaktion $c-C_{8} H_{15}+O$, Wahrscheinlichkeit des Zerfalls vs. $Z_{L J}[M]$ unter der Annahme, dass neben dem $\beta$-C-C-Bindungsbruch nur Stoßstabilisierung auftritt. Für Details der Berechnung siehe Text.

Mit dem hier verwendeten einfachen Ansatz kann die Druckabhängigkeit der Reaktion gut beschrieben werden. So wird anhand der Werte deutlich, dass ein höherer Gesamtdruck und damit die Anzahl der Stöße pro Zeiteinheit zu einer Stabilisierung des offenkettigen 
Isomers und damit zu einer geringeren Ausbeute kleinerer Kohlenwasserstoffe führt. Diese Druckabhängigkeit konnte durch Versuche bei einem Gesamtdruck von 4 mbar und 1 bar experimentell bestätigt werden. Dabei sei an dieser Stelle erwähnt, dass es sich um einen einfachen Ansatz handelt, der zwar eine qualitative Aussage macht, die Druckabhängigkeit der Ausbeute aber nicht quantitativ beschreibt.

Die quantitativen Ergebnisse der quantenchemischen Rechnungen finden sich zusammen mit den Ergebnissen der Experimente in Tabelle 4.12. Dort sind zum Vergleich auch die Ergebnisse der Reaktion Cyclopentyl + O und Cyclohexyl + O aufgeführt.

\subsubsection{Diskussion}

Für die Reaktion Cyclooktyl + O liegen in der Literatur keine Ergebnisse vor. Ein Vergleich, der Auskunft über die Güte und Validität der Experimente geben kann, erfolgt daher durch Vergleich mit den Resultaten der Reaktionen Cyclopentyl + O und Cyclohexyl $+\mathrm{O}$ und den quantenchemischen Rechnungen. Dazu werden die Ergebnisse der einzelnen Reaktionen zunächst in Tabelle 4.12 gegenüber gestellt.

\begin{tabular}{l|ccc|ccc}
\hline & \multicolumn{3}{|c}{ Experiment } & \multicolumn{3}{c}{ Rechnung } \\
Kanal & ${\mathrm{c}-\mathrm{C}_{5} \mathrm{H}_{9}}$ & $\mathrm{c}-\mathrm{C}_{6} \mathrm{H}_{11}$ & $\mathrm{c}-\mathrm{C}_{8} \mathrm{H}_{15}$ & $\mathrm{c}-\mathrm{C}_{5} \mathrm{H}_{9}$ & ${\mathrm{c}-\mathrm{C}_{6} \mathrm{H}_{11}}$ & $\mathrm{c}-\mathrm{C}_{8} \mathrm{H}_{15}$ \\
\hline Cycloketon & $31 \pm 2$ & $19 \pm 3$ & $18 \pm 2$ & 13 & 6,9 & 8,7 \\
Aldehyd & $40 \pm 5$ & $47 \pm 6$ & $20 \pm 2$ & 3 & 1,0 & 0,8 \\
o-C-C & $17 \pm 5$ & $11 \pm 5$ & $29 \pm 4$ & $84-\mathrm{x}$ & 26,3 & 37 \\
H shift & $12 \pm 5$ & $23 \pm 5$ & $33 \pm 4$ & $\mathrm{x}$ & 65,8 & 53,5 \\
\hline Summe & 100 & 100 & 100 & 100 & 100 & 100 \\
\hline
\end{tabular}

Tabelle 4.12: Vergleich der Reaktionen Cycloalkyl + O für Cyclopentyl, Cyclohexyl und Cyclooktyl. Alle hier angegebenen experimentellen Werte sind mit Hilfe der FTIR Apparatur bestimmt. Die quantenchemischen Rechnungen wurden von M. OlzMANn und O. WELZ durchgeführt. Für eine bessere Vergleichbarkeit wurde der Abstraktionskanal, der bei der Reaktion Cyclopentyl + $O$ und $C y c l o o c y t l+O$ beobachtet wurde, nicht aufgeführt. Für weitere Erklärungen siehe Text.

In obiger Tabelle bezeichnet o-C-C weitere C-C-Bindungsbrüche des offenkettigen Isomers, die zur Bildung kleinere Kohlenwasserstoffe oder Aldehyde führen. Aus der Tabelle wird ersichtlich, dass der $\beta$-C-H-Bindungsbruch, der zur Bildung des zyklischen Ketons führt, in allen Fällen eine entscheidende Rolle spielt. Dabei ist der Wert für den Fünfring 
um den Faktor 1,5 größer, als für die beiden anderen Cycloalkyl-Radikale. Es wurde vermutet, dass dieser Kanal für die Reaktion Cyclohexyl + O im Vergleich mit den beiden anderen Cycloalkyl-Radikale auf Grund der geringeren Ringspannung stärker ausgeprägt sein müßte. Dies konnte durch die Experimente nicht bestätigt werden. Des Weiteren konnte diese Annahme durch die quantenchemischen Rechnungen widerlegt werden, die für das Cyclohexanon ebenfalls keine erhöhte Ausbeute lieferten. Der Kanal unter Bildung des langkettigen Aldehyds verringert sich in etwa um den Faktor 0,5 vom Sechsring zum Achtring. Dies ist durch die Zunahme an möglichen H-shifts erklärbar. Der Vergleich der experimentellen Daten mit den Werten der quantenchemischen Rechnungen zeigt, dass der Kanal zum langkettigen Aldehyd bei den Rechnungen systematisch unterschätzt wird. Hier ist weitere Arbeit erforderlich.

\subsection{Untersuchung der Reaktion eines zyklischen Al- kens mit Sauerstoff-Atomen}

\subsubsection{Die Reaktion Cyclopenten + O}

\subsubsection{Einleitung}

Die Reaktion Cyclopenten $+\mathrm{O}$ ist sehr schnell $\left(\mathrm{k}=1,25 \cdot 10^{13} \mathrm{~cm}^{3} \cdot \mathrm{mol}^{-1} \mathrm{~s}^{-1}\right.$ [Cve87]). Diese Reaktion wurde im Rahmen der Reaktion Cyclopentyl + O untersucht (siehe dazu Abschnitt 4.1.1), da Cyclopenten als Produkt dieser Reaktion identifiziert werden konnte. Die Reaktion mit O-Atomen ist im Vergleich mit anderen primären Produkten um zwei bis drei Größenordnungen schneller (vgl. Tabelle 4.1). Ein wichtiger Bestandteil der Untersuchung war somit herauszufinden, ob primär gebildetes Cyclopenten mit O-Atomen in einer Folgereaktion abgebaut und so der Abstraktionskanal unterschätzt wird. Daher wurden für die Untersuchung der Reaktion Cyclopenten + O Bedingungen gewählt, die denen bei der Reaktion Cyclopentyl + O entsprechen. Es wurde zwar bei der Reaktion Cyclopentyl + O ein Sauerstoffunterschuss gewählt, doch liegt das Verhältnis [O] : $\left[\mathrm{c}^{-} \mathrm{C}_{5} \mathrm{H}_{8}\right]$ auf Grund des gering gehalteten Umsatzes an Cyclopentan auf Seite der Sauerstoffkonzentration. Die Reaktion Cyclopenten + O wurde schon von Cvetanovic̀ [Cve71] untersucht. Auf Grund einer massenspektrometrischen Untersuchung war auch hier, wie 
bei RoHDE (bei der Untersuchung Cyclopentyl + O) die eindeutige Identifikation einiger Substanzen nur bedingt möglich. Für die Reaktion sind folgende Reaktionskanäle denkbar:

$$
\begin{array}{rlr}
\mathrm{c}-\mathrm{C}_{5} \mathrm{H}_{8}+\mathrm{O} & \longrightarrow \mathrm{c}-\mathrm{C}_{5} \mathrm{H}_{8} \mathrm{O}^{\star} & (a) \\
\mathrm{c}-\mathrm{C}_{5} \mathrm{H}_{8} \mathrm{O}^{\star} & \longrightarrow \mathrm{c}-\mathrm{C}_{5} \mathrm{H}_{8} \mathrm{O} & \left(a_{1}\right) \\
& \longrightarrow \mathrm{C}_{2} \mathrm{H}_{4}+\mathrm{CH}_{2} \mathrm{CHCHO} \\
& \longrightarrow \mathrm{C}_{4} \mathrm{H}_{7} \mathrm{CHO} & \left(a_{3}\right) \\
& \longrightarrow \mathrm{c}-\mathrm{C}_{4} \mathrm{H}_{7} \mathrm{CHO} & \left(a_{4}\right) \\
& \longrightarrow \text { Dihydropyran } & \left(a_{5}\right) \\
& \longrightarrow \text { Cyclopentenoxid }
\end{array}
$$

Im Gegensatz zu den bisher betrachteten Radikal-Reaktionen ist für die Reaktion Cyclopenten $+\mathrm{O}$ eine H-Abstraktion unwahrscheinlich, sodass in einem ersten Schritt das Sauerstoffatom an ein C-Atom der Doppelbindung addiert wird. Cvetanović postuliert dabei die Bildung eines angeregten „triplet Biradikals“, welches weiter zerfallen oder unter Ringbildung weitere Addukte ergeben kann. Durch die Biradikalbildung wird die Bindung geschwächt und das entstandene Biradikal kann unter $H$-shift vom $\alpha$-C-Atom zum $\beta$-C-Atom Cyclopentanon $\left(\mathrm{a}_{1}\right)$ oder unter Ausbildung einer weiteren O-C-Bindung zum Cyclopentenoxid $\left(\mathrm{a}_{6}\right)$ reagieren. Beide Reaktionskanäle erhalten den Fünfring. Die Reaktionen $\left(\mathrm{a}_{2}\right)$ - $\left(\mathrm{a}_{5}\right)$ sind Folge eines C-C-Bindungsbruchs zwischen dem $\alpha$-C-Atom und dem $\beta$-C-Atom. Nach Ringöffnung kann das gebildete Biradikal unter Ausbildung von Ethen und Acrolein weiter zerfallen $\left(\mathrm{a}_{2}\right)$ oder unter H-shift 4-Pentenal $\left(\mathrm{a}_{3}\right)$ oder durch 1,2-Ringkontraktion Cyclobutylcarboxyaldehyd bilden. Aus dem offenen Biradikal kann nach Isomerisierung ein anderes Biradikal gebildet werden, welches dann unter Ringschluss zu Dihydropyran $\left(a_{5}\right)$ isomerisieren kann.

\subsubsection{Qualitative Analyse}

Die stabilen Endprodukte der Reaktion Cyclopenten $+\mathrm{O}$ wurden wie in Abschnitt 3.1.1 beschrieben mit Hilfe FTIR-Spektroskopie nachgewiesen bzw. quantitativ bestimmt. Als Sauerstoffvorläufer diente auch hier $\mathrm{SO}_{2}$. 
Wie schon beschrieben wurde für diese Reaktion ein Verhältnis an $\left[\mathrm{c}-\mathrm{C}_{5} \mathrm{H}_{8}\right]_{0} /\left[\mathrm{SO}_{2}\right]_{0}=$ 5 verwendet, um die bei der Reaktion Cyclopentyl + O herrschenden Bedingungen nachzustellen.

Abbildung 4.22 zeigt ein Reaktionsspektrum bei den Bedingungen $\mathrm{p}\left(\mathrm{c}-\mathrm{C}_{5} \mathrm{H}_{8}\right)=0,02$ mbar, $\mathrm{p}\left(\mathrm{SO}_{2}\right)=0,1$ mbar, $\mathrm{p}_{\text {ges }}=4$ mbar, $\mathrm{T}=298 \mathrm{~K}$ und 400 Photolysen.

Die Identifikation primärer Produkte der Reaktion Cyclopenten $+\mathrm{O}$ wird anhand der zur Verfügung stehenden Reinspektren vorgenommen. Ein Vergleich in ausgezeichneten Bereichen des Reaktionsspektrums mit den Spektren der identifizierten Substanzen wird in Abbildung 4.23 dargestellt, aus der folgt, dass die in der Übersicht vorgeschlagenen Produkte mit Ausnahme von Cyclobutylcarboxyaldehyd und Dihydropyran eindeutig identifiziert werden konnten. Daneben wurde die Bildung von Formaldehyd und Ethin beobachtet. Aus welchem Reaktionskanal Formaldehyd entstammt, ist allerdings unklar.

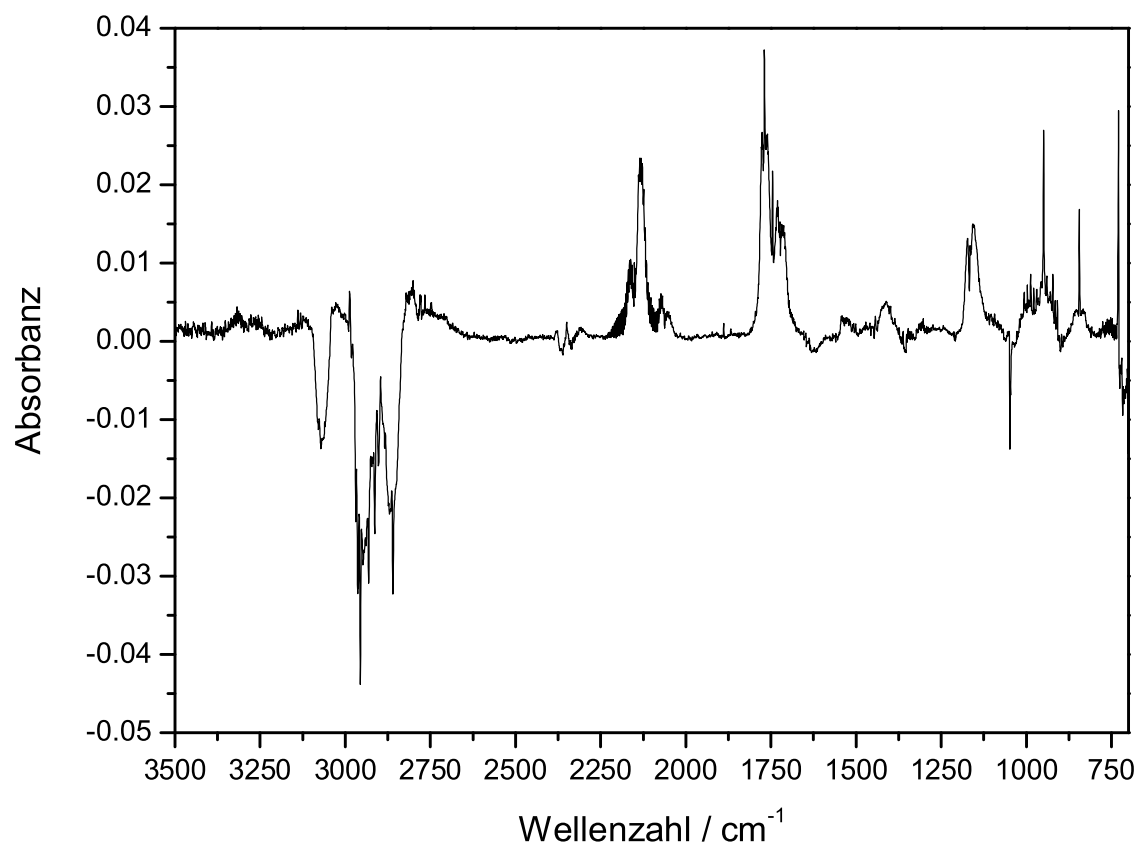

Abbildung 4.22: Reaktion $c-C_{5} H_{8}+O$, Reaktionsspektrum. $p\left(c-C_{5} H_{8}\right)=0,02$ mbar, $p\left(\mathrm{SO}_{2}\right)=$ 0,1 mbar, $p_{g e s}=4$ mbar, $T=298 \mathrm{~K}, 400$ Photolysen. 

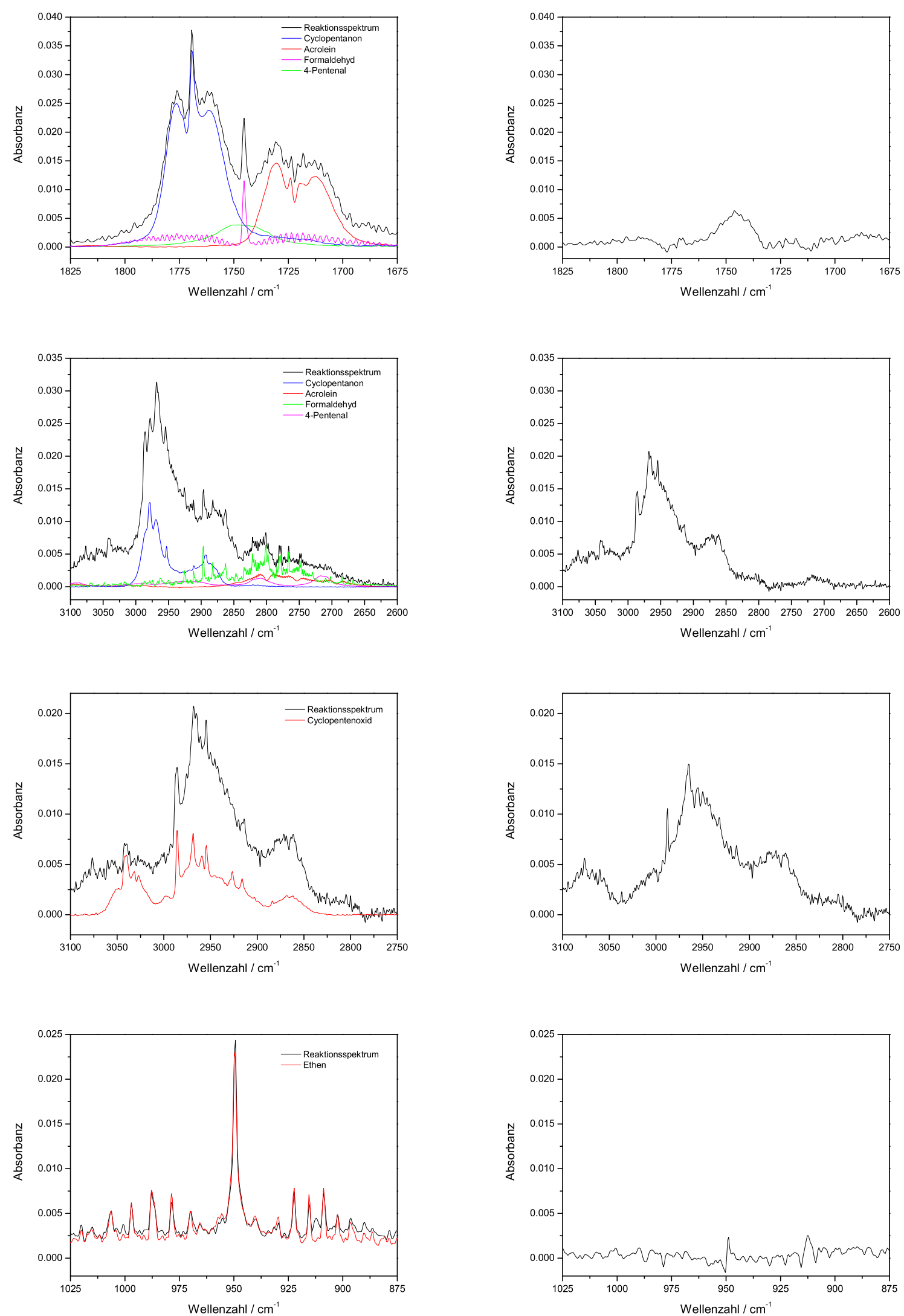

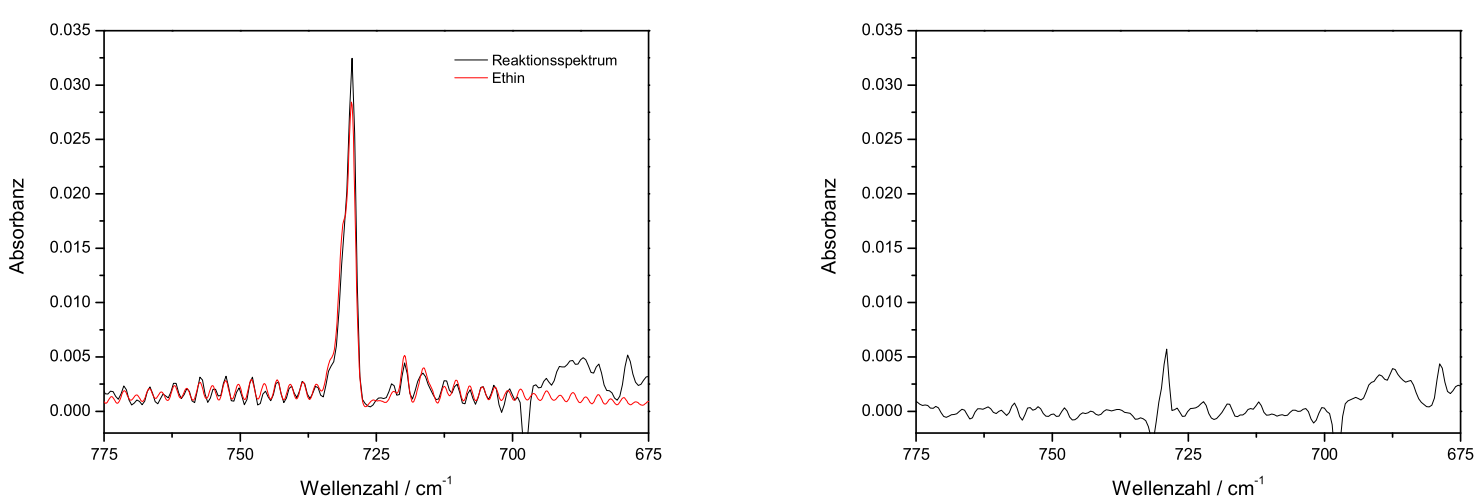

Abbildung 4.23: Reaktion c- $\mathrm{C}_{5} \mathrm{H}_{8}+\mathrm{O}$, qualitative Analyse. $\mathrm{p}\left(\mathrm{c}-\mathrm{C}_{5} \mathrm{H}_{8}\right)=0,02$ mbar, $\mathrm{p}\left(\mathrm{SO}_{2}\right)$ $=0,1 \mathrm{mbar}, p_{\text {ges }}=4 \mathrm{mbar}, \mathrm{T}=298 \mathrm{~K}, 400$ Photolysen. Auf der linken Seite sind jeweils das Reaktionsspektrum (schwarz) zusammen mit den Reinspektren der identifizierten Substanzen (farbig) dargestellt, die rechte Spalte zeigt das zugehörige Residuum.

\subsubsection{Quantitative Analyse}

Die quantitative Analyse der Reaktion wurde wie in Abschnitt 3.3.2. beschrieben durchgeführt. Dazu wurden von den identifizierten Substanzen in einem Partialdruckbereich von 0,01 - 0,1 mbar Reinspektren bei einem Gesamtdruck von 4 mbar aufgenommen. Bei der quantitativen Analyse konnte beobachtet werden, dass sich bei steigender Photolysenanzahl das Verzweigungsverhältnis der Produkte änderte. Diese Änderung des Verzweigungsverhältnisses ist auf eine Sekundärphotolyse primär gebildeter Produkte oder Reaktionen mit O-Atomen zurückzuführen. In Abbildung 4.24 sind die Partialdrücke der identifizierten Substanzen gegen die Abnahme des Vorläufers Cyclopenten aufgetragen. Die zugehörigen Messwerte sind in Tabelle 4.14 angegeben. Aus der Abbildung wird ersichtlich, dass der Partialdruck von Acrolein nicht, wie für die anderen Substanzen, linear mit der Abnahme an Vorläufer ansteigt. Bei steigender Photolysenanzahl ist weiterhin die Bildung von Ethin zu beobachten, was als mögliches Produkt der Photolyse von Acrolein in Frage kommt. Für die quantitative Analyse wird daher das Verhältnis der Produkte bei der geringsten Photolysenanzahl bestimmt. Unter dieser Voraussetzung ergeben sich für das Produktverhältnis die in Tabelle 4.13 angegebenen Werte. 


\begin{tabular}{ccccc}
\hline${\mathrm{c}-\mathrm{C}_{5} \mathrm{H}_{8} \mathrm{O}}$ & $\mathrm{C}_{4} \mathrm{H}_{7} \mathrm{CHO}$ & Acrolein & Ethen & Cyclopentenoxid \\
\hline \hline 29,5 & 5,7 & 18,2 & 23,9 & 22,7 \\
\hline
\end{tabular}

Tabelle 4.13: Reaktion c- $\mathrm{C}_{5} \mathrm{H}_{8}+\mathrm{O}$, Produktverhältnis. Für die Bestimmung des Verhältnisses gingen nur die Werte nach 100 Photolysen ein, da bei einer größeren Anzahl eine Sekundärphotolyse bzw. Reaktionen der Produkte mit O-Atomen beobachtet wurden.

\begin{tabular}{|c||c|c|c|c|c|c|c|}
\hline $\begin{array}{c}\mathrm{c}-\mathrm{C}_{5} \mathrm{H}_{8} \\
\text { Verbrauch }\end{array}$ & $\begin{array}{c}\mathrm{p}\left(\mathrm{C}_{2} \mathrm{H}_{4}\right) \\
{[\mathrm{mbar}]}\end{array}$ & $\begin{array}{c}\mathrm{p}\left(\mathrm{C}_{3} \mathrm{H}_{4} \mathrm{O}\right) \\
{[\mathrm{mbar}]}\end{array}$ & $\begin{array}{c}\mathrm{p}\left(\mathrm{c}-\mathrm{C}_{5} \mathrm{H}_{8} \mathrm{O}\right) \\
{[\mathrm{mbar}]}\end{array}$ & $\begin{array}{c}\mathrm{p}(\mathrm{CPO}) \\
{[\mathrm{mbar}]}\end{array}$ & $\begin{array}{c}\mathrm{p}\left(\mathrm{H}_{2} \mathrm{CO}\right) \\
{[\mathrm{mbar}]}\end{array}$ & $\begin{array}{c}\mathrm{p}\left(\mathrm{c}_{4} \mathrm{H}_{7} \mathrm{CHO}\right) \\
{[\mathrm{mbar}]}\end{array}$ & $\begin{array}{c}\mathrm{p}\left(\mathrm{C}_{2} \mathrm{H}_{2}\right) \\
{[\mathrm{mbar}]}\end{array}$ \\
\hline \hline 0,017 & 0,0046 & 0,0039 & 0,0025 & 0,002 & $7,5 \mathrm{E}-4$ & $4,7 \mathrm{E}-4$ & 0,0033 \\
\hline 0,064 & 0,0124 & 0,0091 & 0,0089 & 0,0058 & 0,0028 & 0,0028 & 0,0028 \\
\hline 0,0058 & 0,0023 & $5 \mathrm{E}-4$ & 0,0014 & $8,7 \mathrm{E}-4$ & $5 \mathrm{E}-4$ & $6,2 \mathrm{E}-4$ & $6,2 \mathrm{E}-4$ \\
\hline
\end{tabular}

Tabelle 4.14: Reaktion c- $\mathrm{C}_{5} \mathrm{H}_{8}+\mathrm{O}$, Messwerte. $\mathrm{p}\left(\mathrm{c}-\mathrm{C}_{5} \mathrm{H}_{8}\right)=0,02$ mbar, $\mathrm{p}\left(\mathrm{SO}_{2}\right)=0,1 \mathrm{mbar}$, $p_{\text {ges }}=4$ mbar $\mathrm{T}=298 \mathrm{~K}(\mathrm{CPO}:=$ Cyclopentenoxid $)$, Badgas Argon.

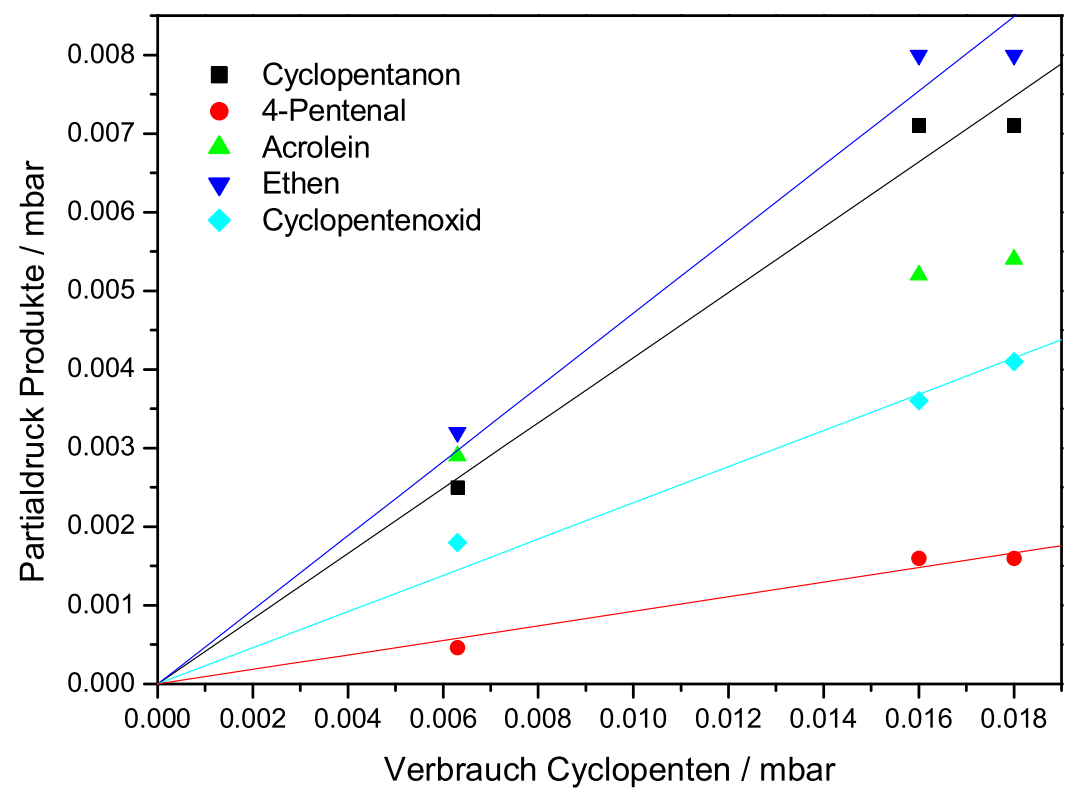

Abbildung 4.24: Reaktion c- $C_{5} \mathrm{H}_{8}+\mathrm{O}$, quantitative Analyse. $p\left(c-\mathrm{C}_{5} \mathrm{H}_{8}\right)=0,02 \mathrm{mbar}, \mathrm{p}\left(\mathrm{SO}_{2}\right)=$ 0,1 mbar, $p_{g e s}=4 \mathrm{mbar}, \mathrm{T}=298 \mathrm{~K}$. Die Abbildung zeigt, dass mit steigender Photolysenanzahl der Partialdruck von Acrolein nicht linear zunimmt. Deshalb wurde die quantitative Analyse auf Grundlage der kleinsten Photolysenanzahl vorgenommen.

\subsubsection{Diskussion}

Die Reaktion von Cyclopenten + O wurde schon von Cvetanović [Cve71] untersucht. Dabei wurden die O-Atome mit einer Quecksilberdampflampe erzeugt, was lange Bestrah- 
lungszeiten (bis zu 150 min) erforderte. Die Reaktion wurde in Hinblick auf eine Druck-, Bestrahlungszeit- und Temperaturabhängigkeit untersucht. Dabei wurden Messungen bei $25^{\circ} \mathrm{C}, 125^{\circ} \mathrm{C}$ und $175^{\circ} \mathrm{C}$ durchgeführt. Der Gesamtdruck wurde von 35 mbar bis 450 mbar variiert. Weiterhin wurde der Cyclopentenpartialdruck bei konstantem $\mathrm{N}_{2} \mathrm{O}$-Druck verändert. $\mathrm{N}_{2} \mathrm{O}$ diente bei den Experimenten als Vorläufer für die Sauerstoffatome. Die Veränderung des Cyclopentenpartialdrucks bei konstantem $\mathrm{N}_{2} \mathrm{O}$-Druck hatte nach CvETANOviĆ keinen Einfluss auf das relative Produktverhältnis. Ebenso hat die Erhöhung keine Änderung des Kanalverzweigungsverhältnisses bewirkt. Die Erhöhung des Gesamtdrucks führte zu einer Steigerung der O-Atom Additionsprodukte bis hin zu einem konstantem Wert. Das konstante Verhältnis wurde ungefähr ab einem Gesamtdruck von 200 mbar erreicht. Die Temperaturveränderung hat nur einen geringen Einfluss auf das relative Produktverhältnis. Der Kanal zum Cyclopentenoxid nimmt mit steigender Temperatur allerdings unter Bildung zweier von Cvetanović nicht identifizierter Produkte ab.

Da die Reaktion innerhalb der vorliegenden Arbeit nur bei einem Gesamtdruck von 4 mbar untersucht wurde, werden die Ergebnisse mit denen von Cvetanović bei dem kleinsten Druck von 36 mbar und einer Temperatur von $25^{\circ} \mathrm{C}$ verglichen. Die Daten sind in Tabelle 4.15 dargestellt.

\begin{tabular}{lcc}
\hline Substanz & Diese Arbeit & Cvetanović \\
\hline $\mathrm{c}-\mathrm{C}_{5} \mathrm{H}_{8} \mathrm{O}$ & 29,5 & 16,3 \\
$\mathrm{C}_{4} \mathrm{H}_{7} \mathrm{CHO}$ & 5,7 & 0,5 \\
Acrolein & 18,2 & 20,9 \\
Ethen & 23,9 & 18,6 \\
Cyclopentenoxid & 22,7 & 36,0 \\
Cyclobutylcarboxyaldehyd & 0 & 7,0 \\
Dihydropyran & 0 & 0,7 \\
\hline
\end{tabular}

Tabelle 4.15: Reaktion c- $\mathrm{C}_{5} \mathrm{H}_{8}+\mathrm{O}$, Produktverhältnisse in \% bezogen auf die Summe aller Produkte. Die Tabelle zeigt die Ergebnisse dieser Arbeit im Vergleich mit den Daten von Cvetanović.

Mit den experimentell bestimmten Werten für das Produktverhältnis ergibt sich unter Berücksichtigung des eingangs dargestellten Reaktionsmechanismus folgende Kanalverzweigung für die Reaktion Cyclopenten $+\mathrm{O}$ : 


$$
\begin{array}{rlr}
\mathrm{c}-\mathrm{C}_{5} \mathrm{H}_{10}+\mathrm{O} & \longrightarrow \mathrm{c}-\mathrm{C}_{5} \mathrm{H}_{8} \mathrm{O}^{\star} & \\
\mathrm{c}-\mathrm{C}_{5} \mathrm{H}_{8} \mathrm{O}^{\star} & \longrightarrow \mathrm{c}-\mathrm{C}_{5} \mathrm{H}_{8} \mathrm{O} & 37,3 \% \\
& \longrightarrow \mathrm{C}_{2} \mathrm{H}_{4}+\mathrm{CH}_{2} \mathrm{CHCHO} & 26,7 \% \\
& \longrightarrow \mathrm{C}_{4} \mathrm{H}_{7} \mathrm{CHO} & 7,2 \% \\
& \longrightarrow \text { Cyclopentenoxid } & 28,8 \%
\end{array}
$$




\subsection{Untersuchung der Reaktionen offenkettiger Koh- lenwasserstoffradikale mit Sauerstoff-Atomen}

Die Reaktionen offenkettiger Alkylradikale mit O-Atomen spielen bei der Verbrennung von Kohlenwasserstoffen unter Hochtemperaturbedingungen und beim Kohlenwasserstoffabbau in der Atmosphäre eine wichtige Rolle. Bei diesen Reaktionen tritt in einem ersten Schritt vorrangig die Bildung eines O-zentrierten Alkoxyradikals auf. O-zentrierte Radikale haben, wie schon in der Einleitung dargestellt, eine herausragende Bedeutung für die radikalische Polymerisation, da sie bei dem thermischen und photolytischen Zerfall von gängigen Initiatoren gebildet werden. In dieser Arbeit wurden die Reaktionssysteme $\mathrm{CH}_{3}$ / $\mathrm{O}$ und $\mathrm{C}_{2} \mathrm{H}_{5} / \mathrm{O}$ untersucht, um die Geschwindigkeitskoeffizienten der Reaktionen $\mathrm{CH}_{3}+$ $\mathrm{CH}_{3}$ und $\mathrm{C}_{2} \mathrm{H}_{5}+\mathrm{C}_{2} \mathrm{H}_{5}$ relativ zu bestimmen. Die relativ bestimmten Geschwindigkeiten wurden zur Ermittlung der Geschwindigkeiten von Kreuzreaktionen verwendet. Diese sind in Abschnitt 4.5 beschrieben. Untersuchungen zu den Radikal-Radikal-Reaktionen in Abwesenheit von O-Atomen finden sich in Abschnitt 4.4.

Die Reaktion Allyl + O wurde auf Grund ihrer herausragenden Bedeutung für die Verbrennung unter Hochtemperaturbedingungen und der Tatsache, dass in der Literatur für diese Reaktion widersprüchliche Angaben über die Reaktionswege angegeben werden, detailliert untersucht.

Die Geschwindigkeitskoeffizienten für die Selbstreaktionen kleiner Alkylradikale liegen in derselben Größenordnung wie die Geschwindigkeitskoffizienten der Reaktionen der Alkylradikale mit Sauerstoffatomen $\left(\sim 5 \times 10^{13} \mathrm{~cm}^{3} /(\mathrm{mol} \cdot \mathrm{s})\right)$. Dadurch konkurrieren die Selbstreaktion und Oxidation. Diese Konkurrenz kann für die Bestimmung von Geschwindigkeitskoeffizienten ausgenutzt werden. Legt man einen Reaktionsmechanismus zu Grunde, der sowohl die Selbstreaktionen als auch die Oxidationsreaktionen umfasst, so kann bei bekannten Radikalanfangskonzentrationen, Geschwindigkeitskoeffizienten der Selbstreaktionen und Produktverhältnissen durch Simulationsrechnungen der Geschwindigkeitskoeffzient der Oxidationsreaktion bestimmt werden. Der allgemeine Reaktionsmechanismus lautet daher: 


$$
\begin{aligned}
\mathrm{R}+\mathrm{O} & \longrightarrow \text { Alken }+\mathrm{OH} \\
\mathrm{R}+\mathrm{O} & \longrightarrow \mathrm{R}-\mathrm{O}^{\star} \rightarrow \text { Produkte } \\
\mathrm{R}+\mathrm{R} & \longrightarrow \mathrm{R}-\mathrm{R} \\
& \longrightarrow \text { Alkan }+ \text { Alken }
\end{aligned}
$$

Der Abbau der Alkylradikale kann über eine direkte H-Abstraktion (a) und die Oxidationsreaktionen (o) oder die Reaktion zweier Alkylradikale ((c) := Kombination, (d) := Disproportionierung) ablaufen.

\subsubsection{Die Reaktion Methyl + O}

\subsubsection{Einleitung}

Methylradikale sind die essentiellen Radikale in der Verbrennung von Kohlenwasserstoffen. Insbesondere bei der Verbrennung von Erdgas, welches hauptsächlich aus Methan besteht $(\geq 80 \%)$, findet ein Abbau durch Reaktionen mit Atomen (H, O) und anderen Radikalen $(\mathrm{OH})$ statt. Auf Grund der schon im vorangehenden Kapitel beschriebenen Tatsache, dass die Geschwindigkeit der Reaktion von Alkylradikalen mit O-Atomen die der Reaktion von Alkylradikalen mit molekularem Sauerstoff um mehrere Größenordnungen unter Hochtemperaturbedingungen übersteigt, ist der Abbau durch molekularen Sauerstoff unter diesen Bedingungen vernachlässigbar [Gri95].

Die Reaktion Methyl + O wurde innerhalb der Arbeitsgruppe schon detailliert von ZEUCH et al. [Zeu03, Hac05] untersucht. Daneben finden sich in der neueren Literatur Ergebnisse von LeOne et al. und Fockenberg [Foc99, Foc00]. Ziel dieser Untersuchung war, die von ZEUCH mit der auch hier verwendeten Apparatur gefundene Kanalverzweigung für die Reaktion $\mathrm{CH}_{3}+\mathrm{O} \rightarrow$ Produkte zu validieren, um im Folgenden die Geschwindigkeitskoeffizienten für Reaktionen vom Typ $\mathrm{R}_{1}+\mathrm{R}_{2} \rightarrow$ Produkte, wobei $\mathrm{R}_{i}=\mathrm{CH}_{3}$, $\mathrm{C}_{2} \mathrm{H}_{5}, 1-\mathrm{C}_{3} \mathrm{H}_{7}, 1-\mathrm{C}_{4} \mathrm{H}_{9}$ und 2- $\mathrm{C}_{3} \mathrm{H}_{7}$, relativ zu bestimmen. Dazu wurden als VorläuferMischungen Aceton / $\mathrm{SO}_{2}$ und Aceton- $\mathrm{d}_{6} / \mathrm{SO}_{2}$ eingesetzt. Aceton wurde als Vorläufer bei der Untersuchung von ZEUCH nicht verwendet, da durch Wahl dieses Vorläufers CO sowohl aus der Photolyse von Aceton als auch aus der Reaktion $\mathrm{CH}_{3}+\mathrm{O} \rightarrow \mathrm{CO}+\mathrm{H}_{2}+$ H stammen kann. Ausgehend von der in der Literatur intensiv untersuchten Oxidations- 
reaktion $\mathrm{CH}_{3}+\mathrm{O} \rightarrow$ Produkte und der dabei gefundenen Geschwindigkeitskoeffizienten wurde der Geschwindigkeitskoeffizient für die Reaktionen $\mathrm{CH}_{3}+\mathrm{CH}_{3} \rightarrow \mathrm{C}_{2} \mathrm{H}_{6}$ anhand von Simulationsrechnungen bestimmt. Der folgende Reaktionsmechanismus wird für die Reaktion zugrunde gelegt:

$$
\begin{aligned}
\mathrm{CH}_{3}+\mathrm{O} & \longrightarrow \mathrm{H}_{2} \mathrm{CO}+\mathrm{H} \\
& \longrightarrow \mathrm{CO}+\mathrm{H}_{2}+\mathrm{H} \\
\mathrm{CH}_{3}+\mathrm{CH}_{3} & \longrightarrow \mathrm{C}_{2} \mathrm{H}_{6} \\
\mathrm{CH}_{3}+\mathrm{H}+\mathrm{M} & \longrightarrow \mathrm{CH}_{4}+\mathrm{M}
\end{aligned}
$$

Sowohl die experimentellen Ergebnisse als auch die Simulationsrechnungen zeigen, dass die Reaktion $\left(\mathrm{c}_{2}\right)$ für eine adäquate Beschreibung des Reaktionssystems nötig ist. Ohne diese Reaktion lässt sich die beobachtete Bildung von Methan nicht erklären. Die Bildung von Methan könnte zwar auch aus einer Disproportionierung stammen, doch ist die Geschwindigkeit der Reaktion $\mathrm{CH}_{3}+\mathrm{CH}_{3} \rightarrow \mathrm{CH}_{4}+\mathrm{CH}_{2}$ unter den gegebenen Bedingungen zu gering $\left(\mathrm{k}\left(\mathrm{CH}_{3}+\mathrm{CH}_{3} \rightarrow \mathrm{CH}_{2}+\mathrm{CH}_{4}\right)=4,3 \times 10^{10} \mathrm{~cm}^{3} /(\mathrm{mol} \cdot \mathrm{s})\right)$ als dass dadurch die Bildung von Methan beschrieben werden könnte. Da diese Reaktion zu langsam abläuft, wurde sie nicht in den obigen Reaktionsmechanismus aufgenommen. Um die Geschwindigkeit der Reaktion $\mathrm{CH}_{3}+\mathrm{CH}_{3}$ zu bestimmen, wurde das Verhältnis der Vorläuferpartialdrücke in dem Bereich variiert, bei dem es sowohl zur Bildung des Kombinationsproduktes als auch zur Bildung der O-Atom-Additionsprodukte kam. Alle Messungen wurden bei einer Temperatur von $298 \mathrm{~K}$ und einem Gesamtdruck von 4 mbar durchgeführt. Die Auswertung erfolgte anhand von 100-fach gemittelten IR-Spektren nach jeweils 100 Photolysen. Wie bei allen untersuchten Reaktionen wurde die Gesamtphotolysenanzahl auf 400 beschränkt (dies entspricht einem Vorläuferumsatz von 15\%), um die Sekundärphotolyse primär gebildeter Produkte zu vermeiden.

\subsubsection{Qualitative Analyse}

Ein Reaktionsspektrum nach 200 Photolysen bei einer Vorläuferzusammensetzung von Aceton $/ \mathrm{SO}_{2}=0,2 / 0,2$ mbar ist in Abbildung 4.25 gegeben. 


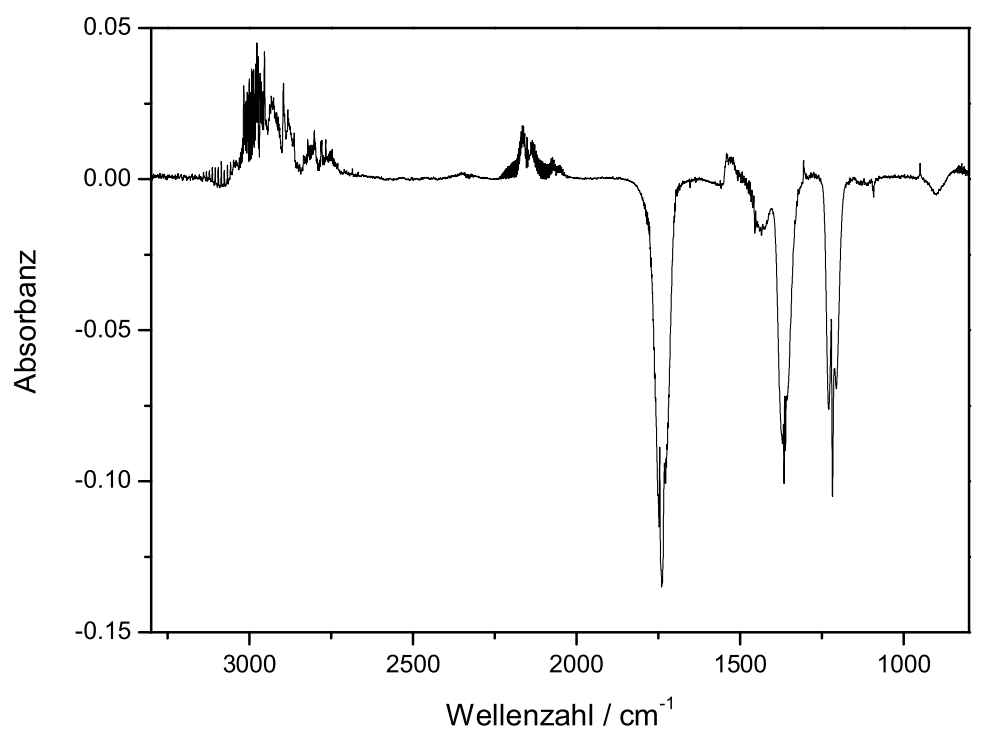

Abbildung 4.25: Reaktionssystem $\mathrm{CH}_{3}$ / O, Reaktionsspektrum nach 200 Photolysen bei einem Gesamtdruck von 4 mbar und $298 \mathrm{~K} ; \mathrm{p}$ (Aceton) $=0,2$ mbar, $\mathrm{p}\left(\mathrm{SO}_{2}\right)=0,2$ mbar.

Für die qualitative Analyse ist der Bereich der CH-Valenzschwingung im Vergleich Reaktionsspektrum mit Spektren der Reinsubstanzen in Abbildung 4.26 exemplarisch vergrößert dargestellt.
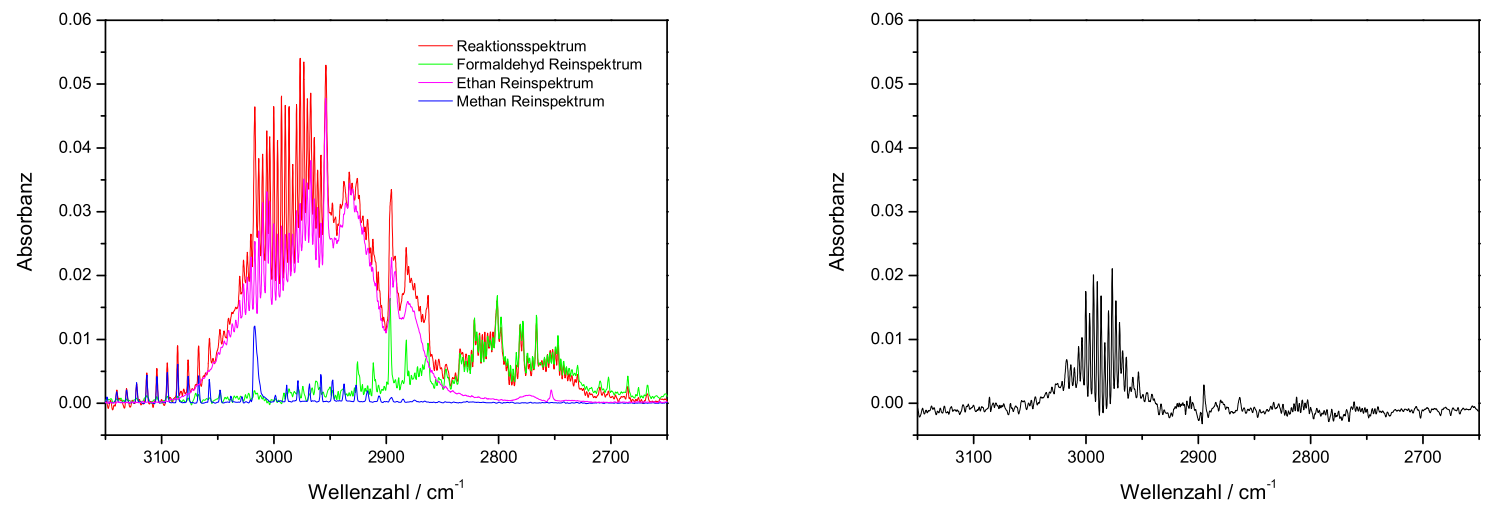

Abbildung 4.26: Reaktionssystem $\mathrm{CH}_{3}$ / O, qualitative Analyse stabiler Endprodukte. Linke Seite: Vergleich des Reaktionsspektrums mit Spektren der Reinsubstanzen; rechte Seite: Residuum. Die Abbildung zeigt, dass die Rotationspeaks von Ethan im Reaktionsspektrum nicht vollständig mit den Rotationspeaks des Ethan Reinspektrums übereinstimmen. p(Aceton) = 0,2 mbar, $p\left(\mathrm{SO}_{2}\right)=0,2$ mbar, Gesamtdruck 4 mbar, $\mathrm{T}=298 \mathrm{~K}, 200$ Photolysen.

Abbildung 4.26 zeigt, dass das Reaktionsspektrum in Bezug auf die rotationsaufgelöste Ethanbande nicht exakt mit dem Reinspektrum von Ethan übereinstimmt. Dies liegt 
wahrscheinlich an der nicht linearen Skalierung der rotationsaufgelösten Bande. Es stand zwar für die quantitative Analyse ein Satz an Kalibrierungsspektren zur Verfügung, doch wurden diese teilweise noch mit einem Faktor multipliziert. Es wird aber deutlich, dass bei den gewählten Bedingungen, neben der Selbstreaktion $\left(c_{1}\right)$ unter Bildung von Ethan, die Reaktion mit atomaren Sauerstoff unter Bildung von Formaldehyd $\left(\mathrm{o}_{1}\right)$ abläuft. Die Entstehung von Methan, was in diesem Umfang nur durch die oben diskutierte Sekundärreaktion $\left(c_{2}\right)$ erklärt werden kann, ist ebenfalls eindeutig feststellbar. Des Weiteren wurde die Bildung von $\mathrm{CO}$ und von Ethen beobachtet. $\mathrm{CO}$ wurde aber auch bei der Photolyse von Aceton gebildet und die Konzentration an Ethen war nur gering.

\subsubsection{Quantitative Analyse}

Wie in der Einleitung dieses Abschnittes beschrieben, war es Ziel, die Geschwindigkeit und Kanalverzweigung der Reaktion $\mathrm{CH}_{3}+\mathrm{CH}_{3} \rightarrow$ Produkte zu untersuchen. In Abwesenheit von Sauerstoffatomen bzw. des O-Atom Vorläufers $\mathrm{SO}_{2}$ konnte ausschließlich die Bildung von Ethan beobachtet werden. Dabei kann 95\% der Abnahme an Aceton durch den Partialdruck an Ethan erklärt werden. Übrige Reaktionen liegen somit unter 5\%. Dabei wurde Methan nur in sehr geringer Konzentration gefunden (vergleiche dazu: Abschnitt 4.4.1). Diese geringe Konzentration rechtfertigt die obige Annahme, dass die Bildung von Methan unter Anwesenheit von O-Atomen auf die Reaktion von Methylradikalen mit H-Atomen zurückzuführen ist, die bei den Reaktionswegen $\left(o_{1}\right)$ und $\left(o_{2}\right)$ gebildet werden.

In Anwesenheit von O-Atomen, die durch Co-Photolyse der Vorläufermischung Aceton / $\mathrm{SO}_{2}$ generiert wurden, hing die Kanalverzweigung stark von dem Anfangsverhältnis $\left[\mathrm{CH}_{3}\right]_{0} /[\mathrm{O}]_{0}$ ab. Somit ist keine generelle Aussage über das Verhältnis von Selbstreaktionsprodukten zu Oxidationsprodukten möglich. Vielmehr muss dieses Verhältnis aus den Anfangskonzentrationen der Radikale $\mathrm{CH}_{3}$ und $\mathrm{O}$ und den Geschwindigkeitskoeffizienten abgeleitet werden.

Sind die Radikalanfangskonzentrationen von $\mathrm{CH}_{3}$ und $\mathrm{O}$ und das Verhältnis von Produkten aus der Reaktion $\mathrm{CH}_{3}+\mathrm{O}$ zu den Produkten der Reaktion $\mathrm{CH}_{3}+\mathrm{CH}_{3}$ bekannt, so kann bei gegebener Geschwindigkeit der Oxidationsreaktion die Geschwindigeit der Selbstreaktion bestimmt werden. Dazu wurden Simulationsrechnungen zu diesen Radikalanfangsverhältnissen und Geschwindigkeitskoeffizienten durchgeführt. Durch Simulation 
der Reaktion mit einem geeigneten Mechanismus konnte die Geschwindigkeit der Kombination in guter Übereinstimmung mit der Literatur bestimmt werden.

\subsubsection{Simulation}

Für die Simulationsrechnungen wurden die aus den Experimenten gewonnene Kanalverzweigung für verschiedene Verhältnisse der Anfangskonzentrationen benutzt. Dabei wurde zum Einen ein Reaktionsmechanismus verwendet, der 13 Reaktionen umfasst, zum Anderen ein reduzierter Mechanismus, der auf die wesentlichen, in der Einleitung dargestellten Reaktionen beschränkt war. Die Reaktionen sind zusammen mit den jeweiligen Geschwindigkeitskoeffizienten in Tabelle 4.16 dargestellt.

\begin{tabular}{|c|c|c|c|c|}
\hline \multicolumn{4}{|c|}{ Reaktion } & \multirow{2}{*}{$\frac{\mathrm{cm}^{3} /(\mathrm{mol} \cdot \mathrm{s})}{2,40 \cdot 10^{13}}$} \\
\hline $\mathrm{CH}_{3}$ & $+\mathrm{CH}_{3}$ & $\rightarrow \mathrm{C}_{2} \mathrm{H}_{6}+$ & + & \\
\hline $\mathrm{CH}_{3}$ & $+\mathrm{CH}_{3}$ & $\rightarrow \mathrm{CH}_{4}+\mathrm{CH}_{2}$ & $2+$ & $4,30 \cdot 10^{10}$ \\
\hline $\mathrm{CH}_{3}$ & $+\mathrm{O}$ & $\rightarrow \mathrm{H}_{2} \mathrm{CO}+\mathrm{H}$ & + & $4,18 \cdot 10^{13}$ \\
\hline $\mathrm{CH}_{3}$ & $+\mathrm{O}$ & $\rightarrow \mathrm{CO} \quad+\mathrm{H}_{2}$ & $+\mathrm{H}$ & $3,42 \cdot 10^{13}$ \\
\hline $\mathrm{CH}_{3}$ & $+\mathrm{H}$ & $\rightarrow \mathrm{CH}_{4}+$ & 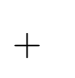 & $1,80 \cdot 10^{13}$ \\
\hline $\mathrm{H}_{2} \mathrm{CC}$ & $+\mathrm{O}$ & $\rightarrow \mathrm{OH}+\mathrm{CO}$ & $+\mathrm{H}$ & $1,00 \cdot 10^{11}$ \\
\hline $\mathrm{H}_{2} \mathrm{CC}$ & $+\mathrm{OH}$ & $\rightarrow \mathrm{H}_{2} \mathrm{O}+\mathrm{CO}$ & $+\mathrm{H}$ & $5,00 \cdot 10^{12}$ \\
\hline $\mathrm{H}_{2} \mathrm{CC}$ & $+\mathrm{H}$ & $\rightarrow \mathrm{CO} \quad+\mathrm{H}_{2}$ & $+\mathrm{H}$ & $3,00 \cdot 10^{10}$ \\
\hline $\mathrm{OH}$ & $+\mathrm{O}$ & $\rightarrow \mathrm{O}_{2} \quad+\mathrm{H}$ & + & $2,00 \cdot 10^{13}$ \\
\hline $\mathrm{OH}$ & $+\mathrm{OH}$ & $\rightarrow \mathrm{H}_{2} \mathrm{O}+\mathrm{O}$ & + & $1,10 \cdot 10^{12}$ \\
\hline $\mathrm{OH}$ & $+\mathrm{H}$ & $\rightarrow \mathrm{H}_{2} \mathrm{O}+$ & + & $1,00 \cdot 10^{10}$ \\
\hline $\mathrm{OH}$ & $+\mathrm{C}_{2} \mathrm{H}_{6}$ & $\rightarrow \mathrm{C}_{2} \mathrm{H}_{5}+\mathrm{H}_{2} \mathrm{O}$ & + & $1,50 \cdot 10^{11}$ \\
\hline $\mathrm{CH}_{3}$ & $+\mathrm{OH}$ & $\rightarrow \mathrm{CH}_{4} \mathrm{O}+$ & + & $5,00 \cdot 10^{13}$ \\
\hline
\end{tabular}

Tabelle 4.16: Reaktionssystem $\mathrm{CH}_{3} / \mathrm{O}$, Reaktionen und Geschwindigkeitskoeffizienten für die Simulationsrechnungen.

Ein Konzentrations-Zeit-Profil für den reduzierten Mechanismus ist in Abbildung 4.27 gegeben. Dabei wurde ein Verhältnis der Anfangskonzentration $\left[\mathrm{CH}_{3}\right]_{0} /[\mathrm{O}]_{0}$ von $8 / 1$ angenommen und die in Tabelle 4.16 dargestellten Geschwindigkeitskoeffizienten benutzt. 


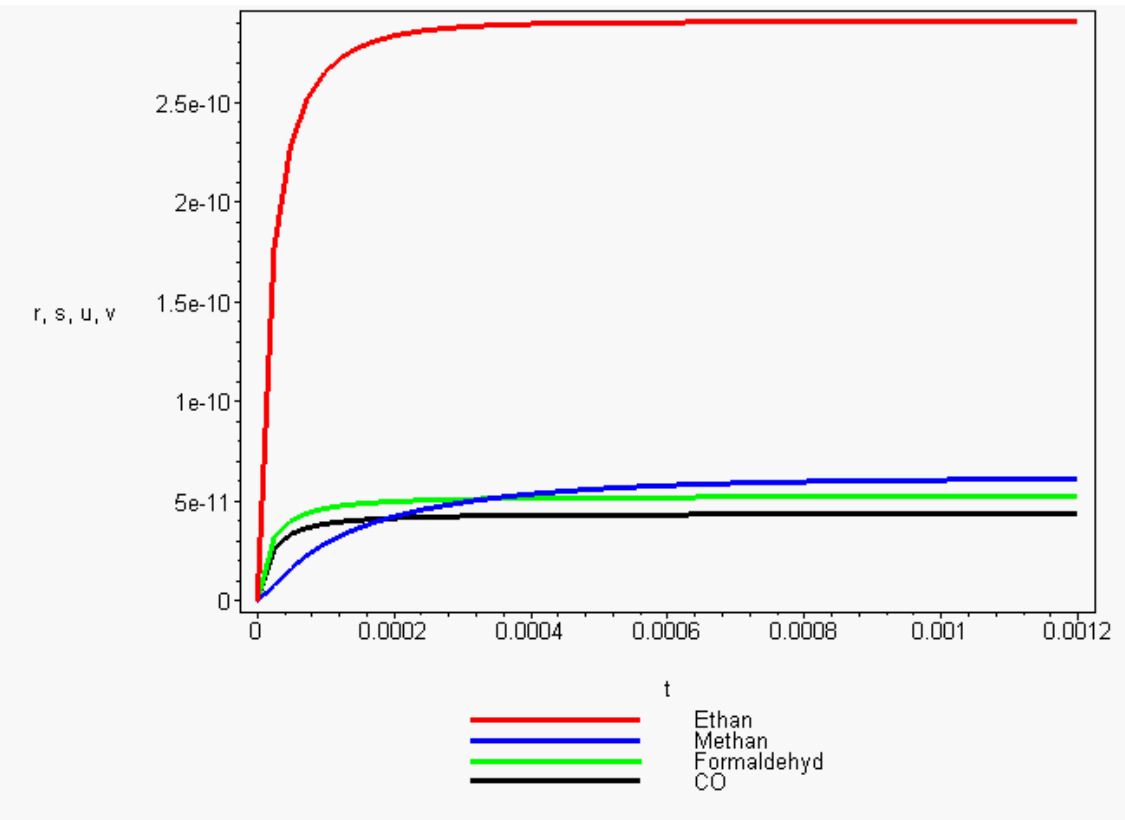

Abbildung 4.27: Reaktionssystem $\mathrm{CH}_{3}$ / O, Simulation der Konzentrations-Zeit-Profile des Reaktionssystems $\mathrm{CH}_{3} / \mathrm{O} ;\left[\mathrm{CH}_{3}\right]_{0}=0,8 \times 10^{-10} \mathrm{~mol} / \mathrm{cm}^{3},[\mathrm{O}]_{0}=0,1 \times 10^{-10} \mathrm{~mol} / \mathrm{cm}^{3}$ (die verwendeten Geschwindigkeitskoeffizienten sind in Tabelle4.16 gegeben; der Reaktionsmechanismus besteht nur aus den 4 Reaktionen der Einleitung, $\left.\mathrm{k}\left(\mathrm{CH}_{3}+\mathrm{CH}_{3} \rightarrow \mathrm{C}_{2} \mathrm{H}_{6}\right)=2,4 \times 10^{13} \mathrm{~cm}^{3} /(\mathrm{mol} \cdot \mathrm{s})\right)$.

Um die Abhängigkeit der Kanalverzweigung von der Geschwindigkeit der Kombinationsreaktion darzustellen, ist in Tabelle 4.17 das Verhältnis des Kombinationsproduktes Ethan zu dem O-Produkt Formaldehyd bei einer Anfangskonzentrationen von $\left[\mathrm{CH}_{3}\right]_{0}=$ $0,8 \times 10^{-10} \mathrm{~mol} / \mathrm{cm}^{3},[\mathrm{O}]_{0}=0,1 \times 10^{-10} \mathrm{~mol} / \mathrm{cm}^{3}$ dargestellt. Die Geschwindigkeit der Reaktion $\left(\mathrm{O}_{2}\right) \mathrm{CH}_{3}+\mathrm{O} \rightarrow \mathrm{H}_{2} \mathrm{CO}+\mathrm{H}$ wurde entsprechend der von HACK et al. [Hac05] für die Reaktion $\mathrm{CH}_{3}+\mathrm{O} \rightarrow$ Produkte bestimmten Kanalverzweigung $\left(\mathrm{o}_{1}\right) /\left(\mathrm{o}_{2}\right)=\mathrm{H}_{2} \mathrm{CO}$ / $\mathrm{CO}=0,55$ / 0,45 angenommen. Das hier experimentell bestimmte Verhältnis bei diesen Bedingungen betrug [Ethan] / $\left[\mathrm{H}_{2} \mathrm{CO}\right]=5,5$. Für die Simulation wurde das folgende Differentialgleichungssystem verwendet:

$$
\begin{aligned}
0 & =\frac{d}{d t} r(t)-k_{1}\left(a_{0}-2 r(t)-2 s(t)-u(t)-v(t)\right)^{2} \\
0 & =\frac{d}{d t} s(t)-k_{2}\left(a_{0}-2 r(t)-2 s(t)-u(t)-v(t)\right)(u(t)+v(t)) \\
0 & =\frac{d}{d t} u(t)-k_{3}\left(a_{0}-2 r(t)-2 s(t)-u(t)-v(t)\right)\left(c_{0}-u(t)-v(t)\right) \\
0 & =\frac{d}{d t} v(t)-k_{4}\left(a_{0}-2 r(t)-2 s(t)-u(t)-v(t)\right)\left(c_{0}-u(t)-v(t)\right),
\end{aligned}
$$

wobei $r(t)=\left[\mathrm{C}_{2} \mathrm{H}_{4}\right], s(t)=\left[\mathrm{CH}_{4}\right], u(t)=\left[\mathrm{H}_{2} \mathrm{CO}\right], v(t)=[\mathrm{CO}]$ und $a_{0}=\left[\mathrm{CH}_{3}\right]_{0}, c_{0}=[\mathrm{O}]_{0}$ und die Geschwindigkeitskoeffizienten $k_{2}-k_{4}$ wie in Tabelle 4.16 dargestellt bezeichnen. 


\begin{tabular}{|c|c||c|c|}
\hline $\mathrm{k}\left(\mathrm{CH}_{3}+\mathrm{CH}_{3} \rightarrow \mathrm{C}_{2} \mathrm{H}_{6}\right)$ & {$\left[\mathrm{C}_{2} \mathrm{H}_{6}\right] /\left[\mathrm{H}_{2} \mathrm{CO}\right]$} & $\mathrm{k}\left(\mathrm{CH}_{3}+\mathrm{CH}_{3} \rightarrow \mathrm{C}_{2} \mathrm{H}_{6}\right)$ & {$\left[\mathrm{C}_{2} \mathrm{H}_{6}\right] /\left[\mathrm{H}_{2} \mathrm{CO}\right]$} \\
\hline \hline $0,5 \times 10^{13}$ & 3,2 & $2,4 \times 10^{13}$ & 5,5 \\
$0,7 \times 10^{13}$ & 3,7 & $2,6 \times 10^{13}$ & 5,7 \\
$1,0 \times 10^{13}$ & 4,3 & $2,8 \times 10^{13}$ & 5,8 \\
$2,0 \times 10^{13}$ & 5,3 & $3,0 \times 10^{13}$ & 5,9 \\
$2,2 \times 10^{13}$ & 5,4 & $4,0 \times 10^{13}$ & 6,5 \\
\hline
\end{tabular}

Tabelle 4.17: Reaktionssystem $\mathrm{CH}_{3} / \mathrm{O}$, Verhältnis $\mathrm{C}_{2} \mathrm{H}_{6} / \mathrm{H}_{2} \mathrm{CO}$ vs. Geschwindigkeit. Eine exakte Übereinstimung von Experiment und Simulationsrechnung wurde für einen Geschwindigkeitskoeffizienten $\mathrm{k}\left(\mathrm{CH}_{3}+\mathrm{CH}_{3} \rightarrow \mathrm{C}_{2} \mathrm{H}_{6}\right)=2,4 \times 10^{13} \mathrm{~cm}^{3} /(\mathrm{mol} \cdot \mathrm{s})$ gefunden. Voraussetzung: $\mathrm{k}\left(\mathrm{CH}_{3}+\mathrm{O} \rightarrow \mathrm{H}_{2} \mathrm{CO}+\mathrm{H}\right)=4,18 \times 10^{13} \mathrm{~cm}^{3} /(\mathrm{mol} \cdot \mathrm{s}),\left[\mathrm{CH}_{3}\right]_{0}=0,8 \mathrm{~mol} / \mathrm{cm}^{3},[\mathrm{O}]_{0}=0,1$ $\mathrm{mol} / \mathrm{cm}^{3}$ (alle Geschwindigkeitskoeffizienten in $\mathrm{cm}^{3} /(\mathrm{mol} \cdot \mathrm{s})$ ).

Die Tabelle 4.17 zeigt, dass eine exakte Übereinstimmung von Experiment und Simulation für eine Geschwindigkeit $\mathrm{k}\left(\mathrm{CH}_{3}+\mathrm{CH}_{3} \rightarrow \mathrm{C}_{2} \mathrm{H}_{6}\right)=2,4 \times 10^{13} \mathrm{~cm}^{3} /(\mathrm{mol} \cdot \mathrm{s})$ gegeben ist. Auf Grund des angenommenen Fehlers für das Verhältnis von [Ethan] / $\left[\mathrm{H}_{2} \mathrm{CO}\right]=(5,5$ $\pm 0,2)$ ergibt sich aus der Simulation für die Geschwindigkeit ein Fehler von $\pm 0,2 \times$ $10^{13} \mathrm{~cm}^{3} /(\mathrm{mol} \cdot \mathrm{s})$.

\subsubsection{Diskussion}

Unter Berücksichtigung der von ZEUCH [Zeu03] und HaCK et al. [Hac05] bestimmten Kanalverzweigung für die Reaktion $\mathrm{CH}_{3}+\mathrm{O}$ konnte, ausgehend von einem konkurrierenden Reaktionsmechanismus, der Geschwindigkeitskoeffizient für die Reaktion $\mathrm{CH}_{3}+\mathrm{CH}_{3} \rightarrow$ $\mathrm{C}_{2} \mathrm{H}_{6}$ bestimmt werden. Mit $\mathrm{k}\left(\mathrm{CH}_{3}+\mathrm{O} \rightarrow\right.$ Produkte $)=7,6 \times 10^{13} \mathrm{~cm}^{3} /(\mathrm{mol} \cdot \mathrm{s})$ konnte relativ bestimmt werden:

$$
k\left(\mathrm{CH}_{3}+\mathrm{CH}_{3} \rightarrow \mathrm{C}_{2} \mathrm{H}_{6}\right)=(2,4 \pm 0,2) \times 10^{13} \mathrm{~cm}^{3} /(\mathrm{mol} \cdot \mathrm{s}) .
$$

Dabei wurde die Fehlergrenze für das Verhältnis der Produkte $\Delta\left([\right.$ Ethan $\left.] /\left[\mathrm{H}_{2} \mathrm{CO}\right]\right)=0,2$ aus der Messungenauigkeit abgeleitet.

Im Folgenden werden kurz die Ergebnisse der vorliegenden Literatur zur Selbstreaktion diskutiert.

In der Literatur finden sich für die Reaktion $\mathrm{CH}_{3}+\mathrm{CH}_{3} \rightarrow \mathrm{C}_{2} \mathrm{H}_{6}$ eine Vielzahl an experimentellen [Mac83, Mac85, Sla88, Cod02] wie auch theoretischen [Wag88, For91, Rob95] Ergebnissen.

Dabei wurde die Reaktion in einem Temperaturbereich von ca. 200 - $1000 \mathrm{~K}$ und einem 
Druckbereich von ca. 1 - 500 mbar intensiv untersucht. Für eine Temperatur von 298 K und einem niedrigen Gesamtdruck ( $\mathrm{p}_{\text {total }} \leq 5$ Torr) wurden dabei Werte für die Geschwindigkeit zwischen $1,3 \times 10^{13} \mathrm{~cm}^{3} /(\mathrm{mol} \cdot \mathrm{s})$ und $3,9 \times 10^{13} \mathrm{~cm}^{3} /(\mathrm{mol} \cdot \mathrm{s})$ gefunden.

Tsang [Tsa86] empfiehlt für einen Gesamtdruck von 4 mbar und einer Temperatur von $300 \mathrm{~K}$ einen Geschwindigkeitskoeffizienten $\mathrm{k}\left(\mathrm{CH}_{3}+\mathrm{CH}_{3} \rightarrow \mathrm{C}_{2} \mathrm{H}_{6}\right)=2,6 \times 10^{13} \mathrm{~cm}^{3} /(\mathrm{mol} \cdot \mathrm{s})$.

Der relativ bestimmte Wert von $\mathrm{k}\left(\mathrm{CH}_{3}+\mathrm{CH}_{3} \rightarrow \mathrm{C}_{2} \mathrm{H}_{6}\right)=(2,4 \pm 0,2) \times 10^{13} \mathrm{~cm}^{3} /(\mathrm{mol} \cdot \mathrm{s})$ wurde für den geringen Druck und das Badgas Argon damit in guter Übereinstimmung mit der Literatur gefunden.

\subsubsection{Die Reaktion Ethyl + O}

\subsubsection{Einleitung}

Die Reaktion von Ethylradikalen mit Sauerstoffatomen ist ebenso wie die vorhergehenden Reaktionen extrem wichtig, um die Verbrennung und den Abbau von Kohlenwasserstoffen in der Atmosphäre zu beschreiben. Die Reaktion von Ethylradikalen mit O-Atomen ist darüber hinaus von besonderem Interesse, weil es sich bei dem Ethylradikal um das am wenigsten komplexe Radikal handelt, dass eine C-C-Bindung enthält. Daher kann diese Reaktion einen ersten Einblick in die Kanalverzweigung höherer Alkoxyradikale gewähren. Ziel dieser Untersuchung ist die Bestimmung der Kanalverzweigung unter Berücksichtigung der Selbstreaktionen und der Oxidationsreaktionen der Ethylradikale. Die Geschwindigkeitskoeffizienten der Kombinationsreaktionen werden, auf der Basis der Anfangs- und Endkonzentrationen aller beteiligter Spezies, relativ zu der Geschwindigkeit der Reaktion $\mathrm{C}_{2} \mathrm{H}_{5}+\mathrm{O}$ mit Hilfe von Simulationsrechnungen bestimmt. Dabei wird für das System $\mathrm{C}_{2} \mathrm{H}_{5}$ / O folgender Reaktionsmechanismus zu Grunde gelegt:

$$
\begin{array}{rlr}
\mathrm{C}_{2} \mathrm{H}_{5}+\mathrm{O} & \longrightarrow \mathrm{C}_{2} \mathrm{H}_{4}+\mathrm{OH} & (\text { abs }) \\
& \longrightarrow \mathrm{CH}_{3} \mathrm{CHO}+\mathrm{H} & \left(o_{1}\right) \\
& \left.\longrightarrow \mathrm{H}_{2} \mathrm{CO}+\mathrm{CH}_{3}\right) \\
\mathrm{C}_{2} \mathrm{H}_{5}+\mathrm{C}_{2} \mathrm{H}_{5} & \longrightarrow \mathrm{C}_{4} \mathrm{H}_{10} & (c) \\
& \longrightarrow \mathrm{C}_{2} \mathrm{H}_{4}+\mathrm{C}_{2} \mathrm{H}_{6} & (d)
\end{array}
$$


Neben der Abstraktion (abs.) können zwei Oxidationsreaktionen $\left(\left(o_{1}\right)\right.$ und $\left.\left(o_{2}\right)\right)$ und die Kombination (c) bzw. Disproportionierung (d) zweier Ethylradikale auftreten. Der Disproportionierung wird dabei auf Grund des um eine Größenordnung kleineren Geschwindigkeitskoeffizienten, eine geringe Bedeutung zugeschrieben $\left(\mathrm{k}\left(\mathrm{C}_{2} \mathrm{H}_{5}+\mathrm{C}_{2} \mathrm{H}_{5} \rightarrow \mathrm{C}_{2} \mathrm{H}_{4}+\right.\right.$ $\left.\left.\mathrm{C}_{2} \mathrm{H}_{6}\right)=1,4 \times 10^{12} \mathrm{~cm}^{3} /(\mathrm{mol} \cdot \mathrm{s})\right)$.

Alle Messungen wurden bei einem Gesamtdruck von 4 mbar und einer Temperatur von 298 K durchgeführt. Die maximale Photolysenanzahl betrug 400, was einem Vorläuferumsatz von ca. $15 \%$ entsprach. Die qualitative und quantitative Analyse wurde anhand von 100-fach gemittelten IR-Spektren nach jeweils 100 Photolysen durchgeführt.

\subsubsection{Qualitative Analyse}

Für die Untersuchung des Reaktionssystems wurden $\mathrm{C}_{2} \mathrm{H}_{5} \mathrm{I} / \mathrm{SO}_{2}$ Mischungen bei $\lambda=$ $193 \mathrm{~nm}$ mit Hilfe von Laserlicht photolysiert. Diethylketon wurde, auf Grund der bei der Photolyse ebenfalls ablaufenden Norrish Typ III Spaltung, nicht als Vorläufer verwendet. Ein Reaktionsspektrum nach 400 Photolysen bei einer Gemischzusammensetzung von $\mathrm{p}\left(\mathrm{C}_{2} \mathrm{H}_{5} \mathrm{I}\right)=0,5, \mathrm{p}\left(\mathrm{SO}_{2}\right)=0,05$ mbar und einem Gesamtdruck von 4 mbar und $\mathrm{T}=298$ $\mathrm{K}$ zeigt Abbildung 4.28. 


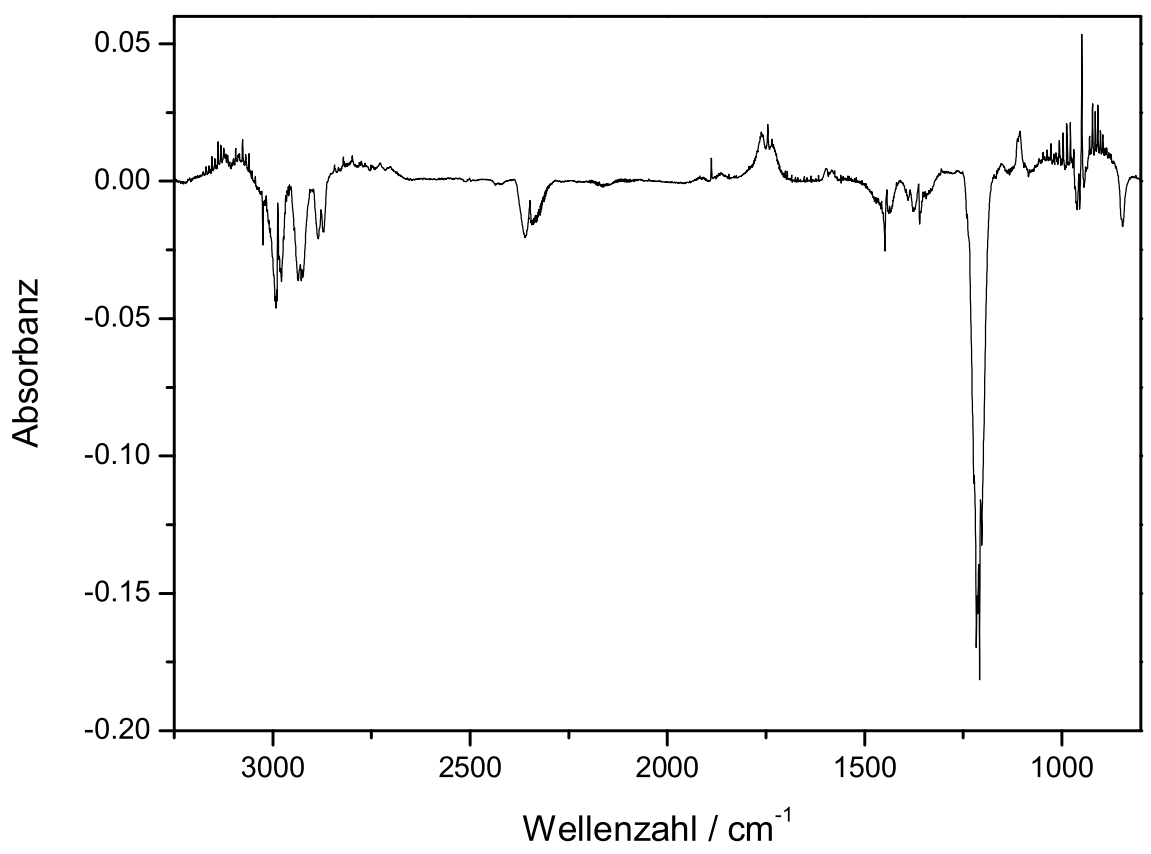

Abbildung 4.28: Reaktionssystem $\mathrm{C}_{2} \mathrm{H}_{5}$ / O, Reaktionsspektrum. $p\left(\mathrm{C}_{2} \mathrm{H}_{5} \mathrm{I}\right)=0,5$ mbar, $p\left(\mathrm{SO}_{2}\right)$ $=0,05$ mbar, $p_{\text {ges }}=4$ mbar, $T=298 \mathrm{~K}, 400$ Photolysen.

Vergrößerte Ausschnitte des um den Vorläufer reduzierten Reaktionsspektrums im Vergleich mit den Reinspektren der identifizierten Substanzen zeigt Abbildung 4.29, Dabei konnten folgende Substanzen eindeutig identifiziert werden: Acetaldehyd, Formaldehyd, Butan, Ethen, Methan. Der sehr große Anteil an Ethen resultiert neben dem Abstraktionsprozess (abs.) aus der direkten HI-Elimination von Ethyliodid bei der Photolyse. Dieser Prozess konnte, wie schon in den vorhergehenden Abschnitten beschrieben, bei allen iodierten Vorläufern beobachtet werden. Es handelt sich dabei nicht um ein Produkt aus der Disproportionierungsreaktion (d). Dies kann durch das Fehlen des dabei entstehenden Gegenstücks Ethan bestätigt werden. Eine Bestimmung des HI-Eliminationskanals erfolgt in Abschnitt 4.4.2 durch Photolyse von Ethyliodid in Abwesenheit von O-Atomen. Bezogen auf die Gesamtabnahme an Ethyliodid wurde der Kanal zu 39\% bestimmt. Die Bildung von Methan ist wahrscheinlich auf die schnelle Reaktion von Methylradikalen mit H-Atomen zurückzuführen $\left(\mathrm{k}\left(\mathrm{CH}_{3}+\mathrm{H}\right)=1,8 \times 10^{13} \mathrm{~cm}^{3} /(\mathrm{mol} \cdot \mathrm{s})\right)$. Methylradikale bzw. H-Atome werden dabei durch die Reaktionen $\left(o_{2}\right)$ bzw. $\left(o_{1}\right)$ gebildet. 

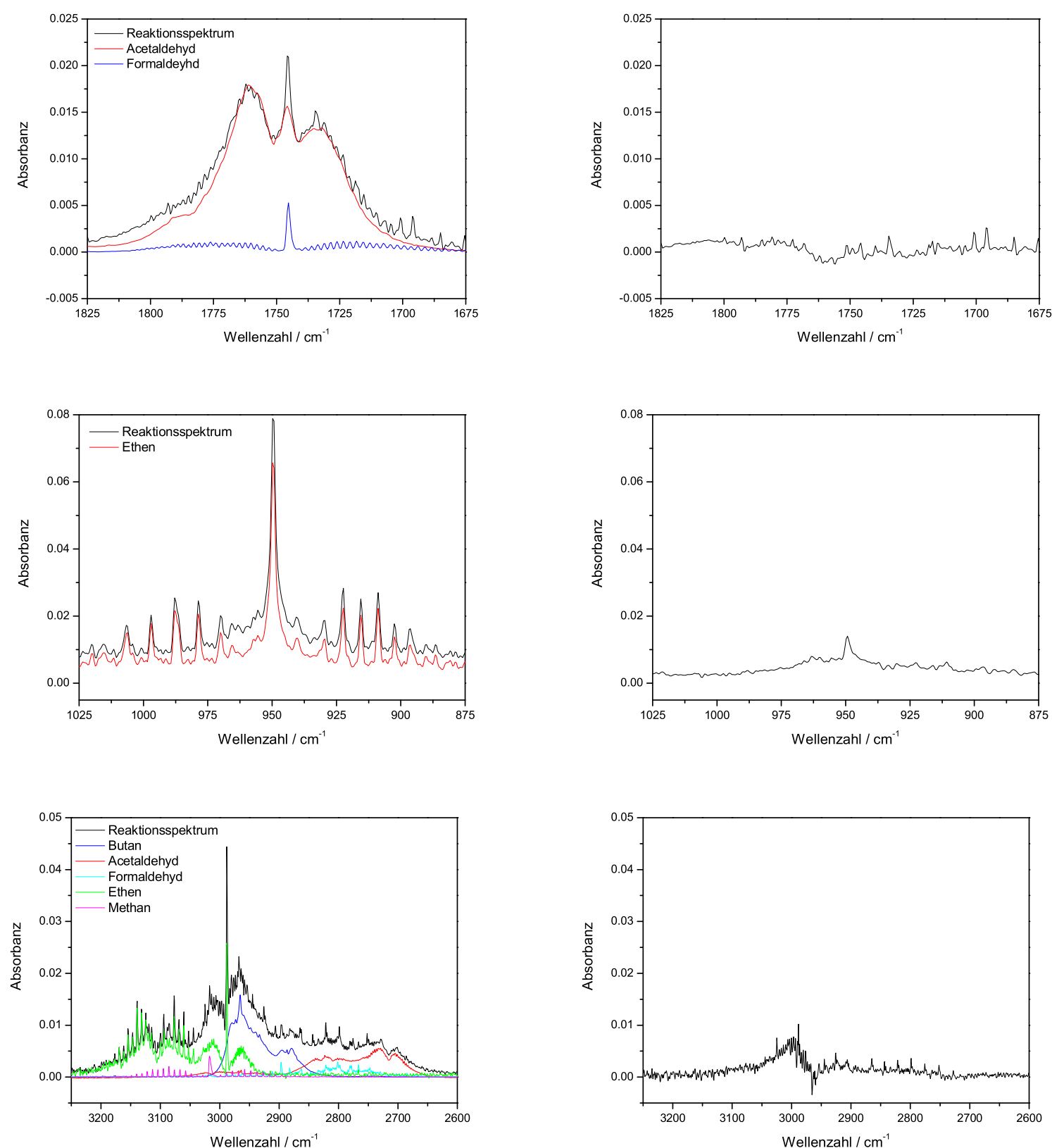

Abbildung 4.29: Reaktionssystem $\mathrm{C}_{2} \mathrm{H}_{5}$ / O, Vorläufer Ethyliodid. Vergleich des Reaktionsspektrums mit Spektren der Reinsubstanzen in vergrößerten Ausschnitten; in der linken Spalte ist jeweils das Reaktionsspektrum (schwarz) mit den Reinspektren (farbig) im Vergleich dargestellt, in der rechten Spalte sind die entsprechenden Residuen in schwarz dargestellt.

\subsubsection{Quantitative Analyse}

Wie auch bei dem Reaktionssystem $\mathrm{CH}_{3}$ / O kann für das Reaktionssystem $\mathrm{C}_{2} \mathrm{H}_{5}$ / O durch eine Bestimmung des Produktverhältnisses von Selbstreaktion zu Oxidation bei bekannten Radikalanfangskonzentrationen die Geschwindigkeit der Selbstreaktion ermittelt 
werden. Für eine Bestimmung des Geschwindigkeitskoeffizienten der Reaktion $\mathrm{C}_{2} \mathrm{H}_{5}+$ $\mathrm{C}_{2} \mathrm{H}_{5} \rightarrow \mathrm{C}_{4} \mathrm{H}_{10}$ wurde daher ein experimentell bestimmtes Verhältnis $\left[\mathrm{C}_{4} \mathrm{H}_{10}\right] /\left[\mathrm{H}_{2} \mathrm{CO}\right]$ auf die Anfangskonzentration der Ethylradikale und O-Atome von $\left[\mathrm{C}_{2} \mathrm{H}_{5}\right]_{0} /[\mathrm{O}]_{0}=6,0$ bezogen. Mit einem auf die wesentlichen konkurrierenden Reaktionen beschränkten Mechanismus wurde dann durch Simulationsrechnungen die Geschwindigkeit der Reaktion $\mathrm{C}_{2} \mathrm{H}_{5}+\mathrm{C}_{2} \mathrm{H}_{5} \rightarrow \mathrm{C}_{4} \mathrm{H}_{10}$ ermittelt.

In Abbildung 4.30 sind die Partialdrücke von Butan und Formaldehyd gegen die Abnahme an Vorläufer aufgetragen. Dabei ist zu beachten, dass ein wesentlicher Teil der Ethyliodidabnahme aus der HI-Elimination resultiert. Die Messdaten sind in Tabelle 4.18 angegeben.

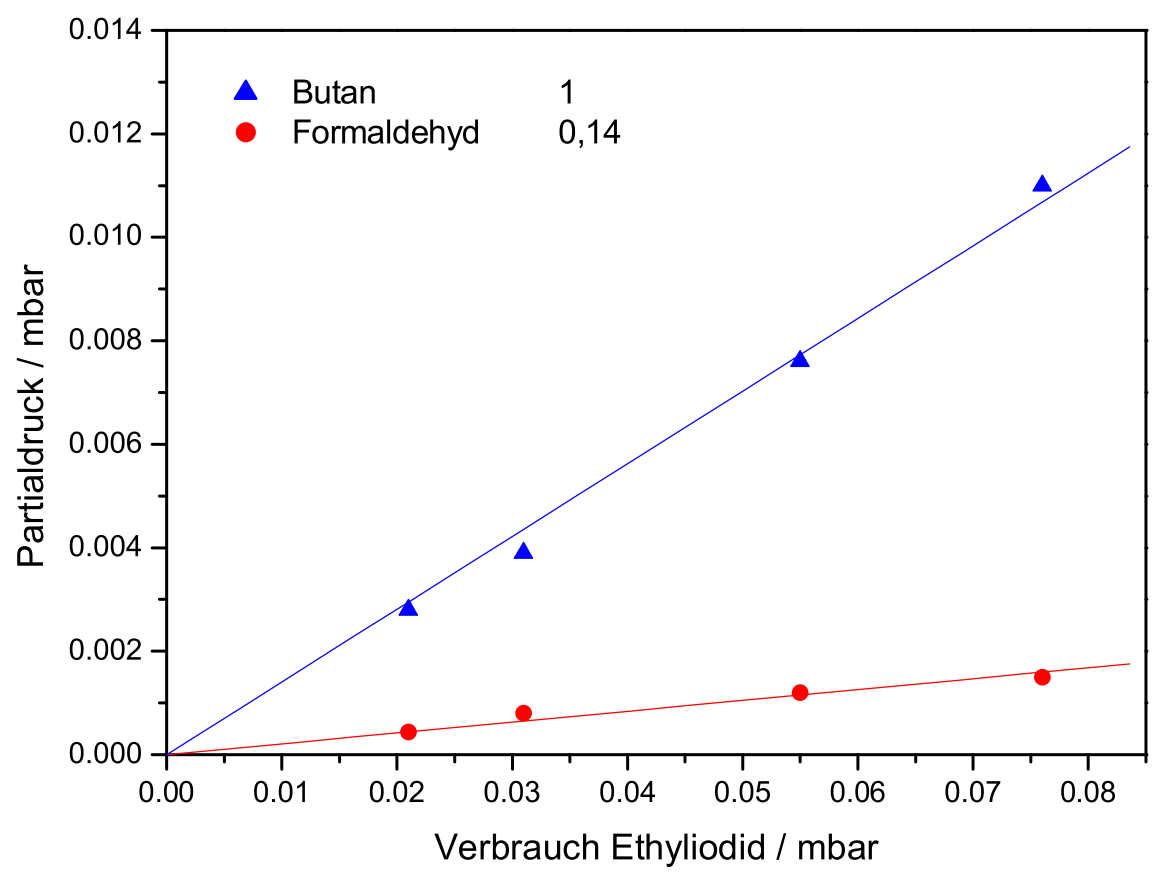

Abbildung 4.30: Reaktionssystem $\mathrm{C}_{2} \mathrm{H}_{5}$ / O , quantitative Analyse für ein Radikal / O-AtomKonzentrationsverhältnis von $\left[\mathrm{C}_{2} \mathrm{H}_{5}\right]_{0} /[\mathrm{O}]_{0}=6,0$. Das bestimmte Verhältnis von Butan / Formaldehyd $=1$ / 0,14 gilt nur für dieses Radikal / O-Atom Verhältnis. 


\begin{tabular}{|c||c|c|c|}
\hline $\begin{array}{c}\mathrm{C}_{2} \mathrm{H}_{5} \mathrm{I} \\
\text { Verbrauch }\end{array}$ & $\begin{array}{c}\mathrm{p} \text { (Ethen) } \\
{[\mathrm{mbar}]}\end{array}$ & $\begin{array}{c}\mathrm{p}\left(\mathrm{H}_{2} \mathrm{CO}\right) \\
{[\mathrm{mbar}]}\end{array}$ & $\begin{array}{c}\mathrm{p} \text { (Butan) } \\
{[\mathrm{mbar}]}\end{array}$ \\
\hline \hline 0,021 & 0,016 & $4,4 \mathrm{E}-4$ & 0,0028 \\
\hline 0,031 & 0,023 & $8 \mathrm{E}-4$ & 0,0039 \\
\hline 0,055 & 0,035 & 0,0012 & 0,0076 \\
\hline 0,076 & 0,038 & 0,0015 & 0,011 \\
\hline
\end{tabular}

Tabelle 4.18: Reaktionssystem $\mathrm{C}_{2} \mathrm{H}_{5} /$ O. Messwerte. $p$ (Ethyliodid) $=0,6$ mbar, $p\left(\mathrm{SO}_{2}\right)=0,05$ mbar, $p_{\text {ges }}=4$ mbar, $\mathrm{T}=298 \mathrm{~K}$, Badgas Argon.

\subsubsection{Simulation}

Für die Simulation des Reaktionssystems $\mathrm{C}_{2} \mathrm{H}_{5}$ / O wurde ein Mechanismus mit 20 Reaktionen zu Grunde gelegt. Die verwendeten Geschwindigkeitskoeffizienten und Reaktionen sind in Tabelle 4.19 dargestellt.

Eine Simulation der Konzentrations-Zeit-Profile erfolgte dabei auf Grundlage der experimentell bestimmten Radikal / O-Atom Anfangskonzentrationen unter Berücksichtigung des in Abschnitt 4.4.2 ermittelten HI-Eliminationsanteils für die Photolyse von Ethyliodid (39\%). Eine Simulation der Konzentrations-Zeit-Profile unter Verwendung des vollständigen Mechanismus aus Tabelle 4.19 und unter Voraussetzung eines Radikal / O-Atom Verhältnisses von $\left[\mathrm{C}_{2} \mathrm{H}_{5}\right]_{0} /[\mathrm{O}]_{0}=6,0$ ist in Abbildung 4.31 dargestellt. 


\begin{tabular}{|c|c|c|c|c|c|}
\hline \multicolumn{5}{|c|}{ Reaktion } & \multirow{2}{*}{$\frac{\mathrm{cm}^{3} /(\mathrm{mol} \cdot \mathrm{s})}{1,10 \cdot 10^{13}}$} \\
\hline $\mathrm{C}_{2} \mathrm{H}_{5}$ & $+\mathrm{C}_{2} \mathrm{H}_{5}$ & $\rightarrow$ Butan & + & + & \\
\hline $\mathrm{C}_{2} \mathrm{H}_{5}$ & $+\mathrm{C}_{2} \mathrm{H}_{5}$ & $\rightarrow \mathrm{C}_{2} \mathrm{H}_{4}$ & $+\mathrm{C}_{2} \mathrm{H}_{6}$ & $6+$ & $1,40 \cdot 10^{12}$ \\
\hline $\mathrm{C}_{2} \mathrm{H}_{5}$ & $+\mathrm{O}$ & $\rightarrow \mathrm{C}_{2} \mathrm{H}_{4}$ & $+\mathrm{OH}$ & + & $3,17 \cdot 10^{13}$ \\
\hline $\mathrm{C}_{2} \mathrm{H}_{5}$ & $+\mathrm{O}$ & \multicolumn{2}{|c|}{$\rightarrow \mathrm{CH}_{3} \mathrm{CHO}+\mathrm{H}$} & + & $5,81 \cdot 10^{13}$ \\
\hline $\mathrm{C}_{2} \mathrm{H}_{5}$ & $+\mathrm{O}$ & $\rightarrow \mathrm{H}_{2} \mathrm{CO}$ & $+\mathrm{CH}_{3}$ & + & $4,22 \cdot 10^{13}$ \\
\hline $\mathrm{C}_{2} \mathrm{H}_{5}$ & $+\mathrm{H}$ & $\rightarrow \mathrm{C}_{2} \mathrm{H}_{6}$ & + & + & $1,80 \cdot 10^{13}$ \\
\hline $\mathrm{C}_{2} \mathrm{H}_{5}$ & $+\mathrm{H}$ & $\rightarrow 2 \mathrm{CH}_{3}$ & + & + & $3,60 \cdot 10^{13}$ \\
\hline $\mathrm{C}_{2} \mathrm{H}_{5}$ & $+\mathrm{O}_{2}$ & \multicolumn{2}{|c|}{$\rightarrow$ Produkte +} & + & $6,02 \cdot 10^{13}$ \\
\hline $\mathrm{H}_{2} \mathrm{CO}$ & $+\mathrm{O}$ & $\rightarrow \mathrm{OH}$ & $+\mathrm{CO}$ & $+\mathrm{H}$ & $1,00 \cdot 10^{11}$ \\
\hline $\mathrm{H}_{2} \mathrm{CO}$ & $+\mathrm{OH}$ & $\rightarrow \mathrm{H}_{2} \mathrm{O}$ & $+\mathrm{CO}$ & $+\mathrm{H}$ & $5,00 \cdot 10^{12}$ \\
\hline $\mathrm{H}_{2} \mathrm{CO}$ & $+\mathrm{H}$ & $\rightarrow \mathrm{CO}$ & $+\mathrm{H}_{2}$ & $+\mathrm{H}$ & $3,00 \cdot 10^{10}$ \\
\hline $\mathrm{OH}$ & $+\mathrm{O}$ & $\rightarrow \mathrm{O}_{2}$ & $+\mathrm{H}$ & + & $2,00 \cdot 10^{13}$ \\
\hline $\mathrm{OH}$ & $+\mathrm{OH}$ & $\rightarrow \mathrm{H}_{2} \mathrm{O}$ & $+\mathrm{O}$ & + & $1,10 \cdot 10^{12}$ \\
\hline $\mathrm{OH}$ & $+\mathrm{H}$ & $\rightarrow \mathrm{H}_{2} \mathrm{O}$ & + & + & $1,00 \cdot 10^{10}$ \\
\hline $\mathrm{O}_{2}$ & $+\mathrm{H}$ & $\rightarrow \mathrm{OH}$ & $+\mathrm{O}$ & + & $7,5 \cdot 10^{13}$ \\
\hline $\mathrm{CH}_{3}$ & $+\mathrm{OH}$ & $\rightarrow \mathrm{CH}_{4} \mathrm{O}$ & + & + & $5,00 \cdot 10^{13}$ \\
\hline $\mathrm{CH}_{3}$ & $+\mathrm{CH}_{3}$ & $\rightarrow \mathrm{C}_{2} \mathrm{H}_{6}$ & + & + & $2,40 \cdot 10^{13}$ \\
\hline $\mathrm{CH}_{3}$ & $+\mathrm{O}$ & $\rightarrow \mathrm{CO}$ & $+\mathrm{H}_{2}$ & $+\mathrm{H}$ & $3,42 \cdot 10^{13}$ \\
\hline $\mathrm{CH}_{3}$ & $+\mathrm{O}$ & $\rightarrow \mathrm{H}_{2} \mathrm{CO}$ & $+\mathrm{H}$ & + & $4,18 \cdot 10^{13}$ \\
\hline $\mathrm{CH}_{3}$ & $+\mathrm{H}$ & $\rightarrow \mathrm{CH}_{4}$ & + & + & $1,80 \cdot 10^{13}$ \\
\hline
\end{tabular}

Tabelle 4.19: Reaktionssystem $\mathrm{C}_{2} \mathrm{H}_{5}$ / O, Reaktionen und Geschwindigkeitskoeffizienten der Simulationsrechnungen für verschiedene Anfangskonzentrationen. 


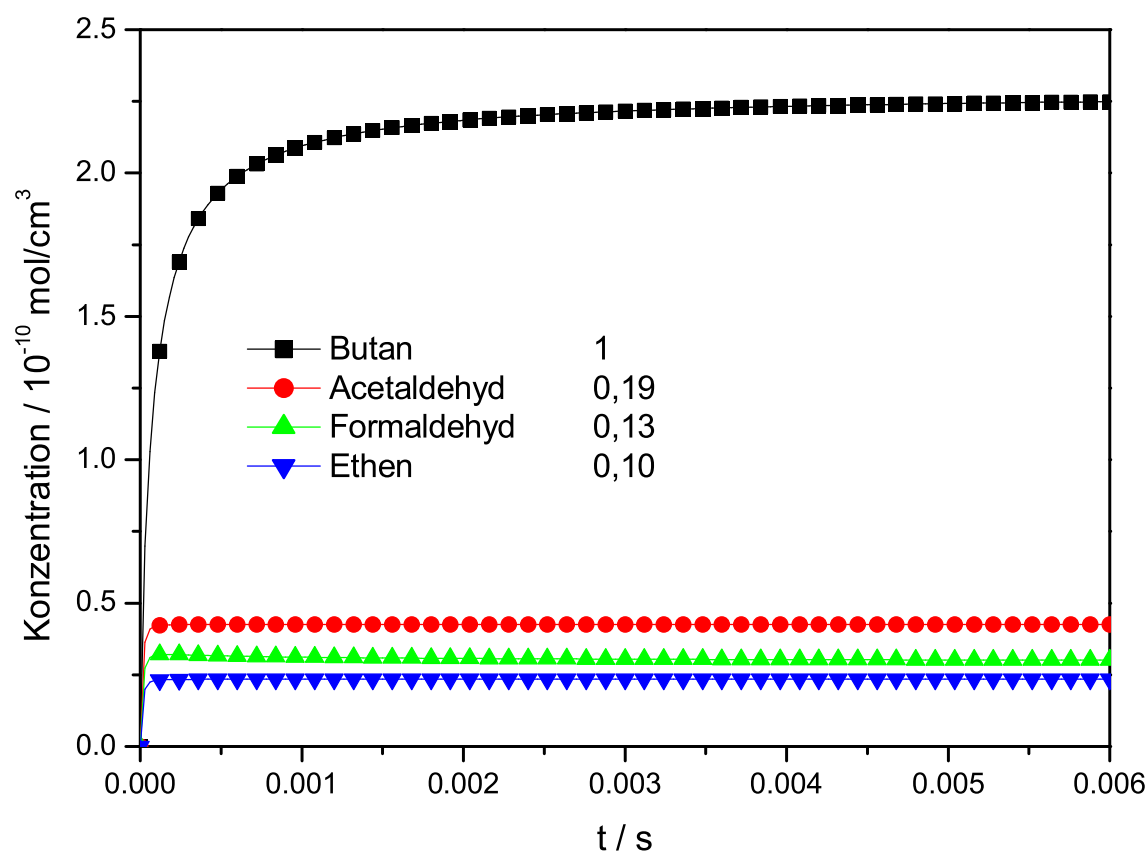

Abbildung 4.31: Reaktionssystem $\mathrm{C}_{2} \mathrm{H}_{5}$ / O, Konzentrations-Zeit-Profile; Radikal / O-AtomKonzentrationsverhältnis von $\left[\mathrm{C}_{2} \mathrm{H}_{5}\right]_{0} /[\mathrm{O}]_{0}=6$. Der Mechanismus ist in Tabelle 4.19 angegeben. Aus der Simulation folgt für die Reaktion $\mathrm{C}_{2} \mathrm{H}_{5}+\mathrm{C}_{2} \mathrm{H}_{5} \rightarrow \mathrm{C}_{4} \mathrm{H}_{10}$ ein Geschwindigkeitskoeffizient von $\mathrm{k}\left(\mathrm{C}_{2} \mathrm{H}_{5}+\mathrm{C}_{2} \mathrm{H}_{5} \rightarrow \mathrm{C}_{4} \mathrm{H}_{10}\right)=1,1 \times 10^{13} \mathrm{~cm}^{3} /(\mathrm{mol} \cdot \mathrm{s})$.

\begin{tabular}{|c|c||c|c|}
\hline$\left[\mathrm{C}_{2} \mathrm{H}_{5}\right]_{0} /[\mathrm{O}]_{0}$ & {$\left[\mathrm{H}_{2} \mathrm{CO}\right] /[$ Butan $]$} & {$\left[\mathrm{C}_{2} \mathrm{H}_{5}\right]_{0} /[\mathrm{O}]_{0}$} & {$\left[\mathrm{H}_{2} \mathrm{CO}\right] /[$ Butan $]$} \\
\hline \hline 1 & 2,20 & 6 & 0,13 \\
2 & 0,67 & 7 & 0,10 \\
3 & 0,32 & 8 & 0,09 \\
4 & 0,21 & 9 & 0,08 \\
5 & 0,16 & 10 & 0,07 \\
\hline
\end{tabular}

Tabelle 4.20: Reaktionssystem $\mathrm{C}_{2} \mathrm{H}_{5} / \mathrm{O}$, Verhältnis $\left[\mathrm{H}_{2} \mathrm{CO}\right] /[$ Butan $]$ vs. $\left[\mathrm{C}_{2} \mathrm{H}_{5}\right]_{0} /[\mathrm{O}]_{0}$. Eine Übereinstimung von Experiment und Simulation wurde für das experimentelle Verhältnis $\left[\mathrm{C}_{2} \mathrm{H}_{5}\right]_{0} /[\mathrm{O}]_{0}=6,0$ gefunden. Die Tabelle verdeutlicht die Sensitivität des Mechanismus bezüglich des Radikal / O-Atom Verhältnisses. Reaktionen und verwendete Geschwindigkeiten sind in Tabelle 4.19 angegeben.

Wie schon beschrieben, konnte eine Übereinstimmung von Experiment und Simulation nur für das experimentell bestimmte Verhältnis der Anfangskonzentrationen der Radikale und O-Atome erzielt werden. Um die Abhängigkeit des Verhältnisses [Butan] / $\left[\mathrm{H}_{2} \mathrm{CO}\right]$ von dem Verhältnis $\left[\mathrm{C}_{2} \mathrm{H}_{5}\right]_{0} /[\mathrm{O}]_{0}$ zu verdeutlichen, wurden Simulationsrechnungen durchgeführt, bei denen von dem Experiment abweichende Anfangskonzentrationen verwendet 
wurden. Die Ergebnisse dieser Simulationen sind in Tabelle 4.20 dargestellt.

Neben dem Einfluss der Radikalanfangskonzentration wurde noch der Einfluss der Reaktion $\mathrm{C}_{2} \mathrm{H}_{5} \mathrm{I}+\mathrm{O} \rightarrow \mathrm{C}_{2} \mathrm{H}_{5}+\mathrm{IO}$ auf das Verhältnis von Selbstreaktionsprodukten zu Oxidationsprodukten untersucht. Die Simulationsrechnungen zeigten, dass unter Berücksichtigung dieser Reaktion das Verhältnis von Selbsreaktionsprodukten zu Oxidationsprodukten zunimmt. Um bei gleichen Radikalanfangskonzentrationen dasselbe Verhältnis von Selbstreaktionsprodukt zu Oxidationsprodukten zu erhalten, müsste demnach die Selbstreaktionsgeschwindigkeit gesenkt werden.

\subsubsection{Diskussion}

Aus der quantitativen Analyse und auf Grundlage der bestimmten Radikal / O-Atom Anfangskonzentrationen und des Verhältnisses von $\left[\mathrm{H}_{2} \mathrm{CO}\right]$ zu [Butan] konnte mittels Simulationsrechnungen die Geschwindigkeit der Reaktion $\mathrm{C}_{2} \mathrm{H}_{5}+\mathrm{C}_{2} \mathrm{H}_{5} \rightarrow$ Butan wie folgt bestimmt werden:

$$
k\left(\mathrm{C}_{2} \mathrm{H}_{5}+\mathrm{C}_{2} \mathrm{H}_{5} \rightarrow \text { Butan }\right)=(1,1 \pm 0,3) \times 10^{13} \mathrm{~cm}^{3} /(\mathrm{mol} \cdot \mathrm{s})
$$

Dabei waren experimentelle Ergebnisse und Simulation innerhalb der angegebenen Fehlergrenze im Einklang.

Im Folgenden werden die experimentellen Ergebnisse mit Resultaten für die Kombinationsreaktion ([Dob91, Atk97])) und Oxidation ([Hoy99, Sla88]) in der Literatur verglichen.

Dobis und Benson untersuchten die Reaktion $\mathrm{C}_{2} \mathrm{H}_{5}+\mathrm{C}_{2} \mathrm{H}_{5}$ bezüglich des Verhältnisses von Rekombination zu Disproportionierung und der Geschwindigkeit in einem Temperaturbereich von 203 - $343 \mathrm{~K}$ unter Verwendung eines Niederdruckreaktors [Dob91]. Das Verhältnis Disproportionierung zu Rekombination wurde zu 0,14 \pm 0,01 bestimmt. Die Geschwindigkeit der Rekombination wurde zu k $\left(\mathrm{C}_{2} \mathrm{H}_{5}+\mathrm{C}_{2} \mathrm{H}_{5} \rightarrow\right.$ Butan $)=8,6 \times$ $10^{12} \mathrm{~cm}^{3} /(\mathrm{mol} \cdot \mathrm{s})$ und der Disproportionierung zu $\mathrm{k}\left(\mathrm{C}_{2} \mathrm{H}_{5}+\mathrm{C}_{2} \mathrm{H}_{5} \rightarrow \mathrm{C}_{2} \mathrm{H}_{4}+\mathrm{C}_{2} \mathrm{H}_{6}\right)=$ $1,2 \times 10^{12} \mathrm{~cm}^{3} /(\mathrm{mol} \cdot \mathrm{s})$ bestimmt.

AtKinson und Hudgens benutzten für die Untersuchung der Selbst-Reaktion Laserphotolysen einer $\mathrm{Cl}_{2} / \mathrm{C}_{2} \mathrm{H}_{6}$ Mischung bei $\lambda=308 \mathrm{~nm}$ zur Erzeugung der Radikale und cavity ring-down Spektroskopie zu Detektion der Produkte. Für eine Temperatur von 295 K und einem Gesamtdruck von 7 mbar wurde die Geschwindigkeit der Selbst-Reaktion 
$\mathrm{zu} \mathrm{k}\left(\mathrm{C}_{2} \mathrm{H}_{5}+\mathrm{C}_{2} \mathrm{H}_{5} \rightarrow\right.$ Produkte $)=(1,20 \pm 0,26) \times 10^{13} \mathrm{~cm}^{3} /(\mathrm{mol} \cdot \mathrm{s})$ bestimmt.

ZEUCH hat die Oxidationsreaktion mit Hilfe der in dieser Arbeit ebenfalls verwendeten Apparatur unter Verwendung von 3 verschiedenen Vorläufern (Diethylketon, Ethyliodid und Ethan $/ \mathrm{CFCl}_{3}$ ) bezüglich der Kanalverzweigung untersucht [Zeu03]. Der Abstraktionskanal unter Bildung von Ethen und $\mathrm{OH}$ wurde dabei nur unter Verwendung des Vorläufers Diethylketon quantitativ bestimmt. Es wurde in Übereinstimmung aller Vorläufer folgendes Kanalverhältnis gefunden:

$$
\begin{array}{rlr}
\mathrm{C}_{2} \mathrm{H}_{5}+\mathrm{O} & \longrightarrow \mathrm{C}_{2} \mathrm{H}_{4}+\mathrm{OH} & 24 \pm 7 \\
& \longrightarrow \mathrm{CH}_{3} \mathrm{CHO}+\mathrm{H} & 44 \pm 4 \\
& \longrightarrow \mathrm{H}_{2} \mathrm{CO}+\mathrm{CH}_{3} & 32 \pm 3
\end{array}
$$

SLAGLE et al. untersuchten die Oxidationsreaktion mittels eines Strömungssytems [Sla88]. Radikale bzw. O-Atome wurden durch Photolyse von Diethylketon bzw. $\mathrm{SO}_{2}$ erzeugt. Der Produktnachweis erfolgte über Photoionisations Massenspektrometrie. Die Kanalverzweigung der Reaktion wurde bestimmt zu:

$$
\begin{array}{rlr}
\mathrm{C}_{2} \mathrm{H}_{5}+\mathrm{O} & \longrightarrow \mathrm{C}_{2} \mathrm{H}_{4}+\mathrm{OH} & 23 \pm 7 \\
& \longrightarrow \mathrm{CH}_{3} \mathrm{CHO}+\mathrm{H} & 40 \pm 4 \\
& \longrightarrow \mathrm{H}_{2} \mathrm{CO}+\mathrm{CH}_{3} & 32 \pm 6
\end{array}
$$

Die Geschwindigkeit der Gesamtreaktion $\mathrm{C}_{2} \mathrm{H}_{5}+\mathrm{O} \rightarrow$ Produkte wurde $\mathrm{zu} \mathrm{k}\left(\mathrm{C}_{2} \mathrm{H}_{5}+\mathrm{O}\right.$ $\rightarrow$ Produkte $)=1,32 \times 10^{14} \mathrm{~cm}^{3} /(\mathrm{mol} \cdot \mathrm{s})$ bestimmt. Dieser Wert wurde auch in den Simulationsrechnungen verwendet.

Hoyermann et al. untersuchten die Oxidationsreaktion in einem Druckbereich von 1 mbar - 3 mbar mittels eines Strömungssystems bei 298 K [Hoy99]. Eine Analyse der Endprodukte wurde mittels Massenspektrometrie mit EI (electron impact) durchgeführt. Dabei wurde eine Kanalverzweigung gefunden, die mit der von SLAGLE annähernd übereinstimmt. Quantenchemische Rechnungen unterstützen die experimentell gefundene Kanalverzweigung. 


\subsubsection{Die Reaktion Allyl + O}

\subsubsection{Einleitung}

Die Reaktion von Allylradikalen mit Sauerstoffatomen ist eine der entscheidenden Reaktionen bei der Verbrennung von Kohlenwasserstoffen, da das Radikal auf Grund seiner außergewöhnlichen Stabilität in signifikanten Konzentrationen sowohl in Flammen als auch in der Hochtemperatur-Pyrolyse vorliegt [Cat81, Wes84, Sla90, Dav99, Sim03]. Die Stabilität resultiert aus der $\pi$-Elektronenkonjugation des Radikals, das damit zu einem der kleinsten resonanzstabilisierten Radikale gehört. Diese hohe Allylradikalkonzentration wurde in neueren experimentellen Untersuchungen und Rechnungen für Propen und Propen / Ethin Flammen bestätigt Ata98, Ata03, Hoy04. Generell sind $\mathrm{C}_{3}$-Spezies wie $\mathrm{C}_{3} \mathrm{H}_{6}, \mathrm{C}_{3} \mathrm{H}_{5}, \mathrm{C}_{3} \mathrm{H}_{4}$ (Allen und Propin) und $\mathrm{C}_{3} \mathrm{H}_{3}$ dabei Schlüsselstellen für die Beschreibung von Kohlenwasserstoffverbrennungen unter Hochtemperartur-Bedingungen mittels eines kinetischen Reaktionsmechanismus [War97, Smi00, Hoy04]. Modellierungen zeigen, dass genaue Kenntnisse der kinetischen Daten für diese $\mathrm{C}_{3}$-Spezies notwendig sind, um die Rußbildung, Flammengeschwindigkeiten und andere Verbrennungsbedingungen akkurat zu beschreiben [Hoy04, Qin00]. Auf Grund der Resonanz-Stabilisierung wird für das freie Radikal als Hauptreaktionsmechanismus die Reaktion mit anderen, in Verbrennungsprozessen vorliegenden Radikalen wie $\mathrm{HO}_{2}, \mathrm{CH}_{3}, \mathrm{CH}_{3} \mathrm{O}_{2}, \mathrm{OH}$ und Atomen wie $\mathrm{O}$ und H, vorgeschlagen. Die Konzentrationen dieser Radikale liegen zwar weit unter der Konzentration von molekularem Sauerstoff, doch sind die Radikalreaktionen unter Hochtemperatur-Bedingungen um mehrere Größenordnungen schneller, als die Reaktion mit $\mathrm{O}_{2}$ [Dea85, Bal91, Sto91]. Somit sind die Pyrolyse und Reaktion mit molekularem Sauerstoff im Vergleich dazu von geringerer Bedeutung [Sla90]. Die Reaktion Allyl + O wurde auf Grund ihrer außerordentlichen Bedeutung für Verbrennungsprozesse schon mehrfach untersucht [Trö97, Ker99, Sla90, Par02, Par03, Joo04]. Auf Grundlage dieser Untersuchungen kann von einem Mechanismus analog zu der Reaktion von Alkylradikalen

+ $\mathrm{O}$ über ein energetisch hoch angeregtes Alkoxyradikal und eine direkte H-Abstraktion ausgegangen werden: 


$$
\begin{array}{rlr}
\mathrm{C}_{3} \mathrm{H}_{5}+\mathrm{O} & \longrightarrow \mathrm{C}_{3} \mathrm{H}_{5} \mathrm{O}^{\star} & (a) \\
\mathrm{C}_{3} \mathrm{H}_{5} \mathrm{O}^{\star} & \longrightarrow \mathrm{C}_{3} \mathrm{H}_{4} \mathrm{O}+\mathrm{H} & \left(a_{1}\right) \\
& \left.\longrightarrow \mathrm{C}_{2} \mathrm{H}_{4}+\mathrm{H}+\mathrm{CO}\right) \\
& \longrightarrow \mathrm{H}_{2} \mathrm{CO}+\mathrm{C}_{2} \mathrm{H}_{2}+\mathrm{H} & \left(a_{3}\right) \\
& \longrightarrow \mathrm{H}_{2} \mathrm{CCO}+\mathrm{CH}_{3} \\
\mathrm{C}_{3} \mathrm{H}_{5}+\mathrm{O} & \longrightarrow \mathrm{CH}_{3} \mathrm{CCH}+\mathrm{OH} & \left(b_{1}\right) \\
& \longrightarrow \mathrm{CH}_{2} \mathrm{CCH}+\mathrm{OH} & \left(b_{2}\right)
\end{array}
$$

Wie der Reaktionsmechanismus zeigt, sind neben den Reaktionen, die über ein energetisch hoch angeregtes Allyloxy Radikal $(\mathrm{E} \approx 420 \mathrm{~kJ} / \mathrm{mol}$ [Sla90]) ablaufen (a), zwei direkte HAbstraktionskanäle möglich $\left(\left(b_{1}\right)\right.$ und $\left.\left(b_{2}\right)\right)$.

Die Reaktion wurde trotz der großen Anzahl an bereits vorliegenden Ergebnissen untersucht, da die verwendete FTIR Apparatur gegenüber den in der Literatur benutzten Experimenten eine vollständige Analyse aller Reaktionsprodukte möglich macht. Diese ist z.B. durch ausschließliche Beschränkung auf LIF Experimente nicht gegeben. Des Weiteren bietet die Apparatur die Möglichkeit einer quantitativen Analyse.

\subsubsection{Qualitative Analyse}

Für die qualitative Analyse der Reaktion und die Bestimmung der Kanalverzweigung wurde die in Abschnitt 3.1.1 beschriebene FTIR-Apparatur verwendet. Als Radikalvorläufer dienten Allylbromid, Allylchlorid und Diallyl. Dabei wurden alle drei Substanzen jeweils in Anwesenheit und Abwesenheit von $\mathrm{SO}_{2}$ photolysiert, um Produkte der Radikalkombination bzw. Photolysenprozesse wie Halogen-H-Elimination von Produkten der Oxidation unterscheiden zu können. Die Ergebnisse der Photolyse der Vorläufer in Abwesenheit von $\mathrm{SO}_{2}$ finden sich in Abschnitt 4.4.6, in dem die Reaktion Allyl + Allyl detailliert dargestellt wird.

Um optimale Reaktionsbedingungen zu finden, wurde das Verhältnis der Sauerstoffkonzentration zur Allylradikalkonzentration bei allen Vorläufern durch Variation der O-Atom Vorläuferkonzentration $\mathrm{SO}_{2}$ verändert. 
Der Einsatz von Allylbromid und Allylchlorid hatte den Nachteil, dass durch direkte HXElimination Allen entstanden sein könnte $(\mathrm{X}=\mathrm{Br}, \mathrm{Cl})$. Anders als bei dem Gebrauch von Allyliodid als Vorläufer, wie bei Kersten und Tröger, fällt der direkte Eliminationskanal bei Verwendung von Allylbromid und Allylchlorid als Vorläufer aber gering aus (vgl. dazu [Trö97] und [Ker99]). Ein Problem bei der Verwendung von Allylchlorid könnte die schnelle Reaktion von Cl-Atomen mit bereits gebildeten Produkten sein. Diese tritt bei den vergleichsweise reaktionsträgen Br-Atomen nicht auf. Bei der Verwendung von Diallyl trat neben der erwünschten photolytischen Spaltung in 2 Allylradikale eine direkte O-Addition verbunden mit einem 1,2-H-shift unter Bildung von 5-Hexenal auf. Bei der quantitativen Analyse konnte 5-Hexenal eindeutig als Hauptprodukt der direkten Oxidation unter Sauerstoffatom-Überschuss nachgewiesen werden. Dennoch eignete sich Diallyl als eine Allylradikal-Quelle gut, da das direkte Oxidationsprodukt 5-Hexenal durch vorhandene Reinspektren bei den Reaktionsspektren gut identifiziert und das Reaktionsspektrum mit dem Reinspektrum korrigiert werden konnte. 5-Hexenal ist zudem kein Produkt der Reaktion Allyl + O, was, im Gegensatz zum möglichen HBr- / HCl- Eliminationsprodukt Allen bei der Photolyse von Allylbromid / Allylchlorid, die Analyse der Reaktion Allyl + O nicht behindert.

Alle Messungen fanden bei Zimmertemperatur und einem Gesamtdruck von 4 mbar statt. Es wurden 100-fach gemittelte IR-Produktspektren in Intervallen von 50 bis 100 Photolysen aufgenommen. Um die Wahrscheinlichkeit von Sekundärphotolysen durch zu hohe Photolysenanzahlen einzuschränken, betrug die maximale Photolysenanzahl 400. Die Abnahme des Allylradikal-Vorläufers wurde damit auf ca. 10\% des Vorläufers begrenzt.

Die Produktspektren wurden wie in Abschnitt 3.3.1 dargestellt ausgewertet. Abbildung 4.32 zeigt das IR-Spektrum der Reaktion Allyl + O unter Verwendung des AllylradikalVorläufers Diallyl. 


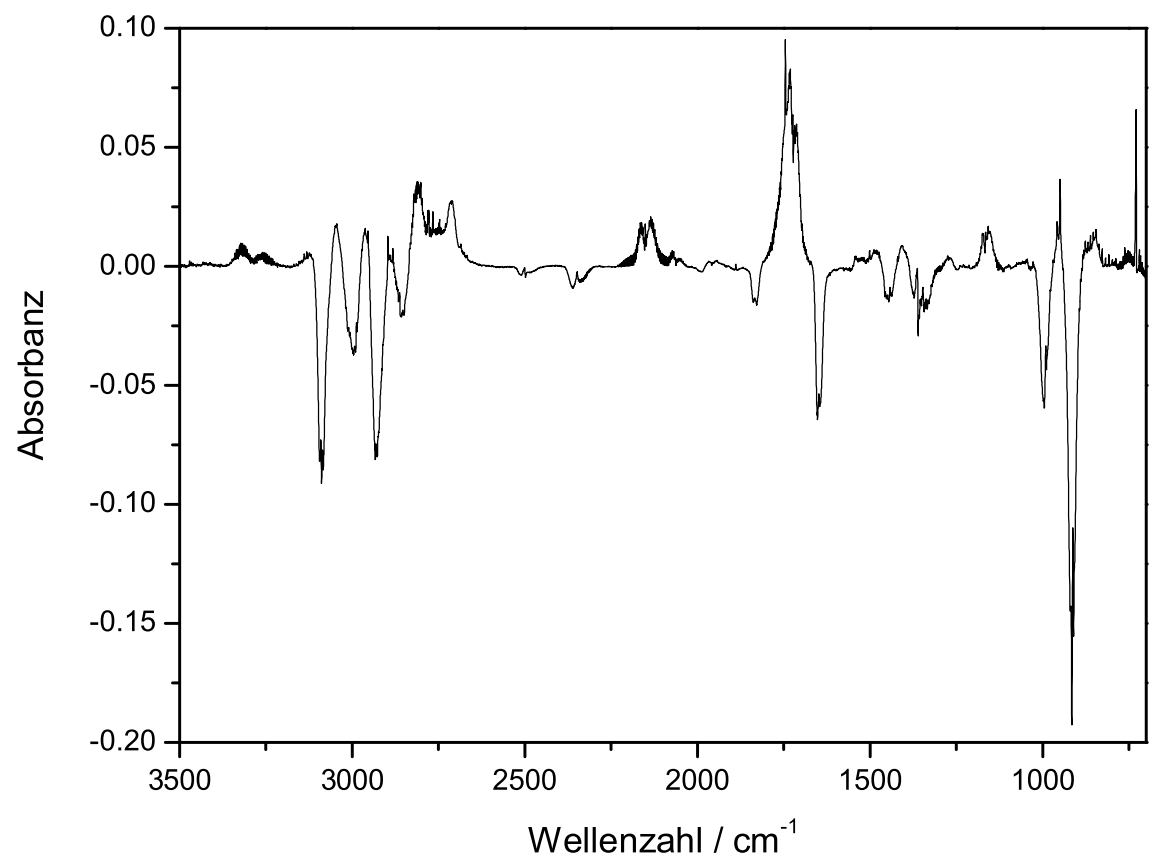

Abbildung 4.32: Reaktion Allyl $+O$, Reaktionsspektrum, Vorläufer Diallyl. $p\left(C_{6} H_{10}\right)=0,4$ mbar, $\mathrm{p}\left(\mathrm{SO}_{2}\right)=0,4$ mbar, $p_{\text {ges }}=4 \mathrm{mbar}, \mathrm{T}=298 \mathrm{~K}, 400$ Photolysen.

Die experimentellen Bedingungen sind dabei: $\mathrm{p}\left(\mathrm{C}_{6} \mathrm{H}_{10}\right)=0,4$ mbar, $\mathrm{p}\left(\mathrm{SO}_{2}\right)=0,4 \mathrm{mbar}$, $\mathrm{p}_{\text {ges }}=4 \mathrm{mbar}, \mathrm{T}=298 \mathrm{~K}$ und 400 Photolysen. Abbildung 4.33 zeigt vergrößerte Ausschnitte des Reaktionsspektrums im Vergleich mit Reinspektren zur Identifikation der Produkte der Reaktion Allyl + O. Anhand des Vergleichs mit Reinspektren konnten folgende Stoffe identifiziert werden: Acrolein, Ethen, Formaldehyd, Allen, Propen, Ethin, Kohlenmonoxid und 5-Hexenal. Dabei ist zu beachten, dass neben der Reaktion Allyl + O auch die schon beschriebene Reaktion Diallyl + O auftritt. Das entsprechende Reaktionsprodukt 5-Hexenal ist in der Abbildung deutlich zu sehen. Des Weiteren konnte die Bildung von Propen beobachtet werden, welches zum Einen aus der Dissproportionierung von 2 Allylradikalen und zum Anderen aus der ebenfalls schnellen Addition eines H-Atoms stammt $\left(\mathrm{k}=1.71 \cdot 10^{14} \mathrm{~cm}^{3} /(\mathrm{mol} \cdot \mathrm{s})\right.$ [Han92]). Das bei der Reaktion ebenfalls beobachtete Allen stammt also nicht nur aus der Reaktion Allyl + O, sondern auch aus diesem Kanal. Weiterhin wurde die Bildung von Ethin beobachtet. Dabei wird davon ausgegangen, dass Ethin aus dem Zerfall des $\mathrm{C}_{2} \mathrm{H}_{3}$-Radikals des Formaldehyd-Kanals $\left(\mathrm{a}_{3}\right)$ gebildet wird. 
KAPITEL 4. MESSERGEBNISSE UND DISKUSSION

4.3. UNTERSUCHUNG DER REAKTIONEN OFFENKETTIGER KOHLENWASSERSTOFFRADIKALE MIT SAUERSTOFF-ATOMEN
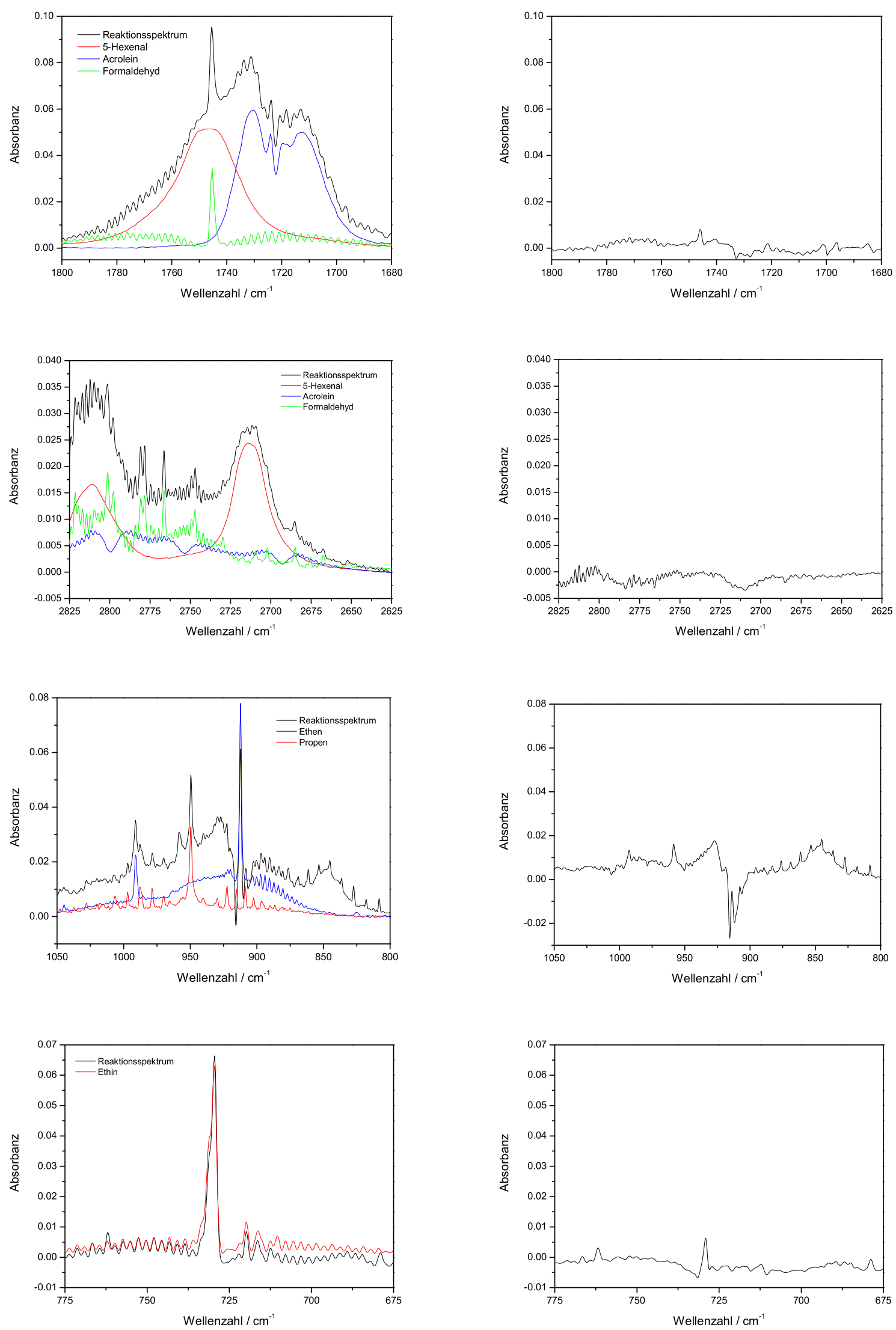

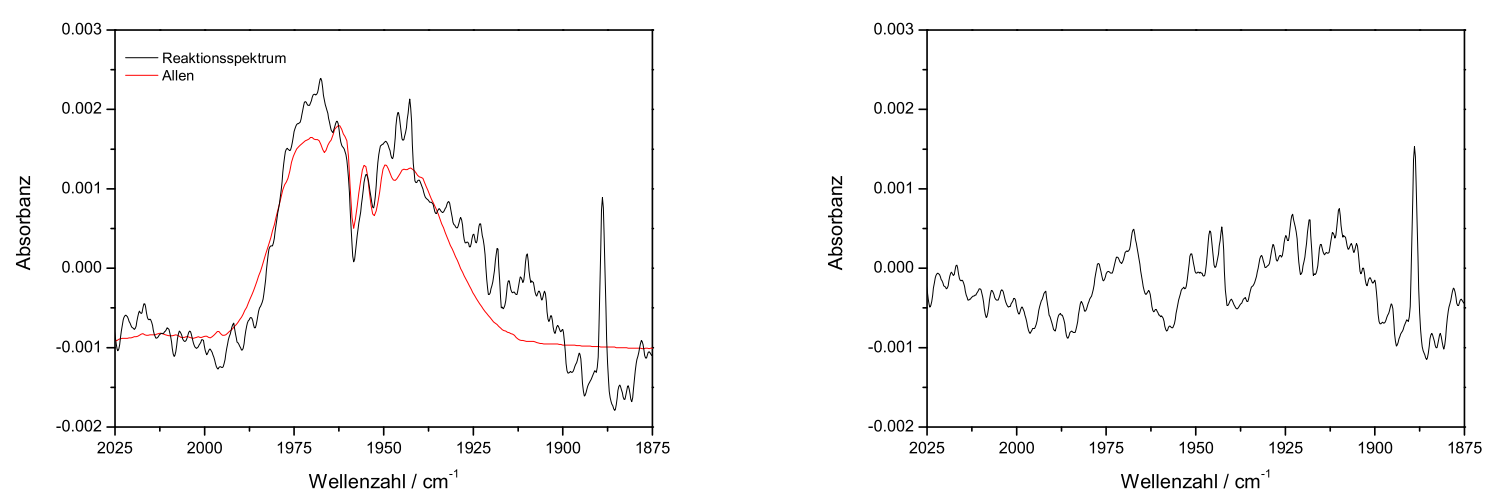

Abbildung 4.33: Reaktion Allyl + O, Vorläufer Diallyl. Vergleich des Reaktionsspektrums mit Spektren der Reinsubstanzen in vergrößerten Ausschnitten; in der linken Spalte ist jeweils das Reaktionsspektrum (schwarz) mit den Reinspektren (farbig) im Vergleich dargestellt, in der rechten Spalte sind die entsprechenden Residuen in schwarz dargestellt. Die negative Bande der Abbildung im Bereich von 1050 - $800 \mathrm{~cm}^{-1}$ resultiert aus der Abnahme an Diallyl, das in diesem Bereich absorbiert.

Bis auf die Bildung des instabilen Ketens und Propin konnte die Bildung aller Produkte der in der Einleitung dargestellten Reaktionskanäle $\left(a_{1}\right)-\left(a_{3}\right)$ und $\left(b_{2}\right)$ für die Reaktion Allyl + O mit dem Vorläufer Diallyl beobachtet werden.

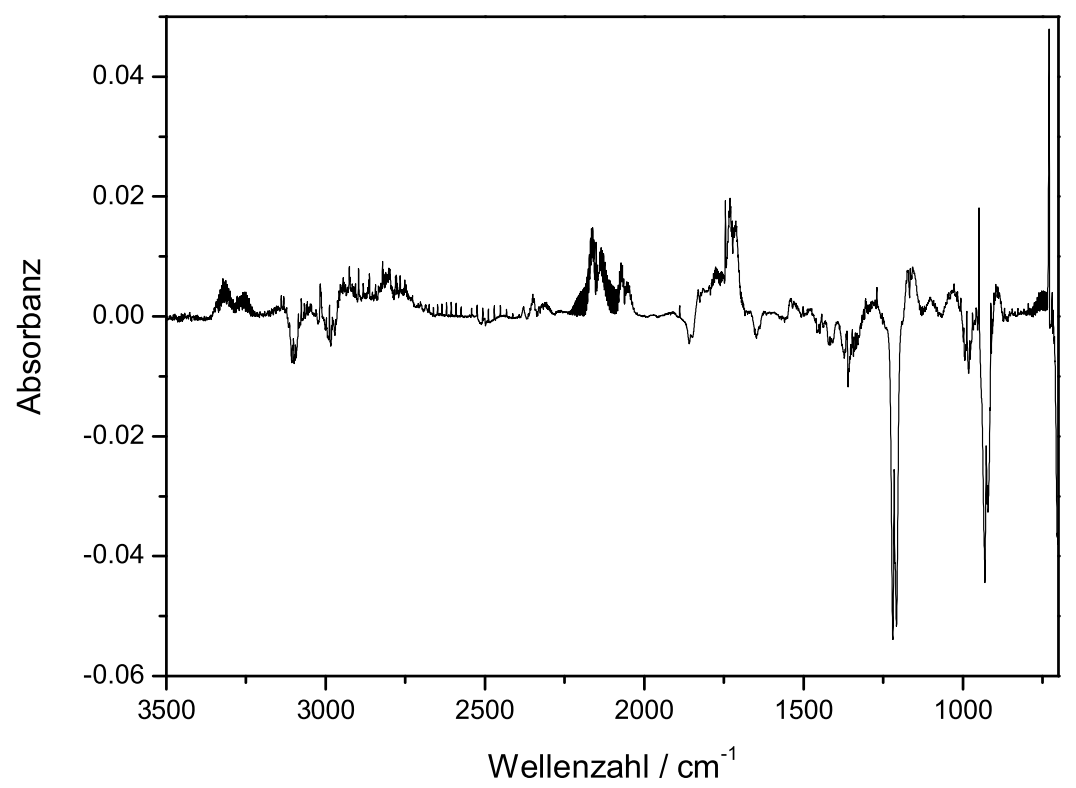

Abbildung 4.34: Reaktion Allyl + O, Reaktionsspektrum, Vorläufer Allylbromid. $p\left(\mathrm{C}_{3} \mathrm{H}_{5} \mathrm{Br}\right)=$ 0,3 mbar, $p\left(\mathrm{SO}_{2}\right)=0,15$ mbar, $p_{\text {ges }}=4 \mathrm{mbar}, \mathrm{T}=298 \mathrm{~K}, 200$ Photolysen.

Im Folgenden wird die qualitative Analyse für den Vorläufer Allylbromid dargestellt. Das 
in Abbildung 4.34 dargestellte Reaktionsspektrum bezieht sich auf folgende experimentelle Bedingungen: $\mathrm{p}\left(\mathrm{C}_{3} \mathrm{H}_{5} \mathrm{Br}\right)=0,3$ mbar, $\mathrm{p}\left(\mathrm{SO}_{2}\right)=0,15$ mbar, $\mathrm{p}_{\text {ges }}=4 \mathrm{mbar}, \mathrm{T}=298$ K, 200 Photolysen.

Vergrößerte Ausschnitte zur Identifizierung einzelner Reaktionsprodukte sind in Abbildung 4.35 dargestellt. Dabei verbleibt nach Abzug von Acrolein und Formaldehyd im Bereich der Carbonylschwingung von 1800 - $1720 \mathrm{~cm}^{-1}$ eine positive Bande, die nicht in Übereinstimmung mit einem vorhandenen Kalibrierspektrum gebracht werden konnte. Da diese Bande nicht bei der Reaktion Allyl + O unter Verwendung des Vorläufers Diallyl auftritt, handelt es sich wahrscheinlich um ein bromierte Verbindung. Ein Vergleich mit den in der Literatur zu findenden Spektren deutet darauf hin, dass es sich bei der Substanz um HCOBr handelt [Yar91]. Es standen aber keine direkten Spektren zum Vergleich zur Verfügung. Die Bildung von 5-Hexenal konnte bei der Wahl von Allylbromid als Vorläufer nicht beobachtet werden.

Produkte der Reaktion Allyl + O unter Verwendung des Vorläufers Allylbromid sind also Acrolein, Ethen, Formaldehyd, Allen, Propen, Ethin und Kohlenmonoxid. Die Bildung von $\mathrm{HCOBr}$ als Produkt der Reaktion $\mathrm{C}_{3} \mathrm{H}_{5} \mathrm{Br}+\mathrm{O}$ wird vermutet. 

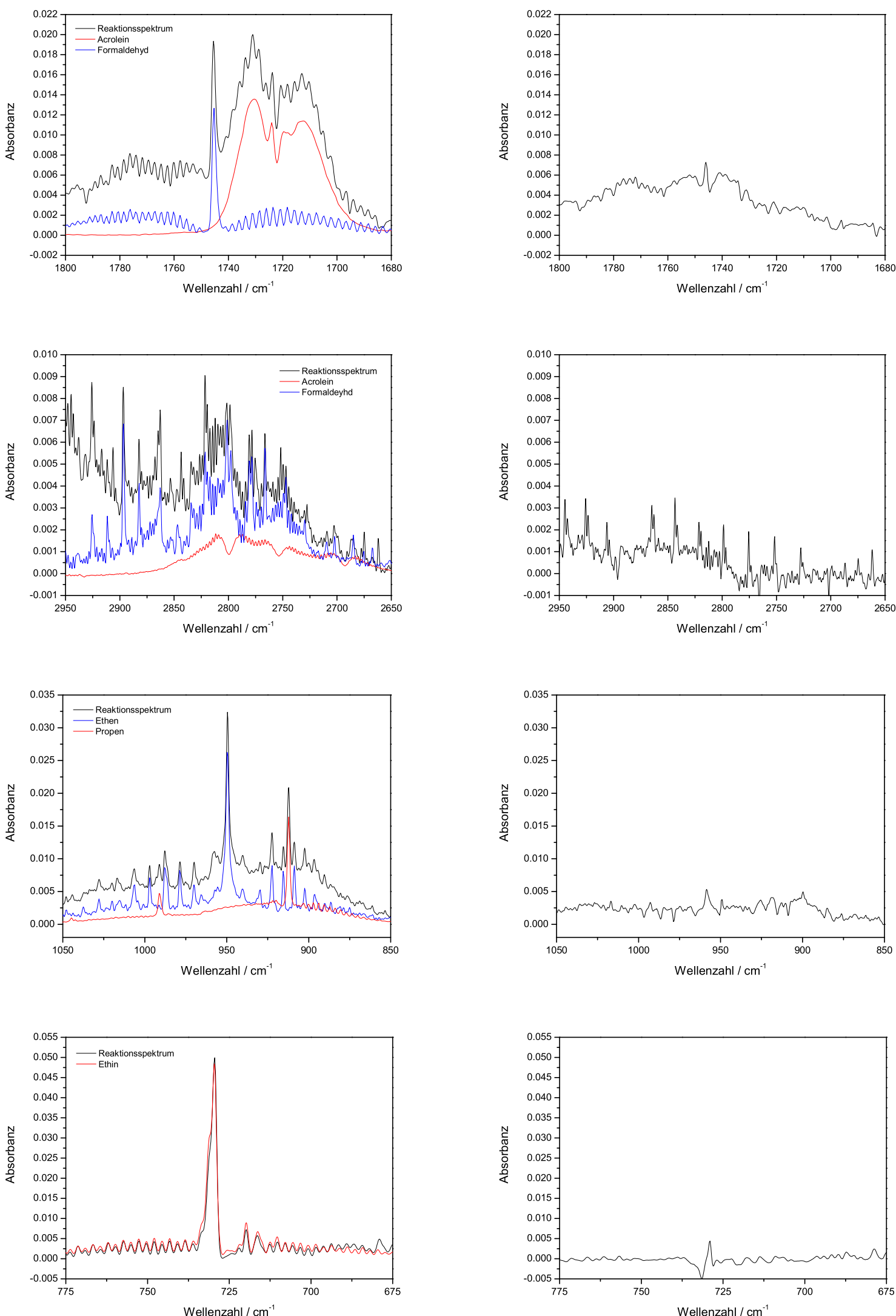

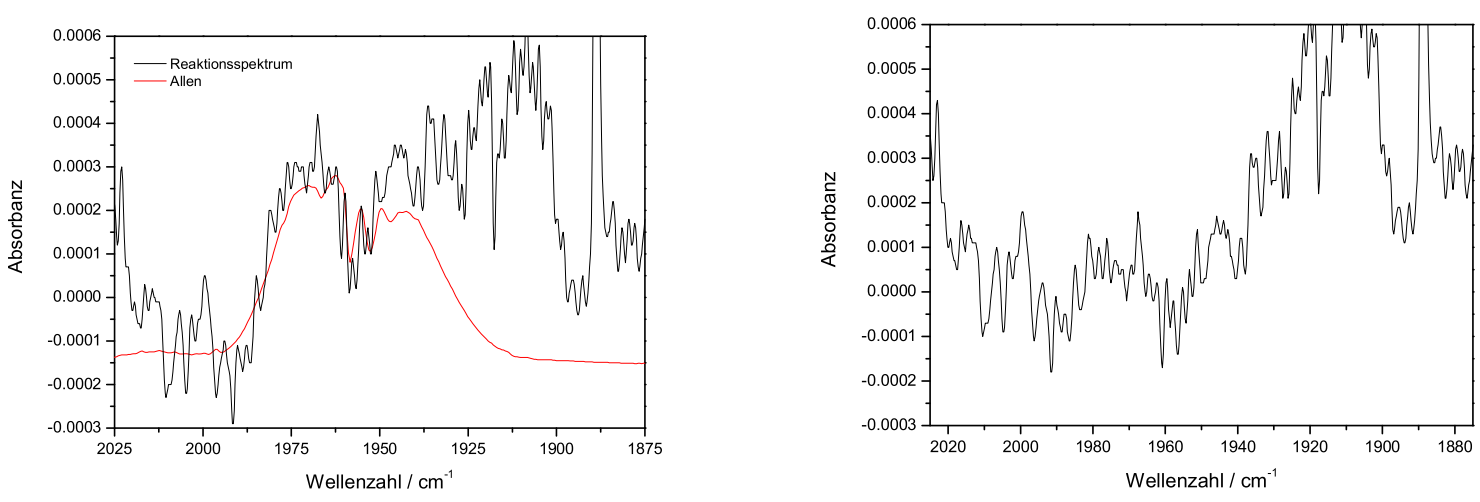

Abbildung 4.35: Reaktion Allyl + O, Vorläufer Allylbromid. Vergleich des Reaktionsspektrums mit Spektren der Reinsubstanzen in vergrößerten Ausschnitten; in der linken Spalte ist jeweils das Reaktionsspektrum (schwarz) mit den Reinspektren (farbig) im Vergleich dargestellt, in der rechten Spalte sind die entsprechenden Residuen in schwarz dargestellt. Die verbleibende positive Bande in Abbildung b) im Bereich der Carbonylschwingung resultiert wahrscheinlich von $\mathrm{HCOBr}$.

Abschließend werden die Ergebnisse der qualitativen Analyse für den Vorläufer Allylchlorid dargestellt. Abbildung 4.36 zeigt ein Reaktionsspektrum nach 200 Photolysen und einer Vorläufermischung von $\mathrm{p}\left(\mathrm{C}_{3} \mathrm{H}_{5} \mathrm{Cl}\right)=\mathrm{p}\left(\mathrm{SO}_{2}\right)=0,3$ mbar.

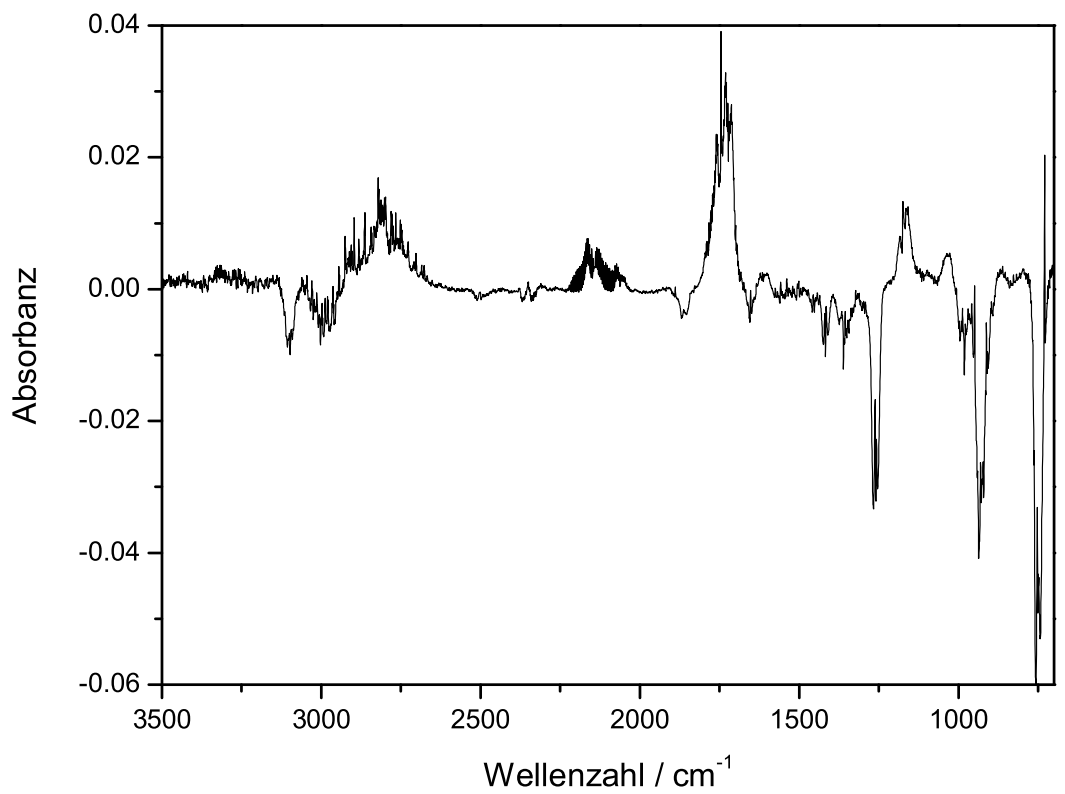

Abbildung 4.36: Reaktion Allyl + O, Reaktionsspektrum, Vorläufer Allylchlorid. $p\left(\mathrm{C}_{3} \mathrm{H}_{5} \mathrm{Cl}\right)=$ 0,3 mbar, $\mathrm{p}\left(\mathrm{SO}_{2}\right)=0,3 \mathrm{mbar}, p_{\text {ges }}=4 \mathrm{mbar}, \mathrm{T}=298 \mathrm{~K}, 200$ Photolysen.

Wie auch für die vorhergende Identifikation primär gebildeter Produkte werden im Fol- 
genden vergrößerte Ausschnitte der einzelnen Absorptionsbereiche vorgestellt, in denen Produktbildung zu beobachten war.
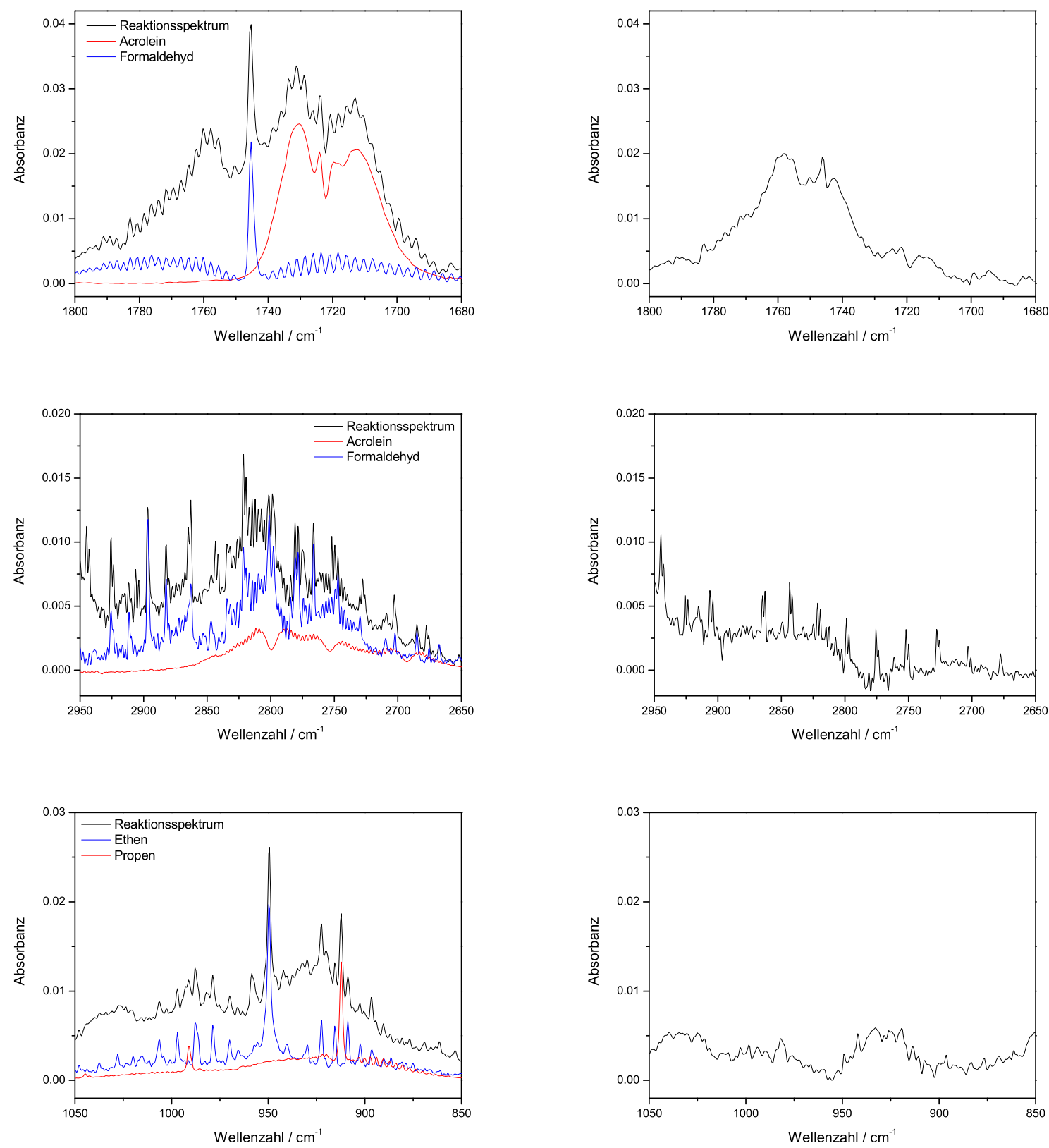

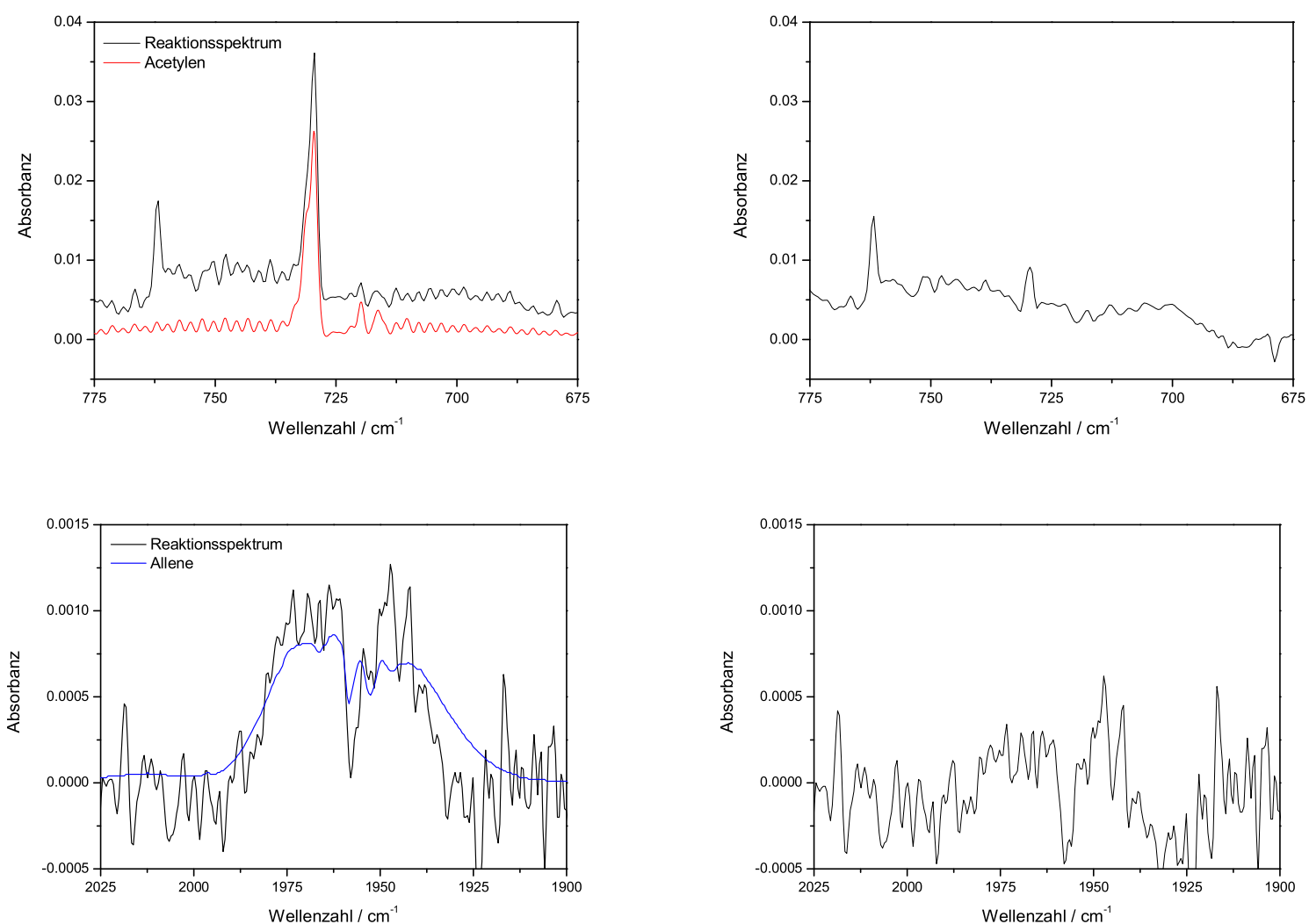

Abbildung 4.37: Reaktion Allyl + O, Vorläufer Allylchlorid. Vergleich des Reaktionsspektrums mit Spektren der Reinsubstanzen in vergrößerten Ausschnitten; in der linken Spalte ist jeweils das Reaktionsspektrum (schwarz) mit den Reinspektren (farbig) im Vergleich dargestellt, in der rechten Spalte sind die entsprechenden Residuen in schwarz dargestellt. Die positive Bande im korrigierten Spektrum, das den Carbonylbereich darstellt, resultiert in diesem Fall von $\mathrm{HCOCl}$.

Bei der in Abbildung 4.37 detailliert dargestellten Reaktion konnten folgende Produkte eindeutig bestimmt werden: Acrolein, Ethen, Formaldehyd, Allen, Propen, Ethin und Kohlenmonoxid. Die Bildung von $\mathrm{HCOCl}$, die entweder aus einer schnellen Reaktion primärer Produkte mit $\mathrm{Cl}$ Atomen oder aus einem Zerfall des möglicherweise gebildeten $\mathrm{C}_{3} \mathrm{H}_{5} \mathrm{ClO}$ Radikals resultiert, konnte ebenfalls beobachtet werden.

Die qualitative Untersuchung der Reaktion ergab für alle 3 Vorläufer übereinstimmend die Bildung folgender Produkte: Acrolein, Ethen, Formaldehyd, Allen, Propen, Ethin und Kohlenmonoxid. Dabei konnte im Fall des Vorläufers Diallyl ein großer Anteil an 5Hexenal und für die Reaktionen halogenierter Kohlenwasserstoffe die Bildung von HCOX $(\mathrm{X}=\mathrm{Br}, \mathrm{Cl})$ beobachtet werden. Die übereinstimmenden Resultate bestärken die Annahme, dass der Mechanismus der Reaktion von Allylradikalen mit O-Atomen nicht allein 
über die Bildung von Acrolein läuft, wie Slagle et al. postulieren [Sla90].

\subsubsection{Quantitative Analyse}

Die quantitative Analyse der Reaktionen wurde wie in Abschnitt 3.3.2 beschrieben durchgeführt. Für alle identifizierten Substanzen wurden in einem Partialdruckbereich von 0,01 - 0,1 mbar Kalibrierspektren aufgenommen, woraus mit Hilfe einer Auftragung die integralen Absorptionskoeffizienten bestimmt wurden. Eine quantitative Analyse der Reaktion erfolgte bei einem Gesamtdruck von 4 mbar.

In Tabelle 4.21 sind die Partialdrücke der Produkte in Bezug auf den Verbrauch des Vorläufers Diallyl für die Reaktion Allyl + O aufgeführt. Die daraus resultierende Auftragung ist in Abbildung 4.38 gegeben.

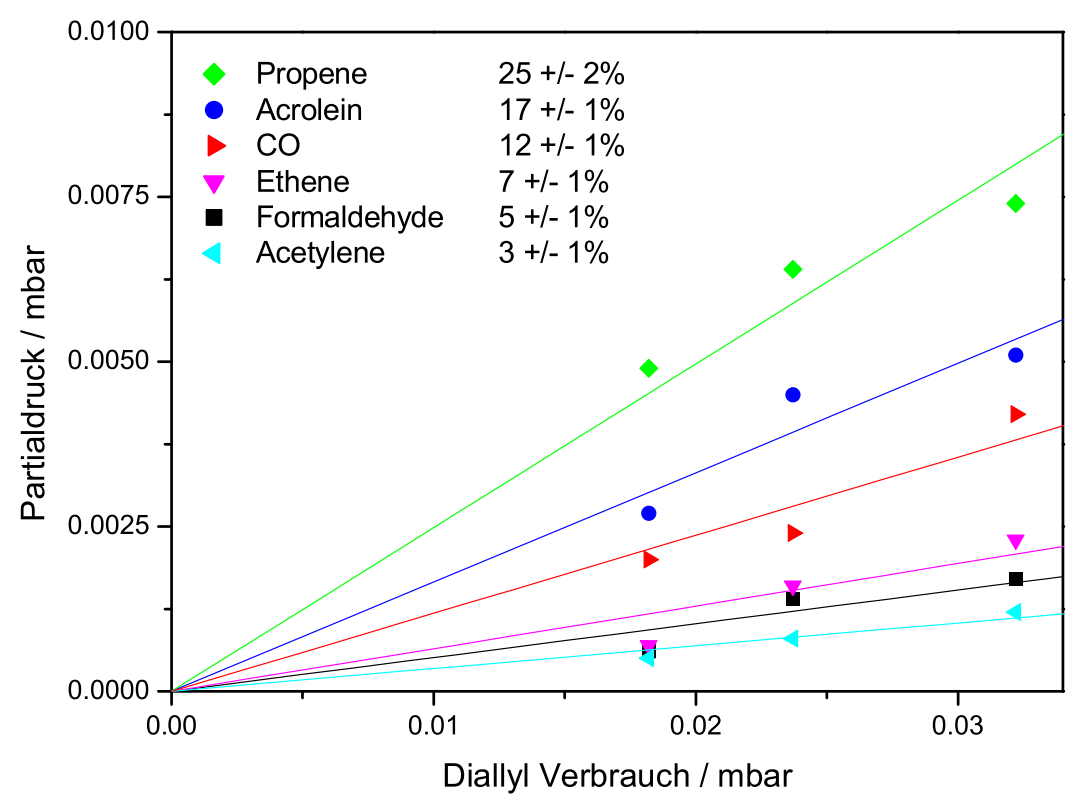

Abbildung 4.38: Reaktion Allyl $+O$, quantitative Analyse, Vorläufer Diallyl. $p\left(\left(C_{3} H_{5}\right)_{2}\right)=0,1$ mbar, $p\left(\mathrm{SO}_{2}\right)=0,3$ mbar $p_{\text {ges }}=4 \mathrm{mbar}, \mathrm{T}=298 \mathrm{~K}$, Badgas Argon. 


\begin{tabular}{|c|c|c|c|c|c|c|}
\hline $\begin{array}{c}\text { Verbrauch } \\
\text { Diallyl }\end{array}$ & $\begin{array}{c}\mathrm{p}\left(\mathrm{H}_{2} \mathrm{CO}\right) \\
{[\mathrm{mbar}]}\end{array}$ & $\begin{array}{c}\mathrm{p}\left(\mathrm{C}_{3} \mathrm{H}_{4} \mathrm{O}\right) \\
{[\mathrm{mbar}]}\end{array}$ & $\begin{array}{c}\mathrm{p}\left(\mathrm{C}_{2} \mathrm{H}_{4}\right) \\
{[\mathrm{mbar}]}\end{array}$ & $\begin{array}{c}\mathrm{p}\left(\mathrm{C}_{3} \mathrm{H}_{6}\right) \\
{[\mathrm{mbar}]}\end{array}$ & $\begin{array}{c}\mathrm{p}\left(\mathrm{C}_{2} \mathrm{H}_{2}\right) \\
{[\mathrm{mbar}]}\end{array}$ & $\begin{array}{c}\mathrm{p}(\mathrm{CO}) \\
{[\mathrm{mbar}]}\end{array}$ \\
\hline \hline 0,0182 & $6 \mathrm{E}-4$ & 0,0027 & $7 \mathrm{E}-4$ & 0,0049 & $5 \mathrm{E}-4$ & 0,002 \\
\hline 0,0237 & 0,0014 & 0,0045 & 0,0016 & 0,0064 & $8 \mathrm{E}-4$ & 0,0024 \\
\hline 0,0322 & 0,0017 & 0,0051 & 0,0023 & 0,0074 & 0,0012 & 0,0042 \\
\hline
\end{tabular}

Tabelle 4.21: Reaktion Allyl $+\mathrm{O}$, Vorläufer Diallyl. Messwerte. $\mathrm{p}\left(\left(\mathrm{C}_{3} \mathrm{H}_{5}\right)_{2}\right)=0,1$ mbar, $\mathrm{p}\left(\mathrm{SO}_{2}\right)$ $=0,3$ mbar $p_{g e s}=4$ mbar, $T=298 \mathrm{~K}$, Badgas Argon .

Die Abbildung 4.38 zeigt ein annähernd lineares Wachstum mit steigender Diallylabnahme für alle Produkte. In der Abbildung 4.38, sowie der zugehörigen Tabelle 4.21 wurde der Partialdruck von 5-Hexenal nicht mit aufgeführt, da dieses Produkt eindeutig aus der Reaktion des Vorläufers mit O-Atomen resultiert. Wie man der quantitativen Analyse entnehmen kann, ensteht zu einem großen Anteil Propen. Bei diesem handelt es sich ebenfalls nicht um ein Produkt der Reaktion Allyl + O. Vielmehr stammt dies aus der Reaktion von 2 Allyl Radikalen, wie auch Simulationsrechnungen für das Reaktionssystem $\mathrm{C}_{3} \mathrm{H}_{5} / \mathrm{O}$ zeigen. Lässt man dementsprechend diesen Kanal unberücksichtigt, ergibt sich für den Vorläufer Diallyl folgendes Produktverhältnis:

Acrolein : Ethen : Formaldehyd : Ethin : CO = $1: 0,41: 0,29: 0,18: 0,71$.

Eine Zuordnung der Produktverhältnisse zu dem Reaktionsmechanismus erfolgt im Anschluß an die quantitative Analyse aller Vorläufer.

Im Folgenden sind die Messwerte und die Auftragung für den Vorläufer Allylbromid zu finden. Tabelle 4.22 enthält die Partialdrücke der identifizierten Substanzen in Abhängigkeit vom Verbrauch an Allylbromid. Die entsprechende Auftragung ist in Abbildung 4.39 zu sehen. 


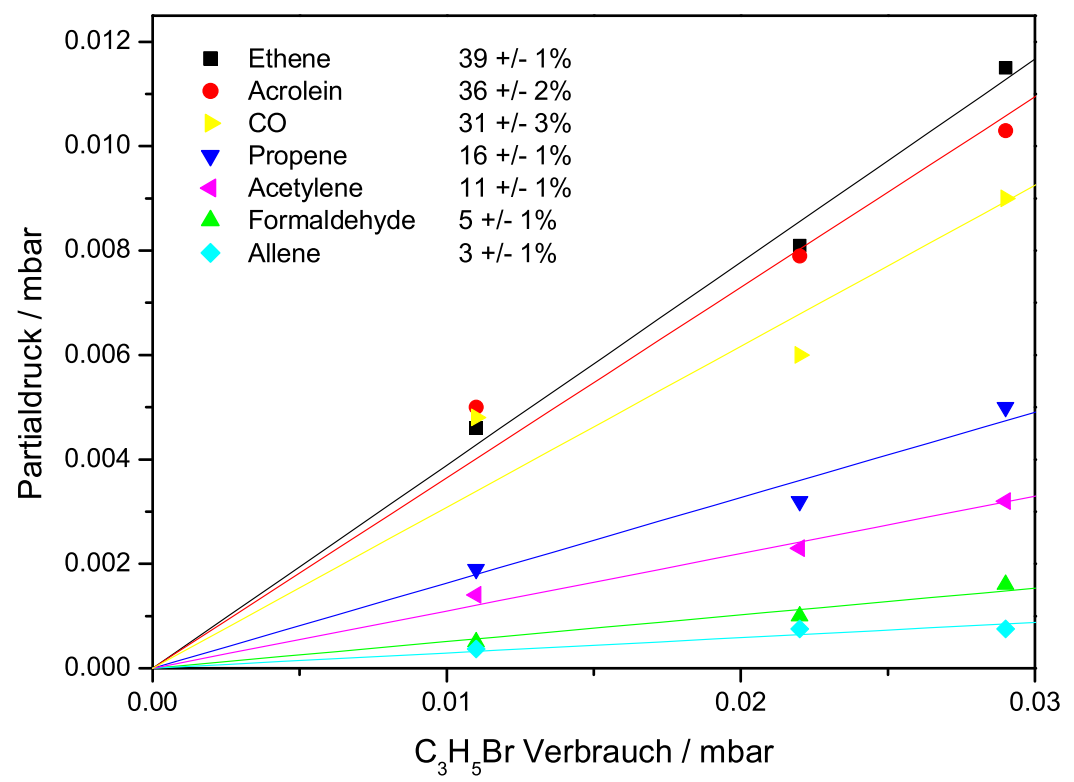

Abbildung 4.39: Reaktion Allyl $+O$, quantitative Analyse, Vorläufer Allylbromid. $p\left(C_{3} H_{5} \mathrm{Br}\right)$ $=0,3$ mbar, $p\left(\mathrm{SO}_{2}\right)=0,3$ mbar pges $=4 \mathrm{mbar}, \mathrm{T}=298 \mathrm{~K}$, Badgas Argon .

\begin{tabular}{|c|c|c|c|c|c|c|c|}
\hline $\begin{array}{c}\text { Verbrauch } \\
\text { Allylbromid }\end{array}$ & $\begin{array}{c}\mathrm{p}\left(\mathrm{C}_{2} \mathrm{H}_{4}\right) \\
{[\mathrm{mbar}]}\end{array}$ & $\begin{array}{c}\mathrm{p}\left(\mathrm{C}_{3} \mathrm{H}_{4} \mathrm{O}\right) \\
{[\mathrm{mbar}]}\end{array}$ & $\begin{array}{c}\mathrm{p}\left(\mathrm{H}_{2} \mathrm{CO}\right) \\
{[\mathrm{mbar}]}\end{array}$ & $\begin{array}{c}\mathrm{p}\left(\mathrm{C}_{3} \mathrm{H}_{6}\right) \\
{[\mathrm{mbar}]}\end{array}$ & $\begin{array}{c}\mathrm{p}\left(\mathrm{CH}_{2} \mathrm{CCH}_{2}\right) \\
{[\mathrm{mbar}]}\end{array}$ & $\begin{array}{c}\mathrm{p}\left(\mathrm{C}_{2} \mathrm{H}_{2}\right) \\
{[\mathrm{mbar}]}\end{array}$ & $\begin{array}{c}\mathrm{p}(\mathrm{CO}) \\
{[\mathrm{mbar}]}\end{array}$ \\
\hline \hline 0,011 & 0,0046 & 0,005 & $5 \mathrm{E}-4$ & 0,0019 & $3,7 \mathrm{E}-4$ & 0,0014 & 0,0048 \\
\hline 0,022 & 0,0081 & 0,0079 & $1 \mathrm{E}-3$ & 0,0032 & $7,5 \mathrm{E}-4$ & 0,0023 & 0,006 \\
\hline 0,029 & 0,0115 & 0,0103 & 0,0016 & 0,005 & $7,5 \mathrm{E}-4$ & 0,0032 & 0,009 \\
\hline
\end{tabular}

Tabelle 4.22: Reaktion Allyl + O, Vorläufer Allylbromid. Messwerte. $p\left(\mathrm{C}_{3} \mathrm{H}_{5} \mathrm{Br}\right)=0,3 \mathrm{mbar}$, $p\left(\mathrm{SO}_{2}\right)=0,3$ mbar $p_{\text {ges }}=4$ mbar, $\mathrm{T}=298 \mathrm{~K}$, Badgas Argon.

Anhand der Auftragung in Abbildung 4.39 kann die Photolyse primär gebildeter Produkte ausgeschlossen werden, da die Partialdrücke der Produkte mit steigender Photolysenanzahl (Verbrauch an Vorläufer) linear anwachsen. In diesem Fall ist ein sehr hoher Anteil an Ethen beobachtbar, der in der selben Größenordnung liegt, wie der Anteil an Acrolein. Ebenfalls hohe Anteile findet man für CO, Propen und Ethin. Dabei wird Propen wieder aus der Reaktion zweier Allyl Radikale stammen. Unter Vernachlässigung dieses Kanals ergibt sich für das Verhältnis der weiteren Produkte:

Acrolein : Ethen : Formaldehyd : Ethin : CO : Allen = $1: 1,08: 0,14: 0,31: 0,86: 0,08$. Eine Ableitung der Kanalverzweigung erfolgt, wie für den vorhergehenden Vorläufer, am Ende der quantitativen Analyse aller Vorläufer. 
Die nachfolgende Tabelle 4.23 und Abbildung 4.40 zeigt die Messwerte und die daraus resultierende Auftragung der Reaktion Allyl + O unter Verwendung des Vorläufers Allylchlorid. Wie auch für die beiden anderen Vorläufer konnte ein linearer Anstieg der Partialdrücke in Bezug auf die Abnahme an Vorläufer festgestellt werden. Dies berechtigt zu der Annahme, dass Sekundärphotolyse primärer Produkte für die Ausbeute nur eine geringe Bedeutung hat.

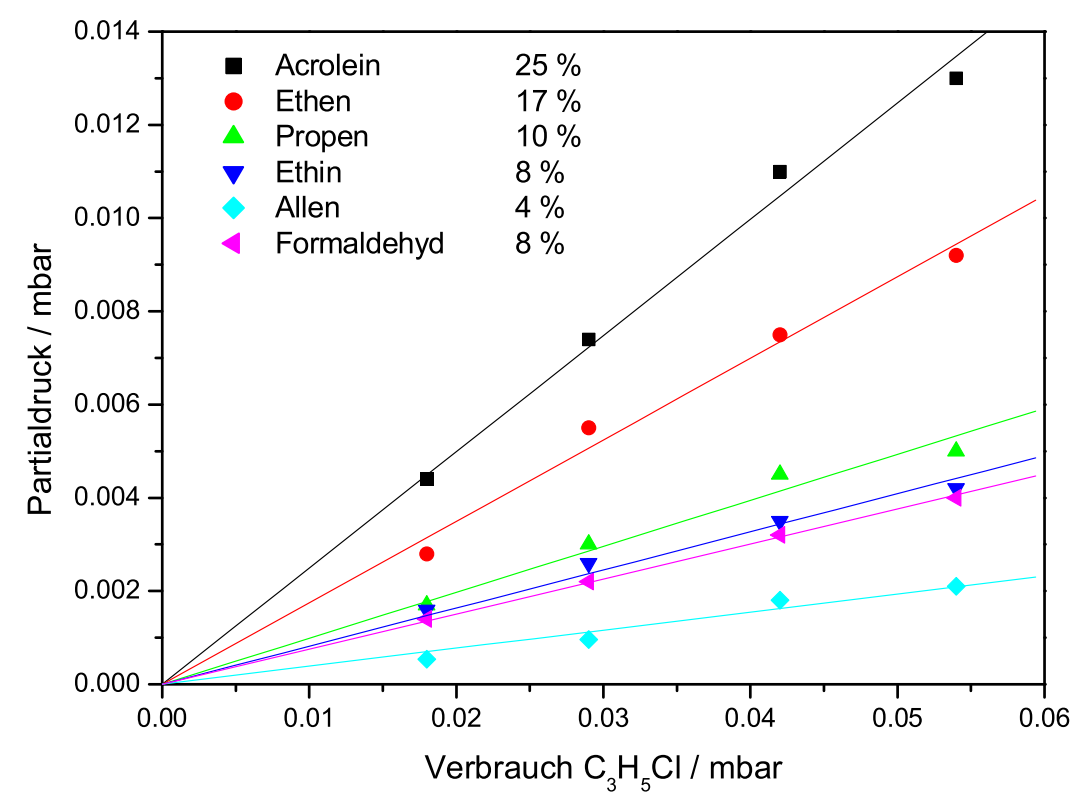

Abbildung 4.40: Reaktion Allyl $+O$, quantitative Analyse, Vorläufer Allylchlorid. $p\left(\mathrm{C}_{3} \mathrm{H}_{5} \mathrm{Cl}\right)=$ 0,3 mbar, $p\left(\mathrm{SO}_{2}\right)=0,3$ mbar $p_{\text {ges }}=4 \mathrm{mbar}, \mathrm{T}=298 \mathrm{~K}$, Badgas Argon.

\begin{tabular}{|c|c|c|c|c|c|c|c|}
\hline $\begin{array}{c}\text { Verbrauch } \\
\text { Allylchlorid }\end{array}$ & $\begin{array}{c}\mathrm{p}\left(\mathrm{C}_{2} \mathrm{H}_{4}\right) \\
{[\mathrm{mbar}]}\end{array}$ & $\begin{array}{c}\mathrm{p}\left(\mathrm{C}_{3} \mathrm{H}_{6}\right) \\
{[\mathrm{mbar}]}\end{array}$ & $\begin{array}{c}\mathrm{p}\left(\mathrm{C}_{3} \mathrm{H}_{4} \mathrm{O}\right) \\
{[\mathrm{mbar}]}\end{array}$ & $\begin{array}{c}\mathrm{p}\left(\mathrm{H}_{2} \mathrm{CO}\right) \\
{[\mathrm{mbar}]}\end{array}$ & $\begin{array}{c}\mathrm{p}\left(\mathrm{C}_{2} \mathrm{H}_{2}\right) \\
{[\mathrm{mbar}]}\end{array}$ & $\begin{array}{c}\mathrm{p}\left(\mathrm{CH}_{2} \mathrm{CCH}_{2}\right) \\
{[\mathrm{mbar}]}\end{array}$ & $\begin{array}{c}\mathrm{p}(\mathrm{CO}) \\
{[\mathrm{mbar}]}\end{array}$ \\
\hline \hline 0,03 & 0,0051 & 0,0031 & 0,0079 & 0,0024 & 0,0027 & $4,5 \mathrm{E}-4$ & 0,0085 \\
\hline 0,0375 & 0,0063 & 0,0042 & 0,01 & 0,0031 & 0,0036 & $6 \mathrm{E}-4$ & 0,012 \\
\hline 0,04725 & 0,008 & 0,0052 & 0,0129 & 0,004 & 0,0042 & $8,9 \mathrm{E}-4$ & 0,015 \\
\hline
\end{tabular}

Tabelle 4.23: Reaktion Allyl + O, Vorläufer Allylchlorid. Messwerte. $p\left(\mathrm{C}_{3} \mathrm{H}_{5} \mathrm{Cl}\right)=0,3 \mathrm{mbar}$, $p\left(\mathrm{SO}_{2}\right)=0,3$ mbar $p_{\text {ges }}=4 \mathrm{mbar}, \mathrm{T}=298 \mathrm{~K}$, Badgas Argon.

Unter Verwendung des Vorläufers Allylchlorid konnte, ähnlich zu den beiden anderen Vorläufern, Acrolein, Ethen und Propen zu hohen Anteilen bestimmt werden. Im Unterschied zum Vorläufer Allylbromid ist dabei aber der Anteil an Ethen geringer als der 
Anteil an Acrolein. Bezogen auf den Anteil an Acrolein und unter Vernachlässigung des Anteils von Propen ergibt sich folgendes Produktverhältnis:

Acrolein : Ethen : Formaldehyd : Ethin : Allen = $1: 0,68: 0,32: 0,32: 0,16$.

Die Ergebnisse aller drei Vorläufer sind im Folgenden zusammenfassend in Tabelle 4.24 dargestellt. Für die Werte in Tabelle 4.24 sind die Ergebnisse der Photolyse der Vorläufer in Abwesenheit von $\mathrm{SO}_{2}$ eingeflossen. Die Ergebnisse dazu finden sich in Abschnitt 4.3.1. Dabei wurde das Verhältnis an Propen zu Ethen und Propen zu Ethin ermittelt und die Ergebnisse der Photolyse in Anwesenheit von $\mathrm{SO}_{2}$ mit diesen Werten korrigiert. Dadurch wurde die Bildung von Ethen und Ethin aus der Reaktion Allyl + Allyl berücksichtigt. Bei der Photolyse ist in Anwesenheit von $\mathrm{SO}_{2}$ Allen entstanden. Dies liegt in so geringen Mengen vor, dass nicht klar festgestellt werden konnte, ob es aus der Reaktion zweier Allylradikale oder aus dem Abstraktionskanal entstanden ist. Daher bleibt dies im Folgenden unberücksichtigt. Die Tabelle zeigt, dass für die verschiedenen Vorläufer Diskrepanzen in den unterschiedlichen Produktanteilen bestehen. Diese resultieren aus den jeweiligen Nebenreaktionen. Der für die quantitative Reaktion verlässlichste Vorläufer ist Allylbromid, da bei diesem Folgereaktionen mit Br-Atomen vernachlässigbar sind. Der Vorläufer Allylchlorid liefert für die qualitative Analyse des Reaktionssystems gute Informationen. Für Aussagen über die Kanalverzweigung eignet sich der Vorläufer aber nur bedingt, da die auftretenden Nebenreaktionen wie Allyl $+\mathrm{Cl}$ und Reaktionen von Produkten der Allyl + O Reaktion mit Cl-Atomen zu schnell sind. Des Weiteren ist das Verhältnis von Acrolein zu Ethen unter Verwendung des Vorläufers Diallyl höher als bei den beiden anderen Vorläufern. Dies resultiert wahrscheinlich aus der direkten Reaktion von Diallyl mit Sauerstoffatomen $\left(\mathrm{C}_{6} \mathrm{H}_{10}+\mathrm{O} \rightarrow \mathrm{C}_{3} \mathrm{H}_{4} \mathrm{O}+\mathrm{C}_{3} \mathrm{H}_{6}\right)$, welche nicht unterdrückt werden kann. 


\begin{tabular}{lccc}
\hline Substanz & Allylchlorid & Allylbromid & Diallyl \\
\hline Acrolein & 1 & 1 & 1 \\
Ethen & 0,56 & 0,99 & 0,18 \\
$\mathrm{CO}$ & - & 0,86 & 0,71 \\
$\mathrm{H}_{2} \mathrm{CO}$ & 0,32 & 0,14 & 0,29 \\
Ethin & 0,09 & 0,11 & - \\
Allen & - & 0,004 & - \\
\hline
\end{tabular}

Tabelle 4.24: Reaktion Allyl + O, Vergleich der Produktanteile für die verschiedenen Vorläufer. Für eine bessere Vergleichbarkeit sind die Anteile aller Produkte auf den Anteil an Acrolein normiert. Die Beschreibung der Berechnung erfolgt im Text.

Auf Grund der quantitativen Analyse der verschiedene Vorläufer wird folgende Kanalverzweigung vorgeschlagen. Dabei wurde die Kanalverzweigung unter Verwendung des Vorläufers Allylbromid zu Grunde gelegt und die Fehlergrenzen bewußt so groß gewählt, dass sie die Ergebnisse des Vorläufers Diallyl mit einschließen.

$$
\begin{array}{rlr}
\mathrm{C}_{3} \mathrm{H}_{5}+\mathrm{O} & \longrightarrow \mathrm{C}_{3} \mathrm{H}_{5} \mathrm{O}^{\star} & \\
\mathrm{C}_{3} \mathrm{H}_{5} \mathrm{O}^{\star} & \longrightarrow \mathrm{C}_{3} \mathrm{H}_{4} \mathrm{O}+\mathrm{H} & (49 \pm 15) \% \\
& \longrightarrow \mathrm{C}_{2} \mathrm{H}_{4}+\mathrm{H}+\mathrm{CO} & (45 \pm 15) \% \\
& \longrightarrow \mathrm{H}_{2} \mathrm{CO}+\mathrm{C}_{2} \mathrm{H}_{2}+\mathrm{H} & (6 \pm 5) \%
\end{array}
$$

\subsubsection{Simulation}

Auf Grundlage der experimentellen Ergebnisse des Vorläufers Allylbromid werden im Folgenden die Ergebnisse der Simulationrechnung für das Reaktionssystem $\mathrm{C}_{3} \mathrm{H}_{5} / \mathrm{O}$ vorgestellt. Dabei wurden die experimentell bestimmten Radikalanfangskonzentrationen von $\left[\mathrm{C}_{3} \mathrm{H}_{5}\right]_{0}=9,0 \times 10^{-10} \mathrm{~mol} / \mathrm{cm}^{3}$ und $[\mathrm{O}]_{0}=4,8 \times 10^{-10} \mathrm{~mol} / \mathrm{cm}^{3}$ zu Grunde gelegt. Unter Verwendung der in Tabelle 4.25 angegebenen Reaktionsgleichungen und Geschwindigkeiten wurden die in Abbildung 4.41 dargestellten Konzentrations-Zeit-Profile erhalten. 


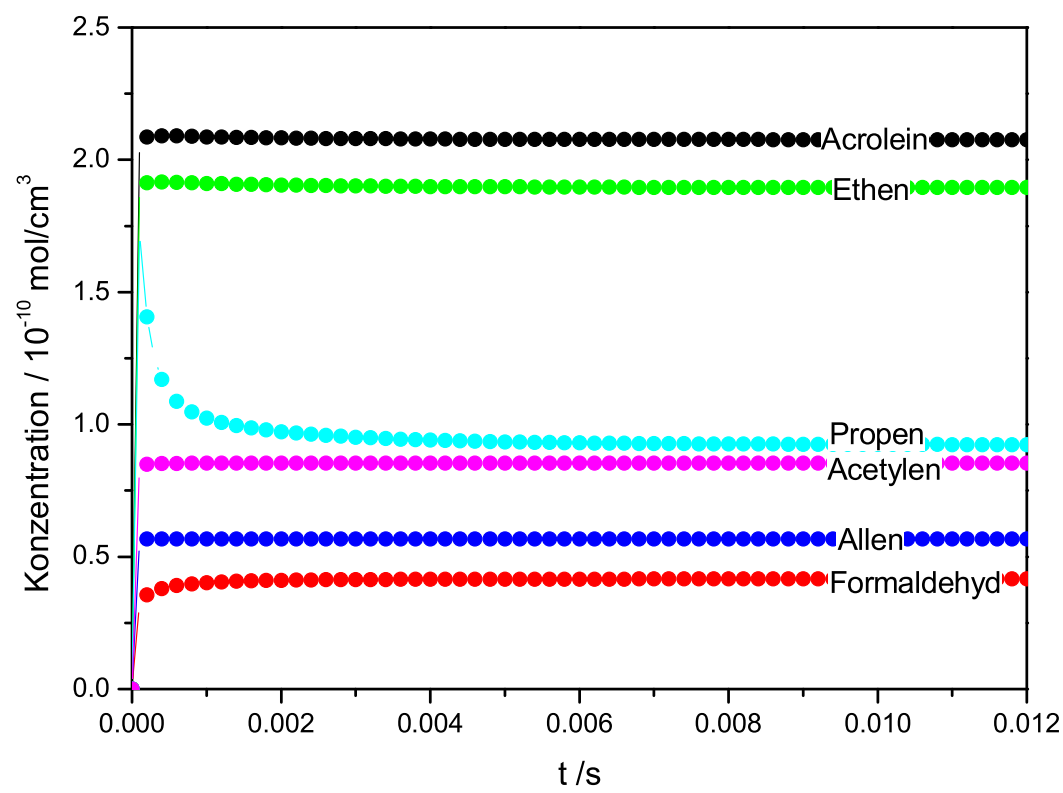

Abbildung 4.41: Reaktion Allyl $+O$, Simulation der Konzentrations-Zeit-Profile. Die verwendeten Reaktionen und Geschwindigkeiten sind in Tabelle 4.25 angegeben.

Ein Vergleich der experimentellen Produktanteile mit den Ergebnissen der Simulationsrechnung zeigt, dass die Resultate nicht vollständig übereinstimmen. Die Ergebnisse des Experiments zusammen mit den Produktanteilen der Simulation sind in Tabelle 4.26 angegeben. 


\begin{tabular}{|c|c|c|c|c|c|}
\hline \multicolumn{5}{|l|}{ Reaktion } & \multirow{2}{*}{$\begin{array}{l}\mathrm{cm}^{3} /(\mathrm{mol} \cdot \mathrm{s}) \\
8,82 \cdot 10^{13}\end{array}$} \\
\hline $\mathrm{C}_{3} \mathrm{H}_{5}$ & $+\mathrm{O}$ & $\rightarrow$ Acrolein & $+\mathrm{H}$ & + & \\
\hline $\mathrm{C}_{3} \mathrm{H}_{5}$ & $+\mathrm{O}$ & $\rightarrow \mathrm{H}_{2} \mathrm{CO}$ & $+\mathrm{C}_{2} \mathrm{H}_{3}$ & + & $1,08 \cdot 10^{13}$ \\
\hline $\mathrm{C}_{3} \mathrm{H}_{5}$ & $+\mathrm{O}$ & $\rightarrow \mathrm{C}_{2} \mathrm{H}_{4}$ & $+\mathrm{H}$ & $+\mathrm{CO}$ & $8,10 \cdot 10^{13}$ \\
\hline $\mathrm{C}_{3} \mathrm{H}_{5}$ & $+\mathrm{C}_{3} \mathrm{H}_{5}$ & $\rightarrow \mathrm{C}_{6} \mathrm{H}_{10}$ & + & + & $2,80 \cdot 10^{12}$ \\
\hline $\mathrm{C}_{3} \mathrm{H}_{5}$ & $+\mathrm{C}_{3} \mathrm{H}_{5}$ & $\rightarrow$ Allen & + Proper & & $5,60 \cdot 10^{12}$ \\
\hline $\mathrm{C}_{3} \mathrm{H}_{5}$ & $+\mathrm{C}_{3} \mathrm{H}_{5}$ & $\rightarrow$ Propin & + Proper & & $5,60 \cdot 10^{12}$ \\
\hline $\mathrm{C}_{3} \mathrm{H}_{5}$ & $+\mathrm{H}$ & $\rightarrow$ Propen & + & + & $1,36 \cdot 10^{14}$ \\
\hline $\mathrm{C}_{3} \mathrm{H}_{5}$ & $+\mathrm{H}$ & $\rightarrow \mathrm{C}_{2} \mathrm{H}_{3}$ & $+\mathrm{CH}_{3}$ & + & $3,4 \cdot 10^{13}$ \\
\hline $\mathrm{C}_{3} \mathrm{H}_{5}$ & $+\mathrm{H}$ & $\rightarrow$ Allen & $+\mathrm{H}_{2}$ & + & $1,80 \cdot 10^{13}$ \\
\hline $\mathrm{C}_{3} \mathrm{H}_{5}$ & $+\mathrm{CH}_{3}$ & $\rightarrow$ Allen & $+\mathrm{CH}_{4}$ & + & $3,60 \cdot 10^{11}$ \\
\hline $\mathrm{C}_{3} \mathrm{H}_{5}$ & $+\mathrm{CH}_{3}$ & $\rightarrow$ Buten & + & + & $6,30 \cdot 10^{13}$ \\
\hline $\mathrm{C}_{2} \mathrm{H}_{3}$ & + & $\rightarrow \mathrm{C}_{2} \mathrm{H}_{2}$ & $+\mathrm{H}$ & + & $1,10 \cdot 10^{10}$ \\
\hline $\mathrm{C}_{2} \mathrm{H}_{3}$ & $+\mathrm{H}$ & $\rightarrow \mathrm{C}_{2} \mathrm{H}_{4}$ & + & + & $1,21 \cdot 10^{14}$ \\
\hline $\mathrm{C}_{2} \mathrm{H}_{3}$ & $+\mathrm{H}$ & $\rightarrow \mathrm{C}_{2} \mathrm{H}_{2}$ & $+\mathrm{H}_{2}$ & + & $1,21 \cdot 10^{13}$ \\
\hline $\mathrm{C}_{2} \mathrm{H}_{3}$ & $+\mathrm{CH}_{3}$ & $\rightarrow$ Propen & + & + & $7,23 \cdot 10^{13}$ \\
\hline $\mathrm{C}_{2} \mathrm{H}_{3}$ & $+\mathrm{CH}_{3}$ & $\rightarrow \mathrm{C}_{2} \mathrm{H}_{2}$ & $+\mathrm{CH}_{4}$ & + & $1,81 \cdot 10^{13}$ \\
\hline $\mathrm{CH}_{3}$ & $+\mathrm{CH}_{3}$ & $\rightarrow \mathrm{C}_{2} \mathrm{H}_{6}$ & + & + & $2,40 \cdot 10^{13}$ \\
\hline $\mathrm{C}_{3} \mathrm{H}_{6}$ & $+\mathrm{O}$ & $\rightarrow$ Produkte- & + & + & $2,90 \cdot 10^{12}$ \\
\hline $\mathrm{C}_{3} \mathrm{H}_{6}$ & $+\mathrm{H}$ & $\rightarrow \mathrm{C}_{3} \mathrm{H}_{7}$ & + & + & $5,56 \cdot 10^{13}$ \\
\hline $\mathrm{CH}_{3}$ & $+\mathrm{O}$ & $\rightarrow \mathrm{H}_{2} \mathrm{CO}$ & $+\mathrm{H}$ & + & $4,18 \cdot 10^{13}$ \\
\hline $\mathrm{CH}_{3}$ & $+\mathrm{O}$ & $\rightarrow \mathrm{CO}$ & $+\mathrm{H}_{2}$ & $+\mathrm{H}$ & $3,42 \cdot 10^{13}$ \\
\hline $\mathrm{CH}_{3}$ & $+\mathrm{H}$ & $\rightarrow \mathrm{CH}_{4}$ & + & + & $1,80 \cdot 10^{13}$ \\
\hline $\mathrm{C}_{2} \mathrm{H}_{3}$ & $+\mathrm{O}$ & $\rightarrow$ Produkte- & & + & $9,64 \cdot 10^{13}$ \\
\hline Allen & $+\mathrm{O}$ & $\rightarrow$ Produkte- & & + & $3,90 \cdot 10^{10}$ \\
\hline Propin & $+\mathrm{O}$ & $\rightarrow$ Produkte- & & + & $4,05 \cdot 10^{11}$ \\
\hline Acrolein & $+\mathrm{O}$ & $\rightarrow$ Produkte- & & + & $2,48 \cdot 10^{11}$ \\
\hline $\mathrm{C}_{2} \mathrm{H}_{4}$ & $+\mathrm{O}$ & $\rightarrow$ Produkte- & & + & $3,76 \cdot 10^{11}$ \\
\hline
\end{tabular}

Tabelle 4.25: Reaktionssystem $\mathrm{C}_{3} \mathrm{H}_{5} / \mathrm{O}$, Reaktionen und Geschwindigkeitskoeffizienten für die Simulationsrechnung. 


\begin{tabular}{lcc}
\hline Substanz & Experiment & Simulation \\
\hline Ethen & 1 & 1 \\
Acrolein & 0,92 & 1,09 \\
Propen & 0,41 & 0,48 \\
Ethin & 0,28 & 0,45 \\
Formaldehyd & 0,13 & 0,22 \\
Allen & 0,08 & 0,29 \\
\hline
\end{tabular}

Tabelle 4.26: Reaktion Allyl + O, Vergleich Experiment mit Simulation.

Die Werte von Experiment und Simulation weichen teilweise stark voneinander ab. Eine bessere Übereinstimmung konnte durch Variation der Geschwindigkeitskoeffizienten der Selbstreaktion nicht erreicht werden. Des Weiteren ist das Verhältnis von Oxidations- und Selbstreaktionsprodukten stark von den Radikalanfangskonzentrationen abhängig.

Für eine Bestimmung der Selbstreaktionsgeschwindigkeit sollte daher ein zweites Reaktionssystem untersucht werden. Dazu bietet sich eine Untersuchung des Systems $\mathrm{C}_{3} \mathrm{H}_{5} /$ $\mathrm{CH}_{3}$ an, da für dieses schon von GaRland und BAYEs Ergebnisse vorliegen [Gar90]. Mittels der hier durchgeführten Simulationsrechnungen wurde der Geschwindigkeitskoeffzient der Gesamtreaktion zu k $\left(\mathrm{C}_{3} \mathrm{H}_{5}+\mathrm{C}_{3} \mathrm{H}_{5} \rightarrow\right.$ Produkte $)=1,4 \times 10^{13} \mathrm{~cm}^{3} /(\mathrm{mol} \cdot \mathrm{s})$ bestimmt.

\subsubsection{Quantenchemische Rechnungen}

Im Rahmen einer Kooperation wurden von Olzmann (TU Karlsruhe) quatenchemische Rechnungen für den Zerfall der Allyloxy Radikals durchgeführt. Ältere Ergebnisse (1996) wiesen zunächst eine Verteilung von $\left[\mathrm{C}_{3} \mathrm{H}_{4} \mathrm{O}\right] /\left[\mathrm{C}_{2} \mathrm{H}_{4}\right] /\left[\mathrm{H}_{2} \mathrm{CO}\right]=0,4 / 0,4 / 0,2$ auf. Dieses Ergebnis ist in relativ guter Übereinstimmung mit dem experimentellen Ergebnissen dieser Arbeit. Allerdings lieferten Rechnungen 1998 eine Kanalverteilung von $\left[\mathrm{C}_{3} \mathrm{H}_{4} \mathrm{O}\right]$ $/\left[\mathrm{C}_{2} \mathrm{H}_{4}\right] /\left[\mathrm{H}_{2} \mathrm{CO}\right]=0,15 / 0,8 / 0,05$. Im Rahmen einer geplanten Veröffentlichung der experimentellen Ergebnisse dieser Arbeit sind quantenchemische Rechnungen durch Olzmann in Arbeit, um die widersprüchlichen Resultate zu klären. Erste Rechnungen ergaben die in Abbildung 4.42 dargestellten Geschwindigkeiten in Abhängigkeit von der Anregungsenergie des Allyloxy-Radikals. 


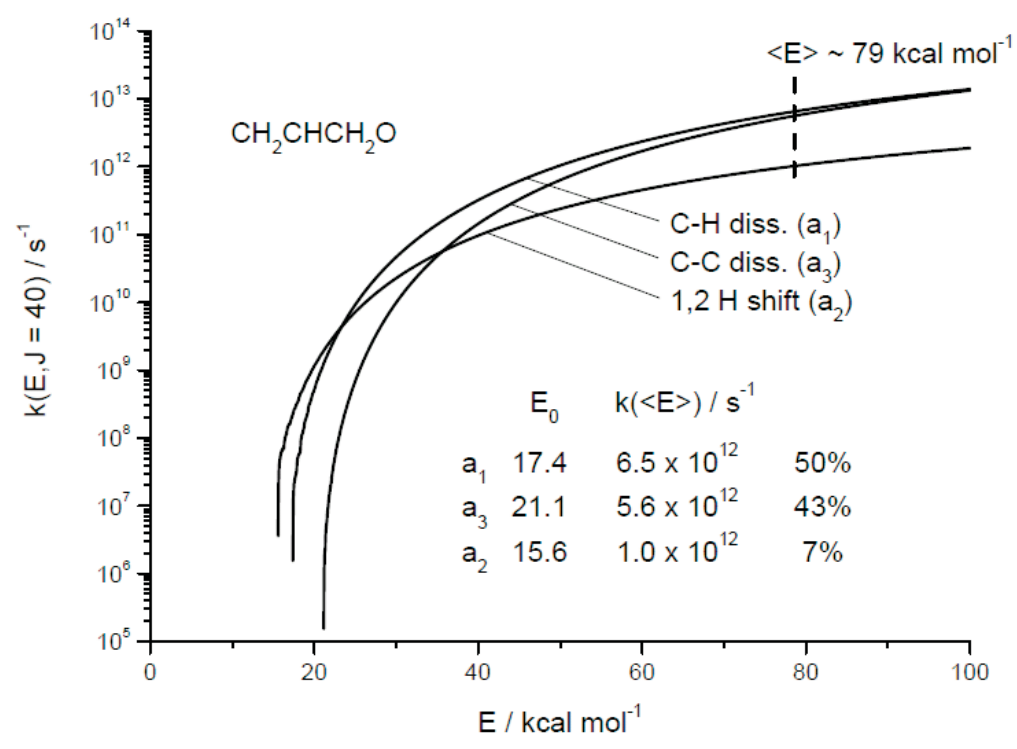

Abbildung 4.42: Reaktion Allyl + O, RRKM-Rechnungen für den Zerfall des Allyloxy-Radikals

Wie die Abbildung 4.42 zeigt, sind die Bildung von Acrolein unter $\beta$-C-H-Bindungsbruch und die Reaktion zu $\mathrm{C}_{2} \mathrm{H}_{3}+\mathrm{H}_{2} \mathrm{CO}$ ungefähr gleich schnell. Die Reaktion zu Ethen, $\mathrm{CO}$ und $\mathrm{H}$ unter 1,2-H-shift ist deutlich langsamer. Die quantenchemischen Rechnungen ergeben ausgehend von dem eingangs dargestellten Mechanismus eine Kanalverzweigung $\operatorname{von}\left(a_{1}\right) /\left(a_{2}\right) /\left(a_{3}\right)=0,5 / 0,07 / 0,43$.

\subsubsection{Diskussion}

Die Ergebnisse dieser Untersuchung werden mit mehreren Arbeiten in der Literatur verglichen. Die Reaktion wurde innerhalb der Arbeitsgruppe schon von TröGER, NACKE und Kersten untersucht [Trö97, Nac98, Ker99]. Dabei untersuchte NACKE die Geschwindigkeit der Reaktion Allyl + O. TRÖGER und Kersten führten eine Produktuntersuchung der Reaktion zur Bestimmung der Kanalverzweigung mittels der ebenfalls für diese Arbeit verwendeten FTIR Apparatur durch. Im Gegensatz zu diesen wurde anstatt Allyliodid als Radikalvorläufer Allylbromid, Allylchlorid und Diallyl benutzt. Es ist bekannt, dass bei Verwendung von Allyliodid als Vorläufer durch Reaktion von Iodatomen IO entsteht, welches wahrscheinlich Einfluss auf den Reaktionsmechanismus hat. Bei diesem Vorläufer konnte weiterhin ein sehr großer HI-Eliminationsanteil festgestellt werden, der zur Bildung von Allen führt. TRÖGER bestimmte folgendes Kanalverzweigungsverhältnis $\left(\mathrm{a}_{1}\right) /$ 
$\left(\mathrm{a}_{2}\right) /\left(\mathrm{a}_{3}\right)=1 / 0,9 / 0,6$. KERSTEN fand für die Reaktion folgendes Kanalverzweigungsverhältnis: $\left(\mathrm{a}_{1}\right) /\left(\mathrm{a}_{2}\right) /\left(\mathrm{a}_{3}\right) /\left(\mathrm{b}_{2}\right)=1 / 3,33 / 2,06 / 5,24$. Der außerordentlich hohe Anteil des Allenkanals $\left(b_{2}\right)$ ist auf die schon beschriebene HI-Elimination zurück zuführen und somit nicht Folge der Reaktion Allyl + O.

SLAGLE et al. untersuchten die Reaktion hinsichtlich der Kinetik mit Hilfe Laserphotolyse einer $\mathrm{C}_{3} \mathrm{H}_{5} \mathrm{Br} / \mathrm{SO}_{2} / \mathrm{He}$ Mischung und einer $\left(\mathrm{C}_{3} \mathrm{H}_{5}\right)_{2} / \mathrm{N}_{2} \mathrm{O} / \mathrm{N}_{2}$ Mischung bei $\lambda=$ 193 nm und Detektion via Photoionisations-Massenspektrometrie und Resonanzfluoreszenz [Sla90]. Die massenspektrometrische Analyse ergab als einziges Produkt der Reaktion Acrolein. Eine direkte Suche nach den möglichen Produkten Formaldehyd und $\mathrm{C}_{2} \mathrm{H}_{3}$ verlief negativ.

Bei neueren Untersuchungen verwendeten PARK et al. [Par02, Par03] zum Einen auf experimenteller Ebene eine laserinduzierte Fluoreszenztechnik zur Detektion von OH Radikalen und zum Anderen quantenchemische Rechnungen. In der kombinierten experimentellen und theoretischen Untersuchung [Par02] konnte dabei mittels Fluoreszenzanalyse ausschließlich der Abstraktionskanal $\left(\mathrm{b}_{2}\right)$ bestätigt werden. Aussagen über die übrigen Reaktionswege waren damit nicht möglich. Ab initio Rechnungen auf Basis des CBS-QB3 Niveaus und RRKM Rechnungen sagen als Hauptkanal der Reaktion zudem die Bildung von Acrolein voraus. Als Gegenstück zu den experimentell gefundenen $\mathrm{OH}$ Radikalen wurde anhand von Rechnungen Allen vorgeschlagen. Die quantenchemischen Rechnungen zeigen dabei, dass es eine Vielzahl an Reaktionswegen gibt, die über drei unterschiedliche Übergangszustände des Allyloxy Radikals ablaufen. Die RRKM Rechnungen ergeben folgende Geschwindigkeitskoeffzienten für den wahrscheinlichsten Übergangszustand $\mathrm{k}\left(\mathrm{a}_{1}\right)=8,1 \times 10^{12} \mathrm{~s}^{-1}, \mathrm{k}\left(\mathrm{b}_{2}\right)=1,2 \times 10^{10} \mathrm{~s}^{-1}, \mathrm{k}\left(\mathrm{b}_{1}\right)=1,5 \times 10^{6} \mathrm{~s}^{-1}$. Die Untersuchung [Par03] diskutiert die Geschwindigkeitskoeffizienten der Reaktion in Abhängigkeit der unterschiedlichen Übergangszuständen. Allgemein wird dabei die Bildung von Acrolein als Hauptkanal vorgeschlagen.

Eine weitere experimentelle und theoretische Untersuchung der Reaktion von Joo et al. Joo04 befasst sich mit dem LIF Nachweis von H Atomen in der Lyman- $\alpha$-Region mit einem Zentrum bei $\lambda=121,6 \mathrm{~nm}$ und ab initio Rechnungen (CBS-QB3 Niveau). Experiment und Rechnung liefern übereinstimmend als Hauptkanal der Reaktion die Bildung von Acrolein $+\mathrm{H}$ aus einem kurzlebigen, hochangeregten Additionskomplex. 
In dieser Arbeit konnte die Bildung von Acrolein als Hauptkanal in Übereinstimmung mit der Literatur bestätigt werden. Die Reaktionskanäle $\left(\mathrm{a}_{2}\right)$ und $\left(\mathrm{a}_{3}\right)$, die Ethen / CO bzw. $\mathrm{H}_{2} \mathrm{CO} /$ Ethin liefern, werden aber ebenfalls für wichtig befunden. Dies konnte unter Verwendung aller Vorläufer festgestellt werden. An dieser Stelle sei aber darauf hingewiesen, dass eine genaue Übereinstimmung der Produktverhältnisse nicht gegeben war.

Die Geschwindigkeit der Selbstreaktion wurde mittels Simulationsrechnungen basierend auf den experimentellen Ergebnissen und einer Geschwindigkeit der Reaktion $\mathrm{C}_{3} \mathrm{H}_{5}+\mathrm{O}$ von $\mathrm{k}\left(\mathrm{C}_{3} \mathrm{H}_{5}+\mathrm{O} \rightarrow\right.$ Produkte $)=1,8 \times 10^{14} \mathrm{~cm}^{3} /(\mathrm{mol} \cdot \mathrm{s})$ bestimmt $\mathrm{zu}$

$$
k\left(\mathrm{C}_{3} \mathrm{H}_{5}+\mathrm{C}_{3} \mathrm{H}_{5} \rightarrow \text { Produkte }\right)=1,4 \times 10^{13} \mathrm{~cm}^{3} /(\mathrm{mol} \cdot \mathrm{s}) .
$$

Beachtet man, dass in der vorliegenden Arbeit der Anteil der Kombination zum Reaktionsmechanismus $20 \%$ beträgt, so ergibt sich für die Geschwindigkeit der Kombinationsreaktion bei einem Gesamtdruck von 4 mbar ein Wert von

$$
k\left(\mathrm{C}_{3} \mathrm{H}_{5}+\mathrm{C}_{3} \mathrm{H}_{5} \rightarrow \mathrm{C}_{6} \mathrm{H}_{10}\right)=2,8 \times 10^{12} \mathrm{~cm}^{3} /(\mathrm{mol} \cdot \mathrm{s}) .
$$

TsAnG empfiehlt für die Selbstreaktion von Allylradikalen einen Wert von $\mathrm{k}\left(\mathrm{C}_{3} \mathrm{H}_{5}+\right.$ $\mathrm{C}_{3} \mathrm{H}_{5} \rightarrow$ Produkte $)=1,6 \times 10^{13} \mathrm{~cm}^{3} /(\mathrm{mol} \cdot \mathrm{s})$ und ein Verhältnis von Disproportionierung zu Kombination von $k(d) / k(c)=0,008$ [Tsa91]. Daher ist die Geschwindigkeit der Kombinationsreaktion gegenüber der vorliegenden Arbeit um den Faktor 5,7 zu hoch. 


\subsection{Untersuchung der Reaktionen offenkettiger Koh- lenwasserstoffradikale}

Die Reaktionen offenkettiger Kohlenwasserstoffradikale sind in der Literatur teilweise detailliert untersucht. Zusammenfassungen der Ergebnisse finden sich z. B. in [Bau05, Tsa86]. Für die im Rahmen dieser Arbeit untersuchten Reaktionen $\mathrm{CH}_{3}+\mathrm{CH}_{3}, \mathrm{C}_{2} \mathrm{H}_{5}+$ $\mathrm{C}_{2} \mathrm{H}_{5}, 1-\mathrm{C}_{3} \mathrm{H}_{7}+1-\mathrm{C}_{3} \mathrm{H}_{7}, 2-\mathrm{C}_{3} \mathrm{H}_{7}+2-\mathrm{C}_{3} \mathrm{H}_{7}$ und 1- $\mathrm{C}_{4} \mathrm{H}_{9}+1-\mathrm{C}_{4} \mathrm{H}_{9}$ liegen einige Ergebnisse für die Kanalverzweigung und die Geschwindigkeitskoeffizienten vor. Vorrangiges Ziel der vorliegenden Untersuchungen war es, jeweils die Kanalverzweigung erneut mit unserer neuen Methode zu bestimmen und geeignete Vorläufer für die Untersuchungen zu den Kreuzkombinationen von Alkylradikalen bereit zu stellen und Nebenreaktionen quantitativ zu bestimmen (s. Kapitel 4.5). Als Radikalvorläufer wurden zum Einen iodierte Kohlenwasserstoffe und zum Anderen Ketone verwendet. Der Einsatz iodierter Kohlenwasserstoffe hat dabei den schon mehrfach beschriebenen Nachteil einer HI-Elimination bei Laserphotolyse $(\lambda=193 \mathrm{~nm})$. Bei der Photolyse von Ketonen tritt dagegen das Problem auf, dass neben der erwünschten Spaltung in 2 Alkylradikale und CO (Norrish Typ I Spaltung) auch eine teilweise Spaltung, bzw. ein Zerfall gemäß Norrish Typ III Spaltung unter $H$-shift zu beobachten ist. Ziel der im Folgenden dargestellten Untersuchungen war, die photolytischen Zerfallsprozesse der bei der Analyse der Kreuzreaktionen eingesetzten Vorläufer zu klären.

\subsubsection{Die Reaktion Methyl + Methyl}

\subsubsection{Einleitung}

Als Methylradikalvorläufer wurde bei allen Untersuchungen der Kreuzreaktionen Aceton$\mathrm{d}_{6}$ benutzt. Dieser Vorläufer hat den Vorteil, dass neben der Spaltung in 2 Methylradikale und CO kaum Nebenreaktionen auftreten. Das Kombinationsprodukt Ethan- $\mathrm{d}_{6}$ ist auf Grund fehlender C-H-Bindungen weiterhin einfach von Produkten der Reaktionen undeuterierter Kohlenwasserstoffe zu unterscheiden. 


\subsubsection{Qualitative Analyse}

Ein Photolysespektrum nach 400 Laserpulsen bei $\mathrm{p}\left(\right.$ Aceton- $\left.\mathrm{d}_{6}\right)=0,2$ mbar und einem Gesamtdruck von 4 mbar und $\mathrm{T}=298 \mathrm{~K}$ ist in Abbildung 4.43 dargestellt. Ein vergrößerter Ausschnitt des Spektrums im Bereich der identifizierten Substanzen Ethan- $\mathrm{d}_{6}$ und CO ist in Abbildung 4.44 gegeben.

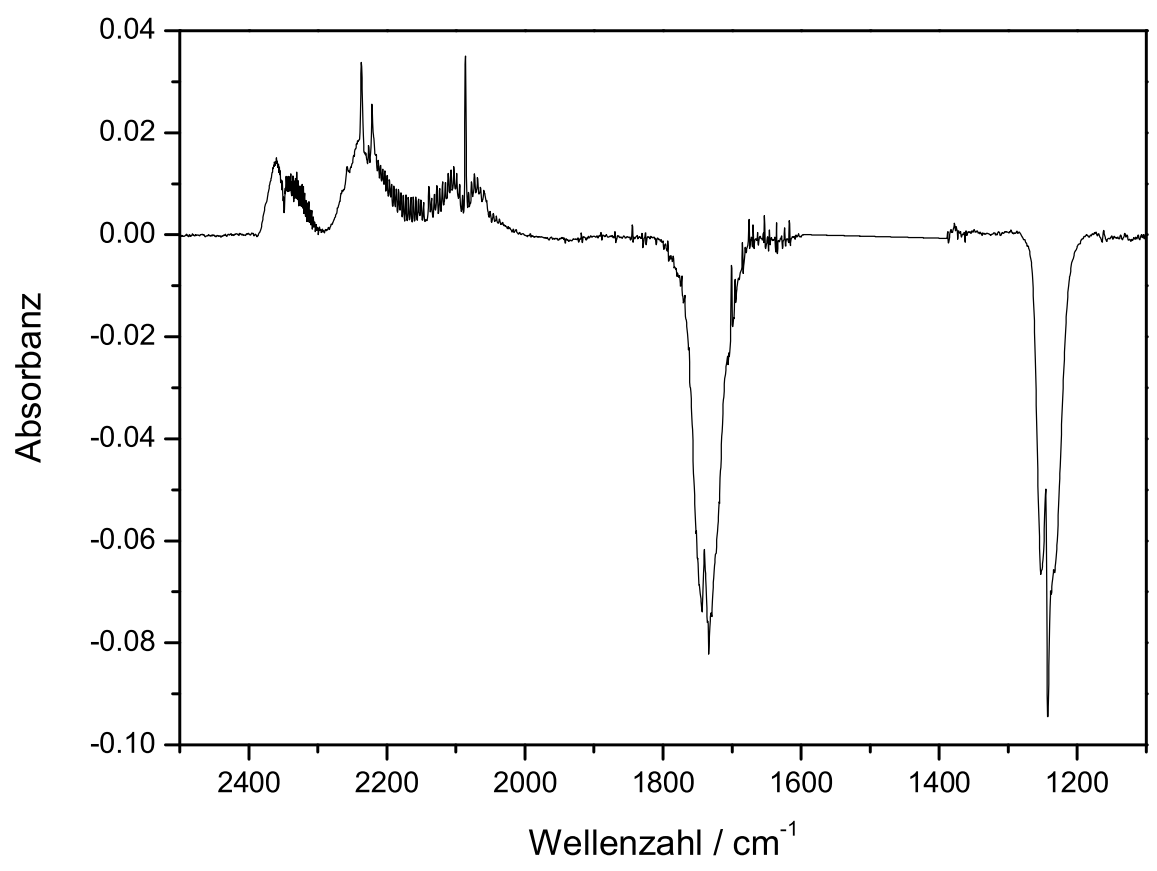

Abbildung 4.43: Reaktion $C D_{3}+C D_{3}$, Vorläufer Aceton- $d_{6} \cdot p\left(C_{3} D_{6} O\right)=0,2$ mbar, $p_{\text {ges }}=4$ mbar, $T=298 \mathrm{~K}, 400$ Photolysen.
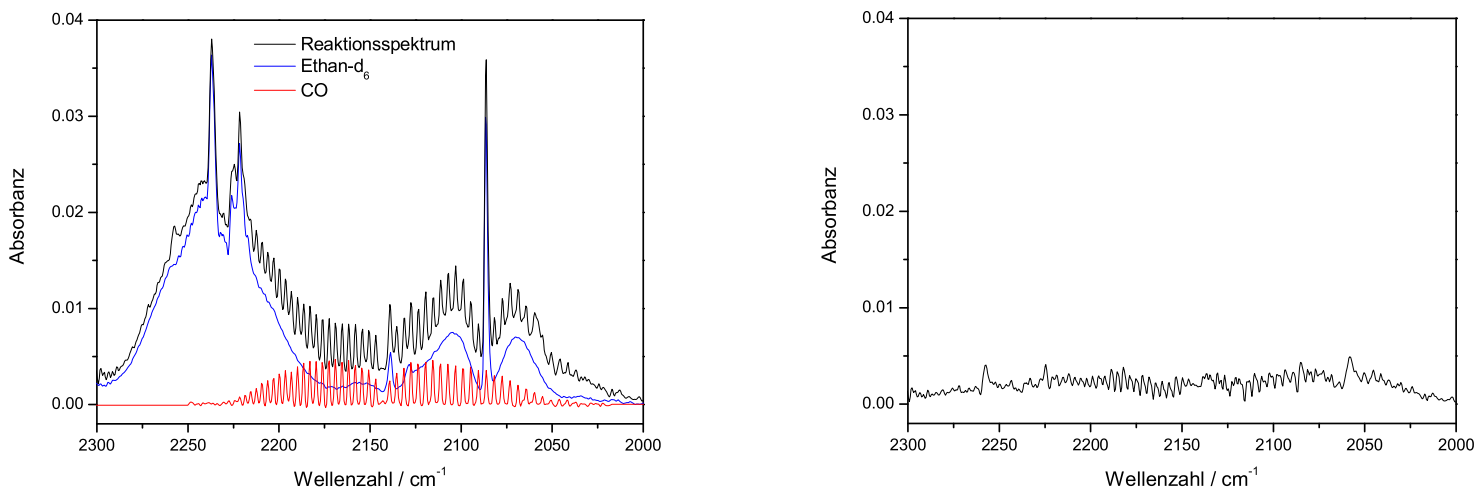

Abbildung 4.44: Reaktion $C D_{3}+C D_{3}$, qualitative Analyse, Vorläufer Aceton-d $d_{6}$ (p (Aceton- $d_{6}$ $=0.2$ mbar, $p_{g e s}=4$ mbar, 400 Photolysen); links: Das schwarz dargestellte Spektrum ist das Reaktionsspektrum, die farbig dargestellten Spektren die der Reinsubstanzen; rechts: Residuum. 
Neben diesen beiden Substanzen konnten keine weiteren Produkte gefunden werden. Damit verläuft die Photolyse von Aceton zu nahezu 100\% zu der Bildung zweier Methylradikale, was auch in der folgenden quantitativen Analyse bestätigt wird.

\subsubsection{Quanitative Analyse}

Für die quantitative Analyse wurden die Partialdrücke der identifizierten Substanzen Ethan- $\mathrm{d}_{6}$ und CO gegen die Abnahme des Radikalvorläufers Aceton- $\mathrm{d}_{6}$ aufgetragen. Die Auftragung ist in Abbildung 4.45 dargestellt. Die zugehörigen Messwerte sind in Tabelle 4.27 angegeben.

\begin{tabular}{|c|c|c|}
\hline $\begin{array}{c}\text { Aceton- } \mathrm{d}_{6} \text {-Verbrauch } \\
{[\mathrm{mbar}]}\end{array}$ & $\begin{array}{c}\text { Ethan- } \mathrm{d}_{6} \\
{[\mathrm{mbar}]}\end{array}$ & $\begin{array}{c}\mathrm{CO} \\
{[\mathrm{mbar}]}\end{array}$ \\
\hline \hline 0,006 & 0,0058 & 0,006 \\
\hline 0,01 & 0,01 & 0,01 \\
\hline 0,016 & 0,017 & 0,015 \\
\hline 0,022 & 0,023 & 0,02 \\
\hline
\end{tabular}

Tabelle 4.27: Reaktion $C D_{3}+C D_{3}$, Vorläufer Aceton- $d_{6}$. Messwerte. $p\left(\right.$ Aceton- $\left.d_{6}\right)=0,2$ mbar, $p_{\text {ges }}=4$ mbar, $\mathrm{T}=298 \mathrm{~K}$, Badgas Argon. 


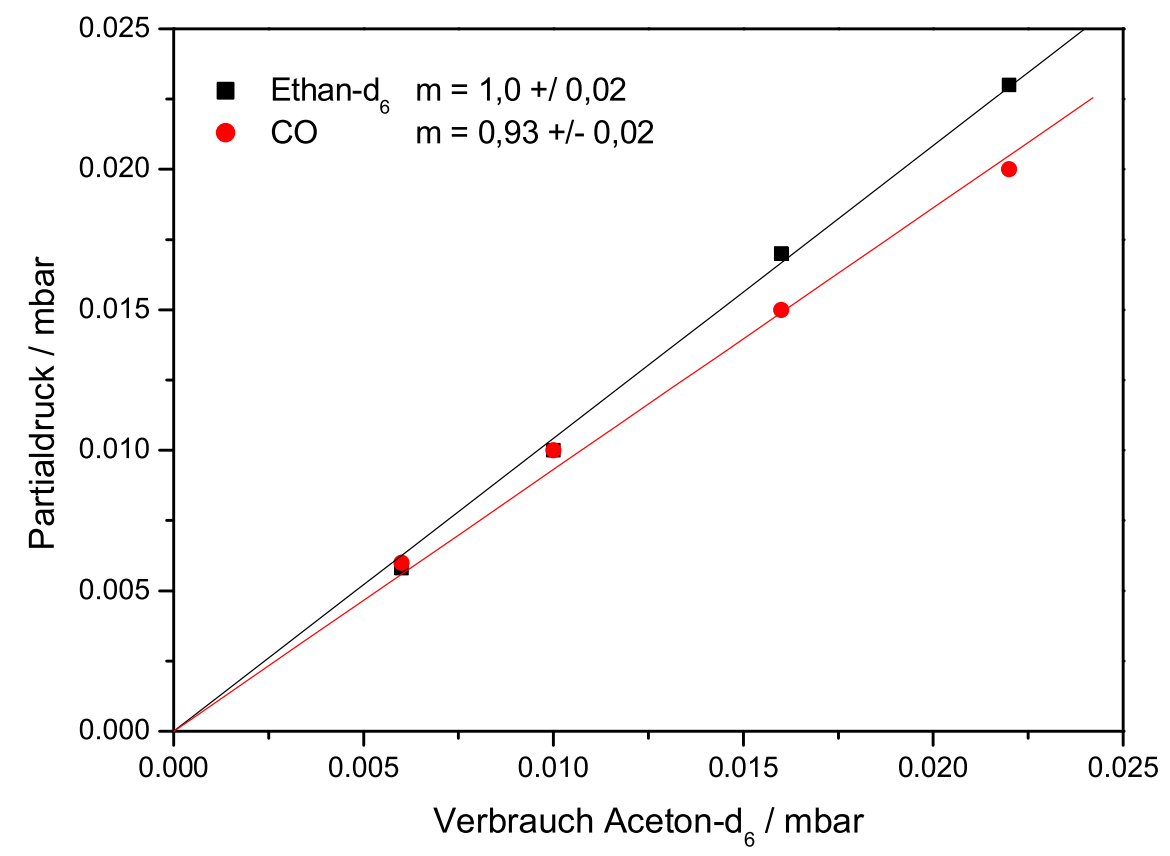

Abbildung 4.45: Reaktion $C D_{3}+C D_{3}$, quantitative Analyse. $p\left(C_{3} D_{6} O\right)=0,2$ mbar, $p_{\text {ges }}=4$ mbar, $T=298 \mathrm{~K}, 400$ Photolysen.

Wie die Abbildung 4.45 zeigt, nehmen die Partialdrücke von Ethan- $\mathrm{d}_{6}$ und CO linear mit dem Verbrauch des Vorläufers zu. Dabei ergibt eine lineare Regression auf Basis eines Konfidenzintervalls von $95 \%$ eine Steigung von $\mathrm{m}\left(\right.$ Ethan- $\left.\mathrm{d}_{6}\right)=1,0 \pm 0,02$ und $\mathrm{m}(\mathrm{CO})=$ $0,93 \pm 0,02$. Für die Photolyse von Aceton- $\mathrm{d}_{6}$ kann also ein 100\%-iger Umsatz zu Ethan$\mathrm{d}_{6}$ bestätigt werden. Die Abweichung von CO resultiert aus der schwierigen Eichung bei höheren Umsätzen, da die rotationsaufgelöste Bande kein lineares Verhalten aufweist. Damit erfolgt bei der Photolyse nur die Reaktion

$$
\mathrm{CD}_{3} \mathrm{COCD}_{3} \stackrel{h \nu}{\longrightarrow} 2 \mathrm{CD}_{3}+\mathrm{CO}
$$

\subsubsection{Diskussion}

Das Ziel dieser Untersuchung, die Kanalverzweigung für die Photolyse von Aceton- $\mathrm{d}_{6}$ zu bestimmen, wurde in doppelter Hinsicht erreicht. Zum Einen beweist die quantitative Analyse und die Massenbilanz, dass alle Produkte der Photolyse identifiziert wurden, zum Anderen zeigt sie, dass der Vorläufer eine sehr saubere $\mathrm{CD}_{3}$-Quelle ist. Eine Kanalverzweigung existiert auf Grund nur eines Reaktionskanals daher nicht. 


\subsubsection{Die Reaktion Ethyl + Ethyl}

\subsubsection{Einleitung}

In diesem Abschnitt werden Ergebnisse der Photolyse von Ethyliodid vorgestellt. Ethyliodid wurde bei den Untersuchungen der Kreuzreaktionen von Alkylradikalen als Ethylradikalvorläufer verwendet. Auf den Einsatz von Diethylketon als Vorläufer wurde auf Grund ähnlicher Bandenlagen wie Dipropylketon und Diisopropylketon verzichtet. Eine quantitative Bestimmung der Radikalanfangskonzentrationen unter Kreuzreaktionsbedingungen wäre im Fall des Einsatzes von zwei Dialkylketonen als Vorläufer erschwert. Bei der Photolyse von Ethyliodid wird zunächst die Konkurrenz von C-I-Spaltung und HIElimination angenommen, deren Verhältnis von [Zeu03] zu (C-I-Split)/(HI-Elim.) = 1,7 / 1 bestimmt wurde.

Für die Reaktion von 2 Ethylradikalen ist eine Disproportionierung und Kombinationsreaktion möglich.

$$
\begin{aligned}
\mathrm{C}_{2} \mathrm{H}_{5}+\mathrm{C}_{2} \mathrm{H}_{5} & \longrightarrow \mathrm{C}_{4} \mathrm{H}_{10} \\
& \longrightarrow \mathrm{C}_{2} \mathrm{H}_{4}+\mathrm{C}_{2} \mathrm{H}_{6}
\end{aligned}
$$

Die in der Literatur vorliegenden Geschwindigkeitskoeffizienten für beide Reaktionswege lassen eine stark favorisierte Kombinationsreaktion erwarten $k(d) / k(c)=0,14$ [Bau05, Tsa86]. Dabei handelt es sich bei der Angabe von BAulch et al. aber nur um eine Abschätzung.

\subsubsection{Qualitative Analyse}

Ein Reaktionsspektrum nach 400 Photolysen von Ethyliodid zeigt Abbildung 4.46 bei $\mathrm{p}\left(\mathrm{C}_{2} \mathrm{H}_{5} \mathrm{I}\right)=0,5$ mbar; $\mathrm{T}=298 \mathrm{~K} ; \mathrm{p}_{\text {ges }}=4$ mbar. 


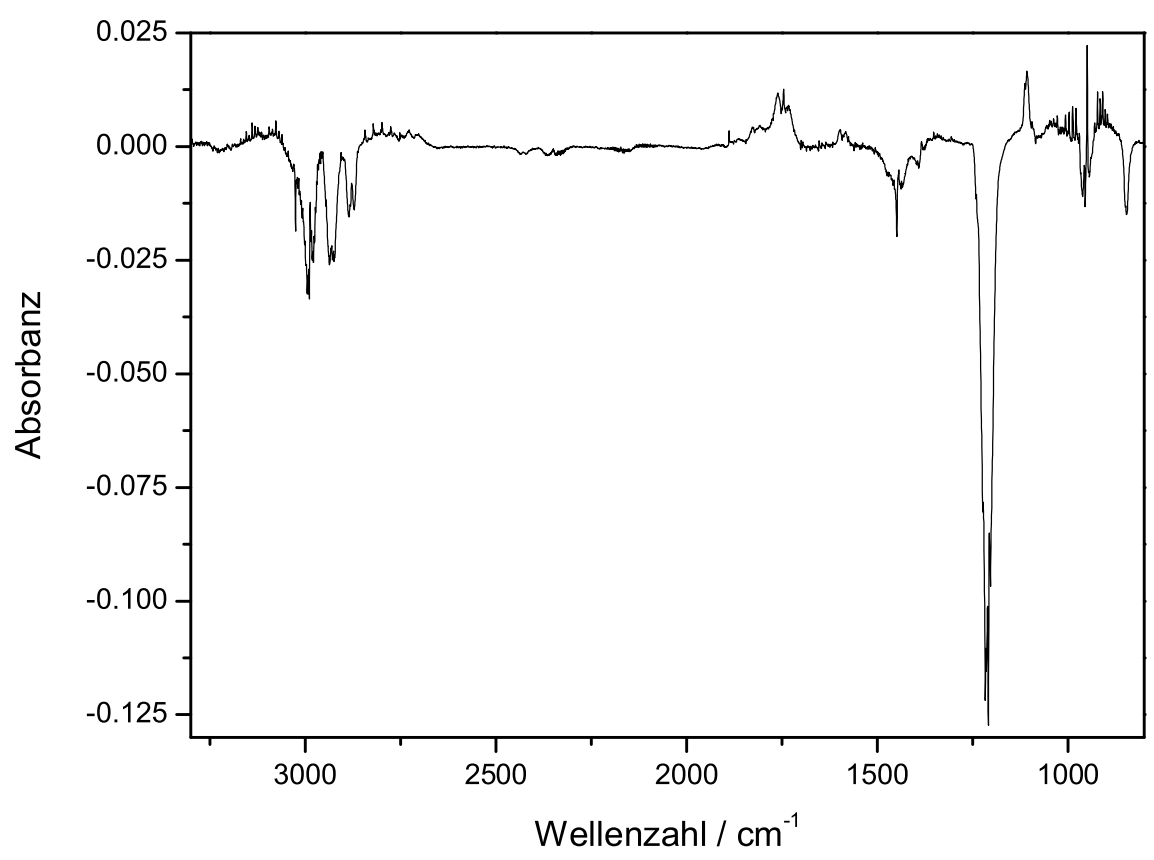

Abbildung 4.46: Reaktion $\mathrm{C}_{2} \mathrm{H}_{5}+\mathrm{C}_{2} \mathrm{H}_{5}$, Reaktionsspektrum, Vorläufer Ethyliodid. $\mathrm{p}\left(\mathrm{C}_{2} \mathrm{H}_{5} \mathrm{I}\right)$ $=0,5$ mbar, $p_{\text {ges }}=4$ mbar, $T=298 \mathrm{~K}, 400$ Photolysen.

In dem dort dargestellten Reaktionsspektrum sieht man deutlich eine positive Bande im Bereich der Carbonylschwingung um $\tilde{\nu}=1750 \mathrm{~cm}^{-1}$. Diese Bande konnte eindeutig Acetaldehyd zugeordnet werden. Die Bildung von Acetaldehyd kann durch Reaktion von Ethylradikalen mit in der Zelle befindlichem molekularem Sauerstoff erklärt werden. Der Eintrag von Luft und damit von Sauerstoff in die Zelle trat teilweise nach Einbau neuer Fenster auf. Neben diesem unerwünschten Produkt konnte die Bildung von Ethen, Butan und Ethan nachgewiesen werden. Die Identifikation dieser Substanzen und von Acetaldehyd ist in Abbildung 4.47 aufgezeigt. Dabei wird das Reaktionsspektrum in vergrößerten Ausschnitten mit den Reinspektren der jeweiligen Substanzen verglichen.

Bei der qualitativen Analyse konnten somit Produkte der Kombinationsreaktion (Butan), wie auch der Disproportionierung (Ethan und Ethen) gefunden werden. Dabei ist allerdings zu beachten, dass die Bildung von Ethen vorrangig über die direkte HI-Elimination geschieht und somit die quantitative Bestimmung dieses Kanals über die Analyse des Ethanpartialdrucks vorgenommen werden muss. 

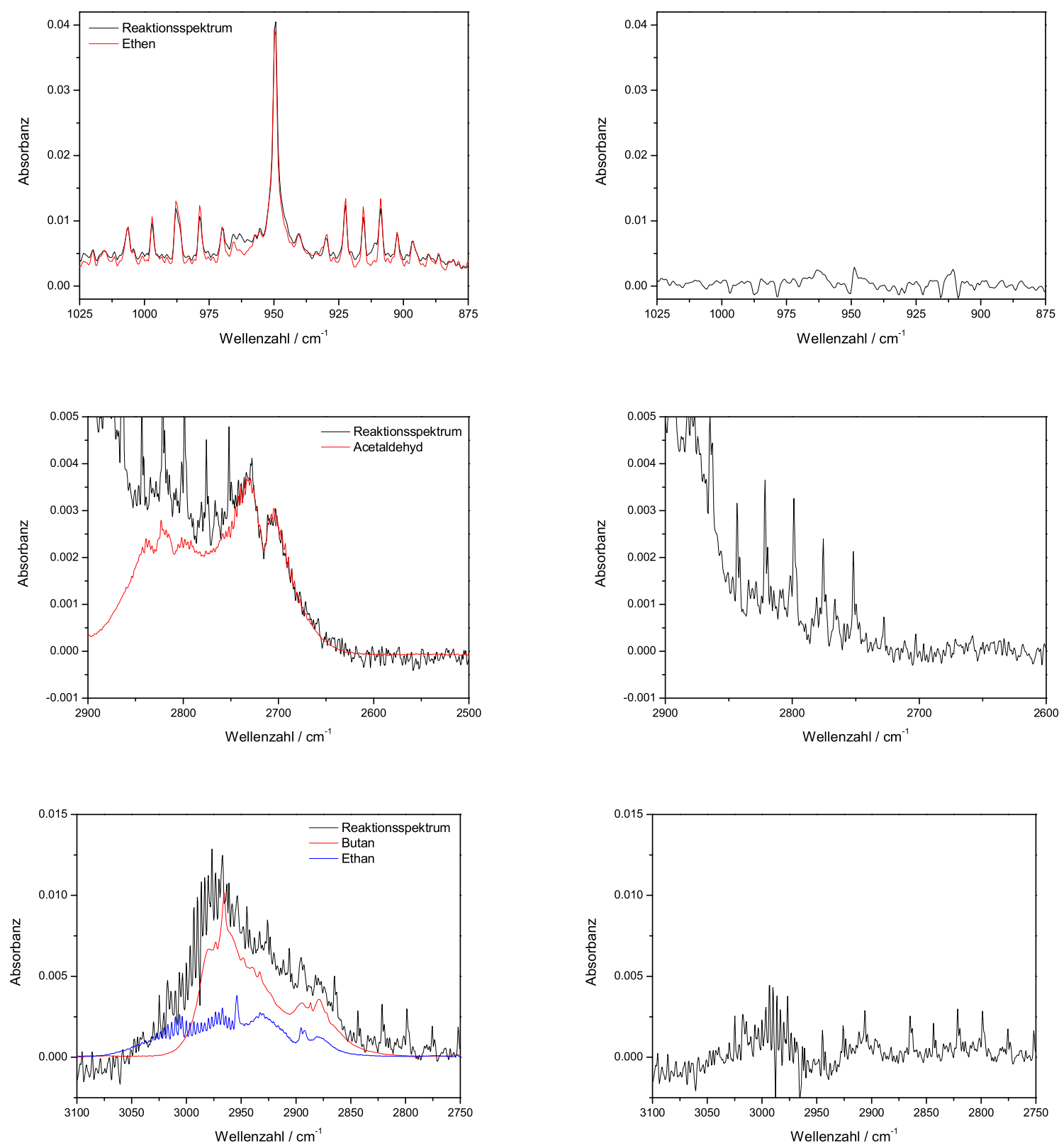

Abbildung 4.47: Reaktion $\mathrm{C}_{2} \mathrm{H}_{5}+\mathrm{C}_{2} \mathrm{H}_{5}$, qualitative Analyse, Vorläufer Ethyliodid, $\left(\mathrm{p}\left(\mathrm{C}_{2} \mathrm{H}_{5} \mathrm{I}\right.\right.$ $=0.5$ mbar, $p_{\text {ges }}=4 \mathrm{mbar}, 400$ Photolysen); links: Das schwarz dargestellte Spektrum ist das Reaktionsspektrum, die farbig dargestellten Spektren die der Reinsubstanzen; rechts: Residuum.

\subsubsection{Quantitative Analyse}

Die quantitative Analyse der Reaktion wurde, wie auch bei den übrigen Reaktionen, anhand einer Auftragung der geeichten Partialdrücke der identifizierten Substanzen gegen die Abnahme an Vorläufer vorgenommen. Dabei wurde in diesem Fall die Reaktion der Ethylradikale mit dem in der Zelle befindlichen molekularen Sauerstoff unter Bildung von 
Acetaldehyd berücksichtigt. Die ursprünglich bestimmte Abnahme an Vorläufer wurde also mit der Zunahme an Acetaldehyd nach unten korrigiert. Die resultierende Abnahme an Ethyliodid entspricht also der Stoffmenge, die für die Kombinations- und Disproportionierungsreaktion sowie für die HI-Elimination zur Verfügung stand. Die Auftragung der Partialdrücke der Produkte gegen die korrigierte Abnahme an Ethyliodid zeigt die Abbildung 4.48. Die zugehörigen Messwerte sind in Tabelle 4.28 gegeben.

\begin{tabular}{|c|c|c|c|c|}
\hline $\begin{array}{c}\text { Verbrauch Ethyliodid } \\
{[\mathrm{mbar}]}\end{array}$ & $\begin{array}{c}\mathrm{p}\left(\mathrm{C}_{2} \mathrm{H}_{4}\right) \\
{[\mathrm{mbar}]}\end{array}$ & $\begin{array}{c}\mathrm{p}\left(\mathrm{C}_{4} \mathrm{H}_{10}\right) \\
{[\mathrm{mbar}]}\end{array}$ & $\begin{array}{c}\mathrm{p}\left(\mathrm{C}_{2} \mathrm{H}_{4} \mathrm{O}\right) \\
{[\mathrm{mbar}]}\end{array}$ & $\begin{array}{c}\mathrm{p}\left(\mathrm{C}_{2} \mathrm{H}_{5} \mathrm{I}\right)_{\text {korr. }} \\
{[\mathrm{mbar}]}\end{array}$ \\
\hline \hline 0,0085 & 0,0029 & $9 \mathrm{E}-4$ & $1 \mathrm{E}-3$ & 0,0075 \\
\hline 0,011 & 0,0046 & 0,0016 & 0,0022 & 0,0088 \\
\hline 0,013 & 0,0058 & 0,002 & 0,003 & 0,01 \\
\hline 0,016 & 0,007 & 0,0022 & 0,002 & 0,014 \\
\hline
\end{tabular}

Tabelle 4.28: Reaktion $\mathrm{C}_{2} \mathrm{H}_{5}+\mathrm{C}_{2} \mathrm{H}_{5}$, Vorläufer Ethylidodid, Messwerte. $p\left(\mathrm{C}_{2} \mathrm{H}_{5} \mathrm{I}\right)=0,3 \mathrm{mbar}$, $p_{\text {ges }}=4$ mbar, $\mathrm{T}=298 \mathrm{~K}$, Badgas Argon.

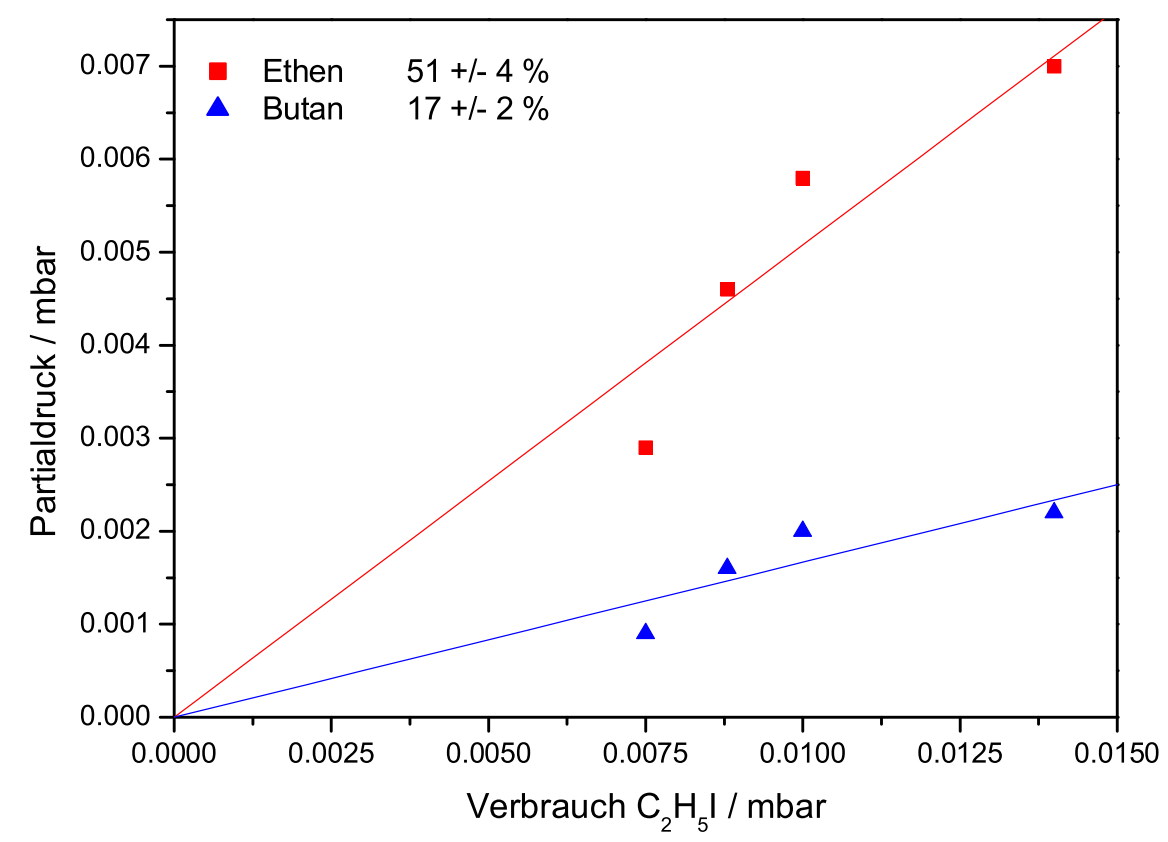

Abbildung 4.48: Reaktion $\mathrm{C}_{2} \mathrm{H}_{5}+\mathrm{C}_{2} \mathrm{H}_{5}$, quantitative Analyse, Vorläufer Ethyliodid. $\mathrm{p}\left(\mathrm{C}_{2} \mathrm{H}_{5} \mathrm{I}\right)$ $=0,5$ mbar, $p_{\text {ges }}=4$ mbar, $T=298 \mathrm{~K}, 400$ Photolysen.

Dabei sind in Abbildung 4.48 nur die Produkte Ethen und Butan aufgeführt, da die Bildung von Ethan erst bei sehr hohen Photolysenanzahlen quantitativ messbar war. Hohe 
Photolysenanzahlen begünstigten allerdings Sekundärrekationen, die zu einer Spaltung von Butan führten. So war mit steigender Photolysenanzahl eine Änderung des Verhältnisses [Ethen] / [Butan] von 3 / 1 (100 Photolysen) zu 4,3 / 1 (400 Photolysen) zu beobachten. Der absolute Anteil an Ethen stieg bezogen auf die Abnahme an Ethyliodid von 39\% (100 Photolysen) auf 78\% (400 Photolysen). Der Wert von 39\% bezogen auf die Abnahme an Ethyliodid stimmt gut mit dem von ZEUCH gefundenen Wert von $36 \%$ überein [Zeu03]. Daher wird für den photolytischen Zerfall von Ethyliodid von der folgenden Kanalverzweigung ausgegangen:

$$
\begin{array}{rlr}
\mathrm{C}_{2} \mathrm{H}_{5} \mathrm{I} \stackrel{h \nu}{\longrightarrow} \mathrm{C}_{2} \mathrm{H}_{5}+\mathrm{I} & 61 \% \\
\stackrel{h \nu}{\longrightarrow} \mathrm{C}_{2} \mathrm{H}_{4}+\mathrm{HI} & 39 \%
\end{array}
$$

Der in Abbildung 4.48 angegebene Wert von 51\% bezieht sich auf einen Bereich der Ethyliodidabnahme, in dem Nebenreaktionen und Sekundärphotolyse schon nicht mehr ausgeschlossen werden können. Die Abbildung zeigt aber auch, dass, unter Berücksichtigung der Bildung von Butan aus 2 Ethylradikalen, 85\% der Ethyliodidabnahme durch die Kanäle Ethen (51\%) und Butan (17\%) erklärt werden.

\subsubsection{Diskussion}

Die Untersuchung zeigt, dass Ethyliodid als Ethylradikalvorläufer geeingnet ist. Allerdings muss bei der Verwendung die direkte HI-Elimination beachtet werden. Der Anteil der HI-Elimination bezogen auf die Abnahme an Vorläufer wurde zu $39 \%$ bestimmt. Des Weiteren konnte ausgehend von dem in der Einleitung dargestellten Reaktionsmechanismus sowohl die Kombinationsreaktion als auch die Disproportionierung nachgewiesen werden. Dabei war der durch Disproportionierung gebildete Ethananteil bei niedriger Photolysenanzahl allerdings so gering, dass eine quantitative Bestimmung der Kanalverzweigung nicht möglich war. Ein Anteil von $\leq 10 \%$ bezogen auf die Gesamtreaktion wird auf Grund einer Messung nach hoher Photolysenanzahl für realistisch erachtet. Das in der Literatur angegebene Verhältnis von Disproportionierung zu Rekombination von 0,14 wird aber auf Grund des in diesem Experiment als gering eingeschätzten Ethananteils als verläßlich angesehen. Setzt man die von BAULCH et al. empfohlenen Geschwindigkeiten und zugehörigen Fehlergrenzen voraus, so ergibt sich für die Disproportionierung keine 
Aussage, da aus der Fehlerangabe ein Kanalanteil von minimal 2\% bis zu maximal 55\% folgt.

Eine Diskussion der Selbstreaktion wurde schon in Abschnitt 4.3.2 vorgenommen. Daher sei hier auf diese Stelle verwiesen.

\subsubsection{Die Reaktion 1-Propyl + 1-Propyl}

\subsubsection{Einleitung}

Für die Reaktion $\mathrm{C}_{3} \mathrm{H}_{7}+\mathrm{C}_{3} \mathrm{H}_{7}$ liegen, im Vergleich zu den in den beiden vorherigen Abschnitten beschriebenen Reaktionen, wenig experimentelle Daten vor. Daher ist eine genaue Untersuchung dieser Reaktion und der Kreuzreaktionen unter Einsatz von Propylradikalen nötig, um mit Datensätzen (wie von [Bau05]) Verbrennungsprozesse adäquat modellieren und Voraussagen über das Verhältnis von Terminierung durch Kombination und Disproportionierung bei der radikalischen Polymerisation machen zu können.

Für die Untersuchung der Reaktion Propyl + Propyl und den in Abschnitt 4.5 folgenden Kreuzreaktionen mit Propylradikalen wurden als Propylradikalvorläufer Dipropylketon und 1-Propyliodid verwendet.

Ziel dieser Untersuchung ist nun, Photolyseprozesse der Vorläufer zu analysieren, um direkte Photolysereaktionen wie HI-Elimination und Norrish Typ III Spaltungen, die zur Bildung stabiler Kohlenwasserstoffe ohne Erzeugung von freien Radikalen führen, von den Radikalreaktionen unterscheiden zu können. Dabei sollen die unterschiedlichen Photolysekanäle quantifiziert werden, damit bei den Untersuchungen der Kreuzreaktionen die Radikalanfangskonzentrationen und die Nebenreaktionen zu bestimmen sind.

Für die Photolyse von 1-Propyliodid wird von folgenden Photolyseprozessen ausgegangen:

$$
\begin{array}{rlr}
\mathrm{CH}_{3} \mathrm{CH}_{2} \mathrm{CH}_{2} \mathrm{I} & \stackrel{h \nu}{\longrightarrow} \mathrm{CH}_{3} \mathrm{CH}_{2} \mathrm{CH}_{2}+\mathrm{I} & \mathrm{C}-\mathrm{I}-\text { Bruch } \\
& \stackrel{h \nu}{\longrightarrow} \mathrm{CH}_{3} \mathrm{CHCH}_{2}+\mathrm{HI} & \mathrm{H}-\mathrm{I}-\text { Elimin. } \\
& \stackrel{h \nu}{\longrightarrow} \mathrm{CH}_{3}+\mathrm{C}_{2} \mathrm{H}_{4} \mathrm{I} & \mathrm{C}-\mathrm{C}-\text { Bruch }
\end{array}
$$

Bei der Photolyse von Diropylketon tritt neben der erwünschten Bildung von 2 Propylradikalen der Zerfall zu Ethen und Methylpropylketon über einen „radikalfreien“ Reaktionsweg, wie er von MAsson beschrieben ist [Mas52], auf: 


$$
\begin{aligned}
& \mathrm{C}_{3} \mathrm{H}_{7} \mathrm{COC}_{3} \mathrm{H}_{7} \stackrel{h \nu}{\longrightarrow} 2 \mathrm{C}_{3} \mathrm{H}_{7}+\mathrm{CO} \quad \text { Norrish I } \\
& \stackrel{h \nu}{\longrightarrow} \mathrm{CH}_{3} \mathrm{COC}_{3} \mathrm{H}_{7}+\mathrm{C}_{2} \mathrm{H}_{4} \quad \text { Norrish III }
\end{aligned}
$$

Bei der eigentlichen Reaktion der 1-Propylradikale ist wiederum die Kombination unter Bildung von Hexan und die Disproportionierung zu Propan und Propen zu erwarten:

$$
\begin{aligned}
1-\mathrm{C}_{3} \mathrm{H}_{7}+1-\mathrm{C}_{3} \mathrm{H}_{7} & \longrightarrow \mathrm{C}_{6} \mathrm{H}_{14} \\
& \longrightarrow \mathrm{C}_{3} \mathrm{H}_{8}+\mathrm{C}_{3} \mathrm{H}_{6}
\end{aligned}
$$

Untersuchungen in der Literatur in dem selben Druckbereich und $300 \mathrm{~K}$ favorisieren stark die Kombinationsreaktion $k(d) / k(c)=0,15$ [Tsa88].

\subsubsection{Qualitative Analyse}

Im Folgenden werden die Photolysenspektren der Vorläufer und vergrößerte Ausschnitte der Photolysenspektren im Vergleich mit Reinspektren der identifizierten Substanzen abgebildet. Abbildung 4.49 zeigt ein Photolysespektrum von Propyliodid nach 400 Photolysen bei einem Partialdruck von $\mathrm{p}\left(\mathrm{C}_{3} \mathrm{H}_{7} \mathrm{I}\right)=0,3$ mbar, einem Gesamtdruck von 4 mbar und einer Temperatur von $298 \mathrm{~K}$. 


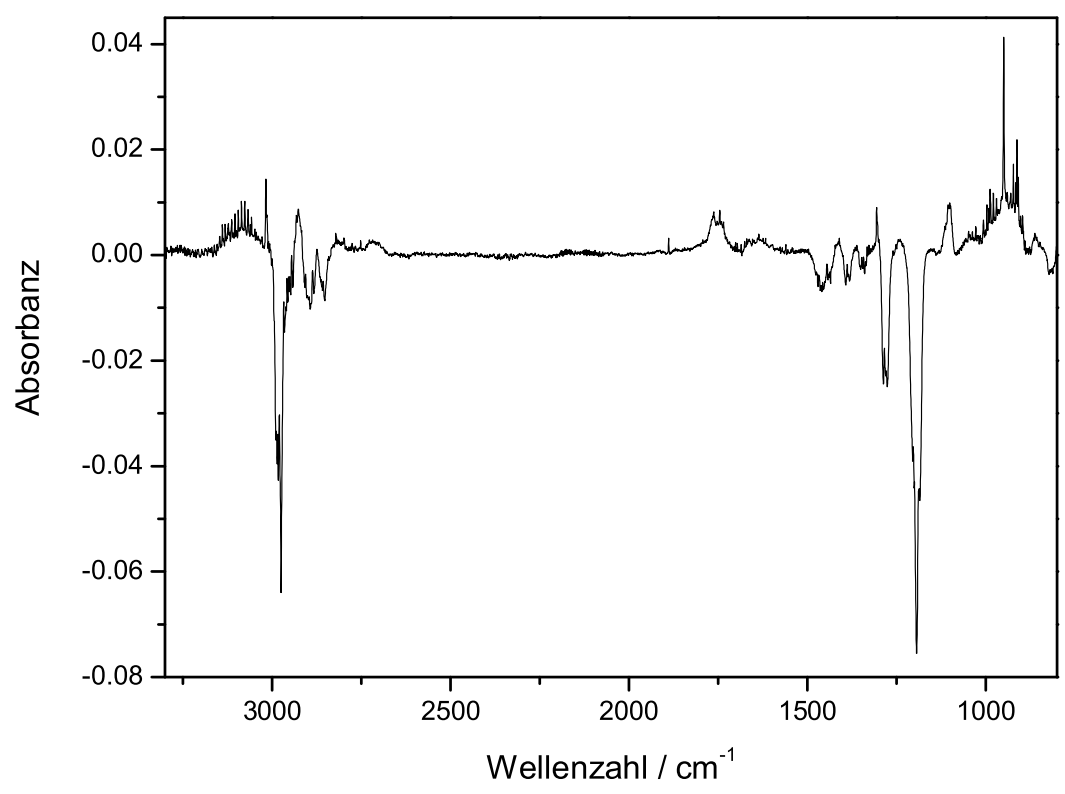

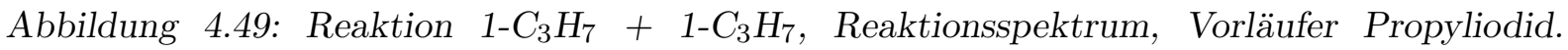
$p\left(C_{3} H_{7} I\right)=0,3$ mbar; $p_{g e s}=4$ mbar; $T=298 \mathrm{~K} ; 400$ Photolysen.

Der in Abbildung 4.50 dargestellte Vergleich des Reaktionsspektrums mit den Spektren der Reinsubstanzen zeigt deutlich die Bildung von Hexan, Propan, Propen, Ethen, Methan, Ethin, Propanal und Formaldehyd. Die sauerstoffhaltigen Substanzen resultieren dabei aus einer Reaktion mit in der Zelle befindlichen Spuren von molekularem Sauerstoff. Dabei kann eine direkte Reaktion mit diesem ablaufen oder eine Reaktion mit O-Atomen, die durch Laserphotolyse des molekularen Sauerstoffs gebildet werden. Der Absorptionsquerschnitt von molekularem Sauerstoff bei $193 \mathrm{~nm}$ ist allerdings sehr gering. Die Bildung von Methan und Ethin kann durch einen direkten Bruch von Propyliodid erklärt werden (Photolyseweg (C-C-Bruch)). Die Bildung von Ethen wird ebenfalls aus diesem Photolyseprozess stammen. 

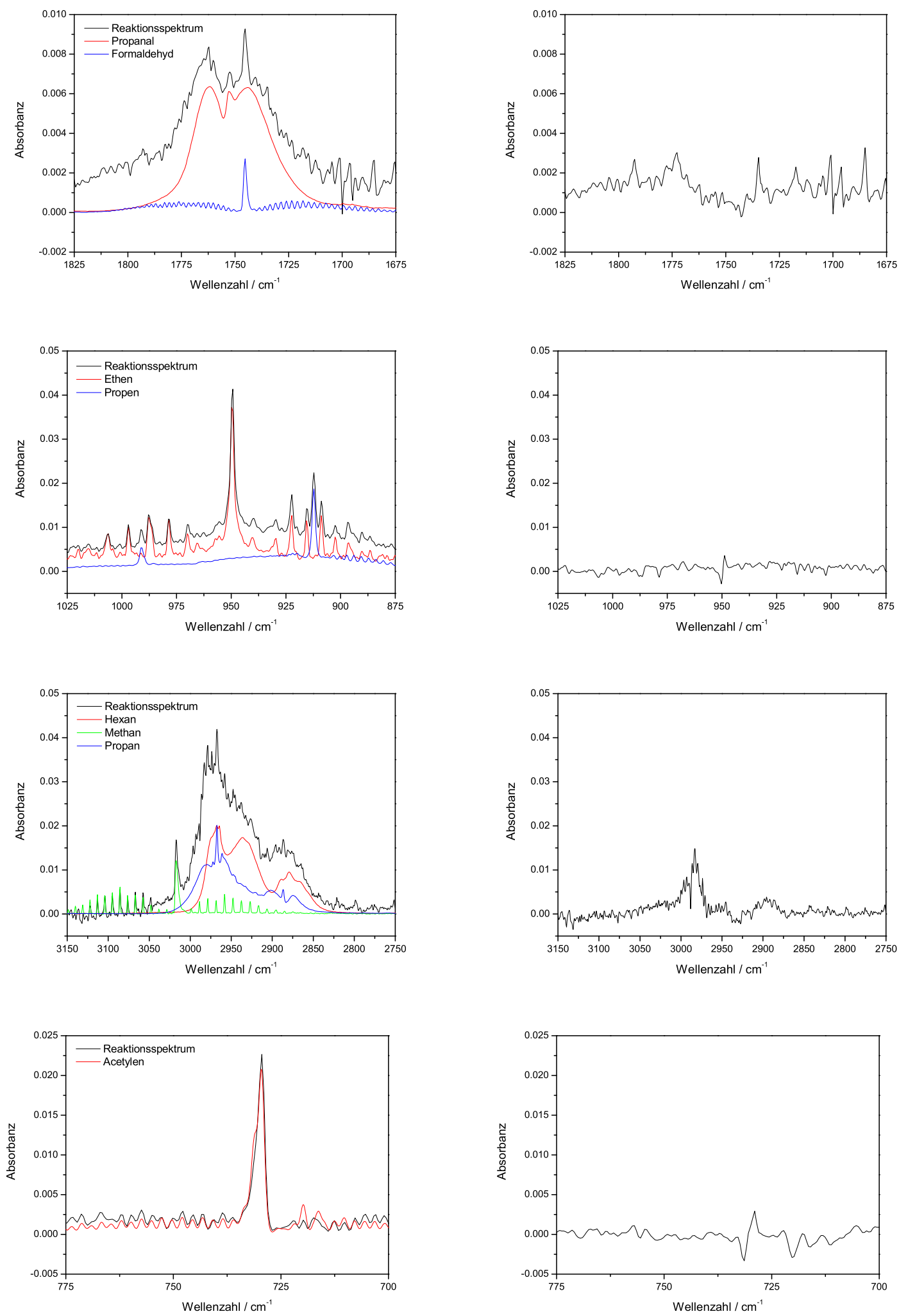

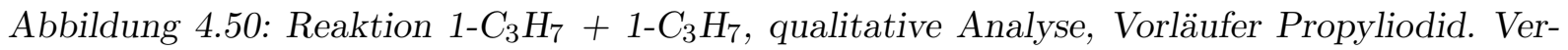
gleich des Reaktionsspektrums mit Spektren der Reinsubstanzen in vergrößerten Ausschnitten; in der linken Spalte ist jeweils das Reaktionsspektrum (schwarz) mit den Reinspektren (farbig) im Vergleich dargestellt, in der rechten Spalte sind die entsprechenden Residuen in schwarz dargestellt. 
Das Photolysespektrum des Vorläufers Dipropylketon nach 400 Photolysen bei einem Anfangsdruck von 0,2 mbar und einem Gesamtdruck von 4 mbar und $\mathrm{T}=298 \mathrm{~K}$ ist in Abbildung 4.51 dargestellt.

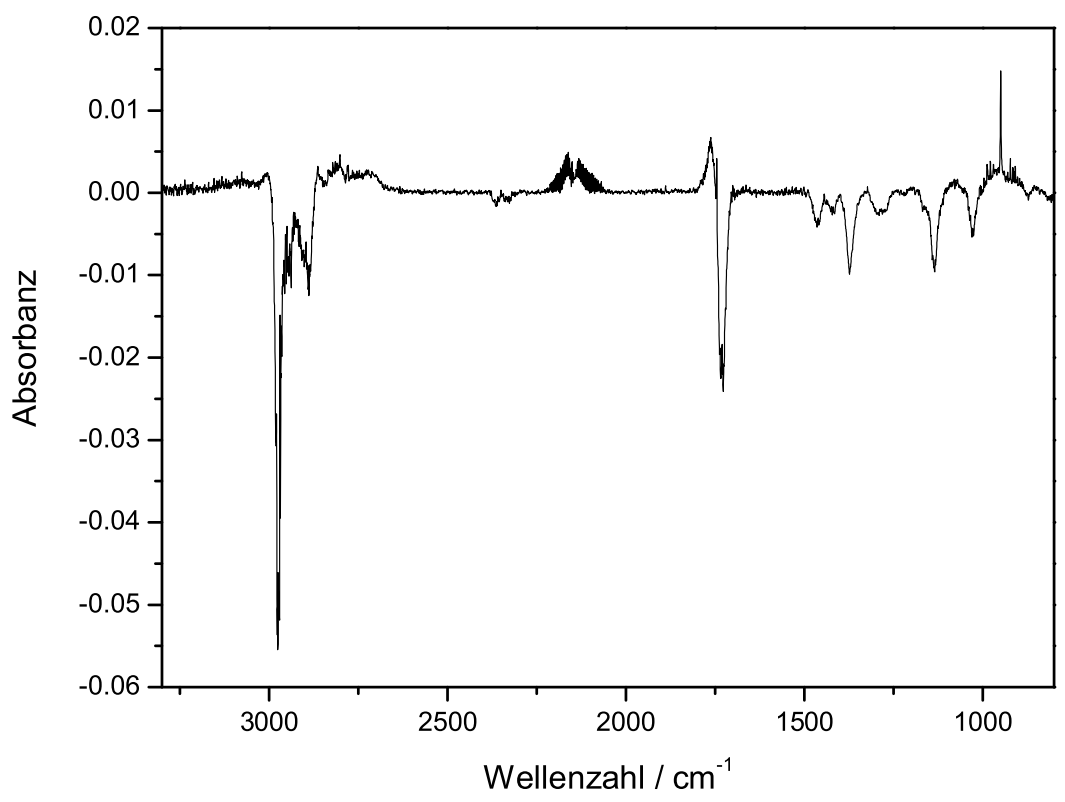

Abbildung 4.51: Reaktion 1- $\mathrm{C}_{3} \mathrm{H}_{7}+1-\mathrm{C}_{3} \mathrm{H}_{7}$, Reaktionsspektrum, Vorläufer Dipropylketon. $p\left(\left(\mathrm{C}_{3} \mathrm{H}_{7}\right)_{2} \mathrm{CO}\right)=0,2 \mathrm{mbar} ; p_{\text {ges }}=4 \mathrm{mbar}, \mathrm{T}=298 \mathrm{~K} ; 400$ Photolysen.

Für die Identifikation sind Bereiche des Reaktionsspektrums zusammen mit Spektren der Reinsubstanzen in Abbildung 4.52 vergrößert dargestellt.

Dabei können folgende Substanzen eindeutig identifiziert werden: Hexan, Propen, Ethen, Methylpropylketon, Propanal und Formaldehyd. Die Bildung von Propan, welches das Gegenstück von Propen aus dem Disproportionierungskanal darstellt, konnte auf Grund von spektraler Interferenz im Bereich der C-H-Schwingung nicht eindeutig identifiziert werden. Die Bildung von Ethen und Methylpropylketon kann durch den Photolyseweg Norrish III erklärt werden. Dass Propanal und Formaldehyd gefunden werden konnten, ist wieder durch eine Reaktion der Propylradikale mit molekularem Sauerstoff, bzw. durch eine Reaktion mit durch Laserphotolyse aus $\mathrm{O}_{2}$ gebildeten O-Atomen zu erklären. 

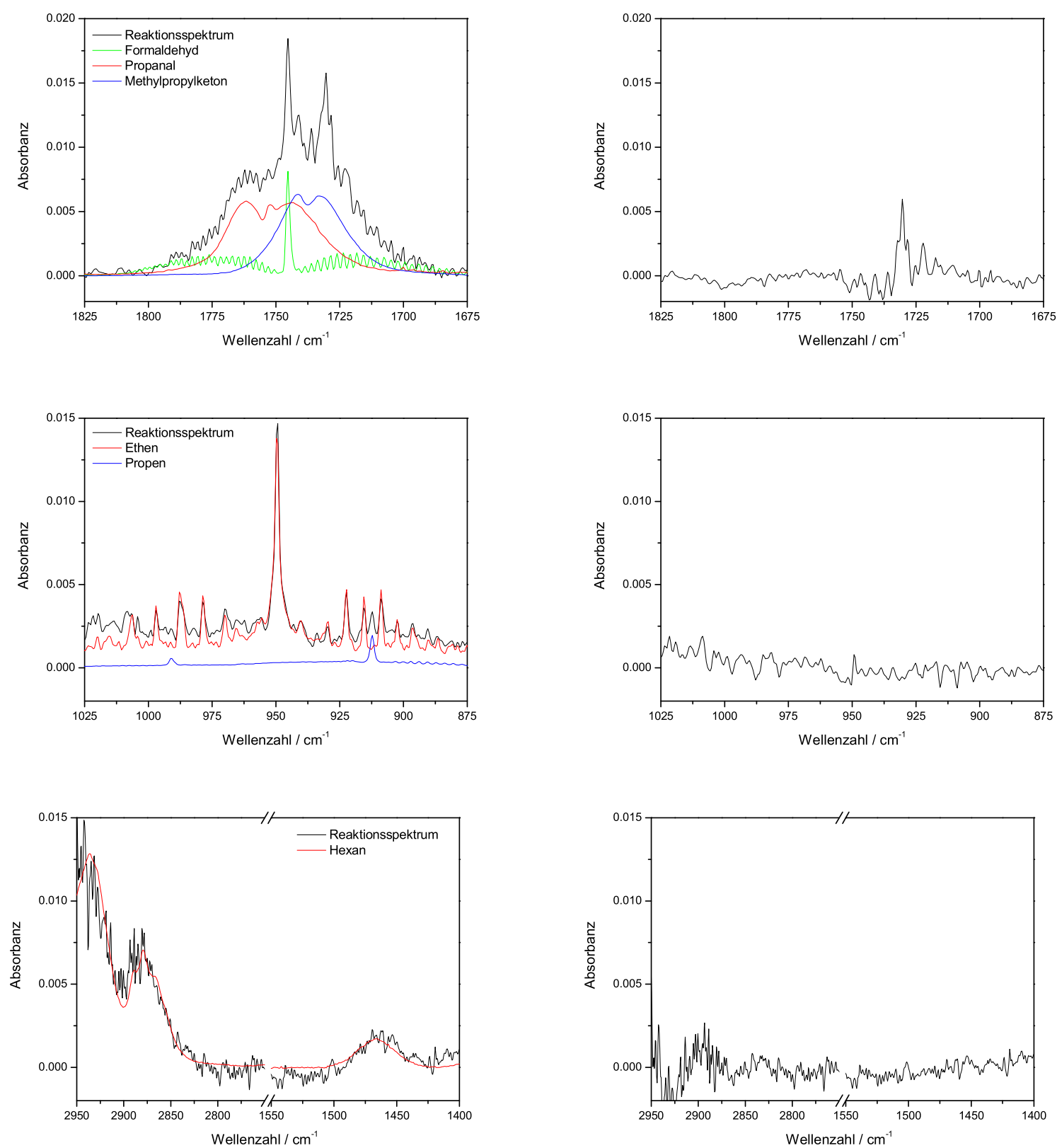

Abbildung 4.52: Reaktion 1- $\mathrm{C}_{3} \mathrm{H}_{7}+1-\mathrm{C}_{3} \mathrm{H}_{7}$, qualitative Analyse, Vorläufer Dipropylketon. Vergleich des Reaktionsspektrums mit Spektren der Reinsubstanzen in vergrößerten Ausschnitten; in der linken Spalte ist jeweils das Reaktionsspektrum (schwarz) mit den Reinspektren (farbig) im Vergleich dargestellt, in der rechten Spalte sind die entsprechenden Residuen in schwarz dargestellt.

\subsubsection{Quantitative Analyse}

Für die quantitative Analyse wurden die mittels der Eichkoeffizienten berechneten Partialdrücke der Produkte gegen den Verbrauch an Vorläufer aufgetragen. Wie auch bei 
der vorhergehenden Analyse von Ethyliodid wurden dabei ablaufende Oxidationsreaktionen berücksichtigt. Dies wurde durch Subtraktion des Propanalpartialdrucks vom Propyliodidpartialdruck erreicht. Damit handelt es sich bei der in Abbildung 4.53 mit Verbrauch $\mathrm{C}_{3} \mathrm{H}_{7} I$ bezeichneten Achse um den mit Propanal korrigierten Wert. Aus der Abbildung wird ersichtlich, dass der Propen- und Propananteil nicht übereinstimmen. Der um den Faktor 1,6 höhere Wert für Propen resultiert aus der direkten HI-Elimination. Für den Disproportionierungskanal wird also der Anteil an Propan zu Grunde gelegt. Mit den Resultaten der quantitativen Analyse ergibt sich für die Kanalverzeigung $k(d) / k(c)=0,42$. Die Messwerte zu Abbildung 4.53 sind in Tabelle 4.29 angegeben.

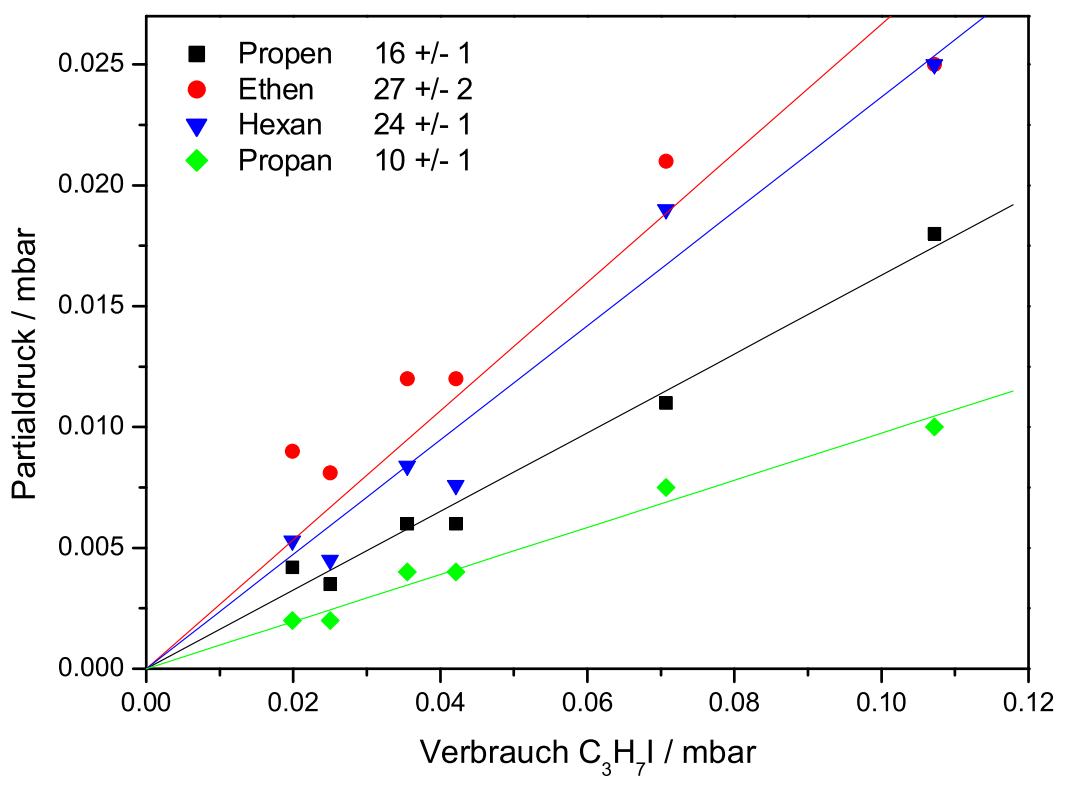

Abbildung 4.53: Reaktion 1- $\mathrm{C}_{3} \mathrm{H}_{7}+1-\mathrm{C}_{3} \mathrm{H}_{7}$, quantitative Analyse, Vorläufer Propyliodid. Die Abbildung zeigt die Partialdrücke der Produkte vs. Verbrauch Propyliodid. Dabei ist der Verbrauch an Propyliodid um die Werte des Oxidationsprodukts Propanal korrigiert; $p_{\text {ges }}=4 \mathrm{mbar}$, $T=298 \mathrm{~K}$. 


\begin{tabular}{|c|c|c|c|c|c|c|}
\hline $\begin{array}{c}\text { Verbrauch } \mathrm{C}_{3} \mathrm{H}_{7} \mathrm{I} \\
{[\mathrm{mbar}]}\end{array}$ & $\begin{array}{c}\mathrm{p}\left(\mathrm{C}_{3} \mathrm{H}_{6}\right) \\
{[\mathrm{mbar}]}\end{array}$ & $\begin{array}{c}\mathrm{p}\left(\mathrm{C}_{2} \mathrm{H}_{4}\right) \\
{[\mathrm{mbar}]}\end{array}$ & $\begin{array}{c}\mathrm{p}\left(\mathrm{C}_{4} \mathrm{H}_{10}\right) \\
{[\mathrm{mbar}]}\end{array}$ & $\begin{array}{c}\mathrm{p}\left(\mathrm{C}_{3} \mathrm{H}_{8}\right) \\
{[\mathrm{mbar}]}\end{array}$ & $\begin{array}{c}\mathrm{p}\left(\mathrm{C}_{3} \mathrm{H}_{6} \mathrm{O}\right) \\
{[\mathrm{mbar}]}\end{array}$ & $\begin{array}{c}\mathrm{p}\left(\mathrm{C}_{3} \mathrm{H}_{7} \mathrm{I}\right)_{\text {korr. }} \\
{[\mathrm{mbar}]}\end{array}$ \\
\hline \hline 0,021 & 0,0042 & 0,009 & 0,0053 & 0,002 & 0,0011 & 0,02 \\
\hline 0,026 & 0,0035 & 0,0081 & 0,0045 & 0,002 & 0,001 & 0,025 \\
\hline 0,037 & 0,006 & 0,012 & 0,0084 & 0,004 & 0,0015 & 0,0355 \\
\hline 0,044 & 0,006 & 0,012 & 0,0076 & 0,004 & 0,0019 & 0,0421 \\
\hline 0,073 & 0,011 & 0,021 & 0,19 & 0,0075 & 0,0023 & 0,071 \\
\hline 0,11 & 0,018 & 0,0025 & 0,025 & 0,01 & 0,0025 & 0,107 \\
\hline
\end{tabular}

Tabelle 4.29: Reaktion 1- $\mathrm{C}_{3} \mathrm{H}_{7}+1-\mathrm{C}_{3} \mathrm{H}_{7}$, Vorläufer Propyliodid, Messwerte. $p\left(\mathrm{C}_{3} \mathrm{H}_{7} \mathrm{I}\right)=0,3$ mbar, $p_{\text {ges }}=4$ mbar, $\mathrm{T}=298 \mathrm{~K}$, Badgas Argon.

Für den Vorläufer Propyliodid wird damit die folgende Verzweigung für die Photolyse vorgeschlagen. Der Anteil des Kanals zu Methylradikalen und Ethyliodid wurde dabei auf Grundlage einer Kohlenstoffmassenbilanz bestimmt:

$$
\begin{array}{rlr}
\mathrm{CH}_{3} \mathrm{CH}_{2} \mathrm{CH}_{2} \mathrm{I} & \stackrel{h \nu}{\longrightarrow} \mathrm{CH}_{3} \mathrm{CH}_{2} \mathrm{CH}_{2}+\mathrm{I} & 67 \% \\
\stackrel{h \nu}{\longrightarrow} \mathrm{CH}_{3} \mathrm{CHCH}_{2}+\mathrm{HI} & 6 \% \\
\stackrel{h \nu}{\longrightarrow} \mathrm{CH}_{3}+\mathrm{C}_{2} \mathrm{H}_{5} \mathrm{I} & 27 \%
\end{array}
$$

Bezogen auf die durch den obigen Photolysemechanismus bestimmte Radikalkonzentration ergibt sich für die Kanalverzweigung der Reaktion $\mathrm{C}_{3} \mathrm{H}_{7}+\mathrm{C}_{3} \mathrm{H}_{7}$ :

$$
\begin{array}{rlr}
1-\mathrm{C}_{3} \mathrm{H}_{7}+1-\mathrm{C}_{3} \mathrm{H}_{7} & \longrightarrow \mathrm{C}_{6} \mathrm{H}_{14} & 71 \% \\
& \longrightarrow \mathrm{C}_{3} \mathrm{H}_{8}+\mathrm{C}_{3} \mathrm{H}_{6} & 29 \%
\end{array}
$$

Die Abbildung für die quantitative Analyse des Vorläufers Dipropylketon ist in Abbildung 4.54 gegeben. Die zugehörigen Messwerte sind in Tabelle 4.30 dargestellt. Wie bei der vorhergehenden Analyse wurde dabei die Partialdruckabnahme des Vorläufers durch Abzug des Partialdrucks des oxidierten Produkts Propanal korrigiert. Die Tabelle zeigt außerdem die ursprüngliche Dipropylketonabnahme. 


\begin{tabular}{|c|c|c|c|c|c|c|}
\hline $\begin{array}{c}\text { Verbrauch DIPROP } \\
{[\mathrm{mbar}]}\end{array}$ & $\begin{array}{c}\mathrm{p}\left(\mathrm{C}_{6} \mathrm{H}_{14}\right) \\
{[\mathrm{mbar}]}\end{array}$ & $\begin{array}{c}\mathrm{p}\left(\mathrm{C}_{2} \mathrm{H}_{4}\right) \\
{[\mathrm{mbar}]}\end{array}$ & $\begin{array}{c}\mathrm{p}(\mathrm{MPK}) \\
{[\mathrm{mbar}]}\end{array}$ & $\begin{array}{c}\mathrm{p}\left(\mathrm{C}_{3} \mathrm{H}_{6}\right) \\
{[\mathrm{mbar}]}\end{array}$ & $\begin{array}{c}\mathrm{p}\left(\mathrm{C}_{3} \mathrm{H}_{6} \mathrm{O}\right) \\
{[\mathrm{mbar}]}\end{array}$ & $\begin{array}{c}\mathrm{p}\left(\left(\mathrm{C}_{3} \mathrm{H}_{7}\right)_{2} \mathrm{CO}\right)_{\text {korr. }} \\
{[\mathrm{mbar}]}\end{array}$ \\
\hline \hline 0,003 & 0,0011 & 0,0016 & 0,0015 & 0,0004 & 0,0009 & 0,0021 \\
\hline 0,01 & 0,0026 & 0,0029 & 0,0025 & 0,0005 & 0,0028 & 0,0072 \\
\hline 0,0154 & 0,0042 & 0,041 & 0,004 & 0,00063 & 0,0041 & 0,0113 \\
\hline 0,0221 & 0,0074 & 0,0051 & 0,0053 & 0,00092 & 0,0056 & 0,0165 \\
\hline
\end{tabular}

Tabelle 4.30: Reaktion 1- $\mathrm{C}_{3} \mathrm{H}_{7}+1-\mathrm{C}_{3} \mathrm{H}_{7}$, Vorläufer Dipropylketon, Messwerte. $p\left(\left(\mathrm{C}_{3} \mathrm{H}_{7}\right)_{2} \mathrm{CO}\right)$ $=0,3 \mathrm{mbar}, p_{\text {ges }}=4 \mathrm{mbar}, \mathrm{T}=298 \mathrm{~K}$, Badgas Argon (DIPROP $:=$ Dipropylketon, MPK $:=$ Methylpropylketon).

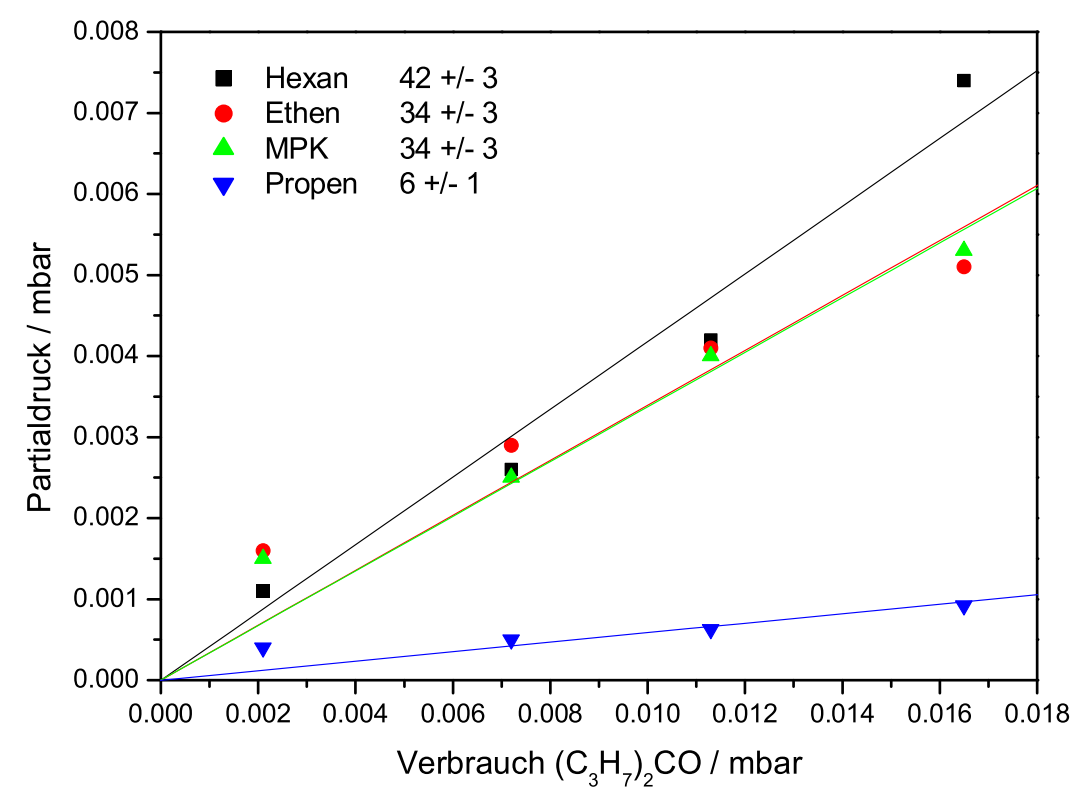

Abbildung 4.54: Reaktion 1- $\mathrm{C}_{3} \mathrm{H}_{7}+1-\mathrm{C}_{3} \mathrm{H}_{7}$, quantitative Analyse, Vorläufer Dipropylketon. Die Abbildung zeigt die Partialdrücke der Produkte vs. Verbrauch Dipropylketon (MPK := Methylpropylketon). Dabei ist der Verbrauch an Dipropylketon um die Werte des Oxidationsprodukts Propanal korrigiert; $\mathrm{p}\left(\left(\mathrm{C}_{3} \mathrm{H}_{7}\right)_{2} \mathrm{CO}\right)=0,6 \mathrm{mbar}, \mathrm{p}_{\text {ges }}=4 \mathrm{mbar}, \mathrm{T}=298 \mathrm{~K}$.

Die Abbildung 4.54 zeigt eine annähernd lineare Zunahme aller Substanzen mit steigender Abnahme an Vorläufer. Sekundärreaktionen und Photolyse primärer Produkte können damit als unbedeutend angesehen werden. Die in der Abbildung angegebenen Produkte erklären anhand einer Massenbilanz 80\% der Vorläuferabnahme. Die Abbildung zeigt weiterhin, dass die Gegenstücke Methylpropylketon und Ethen aus der photolytischen Spaltung, wie zu erwarten war, im Verhältnis 1:1 gebildet werden. Damit kann für die Photolyse von Dipropylketon von der folgenden Verzweigung ausgegangen werden: 


$$
\begin{array}{rlr}
\mathrm{C}_{3} \mathrm{H}_{7} \mathrm{COC}_{3} \mathrm{H}_{7} \stackrel{h \nu}{\longrightarrow} 2 \mathrm{C}_{3} \mathrm{H}_{7}+\mathrm{CO} & 59 \% \\
& \stackrel{h \nu}{\longrightarrow} \mathrm{CH}_{3} \mathrm{COC}_{3} \mathrm{H}_{7}+\mathrm{C}_{2} \mathrm{H}_{4} & 41 \%
\end{array}
$$

Im Gegensatz zur Verwendung des Vorläufers Propyliodid wird hier allerdings ein niedrigerer Anteil für die Disproportionierung festgestellt. Da in diesem Fall die quantitative Analyse von Propan auf Grund der geringen Konzentration an Propan und spektraler Interferenz im Bereich der C-H-Valenzschwingung wieder nicht möglich war, kann für die Bestimmung der Kanalverzweigung der Reaktion $\mathrm{C}_{3} \mathrm{H}_{7}+\mathrm{C}_{3} \mathrm{H}_{7}$ nur der Anteil an Propen herangezogen werden. Mit diesem ergibt sich folgendes Verzweigungsverhältnis:

$$
\begin{array}{rlr}
\mathrm{C}_{3} \mathrm{H}_{7}+\mathrm{C}_{3} \mathrm{H}_{7} & \longrightarrow \mathrm{C}_{6} \mathrm{H}_{14} & 87,5 \% \\
& \longrightarrow \mathrm{C}_{3} \mathrm{H}_{8}+\mathrm{C}_{3} \mathrm{H}_{6} & 12,5 \%
\end{array}
$$

\subsubsection{Diskussion}

Die Verwendung unterschiedlicher Vorläufer zeigt eine große Diskrepanz bezüglich der Kanalverzweigung für die Reaktion $\mathrm{C}_{3} \mathrm{H}_{7}+\mathrm{C}_{3} \mathrm{H}_{7}$. Die quantitative Analyse ergibt zwar bei beiden Vorläufern die Kombinationsreaktion gegenüber der Disproportionierung als favorisiert (Propyliodid: $k(d) / k(c)=0,41$; Dipropylketon: $k(d) / k(c)=0,14$ ), doch ist der Disproportionierungskanal im Fall von Propyliodid im Vergleich mit der Literatur sehr groß [Mas52, Ker61, Tsa88]. Wahrscheinlich wird der Disproportionierungskanal auf Grund der spektralen Interferenz im Bereich der C-H-Streckschwingung für den Vorläufer Propyliodid überschätzt. Neben den Produkten Propan und Hexan resultieren in diesem Bereich auch Banden durch den Radikalvorläufer Propyliodid selbst. Die Verwendung des Kanalverzweigungsverhältnisses, welches durch Einsatz des Vorläufers Dipropylketon erhalten wurde, stimmt mit den in der Literatur angegebenen Werten sehr gut überein. Da außerdem die photolytische Spaltung des Vorläufers durch exakte Übereinstimmung der Gegenstücke Ethen und Methylpropylketon gut beschrieben werden kann, wird dieser Vorläufer als verlässlicher angesehen. Daher wird für die Reaktion $\mathrm{C}_{3} \mathrm{H}_{7}+\mathrm{C}_{3} \mathrm{H}_{7}$ folgende Kanalverzweigung postuliert: 


$$
\begin{array}{rlr}
\mathrm{C}_{3} \mathrm{H}_{7}+\mathrm{C}_{3} \mathrm{H}_{7} \longrightarrow \mathrm{C}_{6} \mathrm{H}_{14} & 87,5 \% \\
& \longrightarrow \mathrm{C}_{3} \mathrm{H}_{8}+\mathrm{C}_{3} \mathrm{H}_{6} & 12,5 \%
\end{array}
$$

Mit der quantitativen Bestimmung der photolytischen Spaltungsreaktionen ist es nun möglich, die Radikalkonzentrationen anzugeben und Kreuzreaktionen zu untersuchen.

Im Folgenden werden die Ergebnisse mit der Literatur verglichen.

MAsson untersuchte die Photolyse von Dipropylketon bei $\lambda=313 \mathrm{~nm}$ und Temperaturen zwischen $55^{\circ} \mathrm{C}$ und $357^{\circ} \mathrm{C}$ [Mas52]. Dabei wurde bis zu einer Temperatur von $161^{\circ} \mathrm{C}$ weder eine Druck- noch eine Temperaturabhängigkeit für die Photolyse gefunden. Das Verhältnis von Disproportionierung zu Kombination wird zu $k(d) / k(c)=0,15$ angegeben.

KerR und Calvert nutzten als Vorläufer für Propylradikale Azo-n-Propan. Dabei wurden die Radikale durch Photolyse bei $\lambda=366$ nm erzeugt und Produkte mittels Gaschromatographie nachgewiesen. Bei Zimmertemperatur $\left(25^{\circ} \mathrm{C}\right)$ wurde das Kanalverhältnis zu $k(d) / k(c)=0,16$ bestimmt [Ker61].

\subsubsection{Die Reaktion 2-Propyl + 2-Propyl}

\subsubsection{Einleitung}

Die Reaktion 2- $\mathrm{C}_{3} \mathrm{H}_{7}+2-\mathrm{C}_{3} \mathrm{H}_{7}$ wurde ebenfalls durch Einsatz eines iodierten Vorläufers (Isopropyliodid) und eines Ketons (Diisopropylketon) untersucht. Für die Reaktion zweier Isopropylradikale liegen 3 Literaturangaben vor [Ana87, Par76, Arr78], aus denen BAulch et al. [Bau05] ihre Empfehlung für die Kanalverzweigung abgeleitet haben. Mit einem Geschwindigkeitskoeffizienten für die Gesamtreaktion von $\mathrm{k}\left(\mathrm{C}_{3} \mathrm{H}_{7}+\mathrm{C}_{3} \mathrm{H}_{7} \rightarrow\right.$ Produkte $)=$ $6,62 \times 10^{12} \mathrm{~cm}^{3} /(\mathrm{mol} \cdot \mathrm{s})$ und einer Kanalverzweigung von $k(d) / k(c)=0,62$ wurden die Geschwindigkeiten der Kombination und Disproportionierung bestimmt. Die Empfehlung von BAulch et al. zeigt, dass im Gegensatz zu den vorherigen Reaktionen die Kombination nicht stark favorisiert ist. Dies ist auf Grund sterischer Effekte auch zu erwarten.

Im Folgenden sind kurz die zu erwartenden Photolyseprozesse dargestellt. Für die Photolyse von Isopropyliodid wird von folgenden Photolyseprozessen ausgegangen: 


$$
\begin{array}{rlr}
\mathrm{CH}_{3} \mathrm{CHICH}_{3} & \stackrel{h \nu}{\longrightarrow} \mathrm{CH}_{3} \mathrm{CHCH}_{3}+\mathrm{I} & \mathrm{C}-\mathrm{I}-\text { Bruch } \\
& \stackrel{h \nu}{\longrightarrow} \mathrm{CH}_{3} \mathrm{CHCH}_{2}+\mathrm{HI} & \mathrm{H}-\mathrm{I}-\text { Elimin. }
\end{array}
$$

Im Gegensatz zu 1-Propyliodid als Vorläufer, ist für die photolytische Spaltung von Isopropyliodid ein C-C-Bruch nicht zu erwarten.

Bei der Photolyse von Diisopropylketon wurde von ZEUCH die Bildung von 2 Isopropylradikalen und CO und der „radikalfreie“ Photolyseweg zu Isopropylvinylketon und Methan gefunden [Zeu03]:

$$
\begin{aligned}
& \left(\mathrm{CH}_{3}\right)_{2} \mathrm{CHCOCH}\left(\mathrm{CH}_{3}\right)_{2} \stackrel{h \nu}{\longrightarrow} 2 \quad 2-\mathrm{C}_{3} \mathrm{H}_{7}+\mathrm{CO} \quad \text { Norrish I } \\
& \stackrel{h \nu}{\longrightarrow} \mathrm{CH}_{2} \mathrm{CHCOCH}\left(\mathrm{CH}_{3}\right)_{2}+\mathrm{CH}_{4} \text { Norrish III }
\end{aligned}
$$

Für die Reaktion von zwei Isopropylradikalen wird von folgendem Reaktionsmechanismus ausgegangen:

$$
\begin{aligned}
2-\mathrm{C}_{3} \mathrm{H}_{7}+2-\mathrm{C}_{3} \mathrm{H}_{7} & \longrightarrow\left(\mathrm{CH}_{3}\right)_{2} \mathrm{CHCH}\left(\mathrm{CH}_{3}\right)_{2} \\
& \longrightarrow \mathrm{C}_{3} \mathrm{H}_{8}+\mathrm{C}_{3} \mathrm{H}_{6}
\end{aligned}
$$

\subsubsection{Qualitative Analyse}

Zunächst erfolgt die qualitative Analyse für den Vorläufer Isopropyliodid. Das in Abbildung 4.55 gezeigte Reaktionsspektrum wurde nach 200 Photolysen von $\mathrm{p}\left(\mathrm{CH}_{3} \mathrm{CHICH}_{3}\right)$ = 0,6 mbar bei einem Gesamtdruck von 4 mbar und $\mathrm{T}=298 \mathrm{~K}$ aufgenommen. 


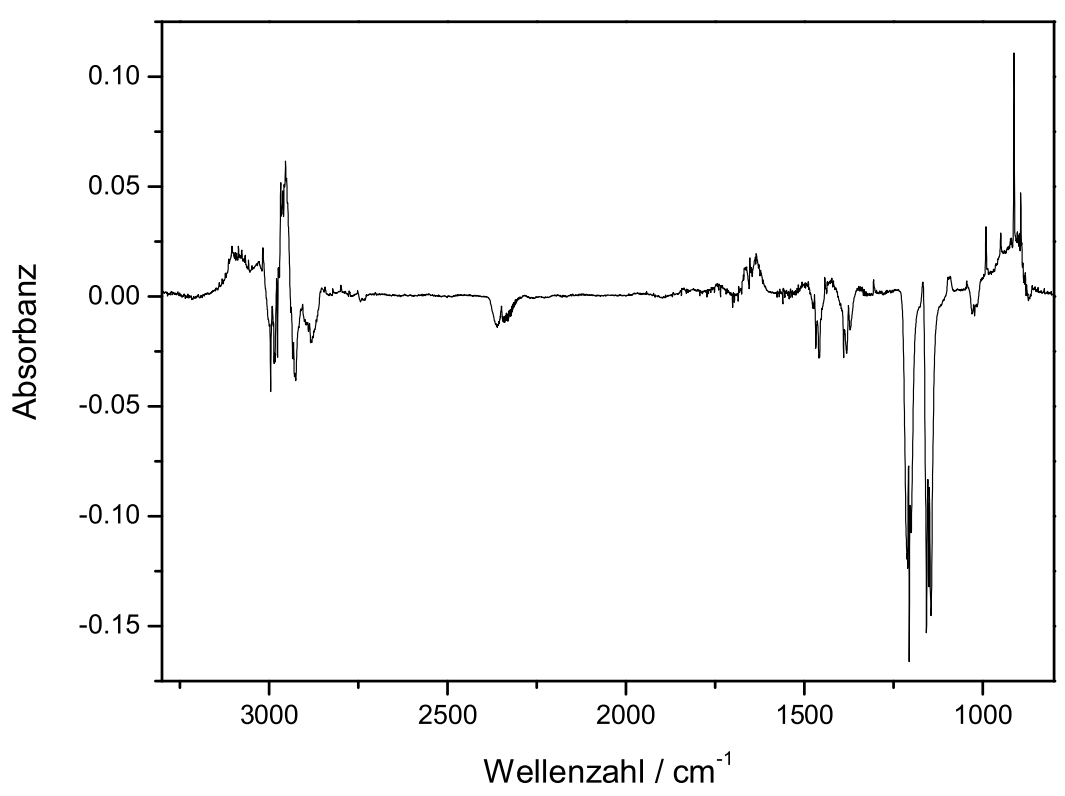

Abbildung 4.55: Reaktion 2- $\mathrm{C}_{3} \mathrm{H}_{7}+2-\mathrm{C}_{3} \mathrm{H}_{7}$, Reaktionsspektrum, Vorläufer Isopropyliodid. $p\left(\mathrm{CH}_{3} \mathrm{CHICH}_{3}\right)=0,6$ mbar; $p_{\text {ges }}=4$ mbar, $\mathrm{T}=298 \mathrm{~K}$; 200 Photolysen.

Für die qualitative Analyse sind Bereiche des Reaktionsspektrums im Vergleich mit Spektren der Reinsubstanzen in Abbildung 4.56 dargestellt. In der Abbildung wird deutlich, dass die Bildung des Kombinationsproduktes Diisopropyl und der Disproportionierungsprodukte Propan und Propen stattgefunden hat. Des Weiteren konnte in geringen Mengen die Bildung von Ethen und Methan beobachtet werden. 

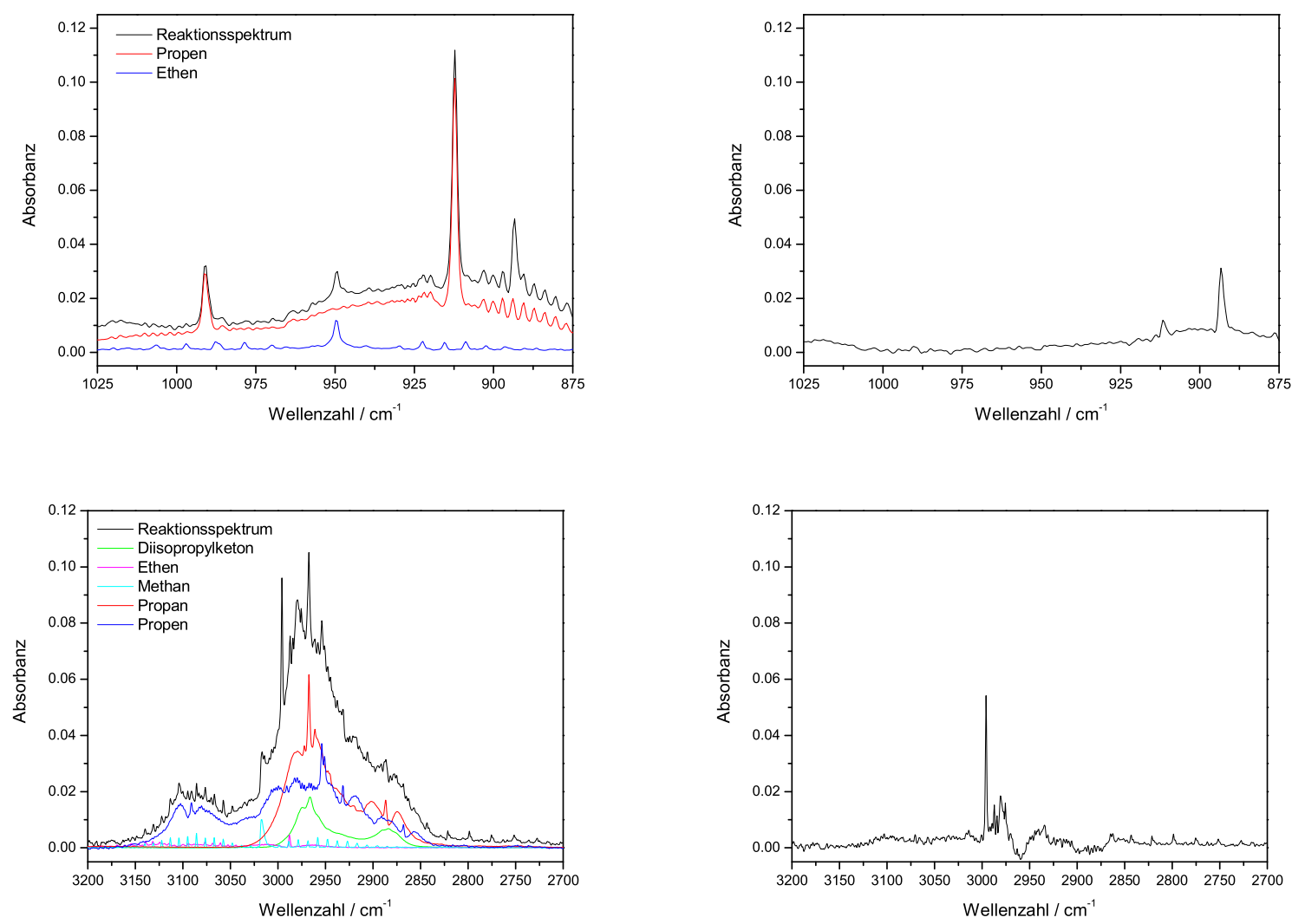

Abbildung 4.56: Reaktion 2- $\mathrm{C}_{3} \mathrm{H}_{7}+2-\mathrm{C}_{3} \mathrm{H}_{7}$, qualitative Analyse, Vorläufer Isopropyliodid. Vergleich des Reaktionsspektrums mit Spektren der Reinsubstanzen in vergrößerten Ausschnitten; in der linken Spalte ist jeweils das Reaktionsspektrum (schwarz) mit den Reinspektren (farbig) im Vergleich dargestellt, in der rechten Spalte sind die entsprechenden Residuen in schwarz dargestellt.

Im Bereich der C-H-Valenzschwingung verbleibt nach Abzug aller identifizierten Substanzen ein scharfer Peak bei $\tilde{\nu}=3000 \mathrm{~cm}^{-1}$. Dieser Peak ist bei dem Vorläufer Isopropyliodid $\mathrm{zu}$ finden und ist somit nicht auf ein unidentifiziertes Produkt zurückzuführen. Solche „Sättigungspeaks“ finden sich bei einigen Substanzen, welche wahrscheinlich auf Grund ausgeprägter Absorbanz bei einer bestimmten Wellenzahl kein Lambert-Beer-Verhalten mehr zeigen, d.h. die Absorbanz nimmt in diesem Bereich nicht linear mit der Konzentration zu.

Der Einsatz des Vorläufers Diisopropylketon lieferte keine zufriedenstellenden Ergebnisse. Zum Einen konnte die Kanalverzweigung auf Grund eines fehlenden Reinspektrums für Isopropylvinylketon nicht quantitativ bestimmt werden, zum Anderen wies der Vorläufer im Bereich der C-H-Valenzschwingung schon bei geringen Konzentrationen eine Sättigung 
auf, die eine quantitative Untersuchung des Verältnisses $k(d) / k(c)$ nicht zuließ. Damit konnte das Ziel dieser Untersuchung für den Vorläufer Diisopropylketon nicht erreicht werden. Auf die Verwendung dieses Vorläufers wurde bei der Analyse daher verzichtet.

\subsubsection{Quantitative Analyse}

Für die quantitative Auswertung der Photolyse von Isopropyliodid wurden die Partialdrücke der Produkte gegen die Abnahme an Vorläufer aufgetragen. Dabei wurde das ebenfalls quantitativ bestimmte Produkt der Oxidationsreaktion (Aceton) um die Abnahme an Vorläufer reduziert. Die Messwerte sind in Tabelle 4.31 und die daraus erstellte Auftragung ist in Abbildung 4.57 dargestellt.

\begin{tabular}{|c|c|c|c|c|c|c|c|}
\hline $\begin{array}{c}\text { Verbrauch ISOP } \\
{[\mathrm{mbar}]}\end{array}$ & $\begin{array}{c}\mathrm{p}\left(\mathrm{C}_{2} \mathrm{H}_{4}\right) \\
{[\mathrm{mbar}]}\end{array}$ & $\begin{array}{c}\mathrm{p}\left(\mathrm{C}_{3} \mathrm{H}_{6}\right) \\
{[\mathrm{mbar}]}\end{array}$ & $\begin{array}{c}\mathrm{p}\left(\mathrm{C}_{3} \mathrm{H}_{8}\right) \\
{[\mathrm{mbar}]}\end{array}$ & $\begin{array}{c}\mathrm{p}(\mathrm{DIISOP}) \\
{[\mathrm{mbar}]}\end{array}$ & $\begin{array}{c}\mathrm{p}\left(\mathrm{CH}_{4}\right) \\
{[\mathrm{mbar}]}\end{array}$ & $\begin{array}{c}\mathrm{p}\left(\mathrm{C}_{3} \mathrm{H}_{6} \mathrm{O}\right) \\
{[\mathrm{mbar}]}\end{array}$ & $\begin{array}{c}\mathrm{p}(\mathrm{ISOP})_{\text {korr. }} \\
{[\mathrm{mbar}]}\end{array}$ \\
\hline \hline 0,018 & 0,0012 & 0,013 & 0,004 & 0,0052 & 0,0012 & 0,00069 & 0,0173 \\
\hline 0,026 & 0,0016 & 0,011 & 0,003 & 0,0072 & 0,0010 & 0,00081 & 0,0252 \\
\hline 0,055 & 0,0039 & 0,032 & 0,010 & 0,0104 & 0,0027 & 0,0007 & 0,054 \\
\hline 0,095 & 0,0046 & 0,040 & 0,014 & - & 0,0030 & 0,001 & 0,094 \\
\hline 0,105 & 0,0070 & 0,062 & 0,020 & 0,012 & 0,0050 & 0,0013 & 0,104 \\
\hline 0,245 & 0,0092 & 0,089 & 0,030 & 0,020 & 0,0070 & 0,002 & 0,243 \\
\hline
\end{tabular}

Tabelle 4.31: Reaktion 2- $\mathrm{C}_{3} \mathrm{H}_{7}+2-\mathrm{C}_{3} \mathrm{H}_{7}$, Vorläufer Isopropyliodid, Messwerte. $p\left(\mathrm{CH}_{3} \mathrm{CHICH}_{3}\right)$ $=0,6$ mbar, $p_{g e s}=4$ mbar, $\mathrm{T}=298 \mathrm{~K}$, Badgas Argon (ISOP:= Isopropyliodid; DIISOP $:=$ Diisopropyl).

Die Abbildung 4.57 zeigt einen linearen Anstieg der Partialdrücke der Produkte mit zunehmender Isopropylabnahme. Die relativ hohen Schwankungen der Messwerte resultieren in diesem Fall aus der Zusammenführung mehrerer Messreihen. Diese wurden aber alle bei den gleichen Bedingungen aufgenommen. Die Abbildung macht deutlich, dass der Hauptkanal der Photolyse die Bildung von Propen ist. Des Weiteren ist ersichtlich, dass der Propananteil um den Faktor 1/3 kleiner ist als der Propenanteil. Daher wird die Bildung von Propen überwiegend aus der direkten HI-Elimination erfolgen. Die Bildung von Ethen und Methan wird wahrscheinlich durch einen C-C-Bruch bei der Photolyse verursacht, allerdings sind die Anteile dieser beiden Produkte relativ gering. 


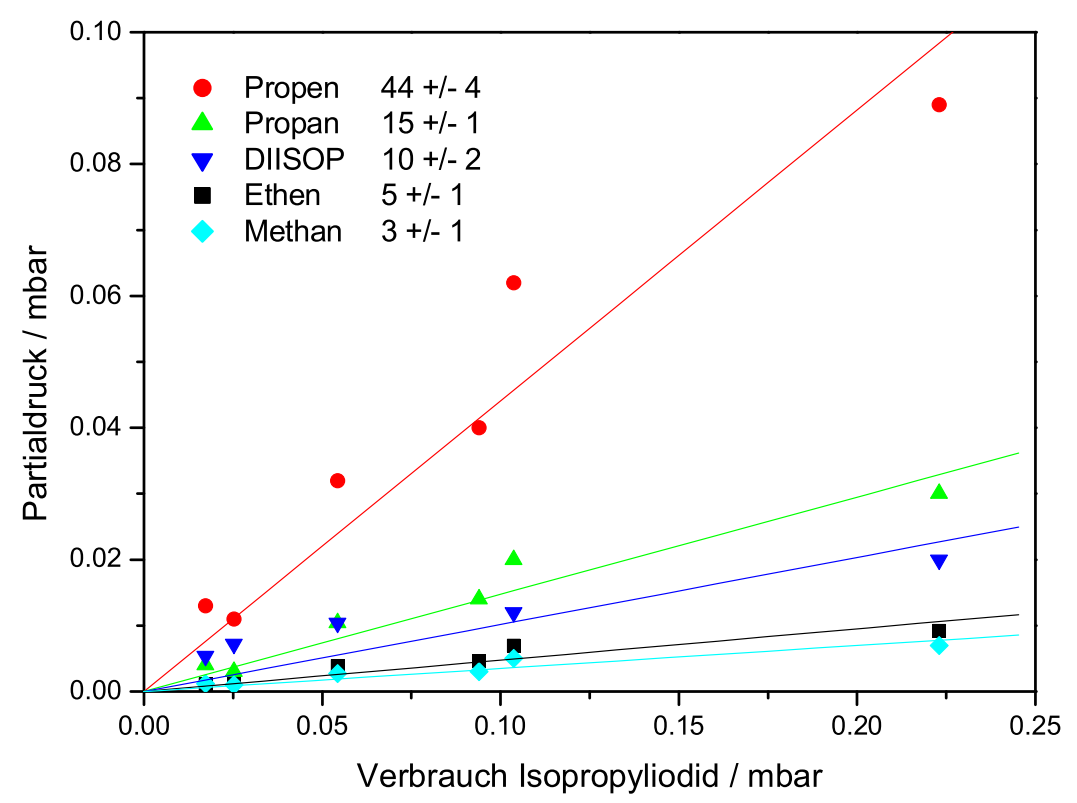

Abbildung 4.57: Reaktion 2- $\mathrm{C}_{3} \mathrm{H}_{7}+2-\mathrm{C}_{3} \mathrm{H}_{7}$, quantitative Analyse. Die Abbildung zeigt die Partialdrücke der Produkte vs. Verbrauch Isoproypyliodid (DIISOP $:=$ Diisopropyl). Dabei ist der Verbrauch an Isopropyliodid um die Werte des Oxidationsprodukts Aceton korrigiert; $p($ Isopropyliodid $)=0,6$ mbar, $p_{\text {ges }}=4 \mathrm{mbar}, \mathrm{T}=298 \mathrm{~K}$.

\subsubsection{Diskussion}

Auf Grund der quantitativen Auswertung der Photolyse von Isopropyliodid wird folgende Verzweigung vorgeschlagen:

$$
\begin{array}{rlr}
\mathrm{CH}_{3} \mathrm{CHICH}_{3} \stackrel{h \nu}{\longrightarrow} \mathrm{CH}_{3} \mathrm{CHCH}_{3}+\mathrm{I} & 48 \% \\
& \stackrel{h \nu}{\longrightarrow} \mathrm{CH}_{3} \mathrm{CHCH}_{2}+\mathrm{HI} & 52 \%
\end{array}
$$

Photolysekanäle, die nicht zur Bildung freier Radikale führen, überwiegen bei diesem Vorläufer. Da sich aber gezeigt hat, dass Diisopropylketon kein geeigneter Vorläufer für die Generierung von Isopropylradikalen ist, wurde dieser Nachteil in Kauf genommen. Unter Berücksichtigung der Photolysekanäle ergibt sich für die Reaktion zweier Isopropylradikale aus den quantitativen Messungen folgende Kanalverzweigung:

$$
\begin{array}{rlr}
2-\mathrm{C}_{3} \mathrm{H}_{7}+2-\mathrm{C}_{3} \mathrm{H}_{7} & \longrightarrow\left(\mathrm{CH}_{3}\right)_{2} \mathrm{CHCH}\left(\mathrm{CH}_{3}\right)_{2} & 40 \% \\
& \longrightarrow \mathrm{C}_{3} \mathrm{H}_{8}+\mathrm{C}_{3} \mathrm{H}_{6} & 60 \%
\end{array}
$$


In der Literatur liegen für die Reaktion mehrere Ergebnisse vor.

GOLDEN et al. untersuchten die Geschwindigkeit der Reaktion mittels einer VLPP-Apparatur (very low-pressure pyrolysis) [Gol74]. Bei Zimmertemperatur fanden sie ein Verhältnis von $k(d) / k(c)_{\infty}=0,69$. Die Geschwindigkeit der Kombination wird dabei im Bereich des fall off-Druckbereichs vermutet, insbesondere wurden Verhältnisse $k(d) / k(c) \geq 0,67$ beobachtet $(0,75 \leq k(d) / k(c) \leq 1,5)$.

AnASTASI et al. geben für das Verhältnis der Kanalverzweigung einen Wert von $k(d) / k(c)$ $=0,62$ an [Ana87. Dabei wurden das Verhältnis ebenfalls durch Messungen bei Zimmertemperatur bestimmt.

\subsubsection{Die Reaktion 1-Butyl + 1-Butyl}

\subsubsection{Einleitung}

Im Rahmen der Untersuchung der Kreuzreaktion von Methylradikalen mit Alkylradikalen wurde die Untersuchung auf längerkettige Radikale ausgedehnt, um einen Einfluss der Kettenlänge auf das Verhältnis von Kombination zu Disproportionierung zu ermitteln. Dieses Verhältnis ist gerade im Hinblick auf die bei der radikalischen Polymerisation ablaufenden Prozesse relevant, da eine Terminierung unter Disproportionierung zum Abbruch des Kettenwachstums führt. Dabei wurde als Vorläufer für 1-Butyl Radikale der iodierte Kohlenwasserstoff verwendet, da ein Einsatz des entsprechenden Diketons zum Einen zu einer Vielzahl von Nebenreaktionen auf Grund des nicht selektiven $\beta$-C-C-Bruchs führt und zum Anderen die Radikalanfangskonzentration weit unter der der iodierten Substanz lag. Im Folgenden ist die Untersuchung der Photolyse des Butylradikal-Vorläufers 1-Butyliodid kurz dargestellt. Erste Ergebnisse zu den Reaktionen von Methylradikalen mit 2-Butylradikalen und n-Pentylradikalen finden sich im Ausblick (Kapitel 5).

Für die Reaktion von zwei 1-Butylradikalen wird analog zu den vorherigen Reaktionen eine Kombination und Disproportionierung erwartet:

$$
\begin{aligned}
\mathrm{C}_{4} \mathrm{H}_{9}+\mathrm{C}_{4} \mathrm{H}_{9} & \longrightarrow \mathrm{C}_{8} \mathrm{H}_{16} \\
& \longrightarrow \mathrm{C}_{4} \mathrm{H}_{8}+\mathrm{C}_{4} \mathrm{H}_{10}
\end{aligned}
$$


Für die Reaktion Butyl + Butyl lagen in der Literatur keine Empfehlung für das Verhältnis von Kombination zu Disproportionierung vor, doch es wird ein ähnliches Verhältnis wie für die Reaktion Propyl + Propyl erwartet.

\subsubsection{Qualitative Analyse}

Ein Photolysespektrum nach 400 Photolysen bei einem Partialdruck von $\mathrm{p}\left(\mathrm{C}_{4} \mathrm{H}_{9} \mathrm{I}\right)=0,3$ mbar und einem Gesamtdruck von 4 mbar und einer Temperatur von 298 K zeigt Abbildung 4.58. Aus der Abbildung geht hervor, dass neben der (Alkyl-Alkyl)-Reaktion auch eine Reaktion mit molekularem Sauerstoff unter Bildung von Butanal stattgefunden hat (positive Bande im Bereich der Carbonylschwingung um $1750 \mathrm{~cm}^{-1}$ ). Die Identifikation von Butanal ist in Abbildung 4.59, neben der Identifikation der übrigen Substanzen, nochmals detailliert dargestellt.

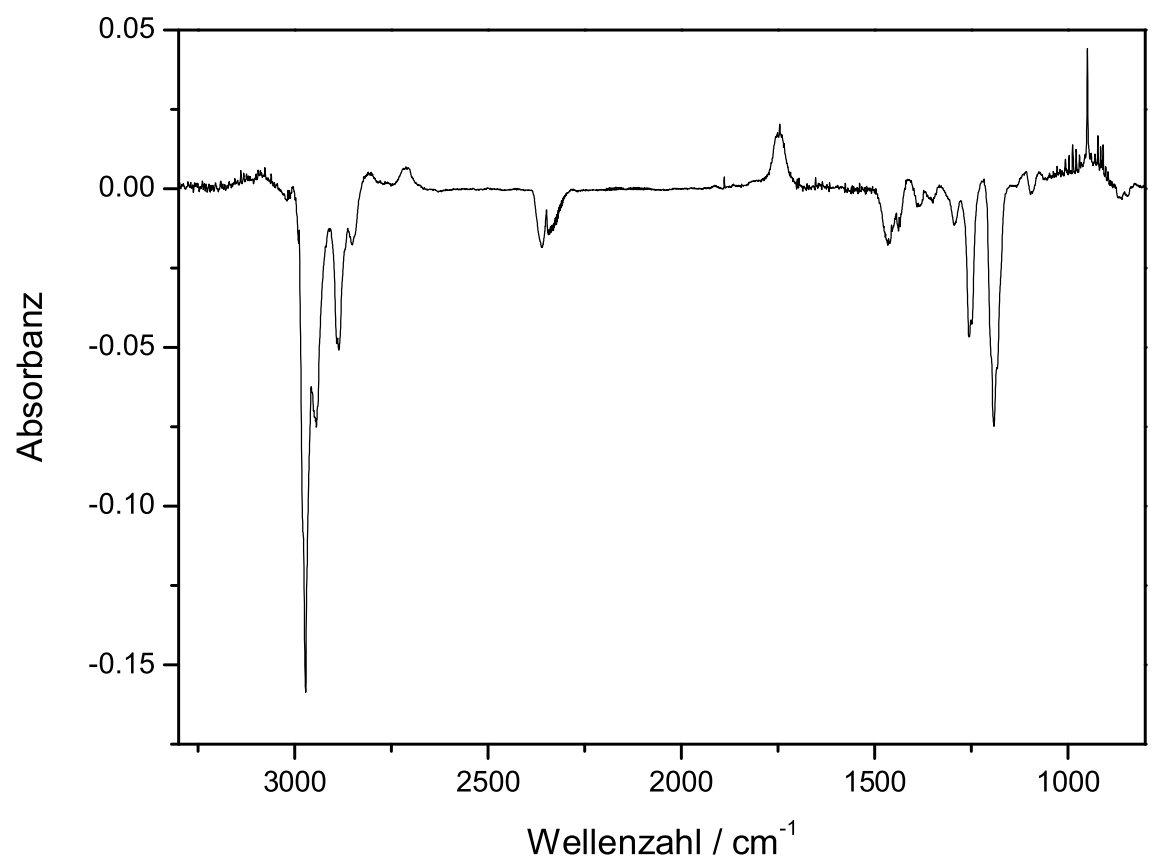

Abbildung 4.58: Reaktion 1- $\mathrm{C}_{4} \mathrm{H}_{9}+1-\mathrm{C}_{4} \mathrm{H}_{9}$, Reaktionsspektrum, Vorläufer Butyliodid. $p\left(C_{4} H_{9} I\right)=0,3$ mbar, $p_{g e s}=4$ mbar, $T=298 \mathrm{~K}, 400$ Photolysen. 

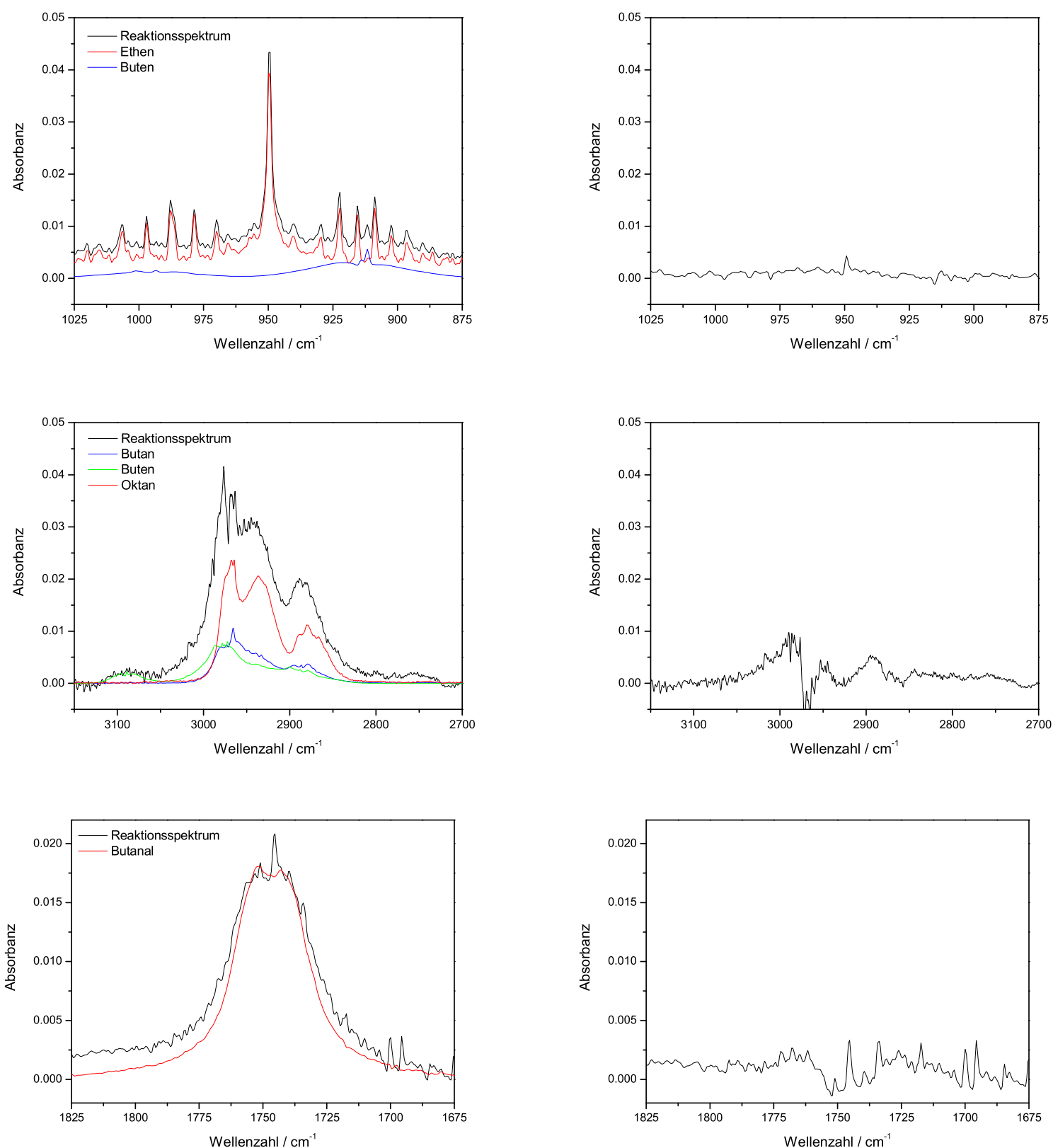

Abbildung 4.59: Reaktion 1- $\mathrm{C}_{4} \mathrm{H}_{9}+1-\mathrm{C}_{4} \mathrm{H}_{9}$, qualitative Analyse, Vorläufer 1-Butyliodid. $p\left(C_{4} H_{9} I\right)=0,3$ mbar, $p_{\text {ges }}=4$ mbar, $T=298 \mathrm{~K}, 400$ Photolysen. Vergleich des Reaktionsspektrums mit Spektren der Reinsubstanzen in vergrößerten Ausschnitten; in der linken Spalte ist jeweils das Reaktionsspektrum (schwarz) mit den Reinspektren (farbig) im Vergleich dargestellt, in der rechten Spalte sind die entsprechenden Residuen in schwarz dargestellt.

Die Abbildung 4.59 zeigt, dass sowohl die Disproportionierungsprodukte Butan und Buten als auch das Kombinationsprodukt Oktan beobachtet werden können. Des Weiteren ist die Bildung von Ethen zu beobachten. Diese ist wahrscheinlich auf einen direkten Zerfall des angeregten Butylradikals zurückzuführen. KNYAZEv und Slagle geben für den Zerfall 
des Butylradikals eine Geschwindigkeit von $k^{\infty}=1.06 \times 10^{13} \exp (-14005 K / T) \mathrm{s}^{-1}$ an Kny96. Für die Reaktion Butyl + Butyl konnten Butan, Buten und Oktan als Produkte nachgewiesen werden.

\subsubsection{Quantitative Analyse}

Die quantitative Analyse der Reaktion 1-Butyl + 1-Butyl lieferte unter Verwendung des Vorläufers Butyliodid das in Tablle 4.32 dargestellte, auf die Abnahme an Butyliodid normierte Verhältnis:

\begin{tabular}{cccccc}
\hline $\mathrm{C}_{4} \mathrm{H}_{9} \mathrm{I}$ & Ethen & Buten & Butan & Oktan & Butanal \\
\hline 1 & 0,24 & 0,08 & 0,09 & 0,11 & 0,43 \\
\hline
\end{tabular}

Tabelle 4.32: Reaktion 1- $\mathrm{C}_{4} \mathrm{H}_{9}+1-\mathrm{C}_{4} \mathrm{H}_{9}$, quantitative Analyse, Vorläufer 1-Butyliodid. Die Partialdrücke der Produkte sind in Bezug zu der Abnahme an Butyliodid dargestellt.

Geht man davon aus, dass die Ethenbildung durch einen Zerfall des angeregten Butylradikals unter H-Abspaltung verläuft, so kann durch eine Massenbilanz 94\% der Abnahme an Butyliodid erklärt werden. Für die Reaktion 1-Butyl + 1-Butyl ergibt sich auf Grund der Messwerte folgende Kanalverzweigung:

$$
\begin{array}{rlr}
1-\mathrm{C}_{4} \mathrm{H}_{9}+1-\mathrm{C}_{4} \mathrm{H}_{9} & \longrightarrow \mathrm{C}_{8} \mathrm{H}_{16} & (56 \pm 5) \% \\
& \longrightarrow \mathrm{C}_{4} \mathrm{H}_{8}+\mathrm{C}_{4} \mathrm{H}_{10} & (44 \pm 5) \%
\end{array}
$$

\subsubsection{Diskussion}

Die Kanalverzweigung der Reaktion 1-Butyl + 1-Butyl liefert einen Wert von $k(d) / k(c)$ $=0,79$. Damit ist der Disproportionierungsanteil im Vergleich mit dem Verhältnis für die Reaktion 1-Propyl + 1-Propyl gestiegen. Ein Vergleich mit der Literatur kann auf Grund fehlender Daten nicht stattfinden. Das Verhältnis von Disproportionierung zu Kombination wird für die Reaktion von 1-Propyl +1 -Propyl mit $k(d) / k(c)=0,16$ angegeben [Tsa88]. Die Untersuchung der Reaktion 1-Propyl + 1-Propyl im Rahmen dieser Arbeit lieferte $k(d) / k(c)=0,14$ (Vorläufer Dipropylketon), bzw. $k(d) / k(c)=0,41$ (Vorläufer Propyliodid). Das Experiment zeigt, dass die gestiegene Kettenlänge zu einem Anstieg 
des Disproportionierungsanteils führt.

Knyazev und Slagle berichten ebenfalls über das Fehlen an Daten für die Reaktion und schlagen daher die Verwendung der Geschwindigkeit der Rekombination von 1-Propyl vor Kny96].

\subsubsection{Die Reaktion Allyl + Allyl}

\subsubsection{Einleitung}

Die Reaktion Allyl + Allyl ist von großer Bedeutung für die Modellierung von Pyrolyseprozessen und Verbrennungsreaktionen. Im Rahmen dieser Arbeit wurde die Reaktion begleitend zu der Reaktion Allyl + O untersucht. Die gewonnen Daten der Reaktion Allyl + Allyl unter Verwendung von verschiedenen Vorläufern gingen dabei in die Bestimmung der Produktverhältnisse der Reaktion Allyl $+\mathrm{O}$ ein. Außerdem erschien eine Untersuchung der Reaktion im Rahmen der systematischen Analyse der Alkylradikalkombinationsreaktionen als sinnvoll.

Die Reaktion wurde schon von Tulloch et al. [Tul82] und JEnkin et al. [Jen93] im Hinblick auf Kanalverzweigung und Kinetik studiert. BoYD et al. bestimmten die Geschwindigkeit der Kombination Boy95].

Für die Reaktion wird folgender Reaktionsmechanismus angenommen:

$$
\begin{aligned}
\mathrm{C}_{3} \mathrm{H}_{5}+\mathrm{C}_{3} \mathrm{H}_{5} & \longrightarrow \mathrm{C}_{6} \mathrm{H}_{10} \\
& \longrightarrow \mathrm{H}_{2} \mathrm{CCCH}_{2}+\mathrm{C}_{3} \mathrm{H}_{6} \\
& \longrightarrow \mathrm{HCCCH}_{3}+\mathrm{C}_{3} \mathrm{H}_{6}
\end{aligned}
$$

Neben der Kombinationsreaktion $\left(\mathrm{a}_{1}\right)$ sind zwei Disproportionierungsreaktionen denkbar $\left(\mathrm{d}_{1}\right)$ und $\left(\mathrm{d}_{2}\right)$.

\subsubsection{Qualitative Analyse}

Bei der Photolyse von Diallyl unter Ausschluß von $\mathrm{SO}_{2}$ wurden neben Allen und Propen, welche aus der Disproportionierungsreaktion stammen, noch Ethen, Ethin und Propin gefunden. Die Darstellung eines Photolysespektrums von Diallyl nach 400 Photolysen bei einem Partialdruck von 0,4 mbar und einem Gesamtdruck von 4 mbar zeigt Abbildung 
4.60. Vergrößerte Ausschnitte des Reaktionsspektrums zusammen mit Spektren der Reinsubstanzen zeigt Abbildung 4.61. An dieser Stelle sei nochmal darauf hingewiesen, dass eine Identifikation von Diallyl, welches als Produkt der Reaktion vermutet wird, unter Verwendung des Vorläufers Diallyl nicht möglich ist.

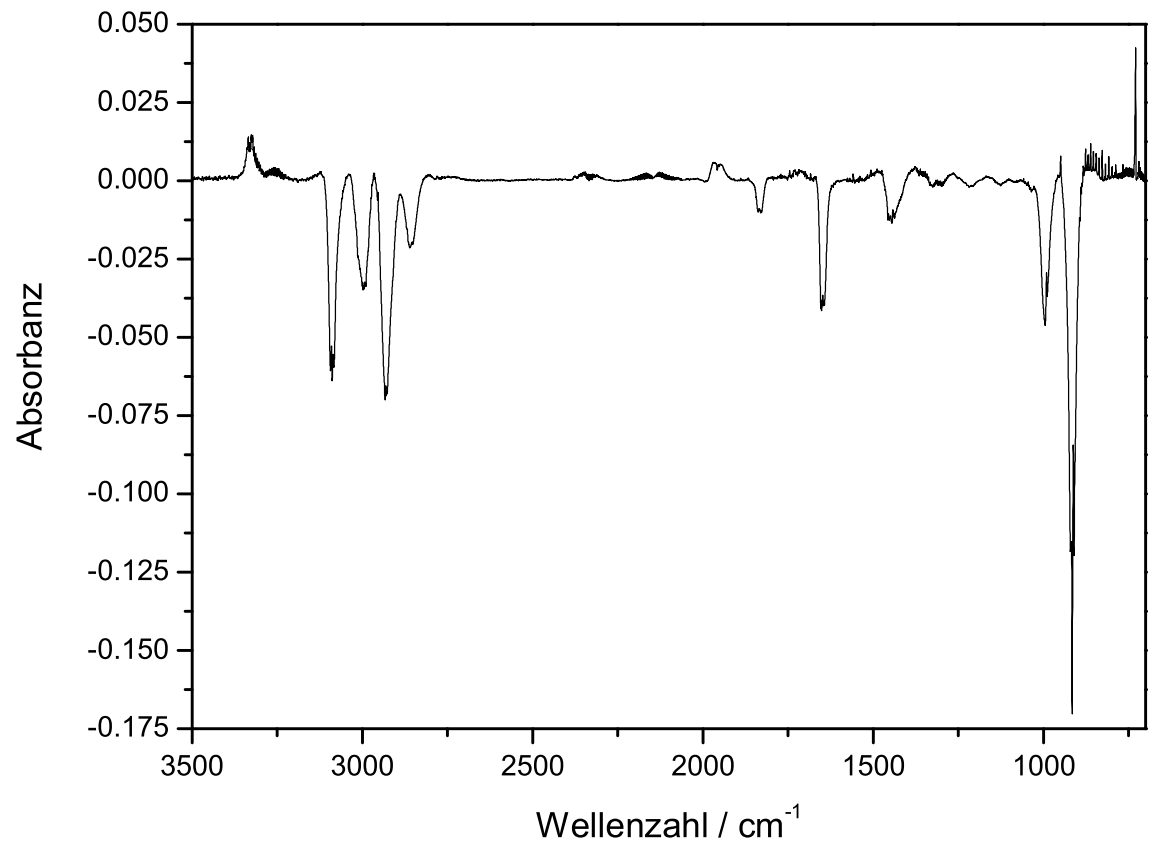

Abbildung 4.60: Reaktion $\mathrm{C}_{3} \mathrm{H}_{5}+\mathrm{C}_{3} \mathrm{H}_{5}$, Reaktionsspektrum, Vorläufer Diallyl. $p\left(\mathrm{C}_{6} \mathrm{H}_{10}\right)=0,4$ mbar, $p_{\text {ges }}=4$ mbar, $T=298 \mathrm{~K}, 400$ Photolysen. 

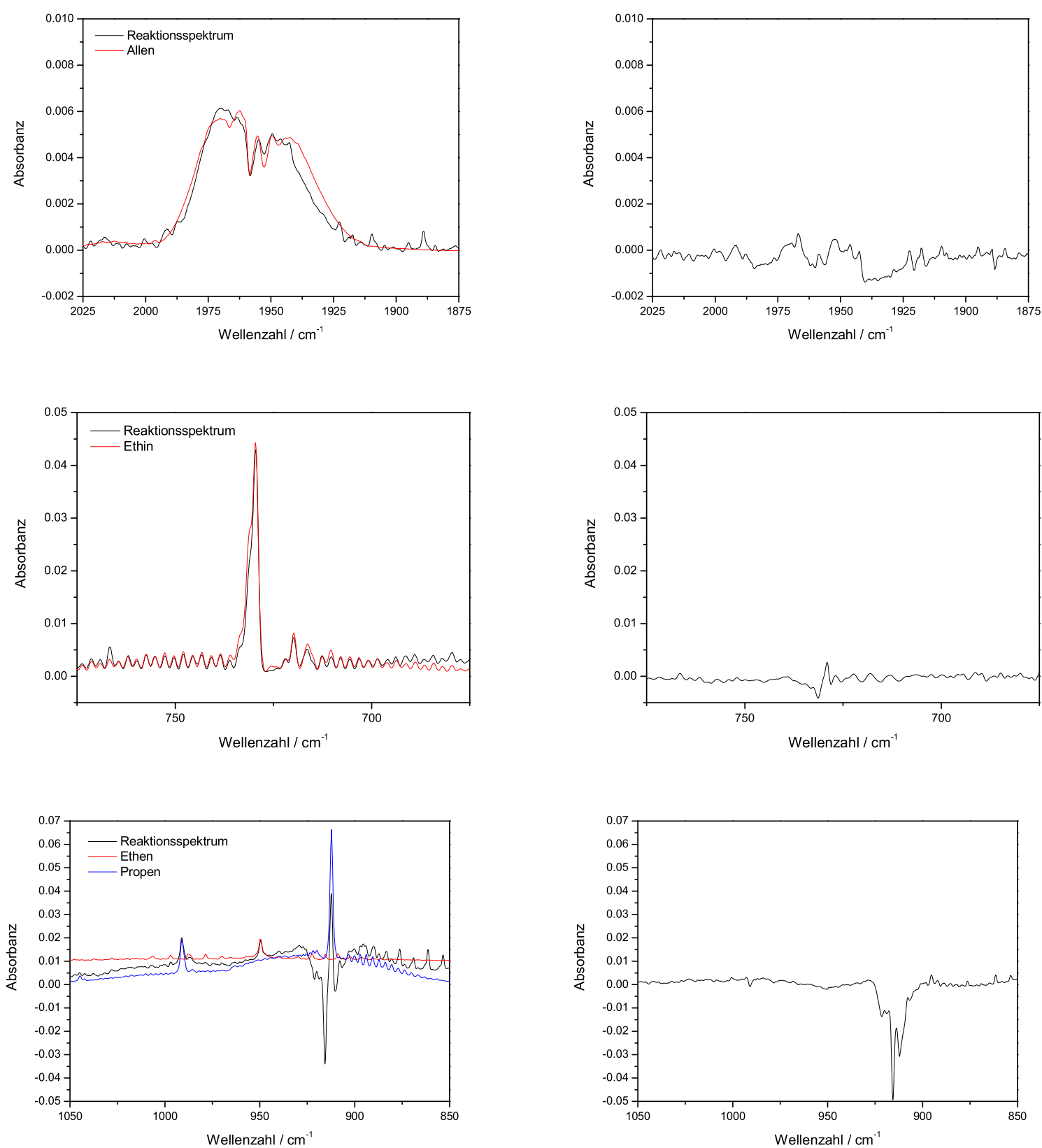

Abbildung 4.61: Reaktion $\mathrm{C}_{3} \mathrm{H}_{5}+\mathrm{C}_{3} \mathrm{H}_{5}$, qualitative Analyse, Vorläufer Diallyl. $p\left(\mathrm{C}_{6} \mathrm{H}_{10}\right)=$ 0,4 mbar, $p_{\text {ges }}=4$ mbar, $T=298 \mathrm{~K}, 400$ Photolysen. Vergleich des Reaktionsspektrums mit Spektren der Reinsubstanzen in vergrößerten Ausschnitten; in der linken Spalte ist jeweils das Reaktionsspektrum (schwarz) mit den Reinspektren (farbig) im Vergleich dargestellt, in der rechten Spalte sind die entsprechenden Residuen in schwarz dargestellt.

Ein Vergleich des Reaktionsspektrums mit dem Spektrum, bei dem die identifizierten Photolyseprodukte abgezogen sind, zeigt Abbildung 4.62. In dem reduzierten, wie auch dem Reaktionsspektrum, ist noch die rotationsaufgelöste Kohlenmonoxidbande bei 2150 $\mathrm{cm}^{-1}$ zu sehen. Die Bildung von CO resultiert aus einer Reaktion von Allylradikalen mit 
dem in der Zelle in geringer Konzentration vorhandenen molekularem Sauerstoff aus der Luft. Die im reduzierten Spektrum noch vorhandene negative Bande bei $915 \mathrm{~cm}^{-1}$ ist aus der Subtraktion des Diallyl Reinspektrums hervorgegangen. Dies kommt durch die in Abschnitt 3.3.2 beschriebene Intensitätsabnahme durch Kondensation an der Zelleninnenwand zustande.

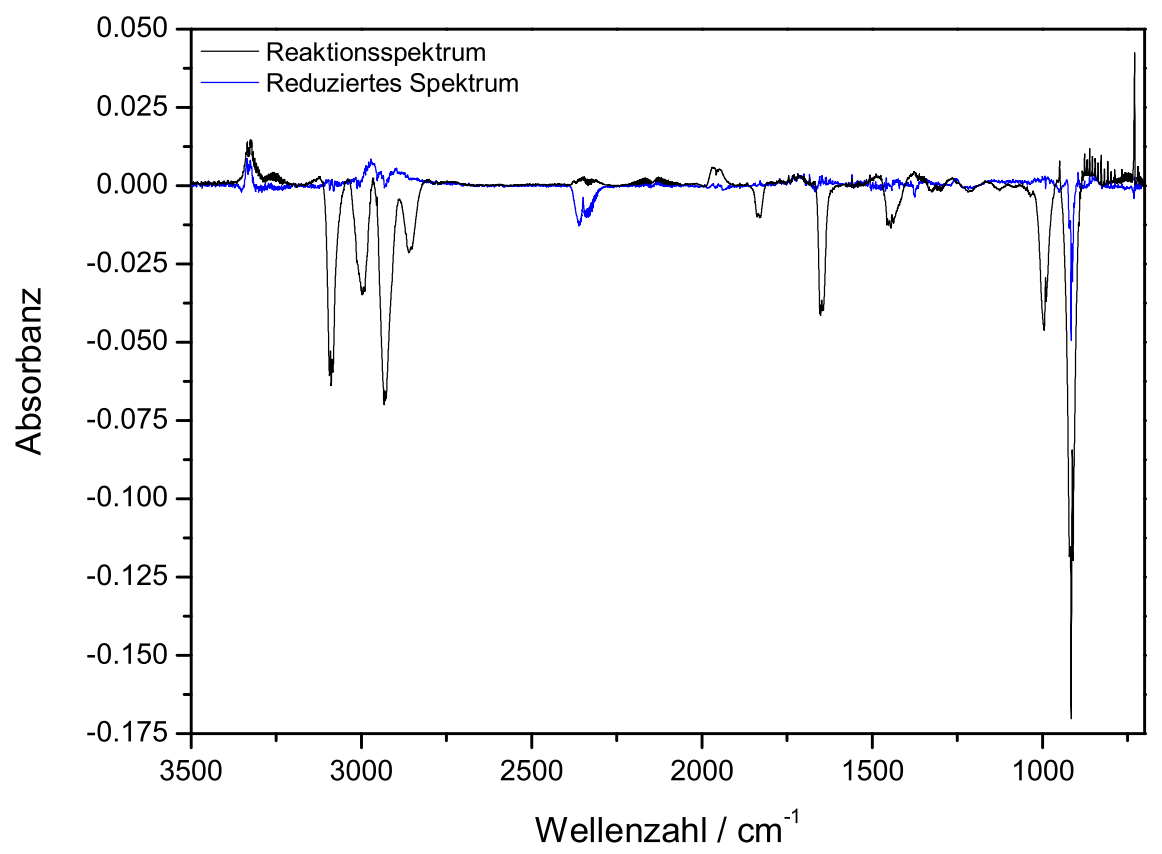

Abbildung 4.62: Reaktion $\mathrm{C}_{3} \mathrm{H}_{5}+\mathrm{C}_{3} \mathrm{H}_{5}$, Vergleich Reaktionsspektrum, Vorläufer Diallyl. $p\left(C_{6} H_{10}\right)=0,4$ mbar, $p_{\text {ges }}=4$ mbar, $T=298 \mathrm{~K}, 400$ Photolysen. Vergleich des Reaktionsspektrums mit dem reduzierten Spektrum, bei dem alle identifizierten Produkte abgezogen wurden.

Die Bildung von 1-Buten, welches das Gegenstück zu dem Photolyseprodukt Ethin sein könnte, wurde nicht beobachtet. TUlLOCH [Tul82] berichtet für die Photolyse von Diallyl bei derselben Photolysenwellenlänge wie in dieser Arbeit und Detektion der Endprodukte mit Hilfe von Flammenionisation nach GC-Analyse die folgenden Reaktionskanäle:

$$
\begin{array}{rlr}
\text { Diallyl } & \longrightarrow \text { Allyl }+ \text { Allyl } & 68 \% \\
& \longrightarrow \text { Propen }+ \text { Allen } & 27 \% \\
& \longrightarrow \text { 1,3-Butadien }+ \text { Ethen } & 2,5 \% \\
& \longrightarrow \text { Methyl }+\mathrm{C}_{5} \mathrm{H}_{7} & 2,5 \%
\end{array}
$$

Da eine Analyse des nicht stabilen Allylradikals mit der verwendeten FTIR-Apparatur 
nicht möglich war, können die von TULLOCH gefundenen prozentualen Reaktionskanäle nicht direkt mit denen dieser Arbeit verglichen werden. Nach Tulloch beträgt der Anteil radikalfreier Reaktionskanäle 32\%. Diese können mit Hilfe der FTIR-Apparatur nicht von dem Allylradikal-Reaktionskanal unterschieden werden, da mit der Apparatur nur eine Analyse stabiler Endprodukte möglich ist. Bei der quantitativen und der qualitativen Analyse wurden Produkte der Reaktion Allyl $+\mathrm{O}_{2}$ beobachtet. Die Anteile dieser Kanäle liegen aber weit unter denen der Reaktion Allyl + Allyl.

Das direkte Produkt der Reaktion Diallyl + O, 5-Hexenal, konnte unter Ausschluß von $\mathrm{SO}_{2}$ nicht gefunden werden.

Ein Reaktionsspektrum der Photolyse von Allylbromid nach 300 Photolysen bei einem Partialdruck von $\mathrm{p}\left(\mathrm{C}_{3} \mathrm{H}_{5} \mathrm{Br}\right)=0,3$ mbar bei $\mathrm{p}_{\text {ges }}=4$ mbar und $\mathrm{T}=298 \mathrm{~K}$ zeigt Abbildung 4.63 .

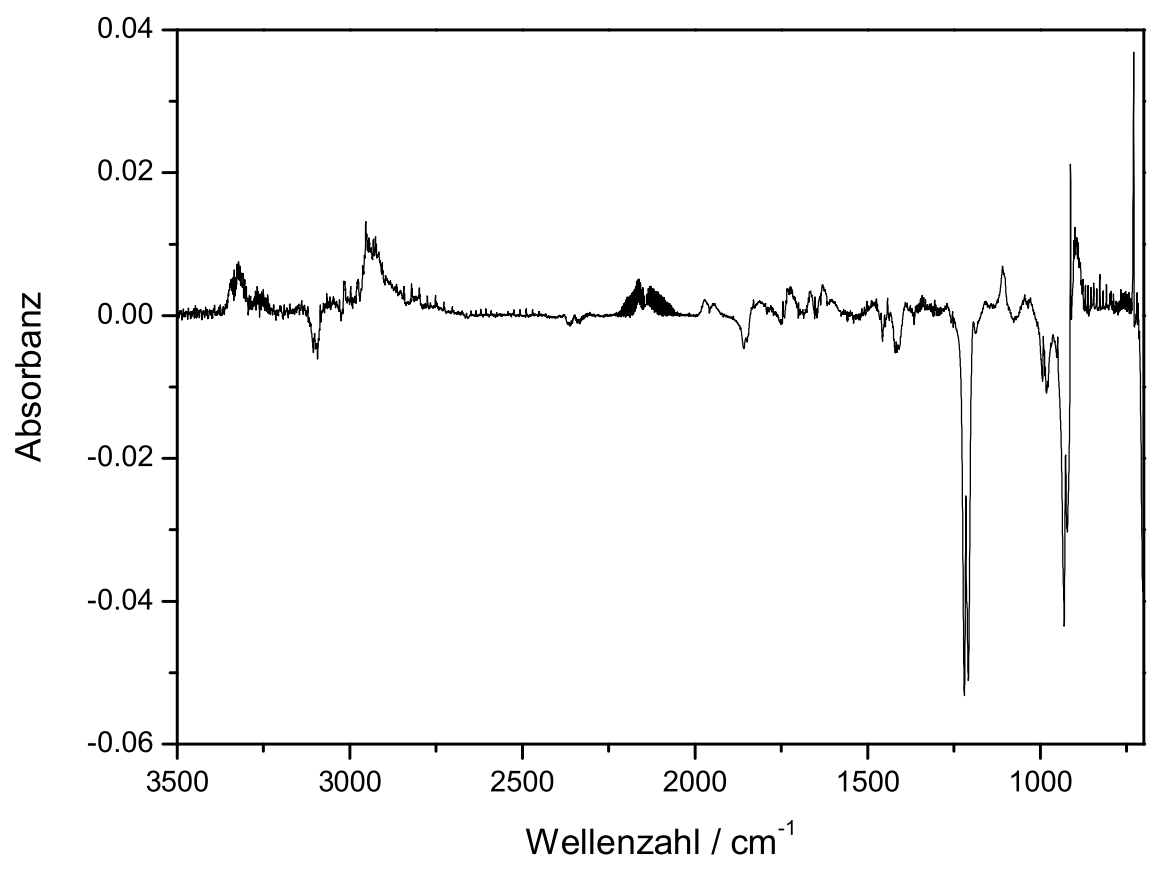

Abbildung 4.63: Reaktion $\mathrm{C}_{3} \mathrm{H}_{5}+\mathrm{C}_{3} \mathrm{H}_{5}$, Reaktionsspektrum, Vorläufer Allylbromid, $\mathrm{p}\left(\mathrm{C}_{3} \mathrm{H}_{5} \mathrm{Br}\right)$ $=0,3$ mbar, $p_{\text {ges }}=4$ mbar, $\mathrm{T}=298 \mathrm{~K}, 400$ Photolysen.

Vergrößerte Ausschnitte des Reaktionsspektrums sind in Abbildung 4.64 zusammen mit den Reinspektren der identifizierten Substanzen dargestellt. Dabei sind Allen, Propen, 
Ethen, Propin und Ethin eindeutig als Produkte zu identifizieren.
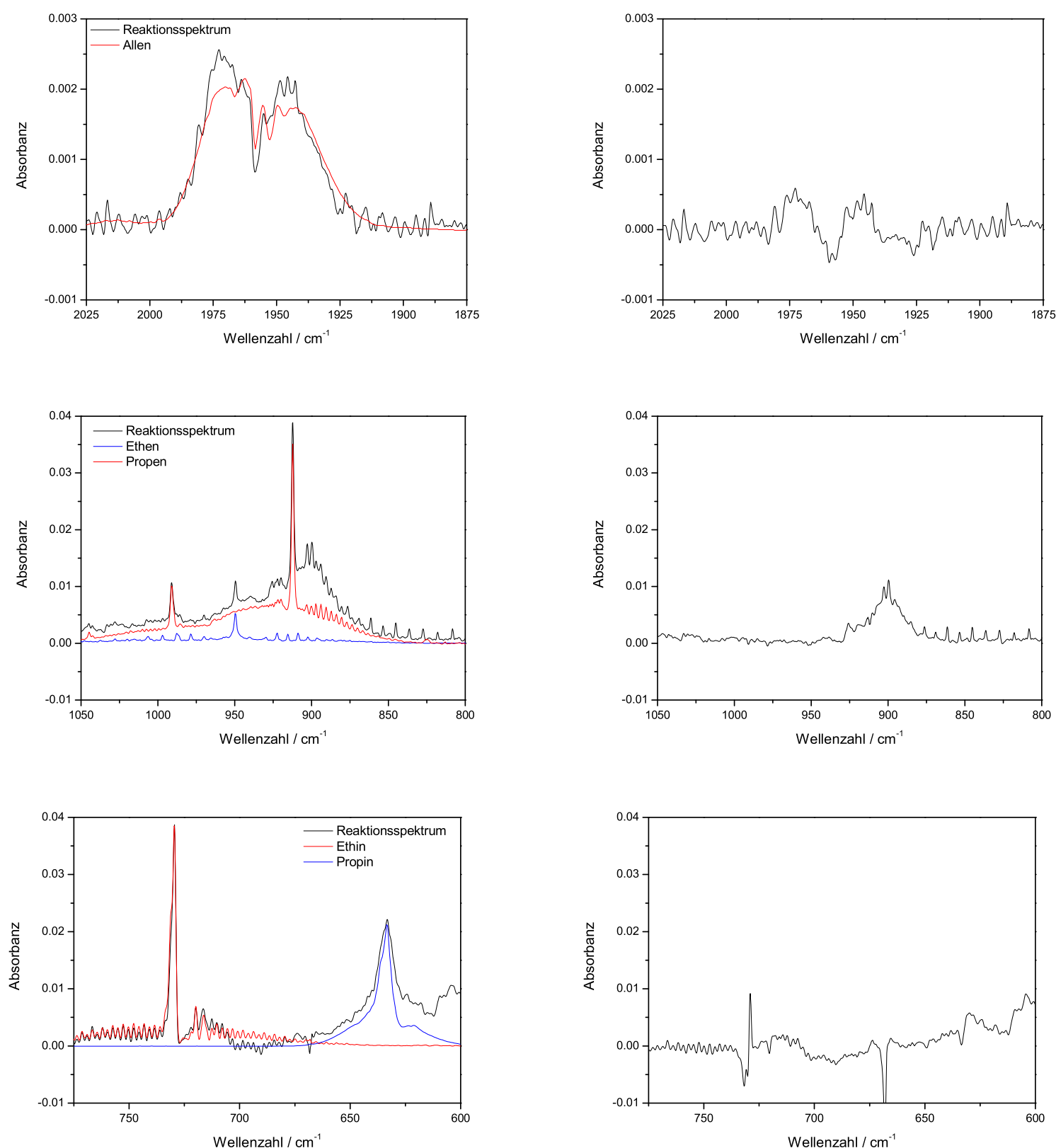

Abbildung 4.64: Reaktion $\mathrm{C}_{3} \mathrm{H}_{5}+\mathrm{C}_{3} \mathrm{H}_{5}$, qualitative Analyse, Vorläufer Allylbromid. $p\left(\mathrm{C}_{3} \mathrm{H}_{5} \mathrm{Br}\right)$ $=0,3$ mbar, $p_{g e s}=4$ mbar, $T=298 \mathrm{~K}, 400$ Photolysen. Vergleich des Reaktionsspektrums mit Spektren der Reinsubstanzen in vergrößerten Ausschnitten; in der linken Spalte ist jeweils das Reaktionsspektrum (schwarz) mit den Reinspektren (farbig) im Vergleich dargestellt, in der rechten Spalte sind die entsprechenden Residuen in schwarz dargestellt.

Ein Reaktionsspektrum nach 300 Photolysen von dem Vorläufer Allylchlorid mit den Anfangsbedingungen $\mathrm{p}\left(\mathrm{C}_{3} \mathrm{H}_{5} \mathrm{Cl}\right)=0,3$ mbar bei $\mathrm{p}_{\text {ges }}=4$ mbar und $\mathrm{T}=298$ zeigt Abbildung 4.65 . 


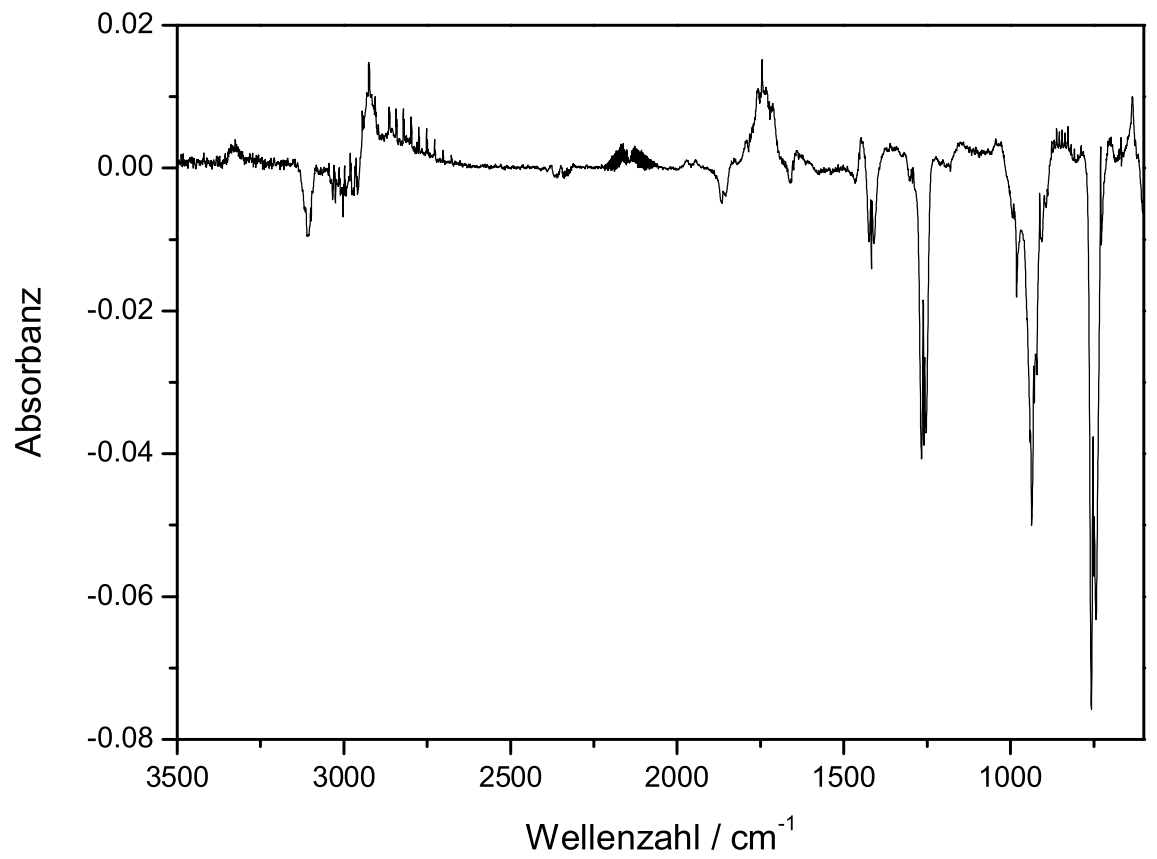

Abbildung 4.65: Reaktion $\mathrm{C}_{3} \mathrm{H}_{5}+\mathrm{C}_{3} \mathrm{H}_{5}$, Reaktionsspektrum, Vorläufer Allylchlorid, $\mathrm{p}\left(\mathrm{C}_{3} \mathrm{H}_{5} \mathrm{Cl}\right)$ $=0,3$ mbar, $p_{g e s}=4 \mathrm{mbar}, \mathrm{T}=298 \mathrm{~K}, 300$ Photolysen.

Für die Identifikation der Produkte sind vergrößerte Ausschnitte des Reaktionsspektrums zusammen mit den Reinspektren der identifizierten Substanzen in Abbildung 4.66 wiedergegeben. Die Abbildung zeigt die Bildung von Allen, Diallyl, Propen, Ethen und Ethin. 

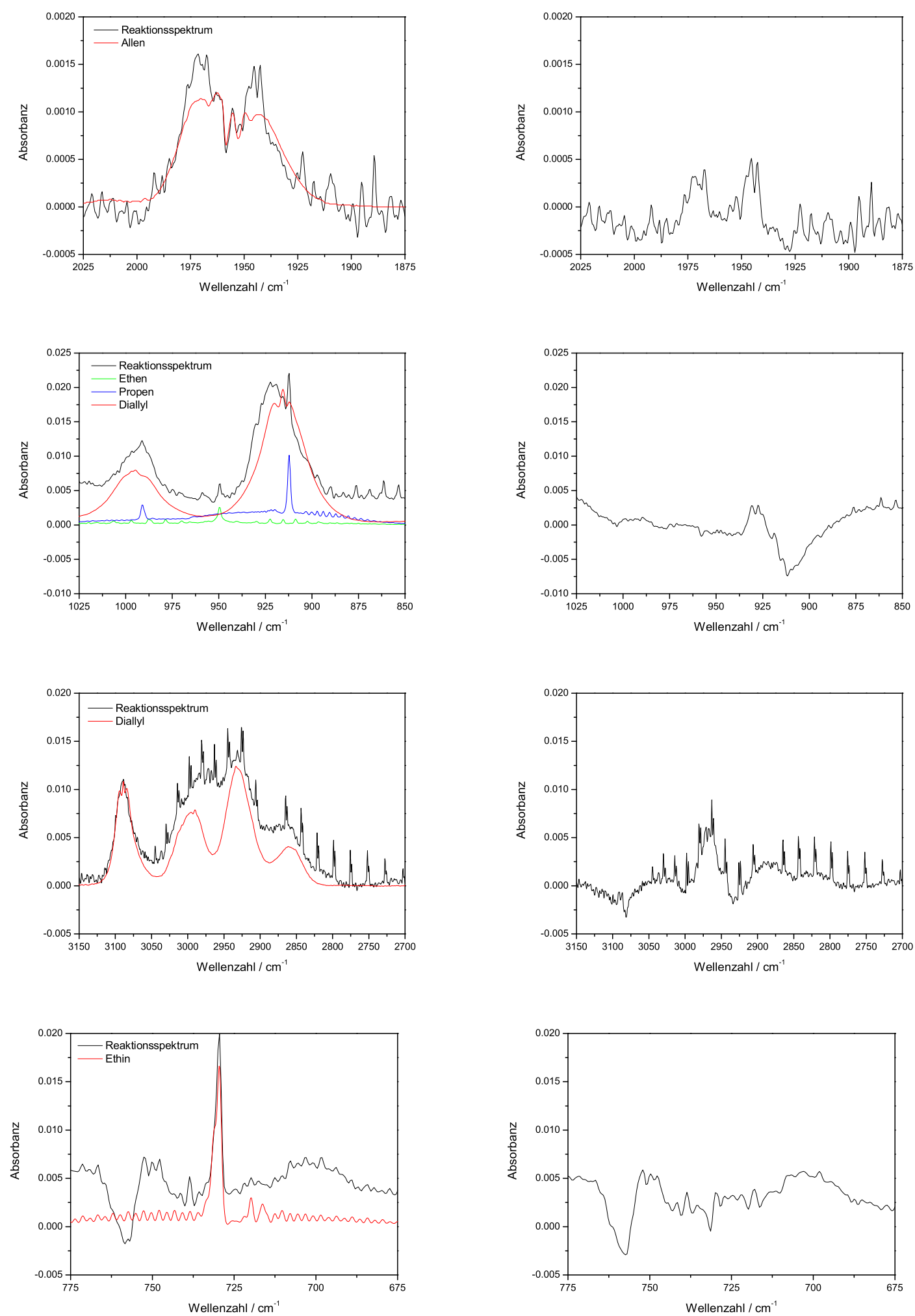

Abbildung 4.66: Reaktion $\mathrm{C}_{3} \mathrm{H}_{5}+\mathrm{C}_{3} \mathrm{H}_{5}$, qualitative Analyse, Vorläufer Allylchlorid. $\mathrm{p}\left(\mathrm{C}_{3} \mathrm{H}_{5} \mathrm{Cl}\right)$ $=0,3$ mbar, $p_{\text {ges }}=4 \mathrm{mbar}, \mathrm{T}=298 \mathrm{~K}, 300$ Photolysen. Vergleich des Reaktionsspektrums mit Spektren der Reinsubstanzen in vergrößerten Ausschnitten; in der linken Spalte ist jeweils das Reaktionsspektrum (schwarz) mit den Reinspektren (farbig) im Vergleich dargestellt, in der rechten Spalte sind die entsprechenden Residuen in schwarz dargestellt. 


\subsubsection{Quantitative Analyse}

Eine quantitative Analyse der Photolyse von Diallyl zeigt Abbildung 4.67. Die zugehörigen Messwerte sind in Tabelle 4.33 angegeben.

\begin{tabular}{|c||c|c|c|c|c|c|c|c|}
\hline $\begin{array}{c}\text { Diallyl Verb. } \\
{[\mathrm{mbar}]}\end{array}$ & $\begin{array}{c}\mathrm{p}\left(\mathrm{C}_{3} \mathrm{H}_{6}\right) \\
{[\mathrm{mbar}]}\end{array}$ & $\begin{array}{c}\mathrm{p}\left(\mathrm{C}_{2} \mathrm{H}_{2}\right) \\
{[\mathrm{mbar}]}\end{array}$ & $\begin{array}{c}\mathrm{p} \text { (Propin) } \\
{[\mathrm{mbar}]}\end{array}$ & $\begin{array}{c}\mathrm{p} \text { (Allen) } \\
{[\mathrm{mbar}]}\end{array}$ & $\begin{array}{c}\mathrm{p}\left(\mathrm{C}_{3} \mathrm{H}_{4} \mathrm{O}\right) \\
{[\mathrm{mbar}]}\end{array}$ & $\begin{array}{c}\mathrm{p}\left(\mathrm{H}_{2} \mathrm{CO}\right) \\
{[\mathrm{mbar}]}\end{array}$ & $\begin{array}{c}\mathrm{p}\left(\mathrm{C}_{2} \mathrm{H}_{4}\right) \\
{[\mathrm{mbar}]}\end{array}$ & $\begin{array}{c}\mathrm{p}(\mathrm{CO}) \\
{[\mathrm{mbar}]}\end{array}$ \\
\hline \hline 0,0068 & 0,0038 & 0,002 & 0,0013 & $1 \mathrm{E}-3$ & $6 \mathrm{E}-4$ & & $4,6 \mathrm{E}-4$ & 0,0012 \\
\hline 0,0092 & 0,0074 & 0,003 & 0,0023 & 0,0024 & 0,0021 & $3 \mathrm{E}-4$ & 0,0012 & 0,0015 \\
\hline 0,0118 & 0,01 & 0,004 & 0,0034 & 0,0037 & 0,0025 & $5 \mathrm{E}-4$ & 0,0018 & 0,003 \\
\hline 0,0183 & 0,015 & 0,0045 & 0,004 & 0,0044 & 0,0024 & $6,1 \mathrm{E}-4$ & 0,0025 & 0,003 \\
\hline
\end{tabular}

Tabelle 4.33: Reaktion $\mathrm{C}_{3} \mathrm{H}_{5}+\mathrm{C}_{3} \mathrm{H}_{5}$, Vorläufer Diallyl, Messwerte. $\mathrm{p}($ Diallyl $)=0,4$ mbar, $p_{\text {ges }}$ $=4$ mbar, $T=298 \mathrm{~K}$, Badgas Argon.

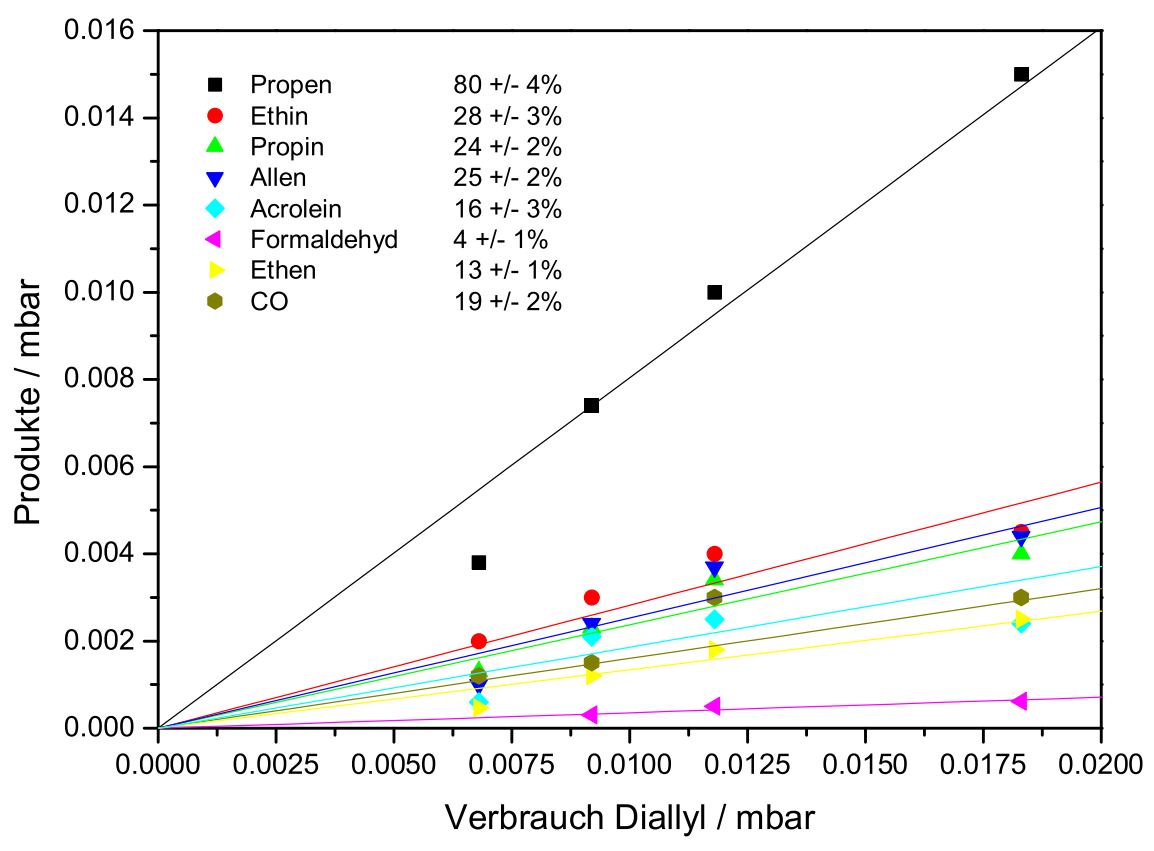

Abbildung 4.67: Reaktion $\mathrm{C}_{3} \mathrm{H}_{5}+\mathrm{C}_{3} \mathrm{H}_{5}$, quantitative Analyse, Vorläufer Diallyl. $p\left(\mathrm{C}_{6} \mathrm{H}_{10}\right)=$ 0,4 mbar, $p_{\text {ges }}=4 \mathrm{mbar}, \mathrm{T}=298 \mathrm{~K}$. Die prozentuale Angabe der Produkte bezieht sich auf den Verbrauch des Vorläufers Diallyl.

Aus der Auftragung 4.67 geht hervor, dass der Hauptkanal der Reaktion Allyl + Allyl die Bildung von Propen ist ( $80 \%$ bezogen auf die Diallylabnahme), daneben scheinen die Kanäle zu Ethin (28\%), Propin (24\%) und Allen (25\%) von Bedeutung zu sein. Ebenso ist die Bildung von Ethen (13\%), Acrolein (16\%) und Kohlenmonoxid (19\%) nicht 
vernachlässigbar, welche aber im Fall der sauerstoffhaltigen Verbindungen auf die schon angesprochene Reaktion mit molekularem Sauerstoff zurückzuführen ist. Bei der Verwendung von Diallyl als Vorläufer ist wie schon oben beschrieben, aber zu beachten, dass die Rückreaktion zweier Allylradikale zu Diallyl nicht detektierbar ist und somit die oben aufgezeigten Kanäle durch den Kanal Allyl + Allyl $\rightarrow$ Diallyl ergänzt werden müssen.

Die quantitativen Ergebnisse der Untersuchung der Photolyse von Allylbromid sind in Abbildung 4.68 dargestellt. Die Messwerte sind in der nachfolgenden Tabelle 4.34 angegeben.

\begin{tabular}{|c||c|c|c|c|c|c|c|c|}
\hline $\begin{array}{c}\mathrm{C}_{3} \mathrm{H}_{5} \mathrm{Br} \\
{[\mathrm{mbar}]}\end{array}$ & $\begin{array}{c}\mathrm{p}\left(\mathrm{C}_{3} \mathrm{H}_{4} \mathrm{O}\right) \\
{[\mathrm{mbar}]}\end{array}$ & $\begin{array}{c}\mathrm{p}\left(\mathrm{H}_{2} \mathrm{CO}\right) \\
{[\mathrm{mbar}]}\end{array}$ & $\begin{array}{c}\mathrm{p}\left(\mathrm{C}_{2} \mathrm{H}_{4}\right) \\
{[\mathrm{mbar}]}\end{array}$ & $\begin{array}{c}\mathrm{p}\left(\mathrm{C}_{3} \mathrm{H}_{6}\right) \\
{[\mathrm{mbar}]}\end{array}$ & $\begin{array}{c}\mathrm{p}\left(\mathrm{C}_{2} \mathrm{H}_{2}\right) \\
{[\mathrm{mbar}]}\end{array}$ & $\begin{array}{c}\mathrm{p}\left(\mathrm{C}_{6} \mathrm{H}_{10}\right) \\
{[\mathrm{mbar}]}\end{array}$ & $\begin{array}{c}\mathrm{p} \text { (Allen) } \\
{[\mathrm{mbar}]}\end{array}$ & $\begin{array}{c}\mathrm{p} \text { (Propin) } \\
{[\mathrm{mbar}]}\end{array}$ \\
\hline \hline 0,011 & $5 \mathrm{E}-4$ & - & $7 \mathrm{E}-4$ & 0,0039 & 0,0023 & $7 \mathrm{E}-4$ & $9,1 \mathrm{E}-4$ & 0,0012 \\
\hline 0,02 & $1 \mathrm{E}-3$ & - & 0,0014 & 0,0071 & 0,0034 & $9 \mathrm{E}-4$ & 0,0015 & 0,002 \\
\hline 0,03 & 0,0013 & $3 \mathrm{E}-4$ & 0,00184 & 0,01 & 0,0043 & 0,0016 & 0,0018 & 0,0028 \\
\hline 0,036 & 0,002 & $5 \mathrm{E}-4$ & 0,0023 & 0,012 & 0,0051 & - & 0,0022 & 0,0036 \\
\hline
\end{tabular}

Tabelle 4.34: Reaktion $\mathrm{C}_{3} \mathrm{H}_{5}+\mathrm{C}_{3} \mathrm{H}_{5}$, Vorläufer Allylbromid, Messwerte. $p$ (Allylbromid) $=0,3$ mbar, $p_{\text {ges }}=4$ mbar, $\mathrm{T}=298 \mathrm{~K}$, Badgas Argon.

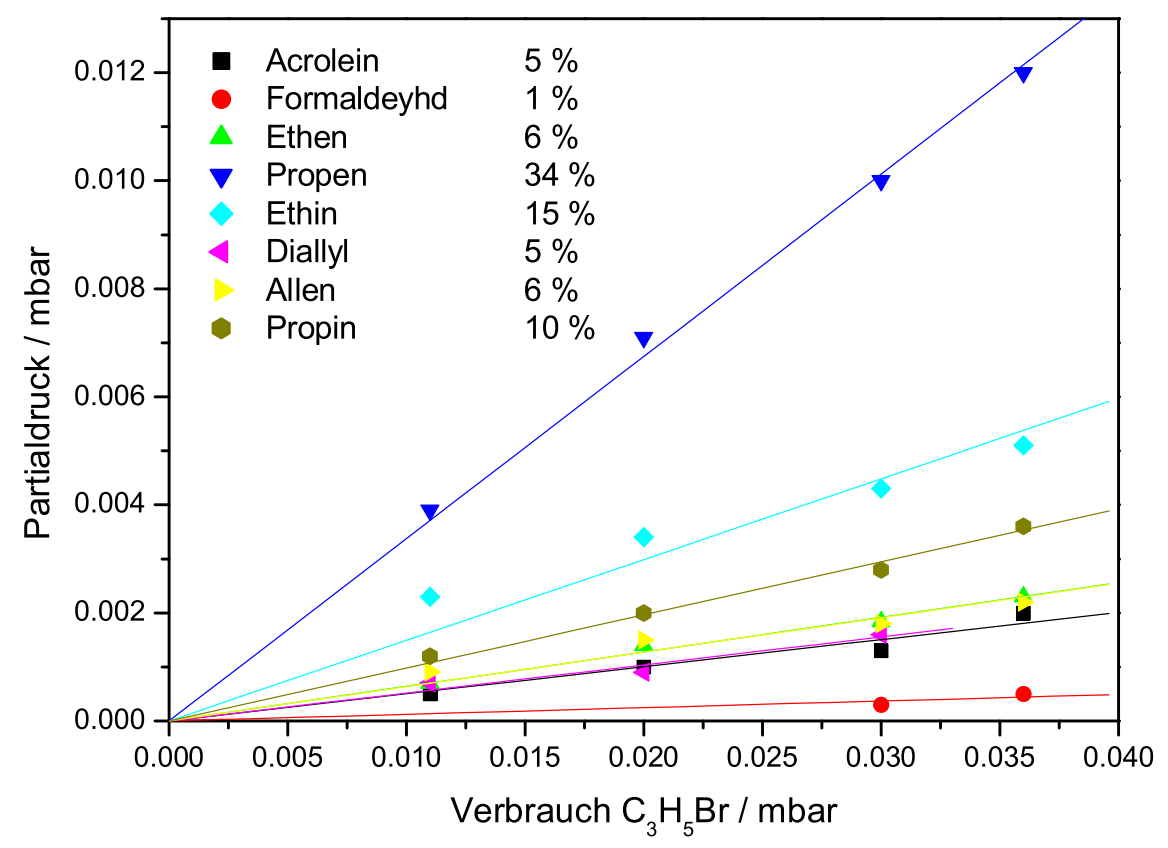

Abbildung 4.68: Reaktion $\mathrm{C}_{3} \mathrm{H}_{5}+\mathrm{C}_{3} \mathrm{H}_{5}$, quantitative Analyse, Vorläufer Allylbromid. $p\left(C_{3} H_{5} \mathrm{Br}\right)=0,3 \mathrm{mbar}, p_{\text {ges }}=4 \mathrm{mbar}, \mathrm{T}=298 \mathrm{~K}$. Die prozentuale Angabe der Produkte bezieht sich auf den Verbrauch des Vorläufers Allylbromid. 
Dabei wurden folgende auf die Abnahme von Allylbromid bezogene Reaktionskanäle festgestellt: Propen 34\%, Propin 10\%, Ethin 15\%, Allen 6\%, Ethen 6\%, Diallyl 5\%. Wie auch bei der Photolyse von Diallyl sind bei dieser Photolyse Produkte der Reaktion Allyl + $\mathrm{O}_{2}$ beobachtet worden.

Bei der Photolyse von Allylchlorid wurden die in Abbildung 4.69 dargestellten Partialdrücke in Abhängigkeit von der Allylchloridabnahme bestimmt. Die Messwerte sind in Tabelle 4.35 dargestellt.

\begin{tabular}{|c||c|c|c|c|c|c|}
\hline $\begin{array}{c}\mathrm{C}_{3} \mathrm{H}_{5} \mathrm{Cl} \\
{[\mathrm{mbar}]}\end{array}$ & $\begin{array}{c}\mathrm{p}\left(\mathrm{C}_{2} \mathrm{H}_{4}\right) \\
{[\mathrm{mbar}]}\end{array}$ & $\begin{array}{c}\mathrm{p}\left(\mathrm{C}_{3} \mathrm{H}_{6}\right) \\
{[\mathrm{mbar}]}\end{array}$ & $\begin{array}{c}\mathrm{p} \text { (Allen) }) \\
{[\mathrm{mbar}]}\end{array}$ & $\begin{array}{c}\mathrm{p}\left(\mathrm{C}_{6} \mathrm{H}_{10}\right) \\
{[\mathrm{mbar}]}\end{array}$ & $\begin{array}{c}\mathrm{p}\left(\mathrm{C}_{2} \mathrm{H}_{2}\right) \\
{[\mathrm{mbar}]}\end{array}$ & $\begin{array}{c}\mathrm{p} \text { (Propin) } \\
{[\mathrm{mbar}]}\end{array}$ \\
\hline \hline 0,017 & $4,6 \mathrm{E}-4$ & - & 0,0012 & 0,003 & $6 \mathrm{E}-4$ & - \\
\hline 0,03 & $6 \mathrm{E}-4$ & 0,0025 & 0,0021 & 0,0046 & 0,00125 & - \\
\hline 0,043 & $9,2 \mathrm{E}-4$ & 0,0032 & 0,0028 & 0,006 & 0,0019 & 0,0012 \\
\hline 0,06 & 0,00138 & 0,0037 & 0,0033 & 0,0082 & 0,0025 & 0,0016 \\
\hline
\end{tabular}

Tabelle 4.35: Reaktion $\mathrm{C}_{3} \mathrm{H}_{5}+\mathrm{C}_{3} \mathrm{H}_{5}$, Vorläufer Allylchlorid, Messwerte. $p$ (Allylchlorid) $=0,3$ mbar, $p_{\text {ges }}=4$ mbar, $\mathrm{T}=298 \mathrm{~K}$, Badgas Argon.

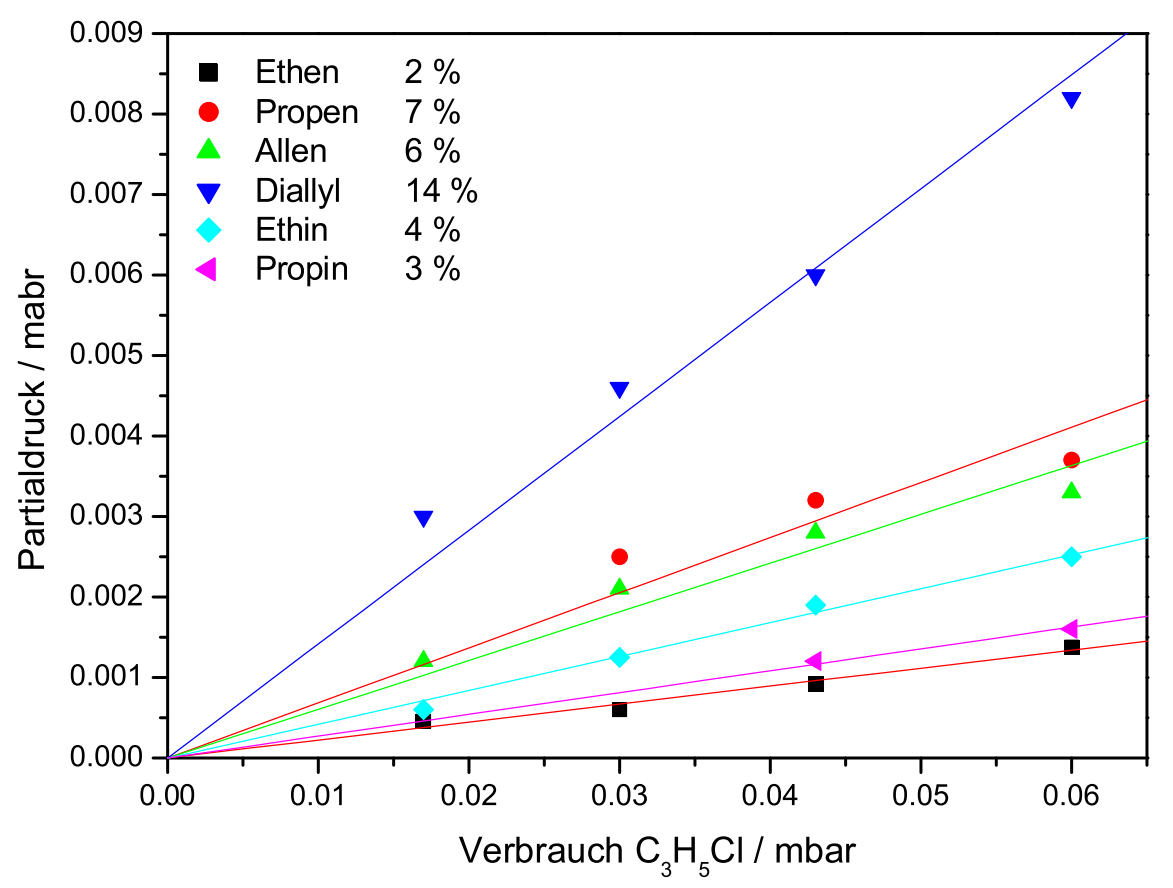

Abbildung 4.69: Reaktion $\mathrm{C}_{3} \mathrm{H}_{5}+\mathrm{C}_{3} \mathrm{H}_{5}$, quantitative Analyse, Vorläufer Allylchlorid. $p\left(\mathrm{C}_{3} \mathrm{H}_{5} \mathrm{Cl}\right)=0,3 \mathrm{mbar}, p_{\text {ges }}=4 \mathrm{mbar}, \mathrm{T}=298 \mathrm{~K}$. Die prozentuale Angabe der Produkte bezieht sich auf den Verbrauch des Vorläufers Allylchlorid. 
Im Gegensatz zu der Verwendung von Diallyl und Allylbromid als Vorläufer konnte Diallyl als Hauptprodukt identifiziert. Diallyl war aber, wie schon eingangs erwähnt, unter Verwendung von Diallyl als Vorläufer nicht detektierbar.

Für einen Vergleich der Reaktion Allyl + Allyl bezüglich der drei verwendeten Vorläufer wurden die Reaktionskanäle jeweils auf den Kanal mit Propen normiert. Der Vergleich ist in Tabelle 4.36 dargestellt. Dabei wurden Produkte, die offensichtlich aus der Reaktion Allyl $+\mathrm{O}_{2}$ stammen, nicht berücksichtigt. Der Vergleich zeigt, dass für die Reaktion Allyl + Allyl eine qualitative Übereinstimmungen für das Kanalverzweigungsverhältnis unter Verwendung verschiedener Vorläufer vorliegt. Dabei stimmen die Ergebnisse für die Vorläufer Diallyl und Allylbromid mit Einschränkungen gut überein. Der Vorläufer Allylchlorid weicht von diesen, speziell für das Verhältnis zu Ethen, relativ stark ab. Bei der Photolyse von Allylchlorid wurden nur geringe Produktpartialdrücke bestimmt, daher würden kleine Abweichungen $(\sim 1 \%)$ schon zu großen Veränderungen der in Tabelle 4.36 angegebenen Werte führen. Des Weiteren ist der Einfluss der bei der Photolyse von Allylchlorid gebildeten Cl-Atome auf das Reaktionssystem sehr hoch. Daher ist die Verwendung dieses Vorläufers für die qualitative Analyse der Reaktion wertvoll, für die Beschreibung der Produktverhältnisse werden aber nur die Ergebnisse der Vorläufer Allylbromid und Diallyl verwendet.

\begin{tabular}{lccc}
\hline Produkt & Allylbromid & Allylchlorid & Diallyl \\
\hline Propen & 1 & 1 & 1 \\
Ethen & 0,18 & 0,29 & 0,16 \\
Allen & 0,18 & 0,86 & 0,31 \\
Ethin & 0,44 & 0,57 & 0,35 \\
Propin & 0,29 & 0,43 & 0,29 \\
Diallyl & 0,15 & 2 & nicht möglich \\
\hline
\end{tabular}

Tabelle 4.36: Reaktion $\mathrm{C}_{3} \mathrm{H}_{5}+\mathrm{C}_{3} \mathrm{H}_{5}$, Vergleich der Vorläufer. Die Tabelle zeigt das Verhältnis der Produkte der Photolyse für die verschiedenen Vorläufer, normiert auf den Propen-Kanal. Die Bestimmung von Diallyl war bei Wahl von Diallyl als Vorläufer nicht möglich. $p_{g e s}=4$ mbar, $\mathrm{T}=298 \mathrm{~K}$. 


\subsubsection{Druckabhängigkeit}

Für die Reaktion Allyl + Allyl wurde die Druckabhängigkeit der Reaktion in einem Bereich von 0,3 - 1000 mbar untersucht. Dabei wurde eine Änderung des Reaktionsmechanismus festgestellt. In Abbildung 4.70 sind die auf den Partialdruck von Propen normierten Partialdrücke der identifizierten Produkte dargestellt. Dabei wurde als Vorläufer Allylbromid verwendet, da bei diesem, im Vergleich zu den anderen Vorläufern, kaum Nebenreaktionen auftraten. Die Suche nach einem Mechanismuswechsel konnte weiterhin nur unter der Voraussetzung durchgeführt werden, dass sowohl das Kombinationsprodukt als auch die Disproportionierungsprodukte quantitativ bestimmt werden können. Dies ist unter Verwendung des Vorläufers Diallyl nicht gegeben.

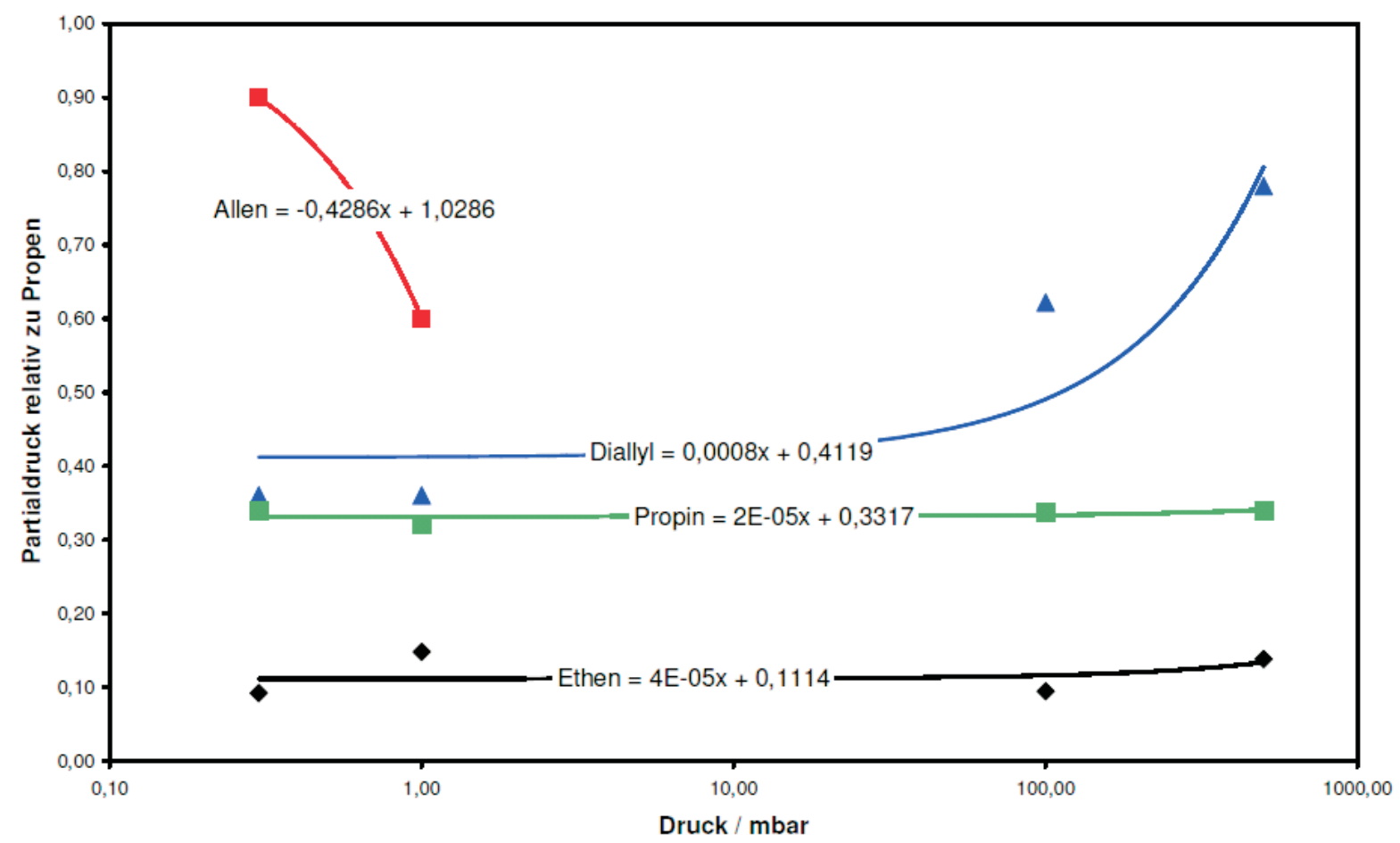

Abbildung 4.70: Reaktion $\mathrm{C}_{3} \mathrm{H}_{5}+\mathrm{C}_{3} \mathrm{H}_{5}$, Druckabhängigkeit der Reaktion. Vorläufer Allylbromid.

Wie die Abbildung 4.70 zeigt, ist bei niedrigen Drücken ein hoher Allenanteil nachweisbar. Die Bildung von Allen nimmt mit steigendem Druck schnell ab. Ab einem Gesamtdruck von 10 mbar ist Allen nicht mehr quantitativ nachweisbar. Der Anteil an Diallyl nimmt dagegen mit steigendem Druck linear zu. Lineare Regressionen für die quantitativ 
bestimmten Produkte sind in der Abbildung 4.70 zusätzlich eingezeichnet. Dabei sollte beachtet werden, dass es sich um eine halb-logarithmische Auftragung handelt und so die Geraden in diesem Fall einen ,gebogenen“ Verlauf aufweisen. Betrachtet man die in der Abbildung angegebenen linearen Anpassung der Daten, so wird ersichtlich, dass sich die Anteile für Ethen und Propin innerhalb des untersuchten Druckbereichs kaum ändern, der Anteil an Diallyl aber um den Faktor 3 zunimmt.

Diese Beobachtungen machen deutlich, dass für ein vollständiges Verständnis dieser Reaktion weitere theoretische Untersuchungen notwendig sind. Diese Druckabhängigkeit deutet aber auf die Bildung eines aktivierten Komplexes $\left(\mathrm{C}_{3} \mathrm{H}_{5}\right)_{2}^{\star}$ hin, der bei niedrigen Drücken schnell zerfällt, bei hohen Drücken dagegen stabilisiert wird.

\subsubsection{Diskussion}

Die Reaktion Allyl + Allyl wurde unter Verwendung verschiedener Vorläufer untersucht (Allylbromid, Allylchlorid, Diallyl). Dabei wurden qualitativ übereinstimmende Ergebnisse erhalten. Eine quantitative Übereinstimmung aller Produktkanäle war aber nicht für alle Vorläufer gegeben. Vor allem der Vorläufer Allylchlorid lieferte von den beiden anderen Vorläufern abweichende Ergebnisse. Dies kann an der schnellen Reaktion von Allylradikalen mit Cl-Atomen liegen. Im Gegensatz zu der Reaktion von Allyl + Br, bei der vorrangig die Rückbildung von Allylbromid stattfindet $\left(k \sim 1,2 \times 10^{14} \mathrm{~cm}^{3} /(\mathrm{mol} \cdot \mathrm{s})\right)$, konkurrieren bei der Reaktion von Allyl $+\mathrm{Cl}$ die Addition zu Allylchlorid und die Abstraktion zu Allen und $\mathrm{HCl}$. Zwar liegt für die Abstraktion keine Geschwindigkeit in der Literatur vor, doch deutet die Abstraktion für $\mathrm{Cl}+\mathrm{C}_{3} \mathrm{H}_{6} \rightarrow \mathrm{C}_{3} \mathrm{H}_{5}+\mathrm{HCl}$ mit k( $\mathrm{Cl}+$ $\left.\mathrm{C}_{3} \mathrm{H}_{6}\right)=2,19 \times 10^{13} \mathrm{~cm}^{3} /(\mathrm{mol} \cdot \mathrm{s})$ bei $\mathrm{T}=300 \mathrm{~K}$ und niedrigem Druck ( $\leq 10$ Torr $)$ auf einen schnellen Abstraktionsprozess für die Reaktion von Allylradikalen mit ChlorAtomen hin [Kai96]. Dies würde auch den gegenüber den anderen Vorläufern höheren Allenanteil erklären. Auf Grund dieser Sekundärreaktion wird für den Vorschlag eines Verzweigungsverhältnisses der Vorläufer Allylchlorid nicht berücksichtigt. Die Untersuchung der Druckabhängigkeit der Reaktion zeigt, dass schon in dem durch die Apparatur zugänglichen Druckbereich ein Mechanismuswechsel beobachtet werden kann. Für den Niederdruckbereich (4 mbar) wird für die Reaktion Allyl + Allyl folgende Kanalverzweigung vorgeschlagen: 


$$
\begin{array}{rlr}
\mathrm{C}_{3} \mathrm{H}_{5}+\mathrm{C}_{3} \mathrm{H}_{5} & \longrightarrow \mathrm{C}_{6} \mathrm{H}_{10} & (20 \pm 5) \% \\
& \longrightarrow \mathrm{H}_{2} \mathrm{CCCH}_{2}+\mathrm{C}_{3} \mathrm{H}_{6} & (40 \pm 5) \% \\
& \longrightarrow \mathrm{HCCCH}_{3}+\mathrm{C}_{3} \mathrm{H}_{6} & (40 \pm 5) \%
\end{array}
$$

Die Ergebnisse dieser Arbeit werden im folgenden mit Ergebnissen aus der Literatur verglichen.

Tulloch et al. verwendeten für die Untersuchung der Reaktion als Vorläufer Diallyl, welches mit Hilfe eines Exciplex-Lasers bei einer Wellenlänge von $\lambda=193 \mathrm{~nm}$ photolytisch gespalten wurde. Die Produktanalyse wurde mittels Gaschromatographie durchgeführt. Die Ergebnisse der photolytischen Spaltung sind in Abschnitt 4.4.5.2 dargestellt. Tulloch et al. geben für den Kanal, der zur Bildung von 2 Allylradikalen führt, einen Anteil von 68\% in Bezug auf die Abnahme an Diallyl an [Tul82]. Eine Bestimmung der Kanalverzweigung für die Reaktion Allyl + Allyl wurde aber nicht durchgeführt. Die Geschwindigkeit der Kombination wird in einem Temperaturbereich von $293 \mathrm{~K}$ - 571 K bei einem Gesamtdruck zwischen 60 mbar und 600 mbar mit $k=(1,02 \pm 0,02) \times 10^{13} \mathrm{~cm}^{3} /(\mathrm{mol} \cdot \mathrm{s})$ angegeben.

JENKIN et al. nutzten für die Untersuchung der Reaktion eine Laser-Photolyse-Technik und Detektion mit Hilfe von UV-Absorptions-Spektroskopie bei 296 K und einem Druck von ca. 1 bar [Jen93]. Radikale wurden aus Diallyl / $\mathrm{N}_{2^{-}}$bzw. Allyliodid / $\mathrm{N}_{2}$-Mischungen bei einer Wellenlänge von $\lambda=193 \mathrm{~nm}$ bzw. $\lambda=248 \mathrm{~nm}$ erzeugt. Durch das Experiment wurde die Geschwindigkeit der Kombination zu $k=(1,8 \pm 0,3) \times 10^{13} \mathrm{~cm}^{3} /(\mathrm{mol} \cdot \mathrm{s})$ bestimmt. Zur Beschreibung der UV-Signale wurde eine Reaktion 2. Ordnung zu Grunde gelegt, die von der Kombination dominiert sein soll. Eine Untersuchung der Kanalverzweigung ist hier ebenfalls nicht zu finden. Die Bildung von Allen wird unter Verwendung von Allyliodid als Vorläufer allerdings bestätigt $(\leq 1 \%)$, doch wird diese der direkten HI-Elimination zu geschrieben.

BOYD et al. untersuchten die Kinetik der Kombination unter Bildung von Diallyl in einem Temperaturbereich von 400 - 540 K mittels Laserphotolyse von Diallyl. Dabei wurde nur von einem Reaktionskanal zu Diallyl ausgegangen. Der Verbrauch 2. Ordnung wurde durch UV Absorption detektiert. Bei einem Gesamtdruck von 1 bar wurde eine mittlere Geschwindigkeit der Rekombination von $k=1,6 \times 10^{13} \mathrm{~cm}^{3} /(\mathrm{mol} \cdot \mathrm{s})$ gefunden.

BAulch et al. empfehlen für Disproportionierungsreaktion zu Propen und Allen eine Ge- 
schwindigkeit von $k=6 \times 10^{10} \mathrm{~cm}^{3} /(\mathrm{mol} \cdot \mathrm{s})$ [Bau05]. Legt man für die Geschwindigkeit der Kombination eine Wert von $k=1,4 \times 10^{13} \mathrm{~cm}^{3} /(\mathrm{mol} \cdot \mathrm{s})$ zu Grunde, so folgt für das Verzweigungsverhältnis nach BAULCH $k(d) / k(c)=0,004$.

Die experimentellen Ergebnisse dieser Arbeit liefern das Verhältnis $k(d) / k(c)=4$ für einen Gesamtdruck von 4 mbar. Die Untersuchung der Druckabhängigkeit zeigt aber, dass bei höheren Drücken ( $\geq 500$ mbar) die Bildung von Diallyl favorisiert wird $k(d) / k(c) \leq 1$. Daher stehen die experimentellen Ergebnisse dieser Arbeit nicht im Widerspruch zu den Ergebnissen in der Literatur, doch ist auch bei hohen Drücken die Bildung von Propen deutlich zu erkennen, die auf eine Disproportionierungsreaktion hindeutet. Allgemein wird der Disproportionierungsanteil in der vorliegenden Literatur systematisch unterschätzt. Die Ergebnisse dieser Arbeit deuten also wie die Resultate der Literatur daraufhin, dass bei der Reaktion ein aktivierter Komplex $\left(\mathrm{C}_{3} \mathrm{H}_{5}\right)_{2}^{\star}$ gebildet wird, der bei niedrigen Drücken schnell zu den Disproportionierungsprodukten zerfällt und bei hohen Drücken stabilisiert werden kann. 


\subsection{Untersuchung der Kreuzreaktionen von Methyl- radikalen mit Kohlenwasserstoffradikalen}

Radikal-Radikal Kreuzkombinationsreaktionen spielen eine entscheidenden Rolle bei der Oxidation und Pyrolyse von Kohlenwasserstoffen [Tsa86] und bei der Terminierung von Makroradikalen und Initiatorradikalen bei der radikalischen Polymerisation. Da für die Kreuzkombinationsreaktionen aber das Verzweigungsverhältnis oft schwierig zu bestimmen ist und entsprechende Daten daher in der Literatur oft unter Verwendung der geometric mean rule abgeschätzt werden, ist ein Ziel dieser Arbeit, das Verzweigungsverhältnis für die Kreuzkombination kleiner unverzweigter und verzweigter Alkylradikale zu bestimmen und die Gültigkeit der geometric mean rule zu prüfen. Die geometric mean rule lautet:

$$
k_{A B}=2 \cdot \sqrt{k_{A A} k_{B B}},
$$

wobei $k_{A B}$ den Geschwindigkeitskoeffizienten der Reaktion $\mathrm{A}+\mathrm{B}$ und $k_{A A}$ bzw. $k_{B B}$ die Geschwindigkeitskoeffizienten der Reaktion A + A bzw. B + B bezeichnen.

Im Folgenden werden die Ergebnisse der Radikal-Radikal Kreuzkombinationsreaktionen für Methyl $+\mathrm{R}$ vorgestellt $\left(\mathrm{R}=\mathrm{C}_{2} \mathrm{H}_{5}, 1-\mathrm{C}_{3} \mathrm{H}_{7}, 2-\mathrm{C}_{3} \mathrm{H}_{7}\right.$ und $\left.1-\mathrm{C}_{4} \mathrm{H}_{9}\right)$.

\subsubsection{Die Reaktion Methyl + Ethyl}

Die Reaktion von Methylradikalen mit Ethylradikalen wurde schon mehrfach untersucht Kny01. Im Rahmen der vorliegenden Dissertation wurde diese Reaktion erneut untersucht, da mit der quantitativen Analyse von FTIR-Spektren eine in der Literatur bisher nicht verwendete Analysemethode zur Verfügung steht. Für das System $\mathrm{CH}_{3} / \mathrm{C}_{2} \mathrm{H}_{5}$ ist dabei folgender Reaktionsmechanismus denkbar: 


$$
\begin{array}{rlr}
\mathrm{CH}_{3}+\mathrm{C}_{2} \mathrm{H}_{5} & \longrightarrow \mathrm{C}_{3} \mathrm{H}_{8} & \left(x_{1}\right) \\
& \longrightarrow \mathrm{CH}_{4}+\mathrm{C}_{2} \mathrm{H}_{4} & \left(x_{2}\right) \\
& \longrightarrow \mathrm{CH}_{2}+\mathrm{C}_{2} \mathrm{H}_{6} & \left(x_{3}\right) \\
\mathrm{CH}_{3}+\mathrm{CH}_{3} & \longrightarrow \mathrm{C}_{2} \mathrm{H}_{6} & \left(b_{1}\right) \\
& \longrightarrow \mathrm{CH}_{4}+\mathrm{CH}_{2} & \left(b_{2}\right) \\
\mathrm{C}_{2} \mathrm{H}_{5}+\mathrm{C}_{2} \mathrm{H}_{5} & \longrightarrow \mathrm{C}_{4} \mathrm{H}_{10} & \left(c_{1}\right) \\
& \longrightarrow \mathrm{C}_{2} \mathrm{H}_{4}+\mathrm{C}_{2} \mathrm{H}_{6} & \left(c_{2}\right)
\end{array}
$$

Dabei sind Produkte der Kreuzreaktionskanäle $\left(x_{i}\right)$ teilweise identisch mit Produkten der Kombinations- bzw. Disproportionierungsreaktionen $\left(b_{i}\right)$ und $\left(c_{i}\right)$. Der Einsatz von vollständig deuteriertem $\mathrm{CD}_{3}$ (Vorläufer Aceton- $\mathrm{d}_{6}$ ) ermöglichte aber die Unterscheidung von Ethan aus den Kanälen $\left(\mathrm{x}_{3}\right),\left(\mathrm{b}_{1}\right)$ und $\left(\mathrm{c}_{2}\right)$.

\subsubsection{Qualitative Analyse}

Als Methylradikalquelle wurde vollständig deuteriertes Aceton eingesetzt. Der Vorteil bei dem Einsatz von deuteriertem Aceton besteht, wie schon in Abschnitt 3.2.1 beschrieben, in der Lage der Absorptionsbanden von deuteriertem Ethan und der Kreuzkombinationsprodukte. Dadurch sind diese Produkte eindeutig von den Produkten der Reaktion Ethyl + Ethyl zu unterscheiden.

Zwar lagen für die teildeuterierten Kreuzkombinationsprodukte größtenteils keine Reinspektren vor, doch konnten diese durch Vergleich mit Tabellen [Shi72], in denen die zugehörigen (infrarotaktiven) Molekülschwingungen aufgeführt sind, identifiziert werden. Eine quantitative Analyse dieser Produkte wurde auf Grund einer Kohlenstoffmassenbilanz erreicht. Eine Kohlenstoffmassenbilanz ist durch die eindeutige Identifikation und quantitative Analyse der undeuterierten und vollständig deuterierten Kohlenwasserstoffe möglich.

Als Radikalvorläufer für Ethylradikale wurde Ethyliodid verwendet. Der Einsatz von Ethyliodid hatte allerdings den Nachteil der direkten HI-Elimination unter Bildung eines Alkens (hier Ethen), der bei allen iodierten Kohlenwasserstoffen zu beobachten ist. Durch eine Quantifizierung dieses Anteils konnte dieser Nachteil aber gemindert werden. 
Des Weiteren wurde das Verhältnis der Anfangskonzentrationen der Ethyl- und Methylradikale durch Variation der Radikalvorläuferkonzentrationen in einem gewissen Rahmen verändert. Alle Messungen wurden bei Zimmertemperatur und einem Gesamtdruck von 4 mbar druchgeführt. Die Analyse erfolgte von 100-fach gemittelten IR-Produktspektren nach jeweis 100 Photolysen. Die maximale Photolysenanzahl betrug höchstens 400, um eine Sekundärphotolyse zuvor gebildeter Produkte zu vermeiden. Der prozentuale Umsatz an Radikalvorläufern betrug nach maximaler Photolysenanzahl ca. 10\%.

Abbildung 4.71 zeigt ein Reaktionsspektrum der Vorläufer Mischung Aceton- $\mathrm{d}_{6}$ / Ethyliodid $=0,2 / 0,55$ mbar nach 400 Photolysen bei einem Gesamtdruck von 4 mbar und einer Temperatur von $298 \mathrm{~K}$.

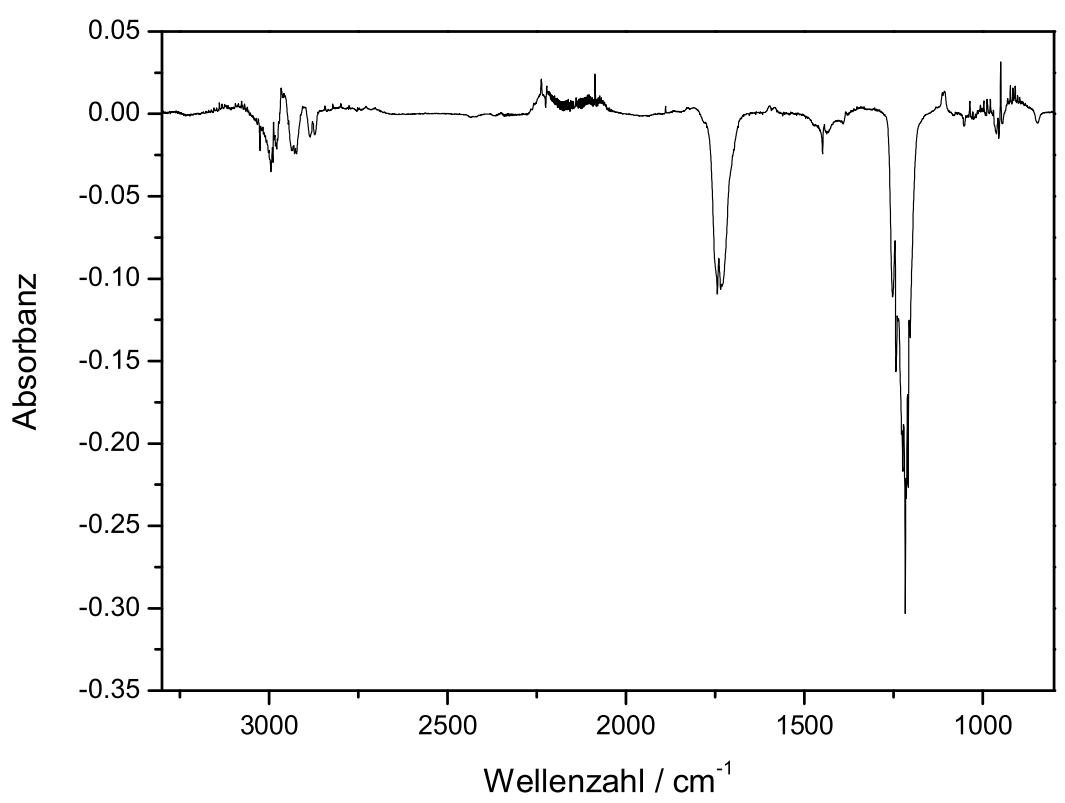

Abbildung 4.71: Reaktionssystem $\mathrm{CH}_{3} / \mathrm{C}_{2} \mathrm{H}_{5}$, Reaktionsspektrum. $p$ (Aceton- $\left.\mathrm{d}_{6}\right)=0,2$ mbar, $p($ Ethyliodid $)=0,55$ mbar, $p_{\text {ges }}=4$ mbar, $T=298 \mathrm{~K}$.

Die Produkte wurden anhand von charakteristischen Banden identifiziert. In Abbildung 4.72 sind Ausschnitte des Reaktionsspektrums im Vergleich mit Spektren der Reinsubstanzen angegeben. In der Abbildung sind sowohl das Kombinationsprodukt der Reaktion $\mathrm{CD}_{3}$ $+\mathrm{CD}_{3}\left(\right.$ Ethan- $\left.\mathrm{d}_{6}\right)$, als auch das der Reaktion $\mathrm{C}_{2} \mathrm{H}_{5}+\mathrm{C}_{2} \mathrm{H}_{5}$ (Butan) zu erkennen. Für die Identifikation des Kreuzkombinationsprodukts $\mathrm{CD}_{3} \mathrm{C}_{2} \mathrm{H}_{5}$ stand kein Vergleichsspektrum zur Verfügung. Eine quantitative Bestimmung des Kreuzkombinationskanals erfolgt somit 
auf Grundlage einer Massenbilanz.
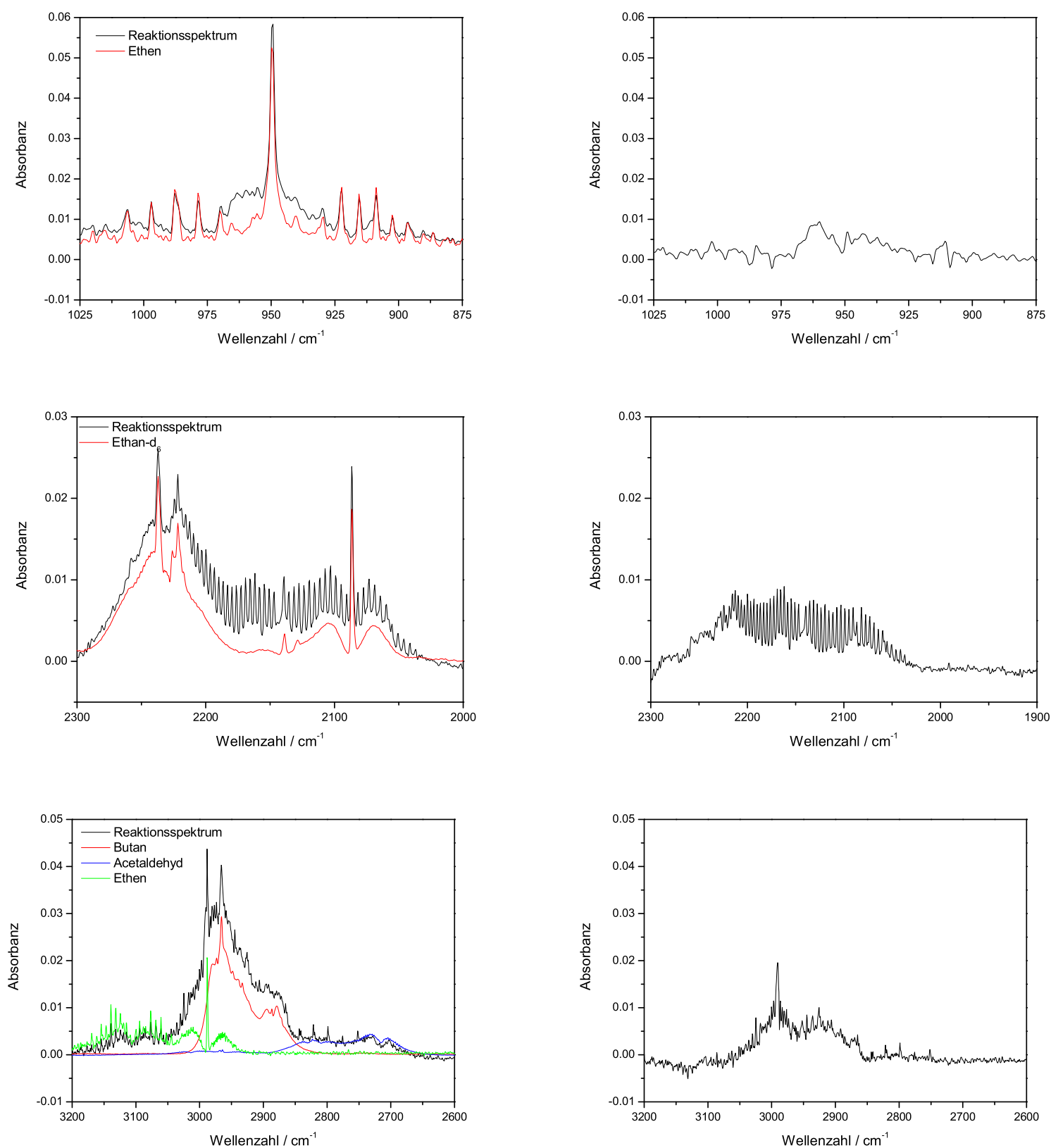

Abbildung 4.72: Reaktionssystem $\mathrm{CH}_{3} / \mathrm{C}_{2} \mathrm{H}_{5}$, qualitative Analyse. $p\left(\right.$ Aceton- $\left.d_{6}\right)=0,2$ mbar, $p($ Ethyliodid $)=0,55$ mbar, $p_{\text {ges }}=4$ mbar $T=298 \mathrm{~K}$. Vergleich des Reaktionsspektrums mit Spektren der Reinsubstanzen in vergrößerten Ausschnitten; in der linken Spalte ist jeweils das Reaktionsspektrum (schwarz) mit den Reinspektren (farbig) im Vergleich dargestellt, in der rechten Spalte sind die entsprechenden Residuen in schwarz dargestellt.

Neben der Bildung der Kombinationsprodukte können noch Ethen und Acetaldehyd identifiziert werden. Dabei ist die Bildung von Ethen auf die direkte HI-Elimination von Ethyliodid zurückzuführen. Acetaldehyd resultiert aus der Reaktion von Ethylradikalen mit 
$\mathrm{O}_{2}$. Der Anteil der HI-Elimination für die Photolyse von Ethyliodid wurde in Abschnitt 4.4.2 zu 39\% in Bezug zu der Abnahme an Ethyliodid bestimmt.

\subsubsection{Quantitative Analyse}

Für die Reaktion von Methylradikalen mit Ethylradikalen wurden verschiedene Vorläuferverhältnisse getestet. Eine quantitative Bestimmung der Produkte aus beiden Kombinationsreaktionen war am besten bei einer Vorläuferzusammensetzung von $\mathrm{p}\left(\right.$ Aceton- $\left.\mathrm{d}_{6}\right)=0,2$ mbar, $\mathrm{p}($ Ethyliodid $)=0,55$ mbar möglich. Mit der in Abschnitt 4.4.1. und 4.4.2 beschriebenen Untersuchungen der Photolyse der Vorläufer, die gezeigt haben, dass die Selbstreaktionen ausschließlich durch Kombination erklärt werden können und unter Berücksichtigung des Oxidationsanteils für Ethylradikale zu Acetaldehyd wurde die Summe der nach 400 Photolysen gebildeten Radikale zu p $0\left(\mathrm{CD}_{3}\right)=0,078$ mbar und $\mathrm{p}_{0}\left(\mathrm{C}_{2} \mathrm{H}_{5}\right)=0,063$ mbar bestimmt. Der Ethan- $\mathrm{d}_{6}$-Partialdruck betrug bei diesem Experiment $\mathrm{p}\left(\right.$ Ethan- $\left.\mathrm{d}_{6}\right)$ $=0,013$ mbar und der Butan-Partialdruck $\mathrm{p}($ Butan $)=0,007$ mbar. Mit diesen Werten konnte der erwartete Partialdruck des Kreuzkombinationsprodukts $\mathrm{CD}_{3} \mathrm{C}_{2} \mathrm{H}_{5}$ bezüglich der Radikalanfangskonzentrationen $\left[\mathrm{CD}_{3}\right]_{0}$ und $\left[\mathrm{C}_{2} \mathrm{H}_{5}\right]_{0}$ berechnet werden. Dazu wurden die jeweiligen Radikalanfangsdrücke mit den Partialdrücken an Ethan- $\mathrm{d}_{6}$ bzw. an Butan korrigiert:

$$
\begin{aligned}
{\left[\mathrm{CD}_{3} \mathrm{C}_{2} \mathrm{H}_{5}\right]_{\mathrm{CD}_{3}} } & =\left[\mathrm{CD}_{3}\right]_{0}-2 \cdot\left[\mathrm{C}_{2} \mathrm{D}_{6}\right] \\
{\left[\mathrm{CD}_{3} \mathrm{C}_{2} \mathrm{H}_{5}\right]_{\mathrm{C}_{2} \mathrm{H}_{5}} } & =\left[\mathrm{C}_{2} \mathrm{H}_{5}\right]_{0}-2 \cdot\left[\mathrm{C}_{4} \mathrm{H}_{10}\right]
\end{aligned}
$$

Die Ergebnisse sind in der nachfolgenden Tabelle 4.37 dargestellt:

\begin{tabular}{|c|c|c|}
\hline$\left[\mathrm{CD}_{3}\right]_{0}$ & {$\left[\mathrm{C}_{2} \mathrm{D}_{6}\right]$} & {$\left[\mathrm{CD}_{3} \mathrm{C}_{2} \mathrm{H}_{5}\right]_{\mathrm{CD}_{3}}$} \\
\hline 0,078 & 0,013 & 0,052 \\
\hline \hline$\left[\mathrm{C}_{2} \mathrm{H}_{5}\right]_{0}$ & {$\left[\mathrm{C}_{4} \mathrm{H}_{10}\right]$} & {$\left[\mathrm{CD}_{3} \mathrm{C}_{2} \mathrm{H}_{5}\right]_{\mathrm{C}_{2} \mathrm{H}_{5}}$} \\
\hline 0,063 & 0,007 & 0,049 \\
\hline
\end{tabular}

Tabelle 4.37: Reaktionssystem $\mathrm{CH}_{3} / \mathrm{C}_{2} \mathrm{H}_{5}$, quantitative Auswertung. Die Tabelle zeigt die aus der Abnahme der Vorläufer und unter Berücksichtigung von Nebenreaktionen bestimmten Radikalanfangskonzentrationen und die Konzentrationen der Kombinationsprodukte. Der zur Abnahme fehlende Anteil wurde jeweils der Kreuzkombination zugerechnet. Die gute Übereinstimmung der beiden Ergebnisse bestätigt diese Methode. Meßbedingungen: $p\left(\right.$ Aceton- $\left.d_{6}\right)=0,2$ mbar, $p($ Ethyliodid $)=0,55$ mbar, $p_{\text {ges }}=4 \mathrm{mbar}, T=298 \mathrm{~K}$. Alle Angaben sind in mbar. 
Die sehr gute Übereinstimmung der in Tabelle 4.37 berechneten Werte für das Kreuzkombinationsprodukt bezüglich der Radikale $\mathrm{CD}_{3}$ und $\mathrm{C}_{2} \mathrm{H}_{5}$ verdeutlichen sowohl die Genauigkeit der quantitativen Analyse mit Hilfe der FTIR-Apparatur als auch die Anwendbarkeit dieser Methode für die Bestimmung von Kreuzreaktionsgeschwindigkeiten. Alternativ hätte an Stelle dieser Methode auch eine Berechnung der Radikalanfangskonzentrationen über die Absorptionsquerschnitte der Vorläufer erfolgen können. Die mit der hier verwendeten Methode berechneten Werte rechtfertigen aber die dargestellte Vorgehensweise.

Der H-Abstraktionskanal der Reaktion $\mathrm{CD}_{3}+\mathrm{C}_{2} \mathrm{H}_{5} \rightarrow \mathrm{CD}_{3} \mathrm{H}+\mathrm{C}_{2} \mathrm{H}_{4}$ wurde nicht beobachtet und geht somit nicht in die quantitative Analyse ein.

\subsubsection{Simulation}

Auf Grund der Ergebnisse der quantitativen Analyse wurde eine Simulation des Reaktionssystems $\mathrm{CD}_{3} / \mathrm{C}_{2} \mathrm{H}_{5}$ durchgeführt. Dabei wurden folgende Radikalanfangskonzentrationen verwendet $\left[\mathrm{CD}_{3}\right]_{0}=7,8 \times 10^{-10} \mathrm{~mol} / \mathrm{cm}^{3},\left[\mathrm{C}_{2} \mathrm{H}_{5}\right]_{0}=6,3 \times 10^{-10} \mathrm{~mol} / \mathrm{cm}^{3}$. Die verwendeten Geschwindigkeitskoeffzienten und Reaktionen sind in Tabelle 4.38 angegeben.

\begin{tabular}{lll}
\hline Reaktion & & $\mathrm{cm}^{3} /(\mathrm{mol} \cdot \mathrm{s})$ \\
\hline $\mathrm{CD}_{3}+\mathrm{CD}_{3} \rightarrow \mathrm{C}_{2} \mathrm{D}_{6}+$ & $\mathrm{k}=2.40 \mathrm{e} 13$ \\
$\mathrm{CD}_{3}+\mathrm{C}_{2} \mathrm{H}_{5} \rightarrow \mathrm{CD}_{3} \mathrm{C}_{2} \mathrm{H}_{5}+$ & $\mathrm{k}=7.40 \mathrm{e} 13$ \\
$\mathrm{CD}_{3}+\mathrm{C}_{2} \mathrm{H}_{5} \rightarrow \mathrm{CD}_{3} \mathrm{H}+\mathrm{C}_{2} \mathrm{H}_{4}$ & $\mathrm{k}=9.00 \mathrm{e} 11$ \\
$\mathrm{C}_{2} \mathrm{H}_{5}+\mathrm{C}_{2} \mathrm{H}_{5} \rightarrow \mathrm{C}_{4} \mathrm{H}_{10}+$ & $\mathrm{k}=1.10 \mathrm{e} 13$ \\
$\mathrm{C}_{2} \mathrm{H}_{5}+\mathrm{C}_{2} \mathrm{H}_{5} \rightarrow \mathrm{C}_{2} \mathrm{H}_{4}+\mathrm{C}_{2} \mathrm{H}_{6}$ & $\mathrm{k}=1.20 \mathrm{e} 12$ \\
\hline
\end{tabular}

Tabelle 4.38: Reaktionssystem $\mathrm{CD}_{3} / \mathrm{C}_{2} \mathrm{H}_{5}$, Reaktionen und Geschwindigkeitskoeffizienten für die Simulation. $\left[\mathrm{CD}_{3}\right]_{0}=7,8 \times 10^{-10} \mathrm{~mol} / \mathrm{cm}^{3},\left[C_{2} H_{5}\right]_{0}=6,3 \times 10^{-10} \mathrm{~mol} / \mathrm{cm}^{3}$.

Ein Konzentrations-Zeit-Profil unter Verwendung der Daten aus Tabelle 4.38 zeigt Abbildung 4.73 . 


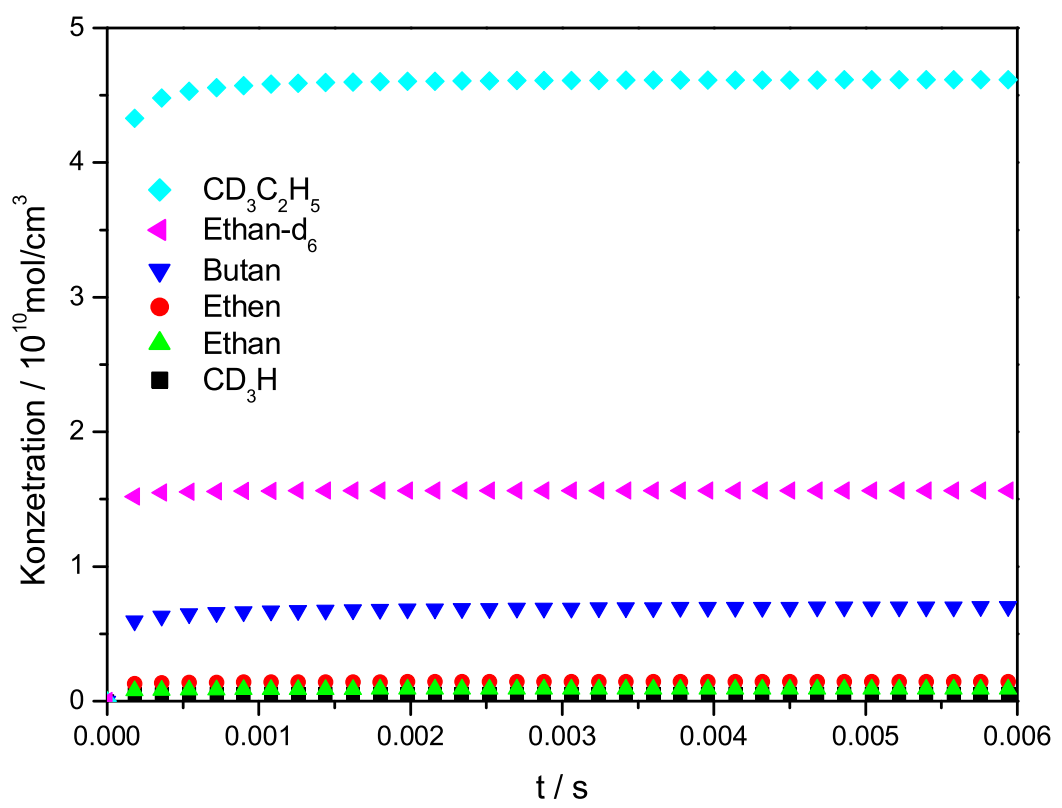

Abbildung 4.73: Reaktionssystem $\mathrm{CH}_{3} / \mathrm{C}_{2} \mathrm{H}_{5}$, Simulation der Konzentrations-Zeit-Profile. $p\left(\right.$ Aceton- $\left.d_{6}\right)=0,2$ mbar, $p($ Ethyliodid $)=0,55 \mathrm{mbar}, p_{\text {ges }}=4 \mathrm{mbar}, \mathrm{T}=298 \mathrm{~K}$. Die übrigen Daten befinden sich in Tabelle 4.38 .

Für eine leichtere Vergleichbarkeit sind die experimentell bestimmten Anteile zusammen mit den Ergebnissen der Simulation in Tabelle 4.39 dargestellt.

\begin{tabular}{lcc}
\hline Substanz & Experiment & Simulation \\
\hline Ethan- $\mathrm{d}_{6}$ & 1 & 1 \\
Butan & 0,54 & 0,45 \\
$\mathrm{CD}_{3} \mathrm{C}_{2} \mathrm{H}_{5}$ & 3,8 & 3 \\
\hline
\end{tabular}

Tabelle 4.39: Reaktionssystem $\mathrm{CH}_{3} / \mathrm{C}_{2} \mathrm{H}_{5}$, Vergleich Experiment mit Simulation. Für eine bessere Vergleichbarkeit sind die Anteile der Produkte auf das Produkt Ethan-d $d_{6}$ normiert.

Mit den in Tabelle 4.38 angegebenen Geschwindigkeitskoeffizienten wurde die beste Übereinstimmung zwischen Experiment und Simulation erzielt. Allerdings ist die Reaktion sehr empfindlich auf das Verhältnis der Anfangskonzentrationen. Simulationen für die von den experimentell bestimmten Anfangskonzentrationen abweichenden Radikalkonzentrationen sind in Abbildung 4.74 dargestellt. 


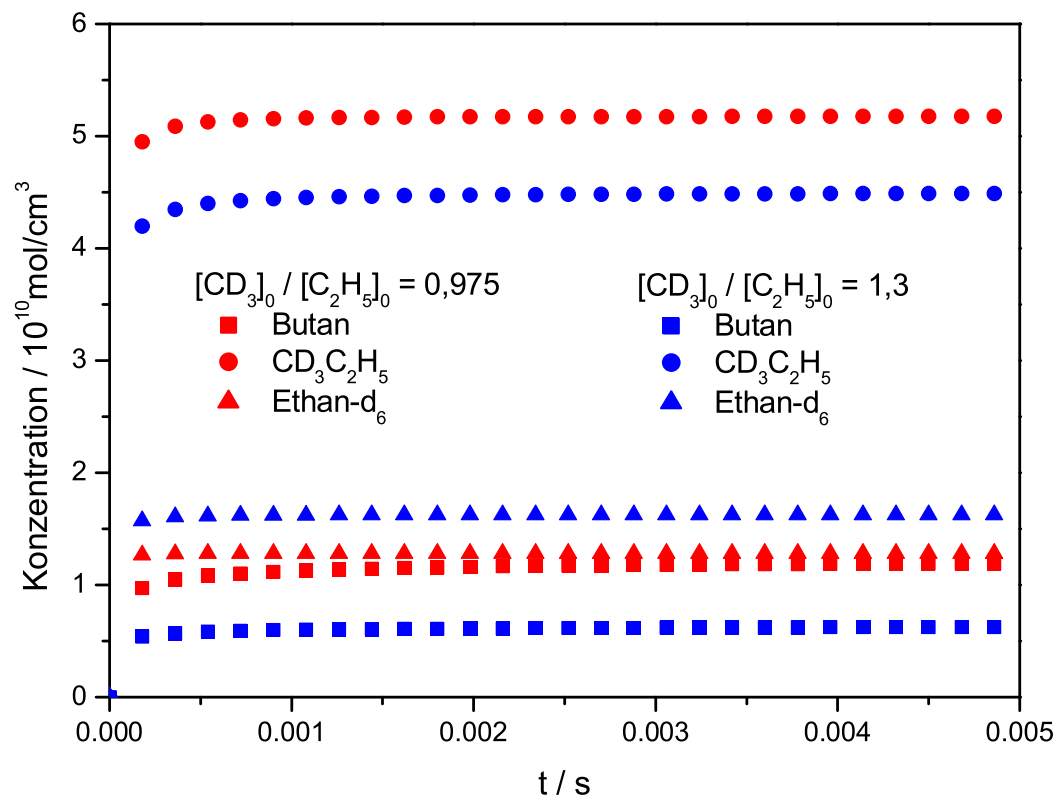

Abbildung 4.74: Reaktionssystem $\mathrm{CH}_{3} / \mathrm{C}_{2} \mathrm{H}_{5}$, Simulation der Konzentrations-Zeit-Profile für verschiedene Radikalkonzentrationen. $p\left(\right.$ Aceton $\left.d_{6}\right)=0,2$ mbar, $p($ Ethyliodid $)=0,55$ mbar, $p_{\text {ges }}$ $=4 \mathrm{mbar}, \mathrm{T}=298 \mathrm{~K}$. Die übrigen Daten befinden sich in Tabelle 4.38.

Für die in Abbildung 4.74 dargestellten Konzentrations-Zeit-Profile ergaben sich die in Tabelle 4.40 dargestellten Kanalverhältnisse.

\begin{tabular}{lccc}
\hline Substanz & {$\left[\mathrm{CD}_{3}\right]_{0} /\left[\mathrm{C}_{2} \mathrm{H}_{5}\right]_{0}=0,975$} & {$\left[\mathrm{CD}_{3}\right]_{0} /\left[\mathrm{C}_{2} \mathrm{H}_{5}\right]_{0}=1,3$} & Experiment \\
\hline Ethan- $\mathrm{d}_{6}$ & 1 & 1 & 1 \\
$\mathrm{CD}_{3} \mathrm{C}_{2} \mathrm{H}_{5}$ & 4 & 2,8 & 3,8 \\
Butan & 0,93 & 0,38 & 0,54
\end{tabular}

Tabelle 4.40: Reaktion Methyl + Ethyl, Vorläufersensitivität. Die angegebenen Werte zeigen die Sensitivität der Reaktion bezüglich der Anfangskonzentrationen der Radikale bei unveränderten Geschwindigkeiten.

\subsubsection{Diskussion}

Mit den Ergebnissen der quantitativen Analyse und der Simulationsrechnungen wird für die Kreuzkombination $\mathrm{CH}_{3}+\mathrm{C}_{2} \mathrm{H}_{5} \rightarrow \mathrm{C}_{3} \mathrm{H}_{8}$ folgende Geschwindigkeit vorgeschlagen:

$$
k_{\mathrm{kk}}=(7,4 \pm 2,0) \times 10^{13} \mathrm{~cm}^{3} /(\mathrm{mol} \cdot \mathrm{s})
$$


Mit der geometric mean rule erhält man mit $\mathrm{k}\left(\mathrm{CH}_{3}+\mathrm{CH}_{3} \rightarrow \mathrm{C}_{2} \mathrm{H}_{6}\right)=2,4 \times 10^{13}$ $\mathrm{cm}^{3} /(\mathrm{mol} \cdot \mathrm{s})$ und $\mathrm{k}\left(\mathrm{C}_{2} \mathrm{H}_{5}+\mathrm{C}_{2} \mathrm{H}_{5} \rightarrow \mathrm{C}_{4} \mathrm{H}_{10}\right)=1,1 \times 10^{13} \mathrm{~cm}^{3} /(\mathrm{mol} \cdot \mathrm{s}):$

$$
k_{A B}=2 \cdot \sqrt{\left(k_{A A} k_{B B}\right)}=3,2 \times 10^{13} \mathrm{~cm}^{3} /(\mathrm{mol} \cdot s)
$$

Der über die geometric mean rule erhaltene Wert ist also um den Faktor 2 kleiner als der experimentell bestimmte Geschwindigkeitskoeffzient.

Im Folgenden werden die Ergebnisse der Arbeit mit Resultaten aus der Literatur verglichen. Für die Reaktion $\mathrm{CH}_{3}+\mathrm{C}_{2} \mathrm{H}_{5}$ liegen mehrere Studien vor, die aber zumeist die Geschwindigkeit der Reaktion lediglich mittels der geometric mean rule abschätzen. Eine Sammlung bisheriger Ergebnisse zusammen mit einer Empfehlung für das Kanalverhältnis ist in [Bau05] gegeben. Für eine Temperatur von $300 \mathrm{~K}$ wird das Verhältnis von Disproportionierung zu Kombination zu $k(d) / k(c)=0,04$ empfohlen. Dies stimmt mit dem Ergebnis dieser Arbeit überein, bei der Produkte der Kreuzreaktions-Disproportionierung in sehr geringen Anteilen vorlagen.

Garland und BAYEs untersuchten die Kreuzreaktionsgeschwindigkeit kleiner Alkylradikale mittels Laserphotolyse und massespektrometrischer Detektion [Gar90]. Für die obige Kreuzreaktion wird eine Geschwindigkeit von $k_{\mathrm{kk}}=(5,6 \pm 2,5) \times 10^{13} \mathrm{~cm}^{3} /(\mathrm{mol} \cdot \mathrm{s})$ angegeben. Dabei wurde die Geschwindigkeit wie in dieser Arbeit unter Berücksichtigung der experimentellen Ergebnisse durch Simulationsrechnungen von Konzentrations-ZeitProfilen bestimmt.

Des Weiteren gibt es zwei neuere Untersuchungen von KNyazev und Slagle und von Mousavipour und Homayoon Kny01, Mou03.

KNyazev und Slagle untersuchten die Reaktion mittels Laserphotolyse und Photoionizationsmassenspektrometrie. Dabei wurden die Radikale bei einer Wellenlänge von $\lambda=$ $193 \mathrm{~nm}$ aus den Vorläufern Aceton und Diethylketon generiert. In guter Übereinstimmung mit dieser Arbeit wird dabei der Photolysekanal, der zur Bildung von 2 Methylradikalen führt, für die Spaltung von Aceton mit einem Anteil von mehr als 95\% angegeben. Bei der Untersuchung wurde außerdem immer ein hoher Überschuss an Methylradikalen $\left(\left[\mathrm{CH}_{3}\right]_{0}\right.$ $\left./\left[\mathrm{C}_{2} \mathrm{H}_{5}\right]_{0} \geq 22\right)$ verwendet. Die Geschwindigkeiten der Reaktionen wurden in einem Temperaturbereich von 297 - $800 \mathrm{~K}$ und einem Gesamtdruck von 1 - 10 mbar bestimmt. Als Produkt der Kreuzkombination konnte Propan beobachtet werden. Die Geschwindigkeit der Kreuzkombination wurde $\mathrm{zu} k_{1}^{\infty}=1,42 \times 10^{13} \exp (433 K / T) \mathrm{cm}^{3} /(\mathrm{mol} \cdot \mathrm{s})$ bestimmt. 
Bei einer Temperatur von $298 \mathrm{~K}$ entspricht dies $k_{1}^{\infty}(298 \mathrm{~K})=6,1 \times 10^{13} \mathrm{~cm}^{3} /(\mathrm{mol} \cdot \mathrm{s})$. Der Niederdruck-Wert beträgt $k_{1}(298 K)=5,8 \times 10^{13} \mathrm{~cm}^{3} /(\mathrm{mol} \cdot \mathrm{s})$.

Mousavipour und Homayoon führten für die Reaktion quantenchemische Rechnungen unter Verwendung verschiedener Methoden zur Berechnung der Potentialflächen durch. RRKM-Rechnungen wurden zur Ermittlung der Druckabhängigkeit der Reaktion angefertigt. Dabei wurde für die Kombinationsreaktion eine Geschwindigkeit von $k_{c}=5,5 \times 10^{14} T^{-0,56} \exp \left(0,53 k J \cdot \mathrm{mol}^{-1} /(R \cdot T)\right) \mathrm{cm}^{3} /(\mathrm{mol} \cdot \mathrm{s})$, was $k_{c}(298 K)=2,8 \times$ $10^{13} \mathrm{~cm}^{3} /(\mathrm{mol} \cdot \mathrm{s})$ entspricht und für die Disproportionierung eine Geschwindigkeit von $k_{d}=9,8 \times 10^{11} \mathrm{~cm}^{3} /(\mathrm{mol} \cdot \mathrm{s})$ gefunden. Legt man eine Temperatur von $300 \mathrm{~K}$ zu Grunde so erhält man aus den Geschwindigkeitskoeffzienten ein Kanalverhältnis von $k(d) / k(c)=$ 0, 035. Dies stimmt gut mit der Empfehlung von BAulch et al. [Bau05] überein.

\subsubsection{Die Reaktion Methyl + 1-Propyl}

Für die Reaktion von Methylradikalen mit 1-Propylradikalen wird auf Grund der möglichen Kreuzkombinationsreaktionen und der Selbstreaktionen (Kombination / Disproportionierung) folgender Reaktionsmechanismus angenommen:

$$
\begin{aligned}
& \mathrm{CH}_{3}+\mathrm{CH}_{2} \mathrm{CH}_{2} \mathrm{CH}_{3} \longrightarrow \mathrm{C}_{4} \mathrm{H}_{10} \quad\left(x_{1}\right) \\
& \longrightarrow \mathrm{CH}_{4}+\mathrm{C}_{3} \mathrm{H}_{6} \quad\left(x_{2}\right) \\
& \longrightarrow \mathrm{CH}_{2}+\mathrm{C}_{3} \mathrm{H}_{8} \quad\left(x_{3}\right) \\
& \mathrm{CH}_{3}+\mathrm{CH}_{3} \longrightarrow \mathrm{C}_{2} \mathrm{H}_{6} \quad\left(b_{1}\right) \\
& \longrightarrow \mathrm{CH}_{4}+\mathrm{CH}_{2} \quad\left(b_{2}\right) \\
& \mathrm{CH}_{2} \mathrm{CH}_{2} \mathrm{CH}_{3}+\mathrm{CH}_{2} \mathrm{CH}_{2} \mathrm{CH}_{3} \longrightarrow \mathrm{C}_{6} \mathrm{H}_{12} \quad\left(c_{1}\right) \\
& \longrightarrow \mathrm{C}_{3} \mathrm{H}_{8}+\mathrm{C}_{3} \mathrm{H}_{6} \quad\left(c_{2}\right)
\end{aligned}
$$

Für die Untersuchung der Reaktion von Methylradikalen mit 1-Propylradikalen wurden verschiedene Vorläufer verwendet. Zum Einen wurden Photolysen von Aceton- $\mathrm{d}_{6}$ / Propyliodid, und zum Anderen von Aceton- $\mathrm{d}_{6}$ / Dipropylketon durchgeführt.

Zusätzlich zu diesen zwei Vorläufermischungen wurde die Reaktion $\mathrm{CH}_{3}+1-\mathrm{C}_{3} \mathrm{H}_{7}$ auch durch Photolysen von Methyl-Propylketon untersucht. Neben der Reaktion $\mathrm{CH}_{3} \mathrm{COC}_{3} \mathrm{H}_{7}$ $\rightarrow \mathrm{CH}_{3}+\mathrm{C}_{3} \mathrm{H}_{7}+\mathrm{CO}$ wurde dabei die Bildung von Aceton und Acetaldehyd beobachtet, 
welche durch Norrish Spaltung III erklärt werden können.

Die Vor- und Nachteile der verschiedenen Vorläufer sind schon in Abschnitt 4.2.1 diskutiert, insbesondere die Möglichkeit, dass im Fall des Dipropylketons neben der erwünschten Norrish Typ I Spaltung auch eine Norrish Typ III Spaltung auftreten kann. Für die qualitative und die quantitative Analyse der Reaktion wurden Photolysen der einzelnen Vorläufer separat durchgeführt, um die Mechansismen und Produkte der Selbstreaktionen zu bestimmen.

Um eine genaue Analyse der Reaktion vorzunehmen, wurde das Verhältnis der Radikalanfangskonzentration variiert $\left(\left[\mathrm{CH}_{3}\right]_{0} /\left[\mathrm{C}_{3} \mathrm{H}_{7}\right]_{0} \ll 1 \ll\left[\mathrm{CH}_{3}\right]_{0} /\left[\mathrm{C}_{3} \mathrm{H}_{7}\right]_{0}\right)$. Die Messungen fanden bei einer Temperatur von $298 \mathrm{~K}$ und einem Gesamtdruck von 4 mbar statt. Die Analyse der stabilen Endprodukte wurde anhand von 100-fach gemittelten IR-Spektren nach jeweils 50 - 100 Photolysen vorgenommen. Dabei betrug die maximale Photolysenanzahl einer Reaktionsmischung 400, um die Sekundärphotolyse von primären Produkten gering zu halten. Der Verbrauch der Vorläufer wurde unter diesen Bedingungen auf eine Abnahme von $\sim 10 \%$ begrenzt.

\subsubsection{Qualitative Analyse}

In Abbildung 4.75 ist ein Reaktionsspektrum der Vorläufermischung Aceton- $\mathrm{d}_{6}$ / Propyliodid nach 400 Photolysen und einer Zusammensetzung von $\mathrm{p}\left(\right.$ Aceton- $\left.\mathrm{d}_{6}\right)=0,2$ mbar und $\mathrm{p}($ Propyliodid $)=0,6$ mbar dargestellt. Abbildung 4.77 zeigt ein Reaktionsspektrum nach 300 Photolysen von dem Vorläufergemisch Aceton- $\mathrm{d}_{6}$ / Dipropylketon bei einer Zusammensetzung von $\mathrm{p}\left(\right.$ Aceton- $\left.\mathrm{d}_{6}\right)=0,2$ mbar und $\mathrm{p}$ (Dipropylketon $)=0,4$ mbar. Das Reaktionsspektrum von Methylpropylketon nach 400 Photolysen ist in Abbildung $4.79 \mathrm{zu}$ finden. 


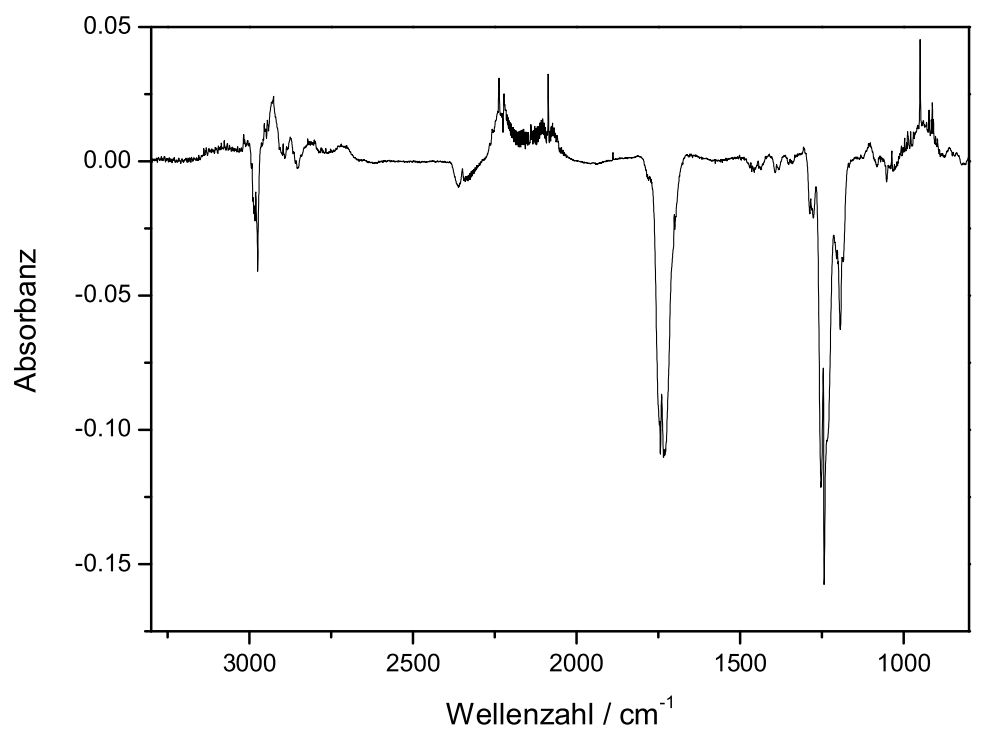

Abbildung 4.75: Reaktionssystem $\mathrm{CH}_{3} / 1-\mathrm{C}_{3} \mathrm{H}_{7}$, Reaktionsspektrum nach 400 Photolysen bei einem Gesamtdruck von 4 mbar und $298 \mathrm{~K} ; \mathrm{p}\left(\right.$ Aceton- $\left._{6}\right)=0,2$ mbar, $p$ (Propyliodid $)=0,6$ mbar.

Für die detaillierte qualitative Analyse wurden Bereiche des Reaktionsspektrums gewählt, in denen die spektrale Interferenz der verschiedenen Substanzen möglichst gering war. Die Abbildung 4.76 zeigt demzufolge ausgewählte Wellenzahlbereiche für die Vorläufer Aceton- $\mathrm{d}_{6} /$ Propyliodid anhand derer eine eindeutige Identifikation der Produkte vorgenommen wurde. 

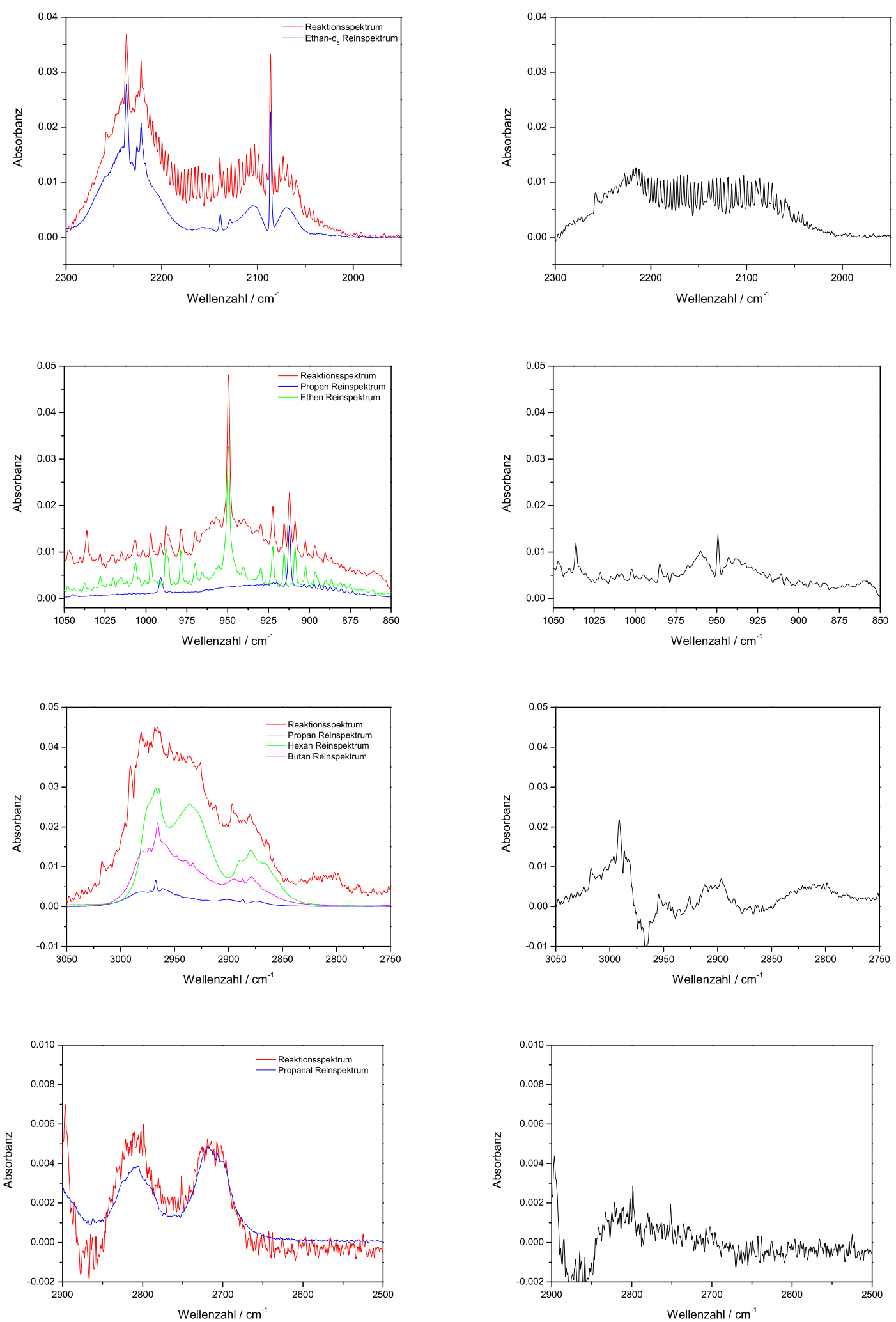

Abbildung 4.76: Reaktionssystem $\mathrm{CH}_{3}$ / 1- $\mathrm{C}_{3} \mathrm{H}_{7}$, qualitative Analyse. Linke Seite: Vergleich des Reaktionsspektrums mit Spektren der Reinsubstanzen; rechte Seite: Residuen. p(Aceton-d $\left.{ }_{6}\right)=$ 0,2 mbar, $p$ (Propyliodid) = 0,6 mbar, Gesamtdruck 4 mbar, T =298 K, 400 Photolysen. 
Als Produkte der Reaktion $\mathrm{CH}_{3}+1-\mathrm{C}_{3} \mathrm{H}_{7}$ wurden unter Verwendung der Vorläufermischung Aceton- $\mathrm{d}_{6}$ / Propyliodid folgende Substanzen identifiziert: Ethan, Ethen, Propen, Hexan, Butan, Propan und Methan. Dabei können einige der Produkte sowohl aus Kreuzkombinationsreaktionen $\left(\mathrm{x}_{i}\right)$ als auch aus Selbstreaktionen $\left(\left(\mathrm{b}_{i}\right)\right.$ oder $\left.\left(\mathrm{c}_{i}\right)\right)$ stammen. Eine Zuordnung der Produkte zu den Kanälen des angegebenen Reaktionsmechanismus war durch Wahl eines deuterierten Radikalvorläufers als $\mathrm{CH}_{3}$-Quelle (genauer $\mathrm{CD}_{3}$-Quelle) aber dennoch möglich, da sich vollständig deuterierte, teildeuterierte und undeuterierte Kohlenwasserstoffe in der Lage der Absorptionsbanden teilweise stark unterscheiden. Neben diesen Reaktionsprodukten konnte noch die Bildung von deuteriertem Formaldehyd $\left(\mathrm{D}_{2} \mathrm{CO}\right)$ und Propanal beobachtet werden. Beide Substanzen werden dabei durch Reaktion mit $\mathrm{O}_{2}$ gebildet und resultieren also nicht aus den Kreuz- oder Selbstreaktionen.

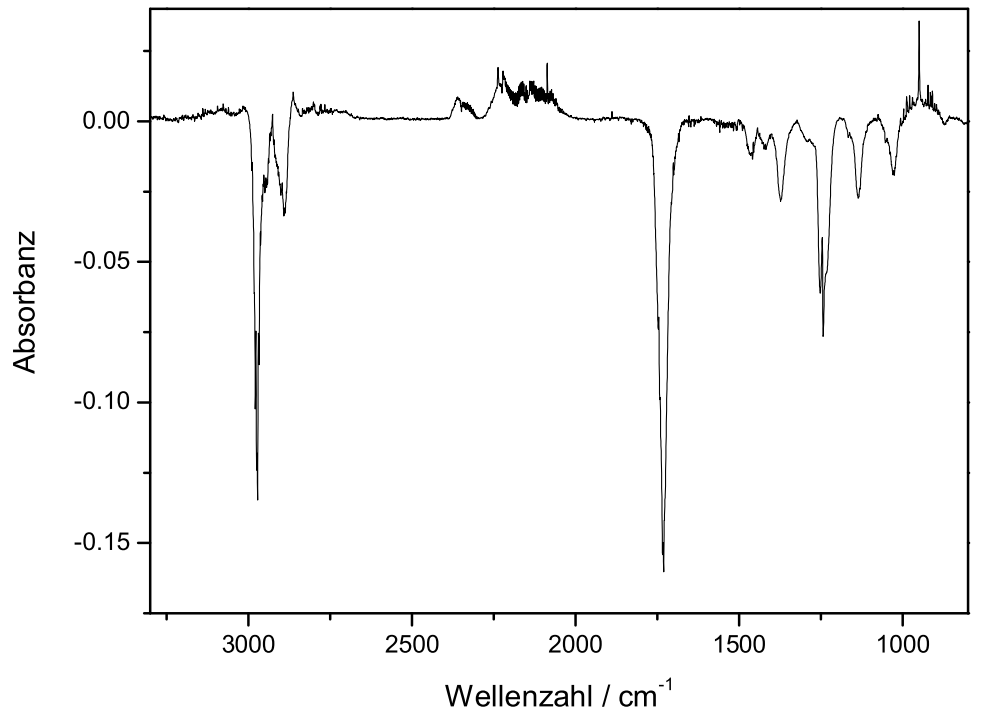

Abbildung 4.77: Reaktionssystem $\mathrm{CH}_{3} / 1-\mathrm{C}_{3} \mathrm{H}_{7}$, Reaktionsspektrum nach 300 Photolysen bei einem Gesamtdruck von 4 mbar und $298 \mathrm{~K} ; \mathrm{p}\left(\right.$ Aceton- $\left._{6}\right)=0,2$ mbar, $p$ (Dipropylketon $)=0,4$ mbar.

Unter Verwendung der Vorläufer Aceton- $\mathrm{d}_{6}$ / Dipropylketon wurden dieselben Endprodukte wie für die obigen Vorläufer festgestellt. Daneben konnte noch die Bildung von Butanon beobachtet werden, welches aus der photolytischen Spaltung von Dipropylketon gemäß einer Norrish Typ III Spaltung erfolgt. In Abwesenheit eines weiteren Eduktes wurde das Verhältnis der photolytischen Spaltung von Dipropylketon zu (Norrish I)/(Norrish III) $=1 / 0,70$ bestimmt. 
Eine detaillierte Darstellung der Identifikation der Produkte für diese Vorläufer ist in Abbildung 4.78 gegeben.
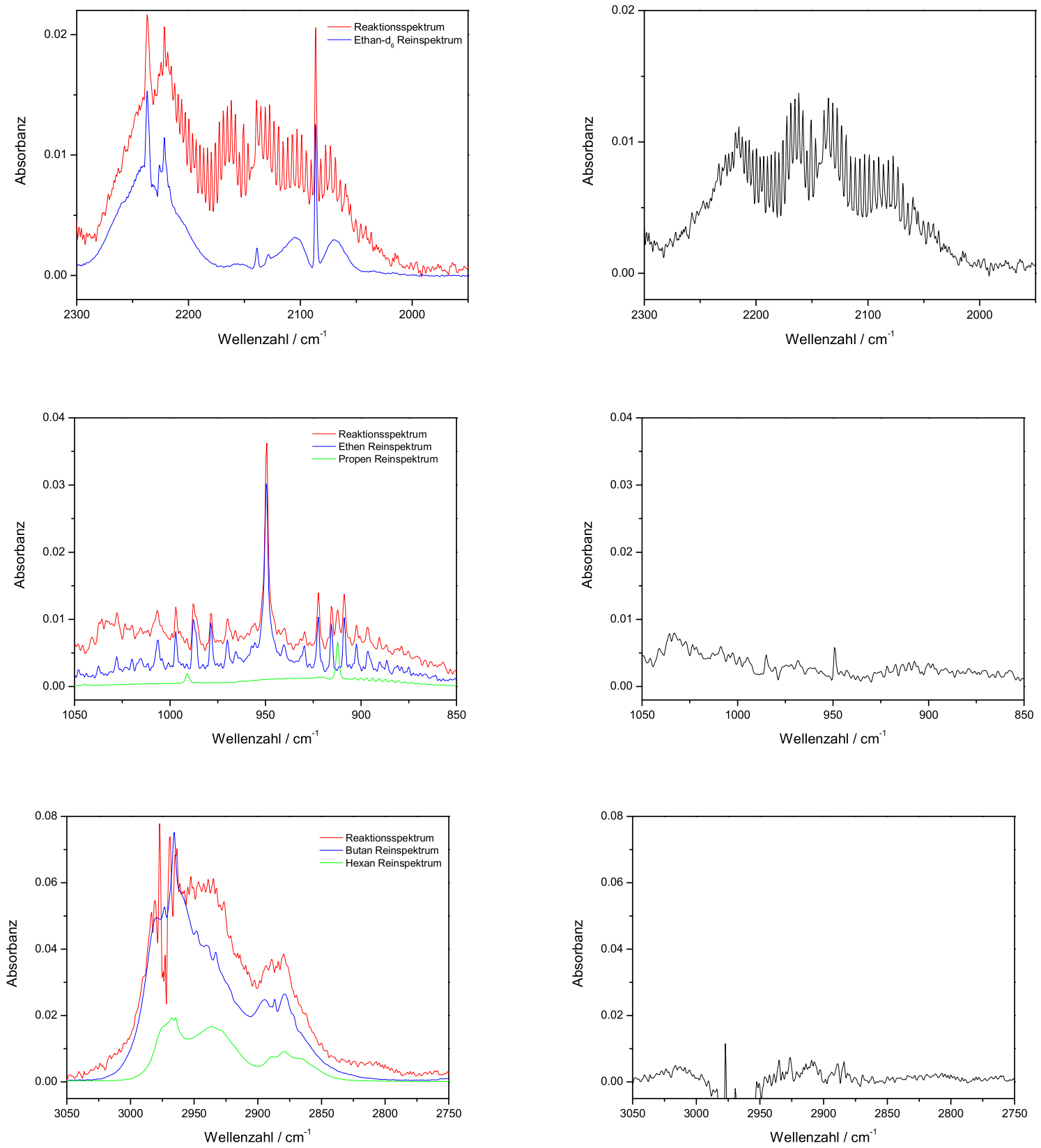

Abbildung 4.78: Reaktionssystem $\mathrm{CH}_{3} /{ }_{1}-\mathrm{C}_{3} \mathrm{H}_{7}$, qualitative Analyse. Linke Seite: Vergleich des Reaktionsspektrums mit Spektren der Reinsubstanzen; rechte Seite: Residuen. $p$ (Aceton- $\left.d_{6}\right)=$ 0,2 mbar, $p$ (Dipropylketon) =0,4 mbar, Gesamtdruck 4 mbar, T=298 K, 300 Photolysen.

Ein Reaktionsspektrum für den Vorläufer Methylpropylketon ist in Abbildung 4.79 gegeben. Das Spektrum wurde nach 400 Photolysen bei einem Gesamtdruck von 4 mbar und einer Temperatur von $298 \mathrm{~K}$ von $\mathrm{p}$ (Methylpropylketon) $=0,2$ mbar aufgenommen. 


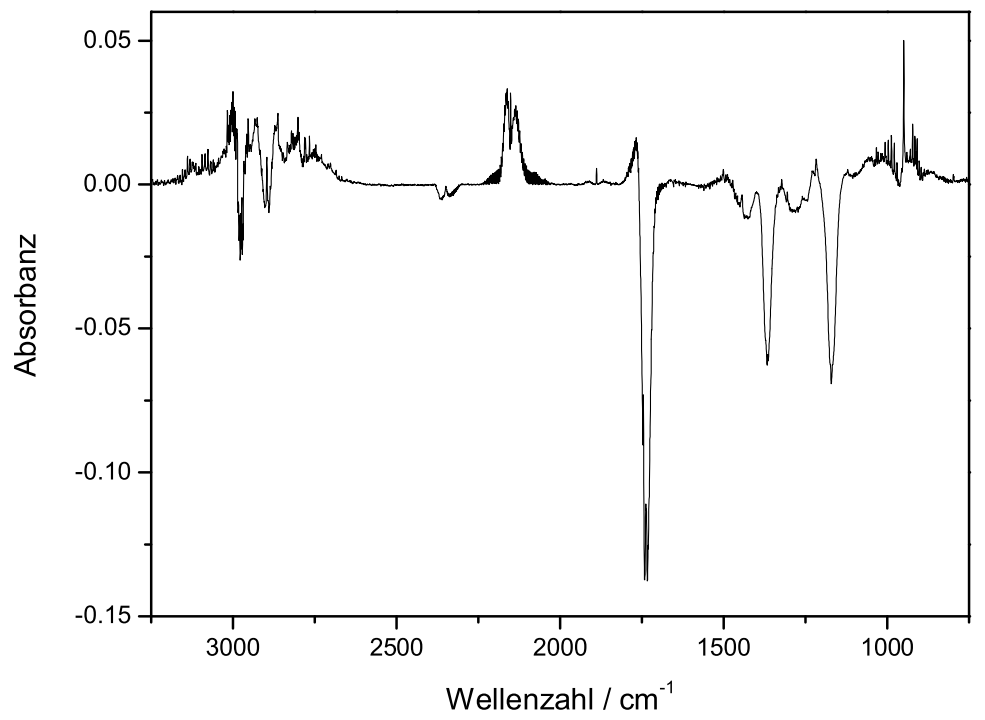

Abbildung 4.79: Reaktionssystem $\mathrm{CH}_{3} / 1-\mathrm{C}_{3} \mathrm{H}_{7}$, Reaktionsspektrum nach 400 Photolysen bei einem Gesamtdruck von 4 mbar und $298 \mathrm{~K}$; $\mathrm{p}$ (Methylpropylketon) = 0,2 mbar.

Vergrößerte Ausschnitte des um den Vorläufer reduzierten Reaktionsspektrums im Vergleich mit den Reinspektren der identifizierten Substanzen sind in Abbildung 4.80 dargestellt. Neben Produkten gemäß des oben angegebenen Reaktionsmechanismus konnten direkte Photolyseprodukte gefunden werden. Für die Photolyse von Methylpropylketon wird dabei folgender Mechanismus vorgeschlagen:

$$
\begin{array}{rlr}
\mathrm{CH}_{3} \mathrm{COC}_{3} \mathrm{H}_{7} & \stackrel{h \nu}{\longrightarrow} \mathrm{CH}_{3}+\mathrm{C}_{3} \mathrm{H}_{7}+\mathrm{CO} & \left(p_{1}\right) \\
& \stackrel{h \nu}{\longrightarrow} \mathrm{C}_{3} \mathrm{H}_{6} \mathrm{O}+\mathrm{C}_{2} \mathrm{H}_{4} & \left(p_{2}\right) \\
& \stackrel{h \nu}{\longrightarrow} \mathrm{CH}_{3} \mathrm{CHO}+\mathrm{C}_{3} \mathrm{H}_{6} & \left(p_{3}\right)
\end{array}
$$

Die Bildung eines Methyl- und eines Propylradikals entlang des Photolyesekanals $\left(p_{1}\right)$ wird auch in [Cal66] et al. angegeben, wobei das Verhältnis der gebildeten Radikale von der Wellenlänge des Photolyselichts abhängt. Diese Wellenlängenabhängigkeit wird dabei detailliert für die Photolyse von Methylethylketon beschrieben, die analog für das hier verwendete Methylpropylketon gilt. Für eine Strahlung der Wellenlänge $\lambda=3,13 \times 10^{-7} \mathrm{~m}$ wird im Fall von Methylethylketon ein Verhältnis $\left[\mathrm{C}_{2} \mathrm{H}_{5}\right] /\left[\mathrm{CH}_{3}\right]=40$ berichtet. Für höhere Energien $\left(\lambda \leq 2,54 \times 10^{-7} \mathrm{~m}\right)$ wird die Photolyse als nicht selektiv berichtet. Identifizierte Produkte, die aus den Photolysewegen $\left(p_{2}\right)$ und $\left(p_{3}\right)$ stammen, sind Ace- 
ton, Acetaldehyd und Ethen. Propen wurde ebenfalls gefunden, allerdings wird dies auch gemäß der Reaktionskanäle $\left(x_{2}\right)$ und $\left(c_{2}\right)$ gebildet. Die quantitative Analyse von Experimenten mit verschiedenen Anfangspartialdrücken lieferten allerdings widersprüchliche Ergebnisse bezüglich der Kanalanteile. Diese werden in dem folgenden Abschnitt diskutiert.
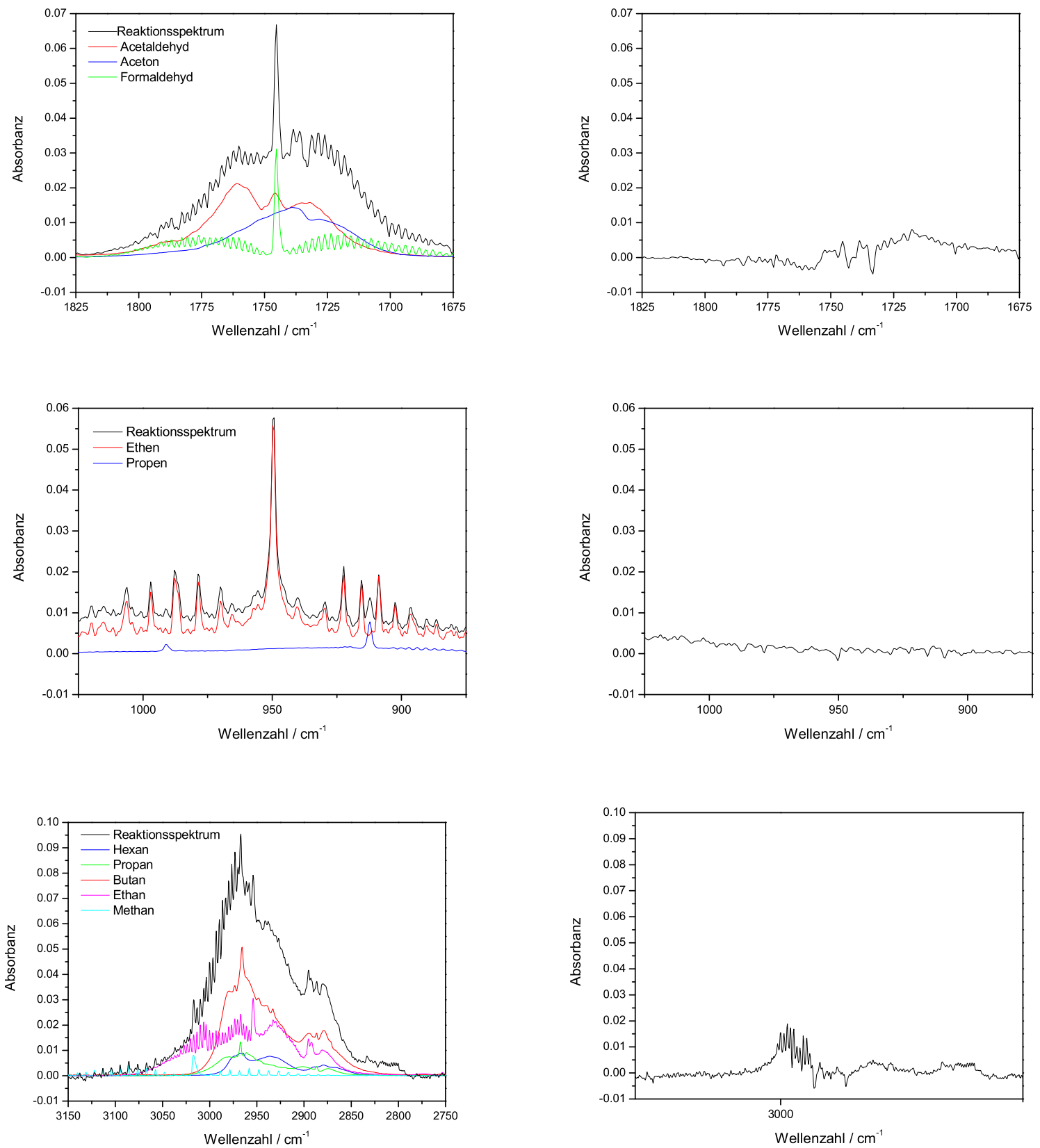

Abbildung 4.80: Reaktionssystem $\mathrm{CH}_{3} /{ }_{1}-\mathrm{C}_{3} \mathrm{H}_{7}$, qualitative Analyse. Linke Seite: Vergleich des Reaktionsspektrums mit Spektren der Reinsubstanzen; rechte Seite: Residuen. $p$ (Methylpropylketon) $=0,2$ mbar, Gesamtdruck 4 mbar, T =298 K, 400 Photolysen. 


\subsubsection{Quantitative Analyse}

Im Folgenden werden die Ergebnisse unter der Verwendung der einzelnen Vorläufer dargestellt. Dazu sind die Partialdrücke der identifizierten Substanzen gegen die Konzentrationsabnahme eines Vorläufers aufgetragen. Die Abbildungen beziehen sich dabei immer auf ein bestimmtes Radikal bzw. auf ein bestimmtes Vorläuferverhältnis, da die Kanalverzweigung bei allen betrachteten Reaktionen sehr sensitiv gegenüber dem Radikalanfangsverhältnis ist. Zunächst erfolgt die Darstellung der Ergebnisse für die Vorläufermischung Aceton- $\mathrm{d}_{6}$ / Propyliodid. Die zugehörigen Messwerte sind in Tabelle 4.41 angegeben.

\begin{tabular}{|c|c||c|c|c|c|}
\hline $\begin{array}{c}\mathrm{C}_{3} \mathrm{H}_{7} \mathrm{I} \text { Verb. } \\
{[\mathrm{mbar}]}\end{array}$ & $\begin{array}{c}\text { Aceton- } \mathrm{d}_{6} \text { Verb. } \\
{[\mathrm{mbar}]}\end{array}$ & $\begin{array}{c}\mathrm{p}\left(\text { Ethan- } \mathrm{d}_{6}\right) \\
{[\mathrm{mbar}]}\end{array}$ & $\begin{array}{c}\mathrm{p} \text { (Propen }) \\
{[\mathrm{mbar}]}\end{array}$ & $\begin{array}{c}\mathrm{p} \text { (Hexan) } \\
{[\mathrm{mbar}]}\end{array}$ & $\begin{array}{c}\mathrm{p} \text { (Propan) } \\
{[\mathrm{mbar}]}\end{array}$ \\
\hline \hline 0,0073 & 0,0077 & 0,0022 & 0,0019 & $9 \mathrm{E}-4$ & - \\
\hline 0,016 & 0,013 & 0,003 & 0,003 & $1 \mathrm{E}-3$ & $5 \mathrm{E}-4$ \\
\hline 0,034 & 0,025 & 0,0056 & 0,005 & 0,0025 & $1 \mathrm{E}-3$ \\
\hline 0,05 & 0,035 & 0,0084 & 0,0064 & 0,0039 & 0,0015 \\
\hline
\end{tabular}

Tabelle 4.41: Reaktionssystem $\mathrm{CH}_{3} /$ 1- $\mathrm{C}_{3} \mathrm{H}_{7}$, Vorläufer Propyliodid, Messwerte. $p$ (Propyliodid) $=0,3$ mbar, $p\left(\right.$ Aceton- $\left.d_{6}\right)=0,3$ mbar, $p_{\text {ges }}=4$ mbar, $T=298 \mathrm{~K}$, Badgas Argon .

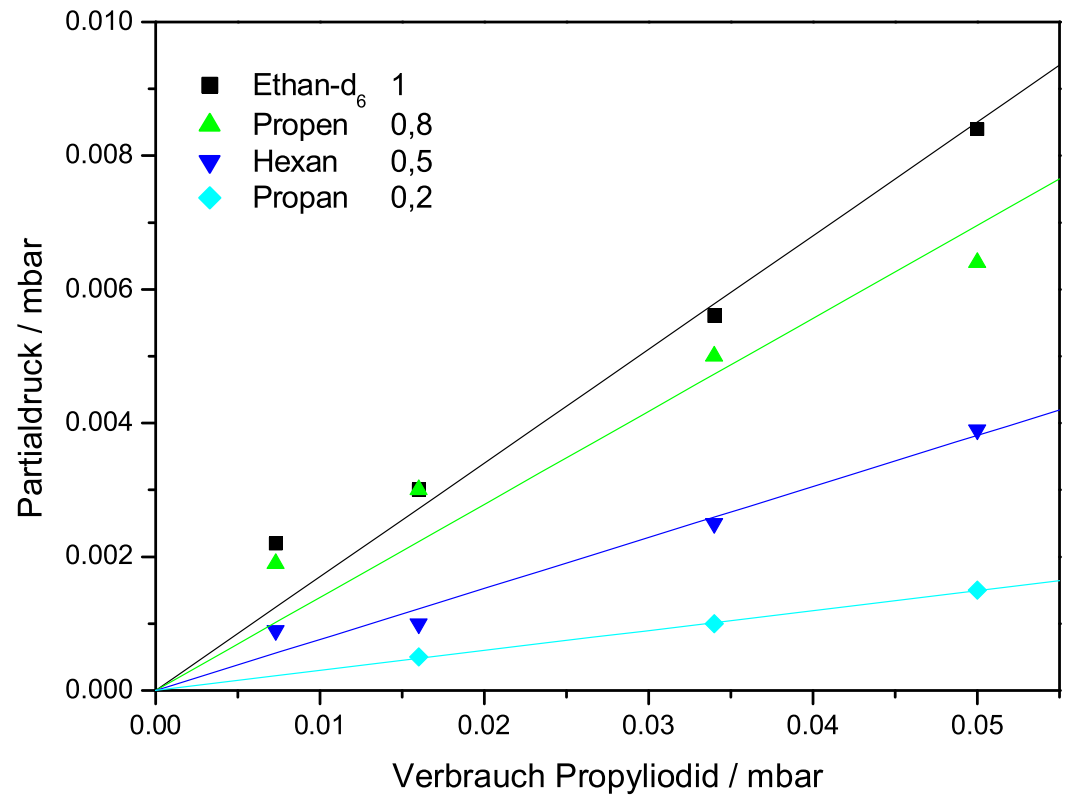

Abbildung 4.81: Reaktionssystem $\mathrm{CH}_{3}$ / 1- $\mathrm{C}_{3} \mathrm{H}_{7}$, quantitative Analyse, Vorläufer Propyliodid. $p\left(\right.$ Aceton- $\left.d_{6}\right)=0,3$ mbar, $p$ (Propyliodid $)=0,3$ mbar, Gesamtdruck 4 mbar, $T=298 \mathrm{~K}$. 
Die Abbildung 4.81 zeigt für alle Produkte mit steigender Propyliodid-Abnahme eine lineare Zunahme. Sekundärreaktionen und Photolyse primärer Produkte können somit vernachlässigt werden. Für das in der Tabelle angegebene Vorläuferverhältnis von 1:1 ergibt sich das in der Abbildung 4.81 angegebene Verhältnis. Eine Zusammenfassung der quantitativen Ergebnisse für die Reaktion Methyl + Propyl erfolgt am Ende des Abschnitts.

Im Folgenden werden die Ergebnisse der quantitativen Analyse unter Verwendung der Vorläufermischung Aceton- $\mathrm{d}_{6}$ / Dipropylketon dargestellt. Dazu ist die Auftragung der Partialdrücke gegen die Abnahme des Vorläufers Dipropylketon in Abbildung 4.82 gegeben. Die Messwerte für die Auftragung befinden sich in Tabelle 4.42.

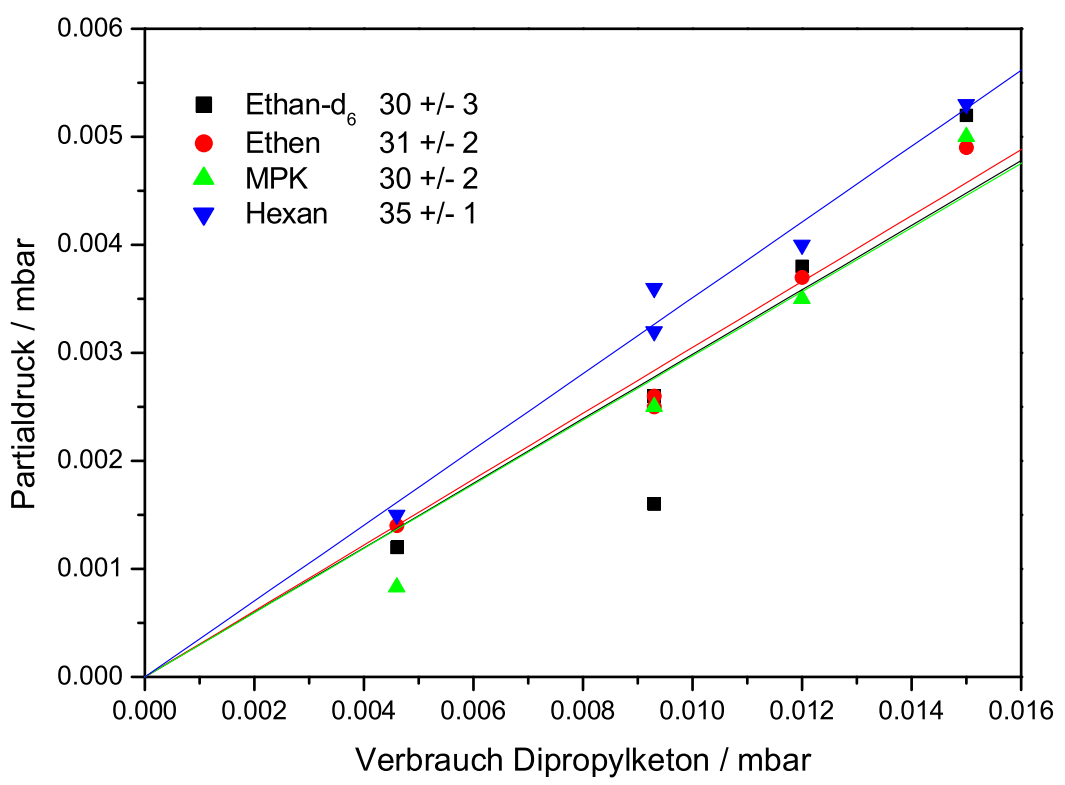

Abbildung 4.82: Reaktionssystem $\mathrm{CH}_{3} / 1-\mathrm{C}_{3} \mathrm{H}_{7}$, quantitative Analyse, Vorläufer Dipropylketon. $p\left(\right.$ Aceton $\left.-d_{6}\right)=0,2$ mbar, $p$ (Dipropylketon) $=0,6$ mbar, Gesamtdruck 4 mbar, $T=298 \mathrm{~K} . \mathrm{MPK}$ $:=$ Methylpropylketon 


\begin{tabular}{|c|c|c|c|c|c|}
\hline $\begin{array}{c}\text { DIPROP Verb. } \\
{[\text { mbar }]}\end{array}$ & $\begin{array}{c}\text { Aceton-d }_{6} \text { Verb. } \\
{[\mathrm{mbar}]}\end{array}$ & $\begin{array}{c}\mathrm{p}\left(\text { Ethan- } \mathrm{d}_{6}\right) \\
{[\text { mbar }]}\end{array}$ & $\begin{array}{c}\mathrm{p}(\text { Ethen }) \\
{[\text { mbar }]}\end{array}$ & $\begin{array}{c}\mathrm{p}(\mathrm{MPK}) \\
{[\mathrm{mbar}]}\end{array}$ & $\begin{array}{c}\mathrm{p}(\text { Hexan }) \\
{[\text { mbar }]}\end{array}$ \\
\hline 0,0046 & 0,0021 & 0,0012 & 0,0014 & $8,3 \mathrm{E}-4$ & 0,0015 \\
\hline 0,0093 & 0,0079 & 0,0026 & 0,0026 & 0,0025 & 0,0036 \\
\hline 0,0093 & 0,0068 & 0,0016 & 0,0025 & 0,0025 & 0,0032 \\
\hline 0,012 & 0,015 & 0,0038 & 0,0037 & 0,0035 & 0,004 \\
\hline 0,015 & 0,02 & 0,0052 & 0,0049 & 0,005 & 0,0053 \\
\hline
\end{tabular}

Tabelle 4.42: Reaktionssystem $\mathrm{CH}_{3} /{ }_{1}-\mathrm{C}_{3} \mathrm{H}_{7}$, Vorläufer Dipropylketon, Messwerte. $p($ Dipropylketon $)=0,6$ mbar, $p\left(\right.$ Aceton $\left.d_{6}\right)=0,2$ mbar, $p_{\text {ges }}=4$ mbar, $T=298 \mathrm{~K}$, Badgas Argon (DIPROP $:=$ Dipropylketon, $M P K:=$ Methylpropylketon).

In der Abbildung 4.82 nehmen alle Produkte mit der Abnahme an Vorläufer linear zu. Daher kann die Sekundärphotolyse primärer Produkte als vernachlässigbar angesehen werden. Der „radikalfreie“ Photolysekanal von Dipropylketon wurde in Abschnitt 4.4.3 zu $41 \%$ in Bezug zu der Dipropylketonabnahme bestimmt. Der dort bestimmte Wert ist in befriedigender Übereinstimmung mit dem Wert, der hier unter Kreuzreaktionsbedingungen gefunden wurde (31\%).

Wie schon in der qualitativen Analyse erwähnt, lieferte die quantitative Analyse des Vorläufers Methylpropylketon widersprüchliche Ergebnisse. Bei einer Messreihe konnte die C-H-Valenzbande allein durch das Reinspektrum des Kreuz-Kombinationsprodukts Butan erklärt werden. Die Massenbilanz zeigte ebenfalls, dass neben der Bildung von Butan und dem Photolyseweg $\left(p_{2}\right)$, der zur Bildung von Ethen und Aceton führt, keine weiteren Reaktionen stattgefunden haben können. Das Reaktionsspektrum von Methylpropylketon, bei welchem eine exakte Übereinstimmung im Bereich der C-H-Valenzbande mit dem Reinspektrum von Butan auftritt, ist in Abbildung 4.83 dargestellt. 


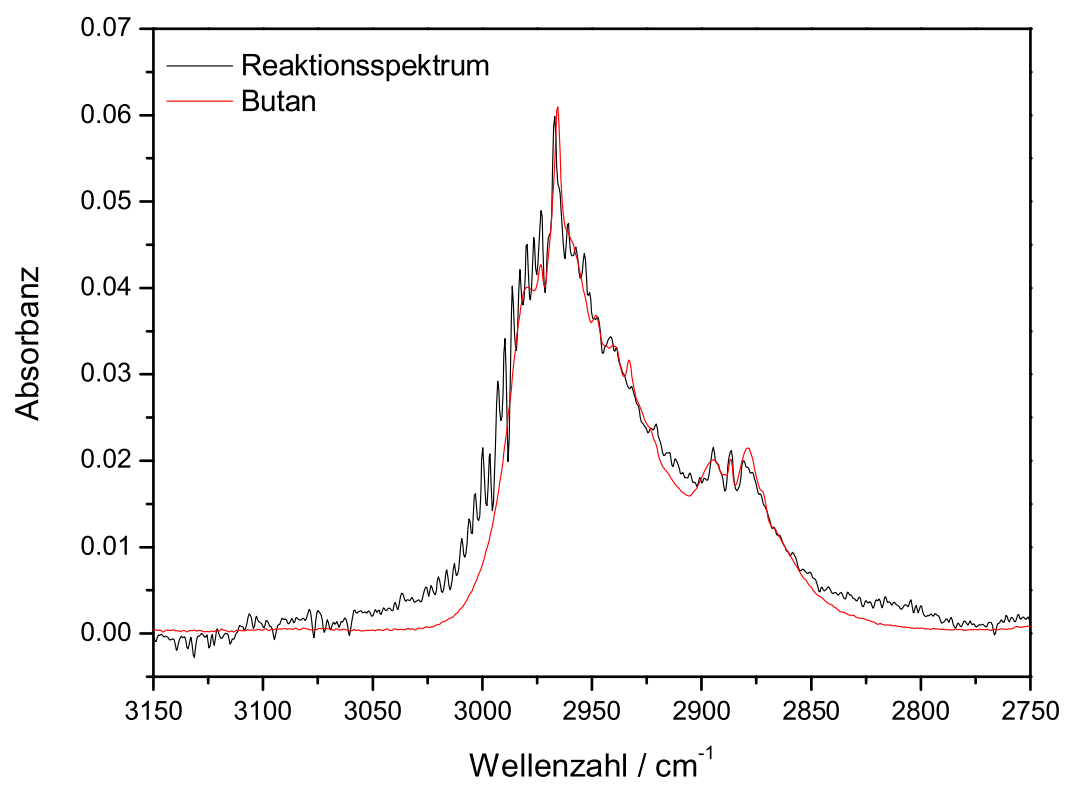

Abbildung 4.83: Reaktionssystem $\mathrm{CH}_{3} / 1-\mathrm{C}_{3} \mathrm{H}_{7}$, quantitative Analyse, Vorläufer Dipropylketon. $p\left(\right.$ Aceton- $\left.d_{6}\right)=0,2$ mbar, $p$ (Dipropylketon) $=0,6$ mbar, Gesamtdruck 4 mbar, $T=298 \mathrm{~K}$.

Die ausschließliche Bildung von Butan bei einer Messreihe ist möglicherweise darauf zurückzuführen, dass die Reaktion nicht über die Bildung freier Radikale abgelaufen ist. Vielmehr könnte es zur Bildung eines angeregten Komplexes gekommen sein, der direkt zu CO und Butan zerfällt. Da die Photolyse von Methylpropylketon nicht abschließend erklärt werden konnte, wird dieser Vorläufer für die quantitative Bestimmung der Kanalverzweigung als ungeeignet erachtet.

Auf Grundlage der Ergebnisse der Vorläufermischung Propyliodid / Aceton- $\mathrm{d}_{6}$ werden im folgenden Abschnitt Simulationsrechnungen durchgeführt. Dabei wird ein Verhältnis der Radikalanfangskonzentrationen von $\left[\mathrm{CD}_{3}\right]_{0} /\left[\mathrm{C}_{3} \mathrm{H}_{7}\right]_{0}=1 / 0,63 \mathrm{zu}$ Grunde gelegt. Dies ergab sich aus der Abnahme an Vorläuferkonzentrationen unter Berücksichtigung des HI-Eliminationskanals zu Propen. Das experimentell bestimmte Verhältnis der Selbstreaktionsprodukte Ethan- $\mathrm{d}_{6}$ und Hexan von $\left[\mathrm{C}_{2} \mathrm{D}_{6}\right] /\left[\mathrm{C}_{6} \mathrm{H}_{10}\right]=2$ war durch Simualtionsrechnungen zu erzielen. 


\subsubsection{Simulation}

Ausgehend von dem auf Seite 185 dargestellten Reaktionsmechanismus wurden für die Reaktion $\mathrm{CH}_{3}+1-\mathrm{C}_{3} \mathrm{H}_{7}$ die Konzentrations-Zeit-Profile simuliert. Dazu wurden die in der Literatur durch eine Vielzahl an Experimenten bestimmten Geschwindigkeitskoeffizienten für die Selbstreaktion von Propylradikalen und die in dieser Arbeit ermittelte Geschwindigkeit der Methyl-Radikal-Kombination verwendet und die Geschwindigkeitskoeffizienten für die Kreuzreaktionen aus den experimentell bestimmten Daten abgeleitet. Die Startkonzentrationen der Radikale wurden aus den Abnahmen der Vorläufer bestimmt. Um mögliche Fehler bei der Bestimmung der Anfangskonzentrationen festzustellen, wurde zunächst das Verhältnis der Anfangskonzentrationen variiert. Dabei zeigte sich, dass die Ergebnisse der Simulationen sehr stark von dem Verhältnis der Radikalanfangskonzentrationen abhängen und eine Übereinstimmung von Simulation und Experiment nur für die aus den Experimenten bestimmten Radikalanfangskonzentrationen gegeben war.

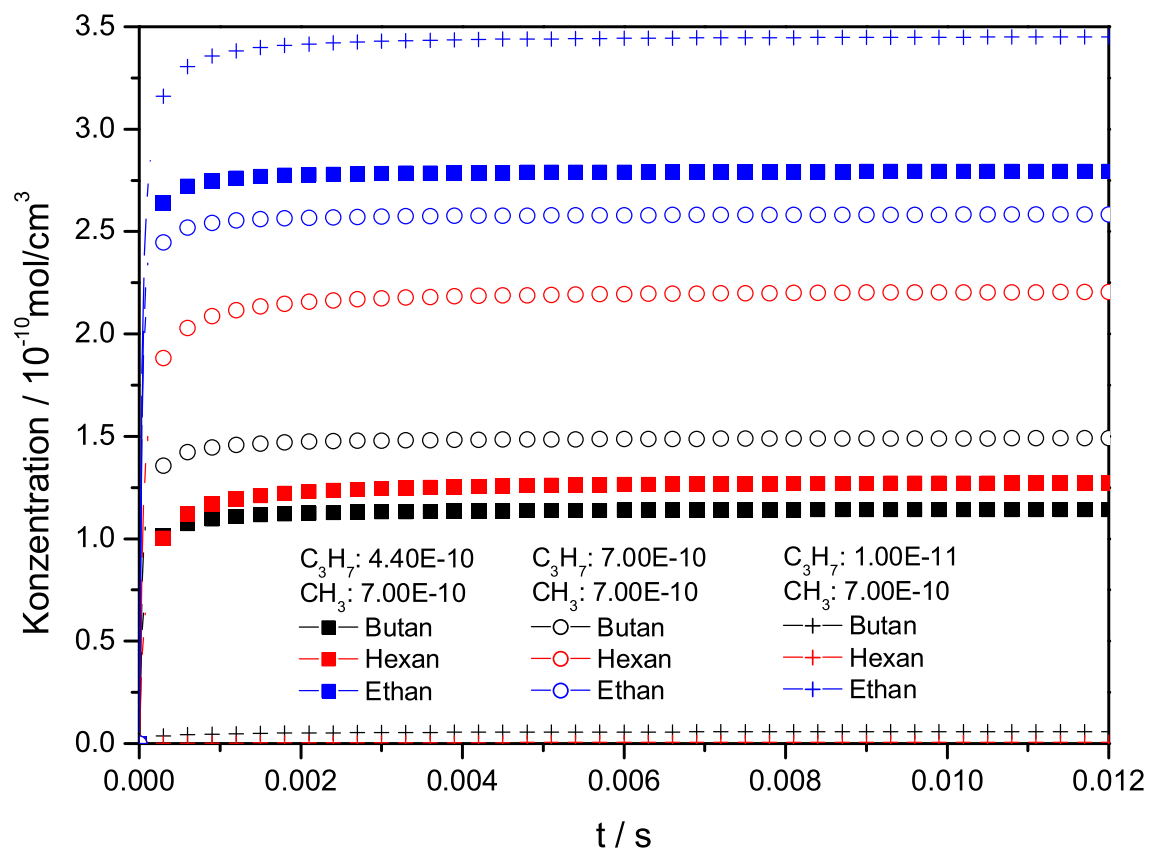

Abbildung 4.84: Reaktionssystem $\mathrm{CH}_{3} /{ }_{1}-\mathrm{C}_{3} \mathrm{H}_{7}$, Einfluss der Startkonzentrationen auf die Simulationsrechnungen. Dargestellt sind das Kreuzreaktionsprodukt Butan (schwarz) und die Kombinationsprodukte der Selbstreaktionen Hexan (rot) und Ethan (blau) für die Verhältnisse $\left[\mathrm{CH}_{3}\right]_{0} /\left[1-\mathrm{C}_{3} \mathrm{H}_{7}\right]_{0}=7 / 4,4$ (Quadrat) (dies ist das experimentelle Verhältnis); $7 / 7$ (Kreis); 7/0,01 (Kreuz). 
Damit ist diese Analysemethode für die Bestimmung von Radikalkonzentrationsverhältnissen sehr zuverlässig. Wie schon erwähnt, hätte das Verhältnis der Radikalanfangskonzentrationen auch über das Verhältnis der Absorptionsquerschnitte berechnet werden können. Der Einfluss des Startkonzentrationsverhältnisses wird in Abbildung 4.84 zusammengefasst.

Neben dem Verhältnis der Radikalanfangskonzentrationen wurden die Geschwindigkeitskoeffizienten der Kreuzkombinations-Reaktionen variiert. Dazu wurde das vorher durch Simulationsrechnungen bestätigte Verhältnis der Radikalanfangskonzentrationen und die in dieser Arbeit bzw. der Literatur entnommenen Selbstreaktionsgeschwindigkeiten verwendet. Dabei wurde ebenfalls eine starke Auswirkung auf die Produktverhältnisse festgestellt. Durch die Bestimmung der stabilen Endproduktverhältnisse und unter Verwendung der Geschwindigkeitskeoffizienten für die Selbstreaktionen konnte auf diesem Weg die Geschwindigkeit der Reaktion $\mathrm{CH}_{3}+1-\mathrm{C}_{3} \mathrm{H}_{7}$ ermittelt werden. Die Simulation der Konzentrations-Zeit-Profile unter Variation der Geschwindigkeit der Kreuzkombination zeigt Abbildung 4.85. 


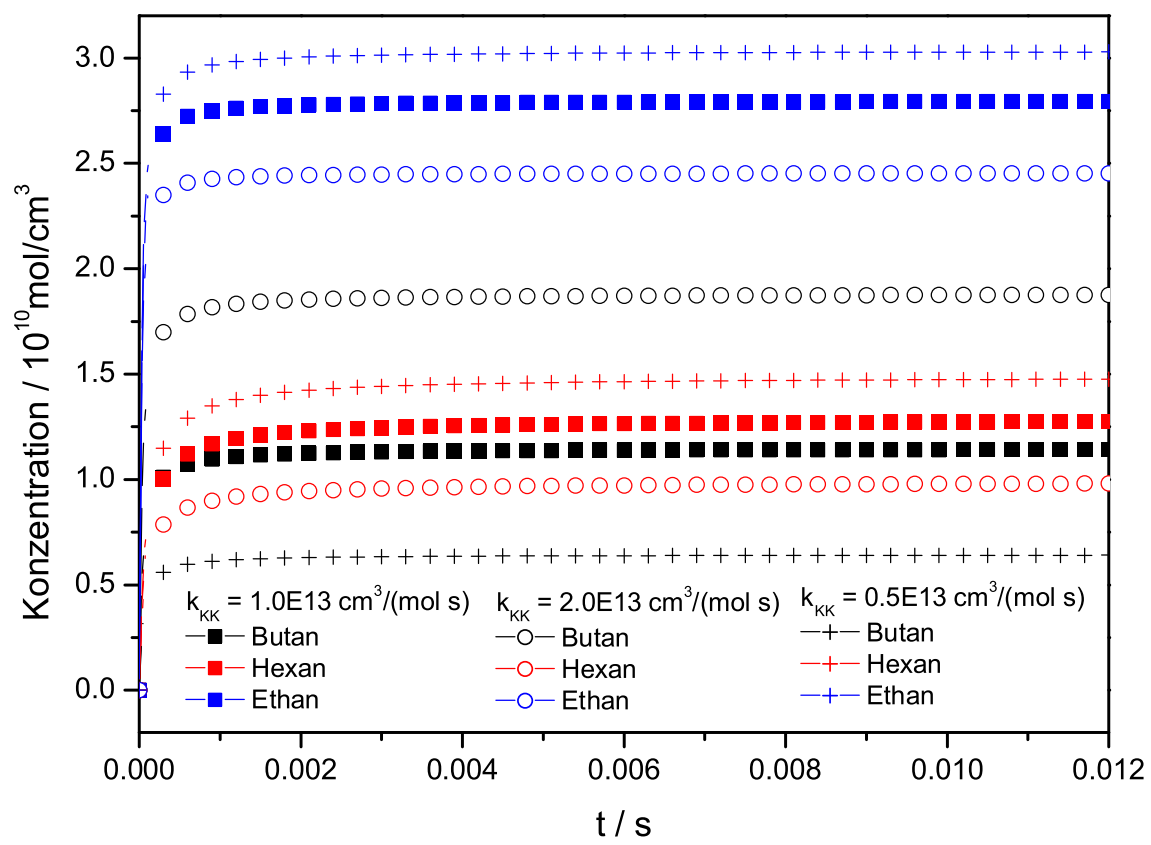

Abbildung 4.85: Reaktionssystem $\mathrm{CH}_{3} / 1-\mathrm{C}_{3} \mathrm{H}_{7}$, Einfluss der Kreuzkombinationsgeschwindigkeit auf die Simulationsrechnungen. Dargestellt sind das Kreuzreaktionsprodukt Butan (schwarz) und die Kombinationsprodukte der Selbstreaktionen Hexan (rot) und Ethan (blau) für die Geschwindigkeiten $\mathrm{k}\left(\mathrm{CH}_{3}+1-\mathrm{C}_{3} \mathrm{H}_{7} \rightarrow\right.$ Butan) $=1,0 \times 10^{13} \mathrm{~cm}^{3} /$ (mol.s) (Quadrat); $2,0 \times 10^{13} \mathrm{~cm}^{3} /(\mathrm{mol} \cdot \mathrm{s})($ Kreis $) ; 0,5 \times 10^{13} \mathrm{~cm}^{3} /(\mathrm{mol} \cdot \mathrm{s})$ (Kreuz).

Die verwendeten Geschwindigkeitskoeffizienten der Reaktionen sind in Tabelle 4.43 gegeben.

Ein experimentell bestimmtes Verhältnis der Radikalanfangskonzentrationen unter Verwendung der Vorläufermischung Propyliodid / Aceton- $\mathrm{d}_{6}$ von $\left[\mathrm{CD}_{3}\right]_{0} /\left[\mathrm{C}_{3} \mathrm{H}_{7}\right]_{0}=1 / 0,63$ wurde dabei für die Simulationen der Konzentrations-Zeit-Profile unter Variation der Geschwindigkeit der Kreuzkombination zu Grunde gelegt. Für die Simulation unter Variation des Verhältnisses der Radikalanfangskonzentrationen wurde eine Geschwindigkeit für die Kreuzkombination von $\mathrm{k}_{K K}=1,0 \times 10^{13} \mathrm{~cm}^{3} /(\mathrm{mol} \cdot \mathrm{s})$ verwendet. Dieser Wert liefert für das experimentell bestimmte Verhältnis der Radikalanfangskonzentrationen die beste Übereinstimmung von Experiment und Simulation bezüglich des Konzentrationsverhältnisses von Ethan-d $6 /$ Hexan $=2$. 


\begin{tabular}{|c|c|c|c|}
\hline \multicolumn{3}{|c|}{ Reaktion } & \multirow{2}{*}{$\begin{array}{r}\mathrm{cm}^{3} /(\mathrm{mol} \cdot \mathrm{s}) \\
1,00 \cdot 10^{13}\end{array}$} \\
\hline$\left(\mathrm{x}_{1}\right)$ & $\mathrm{CH}_{3}$ & $+\mathrm{CH}_{2} \mathrm{CH}_{2} \mathrm{CH}_{3} \rightarrow$ Butan + & \\
\hline$\left(\mathrm{x}_{2}\right)$ & $\mathrm{CH}_{3}$ & $+\mathrm{CH}_{2} \mathrm{CH}_{2} \mathrm{CH}_{3} \rightarrow \mathrm{CH}_{4} \quad+$ Propen & $1,85 \cdot 10^{12}$ \\
\hline$\left(\mathrm{x}_{3}\right)$ & $\mathrm{CH}_{3}$ & $+\mathrm{CH}_{2} \mathrm{CH}_{2} \mathrm{CH}_{3} \rightarrow \mathrm{CH}_{2} \quad+$ Propan & $3,20 \cdot 10^{11}$ \\
\hline$\left(b_{1}\right)$ & $\mathrm{CH}_{3}$ & $\rightarrow$ Ethan + & $2,40 \cdot 10^{13}$ \\
\hline$\left(b_{2}\right)$ & $\mathrm{CH}_{3}$ & $\rightarrow \mathrm{CH}_{4} \quad+\mathrm{CH}_{2}$ & $4,30 \cdot 10^{10}$ \\
\hline$\left(\mathrm{c}_{1}\right)$ & $\mathrm{CH}_{2}$ & $+\mathrm{CH}_{2} \mathrm{CH}_{2} \mathrm{CH}_{3} \rightarrow$ Hexan + & $1,00 \cdot 10^{13}$ \\
\hline$\left(c_{2}\right)$ & $\mathrm{CH}_{2}$ & $+\mathrm{CH}_{2} \mathrm{CH}_{2} \mathrm{CH}_{3} \rightarrow$ Propan + Propen & $1,69 \cdot 10^{12}$ \\
\hline
\end{tabular}

Tabelle 4.43: Reaktionssystem $\mathrm{CH}_{3} / 1-\mathrm{C}_{3} \mathrm{H}_{7}$, Reaktionen und Geschwindigkeitskoeffizienten für die Simulation. Geschwindigkeitskoeffizienten für die Kreuzreaktion wurden aus den experimentellen Ergebnissen abgeleitet.

Aus den Abbildungen 4.84 und 4.85 geht hervor, dass eine gute Übereinstimmung von Simulation und Experiment nur für das experimentell bestimmte Radikalverhältnis gegeben ist. Des Weiteren wurde eine gute Übereinstimmung von Experiment und Simulation für eine Geschwindigkeit der Kreuzkombination von $\mathrm{k}\left(\mathrm{CH}_{3}+\mathrm{C}_{3} \mathrm{H}_{7} \rightarrow\right.$ Butan) $=1,0 \times 10^{13} \mathrm{~cm}^{3} /(\mathrm{mol} \cdot \mathrm{s})$ gefunden. Ein kleinerer Wert von $\mathrm{k}=0,5 \times 10^{13} \mathrm{~cm}^{3} /(\mathrm{mol} \cdot \mathrm{s})$ lieferte noch tolerierbare Ergebnisse, wohingegen eine größere Geschwindigkeit von k = $2,0 \times 10^{13} \mathrm{~cm}^{3} /(\mathrm{mol} \cdot \mathrm{s})$ zu stark von dem Produktverhältnis abwich.

\subsubsection{Diskussion}

Durch Simulation des Reaktionsmechanismus wurde die Geschwindigkeit der Kreuzkombinationsreaktion bestimmt. Dabei wurde folgender Wert gefunden:

$$
k\left(\mathrm{CH}_{3}+\mathrm{C}_{3} \mathrm{H}_{7} \rightarrow \mathrm{C}_{4} \mathrm{H}_{10}\right)=(1,0-0,5 /+0,1) \times 10^{13} \mathrm{~cm}^{3} /(\mathrm{mol} \cdot \mathrm{s})
$$

Aus der geometric mean rule ergibt sich unter Verwendung der experimentell bestimmten Geschwindigkeit der Kombinationsreaktion $\mathrm{k}\left(\mathrm{CH}_{3}+\mathrm{CH}_{3} \rightarrow \mathrm{C}_{2} \mathrm{H}_{6}\right)=2,4 \times 10^{13} \mathrm{~cm}^{3} /(\mathrm{mol} \cdot \mathrm{s})$ und dem aus der Literatur entommenen Wert von $\mathrm{k}\left(\mathrm{C}_{3} \mathrm{H}_{7}+\mathrm{C}_{3} \mathrm{H}_{7} \rightarrow\right.$ Produkte $)=$ $1,17 \times 10^{13} \mathrm{~cm}^{3} /(\mathrm{mol} \cdot \mathrm{s})$ ein Wert von:

$$
k_{\mathrm{KK}}=2 \cdot \sqrt{k_{\mathrm{AA}} k_{\mathrm{BB}}}=3,4 \times 10^{13} \mathrm{~cm}^{3} /(\mathrm{mol} \cdot \mathrm{s})
$$

Für die Geschwindigkeit der Kreuzkombination geben Slagle und KnYazev einen Wert von $6,6 \times 10^{13} \mathrm{~cm}^{3} /(\mathrm{mol} \cdot \mathrm{s})$ an Kny01.

TsANG empfiehlt für die Kreuzkombination einen Wert von $3,1 \times 10^{13} \mathrm{~cm}^{3} /(\mathrm{mol} \cdot \mathrm{s})$ [Tsa88]. 


\subsubsection{Die Reaktion Methyl + 2-Propyl}

Die Reaktion von Methylradikalen mit Isopropylradikalen wurde analog zu den vorhergehenden Reaktionen untersucht. Dabei wird auf Grundlage der vorliegenden Literatur folgender allgemeiner Reaktionsmechanismus angenommen:

$$
\begin{array}{rlr}
\mathrm{CH}_{3}+\mathrm{CH}_{3} \mathrm{CHCH}_{3} & \longrightarrow \mathrm{CH}\left(\mathrm{CH}_{3}\right)_{3} & \left(x_{1}\right) \\
& \longrightarrow \mathrm{CH}_{4}+\mathrm{C}_{3} \mathrm{H}_{6} & \left(x_{2}\right) \\
& \longrightarrow \mathrm{CH}_{2}+\mathrm{C}_{3} \mathrm{H}_{8} & \left(x_{3}\right) \\
\mathrm{CH}_{3}+\mathrm{CH}_{3} & \longrightarrow \mathrm{C}_{2} \mathrm{H}_{6} & \left(b_{1}\right) \\
& \longrightarrow \mathrm{CH}_{4}+\mathrm{CH}_{2} & \left(b_{2}\right) \\
\mathrm{CH}_{3} \mathrm{CHCH}_{3}+\mathrm{CH}_{3} \mathrm{CHCH}_{3} & \left.\longrightarrow\left(\mathrm{CH}_{3}\right)_{2} \mathrm{CHCH}_{2} \mathrm{CH}_{3}\right)_{2} & \left(c_{1}\right) \\
& \longrightarrow \mathrm{C}_{3} \mathrm{H}_{8}+\mathrm{C}_{3} \mathrm{H}_{6} & \left(c_{2}\right)
\end{array}
$$

Neben den Kreuzreaktionsprodukten $\left(\mathrm{x}_{i}\right)$ sind Produkte der Selbstreaktionen zu erwarten, wobei der Beitrag der Kombination von zwei Isopropylradikalen $\left(c_{1}\right)$ auf Grund sterischer Effekte gegenüber der Disproportionierung $\left(c_{2}\right)$ als weniger dominant eingeschätzt wird, als dies bei der Reaktion von zwei 1-Propylradikalen der Fall ist.

Analog zu den vorhergehenden Reaktionen wurde diese Reaktion mit Hilfe verschiedener Vorläufergemische untersucht. Dazu wurden zum Einen Aceton- $\mathrm{d}_{6^{-}}$/ IsopropyliodidMischungen und zum Anderen Aceton- $\mathrm{d}_{6}{ }^{-} /$Diisopropylketon-Mischungen verwendet. Die Vor- und Nachteile der Vorläufer sind Abschnitt 4.2.1 detailliert beschrieben. Wie auch für die vorhergehenden Reaktionen, wurde das Verhältnis der Radikalvorläufer variiert und Photolysen der Vorläufer ohne den zweiten Reaktionspartner zur Bestimmung der Produkte der Selbstreaktionen durchgeführt. Alle Messungen wurden bei einer Temperatur von 298 K und einem Gesamtdruck von 4 mbar vorgenommen. Die maximale Photolysenanzahl betrug zur Vermeidung von Sekundärphotolysen 400, was einem Vorläuferverbrauch von ca. $10 \%$ entspricht. Die Spektren sind 100-fach gemittelt und wurden in Abständen von 50 - 100 Photolysen aufgenommen. 


\subsubsection{Qualitative Analyse}

Reaktionsspektren für die verschiedenen Vorläufermischungen sind in Abbildung 4.86 und 4.87 nach jeweils 400 Photolysen dargestellt. Die Abbildungen zeigen deutlich die Abnahmen der verschiedenen Vorläufer.

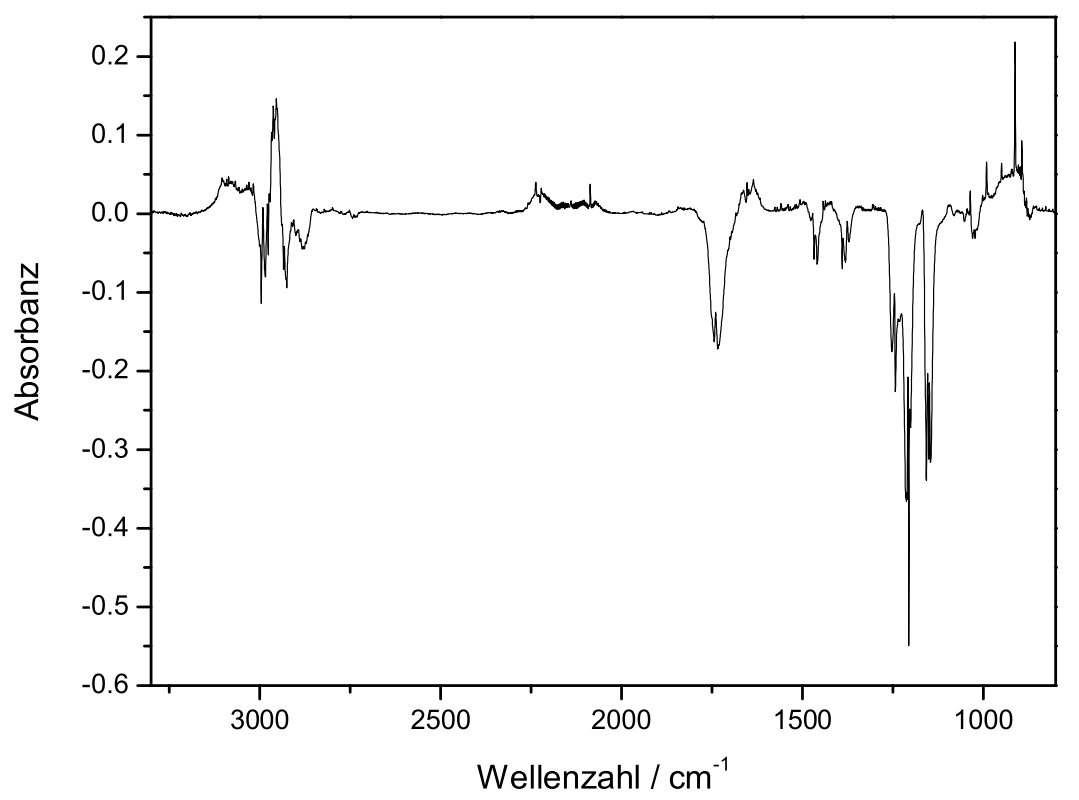

Abbildung 4.86: Reaktionssystem $\mathrm{CH}_{3} / 2-\mathrm{C}_{3} \mathrm{H}_{7}$, Reaktionsspektrum nach 400 Photolysen bei einem Gesamtdruck von 4 mbar und $298 \mathrm{~K} ; \mathrm{p}\left(\right.$ Aceton- $\left._{6}\right)=0,3$ mbar, $\mathrm{p}$ (Isoropyliodid) $=0,6$ mbar. 


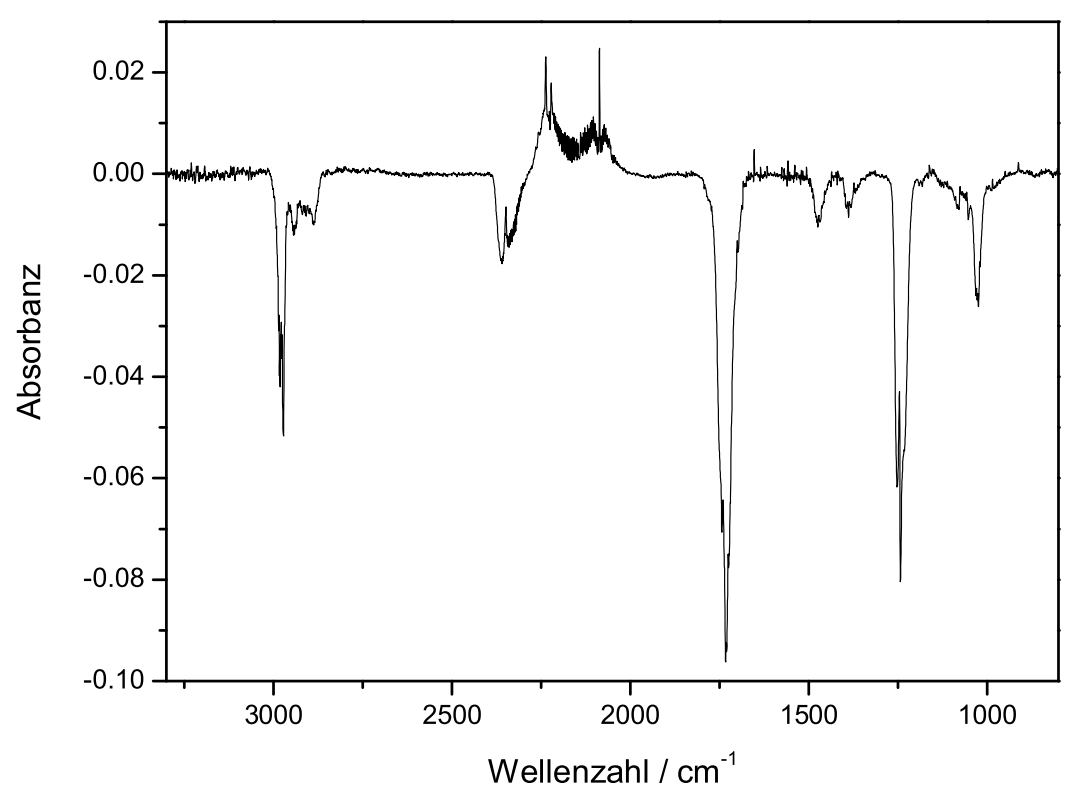

Abbildung 4.87: Reaktion $\mathrm{CH}_{3}+2-\mathrm{C}_{3} \mathrm{H}_{7}$, Reaktionsspektrum nach 400 Photolysen bei einem Gesamtdruck von 4 mbar und $298 \mathrm{~K} ; \mathrm{p}\left(\right.$ Aceton $\left.\mathrm{d}_{6}\right)=0,1$ mbar, $\mathrm{p}$ (Diisopropylketon) $=0,2$ mbar.

Die Identifikation stabiler Endprodukte ist anhand von Ausschnitten des Reaktionsspektrums im Vergleich mit Reinspektren der jeweiligen Substanzen in Abbildung 4.88 dargestellt.

Bei der Photolyse der Vorläufermischung Aceton- $\mathrm{d}_{6} /$ Propyliodid konnten folgende Produkte eindeutig identifiziert werden: Propen, Propan, Diisopropyl, Ethan- $\mathrm{d}_{6}$ und Ethen. Neben diesen Substanzen ist in Abbildung 4.88 das Reinspektrum von Isobutan zum Vergleich angegeben. Dabei ist zu beachten, dass es sich nicht um die teildeuterierte Substanz handelt, die bei der Reaktion gebildet wird. 

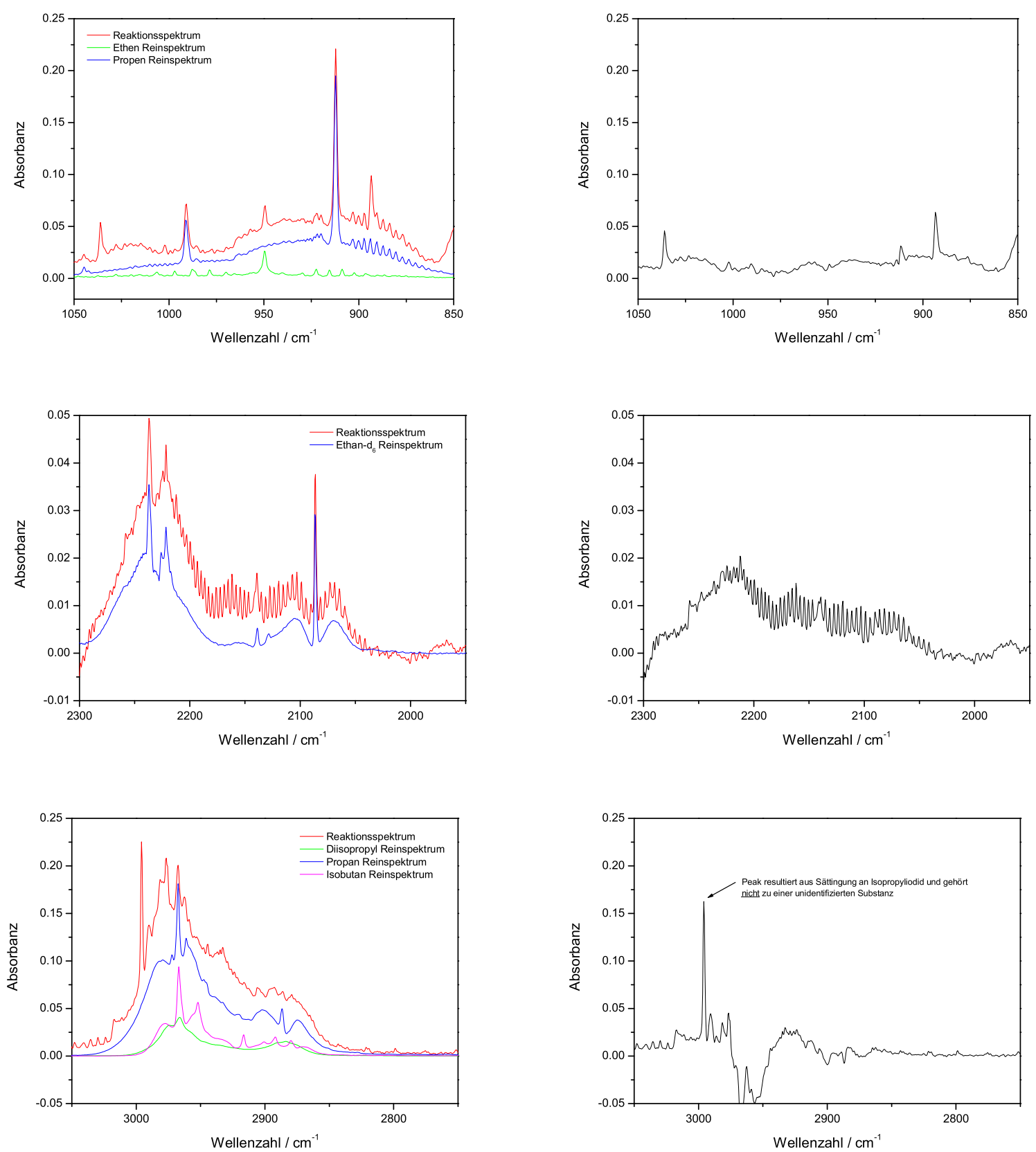

Abbildung 4.88: Reaktionssystem $\mathrm{CH}_{3} /{ }_{2}-\mathrm{C}_{3} \mathrm{H}_{7}$, qualitative Analyse stabiler Endprodukte. Linke Seite: Vergleich des Reaktionsspektrums mit Spektren der Reinsubstanzen; rechte Seite: Residuen. $p\left(\right.$ Aceton $\left.-d_{6}\right)=0,3$ mbar, $p$ (Isopropyliodid $)=0,6$ mbar, Gesamtdruck 4 mbar, $T=$ 298 K, 400 Photolysen.

In Abbildung 4.89 ist die Identifikation von Propen, Propan und Ethan- $\mathrm{d}_{6}$ für die Vorläufermischung Aceton- $\mathrm{d}_{6} /$ Diisopropylketon gezeigt. Da neben diesen Produkten die Identifikation des Rekombinationsproduktes Diisopropyl negativ war, wird auf eine quantitative Auswertung der Vorläufermischung Aceton- $\mathrm{d}_{6} /$ Diisopropylketon, wie schon in Abschnitt 4.4.4 beschrieben, verzichtet. 

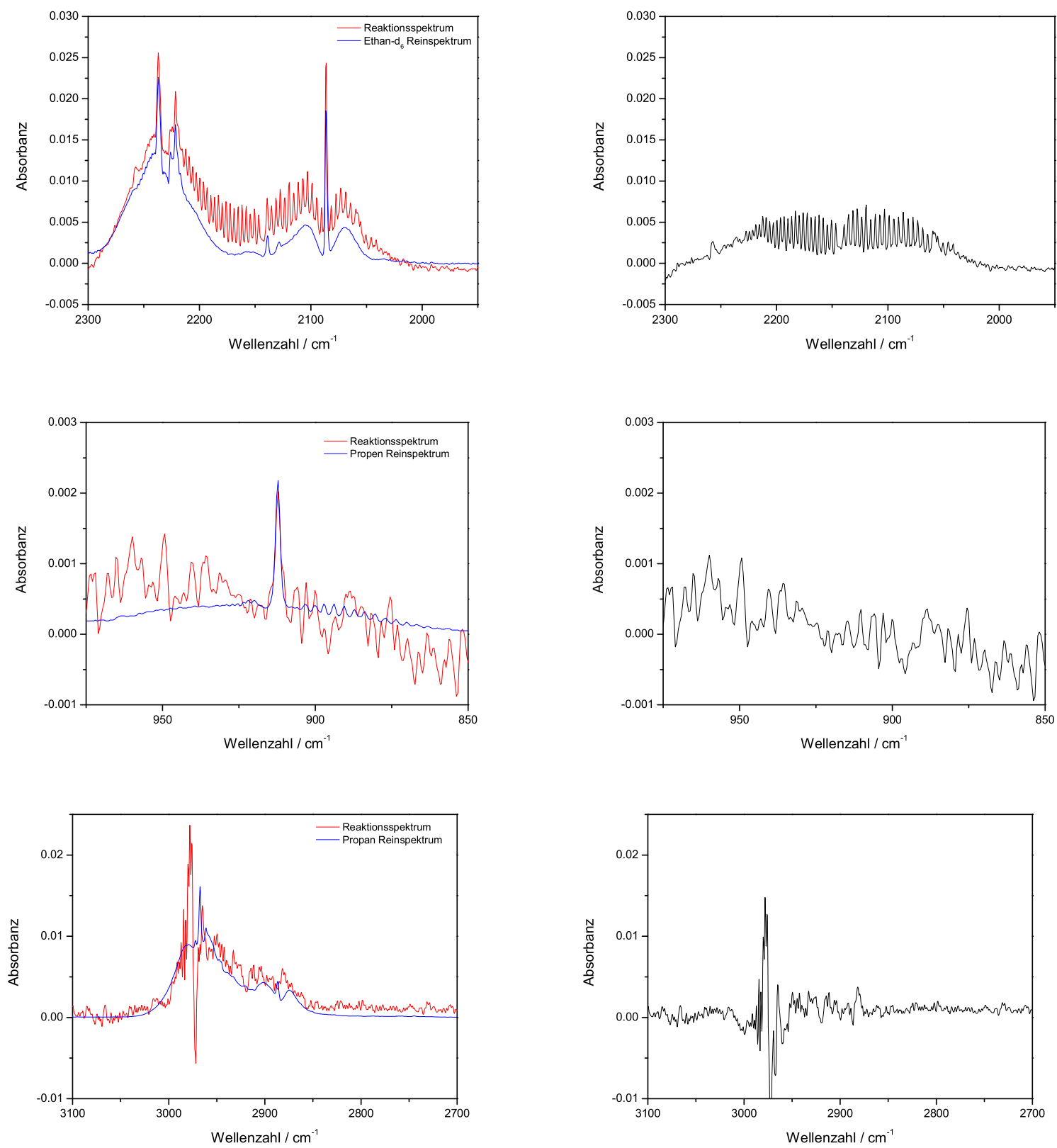

Abbildung 4.89: Reaktionssystem $\mathrm{CH}_{3} / 2-\mathrm{C}_{3} \mathrm{H}_{7}$, qualitative Analyse stabiler Endprodukte. Linke Seite: Vergleich des Reaktionsspektrums mit Spektren der Reinsubstanzen; rechte Seite: Residuen. $p$ (Aceton- $\left.d_{6}\right)=0,1$ mbar, $p$ (Diisopropylketon) $=0,2$ mbar, Gesamtdruck 4 mbar, $T$ $=298 \mathrm{~K}, 400$ Photolysen.

\subsubsection{Quantitative Analyse}

Im Folgenden werden die quantitativen Resultate der Photolyse der Aceton- $\mathrm{d}_{6}$ / IsopropyliodidVorläufermischung präsentiert. In Tabelle 4.44 sind die Partialdrücke der identifizierten

Substanzen für eine Vorläuferzusammensetzung von $\mathrm{p}\left(\right.$ Aceton- $\left.\mathrm{d}_{6}\right)=0,3 \mathrm{mbar}, \mathrm{p}$ (Isopropyliodid) $=0,6$ mbar bei $\mathrm{p}_{\text {ges }}=4 \mathrm{mbar}$ und $\mathrm{T}=298 \mathrm{~K}$ angegeben. Im Rahmen der Arbeit wurden 
auch Messreihen für andere Vorläuferverhältnisse durchgeführt, doch stellte die präsentierte Zusammensetzung das Optimum der Untersuchungen dar, da hier sowohl die Produkte der Reaktion Isopropyl + Isopropyl als auch das Produkt der Rekombination $\mathrm{CD}_{3}+$ $\mathrm{CD}_{3}$ (Ethan- $\left.\mathrm{d}_{6}\right)$ quantitativ bestimmt werden konnten. Eine Auftragung der Messwerte in Abhängigkeit von der Abnahme des Vorläufers Isopropyliodid ist in Abbildung 4.90 dargestellt.

\begin{tabular}{|c|c||c|c|c|c|}
\hline $\begin{array}{c}\text { IPI Verb. } \\
{[\mathrm{mbar}]}\end{array}$ & $\begin{array}{c}\text { Aceton- } \mathrm{d}_{6} \text { Verb. } \\
{[\mathrm{mbar}]}\end{array}$ & $\begin{array}{c}\mathrm{p}\left(\text { Ethan- }_{6}\right) \\
{[\mathrm{mbar}]}\end{array}$ & $\begin{array}{c}\mathrm{p} \text { (Propen }) \\
{[\mathrm{mbar}]}\end{array}$ & $\begin{array}{c}\mathrm{p} \text { (Propan) } \\
{[\mathrm{mbar}]}\end{array}$ & $\begin{array}{c}\mathrm{p} \text { (DIP) } \\
{[\mathrm{mbar}]}\end{array}$ \\
\hline \hline 0,0505 & 0,013 & 0,0079 & 0,0248 & 0,006 & 0,0065 \\
\hline 0,13 & 0,027 & 0,016 & 0,056 & 0,013 & 0,017 \\
\hline 0,175 & 0,038 & 0,022 & 0,078 & 0,018 & 0,024 \\
\hline
\end{tabular}

Tabelle 4.44: Reaktionssystem $\mathrm{CH}_{3} \quad / \quad 2-\mathrm{C}_{3} \mathrm{H}_{7}$, Vorläufer Isopropyliodid, Messwerte. $p($ Isopropyliodid $)=0,6$ mbar, $p\left(\right.$ Aceton $\left.d_{6}\right)=0,3 \mathrm{mbar}, p_{\text {ges }}=4 \mathrm{mbar}, \mathrm{T}=298 \mathrm{~K}$, Badgas Argon (IPI $:=$ Isopropyliodid, DIP $:=$ Diisopropyl).

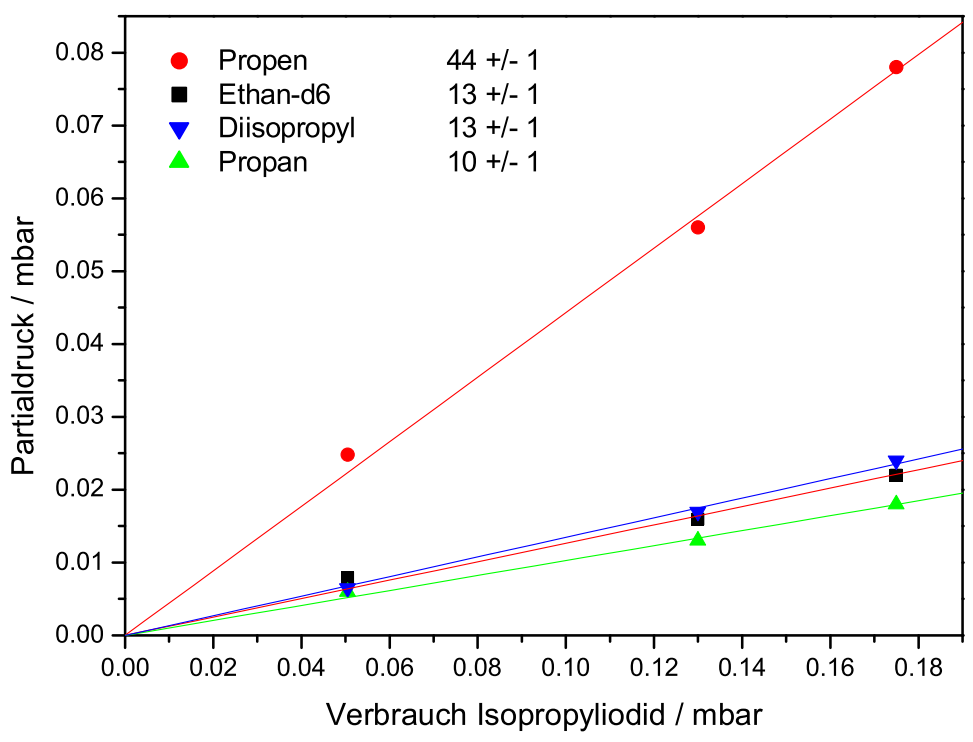

Abbildung 4.90: Reaktionssystem $\mathrm{CH}_{3} / 2-\mathrm{C}_{3} \mathrm{H}_{7}$, quantitative Analyse, Vorläufer Aceton-d 6 / Isopropyliodid. $p$ (Aceton- $\left.d_{6}\right)=0,3$ mbar, $p$ (Isopropyliodid) $=0,6$ mbar, $p_{\text {ges }}=4$ mbar, $T=$ $298 \mathrm{~K}$.

Die Abbildung 4.90 zeigt eine lineare Zunahme der Partialdrücke der Produkte mit Verbrauch des Vorläufers. Daher kann angenommen werden, dass Sekundärreaktionen und die Photolyse primärer Produkte vernachlässigt werden können. Ein Vergleich dieser Auf- 
tragung mit der Auftragung in Abbildung 4.57 zeigt weiterhin eine gute Übereinstimmung bezüglich der Bestimmung des HI-Eliminationskanals, der im Wesentlichen für die Bildung von Propen verantwortlich ist. Außerdem lieferte die dort gemachte Voruntersuchung für das Verhältnis von Propan zu Diisopropyl aus der Reaktion 2- $\mathrm{C}_{3} \mathrm{H}_{7}+2-\mathrm{C}_{3} \mathrm{H}_{7}$ einen Wert von $\mathrm{k}($ Propan $) / \mathrm{k}($ Diisopropyl $)=1,5$. Das bei dieser Reaktion gefundene Verhältins von $\mathrm{k}($ Propan $) / \mathrm{k}($ Diisopropyl $)=0,78$ stimmt also nicht mit der Voruntersuchung überein. Der in der Literatur angegebene Wert von 0,65 deutet darauf hin, dass bei der Voruntersuchung die Reaktion 2- $\mathrm{C}_{3} \mathrm{H}_{7}+\mathrm{H}$ zu einem höheren Propananteil geführt hat.

Der Partialdruck des Kreuzkombinationsprodukts wurde, wie schon in vorherigen Abschnitten, über die Abnahme der Vorläufer und Zunahme der Selbstreaktionsprodukte berechnet. Dabei erhielt man bezogen auf die Vorläufer die in Tabelle 4.45 angegebenen Partialdrücke der nach 100 - 300 Photolysen erzeugten Radikale:

\begin{tabular}{|c|c|c|c|}
\hline$\left[\mathrm{CH}_{3} \mathrm{CHCH}_{3}\right]_{0}$ & {$\left[\mathrm{CD}_{3}\right]_{0}$} & {$\left[\left(\mathrm{CH}_{3}\right)_{2} \mathrm{CHCD}_{3}\right]_{\mathrm{aus}_{3} \mathrm{CHCH}_{3}}$} & {$\left[\left(\mathrm{CH}_{3}\right)_{2} \mathrm{CHCD}_{3}\right]_{\text {aus } \mathrm{CD}_{3}}$} \\
\hline \hline 0,03 & 0,026 & 0,01 & 0,01 \\
\hline 0,087 & 0,054 & 0,027 & 0,022 \\
\hline 0,115 & 0,076 & 0,032 & 0,031 \\
\hline
\end{tabular}

Tabelle 4.45: Reaktionssystem $\mathrm{CH}_{3} /{ }_{2}-\mathrm{C}_{3} \mathrm{H}_{7}$, quantitative Analyse. Die Tabelle zeigt die aus der Abnahme der Vorläufer und unter Berücksichtigung von Nebenreaktionen (HI-Elimination) bestimmten Radikalanfangskonzentrationen. Der nach Abzug der Selbstreaktionsprodukte zur Abnahme fehlende Anteil wurde jeweils der Kreuzkombination zugerechnet. Die gute Übereinstimmung der beiden Ergebnisse bestätigt diese Methode. $p$ (Aceton- $\left.d_{6}\right)=0,3$ mbar , $p($ Isoproypliodid $)=0,6$ mbar, $p_{g e s}=4 \mathrm{mbar}, \mathrm{T}=298 \mathrm{~K}$, Konzentrationsangaben in mbar.

Die Bestimmung der Geschwindigkeit der Kreuzreaktion erfolgt wieder ausgehend von den experimentellen Ergebnissen für eine bestimmte Anfangsradikalkonzentration mit Hilfe von Simulationsrechnungen. Dabei wurde das aus Tabelle 4.45 ermittelte Verhältnis der Radikalanfangskonzentrationen $\left[\mathrm{CD}_{3}\right]_{0} /\left[\mathrm{CH}_{3} \mathrm{CHCH}_{3}\right]_{0}=0,72 / 1$ verwendet. Die Simulationen sind im nachfolgenden Abschnitt dargestellt.

\subsubsection{Simulation}

Ausgehend von den quantitativen Ergebnissen für die experimentell bestimmten Radikalanfangskonzentrationen, wurden Simulationsrechnungen mit einem geeigneten Reaktionsmechanismus durchgeführt. Dazu wurden die in Tabelle 4.46 angegebenen Reak- 
tionen und Geschwindigkeitskoeffizienten verwendet. Die Geschwindigkeit der Kreuzreaktion wurde dabei so angepasst, dass das experimentelle Resultat und die Simulation im Einklang waren. Abbildung 4.91 zeigt die Konzentrations-Zeit-Profile für die SelbstKombinationsprodukte Ethan und Diisopropyl sowie für das Kreuzkombinationsprodukt Isobutan unter Variation der Geschwindigkeit für die Kreuzkombination.

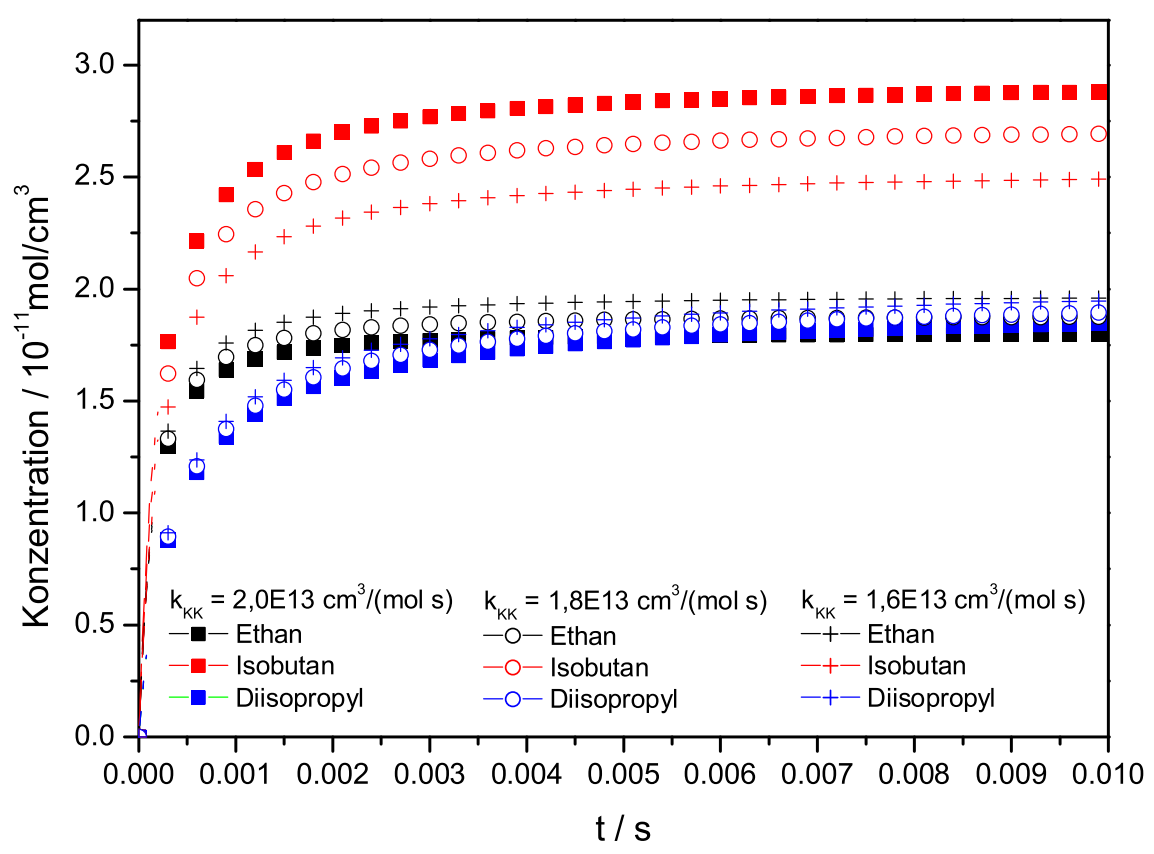

Abbildung 4.91: Reaktionssystem $\mathrm{CH}_{3} / 2-\mathrm{C}_{3} \mathrm{H}_{7}$, Einfluss der Kreuzkombinationsgeschwindigkeit. Dargestellt sind das Kreuzreaktionsprodukt Isobutan (rot) und die Kombinationsprodukte der Selbstreaktionen Diisopropyl (blau) und Ethan (schwarz) für die Geschwindigkeiten $\mathrm{k}\left(\mathrm{CH}_{3}\right.$ $+2-\mathrm{C}_{3} \mathrm{H}_{7} \rightarrow$ Isoutan $)=2,0 \times 10^{13} \mathrm{~cm}^{3} /(\mathrm{mol} \cdot \mathrm{s})$ (Quadrat); $1,8 \times 10^{13} \mathrm{~cm}^{3} /(\mathrm{mol} \cdot \mathrm{s})$ (Kreis); $1,6 \times 10^{13} \mathrm{~cm}^{3} /(\mathrm{mol} \cdot \mathrm{s})($ Kreuz $)$.

Eine hevorragende Übereinstimmung von Experiment und Simulation wurde für einen Geschwindigkeitskoeffizienten für die Kreuzkombination von $\mathrm{k}\left(\mathrm{CH}_{3}+2-\mathrm{C}_{3} \mathrm{H}_{7} \rightarrow\right.$ i-Butan $)=$ $1,8 \times 10^{13} \mathrm{~cm}^{3} /(\mathrm{mol} \cdot \mathrm{s})$ gefunden. Für diesen wurde das experimentell bestimmte Verhältnis von [Ethan] / [Diisopropyl] / [Isobutan] $=1 / 1,1 / 1,4$ gut simuliert. Dabei wurde die Konzentration des Kreuzkombinationsprodukts, wie oben beschrieben, aus den fehlenden Anteilen berechnet. Die Simulation unter Verwendung des angesprochenen Geschwindigkeitskoeffizienten liefert [Ethan] / [Diisopropyl] / [Isobutan] $=1 / 1 / 1,4$. Die Abbildung zeigt weiterhin, dass dies Verhältnis für eine größere bzw. kleinere Geschwindigkeit der Kreuzreaktion nicht erreicht wird. Ein Geschwindigkeitskoeffizient von $\mathrm{k}\left(\mathrm{CH}_{3}+2-\mathrm{C}_{3} \mathrm{H}_{7}\right.$ 
$\rightarrow$ i-Butan $)=2,0 \times 10^{13} \mathrm{~cm}^{3} /(\mathrm{mol} \cdot \mathrm{s})$ liefert ein Verhältnis von [Ethan] / [Diisopropyl] / [Isobutan] $=1 / 1 / 1,5$ und ein Geschwindigkeitskoeffizient von $\mathrm{k}\left(\mathrm{CH}_{3}+2-\mathrm{C}_{3} \mathrm{H}_{7} \rightarrow \mathrm{i}\right.$ Butan $)=1,6 \times 10^{13} \mathrm{~cm}^{3} /(\mathrm{mol} \cdot \mathrm{s})$ resultiert in einem Verhältnis von [Ethan] / [Diisopropyl] $/[$ Isobutan $]=1 / 1 / 1,3$.

Zusätzlich wurden zur Kontrolle der experimentellen Ergebnisse Simulationen für die von dem experimentell bestimmtem Verhältnis abweichende Verhältnisse durchgeführt. Dadurch sollte die Güte der experimentell bestimmten Radikalkonzentrationen überprüft werden. Abbildung 4.92 zeigt Simulationen für verschiedene Verhältnisse der Radikalanfangskonzentrationen unter Verwendung des zuvor bestimmten optimalen Geschwindigkeitskoeffizienten.

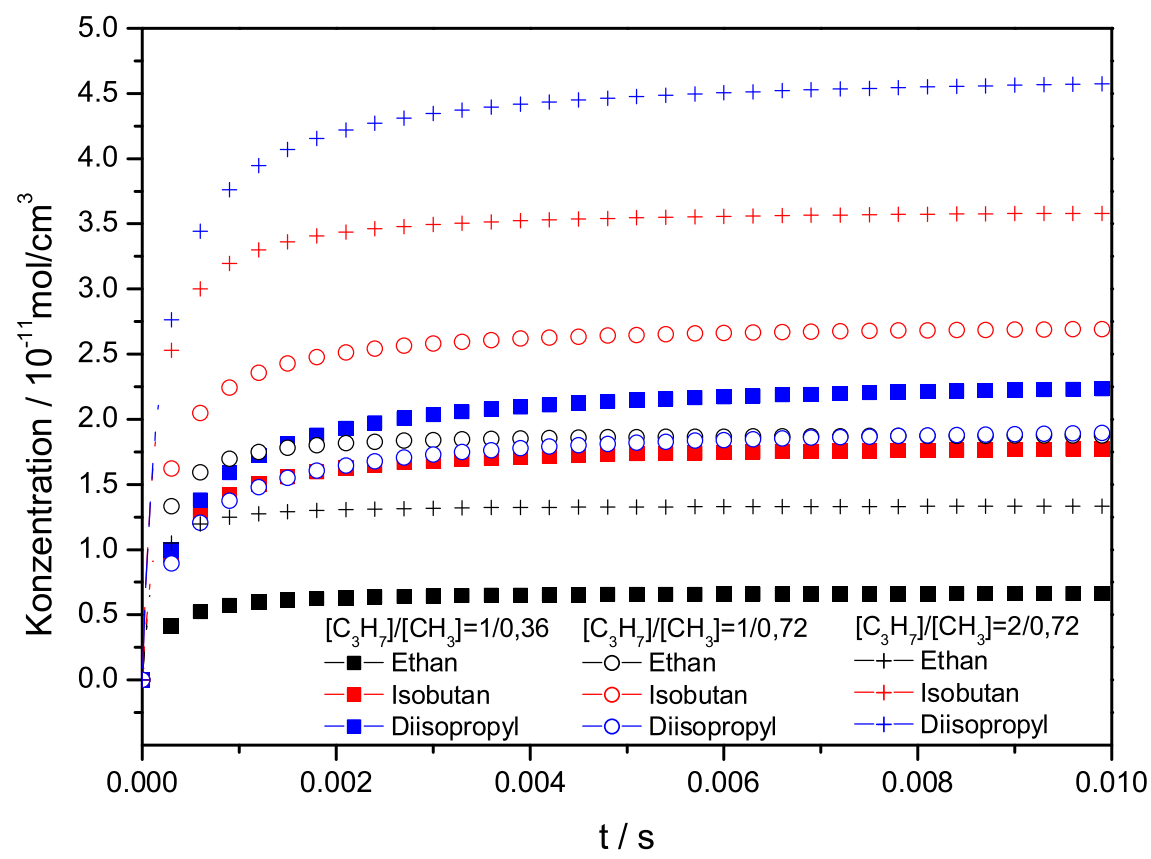

Abbildung 4.92: Reaktionssystem $\mathrm{CH}_{3} / 2-\mathrm{C}_{3} \mathrm{H}_{7}$, Einfluss des Verhältnisses der Radikalanfangskonzentrationen. Dargestellt sind das Kreuzreaktionsprodukt Isobutan (rot) und die Kombinationsprodukte der Selbstreaktionen Diisopropyl (blau) und Ethan (schwarz) für die Radikalverhältnisse $\left[\mathrm{C}_{3} \mathrm{H}_{7}\right]_{0} /\left[\mathrm{CH}_{3}\right]_{0}=1 / 0,36$ (Quadrat); $\left[\mathrm{C}_{3} \mathrm{H}_{7}\right]_{0} /\left[\mathrm{CH}_{3}\right]_{0}=1 / 0,72$ (Kreis); $\left[\mathrm{C}_{3} \mathrm{H}_{7}\right]_{0}$ $/\left[\mathrm{CH}_{3}\right]_{0}=2 / 0,72($ Kreuz$)$.

Die Abbildung 4.92 zeigt, dass eine Übereinstimmung von Experiment und Simulation nur für das experimentell bestimmte Verhältnis erzielt werden kann. 


\begin{tabular}{lllllr}
\hline \multicolumn{2}{l}{ Reaktion } & & & $\mathrm{cm}^{3} /(\mathrm{mol} \cdot \mathrm{s})$ \\
\hline$\left(\mathrm{x}_{1}\right)$ & $\mathrm{CH}_{3}$ & $+\mathrm{CH}_{3} \mathrm{CHCH}_{3}$ & $\rightarrow$ i-Butan & + & $1,80 \cdot 10^{13}$ \\
$\left(\mathrm{x}_{2}\right)$ & $\mathrm{CH}_{3}$ & $+\mathrm{CH}_{2} \mathrm{CH}_{2} \mathrm{CH}_{3} \rightarrow \mathrm{CH}_{4}$ & + Propen & $4,53 \cdot 10^{12}$ \\
$\left(\mathrm{~b}_{1}\right)$ & $\mathrm{CH}_{3}$ & $+\mathrm{CH}_{3}$ & $\rightarrow$ Ethan & + & $2,40 \cdot 10^{13}$ \\
$\left(\mathrm{~b}_{2}\right)$ & $\mathrm{CH}_{3}$ & $+\mathrm{CH}_{3}$ & $\rightarrow \mathrm{CH}_{4}$ & $+\mathrm{CH}_{2}$ & $4,30 \cdot 10^{10}$ \\
$\left(\mathrm{c}_{1}\right)$ & $\mathrm{CH}_{3} \mathrm{CHCH}_{3}+\mathrm{CH}_{3} \mathrm{CHCH}_{3}$ & $\rightarrow$ Diisopropyl+ & $6,05 \cdot 10^{12}$ \\
$\left(\mathrm{c}_{2}\right)$ & $\mathrm{CH}_{3} \mathrm{CHCH}_{3}+\mathrm{CH}_{3} \mathrm{CHCH}_{3}$ & $\rightarrow$ Propan & + Propen & $3,93 \cdot 10^{12}$ \\
\hline
\end{tabular}

Tabelle 4.46: Reaktionssystem $\mathrm{CH}_{3} / 2-\mathrm{C}_{3} \mathrm{H}_{7}$, Reaktionen und Geschwindigkeitskoeffizienten für die Simulation. Geschwindigkeitskoeffizienten für die Kreuzreaktion wurden aus den experimentellen Ergebnissen abgeleitet.

\subsubsection{Diskussion}

Aus den experimentellen Ergebnissen und der Simulationrechnungen wurde die Geschwindigkeit der Kreuzkombination $\mathrm{CH}_{3}+2-\mathrm{C}_{3} \mathrm{H}_{7} \rightarrow$ i-Butan bestimmt. Dabei wurde folgender Geschwindigkeitskoeffizient gefunden:

$$
k\left(\mathrm{CH}_{3}+2-\mathrm{C}_{3} \mathrm{H}_{7} \rightarrow \mathrm{i}-\mathrm{Butan}\right)=(1,8 \pm 0,1) \times 10^{13} \mathrm{~cm}^{3} /(\mathrm{mol} \cdot \mathrm{s})
$$

Mit Hilfe der geometric mean rule und unter Verwendung der Geschwindigkeiten für die Selbstreaktionen von $\mathrm{k}\left(\mathrm{CH}_{3}+\mathrm{CH}_{3} \rightarrow\right.$ Produkte $)=2,4 \times 10^{13} \mathrm{~cm}^{3} /(\mathrm{mol} \cdot \mathrm{s})$ und $\mathrm{k}\left(2-\mathrm{C}_{3} \mathrm{H}_{7}+\right.$ $2-\mathrm{C}_{3} \mathrm{H}_{7} \rightarrow$ Produkte $)=1,0 \times 10^{13} \mathrm{~cm}^{3} /(\mathrm{mol} \cdot \mathrm{s})$ ergibt sich ein Geschwindigkeitskoeffizient von

$$
k_{K K}=2 \cdot \sqrt{k_{\mathrm{AA}} k_{\mathrm{BB}}}=3,1 \times 10^{13} \mathrm{~cm}^{3} /(\mathrm{mol} \cdot \mathrm{s})
$$

Der mittels der geometric mean rule berechnete Wert weicht also um einen Faktor 1,7 nach oben ab. In diesem Fall liefert die geometric mean rule ein tollerierbares Ergebnis.

Im Folgenden werden die experimentellen Ergebnisse mit der Literatur verglichen.

TsANG empfiehlt für die Kreuz-Kombinationsreaktionen einen Wert von $\mathrm{k}_{c}=2,8 \times$ $10^{13}(300 / T)^{0,68} \mathrm{~cm}^{3} /(\mathrm{mol} \cdot \mathrm{s})$ [Tsa88]. Das Verhältnis von Kreuz-Kombination zu Disproportionierung wird mit $\mathrm{k}_{d} / \mathrm{k}_{c}=0,16$ angegeben. Der von TsANG empfohlene Wert liegt nach den experimentellen Ergebnissen zu hoch. Neuere Resultate zu der Reaktion wurden nicht in der Literatur gefunden. 


\subsubsection{Die Reaktion Methyl + 1-Butyl}

\subsubsection{Einleitung}

Die Reaktion von Methylradikalen mit 1-Butylradikalen wurde hinsichtlich der Kanalverzweigung untersucht. Ziel dieser Untersuchung ist, aus der Kanalverzweigung der Reaktion den Geschwindigkeitskoeffizienten der Kreuzreaktion zu bestimmen. Die Analyse der Reaktion geht dabei von folgendem Reaktionsmechanismus aus:

$$
\begin{array}{rlr}
\mathrm{CH}_{3}+\left(\mathrm{CH}_{2}\right)_{3} \mathrm{CH}_{3} & \longrightarrow \mathrm{C}_{5} \mathrm{H}_{12} & \left(x_{1}\right) \\
& \longrightarrow \mathrm{CH}_{4}+\mathrm{C}_{4} \mathrm{H}_{8} & \left(x_{2}\right) \\
& \longrightarrow \mathrm{CH}_{2}+\mathrm{C}_{4} \mathrm{H}_{10} & \left(x_{3}\right) \\
\mathrm{CH}_{3}+\mathrm{CH}_{3} & \longrightarrow \mathrm{C}_{2} \mathrm{H}_{6} & \left(b_{1}\right) \\
& \longrightarrow \mathrm{CH}_{4}+\mathrm{CH}_{2} & \left(b_{2}\right) \\
\left(\mathrm{CH}_{2}\right)_{3} \mathrm{CH}_{3}+\left(\mathrm{CH}_{2}\right)_{3} \mathrm{CH}_{3} & \longrightarrow \mathrm{C}_{8} \mathrm{H}_{18} & \left(c_{1}\right) \\
& \longrightarrow \mathrm{C}_{4} \mathrm{H}_{8}+\mathrm{C}_{4} \mathrm{H}_{10}
\end{array}
$$

Dabei wird der Anteil der Kreuzreaktionskanäle $\left(x_{2}\right)$ und $\left(x_{3}\right)$ analog zu anderen Reaktionen als untergeordnet eingeschätzt. Der Anteil der Disproportionierung $\left(b_{2}\right)$ ist gegenüber der Kombinationsreaktion $\left(b_{1}\right)$, wie auch in dieser Arbeit gezeigt wurde, vernachlässigbar. Wie in Abschnitt 4.4.5 gezeigt, beträgt das Verhältnis der Disproportionierung zu Kombination für die Reaktion von zwei 1-Butylradikalen $k(d) / k(c)=0,78$.

\subsubsection{Qualitative Analyse}

Für die Untersuchung der Reaktion wurden Vorläufermischungen von Aceton-d 6 / 1Butyliodid photolysiert. Der Einsatz eines Diketons als Butylradikalquelle wurde auf Grund einer Vielzahl von möglichen Photolyseprodukten und eines schlechten Umsatzes nicht vorgenommen. Der Vorteil der Verwendung von deuteriertem Aceton liegt in der schon mehrfach beschriebenen günstigen IR-Bandenlage, die eine eindeutige quantitative Identifikation des Selbstkombinationsprodukts Ethan- $\mathrm{d}_{6}$ gestattet. Die Abnahme an Aceton- $\mathrm{d}_{6}$ ist außerdem gut quantifizierbar, womit der Anteil an $\mathrm{CD}_{3}$-Radikalen, der bei der Kreuzreaktion verbraucht wird, berechnet werden kann. 
Alle Messungen wurden bei Zimmertemperatur (298 K) und einem Gesamtdruck von 4 mbar durchgeführt. Die Analyse erfolgte nach jeweils 100 Photolysen aus 100-fach gemittlten IR-Spektren. Die maximale Photolysenanzahl betrug 400, um Sekundärreaktionen und die Photolyse primärer Produkte zu vermeiden. Nach 400 Photolysen waren dabei 18\% des vorgelegten Butyliodids umgesetzt.

Abbildung 4.93 zeigt ein Reaktionsspektrum nach 400 Photolysen bei einer Vorläuferzusammensetzung von $\mathrm{p}\left(\right.$ Aceton- $\left.\mathrm{d}_{6}\right)=0,1$ mbar und $\mathrm{p}(1$-Butyliodid $)=0,3$ mbar.

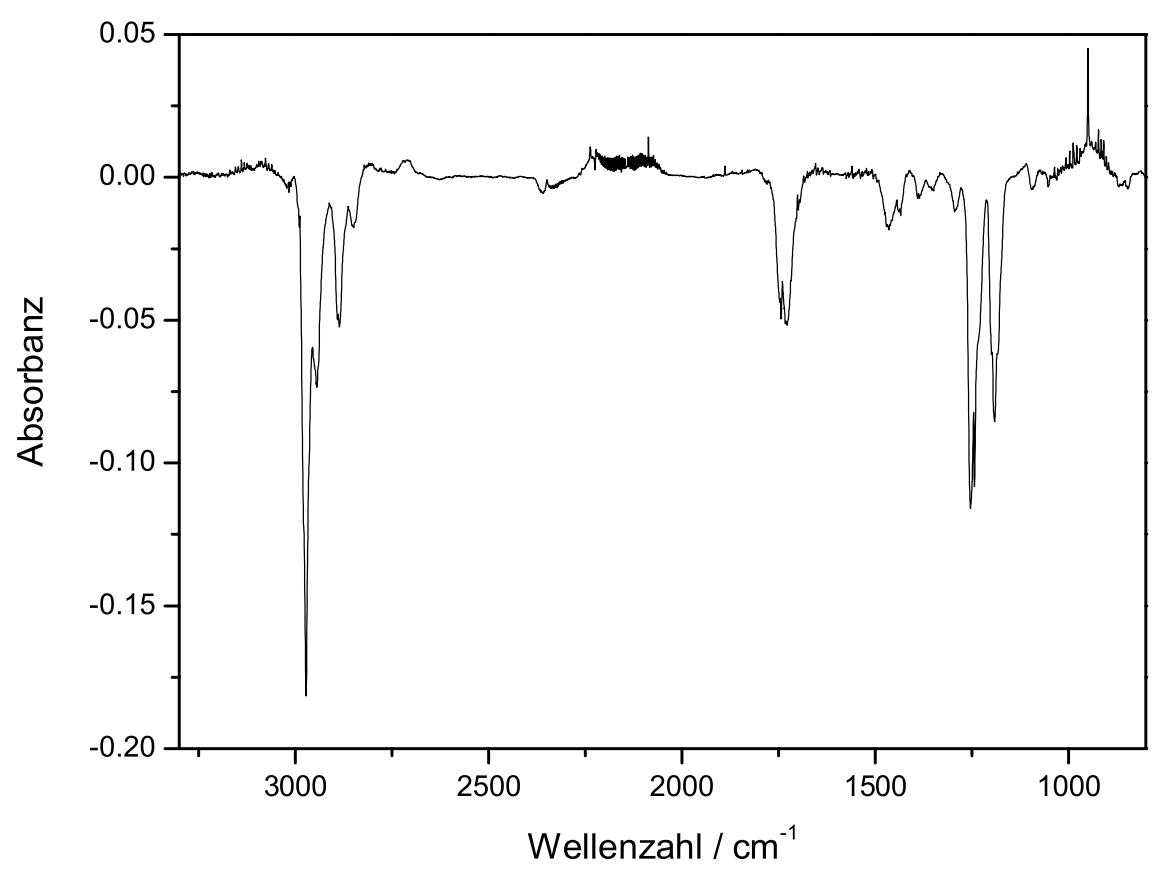

Abbildung 4.93: Reaktionssystem $\mathrm{CH}_{3} / 1-\mathrm{C}_{4} \mathrm{H}_{9}$, Reaktionsspektrum. $p$ (Aceton- $\left.\mathrm{d}_{6}\right)=0,1 \mathrm{mbar}$, $p(1$-Butyliodid $)=0,3$ mbar, $p_{g e s}=4 \mathrm{mbar}, \mathrm{T}=298 \mathrm{~K}$.

Aus Abbildung 4.93 geht die Abnahme an Vorläufern eindeutig hervor. Für die qualitative Analyse sind Bereiche des um die Abnahme an Vorläufern korrigierten Reaktionsspektrums vergrößert in Abbildung 4.94 dargestellt, in denen Produktbildung beobachtet werden konnte. Zum Vergleich sind die Reinspektren der identifizierten Substanzen zusätzlich eingezeichnet. 

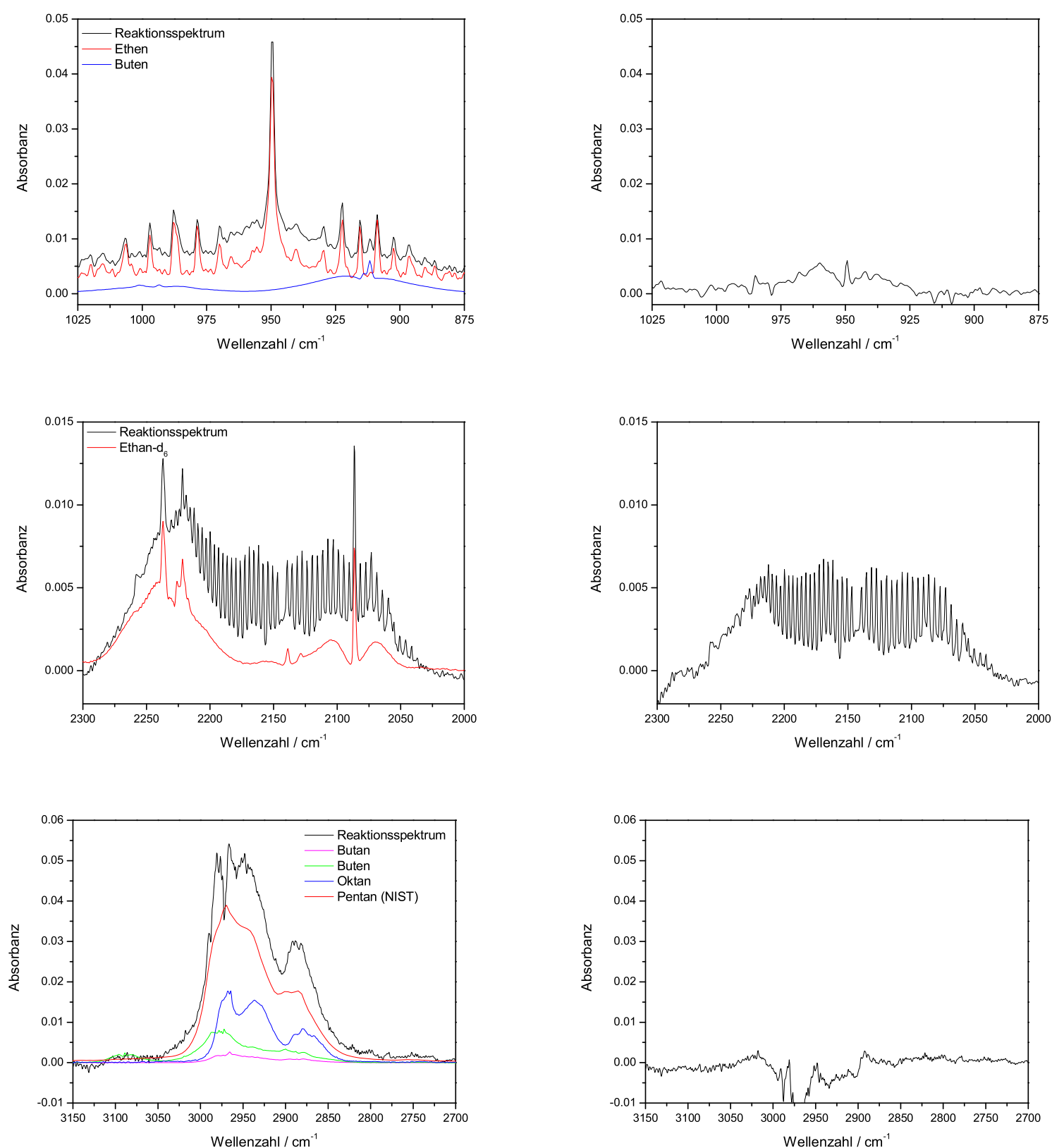

Abbildung 4.94: Reaktionssystem $\mathrm{CH}_{3} /{ }_{1}-\mathrm{C}_{4} \mathrm{H}_{9}$, qualitative Analyse stabiler Endprodukte. Linke Seite: Vergleich des Reaktionsspektrums mit Spektren der Reinsubstanzen; rechte Seite: Residuen. $p\left(\right.$ Aceton- $\left.d_{6}\right)=0,1$ mbar, $p(1$-Butyliodid $)=0,3$ mbar, Gesamtdruck 4 mbar, $T=298$ K, 400 Photolysen. Bei dem Reinspektrum von Pentan handelt es sich nicht um das Spektrum des deuterierten Kreuzkombinationsprodukts. Dies stand nicht zur Verfügung. Das Reinspektrum von Pentan soll hier nur als Anhaltspunkt für die Bandenlage dienen.

Wie aus der Abbildung 4.94 hervorgeht, konnten folgenden Produkte identifiziert werden: Butan, 1-Buten, Ethan- $\mathrm{d}_{6}$, Ethen und Oktan. Das eingezeichnete Spektrum von Pentan dient nur zur Orientierung für das eigentlich gebildete $\mathrm{CD}_{3}\left(\mathrm{CH}_{2}\right)_{3} \mathrm{CH}_{3}$. Die Bildung von Ethen resultiert wahrscheinlich aus einem direkten Zerfall des angeregten Butylradikals. 
Die Bildung konnte auch zu gleichem Anteil (25\% bezogen auf die Butyliodidabnahme) bei Abwesenheit von Aceton- $\mathrm{d}_{6}$ festgestellt werden. Die Kanalverzweigung für die Photolyse von Butyliodid ist in Abschnitt 4.4.5 dargestellt. Damit stammt die Bildung von Ethen eindeutig aus dem Zerfall des Butyl-Radikals und ist nicht Resultat einer Reaktion mit Methylradikalen.

\subsubsection{Quantitative Analyse}

Im Folgenden werden die quantitativen Ergebnisse der Reaktion von Methylradikalen mit 1-Butylradikalen kurz vorgestellt. Dazu sind die Abnahmen an Vorläufern und die Partialdrücke der identifizierten Produkte in Tabelle 4.47 dargestellt. Die Werte sind für eine bessere Übersichtlichkeit auf die Abnahme an Aceton- $\mathrm{d}_{6}$ normiert.

\begin{tabular}{cccccccc}
\hline${\text { Aceton- } \mathrm{d}_{6}}$ & $\mathrm{C}_{4} \mathrm{H}_{9} \mathrm{I}$ & $\mathrm{C}_{4} \mathrm{H}_{8} \mathrm{O}$ & Ethen & Ethan- $\mathrm{d}_{6}$ & Butan & Buten & Oktan \\
\hline 1 & 4,15 & 1 & 1,05 & 0,43 & 0,35 & 0,36 & 0,45 \\
\hline
\end{tabular}

Tabelle 4.47: Reaktionssystem $\mathrm{CH}_{3} / 1-\mathrm{C}_{4} \mathrm{H}_{9}$, quantitative Analyse. Die Abnahme an Butyliodid bzw. die Zunahmen der Produkte sind auf die Abnahme an Aceton- $d_{6}$ normiert.

Unter Berücksichtigung der Oxidationsreaktion zu Butanal und der Annahme, dass die Bildung von Ethen aus einem direkten Zerfall des angeregten Butylradikals erfolgt, ergibt sich für das Verhältnis von Methylradikalen zu 1-Butylradikalen ,die für Selbstreaktionen und Kreuzreaktionen zur Verfügung stehen: $\left[\mathrm{CD}_{3}\right]_{0} /\left[\mathrm{C}_{4} \mathrm{H}_{9}\right]_{0}=1 / 1,3$. Dabei ist noch zu beachten, dass aus der Photolyse von Aceton- $\mathrm{d}_{6}$ zwei Methylradikale entstehen. Die Verhältnisse bezogen auf die tatsächlichen Radikalkonzentrationen sind in Tabelle 4.48 angegeben:

\begin{tabular}{cccccc}
\hline$\left[\mathrm{CD}_{3}\right]_{0}$ & {$\left[\mathrm{C}_{4} \mathrm{H}_{9}\right]_{0}$} & Ethan-d & Butan & Buten & Oktan \\
\hline 1 & 1,3 & 0,22 & 0,18 & 0,18 & 0,23 \\
\hline
\end{tabular}

Tabelle 4.48: Reaktionssystem $\mathrm{CH}_{3} / 1-\mathrm{C}_{4} \mathrm{H}_{9}$, quantitative Analyse. Alle angegebenen Daten sind auf die Anfangsradikalkonzentration von Methyl bezogen. Für die Berechnung der Butylradikalkonzentration wurde der Zerfall zu Ethen und die Oxidationsreaktion zu Butanal berücksichtigt.

Subtrahiert man nun die Selbstreaktionsprodukte von den anfänglichen Radikalkonzentrationen, so erhält man für die Methyl- bzw. 1-Butylradikale, die durch Kreuzreaktion 
verbraucht werden, ein Verhältnis von: $\left[\mathrm{CD}_{3}\right]_{\text {fürKreuzr. }} /\left[1-\mathrm{C}_{4} \mathrm{H}_{9}\right]_{\text {fürKreuzr. }}=0,56 / 0,48$. Dabei ist insbesondere zu beachten, dass bei der Bildung von Ethan- $\mathrm{d}_{6}$ bzw. von Oktan jeweils 2 Radikale verbraucht werden. Das Verhältnis erreicht nicht ganz den „Sollwert“ von 1, doch ist die Abweichung von $\approx 15 \%$ tolerabel. Daher kann das experimentell bestimmte Kanalverhältnis für ein Radikalverhältnis von $\left[\mathrm{CD}_{3}\right]_{0} /\left[1-\mathrm{C}_{4} \mathrm{H}_{9}\right]_{0}=1 / 1,3$ als Basis für eine Simulation genutzt werden, aus der die Geschwindigkeit der Kreuzkombination abgeleitet werden soll.

\subsubsection{Simulation}

Im Folgenden werden die Ergebnisse der Simulationsrechnungen vorgestellt. Dabei wurde zum Einen die Geschwindigkeit der Kreuzreaktion variiert, bis eine optimale Übereinstimmung von Experiment und Simulation gegeben war und zum Anderen wurden Simulationen unter Verwendung von Radikalanfangsverhältnissen durchgeführt, die von den experimentell bestimmten Verhältnis abwichen. Dieses diente zur Validierung des ermittelten Verhältnisses der Radikalanfangskonzentrationen und zur Verdeutlichung der Sensitivität gegenüber dem Verhältnis. Für die Simulation wurde für die Kombinationsreaktion der 1Butylradikale der Geschwindigkeitskoeffizient der 1-Propylkombination verwendet, da für die 1-Butylkombination in der Literatur kein Geschwindigkeitskoeffizient vorliegt (vergleiche dazu [Kny96]). Mit der in Abschnitt 4.4.5 bestimmten Kanalverzweigung für die Reaktion 1-Butyl + 1-Butyl ergeben sich demnach folgende Geschwindigkeitskoffizienten:

$$
\begin{array}{r}
k\left(1-\mathrm{C}_{4} \mathrm{H}_{9}+1-\mathrm{C}_{4} \mathrm{H}_{9} \rightarrow \mathrm{C}_{8} \mathrm{H}_{18}\right)=1,0 \times 10^{13} \mathrm{~cm}^{3} /(\mathrm{mol} \cdot \mathrm{s}) \\
k\left(1-\mathrm{C}_{4} \mathrm{H}_{9}+1-\mathrm{C}_{4} \mathrm{H}_{9} \rightarrow \mathrm{C}_{4} \mathrm{H}_{8}+\mathrm{C}_{4} \mathrm{H}_{10}\right)=7,9 \times 10^{12} \mathrm{~cm}^{3} /(\mathrm{mol} \cdot \mathrm{s})
\end{array}
$$

Abbildung 4.95 zeigt die Konzentrations-Zeit-Profile der Kombinationsprodukte Ethan, Oktan und Pentan. Aus Platzgründen wurde auf die Darstellung der Disproportionierungsprodukte innerhalb der Grafik verzichtet. Wie die Abbildung deutlich zeigt, konnte eine gute Übereinstimmung von Experiment und Simulation für einen Geschwindigkeitskoeffizienten der Kreuzkombination von $k_{k k}=3,8 \times 10^{13} \mathrm{~cm}^{3} /(\mathrm{mol} \cdot \mathrm{s})$ erzielt werden. Das dabei erhaltene Verhältnis von [Ethan] / [Oktan $] /[$ Pentan $]=1 / 0,97 / 2,32$ stimmt gut mit dem experimentell bestimmten Verhältnis von [Ethan] / [Oktan] / [Pentan $]=1$ / 1,05 / 2,36 überein. 


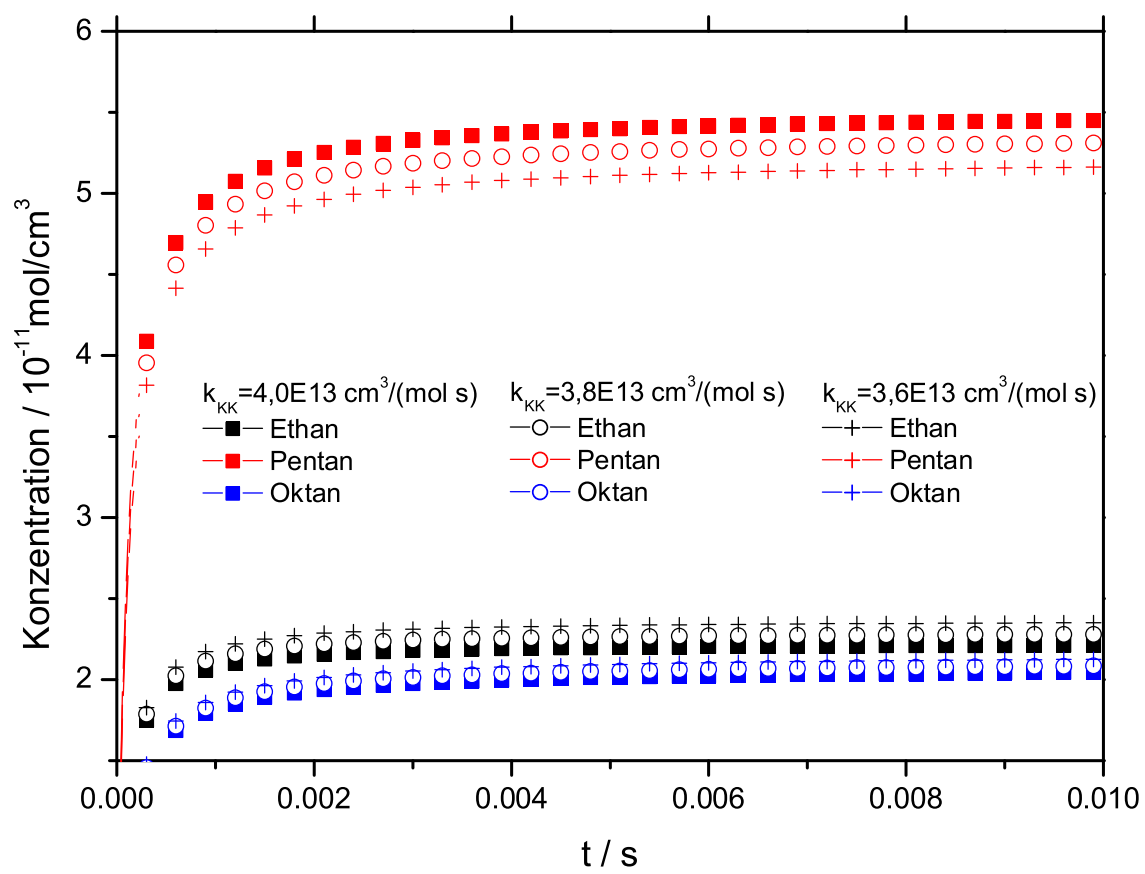

Abbildung 4.95: Reaktionssystem $\mathrm{CH}_{3} / 1-\mathrm{C}_{4} \mathrm{H}_{9}$, Einfluss der Kreuzkombinationsgeschwindigkeit. Dargestellt sind das Kreuzreaktionsprodukt Pentan (rot) und die Kombinationsprodukte der Selbstreaktionen Oktan (blau) und Ethan (schwarz) für die Geschwindigkeiten $\mathrm{k}\left(\mathrm{CH}_{3}\right.$ $+1-C_{4} H_{9} \rightarrow$ Pentan) $=4,0 \times 10^{13} \mathrm{~cm}^{3} /(\mathrm{mol} \cdot \mathrm{s})$ (Quadrat); $3,8 \times 10^{13} \mathrm{~cm}^{3} /(\mathrm{mol} \cdot \mathrm{s})$ (Kreis); $3,6 \times 10^{13} \mathrm{~cm}^{3} /(\mathrm{mol} \cdot \mathrm{s})($ Kreuz$)$.

Um die Empfindlichkeit der Reaktion auf die Radikalkonzentrationen zu überprüfen und um zu testen, ob die experimentell bestimmten Radikalkonzentrationen valide sind, wurde das experimentell bestimmte Verhältnis um den Faktor 2 erhöht bzw. erniedrigt. Die Ergebnisse der Simulationen unter diesen Anfangsbedingungen sind zusammen mit dem experimentell bestimmten Radikalverhältnis in Abbildung 4.96 dargestellt. 


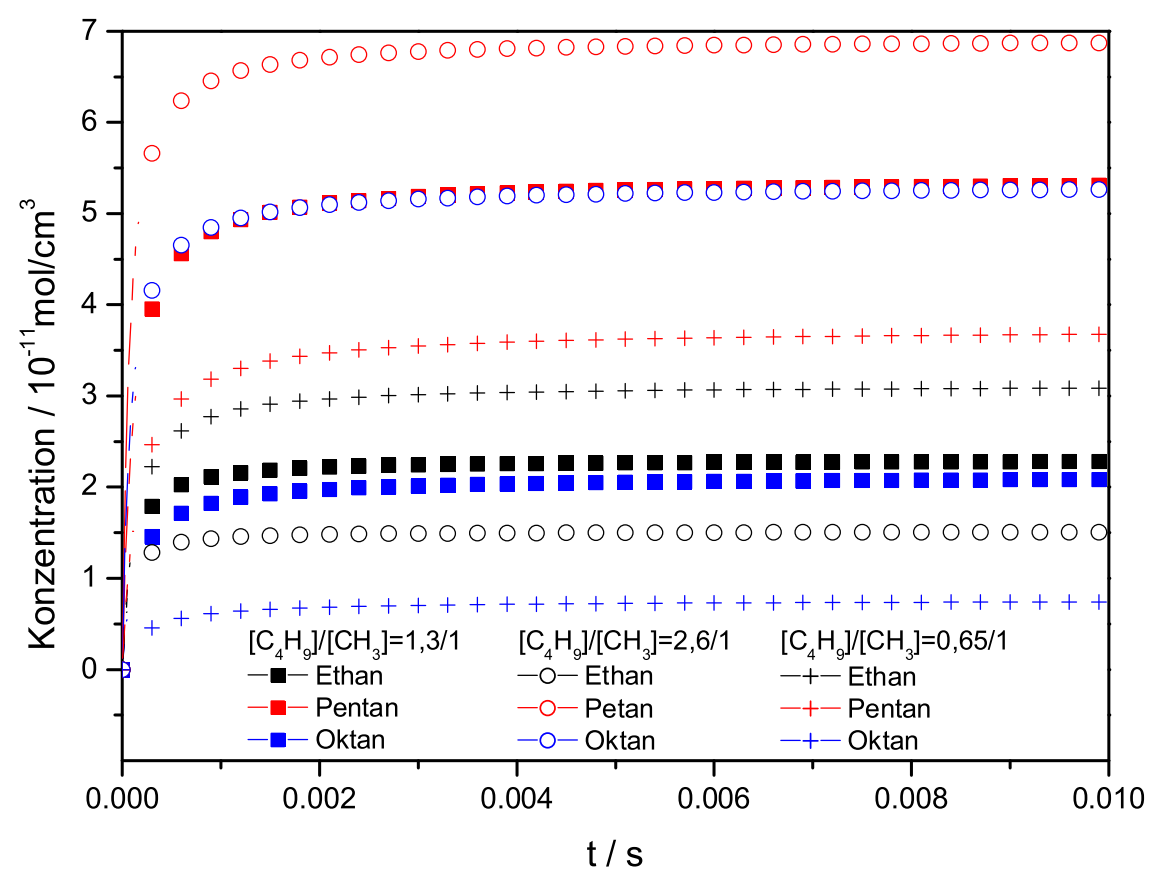

Abbildung 4.96: Reaktionssystem $\mathrm{CH}_{3} / 1-\mathrm{C}_{4} \mathrm{H}_{9}$, Einfluss der Radikalanfangskonzentrationen. Dargestellt sind das Kreuzreaktionsprodukt Pentan (rot) und die Kombinationsprodukte der Selbstreaktionen Oktan (blau) und Ethan (schwarz) für die Verhältnisse der Radikalanfangskonzentrationen $\left[\mathrm{C}_{4} \mathrm{H}_{9}\right]_{0} /\left[\mathrm{CH}_{3}\right]_{0}=1,3 / 1$ (Quadrat); $\left[\mathrm{C}_{4} \mathrm{H}_{9}\right]_{0} /\left[\mathrm{CH}_{3}\right]_{0}=2,6 / 1$ (Kreis); $\left[\mathrm{C}_{4} \mathrm{H}_{9}\right]_{0} /\left[\mathrm{CH}_{3}\right]_{0}=0,65 / 1$ (Kreuz).

Die Abbildung 4.96 zeigt, dass das experimentell bestimmte Verhältnis von Ethan- $\mathrm{d}_{6} \mathrm{zu}$ Oktan von $\left[\right.$ Ethan- $\left.\mathrm{d}_{6}\right] /[$ Oktan $]=1 / 1,05$ nur für das experimentell bestimmte Verhältnis der Radikalanfangskonzentrationen von $\left[1-\mathrm{C}_{4} \mathrm{H}_{9}\right]_{0} /\left[\mathrm{CD}_{3}\right]_{0}=1,3 / 1$ gilt. Ein Verhältnis von $\left[1-\mathrm{C}_{4} \mathrm{H}_{9}\right]_{0} /\left[\mathrm{CD}_{3}\right]_{0}=2,6 / 1$ liefert, unter Verwendung der in Tabelle 4.49 angegebenen Geschwindigkeitskoeffizienten, einen Wert von $\left[\right.$ Ethan- $\left.\mathrm{d}_{6}\right] /[$ Oktan $]=1 / 3,5$ und ein Verhältnis von $\left[1-\mathrm{C}_{4} \mathrm{H}_{9}\right]_{0} /\left[\mathrm{CD}_{3}\right]_{0}=0,65 / 1$ liefert einen Wert von $\left[\right.$ Ethan- $\left.\mathrm{d}_{6}\right] /[$ Oktan] $=1 / 0,24$.

An dieser Stelle sei nocheinmal angemerkt, dass die Geschwindigkeit der Kombinationsreaktion für 1-Butyl nicht vorlag und daher der Wert von 1-Propyl verwendet wurde. Außerdem wurde das in dieser Arbeit bestimmte Verhältnis von Disproportionierung zu Kombination mit in den Mechanismus aufgenommen. 


\begin{tabular}{llllr}
\hline \multicolumn{2}{l}{ Reaktion } & & $\mathrm{cm}^{3} /(\mathrm{mol} \cdot \mathrm{s})$ \\
\hline$\left(\mathrm{x}_{1}\right)$ & $\mathrm{CH}_{3}$ & $+\left(\mathrm{CH}_{2}\right)_{3} \mathrm{CH}_{3} \rightarrow$ Pentan+ & $3,80 \cdot 10^{13}$ \\
$\left(\mathrm{x}_{2}\right)$ & $\mathrm{CH}_{3}$ & $+\left(\mathrm{CH}_{2}\right)_{3} \mathrm{CH}_{3} \rightarrow \mathrm{CH}_{2}+$ Butan & $3,20 \cdot 10^{12}$ \\
$\left(\mathrm{~b}_{1}\right)$ & $\mathrm{CH}_{3}$ & $+\mathrm{CH}_{3}$ & $\rightarrow$ Ethan + & $2,40 \cdot 10^{13}$ \\
$\left(\mathrm{~b}_{2}\right)$ & $\mathrm{CH}_{3}$ & $+\mathrm{CH}_{3}$ & $\rightarrow \mathrm{CH}_{4} \quad+\mathrm{CH}_{2}$ & $4,30 \cdot 10^{10}$ \\
$\left(\mathrm{c}_{1}\right)$ & $\left(\mathrm{CH}_{2}\right)_{3} \mathrm{CH}_{3}+\left(\mathrm{CH}_{2}\right)_{3} \mathrm{CH}_{3} \rightarrow$ Oktan + & $1,00 \cdot 10^{13}$ \\
$\left(\mathrm{c}_{2}\right)$ & $\left(\mathrm{CH}_{2}\right)_{3} \mathrm{CH}_{3}+\left(\mathrm{CH}_{2}\right)_{3} \mathrm{CH}_{3} \rightarrow$ Butan + Buten & $7,90 \cdot 10^{12}$ \\
\hline
\end{tabular}

Tabelle 4.49: Reaktionssystem $\mathrm{CH}_{3} / 1-\mathrm{C}_{4} \mathrm{H}_{9}$, Reaktionen und Geschwindigkeitskoeffizienten für die Simulation. Geschwindigkeitskoeffizienten für die Kreuzreaktion wurden aus den experimentellen Ergebnissen abgeleitet. Für die Geschwindigkeit der Kombination $\mathrm{C}_{4} \mathrm{H}_{9}+\mathrm{C}_{4} \mathrm{H}_{9}$ liegen in der Literatur keine Ergebnisse vor. KNYAZEv und Slagle empfehlen die auch hier verwendete Geschwindigkeit der Reaktion 1-Propyl + 1-Propyl [Kny96]. Das Verhältnis von Kombination zu Disproportionierung wurde in dieser Arbeit experimentell bestimmt.

\subsubsection{Diskussion}

In diesem Abschnitt wurde durch experimentelle Bestimmung der Kanalverzweigung für ein definiertes Verhältnis der Radikalanfangskonzentrationen die Geschwindigkeit der Kreuzreaktion $\mathrm{CH}_{3}+1-\mathrm{C}_{4} \mathrm{H}_{9} \rightarrow$ Pentan bestimmt. Die Simulation des Reaktionssystems lieferte dabei einen Geschwindigkeitskoeffizienten von:

$$
k\left(\mathrm{CH}_{3}+1-\mathrm{C}_{4} \mathrm{H}_{9} \rightarrow \text { Pentan }\right)=(3,8 \pm 0,2) \times 10^{13} \mathrm{~cm}^{3} /(\mathrm{mol} \cdot \mathrm{s})
$$

Mittels der geometric mean rule und unter Verwendung der Geschwindigkeiten für die Selbstreaktionen von $\mathrm{k}\left(\mathrm{CH}_{3}+\mathrm{CH}_{3} \rightarrow\right.$ Produkte $)=2,4 \times 10^{13} \mathrm{~cm}^{3} /(\mathrm{mol} \cdot \mathrm{s})$ und $\mathrm{k}\left(1-\mathrm{C}_{4} \mathrm{H}_{9}\right.$ $+1-\mathrm{C}_{4} \mathrm{H}_{9} \rightarrow$ Produkte $)=1,8 \times 10^{13} \mathrm{~cm}^{3} /(\mathrm{mol} \cdot \mathrm{s})$ ergibt sich ein Geschwindigkeitskoeffizient von

$$
k_{K K}=2 \cdot \sqrt{k_{\mathrm{AA}} k_{\mathrm{BB}}}=4,2 \times 10^{13} \mathrm{~cm}^{3} /(\mathrm{mol} \cdot \mathrm{s})
$$

Der mit der geometric mean rule berechnete Wert ist etwas größer als der experimentell bestimmte Wert. Doch liefert die geometric mean rule in diesem Fall ein gutes Ergebnis. In der Literatur liegt für diese Reaktion lediglich ein Ergebnis vor.

Slagle und KnYAZEV geben für die Kreuzreaktion einen Wert von $6,0 \times 10^{13} \mathrm{~cm}^{3} /(\mathrm{mol} \cdot \mathrm{s})$ an Kny01. 


\subsection{Die Reaktionen von $\mathrm{CH}_{2} \mathrm{~F}$ mit $\mathrm{CHF}_{2}, \mathrm{CH}_{3}$ und $\mathrm{C}_{2} \mathbf{H}_{5}$ Radikalen}

Im Rahmen der Dissertation wurden auch die Reaktionen von $\mathrm{CH}_{2} \mathrm{~F}$ Radikalen mit $\mathrm{CHF}_{2}$, $\mathrm{CH}_{3}$ und $\mathrm{C}_{2} \mathrm{H}_{5}$ Radikalen untersucht. Die Ergebnisse dieser Untersuchungen sollen hier kurz zusammengefasst werden. Eine ausführliche Darstellung ist in der Veröffentlichung Hoy04b angegeben.

Für die oben genannten Reaktionen ergibt sich folgender allgemeiner Reaktionsmechanismus:

$$
\begin{aligned}
\mathrm{R}_{1}+\mathrm{R}_{2} & \longrightarrow \mathrm{R}_{1} \mathrm{R}_{2}^{\star} \\
\mathrm{R}_{1} \mathrm{R}_{2}^{\star} & \longrightarrow \mathrm{R}_{1}+\mathrm{R}_{2} \\
\mathrm{R}_{1} \mathrm{R}_{2}^{\star}+\mathrm{M} & \longrightarrow \mathrm{R}_{1} \mathrm{R}_{2}+\mathrm{M} \\
\mathrm{R}_{1} \mathrm{R}_{2}^{\star} & \longrightarrow \mathrm{HF}+\text { Alken } \\
\mathrm{R}_{1}+\mathrm{R}_{2} & \longrightarrow \mathrm{R}_{3}+\mathrm{R}_{1} \mathrm{H}
\end{aligned}
$$

Dabei bezeichnet (A) die Assoziation, (D) die Redissoziation, (S) die Stabilisierung, (E) die Elimination und (DP) die Disproportionierung.

Ausgehend von einem empirischen Reaktionsmechanismen wurden die Geschwindigkeitskoeffizienten der Titelreaktionen bestimmt. Dazu wurden die mittels Strömungssystem und Massenspektrometrie erhaltenen Produktkanäle verwendet. Die Mechanismen sind in Tabelle 4.50, 4.51 und 4.52 dargestellt. Die zugehörigen Konzentrations-Zeit-Profile sind in den Abbildungen 4.97, 4.98 und 4.99 abgebildet. Die Geschwindigkeitskoeffizienten wurden dabei, wie bei den anderen Simulationen dieser Arbeit, so lange variiert, bis eine gute Übereinstimmung der experimentellen Daten mit den Simulationsrechnungen gegeben war. Auf eine Darstellung der experimentellen Daten wird in diesem Fall verzichtet, da diese mit einer anderen Apparatur von BEIDERHASE gewonnen wurden. Eine Darstellung ist in der oben genannten Veröffentlichung [Hoy04b] zu finden. 


\begin{tabular}{llr}
\hline Reaktion & & $\mathrm{cm}^{3} /(\mathrm{mol} \cdot \mathrm{s})$ \\
\hline $\mathrm{CH}_{3} \mathrm{~F}+\mathrm{F}$ & $\rightarrow \mathrm{CH}_{2} \mathrm{~F}+\mathrm{HF}$ & $1,7 \times 10^{13}$ \\
$\mathrm{CH}_{2} \mathrm{~F}_{2}+\mathrm{F} \rightarrow \mathrm{CHF}_{2}+\mathrm{HF}$ & $5,9 \times 10^{12}$ \\
$\mathrm{CH}_{2} \mathrm{~F}+\mathrm{CH}_{2} \mathrm{~F} \rightarrow \mathrm{C}_{2} \mathrm{H}_{3} \mathrm{~F}+\mathrm{HF}$ & $7,0 \times 10^{12}$ \\
$\mathrm{CHF}_{2}+\mathrm{CHF}_{2} \rightarrow \mathrm{C}_{2} \mathrm{H}_{2} \mathrm{~F}_{4}+$ & $2,6 \times 10^{12}$ \\
$\mathrm{CH}_{2} \mathrm{~F}+\mathrm{F} \rightarrow$ Produkte+ & $5,0 \times 10^{13}$ \\
$\mathrm{CHF}_{2}+\mathrm{F} \rightarrow$ Produkte+ & $7,4 \times 10^{12}$ \\
$\mathrm{CH}_{2} \mathrm{~F}+\mathrm{Wand} \rightarrow$ Produkte+ & $50 \mathrm{~s}^{-1}$ \\
$\mathrm{CHF}_{2}+\mathrm{Wand} \rightarrow$ Produkte+ & $20 \mathrm{~s}^{-1}$ \\
$\mathrm{CH}_{2} \mathrm{~F}+\mathrm{CHF}_{2} \rightarrow \mathrm{C}_{2} \mathrm{H}_{2} \mathrm{~F}_{2}+\mathrm{HF}$ & $2,3 / 3,0 / 4,5 \times 10^{12}$ \\
\hline
\end{tabular}

Tabelle 4.50: Reaktionssystem $\mathrm{CH}_{2} \mathrm{~F} / \mathrm{CHF}_{2}$, Parameter für die Simulationsrechnungen.

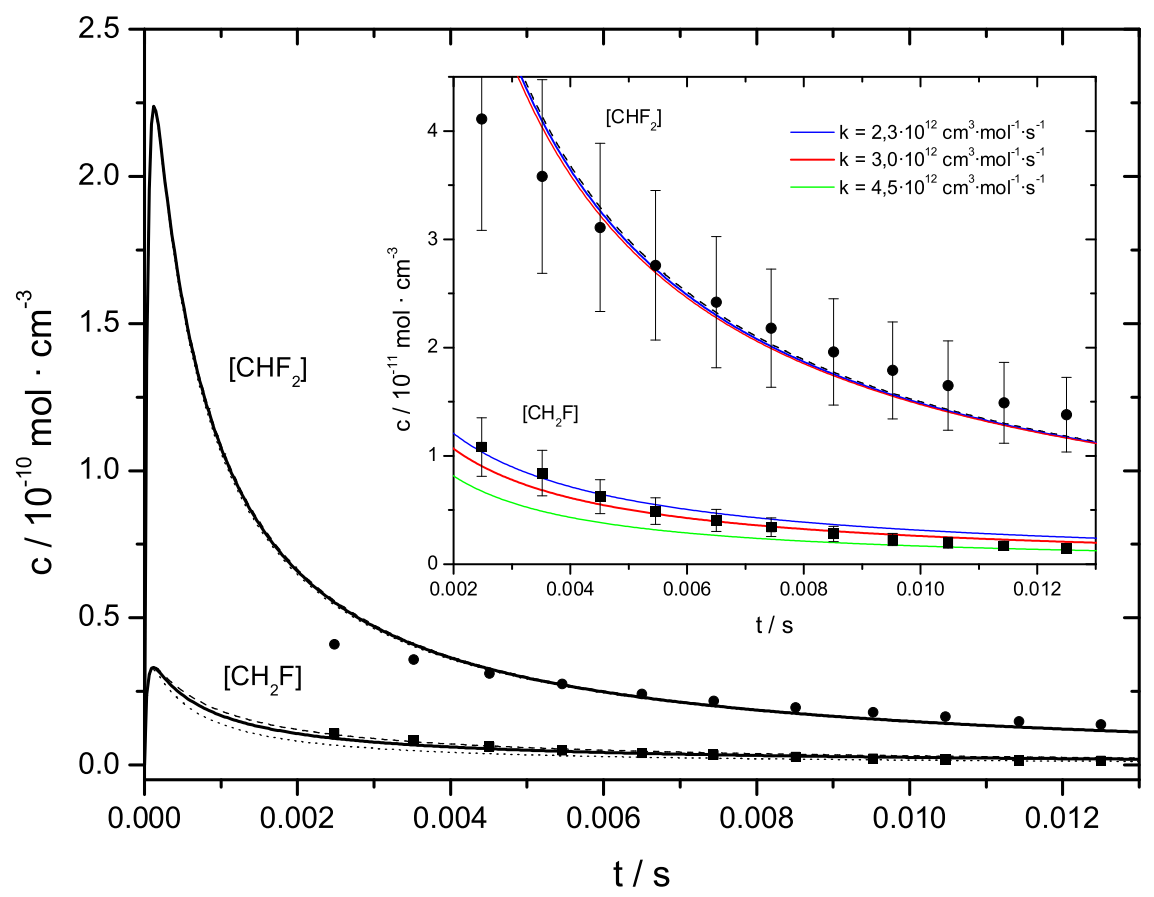

Abbildung 4.97: Reaktionssystem $\mathrm{CH}_{2} \mathrm{~F} / \mathrm{CHF}_{2}$, Simulation der Konzentrations-Zeit-Profile. Die Abbildung zeigt die Konzentrations-Zeit-Profile für verschiedene Geschwindigkeiten der Kreuzreaktion. 


\begin{tabular}{lr}
\hline Reaktion & $\mathrm{cm}^{3} /(\mathrm{mol} \cdot \mathrm{s})$ \\
\hline $\mathrm{CH}_{2} \mathrm{~F}+\mathrm{CH}_{2} \mathrm{~F} \rightarrow \mathrm{C}_{2} \mathrm{H}_{3} \mathrm{~F}+\mathrm{HF}$ & $7,0 \times 10^{12}$ \\
$\mathrm{CH}_{3}+\mathrm{CH}_{3} \rightarrow \mathrm{C}_{2} \mathrm{H}_{6}+$ & $3,6 \times 10^{13}$ \\
$\mathrm{CH}_{2} \mathrm{~F}+$ Wand $\rightarrow$ Produkte + & $50 \mathrm{~s}^{-1}$ \\
$\mathrm{CH}_{3}+$ Wand $\rightarrow$ Produkte+ & $20 \mathrm{~s}^{-1}$ \\
$\mathrm{CH}_{2} \mathrm{~F}+\mathrm{CH}_{3} \rightarrow \mathrm{C}_{2} \mathrm{H}_{4}+\mathrm{HF}$ & $3,0 / 4,0 / 5,0 \times 10^{13}$ \\
\hline
\end{tabular}

Tabelle 4.51: Reaktionssystem $\mathrm{CH}_{2} \mathrm{~F} / \mathrm{CH}_{3}$, Parameter für die Simulationsrechnungen.

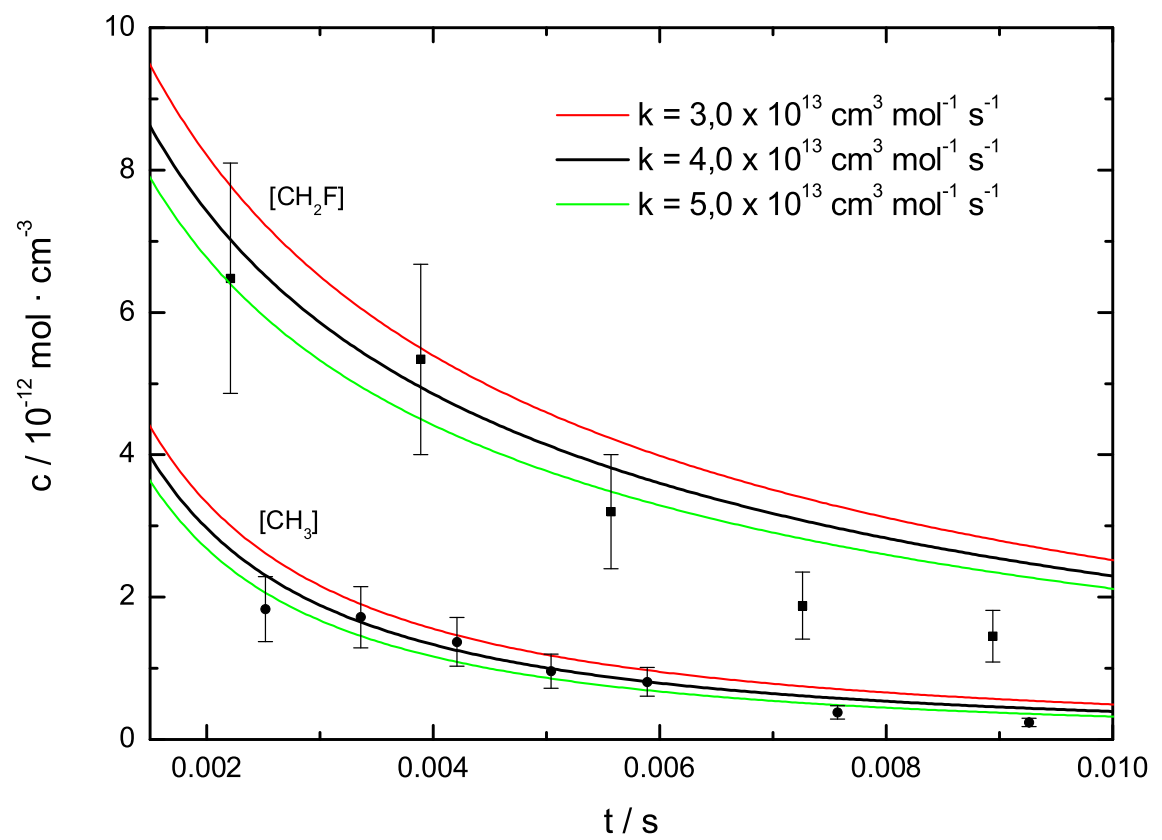

Abbildung 4.98: Reaktionssystem $\mathrm{CH}_{2} \mathrm{~F} / \mathrm{CH}_{3}$, Simulation der Konzentrations-Zeit-Profile. Die Abbildung zeigt die Konzentrations-Zeit-Profile für verschiedene Geschwindigkeiten der Kreuzreaktion.

\begin{tabular}{lr}
\hline Reaktion & $\mathrm{cm}^{3} /(\mathrm{mol} \cdot \mathrm{s})$ \\
\hline $\mathrm{CH}_{2} \mathrm{~F}+\mathrm{CH}_{2} \mathrm{~F} \rightarrow \mathrm{C}_{2} \mathrm{H}_{3} \mathrm{~F}+\mathrm{HF}$ & $7,0 \times 10^{12}$ \\
$\mathrm{C}_{2} \mathrm{H}_{5}+\mathrm{C}_{2} \mathrm{H}_{5} \rightarrow \mathrm{C}_{4} \mathrm{H}_{10}+$ & $1,14 \times 10^{13}$ \\
$\mathrm{CH}_{2} \mathrm{~F}+$ Wand $\rightarrow$ Produkte+ & $20 \mathrm{~s}^{-1}$ \\
$\mathrm{C}_{2} \mathrm{H}_{5}+$ Wand $\rightarrow$ Produkte+ & $20 \mathrm{~s}^{-1}$ \\
$\mathrm{CH}_{2} \mathrm{~F}+\mathrm{C}_{2} \mathrm{H}_{5} \rightarrow \mathrm{C}_{3} \mathrm{H}_{6}+\mathrm{HF}$ & $8,0 / 9,0 / 10,0 \times 10^{12}$ \\
\hline
\end{tabular}

Tabelle 4.52: Reaktionssystem $\mathrm{CH}_{2} \mathrm{~F} / \mathrm{C}_{2} \mathrm{H}_{5}$, Parameter für die Simulationsrechnungen. 


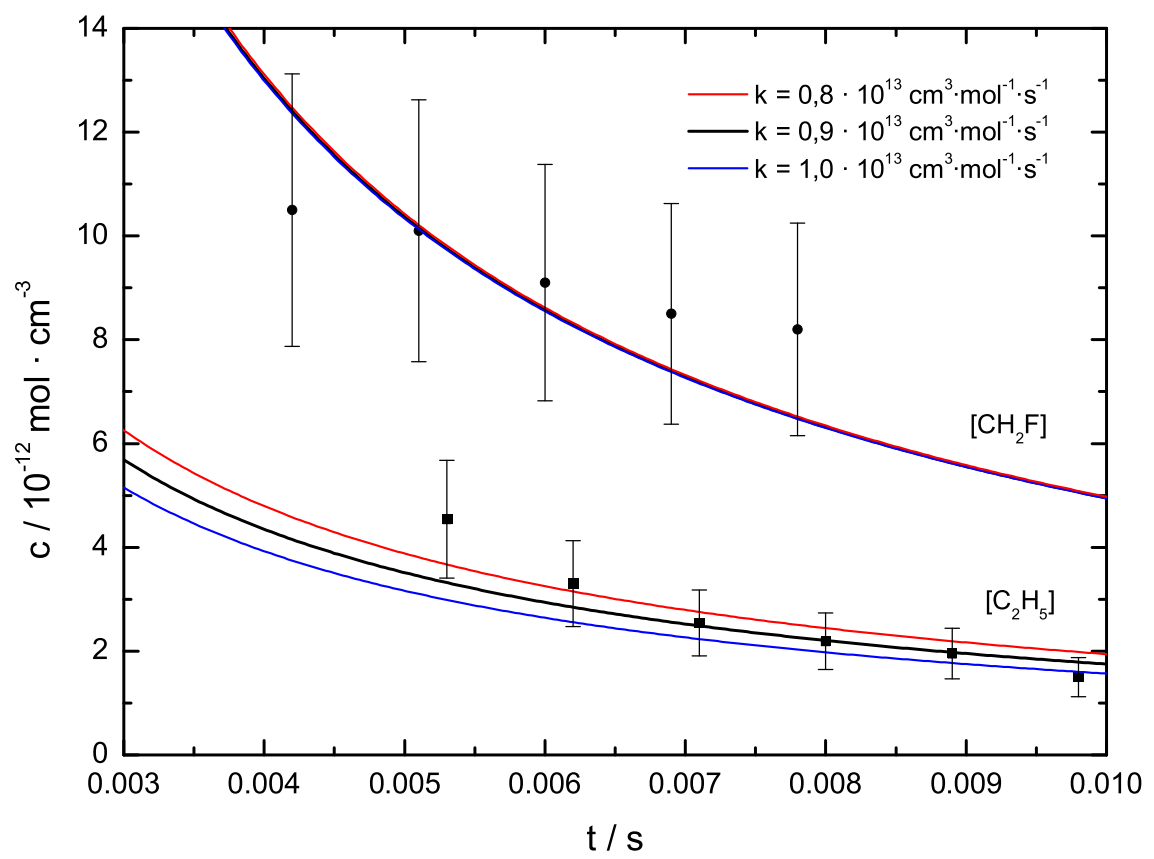

Abbildung 4.99: Reaktionssystem $\mathrm{CH}_{2} \mathrm{~F} / \mathrm{C}_{2} \mathrm{H}_{5}$, Simulation der Konzentrations-Zeit-Profile. Die Abbildung zeigt die Konzentrations-Zeit-Profile für verschiedene Geschwindigkeiten der Kreuzreaktion.

Mit Hilfe der Simulationen konnten, ausgehend von den mit einem Strömungssystem und massenspektrometrischen Nachweis experimentell gewonnenen Daten, folgende Geschwindigkeitskoeffizienten ermittelt werden:

$$
\begin{aligned}
k\left(\mathrm{CH}_{2} \mathrm{~F}+\mathrm{CHF}_{2} \longrightarrow \mathrm{C}_{2} \mathrm{H}_{2} \mathrm{~F}_{2}+\mathrm{HF}\right) & =(3,0+1,5 /-0,7) \times 10^{12} \mathrm{~cm}^{3} /(\mathrm{mol} \cdot \mathrm{s}) \\
k\left(\mathrm{CH}_{2} \mathrm{~F}+\mathrm{CH}_{3} \longrightarrow \mathrm{C}_{2} \mathrm{H}_{4}+\mathrm{HF}\right) & =(4,0 \pm 1,5) \times 10^{13} \mathrm{~cm}^{3} /(\mathrm{mol} \cdot \mathrm{s}) \\
k\left(\mathrm{CH}_{2} \mathrm{~F}+\mathrm{C}_{2} \mathrm{H}_{5} \longrightarrow \mathrm{C}_{3} \mathrm{H}_{6}+\mathrm{HF}\right) & =(9,0 \pm 3,0) \times 10^{12} \mathrm{~cm}^{3} /(\mathrm{mol} \cdot \mathrm{s})
\end{aligned}
$$




\section{Kapitel 5}

\section{Ausblick}

Im Folgenden werden erste Ergebnisse weiterer Reaktionen dargestellt. Für diese Reaktionen wurden dabei noch keine ausgedehnten Messreihen durchgeführt, sodass die Angabe quantitativer Werte für Kanalverzweigung und Geschwindigkeitskoeffizienten noch nicht möglich ist.

\subsection{Die Reaktion Methyl + 2-Butyl}

Für die Untersuchung der Reaktion von Methylradikalen mit 2-Butylradikalen wurden Vorläufermischungen von Aceton- $\mathrm{d}_{6}$ / 2-Butyliodid photolysiert. Ein Reaktionsspektrum nach 400 Photolysen bei einer Vorläuferzusammensetzung von $\mathrm{p}\left(\right.$ Aceton- $\left.\mathrm{d}_{6}\right)=0,15$ und $\mathrm{p}(2$-Butyliodid $)=0,3$ mbar bei $\mathrm{p}_{\text {ges }}=4$ mbar und $\mathrm{T}=298 \mathrm{~K}$ zeigt Abbildung 5.1 . 


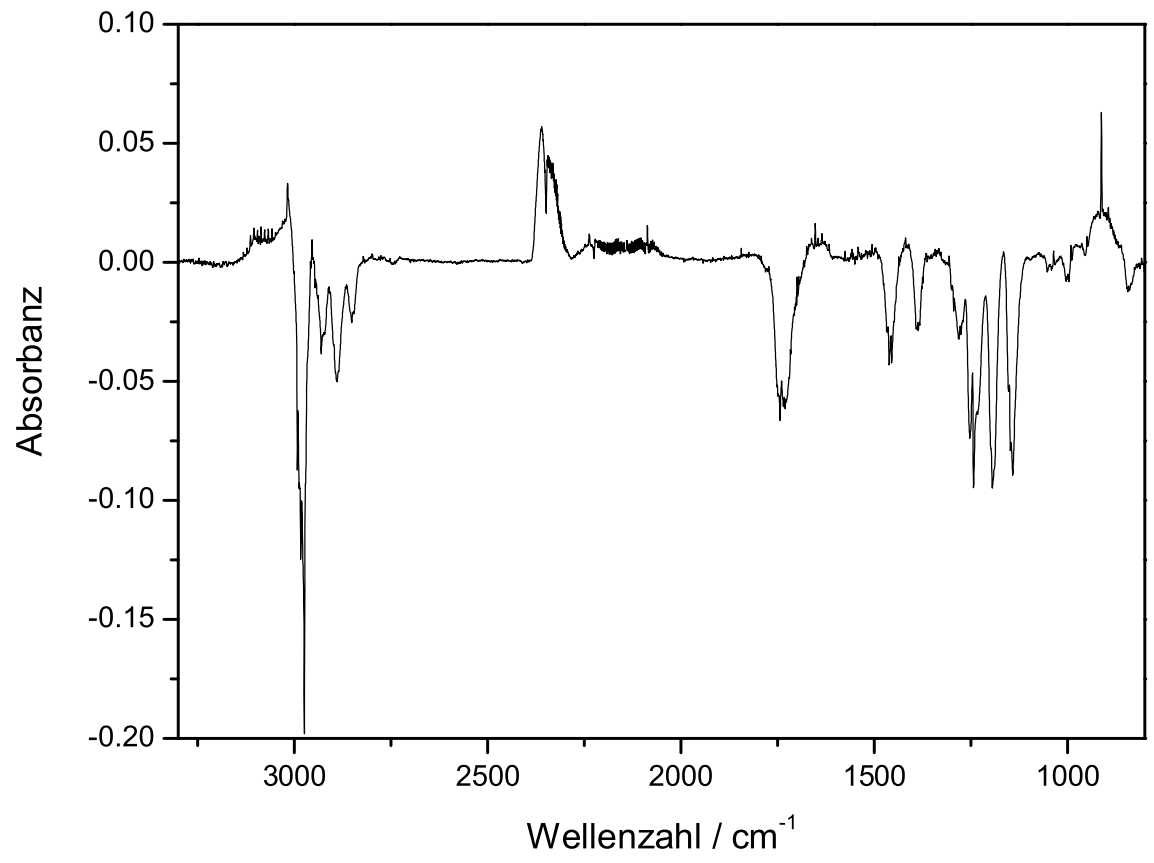

Abbildung 5.1: Reaktionssystem $\mathrm{CH}_{3} / \mathrm{2}_{4} \mathrm{C}_{4} \mathrm{H}_{9}$, Reaktionsspektrum. $p\left(\right.$ Aceton- $\left.\mathrm{d}_{6}\right)=0,15 ; \mathrm{p}(2-$ Butyliodid) $=0,3$ mbar; $p_{\text {ges }}=4$ mbar und $T=298 \mathrm{~K}$.

Vergrößerte Ausschnitte des Reaktionsspektrums im Vergleich mit Spektren der identifizierten Reinsubstanzen zeigt Abbildung 5.2. Aus der Abbildung geht deutlich hervor, dass neben den Selbstreaktionen zu Ethan- $\mathrm{d}_{6}$ und der Bildung von Buten und Butan eine Bildung des Kreuzkombinationsprodukts stattfindet. Dabei sei erwähnt, dass es sich um das Reinspektrum der undeuterierten Substanz handelt. Erste Untersuchungen deuten darauf hin, dass die Disproportionierung im Fall der Reaktion $2-\mathrm{C}_{4} \mathrm{H}_{9}+2-\mathrm{C}_{4} \mathrm{H}_{9}$ gegenüber der Kombinationsreaktion stark favorisiert ist. 

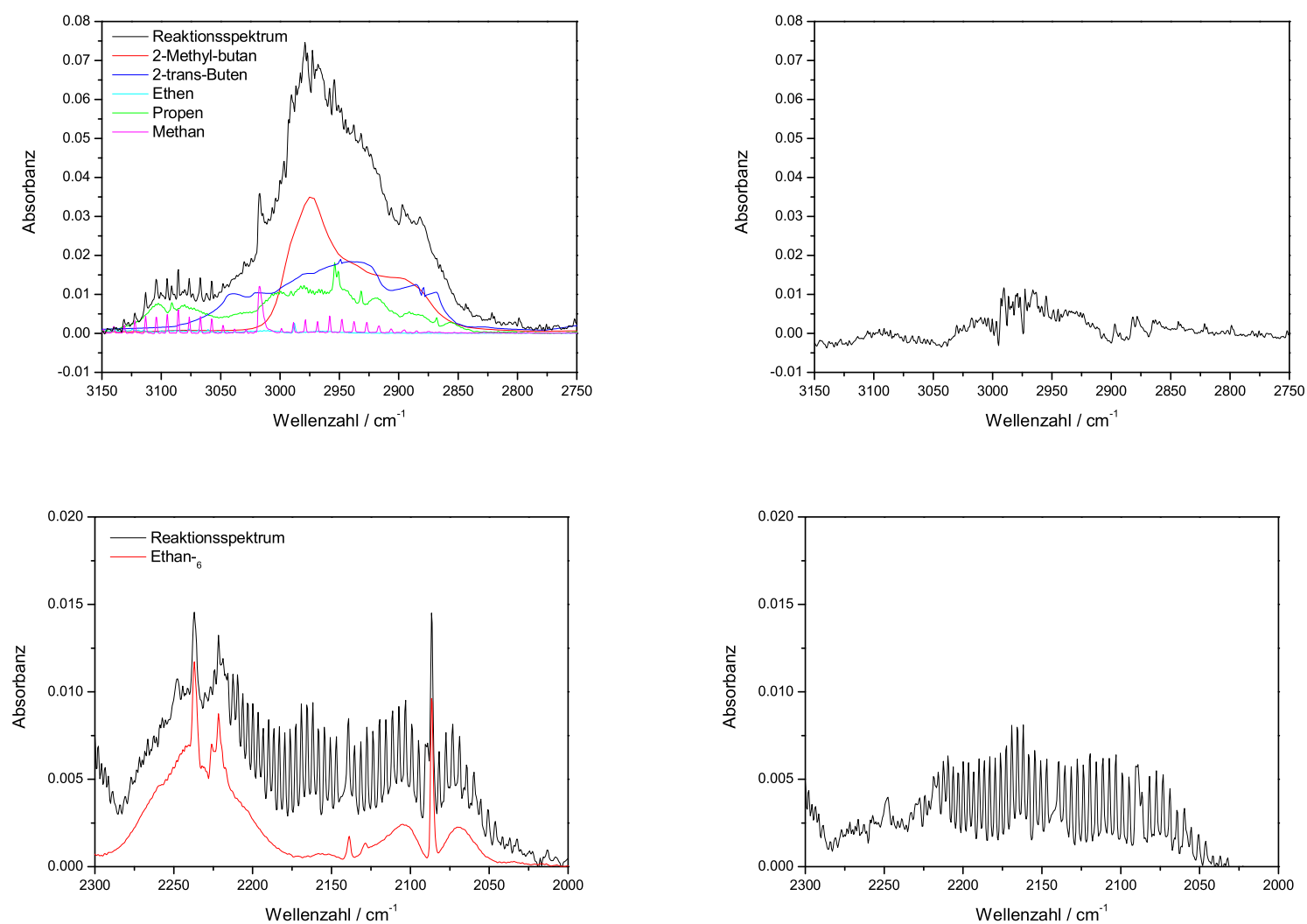

Abbildung 5.2: Reaktionssystem $\mathrm{CH}_{3} / 2-\mathrm{C}_{4} \mathrm{H}_{9}$, qualitative Analyse stabiler Endprodukte. Linke Seite: Vergleich des Reaktionsspektrums mit Spektren der Reinsubstanzen; rechte Seite: Residuen. $p\left(\right.$ Aceton- $\left.d_{6}\right)=0,1$ mbar, $p(1$-Butyliodid $)=0,3$ mbar, Gesamtdruck 4 mbar, $T=298$ K, 400 Photolysen. Bei dem Reinspektrum von 2-Methyl-Butan handelt es sich nicht um das Spektrum des deuterierten Kreuzkombinationsprodukts. Dies stand nicht zur Verfügung. Das Reinspektrum von 2-Methyl-Butan soll hier nur als Anhaltspunkt für die Bandenlage dienen.

Als Produkte der Reaktion konnten 2-trans-Buten, Propen, Ethen, Methan, 2-Metyhlbutan und Ethan- $\mathrm{d}_{6}$ identifiziert werden. Die Bildung von Methan, Propen und Ethen resultiert dabei wahrscheinlich aus einem schnellen Zerfall des angeregten 2-Butylradikals.

Da sehr hohe Anteile der Disproportionierungsprodukte (2-trans-Buten und Butan) der Selbstreaktion von zwei 2-Butylradikalen beobachtet wurden, wird für die Selbstreaktion der folgende Mechanismus vorgeschlagen:

$$
\begin{array}{rlr}
2-\mathrm{C}_{4} \mathrm{H}_{9}+2-\mathrm{C}_{4} \mathrm{H}_{9} & \longrightarrow 2-\mathrm{C}_{4} \mathrm{H}_{8}+\mathrm{C}_{4} \mathrm{H}_{10} & \geq 80 \% \\
& \longrightarrow\left(2-\mathrm{C}_{4} \mathrm{H}_{9}\right)_{2} & \leq 20 \%
\end{array}
$$

Für die Kreuzreaktion wird weiterhin die Kombination gegenüber der Disproportionierung als dominant eingeschätzt, sodass der Mechanismus basierend auf den ersten Ergebnissen 
abgeschätzt wird zu:

$$
\begin{array}{rlr}
\mathrm{CH}_{3}+2-\mathrm{C}_{4} \mathrm{H}_{9} & \longrightarrow\left(\mathrm{CH}_{3}\right)_{2} \mathrm{CH}_{2} \mathrm{CH}_{2} \mathrm{CH}_{2} \mathrm{CH}_{3} & \geq 75 \% \\
& \longrightarrow \mathrm{CH}_{4}+2-\mathrm{C}_{4} \mathrm{H}_{8} & \leq 25 \%
\end{array}
$$

\subsection{Die Reaktion Methyl + 1-Pentyl}

Für die Reaktion von Methylradikalen mit 1-Pentylradikalen liegen bisher ebenfalls nur halb-quantitative Ergebnisse vor. Ein Reaktionsspektrum nach 400 Photolysen von einer Gemischzusammensetzung von $\mathrm{p}\left(\right.$ Aceton- $\left.\mathrm{d}_{6}\right)=0,1$ mbar und $\mathrm{p}(1$-Pentyliodid $)=0,3$ mbar bei einem Gesamtdruck von 4 mbar und einer Temperatur von $298 \mathrm{~K}$ zeigt Abbildung 5.3 .

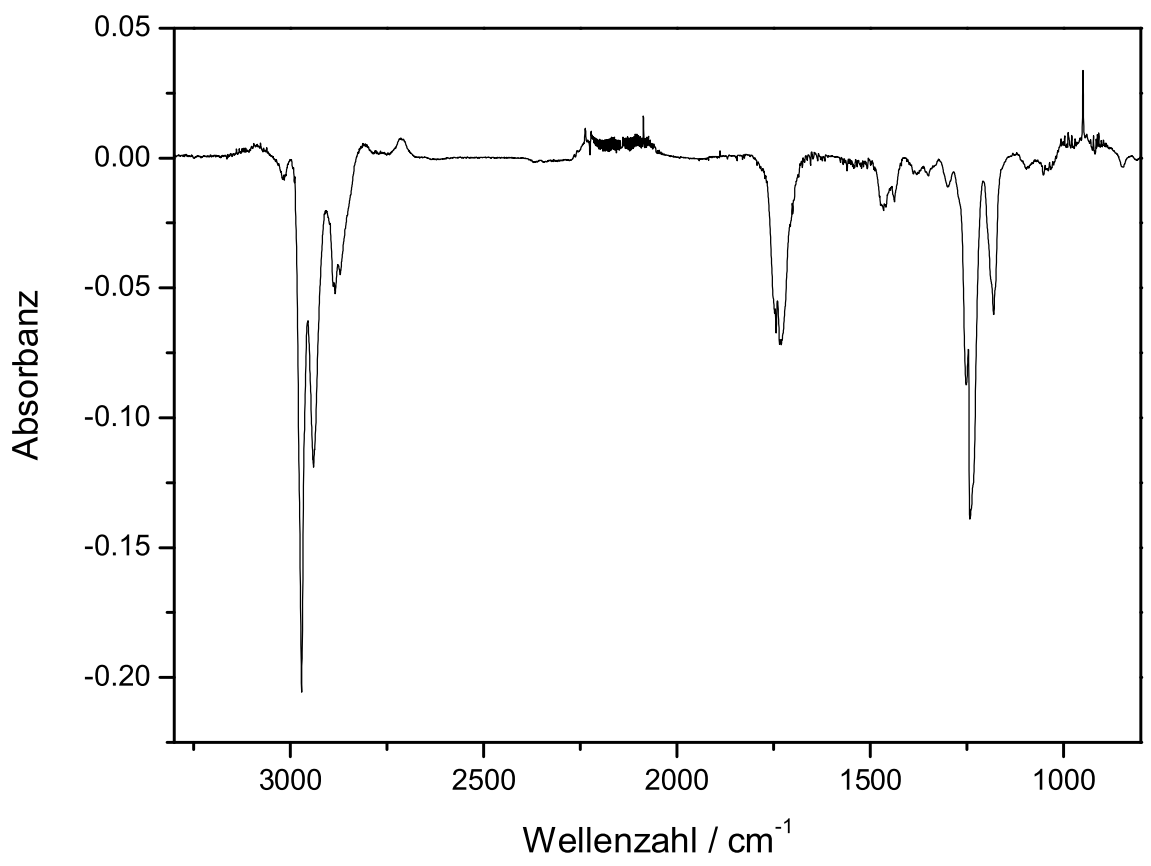

Abbildung 5.3: Reaktionssystem $\mathrm{CH}_{3} /{ }_{1-C_{5} H_{11}}$, Reaktionsspektrum. $p\left(\right.$ Aceton- $\left.d_{6}\right)=0,1 ; p(2-$ Butyliodid) $=0,3$ mbar; $p_{\text {ges }}=4$ mbar und $T=298 \mathrm{~K}$.

Für die qualitative Analyse wurden wieder vergrößerte Ausschnitte des Reaktionsspektrums mit Reinspektren der identifizierten Substanzen verglichen. Eine Darstellung des Vergleichs ist in Abbildung 5.4 angegeben. Aus dieser Darstellung geht die Bildung von 1-Penten, Pentan, Hexan, Ethen, Propen und Ethan- $\mathrm{d}_{6}$ hervor. Damit finden unter diesen 
Versuchsbedingungen sowohl die Selbstreaktionen als auch die Kreuzreaktion statt. Der hohe Anteil an Penten und Pentan deutet darauf hin, dass die Kombinationsreaktion von zwei 1-Pentylradikalen zu Dekan gegenüber der Disproportionierung untergeordnet ist.
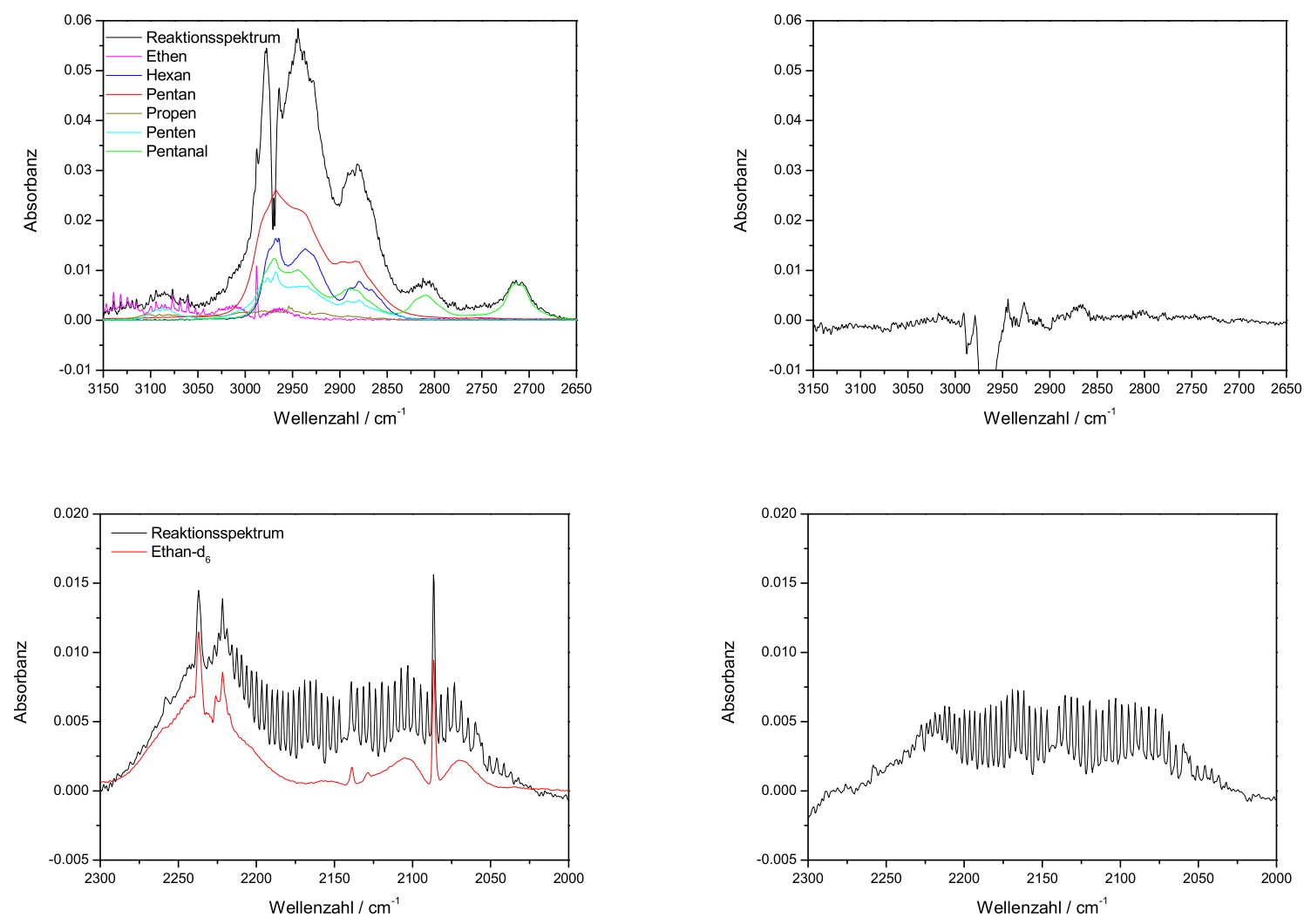

Abbildung 5.4: Reaktionssystem $\mathrm{CH}_{3} / 1-\mathrm{C}_{5} \mathrm{H}_{11}$, qualitative Analyse stabiler Endprodukte. Linke Seite: Vergleich des Reaktionsspektrums mit Spektren der Reinsubstanzen; rechte Seite: Residuen. $p\left(\right.$ Aceton- $\left.d_{6}\right)=0,1$ mbar, $p(1$-Butyliodid $)=0,3$ mbar, Gesamtdruck 4 mbar, $T=298 \mathrm{~K}$, 400 Photolysen. Bei dem Reinspektrum von Hexan handelt es sich nicht um das Spektrum des deuterierten Kreuzkombinationsprodukts. Dies stand nicht zur Verfügung. Das Reinspektrum von Hexan soll hier nur als Anhaltspunkt für die Bandenlage dienen.

Der Mechanismus der Selbstreaktion von zwei 1-Pentylradikalen wird auf Grund der vorliegenden Ergebnisse wie folgt abgeschätzt:

$$
\begin{array}{rlr}
1-\mathrm{C}_{5} \mathrm{H}_{11}+1-\mathrm{C}_{5} \mathrm{H}_{11} \longrightarrow & \longrightarrow \mathrm{C}_{5} \mathrm{H}_{10}+\mathrm{C}_{5} \mathrm{H}_{12} & \geq 70 \% \\
& \longrightarrow \mathrm{C}_{10} \mathrm{H}_{22} & \leq 30 \%
\end{array}
$$

Da die Bildung von $\mathrm{CH}_{3} \mathrm{D}$ nicht beobachtet werden konnte, wird für die Kreuzreaktion ein Mechanismus vorgeschlagen, der das Kombinationsprodukt Hexan favorisiert. 
KAPITEL 5. AUSBLICK

5.2. DIE REAKTION METHYL + 1-PENTYL

$$
\begin{array}{rlr}
\mathrm{CH}_{3}+1-\mathrm{C}_{5} \mathrm{H}_{11} \longrightarrow \mathrm{C}_{6} \mathrm{H}_{14} & \geq 80 \% \\
& \longrightarrow \mathrm{CH}_{4}+\mathrm{C}_{5} \mathrm{H}_{10} & \leq 20 \%
\end{array}
$$




\section{Kapitel 6}

\section{Zusammenfassung}

In dieser Arbeit wurden Reaktionen von Alkylradikalen mit Sauerstoffatomen und Reaktionen von Alkylradikalen mit Alkylradikalen in der Gasphase untersucht. Dabei wurden primäre Produkte, Reaktionsmechanismen und Geschwindigkeitskoeffizienten durch Auswertung von Produktspektren ermittelt, welche mit Hilfe einer FTIR-Apparatur gewonnen wurden. Durch eine Kalibrierungsprozedur war es möglich, identifizierte Produkte quantitativ zu bestimmen. Geschwindigkeitskoeffizienten wurden durch Simulationsrechnungen ausgehend von experimentell bestimmten Radikalanfangskonzentrationen und Produktkonzentrationen berechnet. Für die Simulationsrechnungen wurden Mechanismen verwendet, die alle relevanten Reaktionen beinhalteten. Die Reaktionen fanden in einer stationären Reaktionszelle bei einer Temperatur von 298 K und Gesamtdrücken zwischen 4 mbar und 1000 mbar statt. Die Radikale und Sauerstoffatome wurden aus verschiedenen Vorläufern durch Laserphotolyse bei $\lambda=193 \mathrm{~nm}$ generiert.

\subsection{Reaktionen von zyklischen Alkylradikalen und vom Allylradikal mit Sauerstoffatomen}

Die Reaktionen von zyklischen Alkylradikalen mit Sauerstoffatomen wurden anhand von Laserphotolysen von c-Alkan / $\mathrm{SO}_{2} / \mathrm{CFCl}_{3}$-Mischungen untersucht. Der mögliche Einfluss von Sekundärreaktionen und die Validität der experimentellen Ergebnisse wurde durch zusätzliche experimentelle Untersuchungen der Reaktionen von Alkenen (Cyclopenten, Cyclohexen, Cyclookten) mit Sauerstoffatomen sowie durch Simulationsrechnun- 
gen überprüft. Begleitend zu den experimentellen Ergebnissen wurden von M. OLzMANN (TU Karlsruhe) quantenchemische Rechnungen durchgeführt. Als Mechanismus für den primären Angriff der zyklischen Kohlenwasserstoffradikale durch Sauerstoffatome wurden als Reaktionskanäle die Bildung des chemisch aktivierten Additionskomplexes (a) und die Bildung des zyklischen Alkens (b) nachgewiesen. Die experimentellen Ergebnisse und die Energiebarrieren für die unimolekularen Prozesse des Additionskomplexes sind in der Tabelle 6.1 dargestellt.

\begin{tabular}{|c|c|c|c|c|c|c|c|}
\hline \multicolumn{2}{|c|}{ Kanal } & \multicolumn{2}{|c|}{$\mathrm{c}-\mathrm{C}_{5} \mathrm{H}_{9} \mathrm{O}^{\star}$} & \multicolumn{2}{|c|}{$\mathrm{c}-\mathrm{C}_{6} \mathrm{H}_{11} \mathrm{O}^{\star}$} & \multicolumn{2}{|c|}{$\mathrm{c}-\mathrm{C}_{8} \mathrm{H}_{15} \mathrm{O}^{\star}$} \\
\hline $\begin{array}{c}\text { allgem. } \\
\text { Produkte }\end{array}$ & $\begin{array}{c}\text { Reaktions- } \\
\text { Weg }\end{array}$ & $\begin{array}{c}\text { exp. } \\
\text { Ausbeute }\end{array}$ & $\begin{array}{c}\mathrm{E}_{0} \\
{[\mathrm{~kJ} / \mathrm{mol}]}\end{array}$ & $\begin{array}{c}\text { exp. } \\
\text { Ausbeute }\end{array}$ & $\begin{array}{c}\mathrm{E}_{0} \\
{[\mathrm{~kJ} / \mathrm{mol}]}\end{array}$ & $\begin{array}{c}\text { exp. } \\
\text { Aubeute }\end{array}$ & $\begin{array}{c}\mathrm{E}_{0} \\
{[\mathrm{~kJ} / \mathrm{mol}]}\end{array}$ \\
\hline Cycloketon & $\beta-\mathrm{C}-\mathrm{H}$ & $31 \pm 2$ & 76,0 & $19 \pm 3$ & 74,7 & $18 \pm 2$ & 60,4 \\
\hline Aldehyd & $\beta-\mathrm{C}-\mathrm{H}$ & $40 \pm 5$ & 149,6 & $47 \pm 6$ & 150,4 & $20 \pm 2$ & 146,1 \\
\hline & o-C-C & $17 \pm 5$ & 127,1 & $11 \pm 5$ & 122,6 & $29 \pm 4$ & 122,2 \\
\hline kl. KWs & $H$-shifts & $12 \pm 5$ & - & $23 \pm 5$ & $52,4-101,9$ & $33 \pm 4$ & $69,2-97,8$ \\
\hline
\end{tabular}

Tabelle 6.1: Vergleich der unimolekularen Prozesse zyklischer Alkoxyradikale. Die Energiebarrieren für die Bildung des offenkettigen Aldehyds, des $C$-C-Bindungsbruchs und der $\mathrm{H}$-shifts beziehen sich auf die offenkettigen Isomere. Bei $c-C_{6} H_{11} O^{\star}$ und $c_{-} C_{8} H_{15} O^{\star}$ treten verschiedene $H$-shifts auf; es wurden jeweils die Energiebarrieren des energetisch günstigsten und ungünstigsten $H$-shifts angegeben.

Der direkte H-Abstraktionskanal unter Bildung des Cykloalkens wurde bei der Reaktion Cyclopentyl $+\mathrm{O}$ zu 32\% und bei der Reaktion Cyclooktyl $+\mathrm{O}$ zu $18 \%$ bezogen auf die jeweilige Gesamtreaktion bestimmt. Der direkte H-Abstraktionskanal wurde bei der Reaktion Cyclohexyl + O nicht beobachtet.

Die sich aus den Energiebarrieren ergebenden Hochdruckgrenzwerte für die thermische Aktivierung lauten:

\begin{tabular}{c|cc|cc|cc}
\hline Kanal & \multicolumn{2}{|c}{${\mathrm{c}-\mathrm{C}_{5} \mathrm{H}_{9} \mathrm{O}^{\star}}$} & \multicolumn{2}{c}{${\mathrm{c}-\mathrm{C}_{6} \mathrm{H}_{11} \mathrm{O}^{\star}}^{2}$} & \multicolumn{2}{c}{$\mathrm{c} \mathrm{C}_{8} \mathrm{H}_{15} \mathrm{O}^{\star}$} \\
\hline & $\log \left(\mathrm{A} / \mathrm{s}^{-1}\right)$ & $\mathrm{E}_{a} /[\mathrm{kJ} / \mathrm{mol}]$ & $\log \left(\mathrm{A} / \mathrm{s}^{-1}\right)$ & $\mathrm{E}_{a} /[\mathrm{kJ} / \mathrm{mol}]$ & $\log \left(\mathrm{A} / \mathrm{s}^{-1}\right)$ & $\mathrm{E}_{a} /[\mathrm{kJ} / \mathrm{mol}]$ \\
\hline $\mathrm{c}-\mathrm{C}-\mathrm{H}$ & 13,12 & 77,5 & 13,37 & 77,7 & 13,41 & 63,6 \\
$\mathrm{c}-\mathrm{C}-\mathrm{C}$ & 13,23 & 35,0 & 13,92 & 48,9 & 13,54 & 37,6 \\
\hline
\end{tabular}

Tabelle 6.2: Hochdruckgrenzwerte für den unimolekuaren Zerfall zyklischer Alkoxyradikale.

Der experimentelle Nachweis der Stabilisierung des offenkettigen $\mathrm{C}_{8} \mathrm{H}_{15} \mathrm{O}^{\star}$ bei 1 bar bestätigt die Ergebnisse der quantenchemischen Rechnungen. Diese zeigen, dass die spezifische 
Geschwindigkeit $\mathrm{k}(\mathrm{E})$ des 1,5-H-shifts für den offenkettigen Isomer gegenüber dem C-CBindungsbruch bei diesen Bedingungen favorisiert ist.

\subsection{Geschwindigkeitskoeffizienten für die Selbstreak- tionen der Alkylradikale $\mathrm{CH}_{3}$ und $\mathrm{C}_{2} \mathrm{H}_{5}$ sowie des Allylradikals}

Die Geschwindigkeitskoeffizienten der Reaktionen

$$
\begin{aligned}
\mathrm{CH}_{3}+\mathrm{CH}_{3} & \longrightarrow \mathrm{C}_{2} \mathrm{H}_{6} \\
\mathrm{C}_{2} \mathrm{H}_{5}+\mathrm{C}_{2} \mathrm{H}_{5} & \longrightarrow \mathrm{C}_{4} \mathrm{H}_{10} \\
\mathrm{C}_{3} \mathrm{H}_{5}+\mathrm{C}_{3} \mathrm{H}_{5} & \longrightarrow \text { Produkte }
\end{aligned}
$$

wurden relativ zu den Geschwindigkeitskoffizienten der gut untersuchten Referenz-Reaktionen $\mathrm{CH}_{3}+\mathrm{O} \rightarrow$ Produkte, $\mathrm{C}_{2} \mathrm{H}_{5}+\mathrm{O} \rightarrow$ Produkte durch quantitative Ermittlung der Endprodukte der Reaktionssyteme $\mathrm{CH}_{3} / \mathrm{O}, \mathrm{C}_{2} \mathrm{H}_{5} / \mathrm{O}$ und $\mathrm{C}_{3} \mathrm{H}_{5} / \mathrm{O}$ mittels FTIRAnalyse und Simulation der Konzentrations-Zeit-Profile mit moderat komplexen Mechanismen bestimmt. Dabei wurden folgende Geschwindigkeitskoeffizienten bei einem Gesamtdruck von 4 mbar und einer Temperatur von $298 \mathrm{~K}$ ermittelt:

$$
\begin{aligned}
k\left(\mathrm{CH}_{3}+\mathrm{CH}_{3} \rightarrow \mathrm{C}_{2} \mathrm{H}_{6}\right) & =(2,4 \pm 0,2) \times 10^{13} \mathrm{~cm}^{3} /(\mathrm{mol} \cdot \mathrm{s}) \\
k\left(\mathrm{C}_{2} \mathrm{H}_{5}+\mathrm{C}_{2} \mathrm{H}_{5} \rightarrow \mathrm{C}_{4} \mathrm{H}_{10}\right) & =(1,1 \pm 0,3) \times 10^{13} \mathrm{~cm}^{3} /(\mathrm{mol} \cdot \mathrm{s}) \\
k\left(\mathrm{C}_{3} \mathrm{H}_{5}+\mathrm{C}_{3} \mathrm{H}_{5} \rightarrow \text { Produkte }\right) & =1,4 \times 10^{13} \mathrm{~cm}^{3} /(\mathrm{mol} \cdot \mathrm{s})
\end{aligned}
$$

\subsection{Mechanismus der Reaktion der primären und se- kundären Alkylradikale $\mathrm{C}_{n} \mathrm{H}_{2 n+1}(\mathrm{n}=1,2,3,4)$ und des Allylradikals}

Das Verhältnis von Kombination zu Disproportionierung für Methyl-, Ethyl-, 1-Propyl-, 2-Propyl-, 1-Butyl- und Allylradikale wurde experimentell bestimmt. Dabei wurden bei 
einem Gesamtdruck von 4 mbar und einer Temperatur von $298 \mathrm{~K}$ die in Tabelle 6.3 angegebenen Verhältnisse mittels FTIR-Analyse stabiler Endprodukte gemessen.

\begin{tabular}{l|c|c|c}
\hline Radikal + Radikal & Kombination & Disproportionierung & $k(d) / k(c)$ \\
\hline $\mathrm{CH}_{3}+\mathrm{CH}_{3}$ & $100 \%$ & $0 \%$ & 0 \\
$\mathrm{C}_{2} \mathrm{H}_{5}+\mathrm{C}_{2} \mathrm{H}_{5}$ & $\geq 90 \%$ & $\leq 10 \%$ & $\leq 0,11$ \\
$\mathrm{CH}_{3}+1-\mathrm{C}_{3} \mathrm{H}_{7}$ & $\geq 95 \%$ & $\leq 5 \%$ & $\leq 0,05$ \\
$1-\mathrm{C}_{3} \mathrm{H}_{7}+1-\mathrm{C}_{3} \mathrm{H}_{7}$ & $87,5 \%$ & $12,5 \%$ & 0,14 \\
$1-\mathrm{C}_{4} \mathrm{H}_{9}+1-\mathrm{C}_{4} \mathrm{H}_{9}$ & $56 \%$ & $44 \%$ & 0,78 \\
$2-\mathrm{C}_{3} \mathrm{H}_{7}+2-\mathrm{C}_{3} \mathrm{H}_{7}$ & $40 \%$ & $60 \%$ & 1,5 \\
$\mathrm{C}_{3} \mathrm{H}_{5}+\mathrm{C}_{3} \mathrm{H}_{5}(4$ mbar $)$ & $20 \%$ & $80 \%$ & 4 \\
$\mathrm{C}_{3} \mathrm{H}_{5}+\mathrm{C}_{3} \mathrm{H}_{5}(1$ bar $)$ & $\geq 50 \%$ & $\leq 50 \%$ & $\leq 1$ \\
\hline
\end{tabular}

Tabelle 6.3: Selbstreaktionen von Alkylradikalen: Kombination vs. Disproportionierung.

Die experimentellen Ergebnisse machen folgende Punkte deutlich:

1. Der Anteil des Reaktionskanals Disproportionierung an der Gesamtreaktion nimmt für primäre Radikale mit der Größe des Radikals zu.

2. Der Mechanismus der Selbstreaktionen der isomeren Propylradikale 1- $\mathrm{C}_{3} \mathrm{H}_{7}$ (primäres Radikal) und 2- $\mathrm{C}_{3} \mathrm{H}_{7}$ (sekundäres Radikal) sind stark verschieden; die Verhältnisse Disproportionierung / Kombination unterscheiden sich um eine Größenordnung.

3. Die Reaktionen $\mathrm{C}_{2} \mathrm{H}_{5}+\mathrm{C}_{2} \mathrm{H}_{5}$ und $\mathrm{CH}_{3}+1-\mathrm{C}_{3} \mathrm{H}_{7}$ favorisieren stark die Bildung des Kombinationsproduktes. Die Bildung des stabilisierten Komplexes $\left[\mathrm{C}_{4} \mathrm{H}_{10}\right]^{\ddagger}$ erfolgt daher unabhängig von der Radikal-Radikal-Reaktion.

4. Bei der Reaktion des resonanzstabilisierten Allylradikals $\mathrm{C}_{3} \mathrm{H}_{5}+\mathrm{C}_{3} \mathrm{H}_{5}$ erfolgt im Druckbereich von 1 - 1000 mbar ein Mechanismuswechsel von Disproportionierung zu Kombination. 


\subsection{Kreuzreaktionen kleiner Alkylradikale mit Me- thylradikalen und teilfluorierten Alkylradikalen}

Über die quantitative Bestimmung von Endprodukten und Radikalanfangskonzentrationen mittels FTIR-Analyse wurden die Geschwindigkeitskoeffizienten für Kreuzkombinationsreaktionen durch Simulationsrechnungen auf Basis von moderat komplexen Reaktionsmechanismen bestimmt. Dabei wurden deuterierte Methylradikale durch Photolyse von deuteriertem Aceton und die weiteren Radikale aus geeigneten Vorläufern gewonnen. Die für die Simulation benötigten Geschwindigkeitskoeffizienten wurden im Fall von Methylund Ethylradikalen durch Relativmessungen selbst ermittelt, die übrigen Selbstreaktionsgeschwindigkeiten wurden der Literatur entnommen.

Für die Kreuzreaktionen von $\mathrm{CH}_{3}$-Radikalen mit den primären Radikalen $\mathrm{C}_{2} \mathrm{H}_{5}, 1-\mathrm{C}_{3} \mathrm{H}_{7}$, 1- $\mathrm{C}_{4} \mathrm{H}_{9}$ und 1- $\mathrm{C}_{5} \mathrm{H}_{11}$ sowie den sekundären Radikalen 2- $\mathrm{C}_{3} \mathrm{H}_{7}$ und 2- $\mathrm{C}_{4} \mathrm{H}_{9}$ wurde als Mechanismus die Kombination der Radikale zu den höheren Kohlenwasserstoffen nachgewiesen.

Die Geschwindigkeitskoeffizienten der Reaktionen der $\mathrm{C}_{2^{-}}$bis $\mathrm{C}_{4}$-Radikale sind Hochdruckgrenzwerte im Rahmen der Messgenauigkeit. Die experimentell erhaltenen Geschwindigkeitskoeffizienten sind zusammen mit den Vorhersagen der geometric mean rule (GMR) in Tabelle 6.4 dargestellt.

\begin{tabular}{lc|c|c}
\hline \multirow{2}{*}{ Radikal } & + Radikal & \multicolumn{2}{|c}{$\mathrm{k} / 10^{13} \mathrm{~cm}^{3} /(\mathrm{mol} \cdot \mathrm{s})$} \\
& & Experiment & GMR \\
\hline $\mathrm{CH}_{3}$ & $+\mathrm{C}_{2} \mathrm{H}_{5}$ & $7,4 \pm 2,0$ & 3,2 \\
$\mathrm{CH}_{3}$ & $+1-\mathrm{C}_{3} \mathrm{H}_{7}$ & $1,0-0,5 /+0,1$ & 3,4 \\
$\mathrm{CH}_{3}$ & $+1-\mathrm{C}_{4} \mathrm{H}_{9}$ & $3,8 \pm 0,2$ & 4,2 \\
$\mathrm{CH}_{3}$ & $+2-\mathrm{C}_{3} \mathrm{H}_{7}$ & $1,8 \pm 0,1$ & 3,1 \\
$\mathrm{CH}_{3}$ & $+\mathrm{CH}_{2} \mathrm{~F}$ & $4,0 \pm 1,5$ & 3,2 \\
$\mathrm{C}_{2} \mathrm{H}_{5}$ & $+\mathrm{CH}_{2} \mathrm{~F}$ & $0,9 \pm 0,3$ & 1,8 \\
$\mathrm{CH}_{2} \mathrm{~F}$ & $+\mathrm{CHF}_{2}$ & $0,3-0,07 /+0,15$ & 0,9 \\
\hline
\end{tabular}

Tabelle 6.4: Kreuzreaktionen kleiner Alkylradikale mit Methylradikalen. 
In einer separaten Studie wurden Geschwindigkeitskoeffizienten für die Reaktion von Methyl- und Ethylradikalen mit $\mathrm{CH}_{2} \mathrm{~F}$ und der Reaktion $\mathrm{CH}_{2} \mathrm{~F}+\mathrm{CHF}_{2}$ experimentell bestimmt. Die Werte sind im Vergleich mit Vorhersagen der geometric mean rule ebenfalls in Tabelle 6.4 angegeben.

Die experimentell bestimmten Geschwindigkeiten stimmen für die Kreuzreaktionen von Alkylradikalen mit den Vorhersagen der geometric mean rule bis auf einen Faktor 2,5 überein. Dies gilt insbesondere für die Reaktionen von Methyl- und Ethylradikalen mit $\mathrm{CH}_{2} \mathrm{~F}$. Die Geschwindigkeit der Kreuzreaktion von zwei teilfluorierten Alkylradikalen wird hingegen mit Hilfe der geometric mean rule nicht zufriedenstellend vorhergesagt. Eine generelle Tendenz der Abweichungen bezüglich der Radikalstruktur existiert nicht. Bei Verwendung der geometric mean rule muss daher berücksichtigt werden, dass die Kreuzreaktionsgeschwindigkeit sowohl nach unten als auch nach oben bis um einen Faktor 2,5 von der tatsächlichen abweichen kann. Für eine Abschätzung der Kreuzreaktionsgeschwindigkeit kleiner Alkylradikale ist die geometric mean rule unter Berücksichtigung des Fehlers geeignet. 


\section{Kapitel 7}

\section{Anhang}

\section{$7.1 \quad$ IR-Kalibrierspektren}

Im folgenden sind die für die qualitative und quantitative Analyse mit der Apparatur aufgenommenen IR-Kalibrierspektren abgebildet. Alle hier gezeigten Spektren wurden bei einem Gesamtdruck von 4 mbar und einer Temperatur von $298 \mathrm{~K}$ aufgenommen.

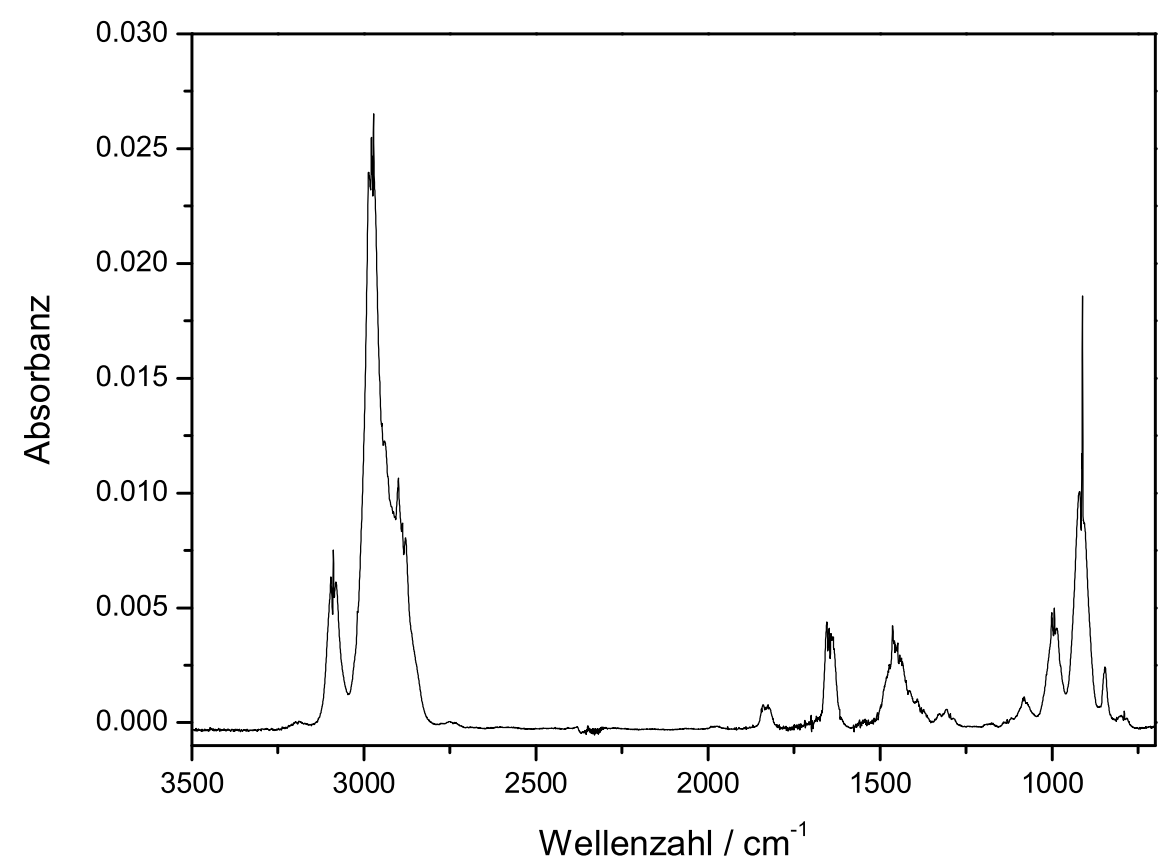

Abbildung 7.1: Kalibrierspektrum 1-Buten 


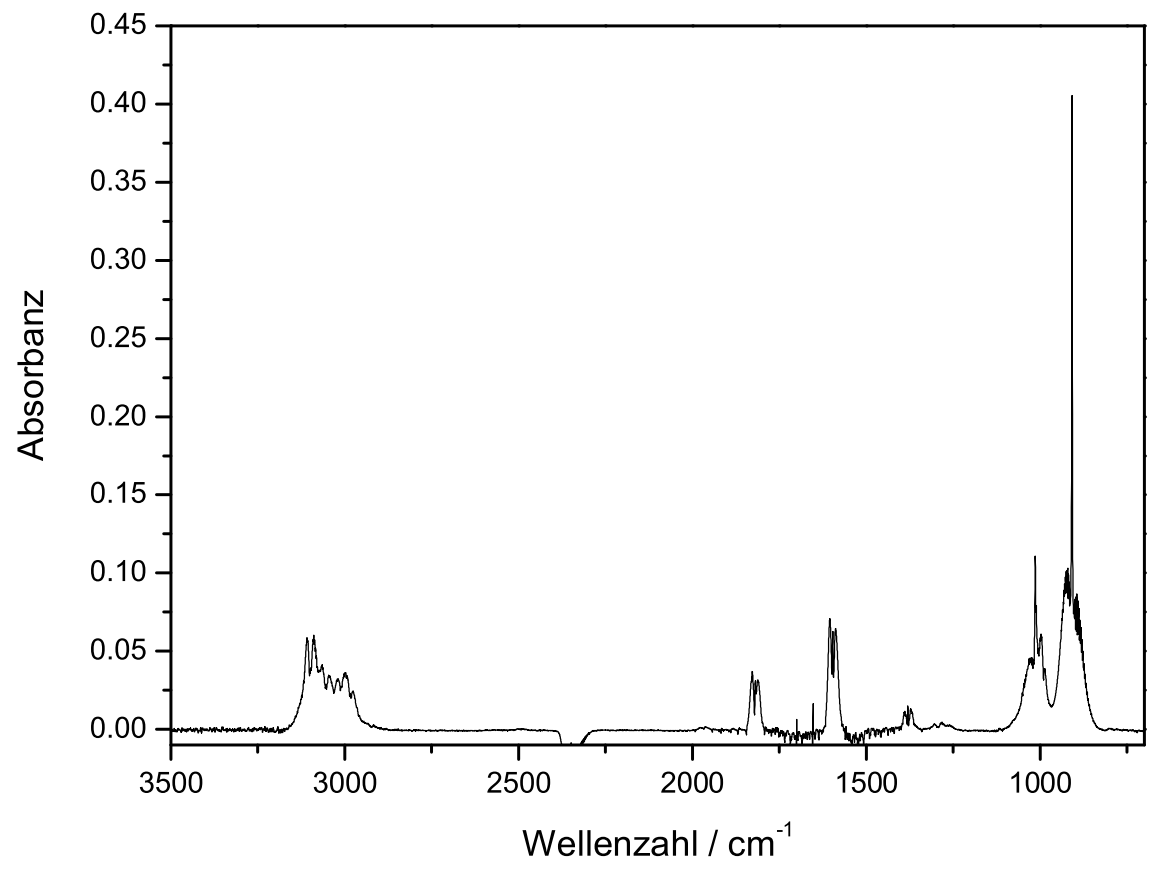

Abbildung 7.2: Kalibrierspektrum 1,3-Butadien

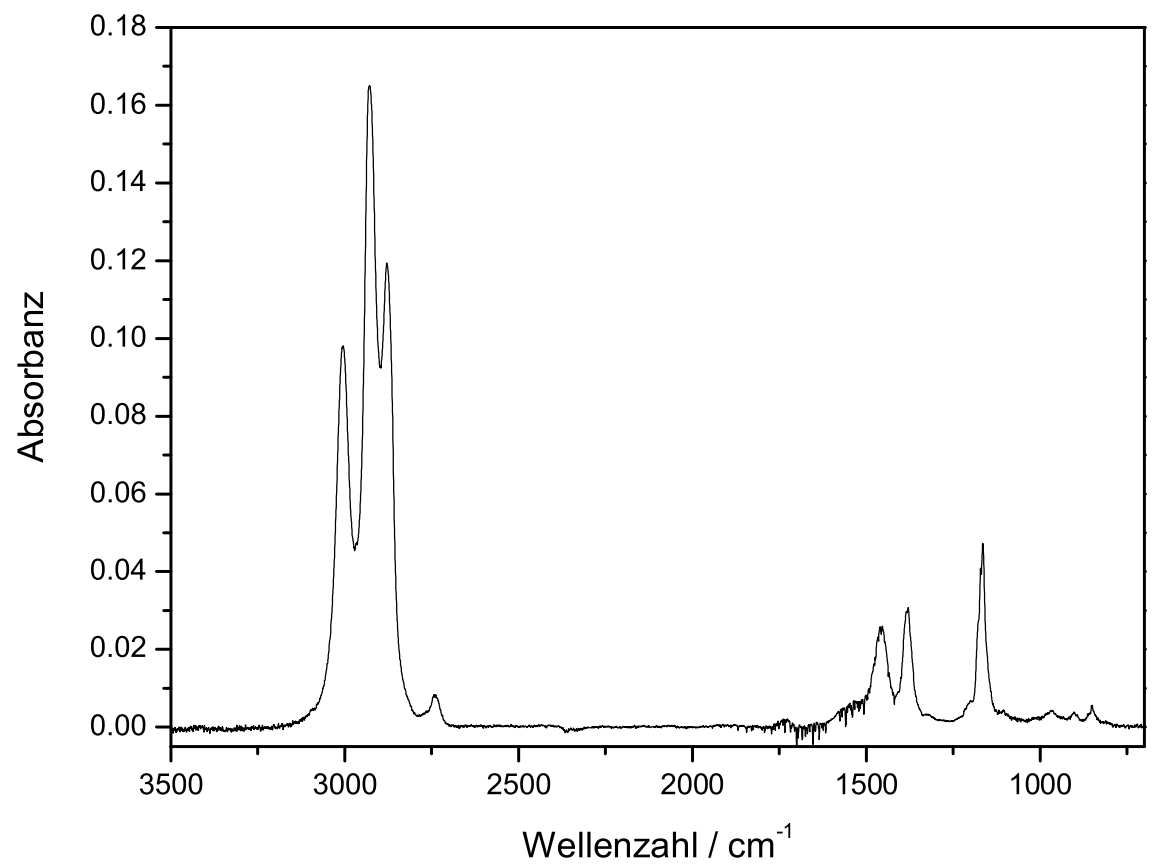

Abbildung 7.3: Kalibrierspektrum 2,3-Dimethyl-2-buten 


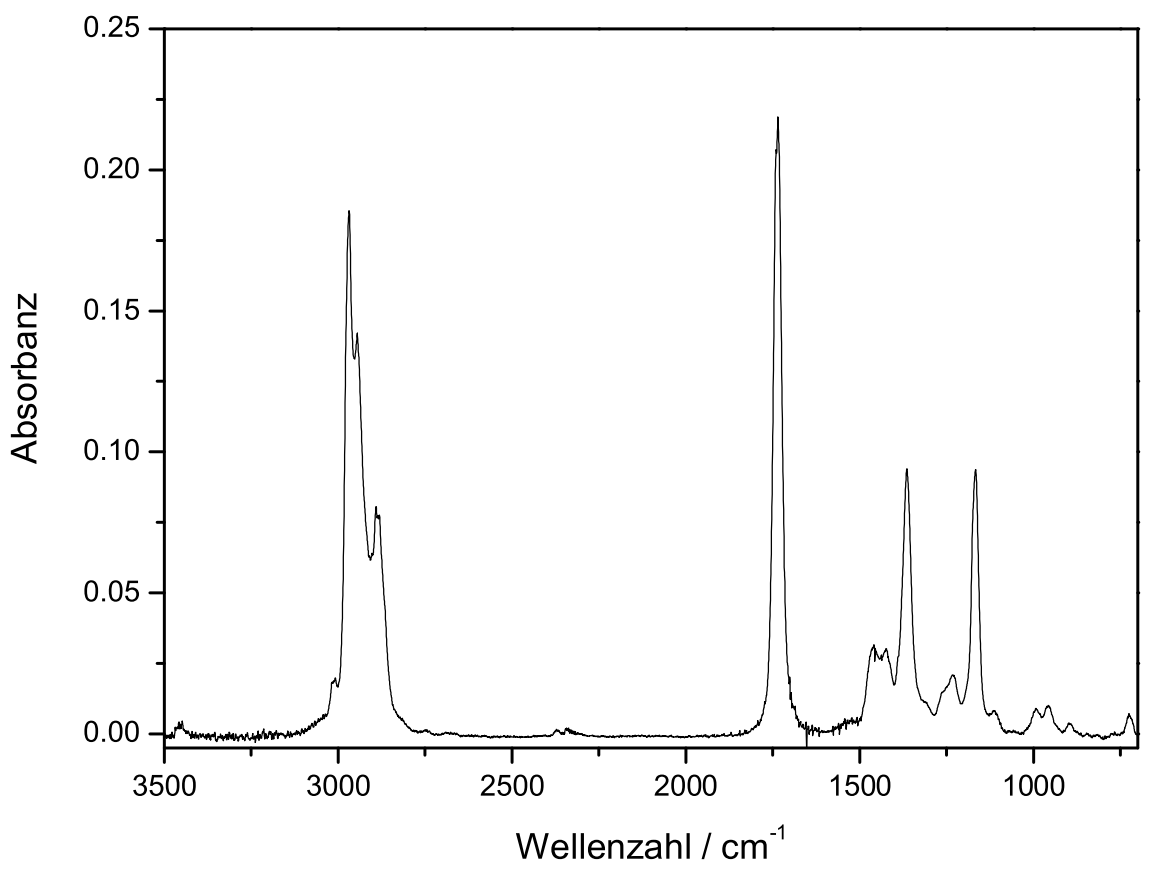

Abbildung 7.4: Kalibrierspektrum 2-Hexanon

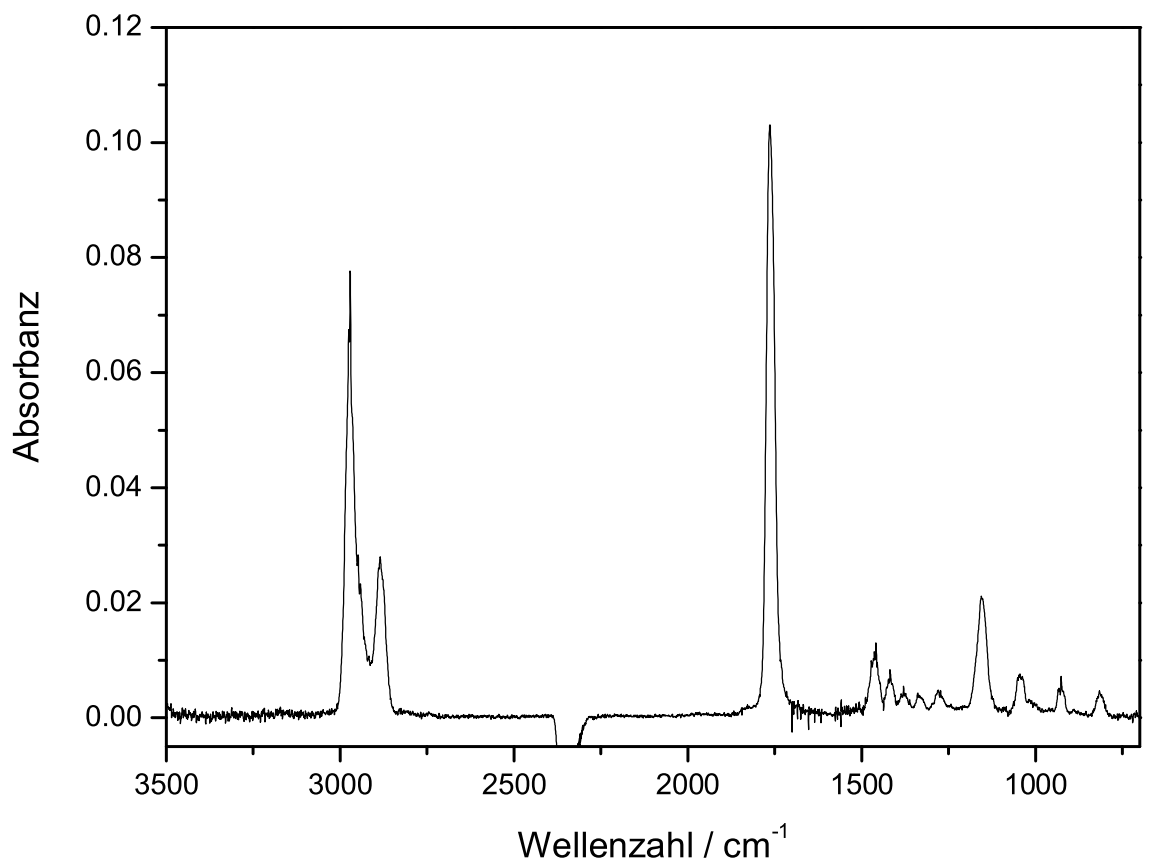

Abbildung 7.5: Kalibrierspektrum 2-Methyl-Cyclopentanon 


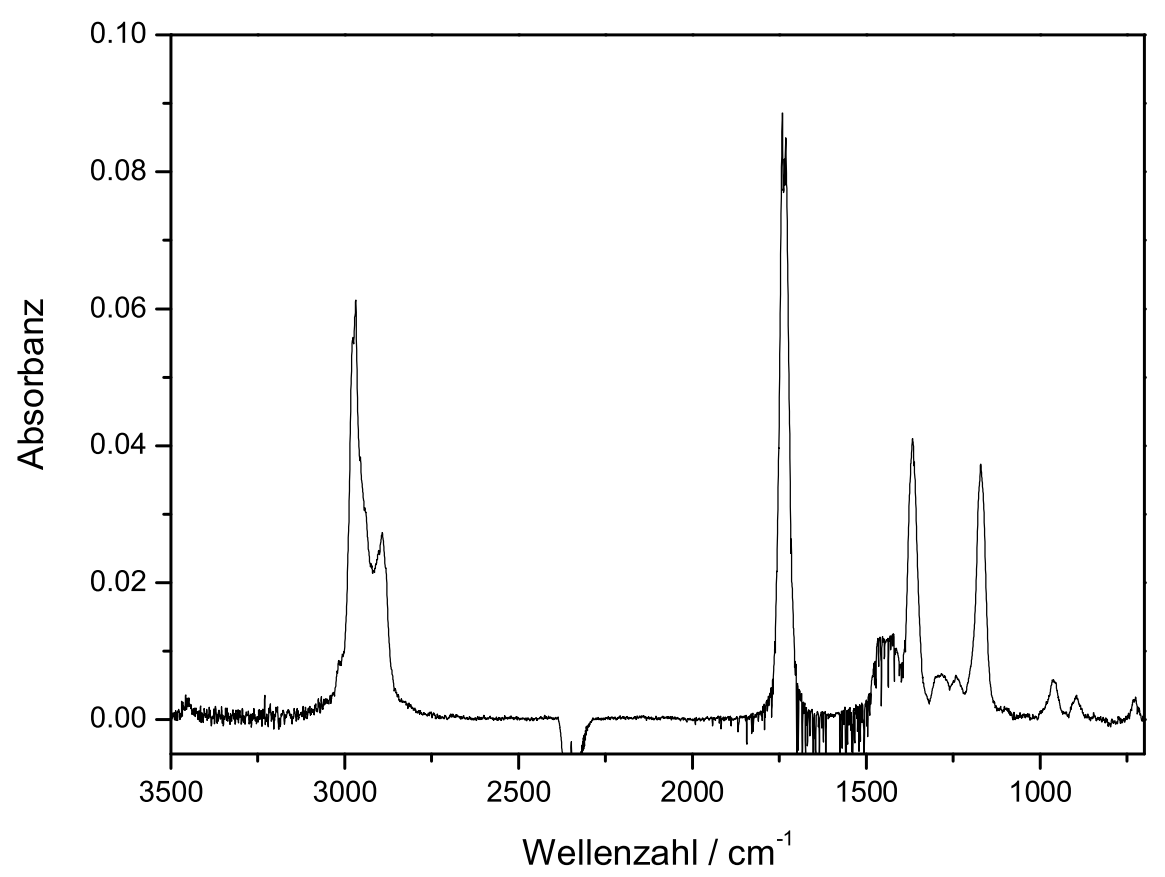

Abbildung 7.6: Kalibrierspektrum 2-Pentanon

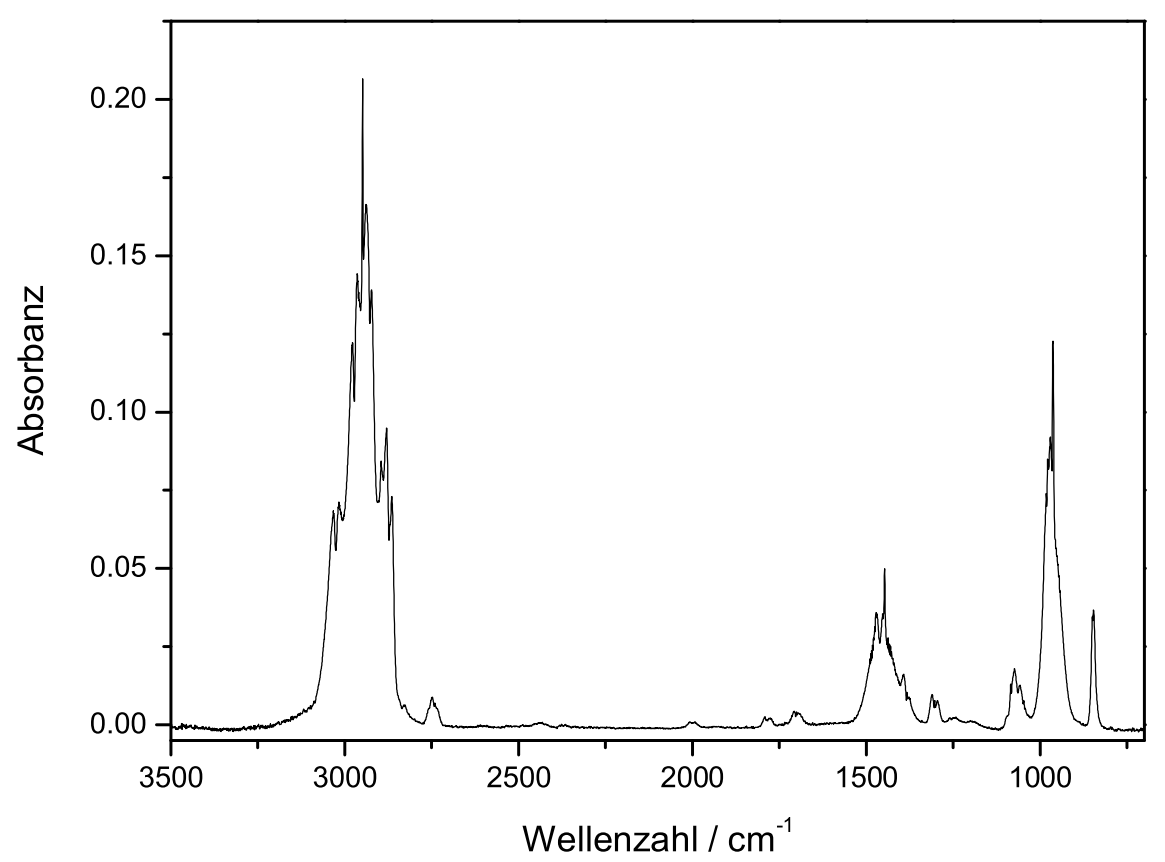

Abbildung 7.7: Kalibrierspektrum 2-trans-Buten 


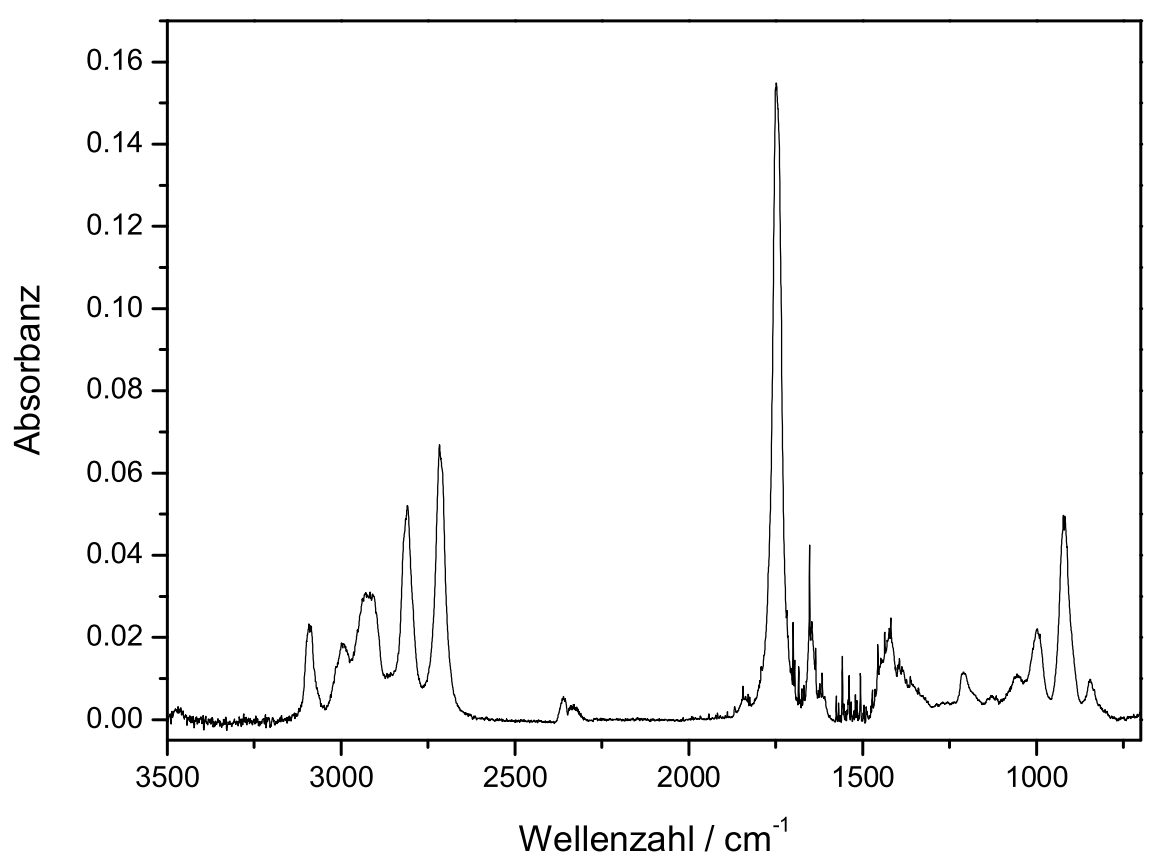

Abbildung 7.8: Kalibrierspektrum 4-Pentenal

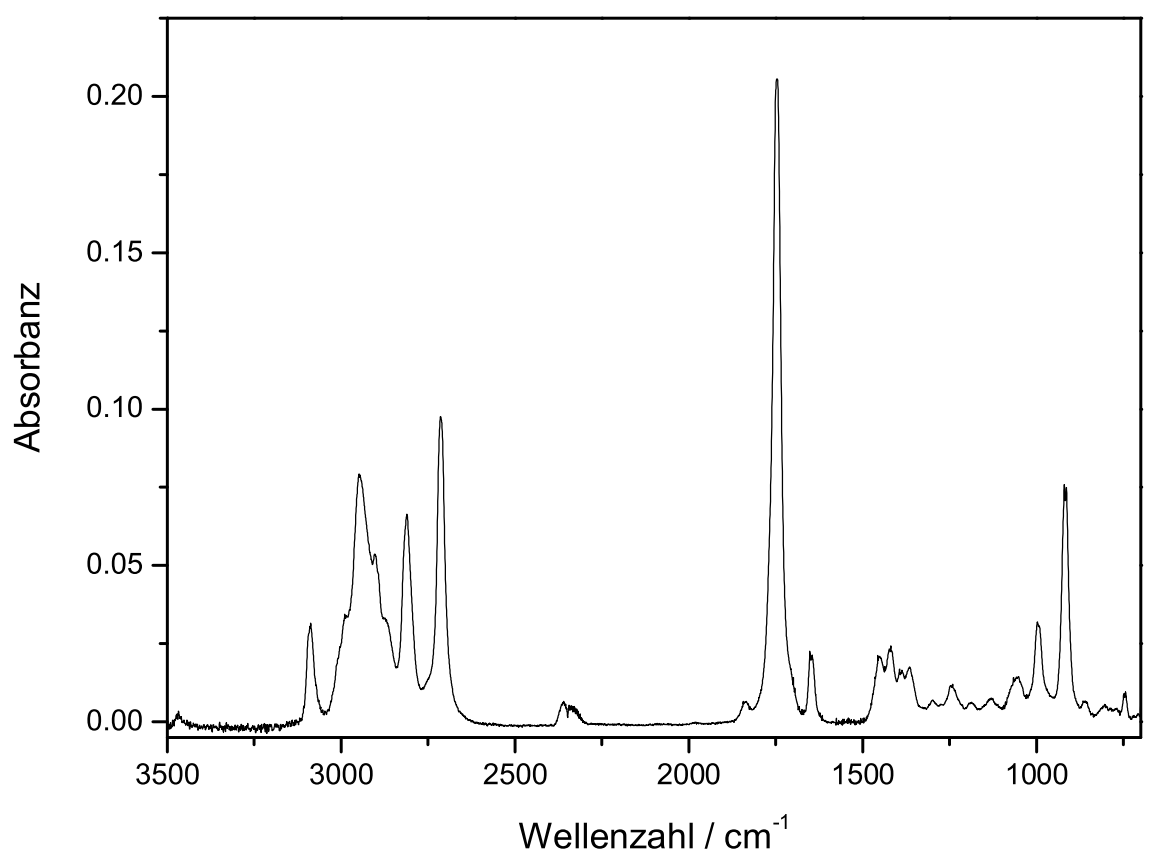

Abbildung 7.9: Kalibrierspektrum 5-Hexenal 


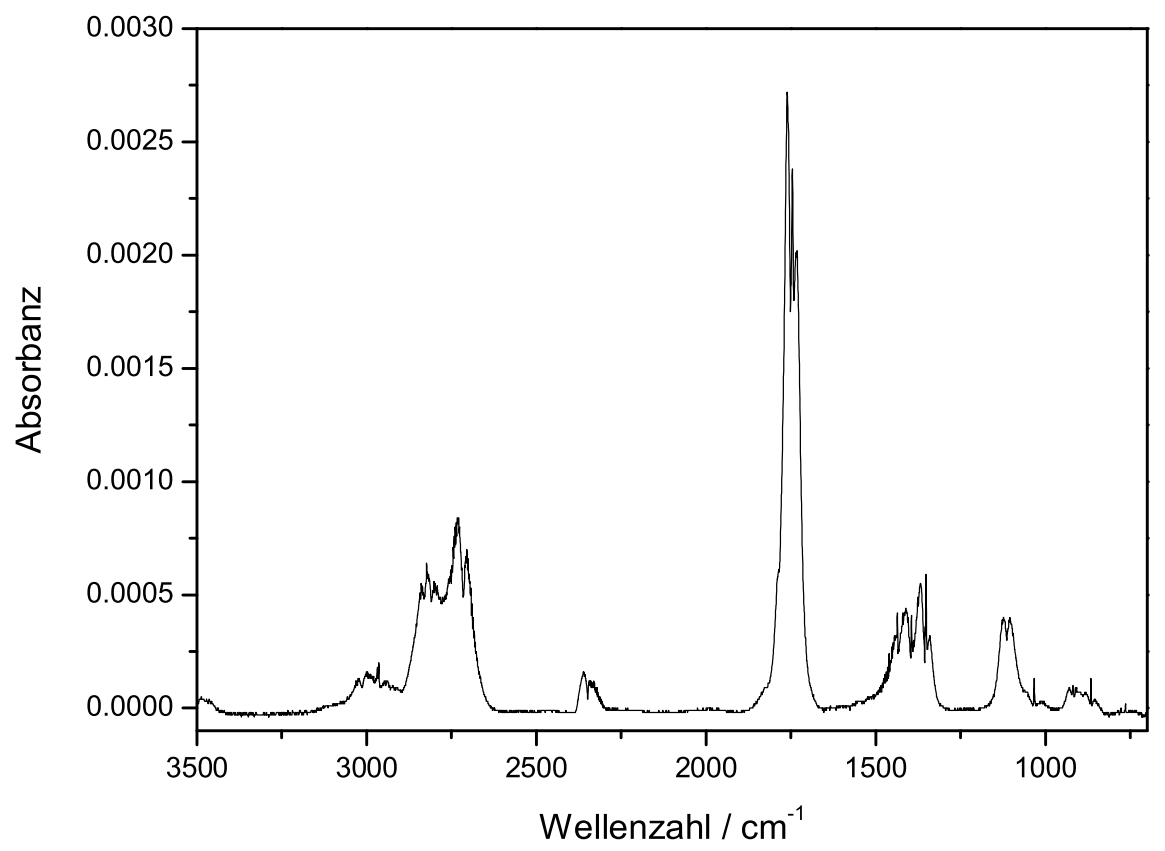

Abbildung 7.10: Kalibrierspektrum Acetaldeyhd

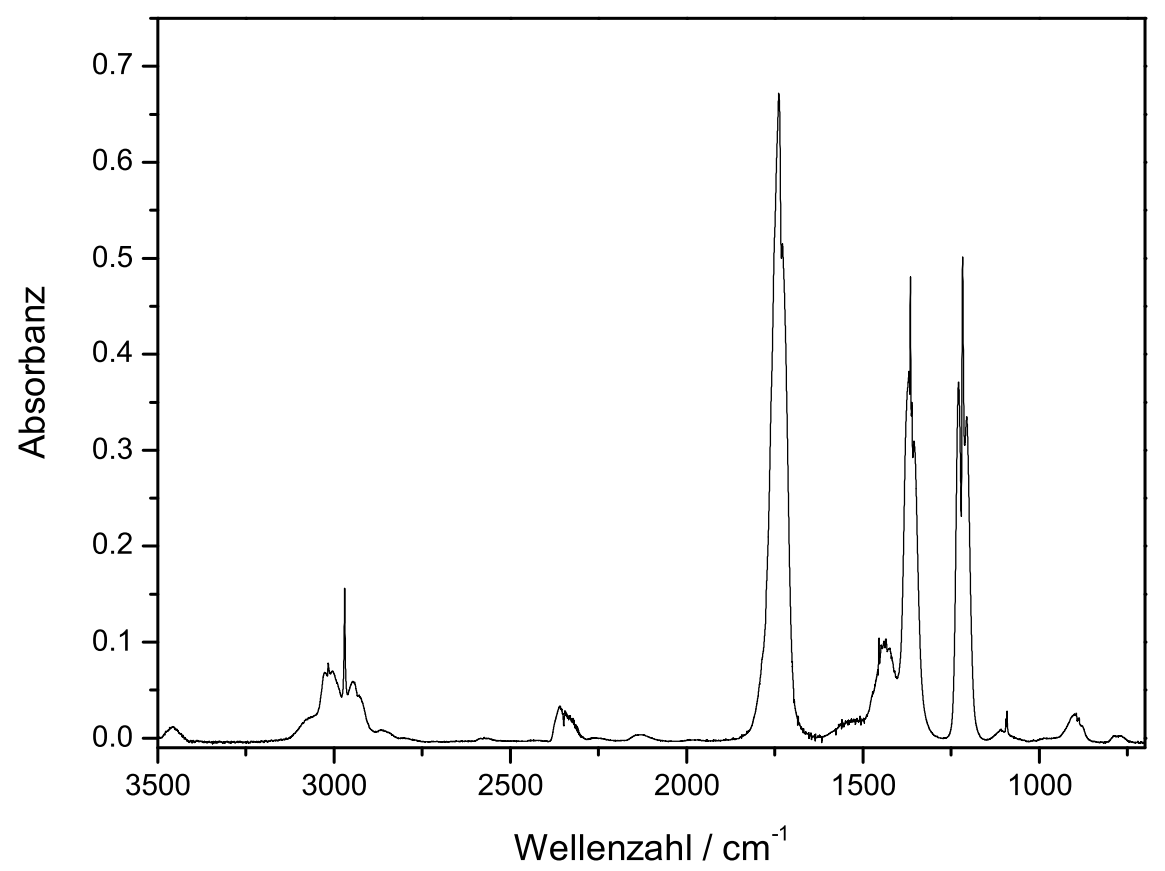

Abbildung 7.11: Kalibrierspektrum Aceton 


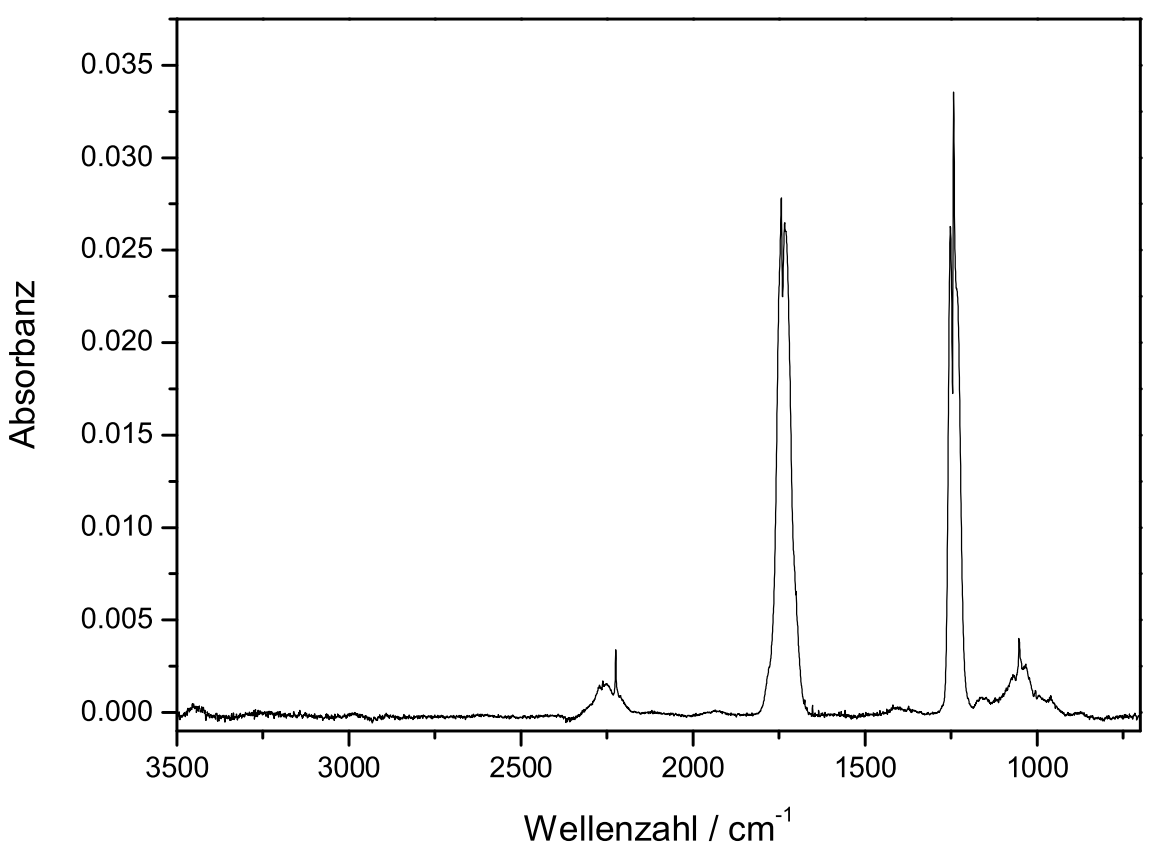

Abbildung 7.12: Kalibrierspektrum Aceton-d ${ }_{6}$

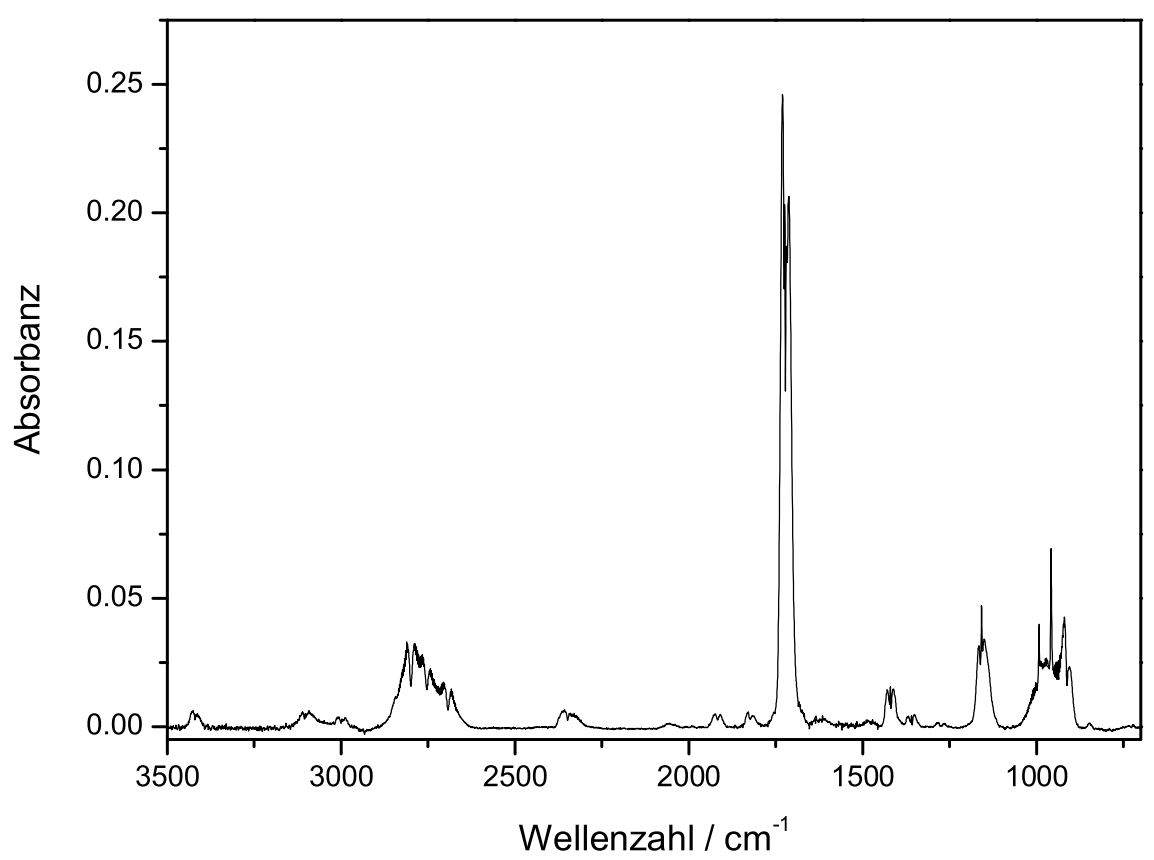

Abbildung 7.13: Kalibrierspektrum Acrolein 


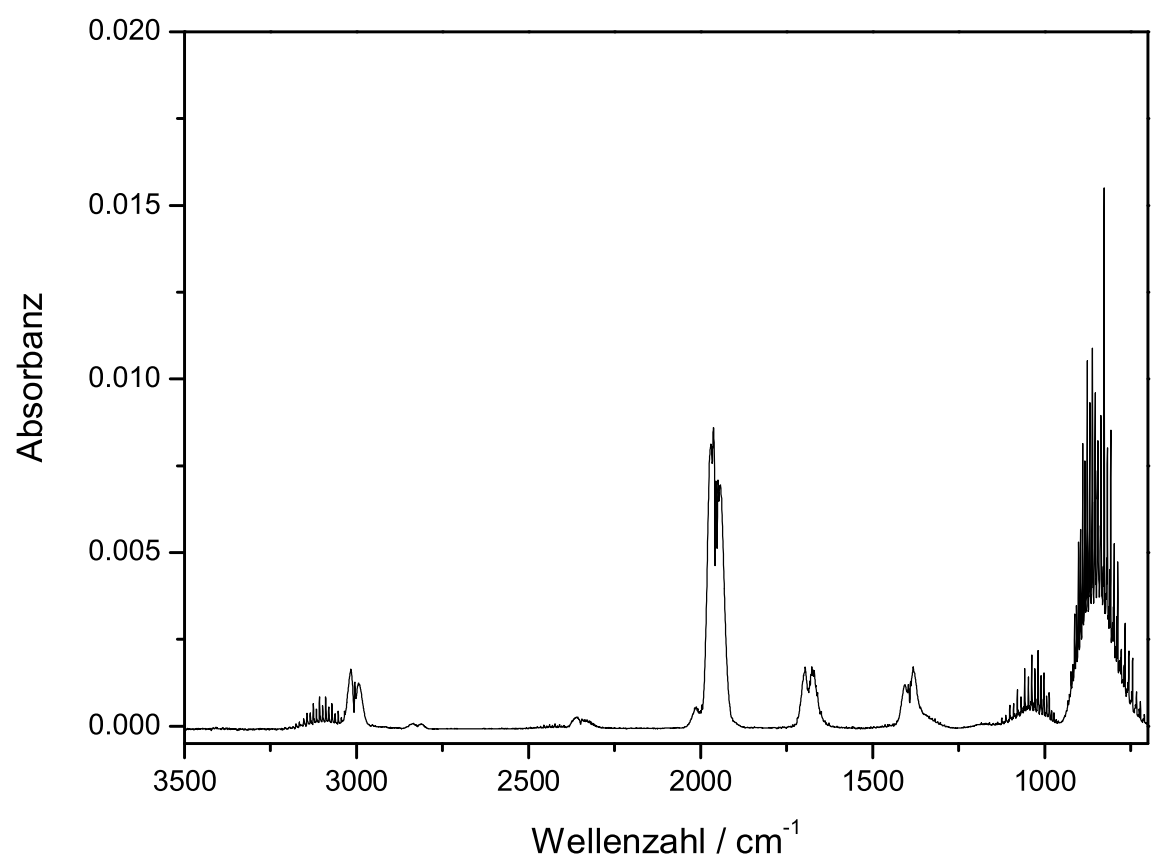

Abbildung 7.14: Kalibrierspektrum Allen

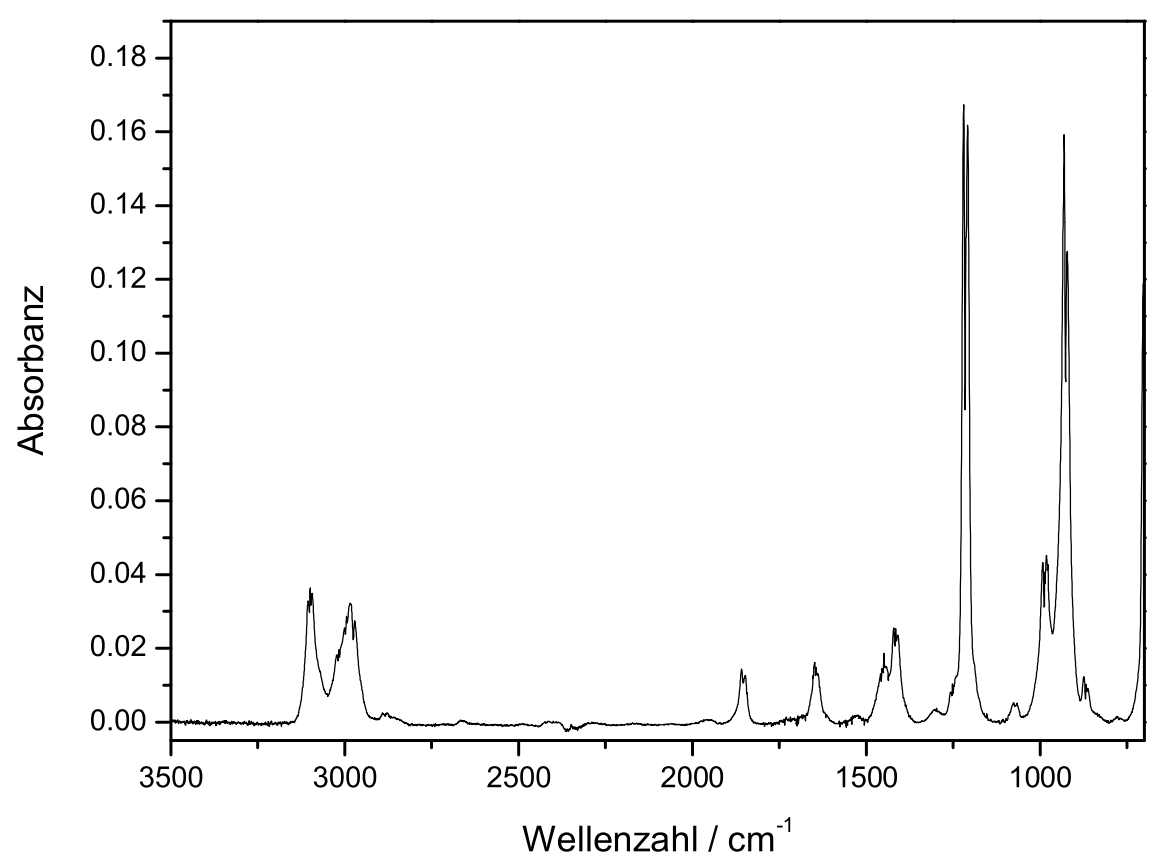

Abbildung 7.15: Kalibrierspektrum Allylbromid 


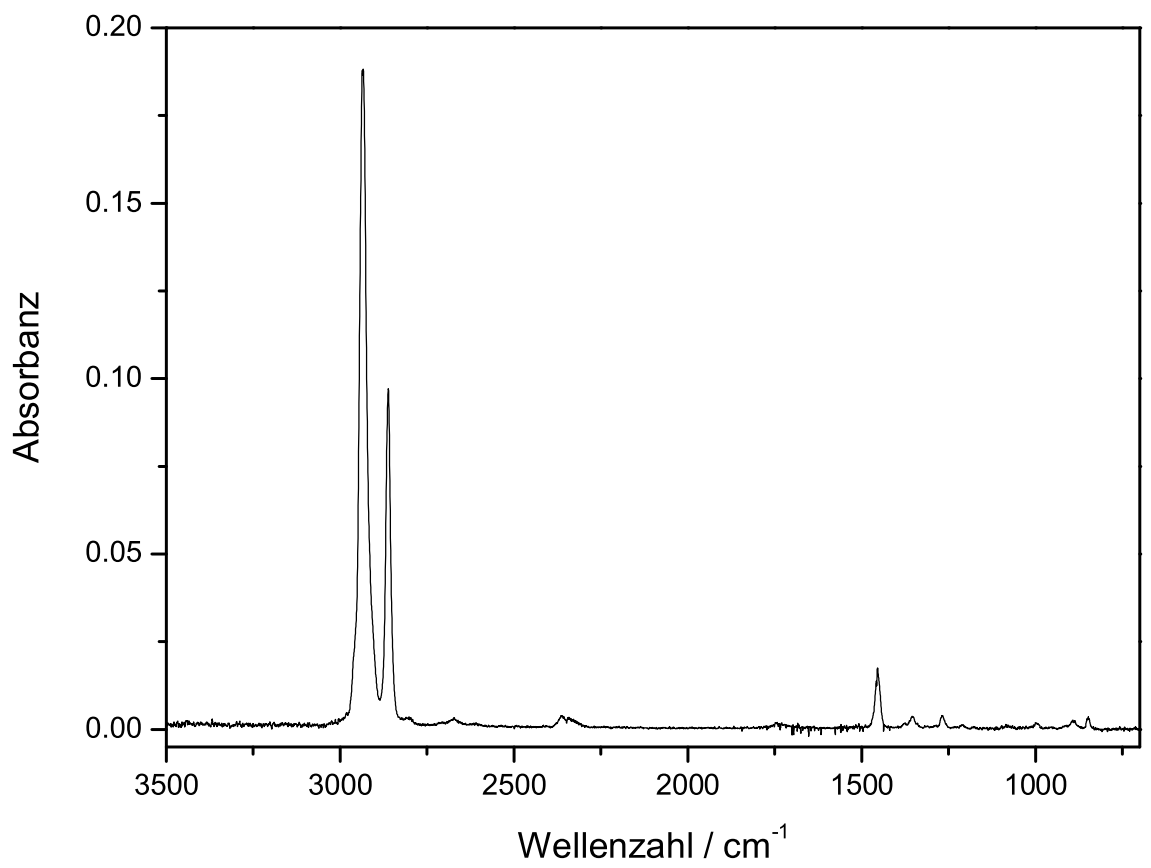

Abbildung 7.16: Kalibrierspektrum Bicyclohexyl

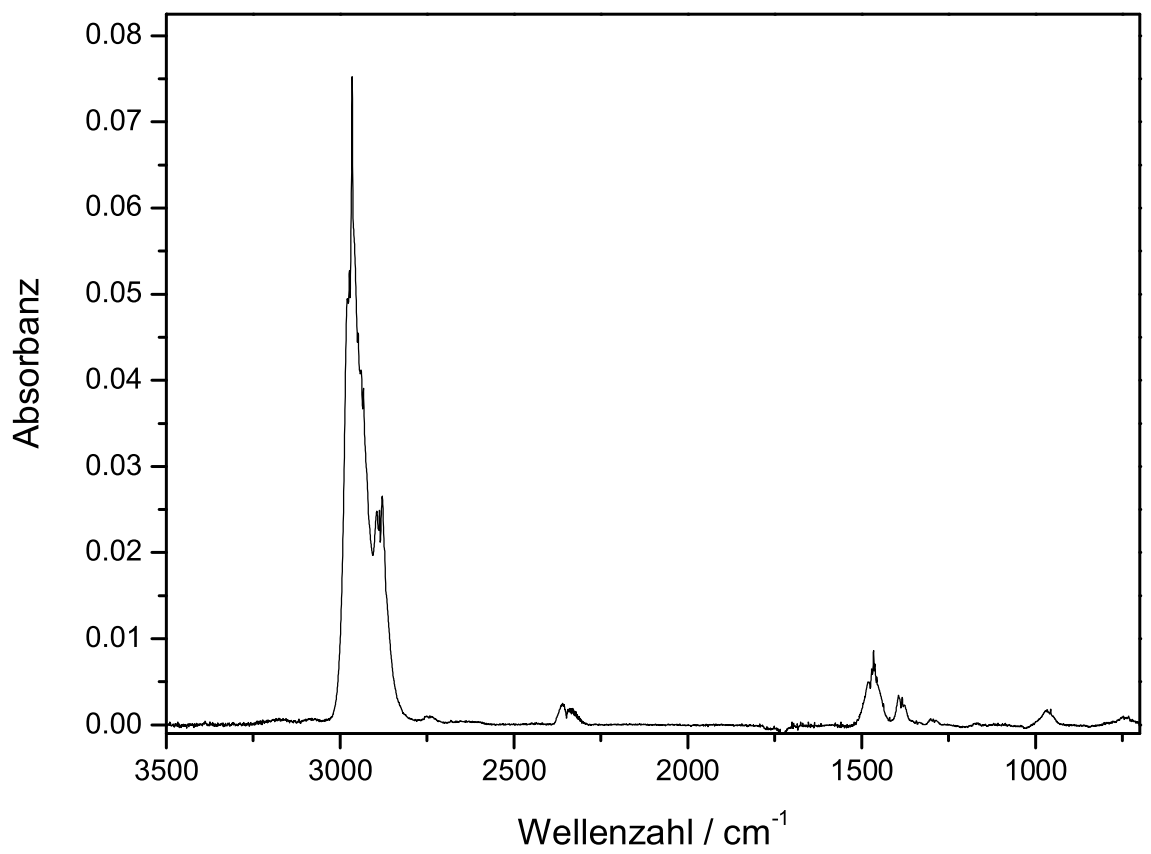

Abbildung 7.17: Kalibrierspektrum Butan 


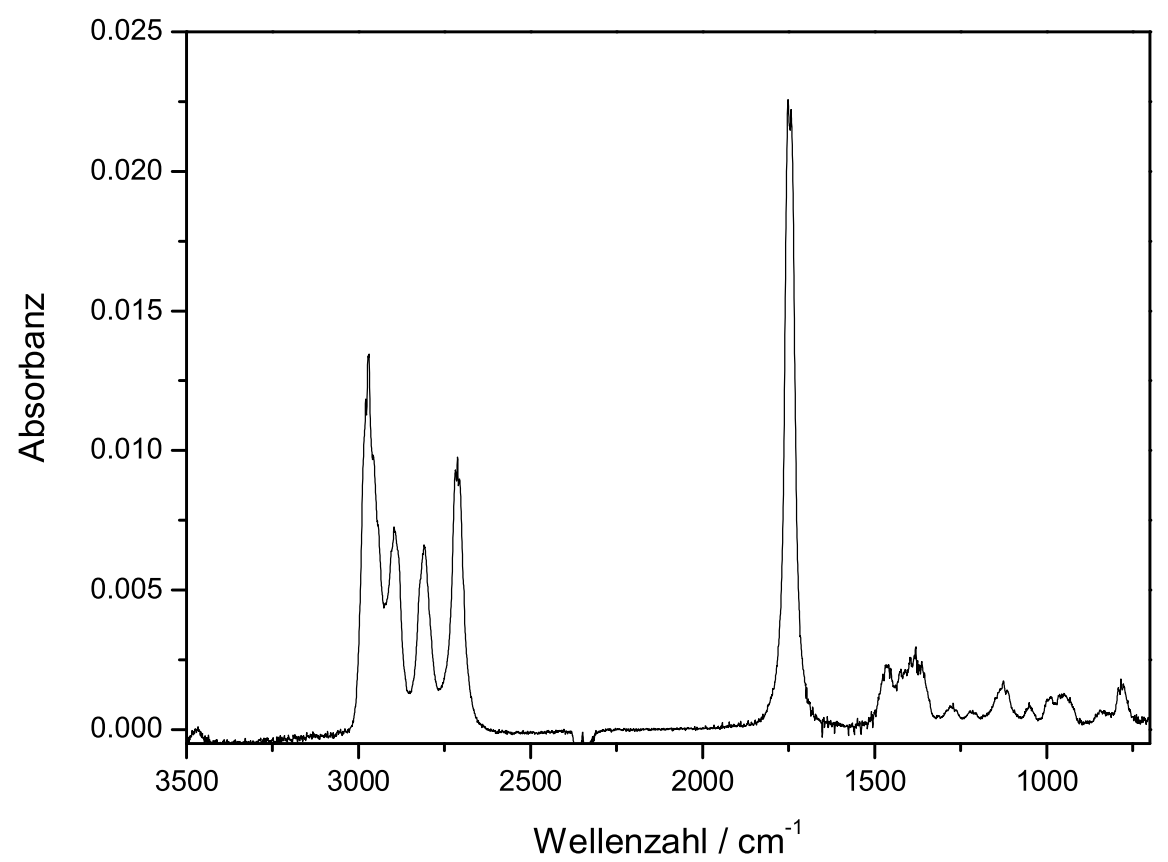

Abbildung 7.18: Kalibrierspektrum Butanal

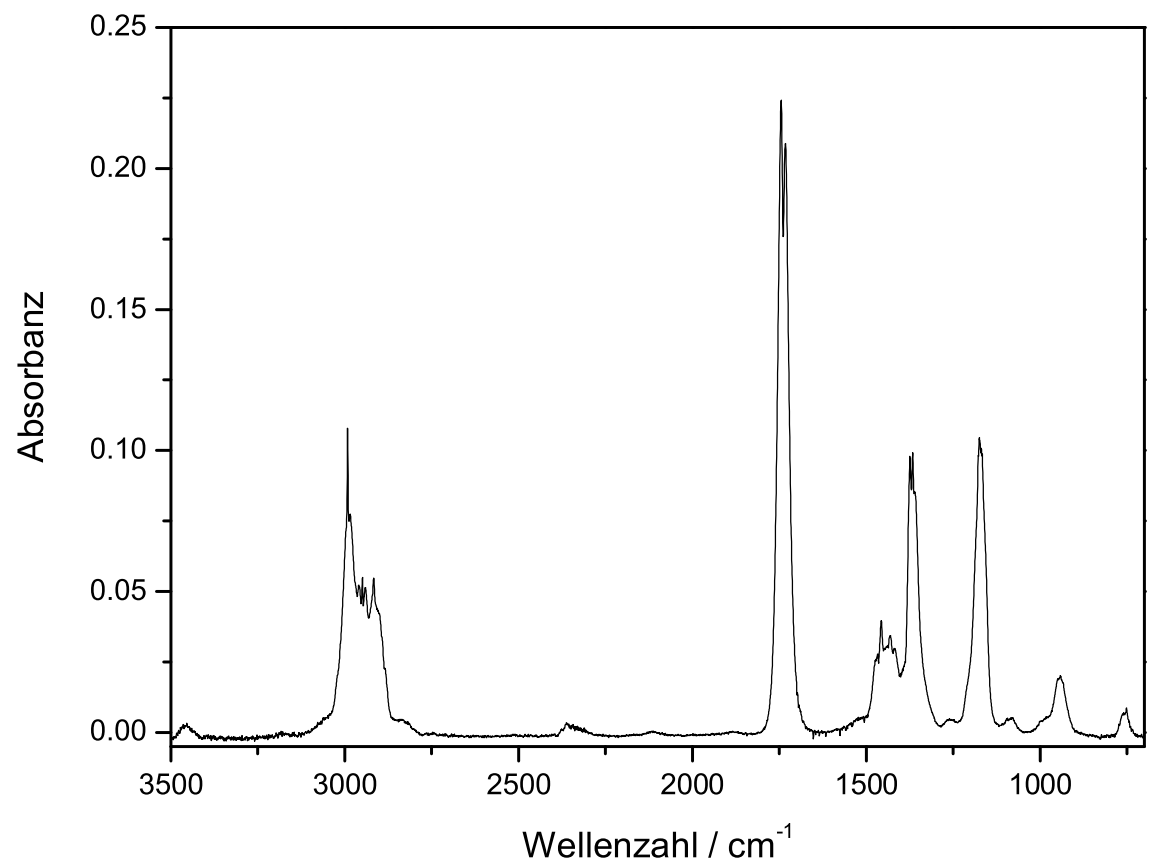

Abbildung 7.19: Kalibrierspektrum Butanon 


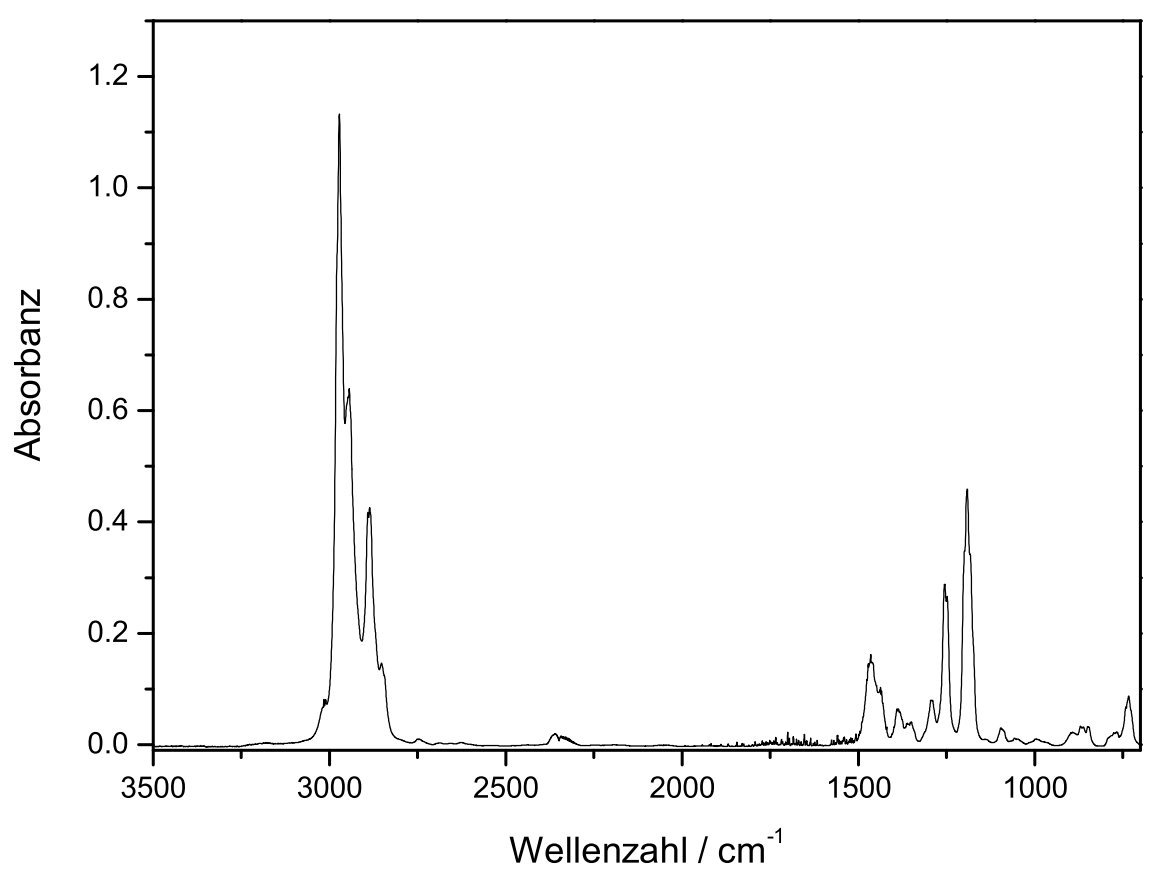

Abbildung 7.20: Kalibrierspektrum Butyliodid

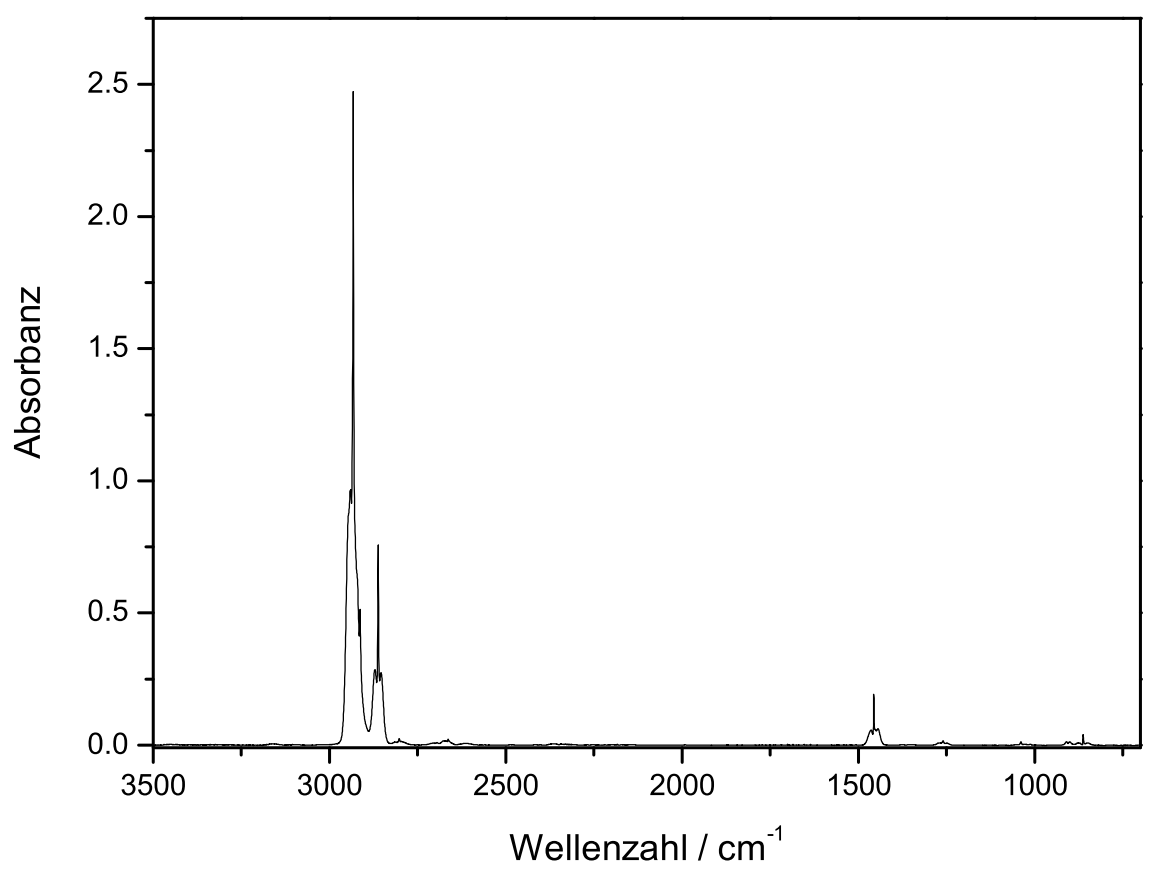

Abbildung 7.21: Kalibrierspektrum Cyclohexan 


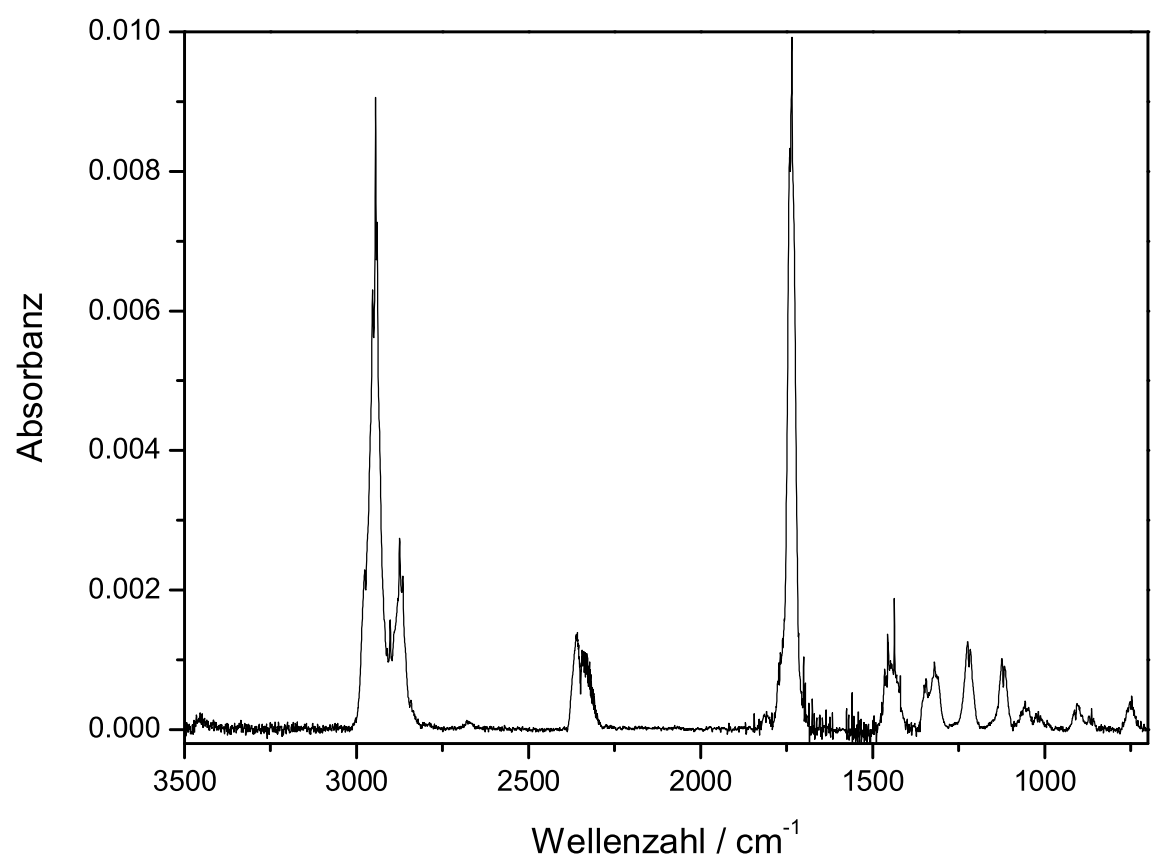

Abbildung 7.22: Kalibrierspektrum Cyclohexanon

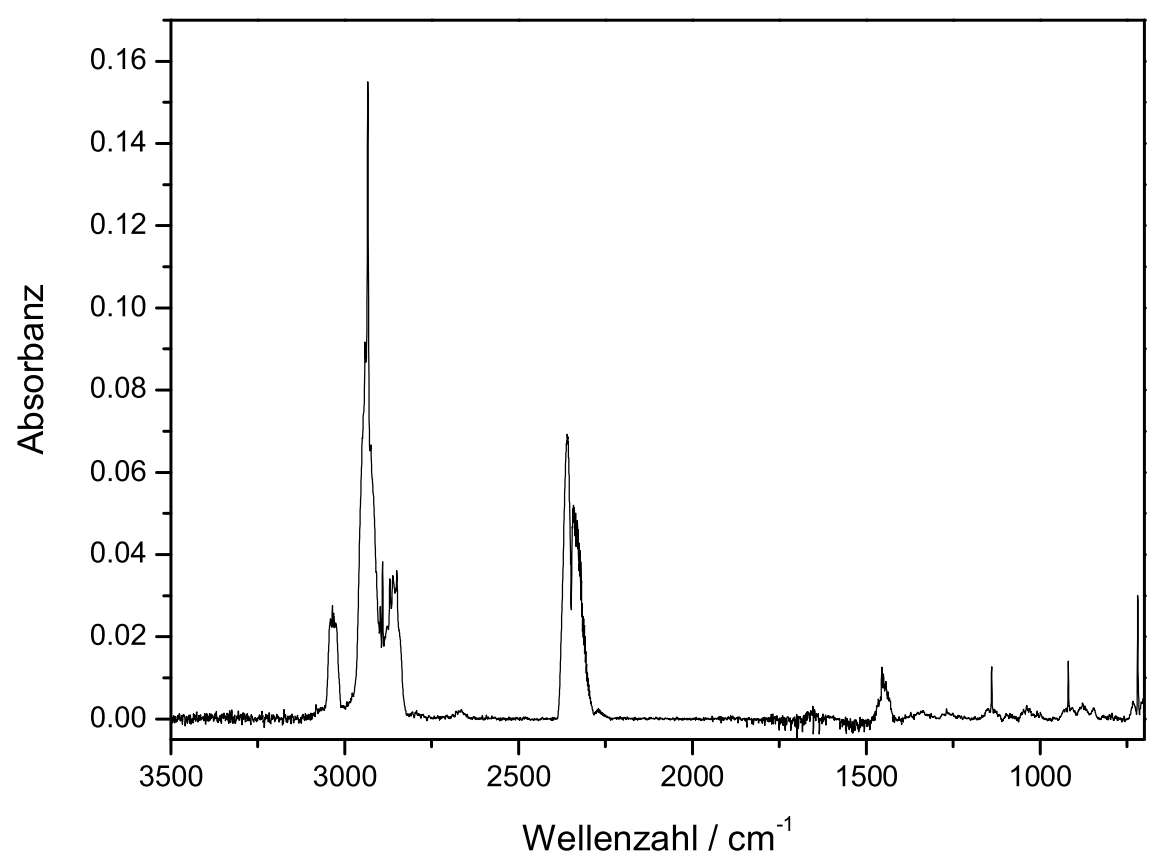

Abbildung 7.23: Kalibrierspektrum Cyclohexen 


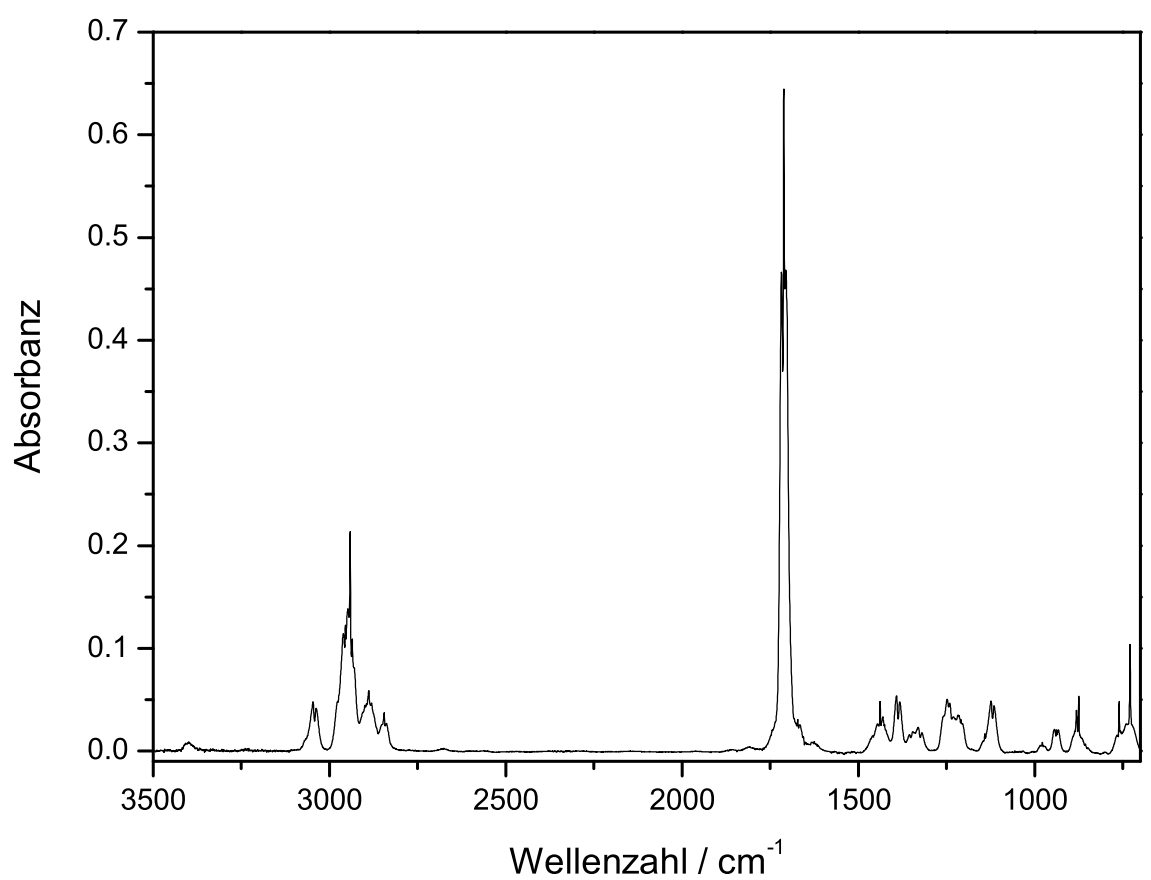

Abbildung 7.24: Kalibrierspektrum Cyclohexenon

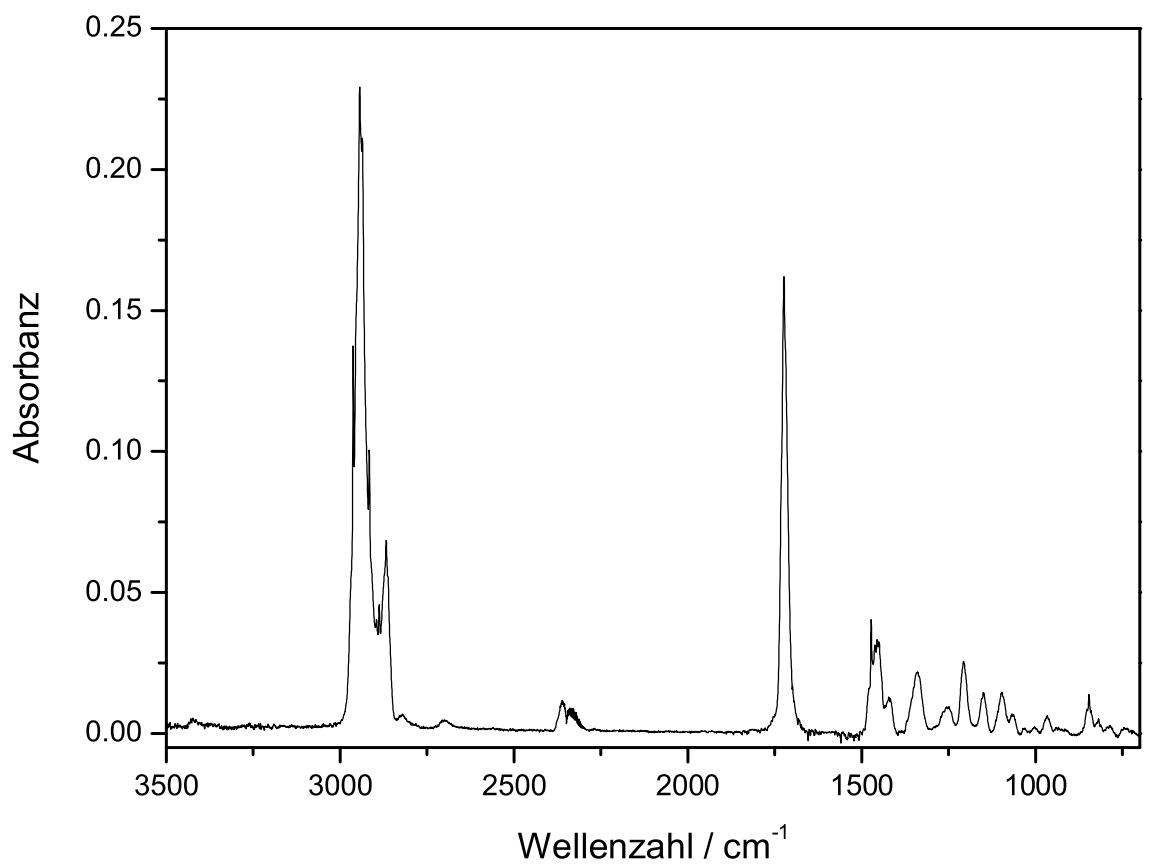

Abbildung 7.25: Kalibrierspektrum Cyclooktanon 


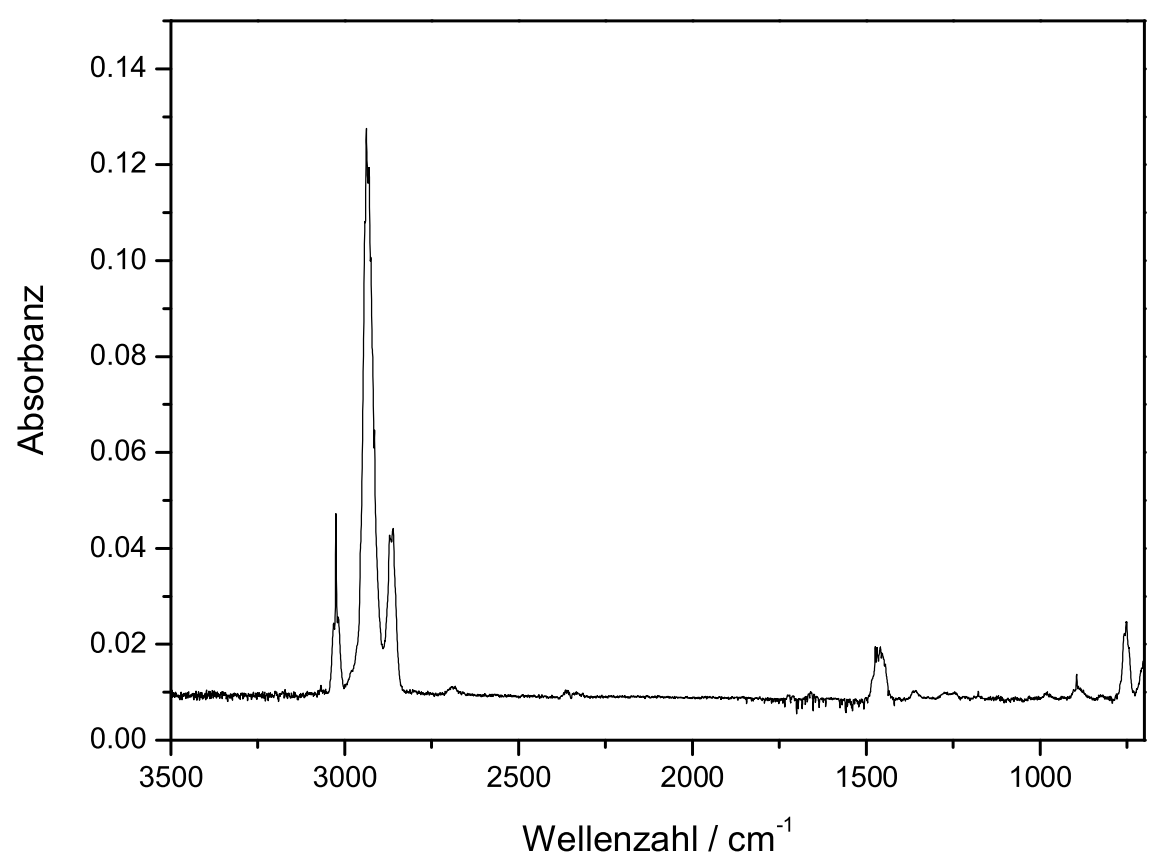

Abbildung 7.26: Kalibrierspektrum Cyclookten

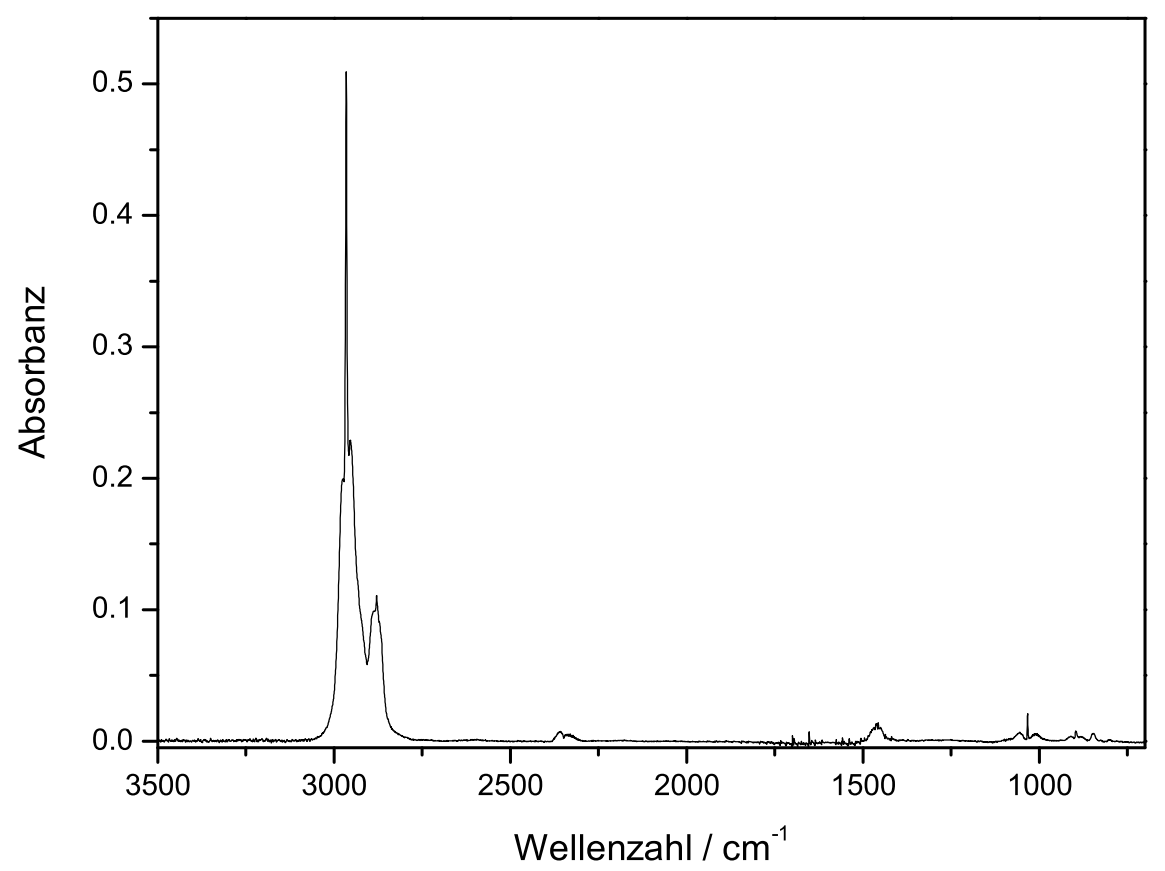

Abbildung 7.27: Kalibrierspektrum Cyclopentan 


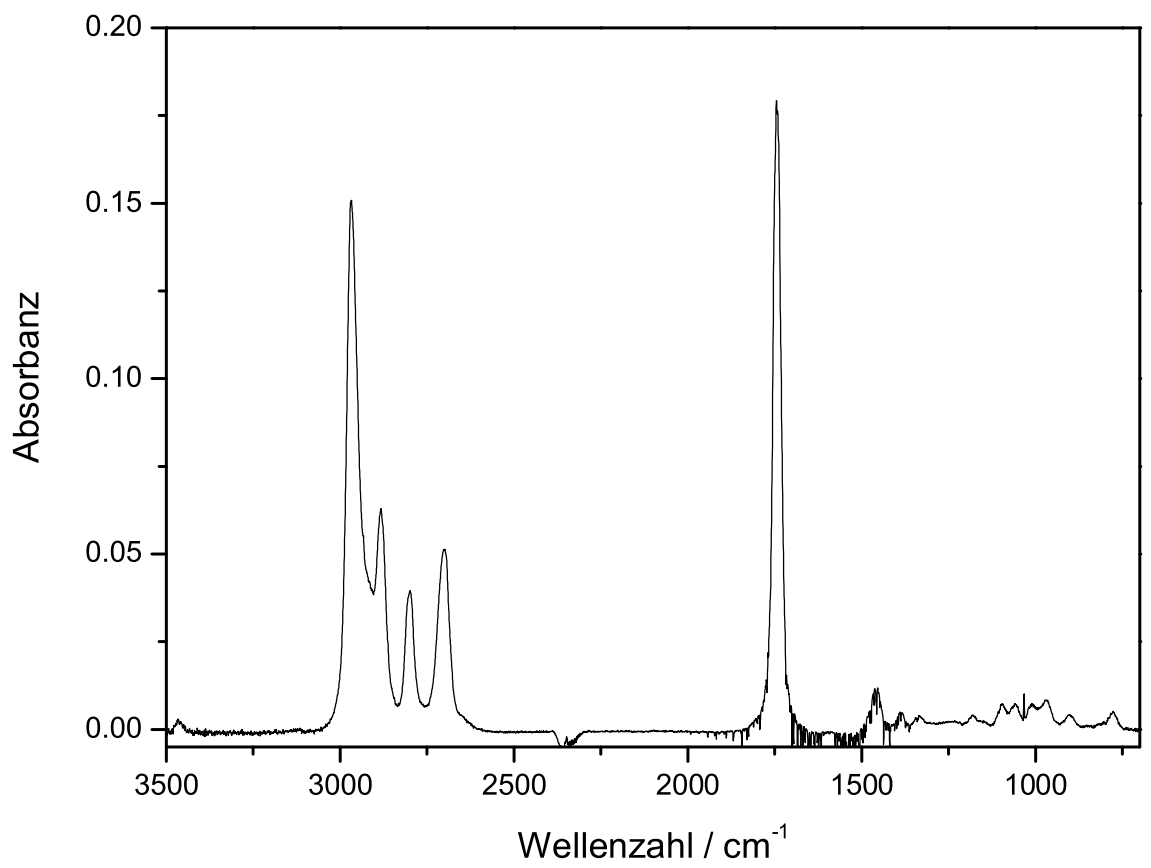

Abbildung 7.28: Kalibrierspektrum Cyclopentancarboxyaldehyd

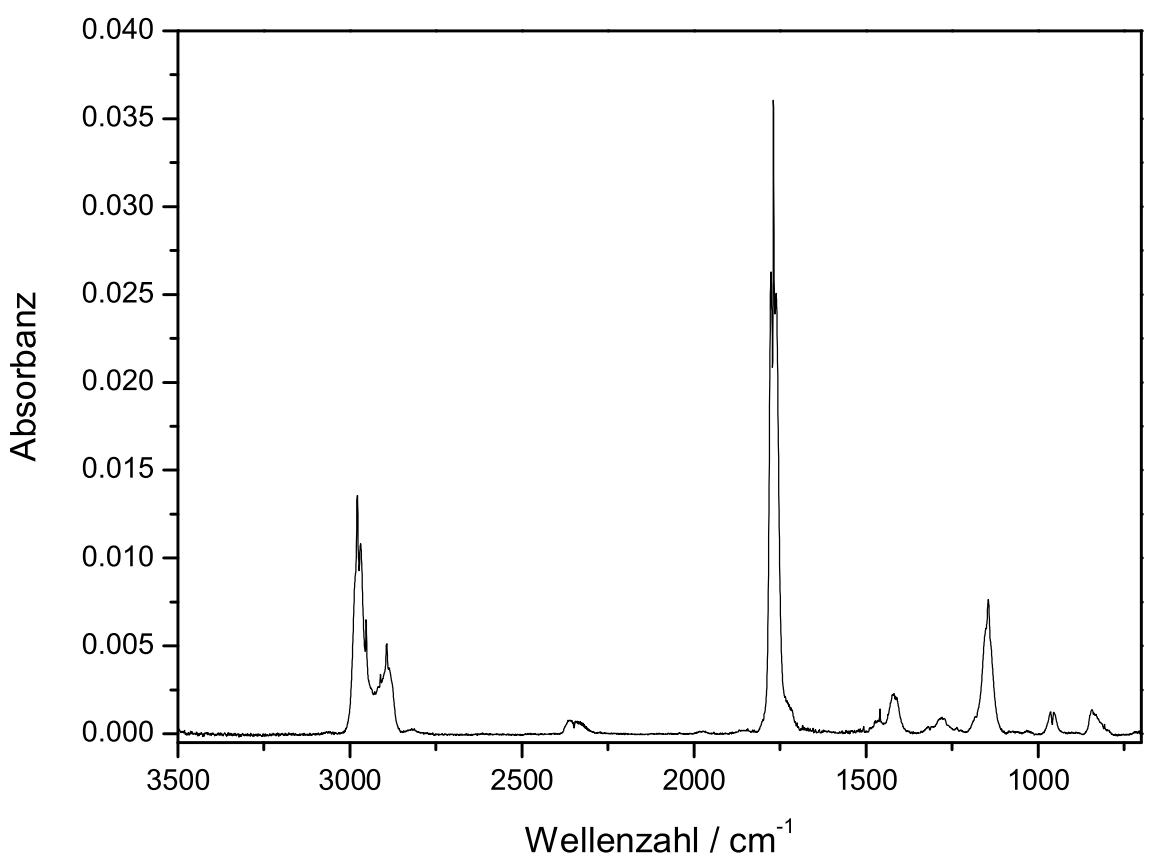

Abbildung 7.29: Kalibrierspektrum Cyclopentanon 


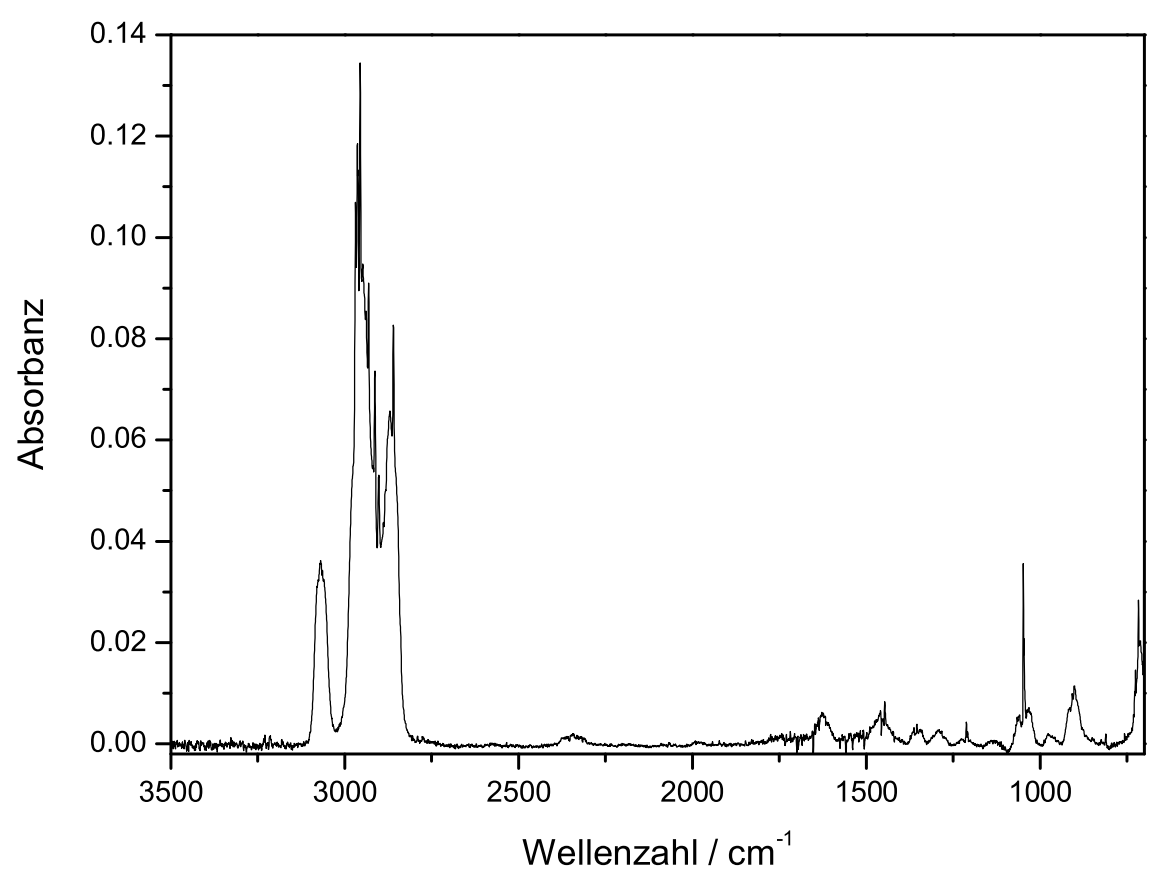

Abbildung 7.30: Kalibrierspektrum Cyclopenten

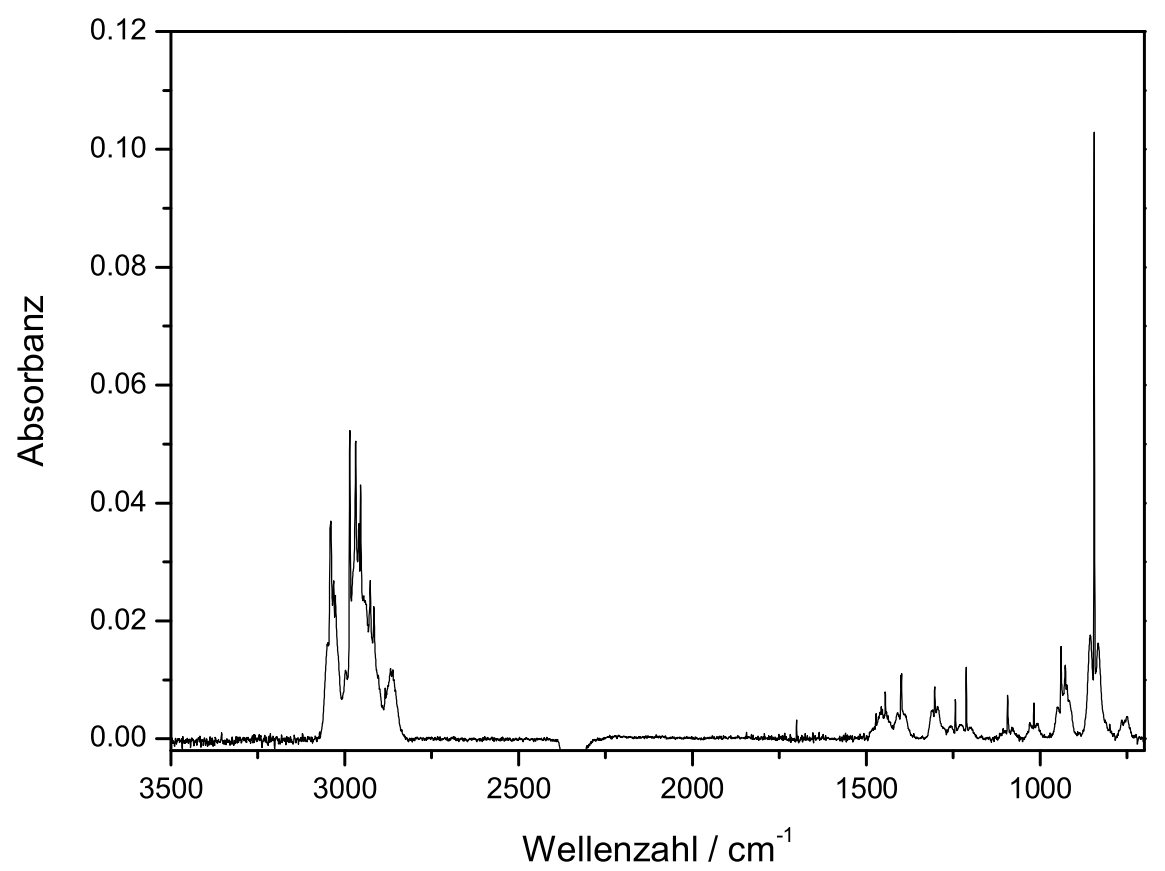

Abbildung 7.31: Kalibrierspektrum Cyclopentenoxid 


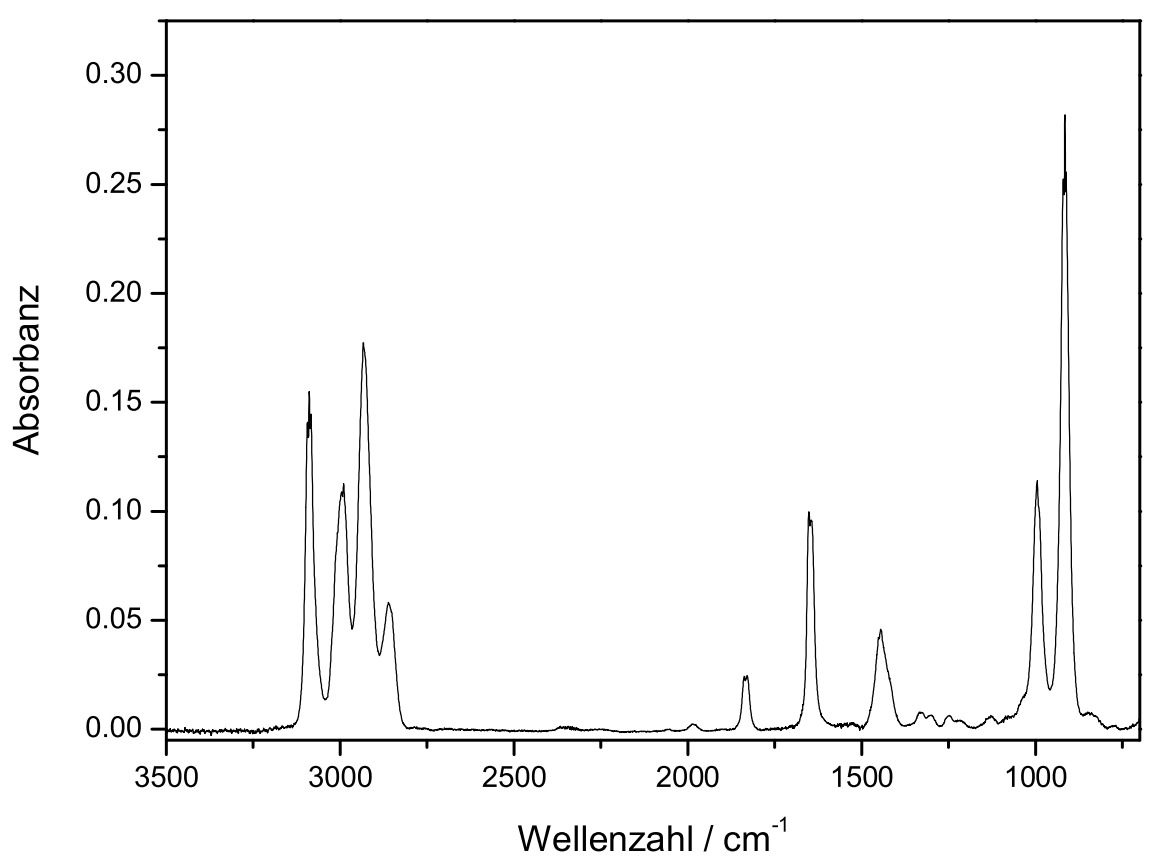

Abbildung 7.32: Kalibrierspektrum Diallyl

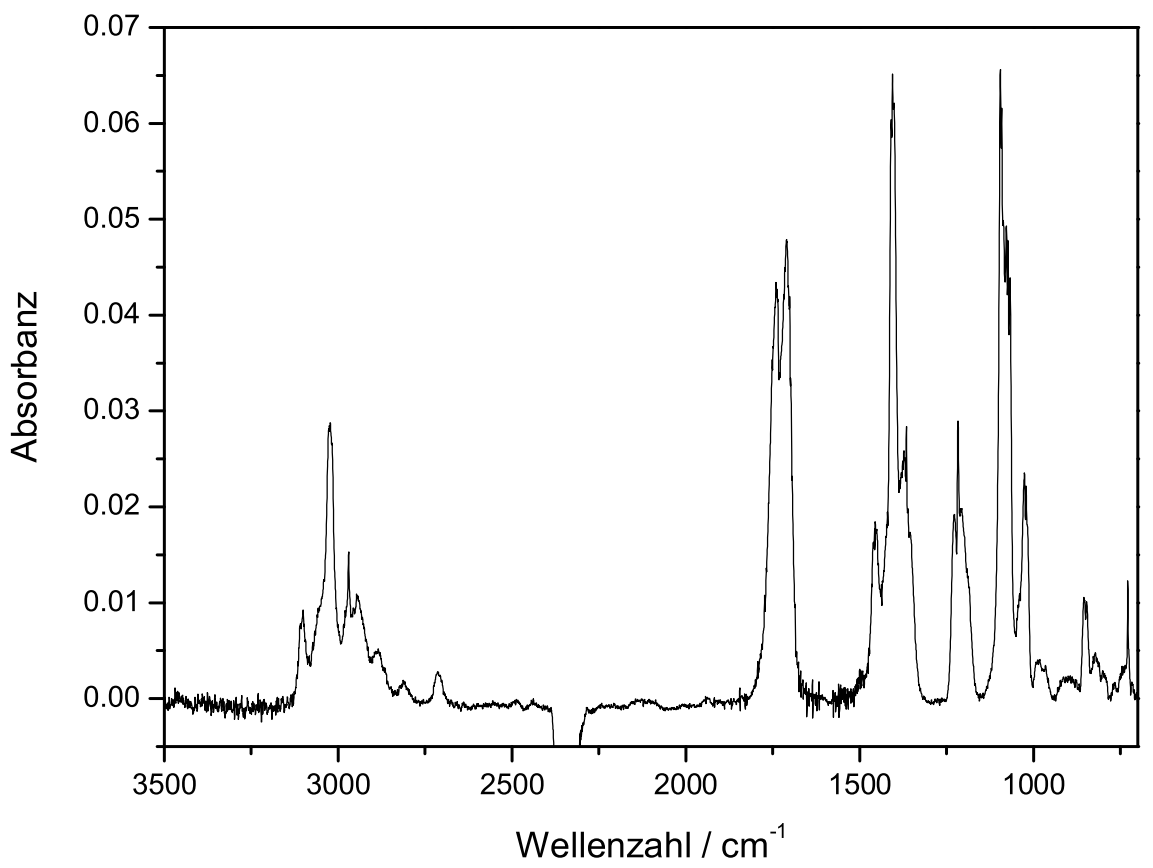

Abbildung 7.33: Kalibrierspektrum Dicyclopropylketon 


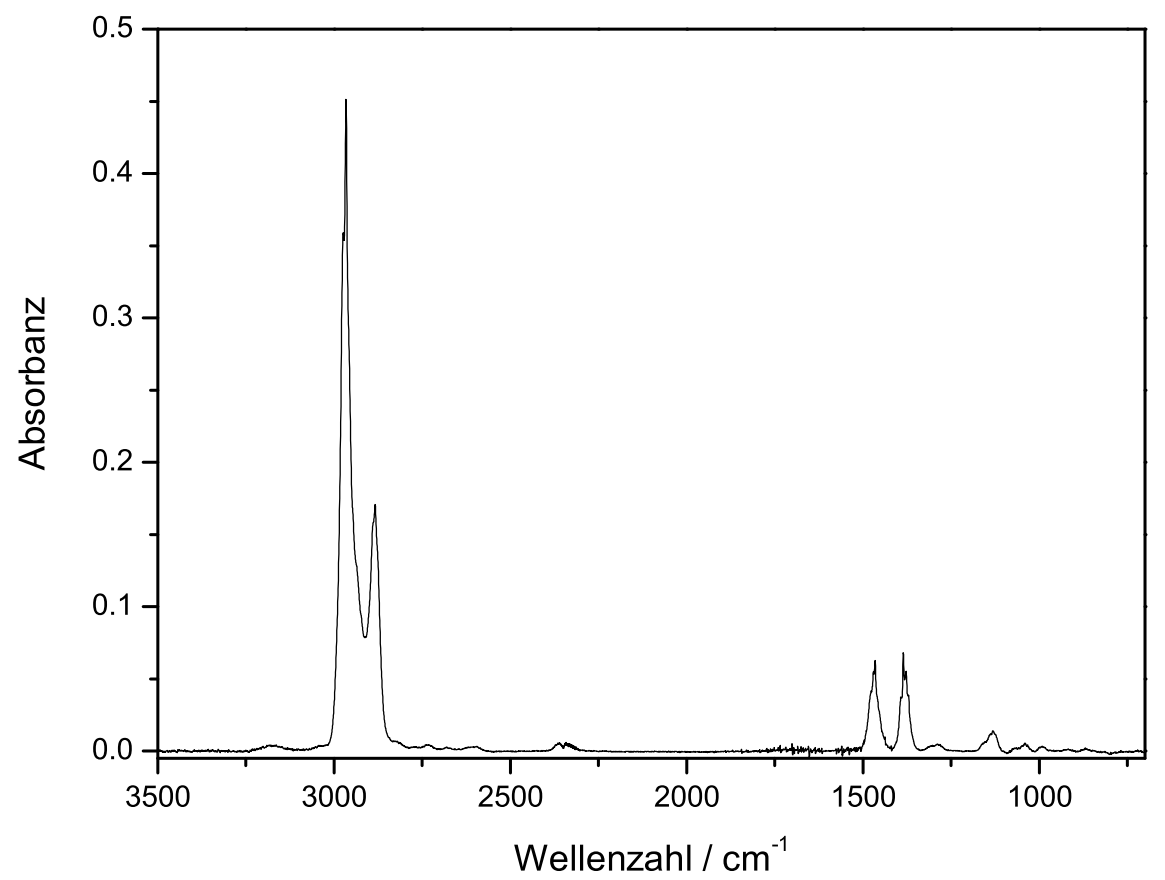

Abbildung 7.34: Kalibrierspektrum Diisopropyl

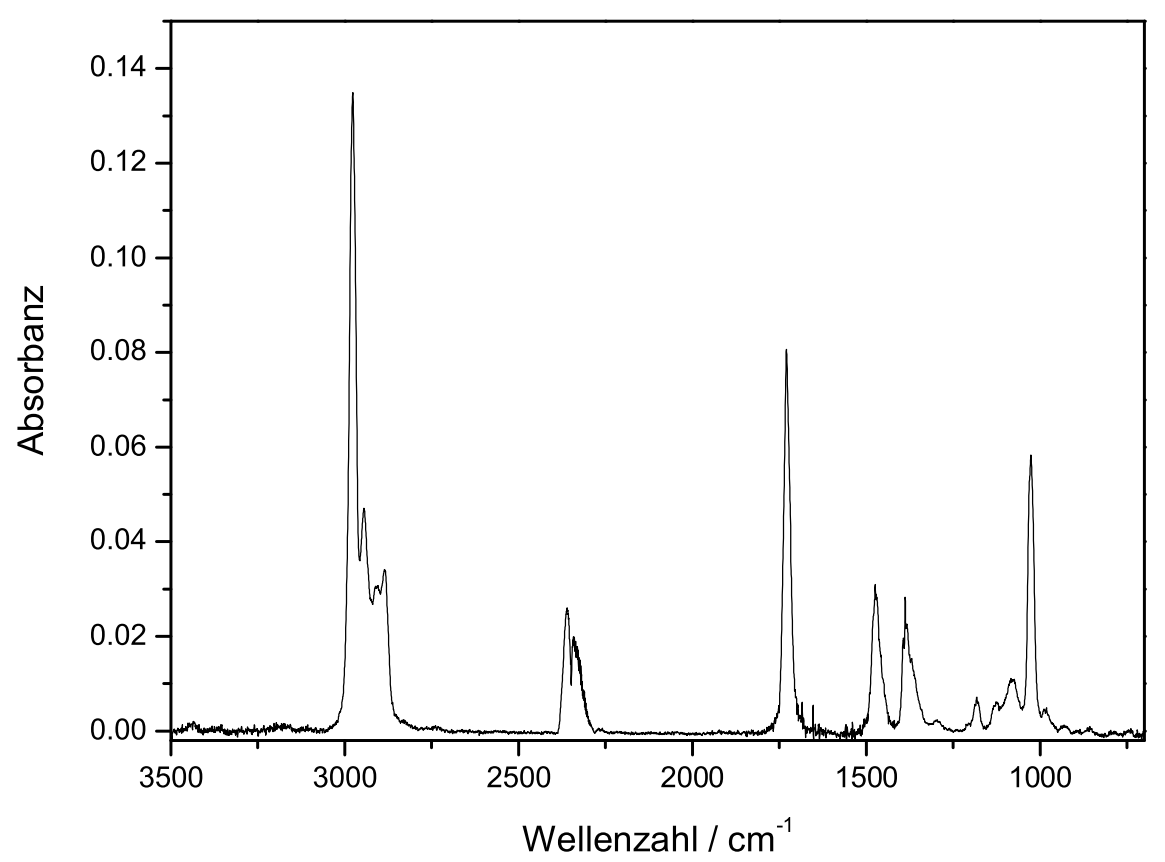

Abbildung 7.35: Kalibrierspektrum Diisopropylketon 


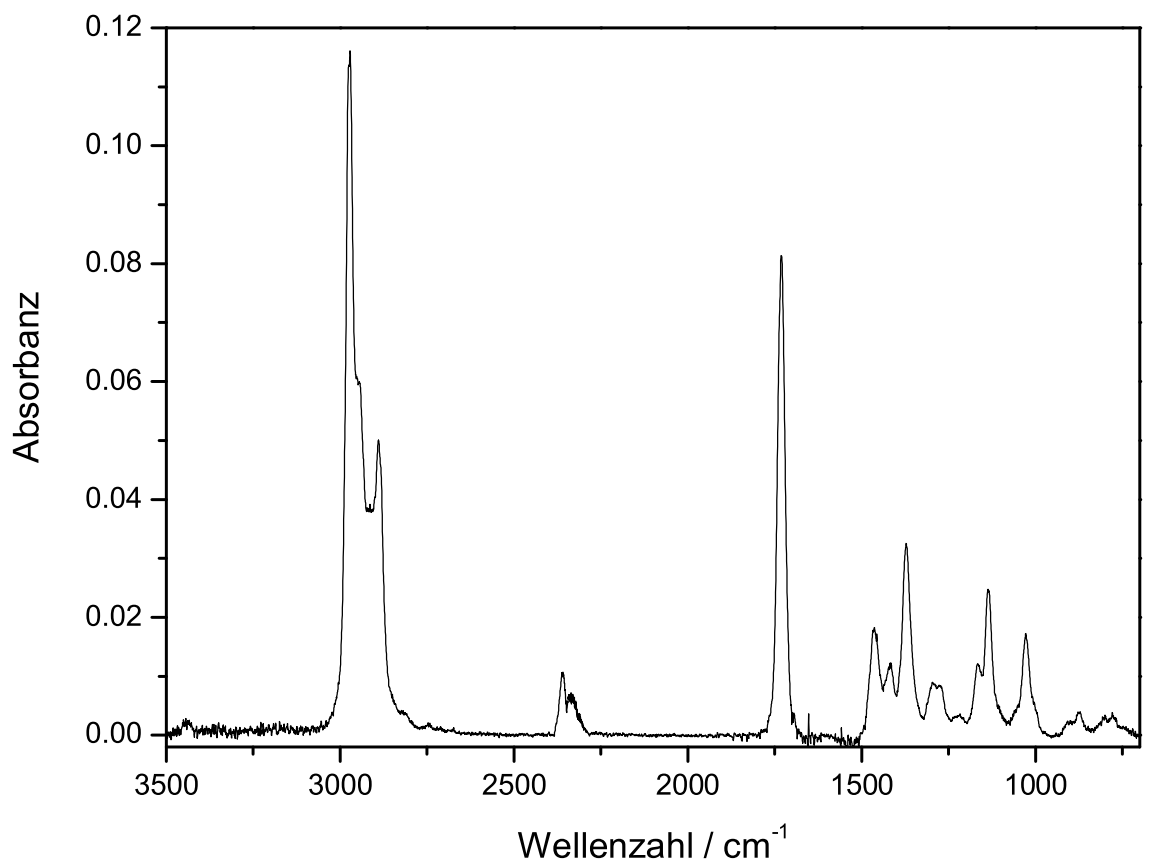

Abbildung 7.36: Kalibrierspektrum Dipropylketon

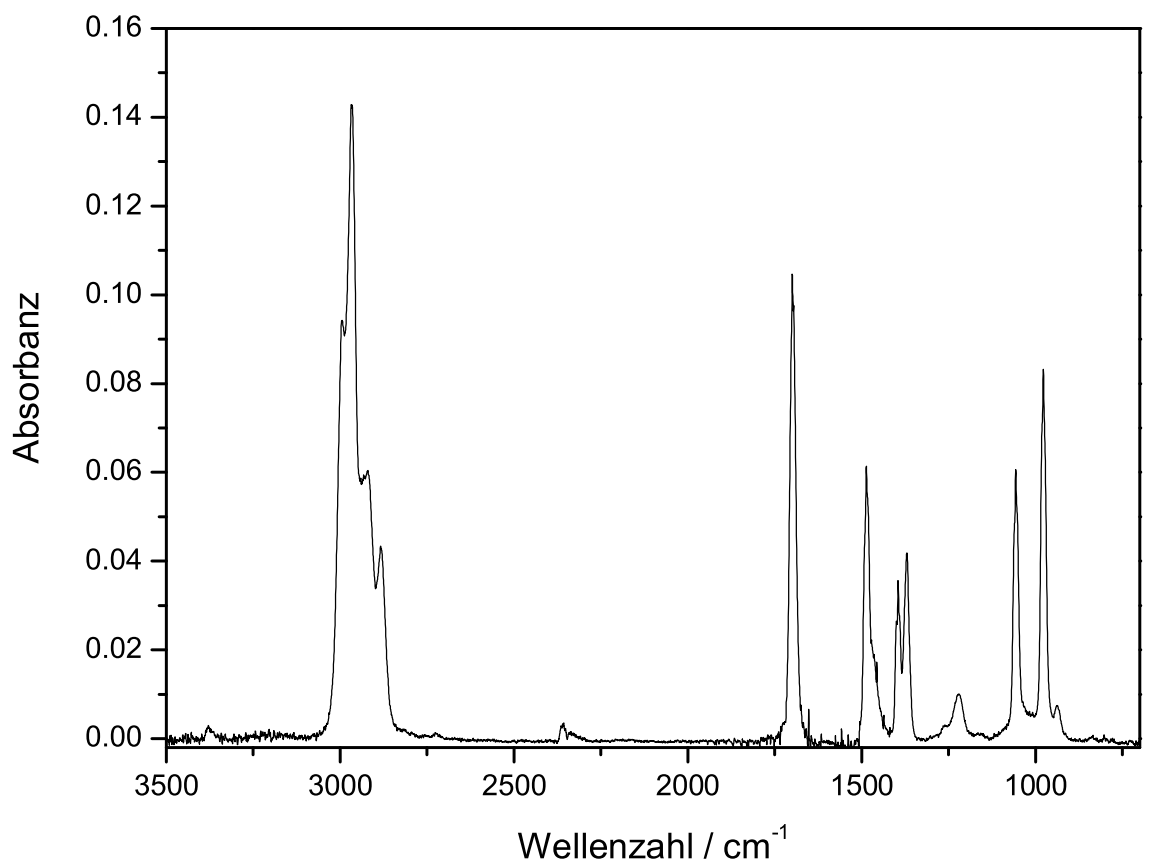

Abbildung 7.37: Kalibrierspektrum Di-tert-Butylketon 


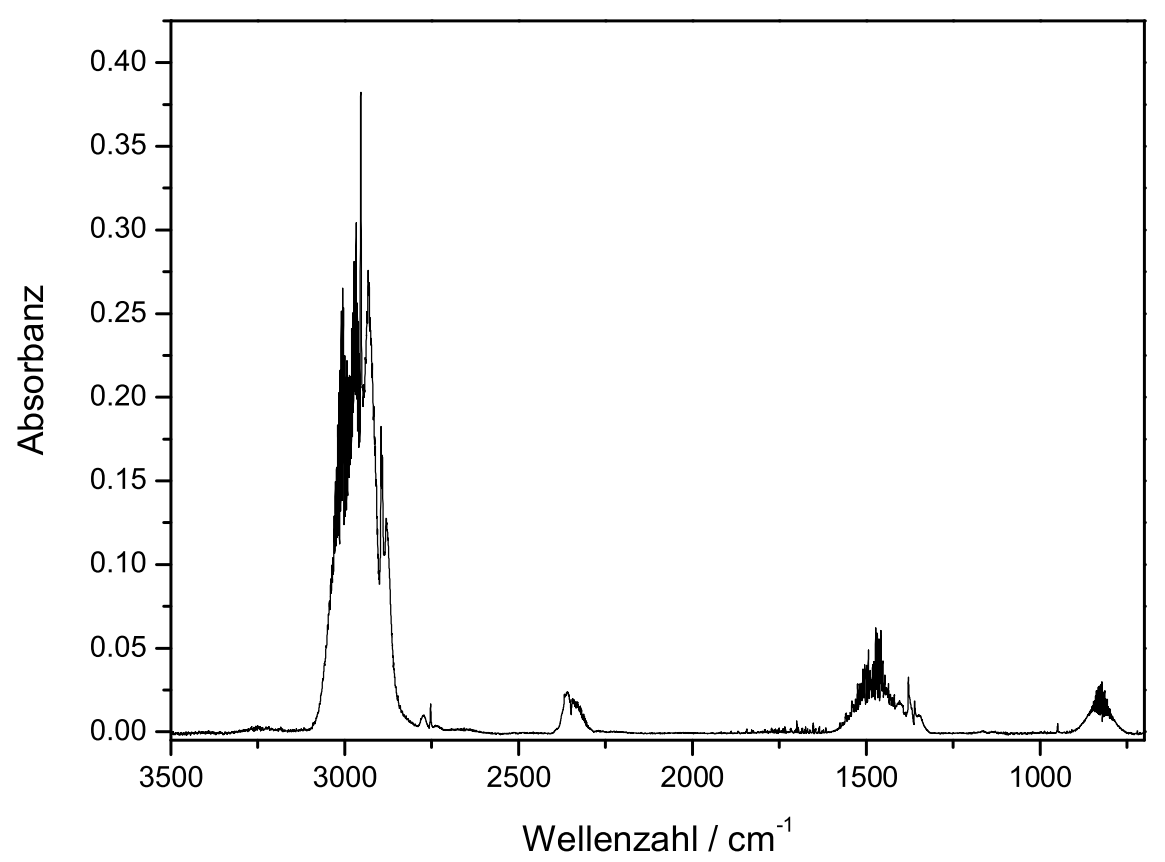

Abbildung 7.38: Kalibrierspektrum Ethan

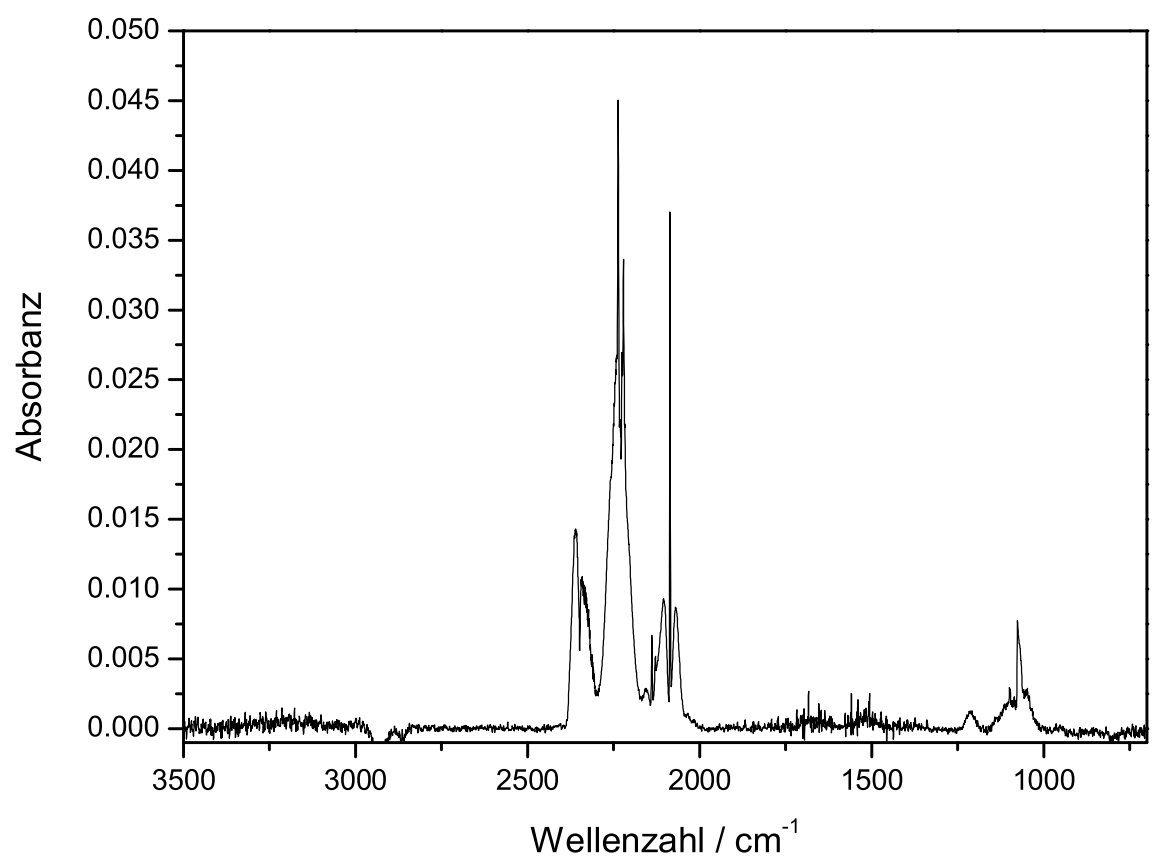

Abbildung 7.39: Kalibrierspektrum Ethan-d 6 


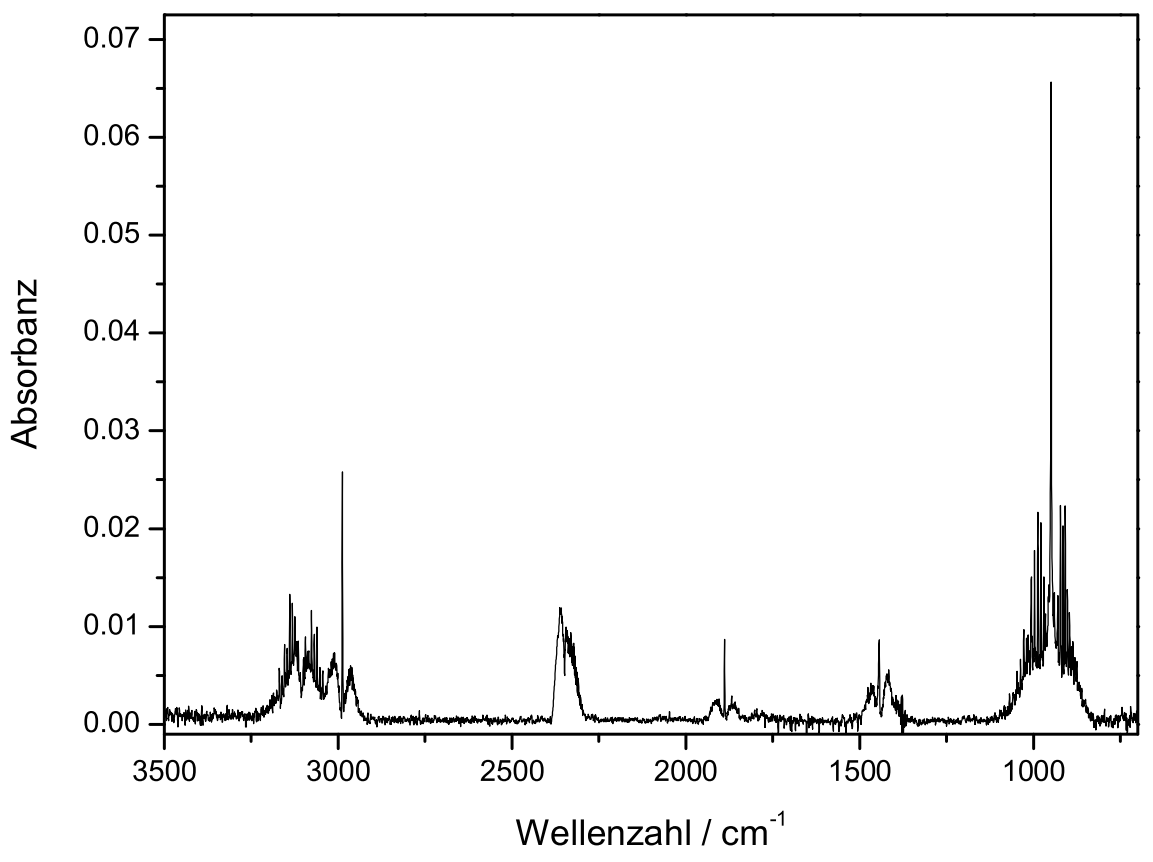

Abbildung 7.40: Kalibrierspektrum Ethen

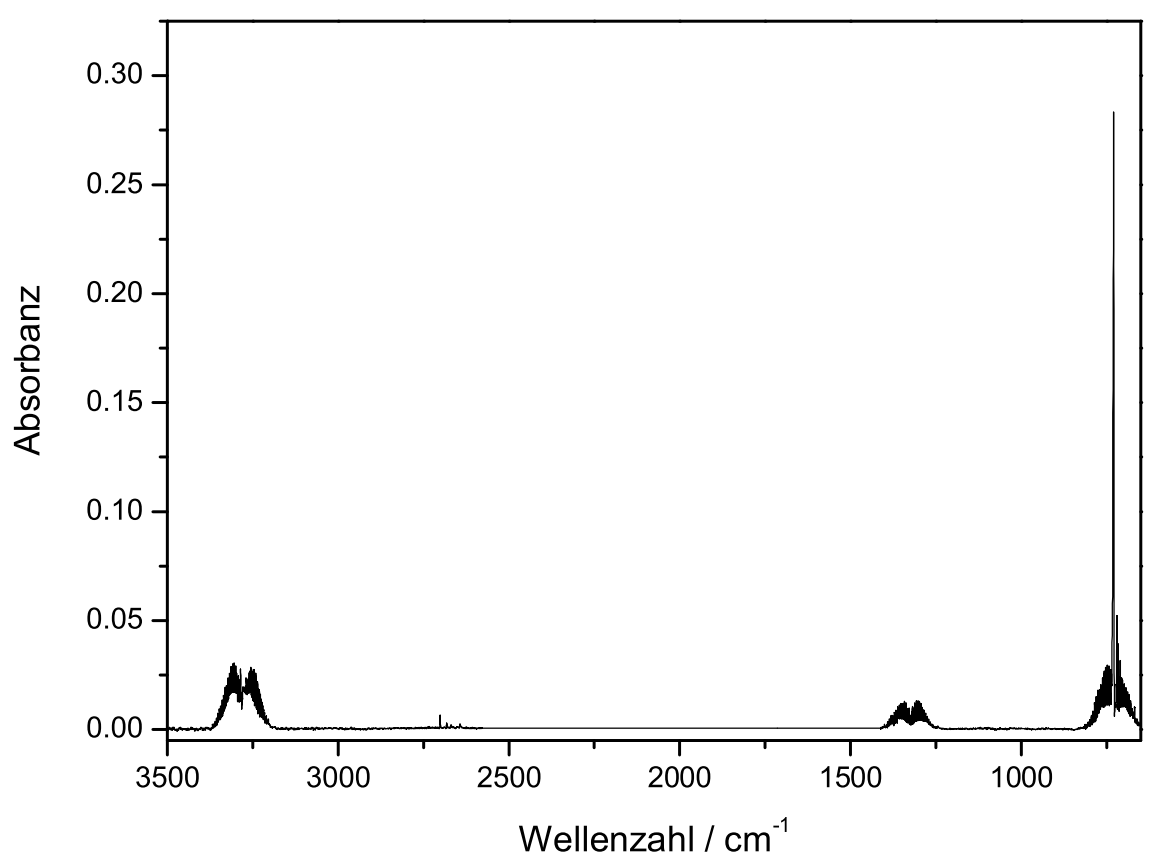

Abbildung 7.41: Kalibrierspektrum Ethin 


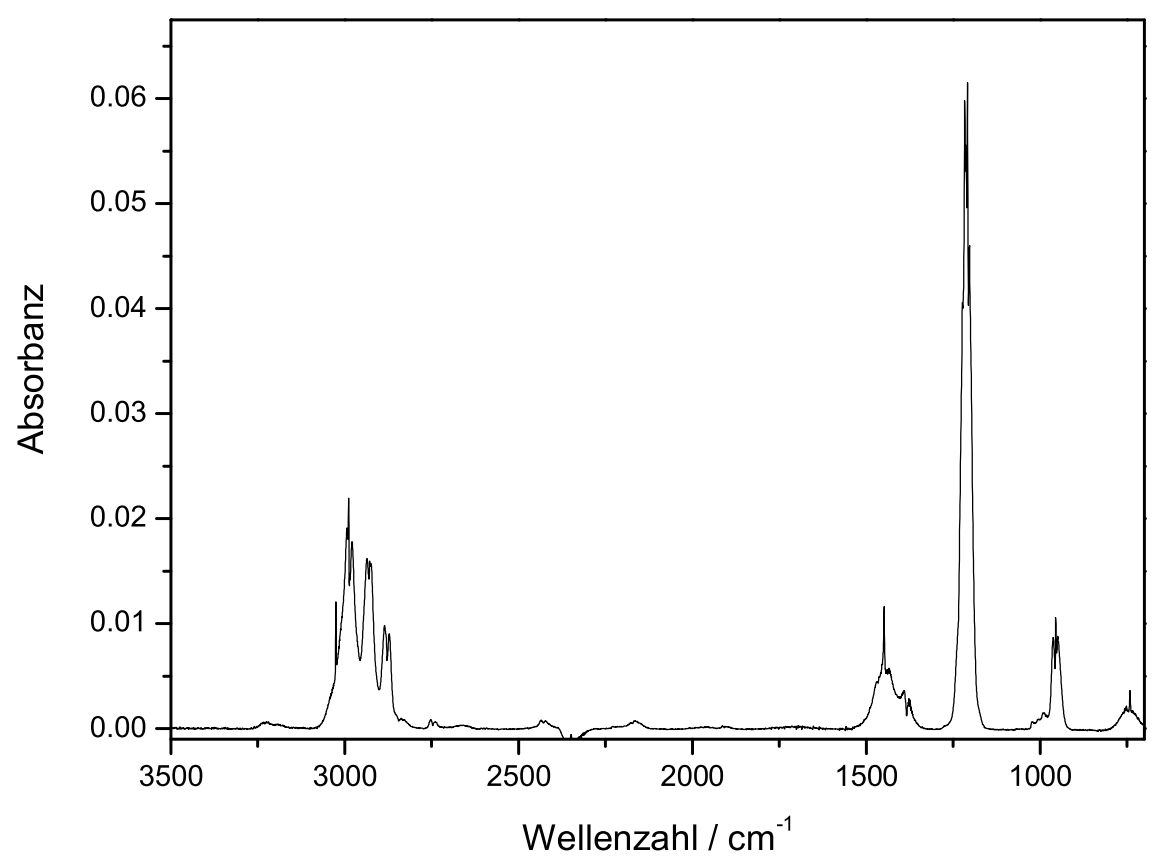

Abbildung 7.42: Kalibrierspektrum Ethyliodid

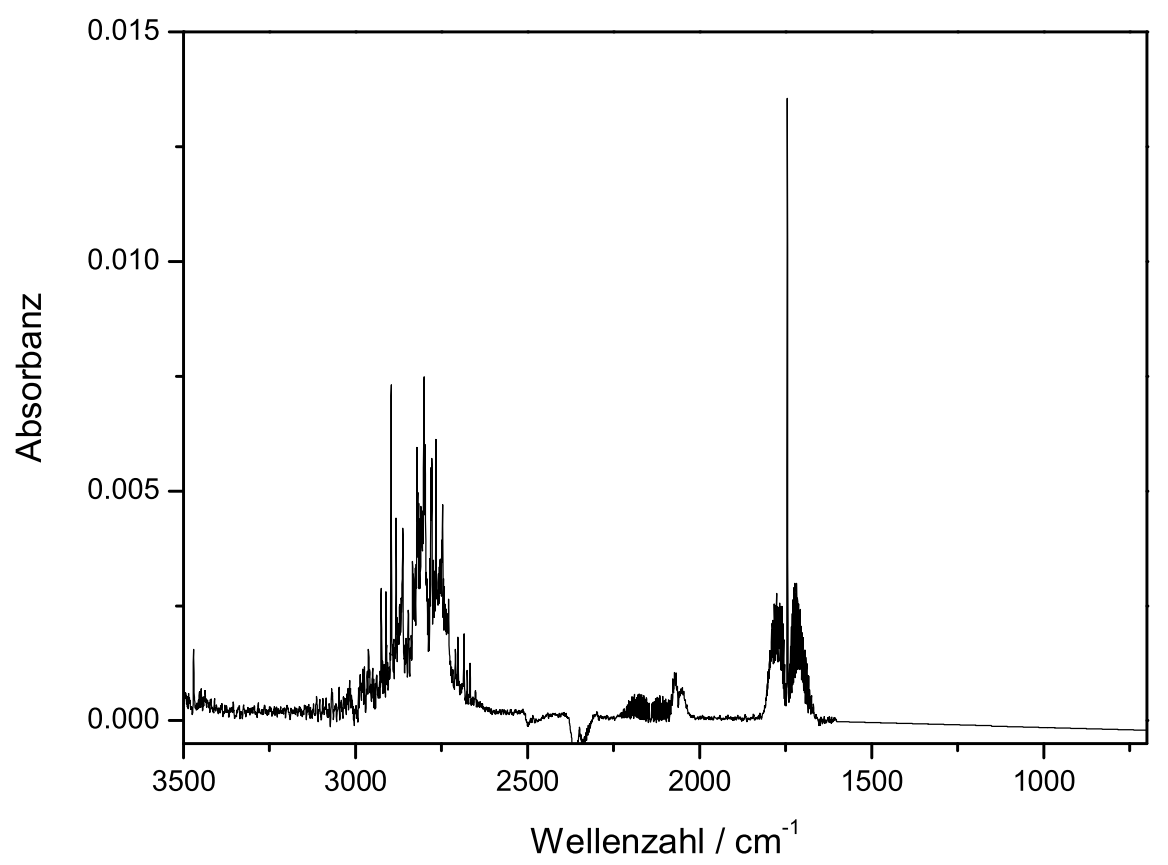

Abbildung 7.43: Kalibrierspektrum Formaldehyd 


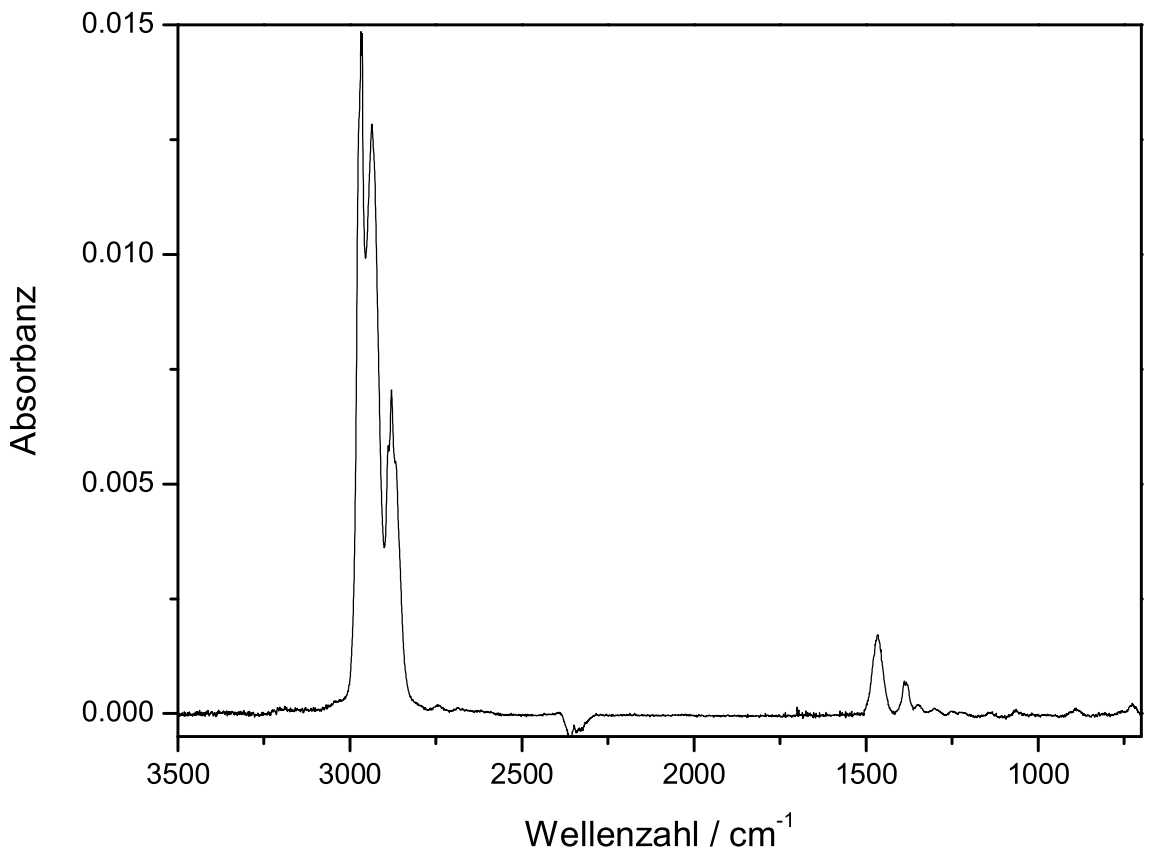

Abbildung 7.44: Kalibrierspektrum Hexan

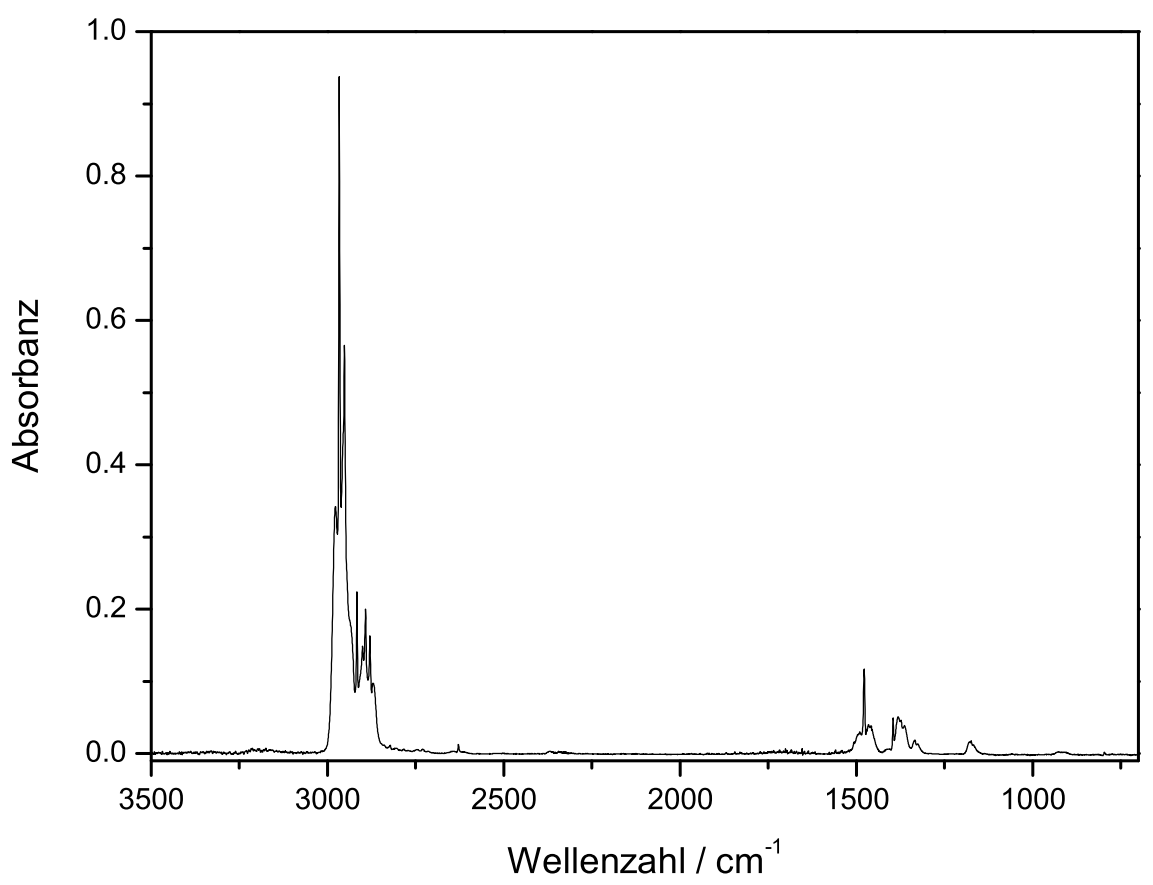

Abbildung 7.45: Kalibrierspektrum Isobutan 


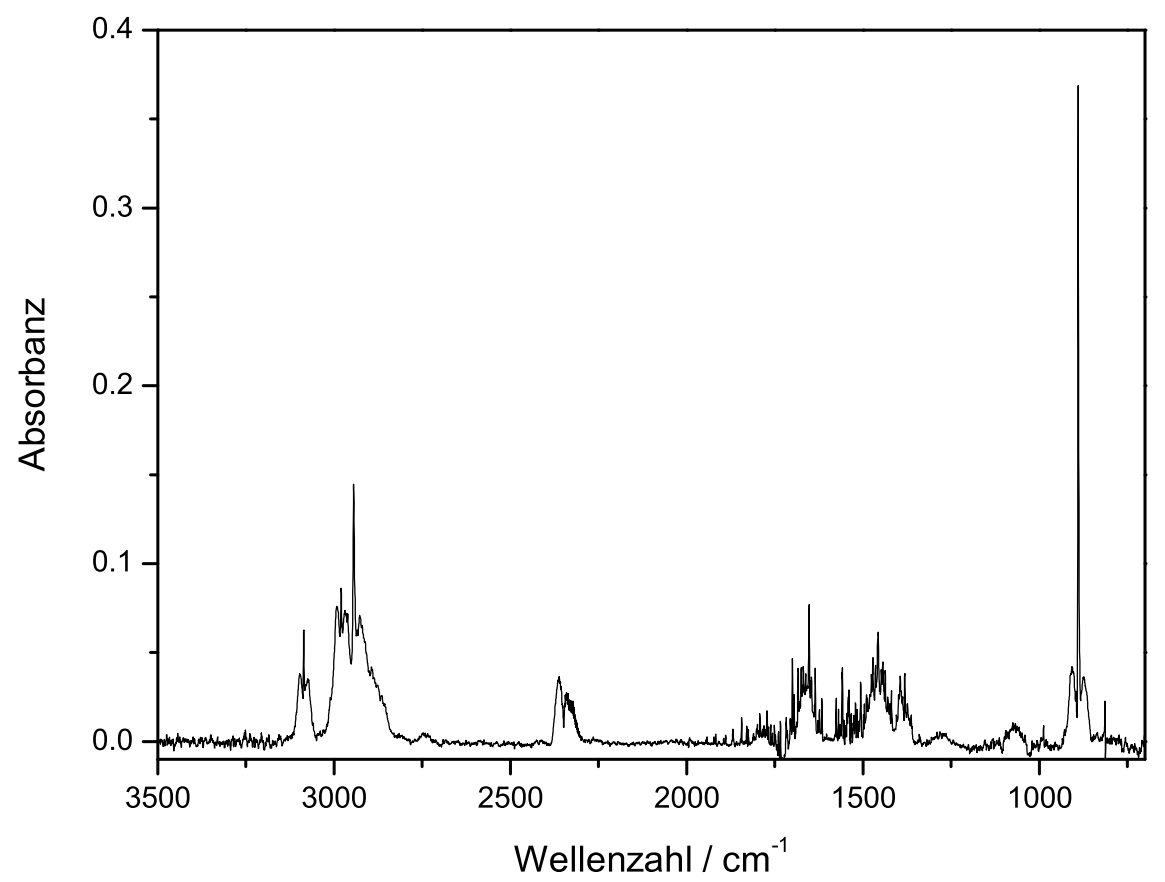

Abbildung 7.46: Kalibrierspektrum Isobuten

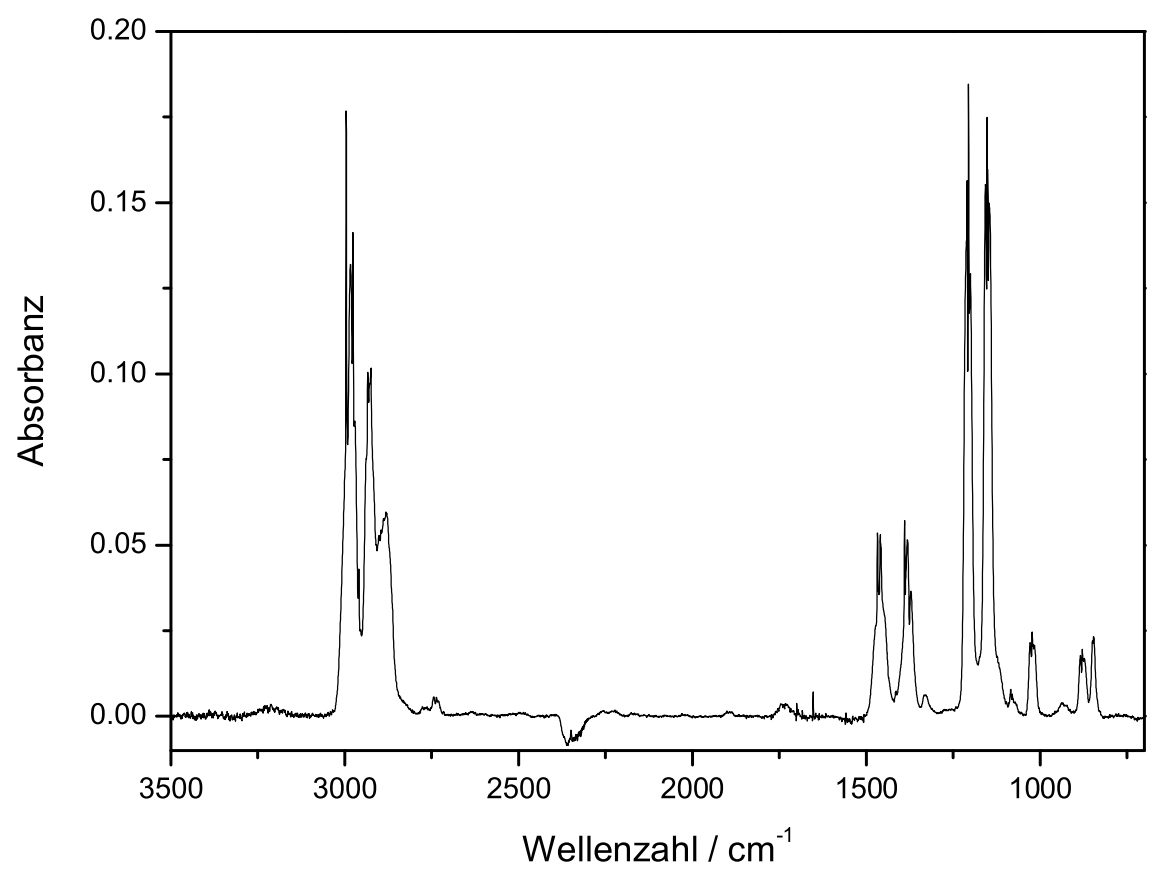

Abbildung 7.47: Kalibrierspektrum Isopropyliodid 


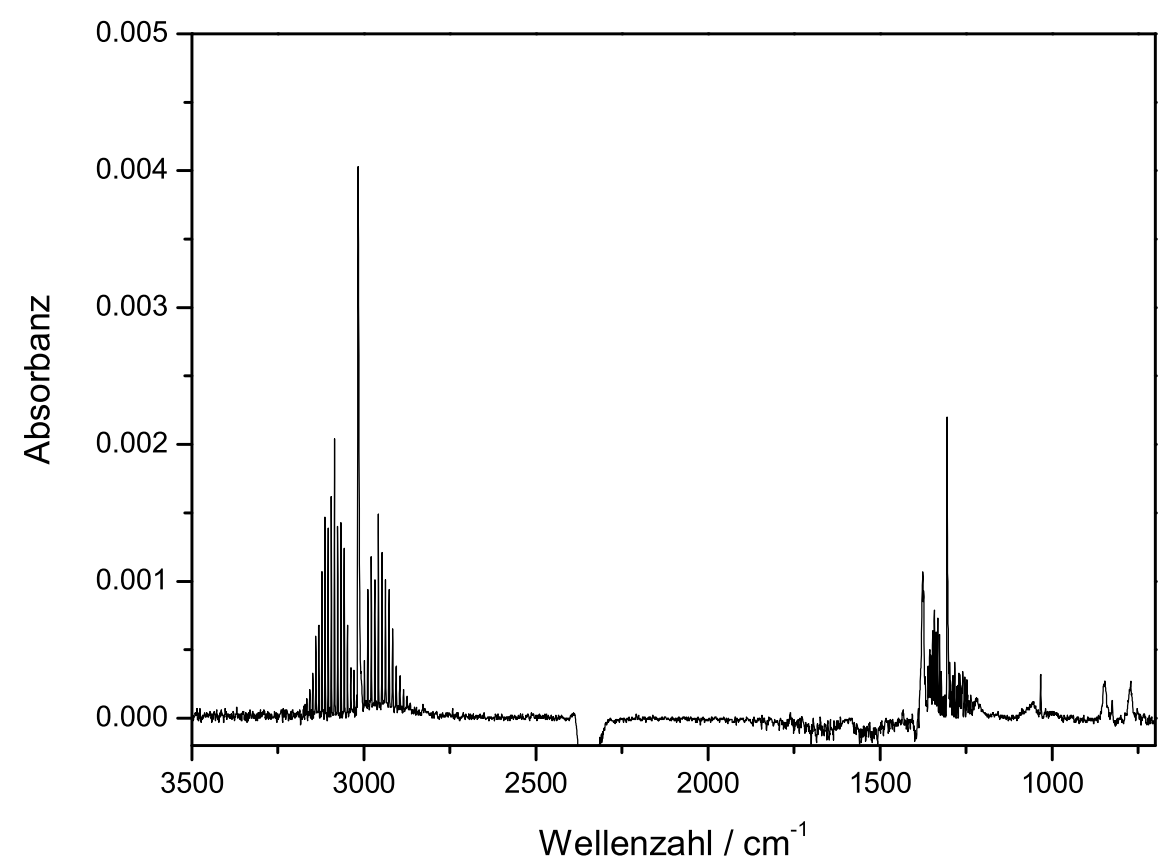

Abbildung 7.48: Kalibrierspektrum Methan

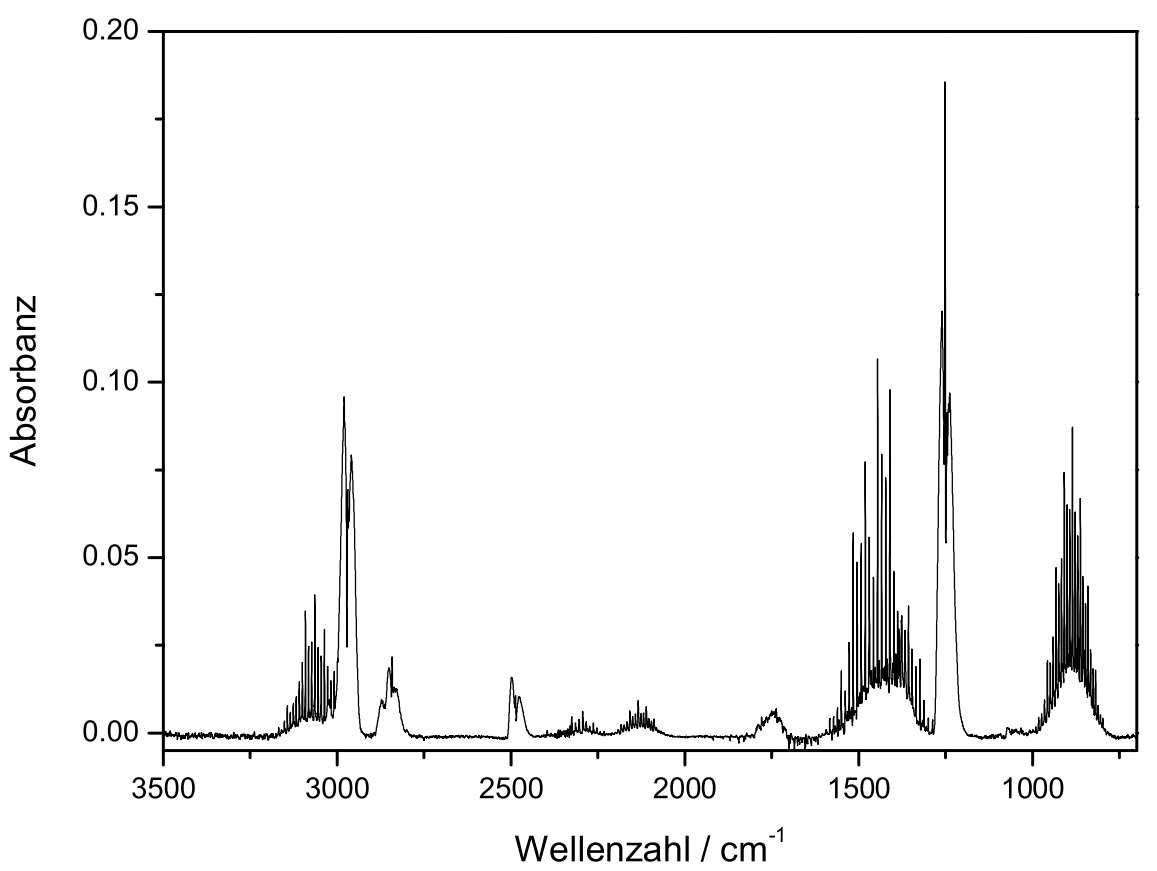

Abbildung 7.49: Kalibrierspektrum Methyliodid 


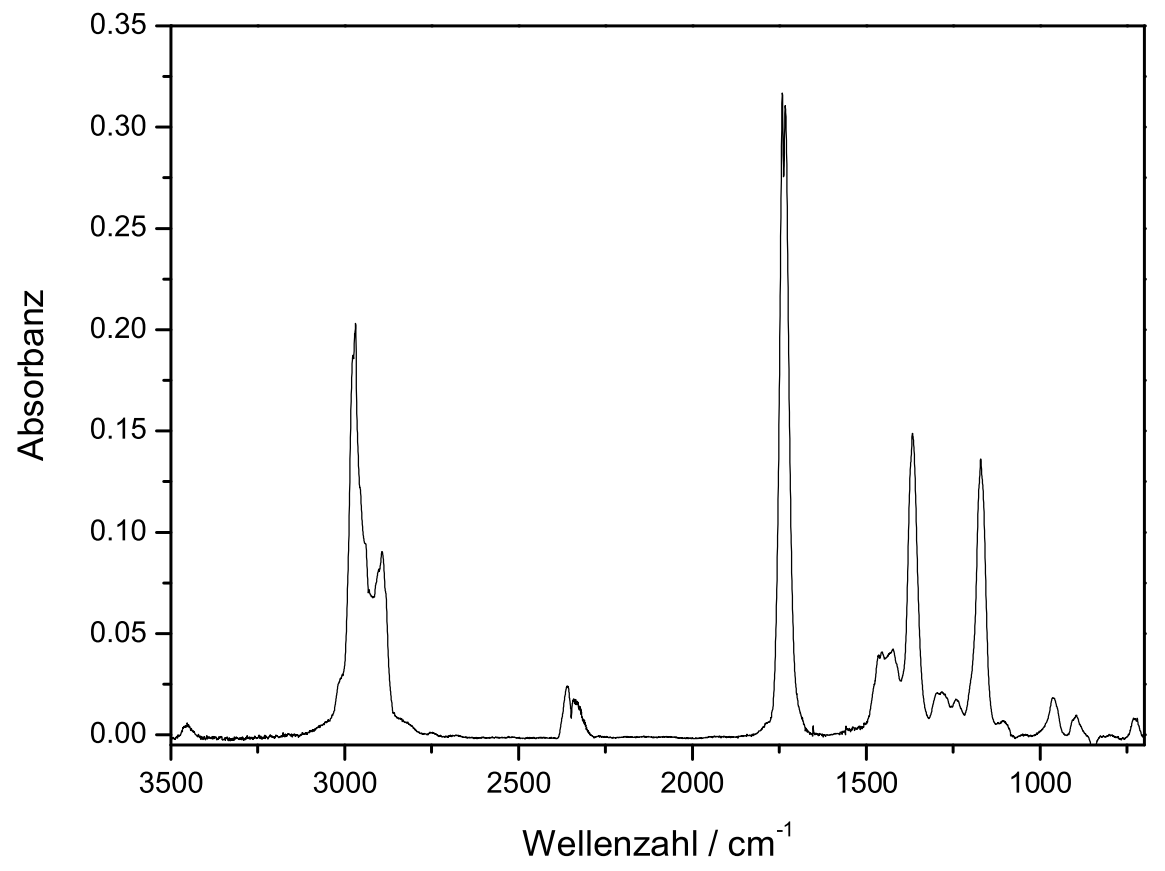

Abbildung 7.50: Kalibrierspektrum Methyl-Propyl-Keton

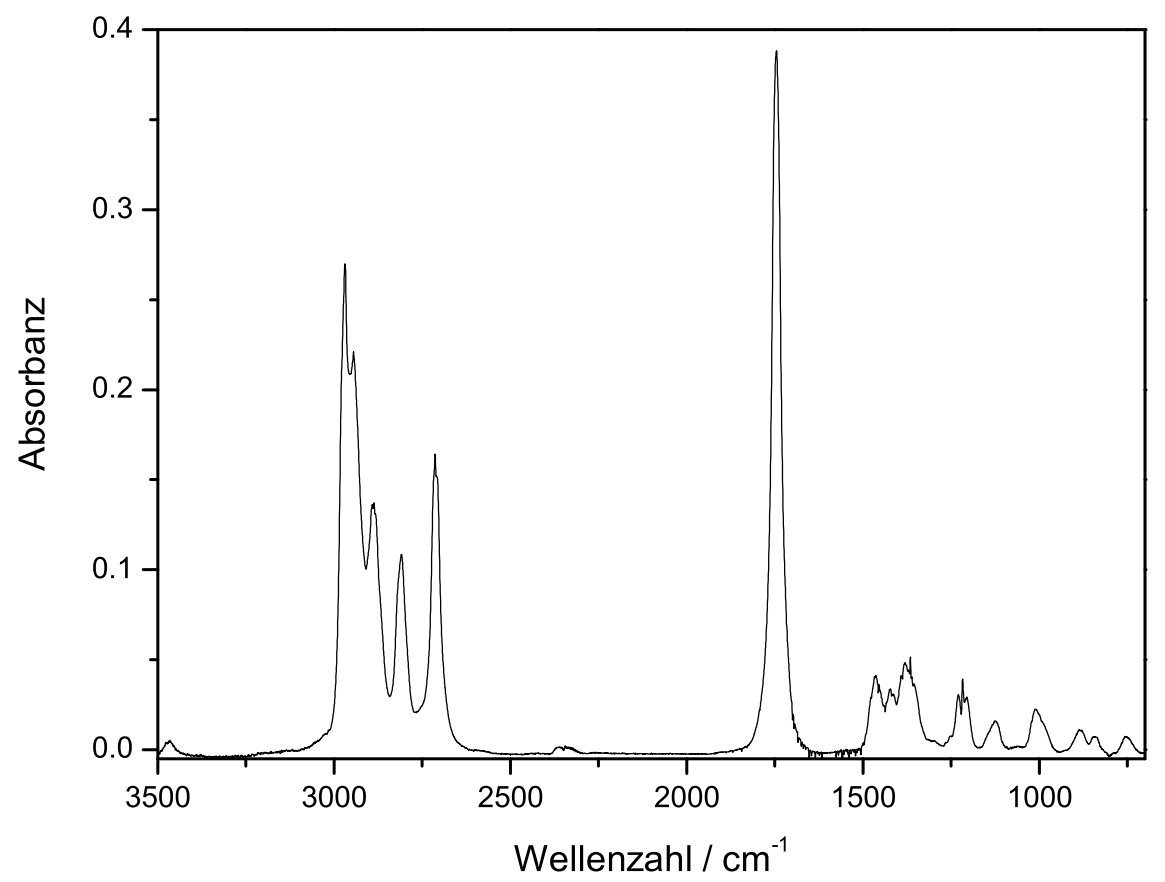

Abbildung 7.51: Kalibrierspektrum Pentanal 


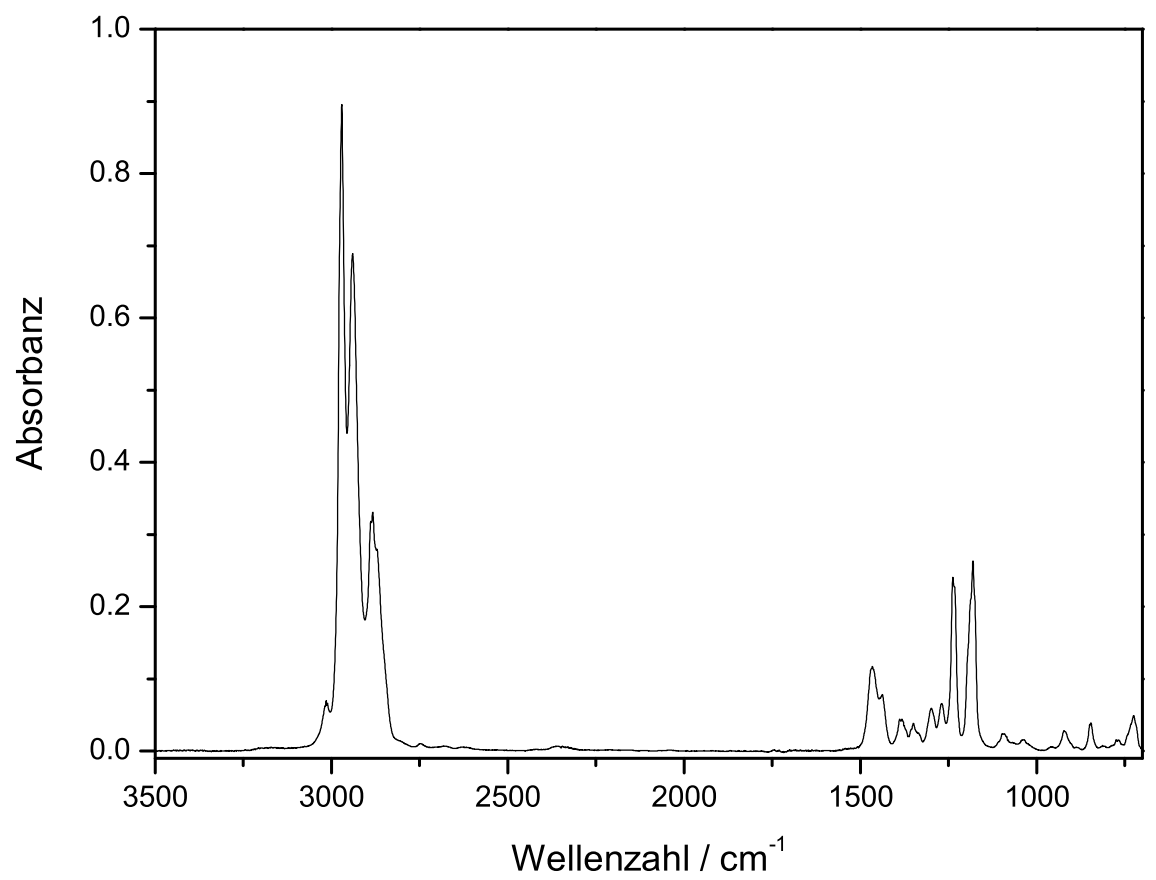

Abbildung 7.52: Kalibrierspektrum Pentyliodid

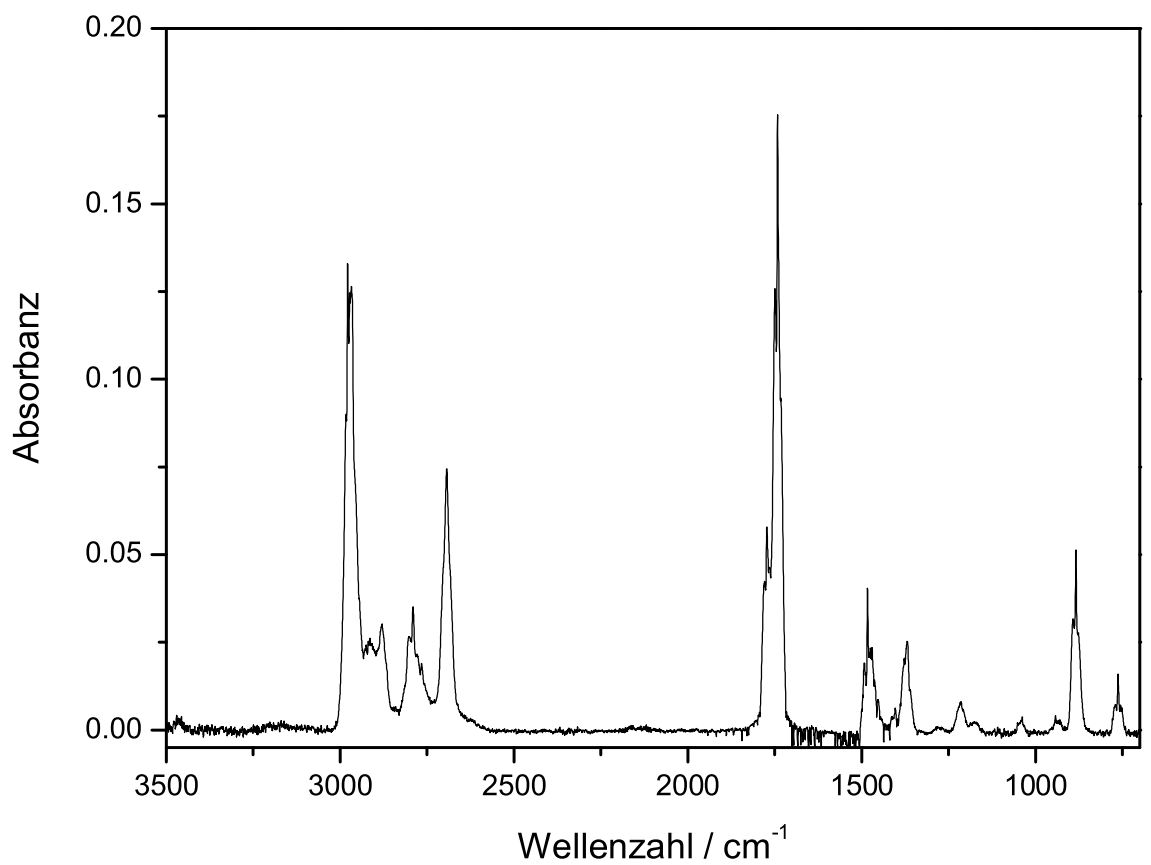

Abbildung 7.53: Kalibrierspektrum Pivaldehyd 


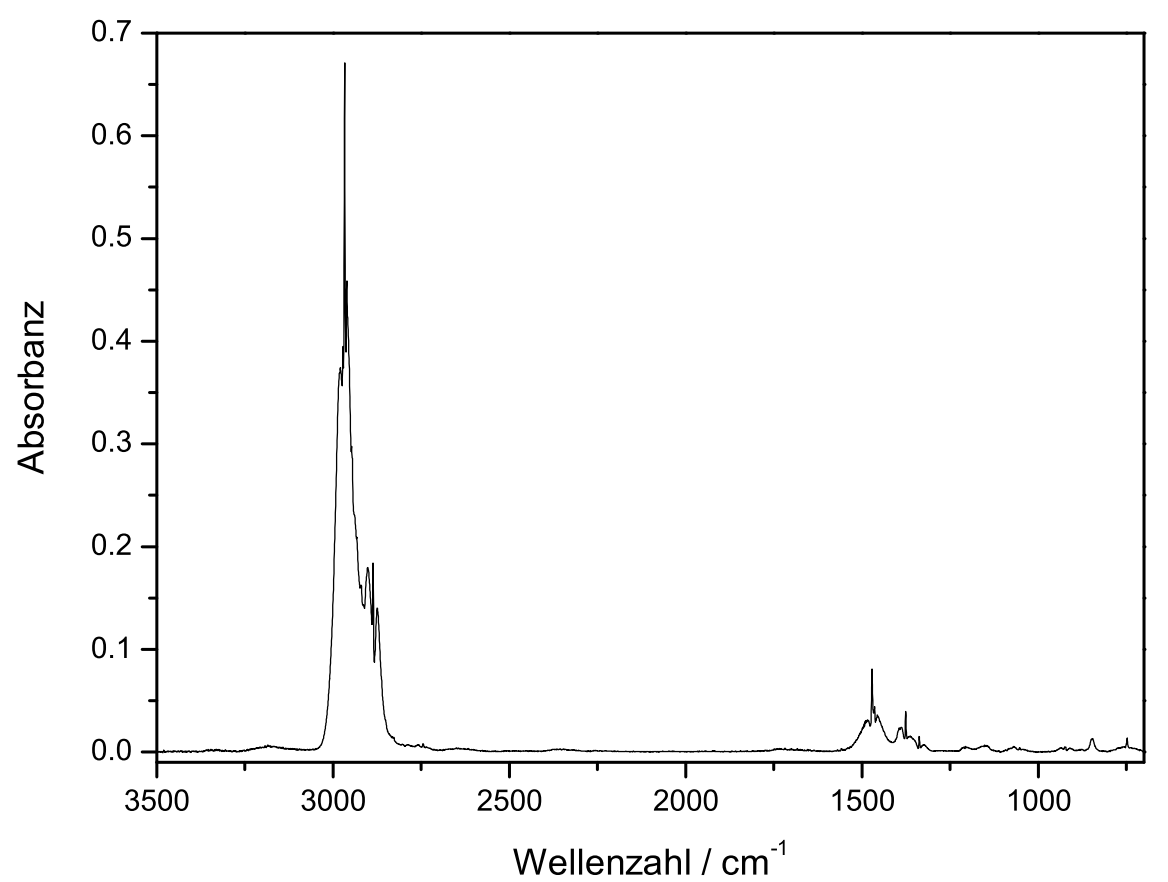

Abbildung 7.54: Kalibrierspektrum Propan

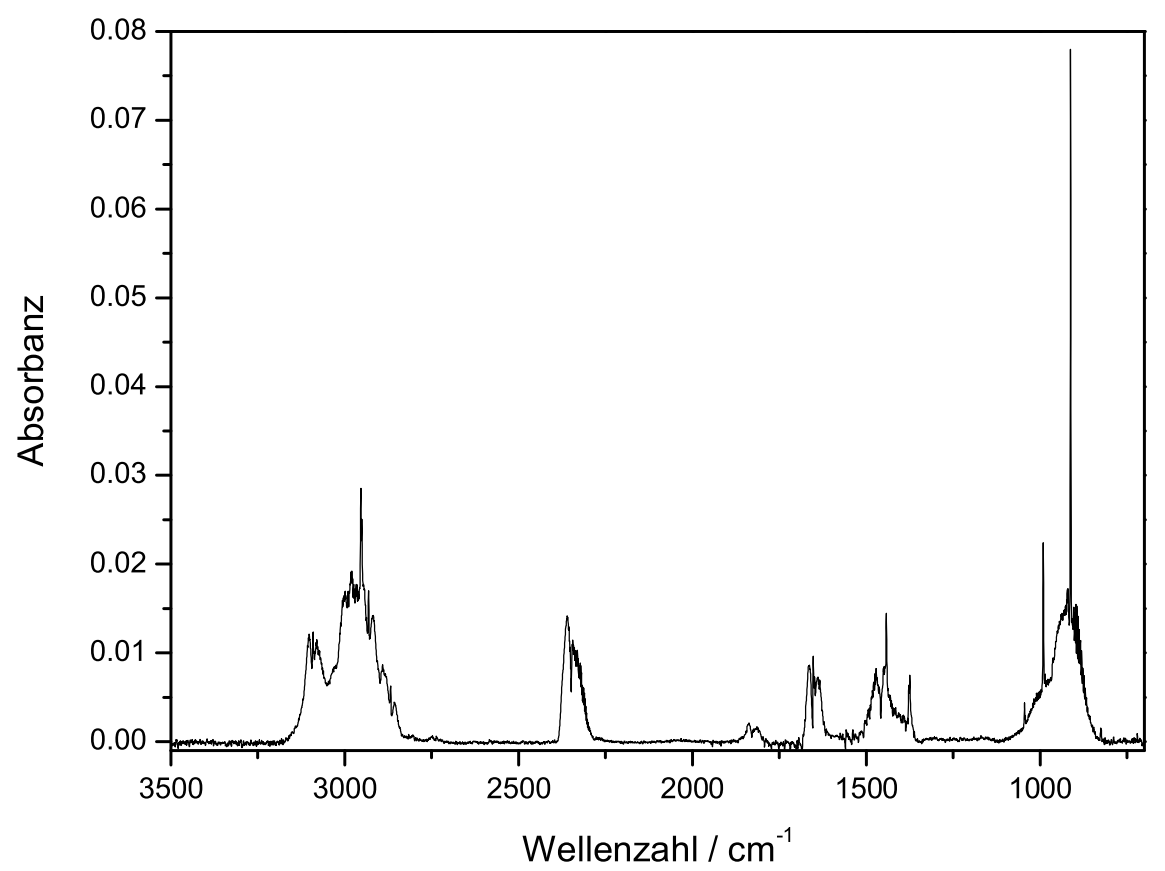

Abbildung 7.55: Kalibrierspektrum Propen 


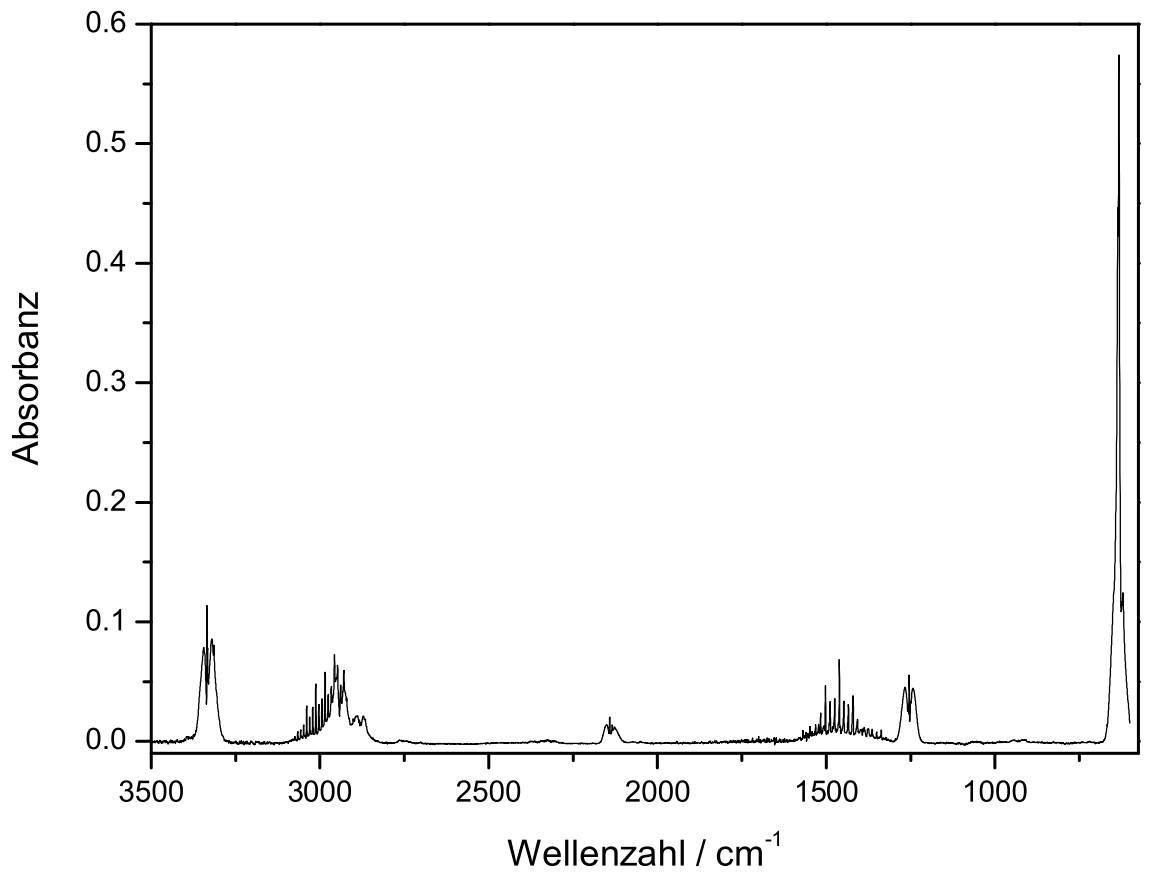

Abbildung 7.56: Kalibrierspektrum Propin

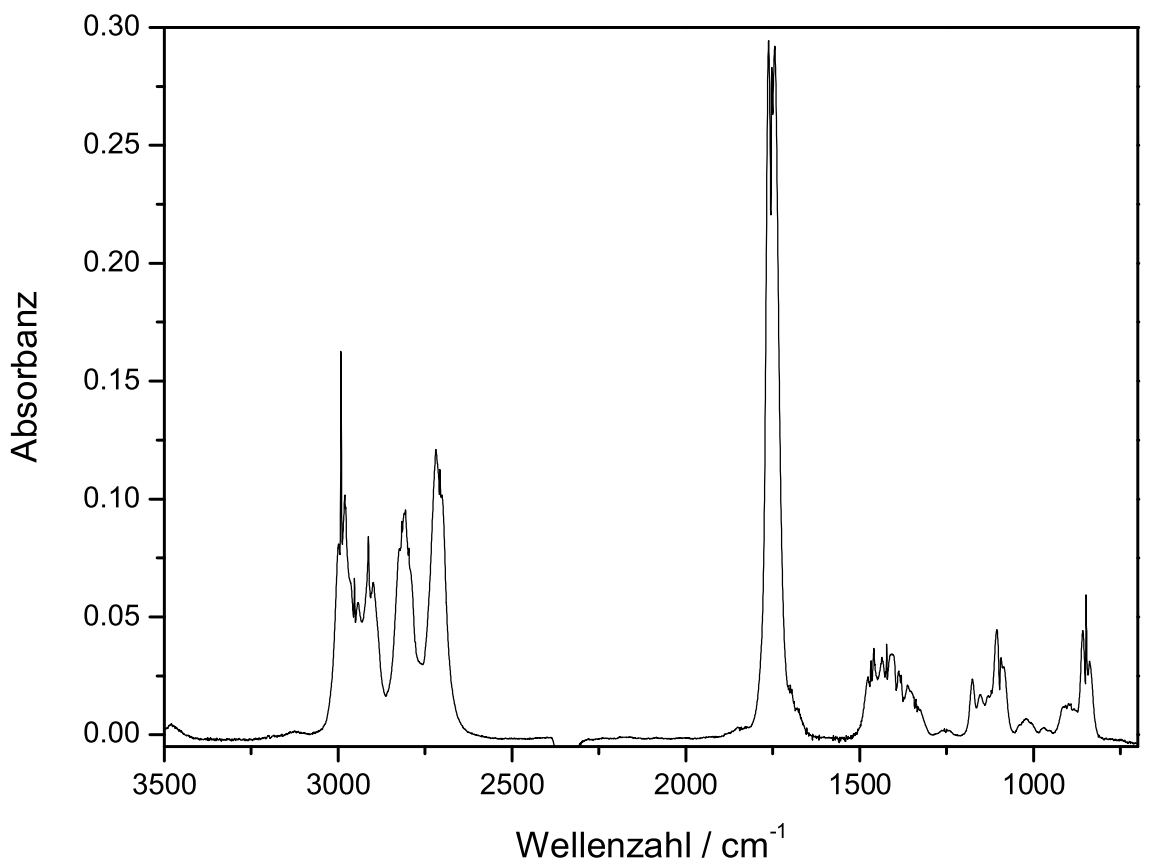

Abbildung 7.57: Kalibrierspektrum Propionaldehyd 


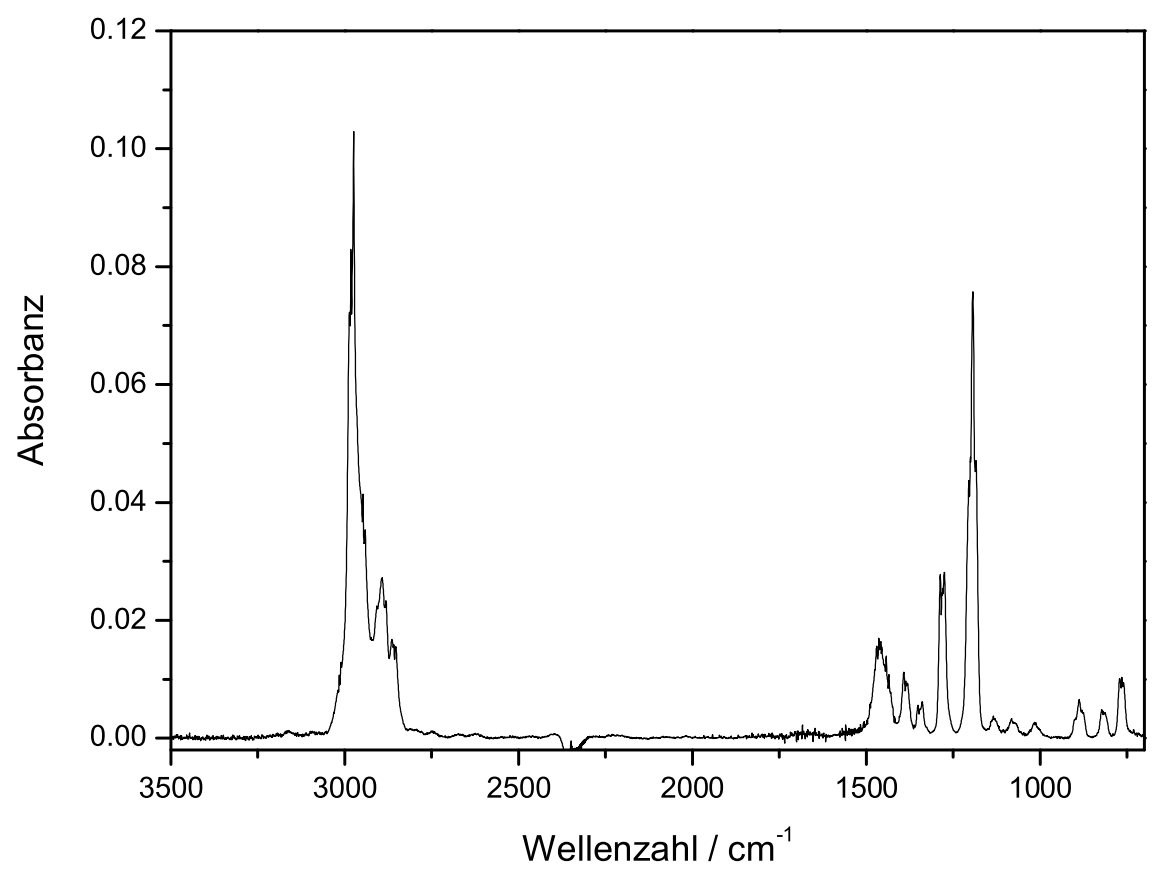

Abbildung 7.58: Kalibrierspektrum Propyliodid 


\subsection{Integrale Absorptionskoeffizienten}

\begin{tabular}{|c|c|c|}
\hline Substanz & Integraler Absorptionskoeffizient / bar $^{-1}$ & Integrationsbereich $/ \mathrm{cm}^{-1}$ \\
\hline 1-Penten & 33,5 & $2990-2920$ \\
\hline 2-Pentanon & 15,4 & $1185-1150$ \\
\hline 2-trans-Buten & 39,2 & $2990-2915$ \\
\hline 4-Pentenal & 14,0 & $2740-2680$ \\
\hline 5-Hexenal & 13,1 & $2725-2700$ \\
\hline Acetaldehyd & 55,7 & $1775-1715$ \\
\hline Aceton & 69 & $1235-1195$ \\
\hline Aceton- $\mathrm{d}_{6}$ & 69,0 & $1760-1710$ \\
\hline Acrolein & 4,85 & $1175-1135$ \\
\hline Allen & 22 & $1983-1929$ \\
\hline Allylbromid & 21,6 & $1227-1200$ \\
\hline Butan & 67 & $2990-2925$ \\
\hline Cyclohexan & 72,0 & $2952-2916$ \\
\hline Cyclohexanon & 43,1 & $2970-2925$ \\
\hline Cyclohexenon & 13,4 & $2966-2927$ \\
\hline Cyclooktan & 196,9 & $2946-2913$ \\
\hline Cyclooktanon & 37,9 & $2957-2927$ \\
\hline Cyclookten & 74,9 & $2947-2917$ \\
\hline Cyclopentan & 353 & $3050-2850$ \\
\hline Cyclopentanon & 48 & $2995-2950$ \\
\hline Cyclopenten & 10,3 & $3090-3050$ \\
\hline Diallyl & 12,5 & $3100-3075$ \\
\hline Diisopropyl & 17,8 & $2900-2865$ \\
\hline Diisopropylketon & 7,3 & $1100-1060$ \\
\hline Dipropylketon & 28,6 & $1150-1120$ \\
\hline Ethan & 7,0 & $2898-2888$ \\
\hline Ethan-d 6 & 50,3 & $2265-2205$ \\
\hline Ethin & 4,0 & $732-728$ \\
\hline Ethyliodid & 29,0 & $1230-1190$ \\
\hline Formaldehyd & 11,2 & $1748-1743$ \\
\hline Hexan & 67 & $2900-2850$ \\
\hline Isopropyliodid & 20,5 & $1163-1138$ \\
\hline Propen & 51,2 & $965-870$ \\
\hline Propionaldehyd & 23,5 & $2740-2690$ \\
\hline Propyliodid & 13,1 & $1210-1180$ \\
\hline
\end{tabular}

Tabelle 7.1: Integrale Absorptionskoeffizienten der für die quantitative Analyse benötigten Substanzen. Die angegebenen Werte beziehen sich auf Spektren, die 3 min nach Befüllung und bei einem Gesamtdruck von 4 mbar und einer Temperatur von $298 \mathrm{~K}$ aufgenommen wurden. Die Werte sind lichtweglängen und damit auch Apparaturspezifisch. 
KAPITEL 7. ANHANG 


\section{Literaturverzeichnis}

[Abe03] Abel, B.; Assmann, J.; Botschwina, P.; Buback, M.; Kling, M.; OsWald, R.; Schmatz, S.; Schroeder, J.; Witte, T.: Experimental and Theoretical Investigations of the Ultrafast Photoinduced Decomposition of Organic Peroxides in Solution: Formation and Decarboxylation of Benzoxyloxy Radicals, J. Phys. Chem. A 107, 5157 (2003)

[Ana87] Anastasi, C.; Arthur, N. L.: J. Chem. Soc. Faraday Trans. 2, 83, 277 (1987)

[Ann04] Annual Energy Review 2004, www.eia.doe.gov/aer

[Arr78] Arrowsmith, P.; Kirsch, L. J.: J. Chem. Soc. Farday Trans. 1, 74, 3016 (1978)

[Ata98] Atakan, B.; Hartlieb, A. T.; Brand, J. Kohse-Höinghaus, K.: Proc. Combust. Inst. 27, 435 (1998)

[Ata03] Atakan, B.; Lamprecht, A.; Kohse-Höinghaus, K.: Combust. Flame $133,431(2003)$

[Atk97] Atkinson. D. B.; Hudgens, J. W.: Chemical Kinetic Studies Using Ultraviolet Cavity-Ringdown Spectroscopic Detection: Self-Reaction of Ethyl and Ethylperoxy Radicals and the Reaction $\mathrm{O}_{2}+\mathrm{C}_{2} \mathrm{H}_{5} \rightarrow \mathrm{C}_{2} \mathrm{H}_{5} \mathrm{O}_{2}$, J. Phys. Chem. A 101, 3901 (1997)

[Bal91] Baldwin, R. R.; Lodi, Z. H.; Stothard, N.; Walker, R. W.: Proc. Combust. Inst. 23, 123 (1991) 
[Bar95] Barbé, P.; Martin, R.; Perrin, D.; Scacchi, G.: Kinetics and Modeling of the Thermal Reaction of Propene at 800 K. Part I. Pure Propene, Int. J. Chem. Kinet. 28, 829 (1996)

[Bau05] Baulch, D. L.; Bowman, C. T.; Cobos, C. J.; Cox, R. A.; Just, Th.; Kerr, J. A.; Pilling M. J.; Stocker, D.; Troe, J.; Tsang, W.; WalKer, R. W.; Warnatz, J.: Evaluated Kinetic Datafor Combustion Modelling: Supplement II, J. Phys. Chem. Ref. Data, Vol. 34, No. 3 (2005)

[Boy95] Boyd, A. A.; Nozière, B.; Lesclaux, R.: Kinetics and Thermochemistry of the Reversible Combination Reactions of the Allyl and Benzyl Radicals with NO, J. Phys. Chem. 99, 10815 (1995)

[Bub00] Buback, M.; Sandmann, J.: Pressure and Temperature Dependence of the Decomposition Rate of Aliphatic tert-butyl Peroxyesters, Z. Phys. Chem. 214, S. $583(2000)$

[Bub04] Buback, M.; Kling, M.; Schmatz, S.; Schroeder, J.: Photo-induced decomposition of organic peroxides: Ultrafast formation and decarboxylation of carbonyloxy radicals, Phys. Chem. Chem. Phys. 6, 5441 (2004)

[Bub05] Buback, M.; Kling, M.; Schmatz, S.: Decomposition of tertiary alkoxy radicals, Z. Phys. Chem. 219, S. 1 (2005)

[Cal66] Calvert, J. G.; Pitts, J. N.: Photochemistry, John Wiley \& Sons, Inc. New York (1966)

[Cat81] Cathonnet, M.; Boetnner, J. C.; James, H.: Proc. Combust. Inst. 18, $903(1981)$

[Cod02] Cody, R. J.; Payne, W. A. Jr.; Thorn, R. P. JR.; Nesbitt, F. L.; Iannone, M. A.; Tardy, D. C.; Stief, L. J.: Rate Constant for the Recombination Reaction $\mathrm{CH}_{3}+\mathrm{CH}_{3} \rightarrow \mathrm{C}_{2} \mathrm{H}_{6}$ at $\mathrm{T}=298$ and $202 \mathrm{~K}$, J. Phys. Chem. A 106, 6060 (2002)

[Cve71] Cvetanovic̀, R. J.; Ring, D. F.; Doyle, L. C.: Reaction of Oxygen Atoms with Cyclopentene, J. Phys. Chem. 75, S. 3056 (1971) 
[Cve87] Cvetanovic̀, R. J.: Evaluated chemical kinetic data for the reactions of atomic oxygen $O\left({ }^{\beta} P\right)$ with unsaturated hydrocarbons, J. Phys. Chem. Ref. Data, $16(1987)$

[Dag02] Dagaut, P.: On the kinetics of hydrocarbons oxidation from natural gas to kerosene and diesel fuels, Phys. Chem. Chem. Phys. 4, S. 2079 (2002)

[Dav99] Davis, S. G.; Law, C. K.; Wang, H.: Combust. Flame 119, 375 (1999)

[Dea85] Dean, A. M.: J. Phys. Chem. 89, 4600 (1985)

[Dob91] Dobis, O.; Benson, S. W.: Temperature Coefficients of the Rates of Cl Atom Reactions with $\mathrm{C}_{2} \mathrm{H}_{6}, \mathrm{C}_{2} \mathrm{H}_{5}$, adn $\mathrm{C}_{2} \mathrm{H}_{4}$. The Rates of Disproportionation and Recombination of Ethyl Radicals, J. Am. Chem. Soc. 113, 6377 (1991)

[Edw03] Edwards, T.: Liquid Fuels and Propellants for Aerospace Propulsion: 19032003, J. Propuls. and Power 19, 6, S. 1089 (2003)

[Foc99] Fockenberg, C.; Hall, G. E.; Preses, J. M.; Sears, T. J.; MuckerMann, J. T.: A Kinetics and Product Study of the Reaction of $\mathrm{CH}_{3}$ Radicals with $O\left({ }^{3} P\right)$ Atoms Using Time Resolved Time-of-Flight Mass Spectrometry, J. Phys. Chem. A 103, 5722 (1999)

[Foc00] Fockenberg, C.; Flynn, W. G.: A Measurement of the Yield of Carbon Monoxide from the Reaction of Methyl Radicals and Oxygen Atoms, J. Phys. Chem. A 104, 6758 (2000)

[Foc02] Fockenberg, C.; Preses, J. M.: Temperature Dependence of the Rate Constant and Product Distribution of the Reaction of $\mathrm{CH}_{3}$ Radicals with $\mathrm{O}\left({ }^{3} \mathrm{P}\right)$ Atoms, J. Phys. Chem. A 106, 2924 (2002)

[For91] Fonst, W.: Microcanonical Variational Theory of Radical Recombination by Inversion of Interpolated Partition Function, with Examples $\mathrm{CH}_{3}+\mathrm{H}, \mathrm{CH}_{3}+$ $\mathrm{CH}_{3}$, J. Phys. Chem. 95, 3612 (1991)

[For99] Forster, O.: Analysis 1, Braunschweig (1999) 
[Gar90] Garland, L. J.; Bayes, K. D.: Rate Constants for Some Radical-Radical Cross-Reactions and the Geometric Mean Rule, J. Phys. Chem. 94, 4941 (1990)

[Gue96] Günzler, H.; HeIse, M.: IR-Spektroskopie: Eine Einführung, Weinheim (1996)

[Gol74] Golden, D. M.; Piszkiewicz, L. W.; Perona, M. J.; Beadle, P. C.: An Absolute Measurement of the Rate Constant for Isopropyl Radical Combination, J. Am. Chem. Soc. 96, 1645 (1974)

[Gri95] Griffiths, J. F.; Barnard, J. A.: Flame and Combustion, 3rd Edition, London (1995)

[Gu05] Gu, Zhihui; Wang, Yixuan; Balbuena, P. B.: Does the Decomposition of Peroxydicarbonates and Diacyl Peroxides Proceed in a Stepwise or Concerted Pathway?, J. Phys. Chem. A XXX, xxx (2005), online erhältlich

[Hac05] Hack, W.; Hold, M.; Hoyermann, K.; Wehmeyer, J.; Zeuch, T.: Mechanism and Rate of the Reaction $\mathrm{CH} 3+\mathrm{O}$ Revisited, Phys. Chem. Chem. Phys., 2005, 7, 1977.

[Han92] Hanning-Lee, M. A., Pilling, M. J.: Kinetics of the reaction between $H$ atoms and allyl radicals, Int. J. Chem. Kinet. 24, S. 271 (1992)

[Hei90] Heinemann-Fiedler, P.; Hoyermann, K.; Rohde, G.: The Reactions of the Cyclic Hydrocarbon Radicals $c-C_{3} H_{5}, c-C_{5} H_{9}$, and $c-C_{6} H_{11}$ with $O$ Atoms in the Gas Phase, Ber. Bunsenges. Phys. Chem. 94, S. 1400 (1990)

[Hol01] Hold, Markus: Dissertation; Universität Göttingen 2001

[Hoy79a] Hoyermann, K.; Sievert, R.: The Reactions of Alkyl Radicals with Oxygen Atoms: Identification of Primary Products at Low Pressure, $17^{\text {th }}$ Symp. (Int.) on Combustion, The Combustion Institute, 17, 517 (1979)

[Hoy99] Hoyermann, K.; Olzmann, M.; Seeba, J.; Viskolcz, B.: Reactions of $\mathrm{C}_{2} \mathrm{H}_{5}$ Radicals with $\mathrm{O}, \mathrm{O}_{3}$, and $\mathrm{NO}_{3}$ : Decomposition Pathways of the Intermediate $\mathrm{C}_{2} \mathrm{H}_{5} \mathrm{O}$ Radical, J. Phys. Chem, A 103, 5692 (1999) 
[Hoy04] Hoyermann, K.; Mauss, F.; Zeuch, T.: A detailed chemical reaction mechanism for the oxidation of hydrocarbons and its application to the analysis of benzene formation in fuel-rich premixed laminar acetylene and propene flames, Phys. Chem. Chem. Phys. 6, 3824 (2004)

[Hoy04b] Beiderhase, T.; Hoyermann, K.; Nothdurft, J.; Olzmann, M.: GasPhase Kinetics of the Reactions of the $\mathrm{CH}_{2} \mathrm{~F}$ Radicals with the Radicals $\mathrm{CHF}_{2}$, $\mathrm{CH}_{3}$, and $\mathrm{C}_{2} \mathrm{H}_{5}$, Z. Phys. Chem. 218, 493 (2004)

[Hoy06a] Hoyermann, K.; Nothdurft, J.; Olzmann, M.; Wehmeyer, J.; ZeUch, T.: Formation and decomposition of chemically activated cyclopentoxy radicals from the $c-C_{5} H_{9}+O$ reaction J. Phys. Chem. A, 110, 3165 (2006)

[Hoy06b] Hoyermann, K.; Maarfeld, S.; Nacke, F.; Nothdurft, J.; Olzmann, M.; Wehmeyer, J.; Welz, O.; Zeuch, T.: Experimental and Theoretical Studies on the Formation and Decomposition of Chemically Activated Cycloalkoxy Radicals, Proc. Combust. Institute, zur Veröffentlichung eingereicht

[Jam95] James, J. F.: A student's guide to Fourier transforms, Cambridge (1995)

[Jen93] Jenkin, M. E.; Murrells, T. P.; Shalliker, S. J.; Hayman, G. D.: Kinetics and product study of the self-reactions of allyl and allyl peroxy radicals at 296 K, J. Chem. Soc. Farad. Trans. 89, S. 433 (1993)

[Joo04] Joo, S. K.; Kwon, L. K.; LeE, H.; Choi, J. H.: Exploring the dynamics of hydrogen atom release from the radical-radical reaction of $O\left({ }^{3} \mathrm{P}\right)$ with $\mathrm{C}_{3} \mathrm{H}_{5}$, J. Chem. Phys. 120, 7976 (2004)

[Kai96] Kaiser, E. W.; Wallington, T. J.: Pressure Dependence of the Reaction $\mathrm{Cl}+\mathrm{C}_{3} \mathrm{H}_{6}$, J. Phys. Chem. 100, 9788 (1996)

[Ker61] KerR, J. A.; CALvert, J. G.: The Photolysis of Azo-n-propane; the Decomposition of the n-Propyl Radical, J. Am. Chem. Soc. 83, 3391 (1961)

[Ker99] Kersten, Christoph: Dissertation; Universität Göttingen 1999 
[Kim04] Kim, D. S.; KIm, M. Y.; LeE, C. S.: Effect of Premixed Gasoline Fuel on the Combustion Characteristics of Compression Ignition Engine, Energy \& Fuels 18, S. 1213 (2004)

[Kny96] Knyazev, V. D.; Slagle, I. R.:Unimolecular Decomposition of $n-C_{4} H_{9}$ and iso- $C_{4} H_{9}$ Radicals, J. Phys. Chem. 100, 5318 (1996)

[Kny01] Knyazev, V. D.; Slagle, I. R.: Kinetics of the Reactions of $n$-Alkyl $\left(C_{2} H_{5}\right.$, ${ }_{n}-\mathrm{C}_{3} \mathrm{H}_{7}$, and $\left.{ }_{n}-\mathrm{C}_{4} \mathrm{H}_{9}\right)$ Radicals with $\mathrm{CH}_{3}$, J. Phys. Chem. A 105, S. 6490 (2001)

[Lec93] Lechner, M. D.; Gehrke, K.; Nordmeier, E. H.: Makromolekulare Chemie, Basel (1993)

[Maa06] MaArfeld, Sven: Staatsexamensarbeit für das Lehramt an Gymnasien, Universität Göttingen 2006

[Mac83] Macpherson, M. T.; Pilling, M. J.; Smith, M. J. C.: The pressure and temperature dependence of the rate constant for methyl radical recombination over the temperature range 296577 K, Chem. Phys. Lett. 94, 430 (1983)

[Mac85] Macpherson , M. T.; Pilling, M. J.; Smith, M. J. C.: Dtermination of the Absorption Cross Section for $\mathrm{CH}_{3}$ at $216.36 \mathrm{~nm}$ and the Absolute Rate Constant for Methyl Radical Recombination over the Temperature Range 296 - 577 K, J. Phys. Chem. 89, 2268 (1985)

[Mar51] Marcus, R.A.; Rice, O.K.: J. Phys. Colloid Chem. 55, S. 894 (1951)

[Mar52] Marcus, R.A.: J. Chem. Phys. 20, S. 359 (1952)

[Mas52] Masson, C. R.: The Photolysis of Di-n-propyl Ketone, J. Am. Chem. Soc. $74,4731(1952)$

[McE05] McEnally, C. S.; Pfefferle, L. D.: Fuel decomposition and hydrocarbon growth processes for substituted cyclohexanes and for alkenes in nonpremixed flames, Proc. Combust. Inst. 30, S. 1425 (2005)

[Moa95] MoAd, G.; Solomon, D. H.: The Chemistry of Free Radical Polymerization, Elsevier (1995) 
[Mou03] Mousavipour, S. H.; Homayoon, Z.: A Theoretical Study on the Kinetics of Disproportionation versus Association Reaction of $\mathrm{CH}_{3}+\mathrm{C}_{2} \mathrm{H}_{5}$, J. Phys. Chem. A 107, S. 8566 (2003)

[Nac98] NaCke, Frank: Dissertation; Universität Göttingen 1998

[Nis05] Chemical Kinetics Database on the Web, Standard Reference Database 17, Version 7.0, Release 1.3, 2005. http://kinetics.nist.gov/index.php

[Not03] Nothdurft, Jörg: Staatsexamensarbeit für das Lehramt an Gymnasien; Universität Göttingen 2003

[Orl00] Orlando, J. J.; Iraci, L. T.; Tyndall, G. S.: Chemistry of the Cyclopentoxy and Cyclohexoxy Radicals at Subambient Temperatures, J. Phys. Chem. A 104, S. $5072(2000)$

[Par02] Park, J.; Lee, H.; Kwon, H.; Kim, H.; Choi, Y.; ChOI, J.: Atom-radical reaction dynamics of $\mathrm{O}\left({ }^{3} \mathrm{P}\right)+\mathrm{C}_{3} \mathrm{H}_{5} \rightarrow \mathrm{C}_{3} \mathrm{H}_{4}+\mathrm{OH}$ : Nascent rovibrational state distributions of product OH, J. Chem. Phys. 117, S. 2017 (2002)

[Par03] PARK, J.; LeE, H.; ChoI, J.: A theoretical study of the reaction of $O\left({ }^{3} P\right)$ with an allyl radical $C_{3} H_{5}$, J. Chem. Phys. 119, S. 8966 (2003)

[Par76] Parkes. D. A., Quinn, C. P.: J. Chem. Soc. Faraday Trans. 1, 72, 1952 (1976)

[Pla99] Platz, J.; Sehested, J.; Nielsen, J.: Atmospheric Chemistry of Cyclohexane: UV Spectra of $c-C_{6} H_{11}$ and $\left(c-C_{6} H_{11}\right) O_{2}$ Radicals, Kinetics of the Reactions of $\left(\mathrm{c}-\mathrm{C}_{6} \mathrm{H}_{11}\right) \mathrm{O}_{2}$ Radicals with $\mathrm{NO}$ and $\mathrm{NO}_{2}$, and the Fate of the Alkoxy Radical $\left(c-C_{6} H_{11}\right) O$, J. Phys. Chem. A 103, S. 2688 (1999)

[Pot98] Potter, T. L.; Simmons, K. E.: Total Petroleum Hydrocarbon Criteria Working Group Series, Volume 2: Composition of Petroleum Mixtures, Amherst Massachusetts (1998)

[Qin00] Qin, Z.; Lissianski, V.; Yang, H.; Gardiner, W. C.; Davis, S. G.; Wang, H.: Proc. Combust. Inst. 28, 1519 (2000) 
[Rob95] Robertson, S. H.; Pilling, M. J.; Baulch, D. L.; Green, N. J. B.: Fitting of Pressure-Dependent Kinetic Rate Data by Master Equation / Inverse Laplacetransform Analysis, J. Phys. Chem. 99, 13452 (1995)

[Roh88] Rohde, Gabrielle: Diplomarbeit; Universität Göttingen 1988

[Shi72] Shimanouchi, T.: Tables of Molecular Vibrational Frequencies; Consolidated Volume I, NSRDS (1972)

[Sim03] Simmie, J. M.: Detailed chemical kinetic models for the combustion of hydrocarbon fuels, Prog. Energy and Combust. Sci. 29, 599 (2003)

[Sla88] Slagle, I.R.; Sarzynski, D.; Gutman, D.; Miller, J.A.; Melius, C.F.: Kinetics of the Reaction between Oxygen Atoms and Ethyl Radicals, J. Chem. Soc. Farad. Trans. 2, 84, 491-503 (1988)

[Sla88b] Slagle, I. R.; Gutman, D.; Davies, J. W.; Pilling, M. J.: Study of the Recombination Reaction $\mathrm{CH}_{3}+\mathrm{CH}_{3} \rightarrow \mathrm{C}_{2} \mathrm{H}_{6}$. 1. Experiment, J. Phys. Chem. $92,2455(1988)$

[Sla90] Slagle, I. R.; Bernhardt, J. R.; Gutman, D.; Hanning-Lee, M. A.; Pilling, M. J.: Kinetics of the Reaction between Oxygen Atoms and Allyl Radicals, J. Phys. Chem. 94, S. 3652 (1990)

[Smi00] Smith, G. P.; Golden, D. M.; Frenlkch, M.; Eiteneer, B.; Goldenberg, M; Bowman, C. T.; Hanson, R. K.; Song, S.; Gardiner, W. C.; Lissianski, V. V.; Qin, Z.: hhtp://www.me.berkeley.edu/GRI_mech, $3652(2000)$

[Sto91] Stothard, N.; Walker, R. W.: J. Chem. Soc. Faraday. Trans. 81, 241 (1991)

[Tak81] Takagi, H.; Washida, N.; Bandow, H.; Akimoto, H.; Okuda, M.: Photooxidation of $C_{5}-C_{7}$ cycloalkanes in the nitric oxide-water-air system, J. Phys. Chem. 85, S. 2701 (1981) 
[Tul82] Tulloch, J. M.; Macpherson, M. T.; Morgan, C. A.; Pilling, M. J.: Flash Photolysis Studies of Free-Radical Reactions: $\mathrm{C}_{3} \mathrm{H}_{5}+\mathrm{C}_{3} \mathrm{H}_{5}$ (293-691 K) and $\mathrm{C}_{3} \mathrm{H}_{5}+\mathrm{NO}$ (295-400 K), J. Phys. Chem. 86, S. 3812 (1982)

[Tro94] Troe, J.: The Polanyi Lecture. The colourful world of complex-forming bimolecular reactions, J. Chem. Soc. Faraday Trans. 90, 2303 (1994)

[Trö97] TröGER, UwE: Dissertation; Universität Göttingen 1997

[Tsa86] Tsang, W.; Hampson, R. F.: Chemical Kinetic Data Base for Combustion Chemistry. Part I. Methane and Related Compounds, J. Phys. Chem. Ref. Data $15,1087(1986)$

[Tsa88] Tsang, W.: Chemical Kinetic Data Base for Combustion Chemistry. Part 3. Propane, J. Phys. Chem. Ref. Data 17, 887 (1988)

[Tsa91] Tsang, W.: Chemical Kinetic Data Base for Combustion Chemistry. Part 5. Propene, J. Phys. Chem. Ref. Data 20, 221 (1991)

[Wag88] Wagner, A. F.; Wardlaw, D. M.: Study of the Recombination Reaction $\mathrm{CH}_{3}+\mathrm{CH}_{3} \rightarrow \mathrm{C}_{2} \mathrm{H}_{6}$. 2. Theory, J. Phys. Chem. 91, 4864 (1987)

[War97] Warnatz, J.; MaAs, U.; Dibble, R. W.: Combustion, Springer, Heidelberg 1997

[Was82] Washida, N.; TAKagi, H.: Reaction of Cyclohexane and Cyclohexyl Radicals with Atomic and Molecular Oxygen, J. Am. Chem. Soc. 104, S. 168 (1982)

[Weh02] Wehmeyer, Jens: Dissertation; Universität Göttingen 2002

[Wes84] Westbrook, C. K.; Pitz, J.: Combust. Sci. Technol. 37, S. 117 (1984)

[Wes99] Westbrook, C. K.: The Internal Combustion, Modelling Considers All Factors, in: Science \& Technology Review, S. 4 (1999)

[Wed97] Wedler, G.: Lehrbuch der Physikalischen Chemie, Weinheim (1997) 
[Yar91] Yarwood, G.; Niki, H.; Maker, P. D.: Kinetic and IR Spectroscopic Studies of Formyl Bromide (HCOBr) Formed via the Reaction $\mathrm{HCO}+\mathrm{Br}_{2} \rightarrow$ $\mathrm{HCOBr}+\mathrm{Br}$, J. Phys. Chem. 95, S. 4773 (1991)

[Zeu03] Zeuch, Thomas: Dissertation; Universität Göttingen 2003 


\section{Lebenslauf}

Am 09. Mai 1977 wurde ich, Jörg Nothdurft, als zweites Kind der Posthalterin Karin Nothdurft, geborene Schmidt, und des Industriedesigners Jürgen Nothdurft in Nürnberg geboren. Ich besitze die deutsche Staatsangehörigkeit.

Von 1983 bis 1987 besuchte ich in Clausthal-Zellerfeld die Grundschule Bremerhöhe, von 1987 bis 1989 die Orientierungsstufe und anschließend die Robert-Koch-Schule (Gymnasium), an der ich 1996 die allgemeine Hochschulreife erlangte.

Von Oktober 1996 bis Juli 1997 leistete ich meinen Wehrdienst als Gebirgsjäger in Mittenwald. Dabei nahm ich als Angehöriger des Skizugs Mittenwald an nationalen und internationalen Biathlon-Vergleichswettkämpfen der europäischen Streitkräfte teil.

Zum Wintersemester 1997/1998 immatrikulierte ich mich an der Georg-August-Universität Göttingen für das Studium Lehramt an Gymnasien in den Fächern Chemie und Mathematik. Im Oktober 1999 bestand ich die Zwischenprüfung in Mathematik und im April 2000 die Zwischenprüfung in Chemie.

Die Hausarbeit mit dem Titel „Die Oxidation des Cyclopentylradikals in der Gasphase" zur Erlangung des 1. Staatsexamens fertigte ich im Fach Chemie unter der Anleitung von Herrn Prof. Hoyermann am Institut für Physikalische Chemie der Universität Göttingen an. Im Juni 2003 bestand ich die erste Staatsprüfung für das Lehramt an Gymnasien in der Fächerkombination Chemie/Mathematik.

Seit Juli 2003 beschäftige ich mich im Rahmen des europäischen Graduiertenkollegs EGS (European Graduate School) „Microstructural Control in Free Radical Polymerisation“ mit dem Schwerpunkt „Reactions of Hydrocarbon Radicals in the Gasphase: Rates, Primary Products, Mechanisms" mit Reaktionen von Kohlenwasserstoffradikalen und deren Oxidation. In diesem Zusammenhang entstand die vorliegende Arbeit. 